FORSCHUNGSERGEBNISSE DER WIRTSCHAFTSUNIVERSITÄT WIEN

\author{
Youri Tacoun
}

\title{
La théorie de la valeur de Christian von Ehrenfels
}


FORSCHUNGSERGEBNISSE DER

WIRTSCHAFTSUNIVERSITÄT WIEN

Youri Tacoun

\section{La théorie de la valeur de Christian von Ehrenfels}

Ce livre a pour objectif la redécouverte de la théorie de la valeur du philosophe autrichien Christian von Ehrenfels; et l'analyse de la possible utilisation de sa théorie dans les sciences économiques actuelles. Après avoir fait connaissance avec Christian von Ehrenfels, l'accent est mis sur l'analyse des principaux traits de sa théorie. Sa complexité fascinante n'a d'égale que la volonté d'exhaustivité du philosophe autrichien. Le débat induit par la discussion sur son utilisation dans les sciences économiques actuelles conduit à la conclusion que le philosophe autrichien a ouvert la voie à une nouvelle perspective sur le processus de valorisation et qu'il mérite à ce titre de sortir de l'oubli.

Né à l'Ile de la Réunion en 1979, Youri Tacoun vit depuis 2001 en Autriche. Après des études de sciences économiques à l'Université Louis Pasteur à Strasbourg, il effectua ses études doctorales à l'Université d'Economie et de Gestion de Vienne ainsi que des recherches à l'Université de l'Alberta à Edmonton. Il travaille actuellement dans le domaine des échanges linguistiques et culturels a fin de promotion francophonie en Europe. 


\section{La théorie de la valeur de Christian von Ehrenfels}




\section{Forschungsergebnisse der Wirtschaftsuniversität Wien}

Band 23

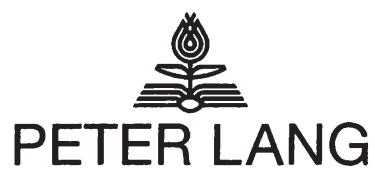

Frankfurt am Main · Berlin · Bern · Bruxelles · New York · Oxford · Wien Youri Tacoun - 978-3-631-75426-9 


\section{Youri Tacoun}

\section{La théorie de la valeur de Christian von Ehrenfels}

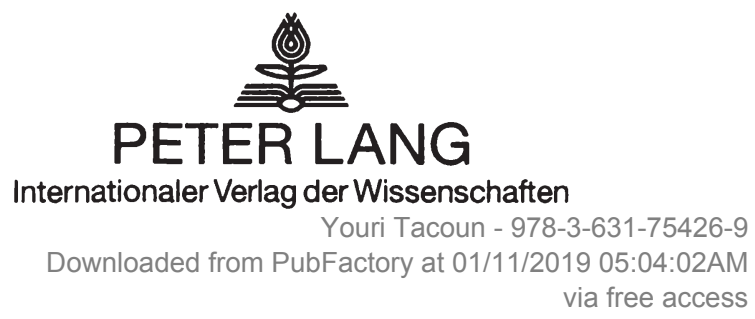


Information bibliographique de la Deutsche Nationalbibliothek La Deutsche Nationalbibliothek a répertorié cette publication dans la Deutsche Nationalbibliographie; les données bibliographiques détaillées peuvent être consultées sur Internet à l'adresse http:// www.d-nb.de.

Open Access: The online version of this publication is published on www.peterlang.com and www.econstor.eu under the international Creative Commons License CC-BY 4.0. Learn more on how you can use and share this work: http://creativecommons.org/licenses/ by/4.0.

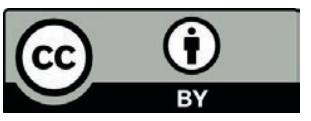

This book is available Open Access thanks to the kind support of ZBW - Leibniz-Informationszentrum Wirtschaft.

Subventionné par l'Université des

Sciences économiques de Vienne.

Couverture:

Atelier Platen, d'après une esquisse de l'agence de publicité Publique.

Logo de l'Université des Sciences économiques de Vienne:

Imprimé avec permission de

l'Université des Sciences économiques de Vienne.

ISSN 1613-3056

ISBN 978-3-631-57073-9

ISBN 978-3-631-75426-9 (eBook)

(c) Peter Lang $\mathrm{GmbH}$

Internationaler Verlag der Wissenschaften

Frankfurt am Main 2008

Tous droits réservés.

L'ouvrage dans son intégralité est placé sous la protection de la loi sur les droits d'auteurs. Toute exploitation en dehors des étroites limites de la loi sur les droits d'auteurs, sans accord de la maison d'édition, est interdite et passible de peines.

Ceci vaut en particulier pour des reproductions, traductions, microfilms, l'enregistrement et le traitement dans des systèmes électroniques.

Imprimé en Allemagne 123457

www.peterlang.de 


\section{REMERCIEMENTS}

Je voudrais adresser mes remerciements aux personnes suivantes, sans qui ce travail n'aurait pu aboutir :

mes directeurs de thèse, $M$. Peter BERGER et M. Werner CLEMENT pour leur soutien et leurs conseils,

mes parents et ma famille,

$>$ ma famille alsacienne,

Rick SZOSTAK pour m'avoir donné la possibilité de compléter mes recherches ainsi que pour ses précieux conseils. 
Youri Tacoun - 978-3-631-75426-9

Downloaded from PubFactory at 01/11/2019 05:04:02AM

via free access 


\section{PRÉFACE}

Issu du milieu intellectuel de l'Europe centrale fin de siècle, Christian von EHRENFELS (né en 1859 à Rodaun, près de Vienne, mort en 1932 à Lichtenau, en Basse-Autriche) figure parmi les fondateurs de la psychologie gestaltiste. Avec la chute de la monarchie des Habsbourg à la fin de la Première Guerre mondiale, il est entré comme beaucoup de ses contemporains dans une profonde crise d'identité, dont il put sortir grâce à son amitié avec le premier président de la République tchécoslovaque, Thomas Garrigue Masaryk. L'université Charles de Prague, de langue allemande, constitua sa deuxième patrie intellectuelle après l'université de Vienne. Dans la première partie de sa vie, il vécut de temps à autre à Berlin et Zurich. Ses centres d'intérêts scientifiques et culturels étaient aussi larges que le réseau social dans lequel il évoluait, si bien décrit par Edward TIMMS. Il s'agissait des cercles intellectuels du tournant du siècle. Ehrenfels admirait Richard Wagner et épousa une amie de Houston Stewart Chamberlain qui comptait, en sa qualité de gendre de Wagner, parmi les étoiles fixes de la scène de Bayreuth. Ehrenfels s'est intéressé au problème des relations entre les sexes, d'un point de vue sexuel aussi bien qu'intellectuel, selon une approche qui, sous certains aspects, rappelle celle d'Otto Weininger. Issu d'une famille aisée, Ehrenfels finança à Berlin une revue culturelle dans laquelle il écrivait régulièrement des articles sur la théorie de l'art. Les sympathisants de Karl Kraus ne voyaient pas d'un mauvais œil ce professeur de Prague puisque Kraus luimême s'était parfois exprimé sur les mérites de l'œuvre scientifique d'Ehrenfels, sans la part habituelle de sarcasme souvent attribuée à ses écrits. Grâce à ses études à Vienne, Ehrenfels fut en contact dès sa jeunesse avec tous les grands noms de l'école viennoise philosophique et économique : Brentano, Meinong, Menger, Wieser etc. C'est de là que vient son ambition de travailler à une théorie psychologique de la valeur qui, par plusieurs aspects, se rapproche des efforts analogues des marginalistes autrichiens, ce qui constitue l'objet de la présente monographie de Youri Tacoun.

Le travail de Tacoun se divise en trois gros chapitres, qui à leur tour sont structurés en cinq sous-parties au plus. Le premier chapitre a pour objet la vie d'Ehrenfels et le milieu intellectuel dans lequel il évolue. Qu'il s'agisse du lecteur intéressé par l'histoire intellectuelle de l'époque fin de siècle et du début du $\mathrm{XX}^{\mathrm{e}}$ siècle, ou de celui qui, dans l'histoire aime "flairer la chair humaine", selon l'expression du grand historien français Marc Bloch, chacun y trouvera son compte. Les héros de Tacoun sont agréablement présentés, dans une narration fluide, comme de brillants excentriques dont les amitiés et les inimitiés servent de stimulants fiables à des présentations scientifiques et artistiques. 
Sans le vouloir, l'auteur nous rappelle que le temps où la science progressait par interactions entre des personnages non-conformistes et insaisissables est bien révolu. $\mathrm{Au}$ contraire, Tacoun veut nous montrer que les biographies des scientifiques, pour peu que celles-ci agissent sur le long terme, sont profondément déterminées par les péripéties de la politique. Ehrenfels s'est toujours trouvé du côté de ceux qui croyaient à une coexistence. pacifique des Slaves et des Allemands en Europe centrale. Il a traité la séparation entre l'Autriche et la Bohème Moravie par l'écriture d'une pièce de théâtre sur le mouvement d'indépendance tchèque. Un destin clément a permis à Ehrenfels de ne pas avoir à vivre ni à commenter l'entrée d'Hitler à Prague.

Le deuxième chapitre de ce travail concerne la théorie de la valeur de Christian von Ehrenfels. Cette théorie est née entre 1894 et 1898 à travers les travaux relevant de plusieurs publications, dont la principale porte comme titre "Système de la théorie de la valeur, éléments d'une éthique ». A n'en pas douter, les travaux sur le problème de la valeur, publiés au même moment par l'école autrichienne de l'utilité marginale, n'ont pu agir que comme stimulants pour l'élève de Brentano qu'était Ehrenfels, de façon à ce qu'il rapproche son sujet des questions philosophiques et psychologiques plus qu'économiques. Le fait de tenter de parvenir à une systématique des catégories de valeur à partir de descriptions de situations pratiques de prises de décision est tout à fait typique de la façon de travailler d'Ehrenfels. Ainsi, imagine-t-il à des fins d'illustration un étudiant saisi d'un désir d'air frais, bien qu'il doive rester à son bureau et travailler. Le désir se transforme en "aspiration" puis en "volonté " ferme d'aller se promener, jusqu'à ce que la valeur que notre étudiant accorde à la rupture imminente de sa séance d'apprentissage soit plus forte que la valeur anticipée de l'examen réussi suite à cet apprentissage. Les exemples de ce type servent chez Ehrenfels à introduire de nombreuses définitions dans sa théorie : la "désirabilité (Begehrbarkeit) » d'un objet, "le relatif encouragement au bonheur " par son acquisition (ou la simple possibilité d'une telle acquisition), le " combat des raisons (Motivenkampf) " au moment de l'acte de choix etc. Réussir à rendre tout ceci compréhensible pour un public du $\mathrm{XXI}^{\mathrm{e}}$ siècle, qui éventuellement ne dispose pas de formation en psychologie, relève pour ainsi dire de l'exploit, accompli ici par Youri Tacoun avec beaucoup de tact et une remarquable capacité de compréhension.

Dans le troisième chapitre du travail de Tacoun, le traitement des théories de la valeur est également magistralement abordé et se laisse comparer à celui d'Ehrenfels. La comparabilité est bien sûr relative. Comme le montre Tacoun de façon convaincante, on peut trouver dans la "Théorie des sentiments moraux " d'Adam Smith des formes représentatives qui apparaissent à nouveau de façon analogue chez Ehrenfels. 
Smith laisse les gens agir en situation de choix comme s'ils comprenaient une instance (fictive) de jugement intrinsèque, le "Impartial Spectator ». L'Impartial Spectator d'Ehrenfels se nomme "créateur de valeur (Wertbildner) " et exerce cette même autorité morale. Quoi qu'il en soit, le traitement du problème de la valeur par Karl Marx a encore cette composante sociale en commun avec l'approche d'Ehrenfels (les jugements de valeur dépendent du contexte social dans lequel elles se développent). Il en va de même de l'importance décisive accordée au fait que la valeur représente une catégorie historique, c'est-à-dire changeante avec le temps. En quoi consiste alors la parenté entre les théories d'Ehrenfeld et l'approche "Welfare-Economics " d'un Vilfredo Pareto ou Léon Walras ? Tacoun est assez honnête pour ne pas les faire apparaitre artificiellement. Il prend plutôt la discussion de l'enseignement de la valeur chez les néoclassiques comme point de départ d'une revendication étonnante. Qu'en serait-il, se demande Tacoun, si Christian von Ehrenfels était arraché de l'oubli non mérité et si ses écrits étaient lus pour servir à la fondation d'une nouvelle compréhension de l'homo oeconomicus basée sur l'introduction de composantes psychologiques?

Suivant cette suggestion, l'éditeur Peter Lang a décidé de publier cette étude de Youri Tacoun et a ainsi franchi un pas important pour le renouveau des échanges intellectuels autour de l'œuvre d'Ehrenfels. Il ne reste qu'à espérer que l'auteur et l'éditeur rencontrent auprès du public le succès qu'ils méritent sans le moindre doute.

Vienne, le 30 janvier 2008

Peter Berger 
Youri Tacoun - 978-3-631-75426-9

Downloaded from PubFactory at 01/11/2019 05:04:02AM

via free access 


\section{SOMMAIRE}

Remerciements 5

Préface $\quad 7$

$\begin{array}{ll}\text { Introduction } & 15\end{array}$

Chapitre Christian von Ehrenfels, biographie et contexte

premier : historique : les origines de sa théorie de la valeur 21

I - Une biographie de Christian von Ehrenfels

II - Le contexte historique : le monde académique austrohongrois à la fin du XIX ${ }^{\text {eme }}$ siècle et ses courants philosophiques et économiques

2.1 - Généralités $\quad 42$

2.2 - L'aristotélisme Autrichien $\quad 45$

2.3 - Franz Brentano 51

2.4 - Carl Menger $\quad 57$

2.5 - Deux développements de la théorie de la valeur de Carl Menger par Friedrich von Wieser et Eugen von Böhm-Bawerk

Chapitre

second: La théorie de la valeur de Christian von Ehrenfels 75

I - Approche et définition du phénomène de la valeur $\quad 80$

1.1 - Généralités $\quad 80$

1.2 - L'origine de la valeur : la relation sujet-objet $\quad 85$

1.3 - Définition de la valeur 93

II - Catégorisation du phénomène de la valeur et ses

notions adjacentes

2.1 - La valeur d'usage et la valeur d'échange 96

2.2 - La valeur intrinsèque et la valeur d'effet 98

2.3 - La relation valeur-utilité et valeur-avantage $\quad 102$

2.4 - Autres types de valeurs 105

2.5 - La notion de non-valeur 107 
III- Les mouvements de valeur et leurs déterminants

3.1 - Les dispositions émotionnelles

3.2 - Les facteurs influençant les dispositions émotionnelles

3.2.1 - Les facteurs psychologiques inconnus

3.2.2 - Les facteurs psychologiques connus

3.2.3 - Les influences extérieures

3.3 - Les mouvements de valeur en fonction du dessein de l'individu

IV- Analyse du désir

4.1 - Le fonctionnement des mécanismes de représentation de la conscience

4.2 - Le phénomène du désir

V- Valeur et éthique : étude psychologique des valorisations conformes aux bonnes mœurs

5.1 - L'éthique en tant que science

5.2 - Analyse empirique et place de la valeur dans l'éthique

5.3 - Le développement de l'éthique, l'évolution des valeurs éthiques et morales

5.4 - Maximes morales, coutumes et droit

5.5 - Conscience morale et sanction éthique

5.6 - Le concept de valeur absolue

186

Résumé et mise au point

Chapitre Discussions sur la valeur avec la théorie de Christian troisième: von Ehrenfels

I- Adam Smith et David Ricardo, la théorie de la valeur de l'Ecole Classique

1.1 - Approche du problème de la valeur par Adam Smith

1.2 - Les apports de David Ricardo à la théorie de la valeur d'Adam Smith

II- La théorie de la valeur ajoutée de Karl Marx

2.1 - L'essentiel de la vie de la Karl Marx

2.2 - La théorie de la valeur de Karl Marx

2.3 - Un rapprochement entre Ehrenfels et Marx est-il possible? 
III- Welfare economics : fondements, théorie du choix et bien être des individus en sciences éonomiques

3.1 - Léon WALRAS et l'adoption de nouvelles hypothèses comportementales en sciences économiques

3.2 - Vilfredo PARETO et la naissance de la microéconomie moderne

3.3 - Le concept de Pareto optimalité comme base de développement de l'économie du bien être ou " welfare economics»

IV- Réflexions sur les limites de la théorie économique actuelle et de la pertinence de la théorie d'Ehrenfels comme alternative dans le domaine de la valeur 4.1 - La perfectibilité des hypothèses sur les comportements humains

4.1.1 - L'individualisme méthodologique 277

4.1.2 - L'hypothèse de rationalité 282

4.1.3 - La recherche du bien être

4.2 - De la philosophie (morale) à l'économie : pour un retour à une approche philosophique, aux valeurs et à l'éthique dans les sciences économiques

4.2.1 - Les théories utilitaristes et la notion d'utilité dans les sciences économiques

4.2.2 - A propos de la dichotomie valeur/fait dans les sciences économiques

4.2.3 - La présence des valeurs dans les théories économiques

4.3 - A la recherche d'une alternative : pour une utilisation de la théorie de Christian von Ehrenfels dans les sciences économiques modernes 
Youri Tacoun - 978-3-631-75426-9

Downloaded from PubFactory at 01/11/2019 05:04:02AM

via free access 


\section{INTRODUCTION}

Les sciences économiques considèrent l'Ecole Autrichienne comme étant un des principaux courants qui ont contribué à son développement. Parmi les principaux économistes de cette école de pensée, on peut citer son fondateur, Carl MENGER, et Friedrich von HAYEK une de ses principales figures de proue au $\mathrm{XX}^{\mathrm{eme}}$ siècle. Nombreux sont les articles et les livres concernant leurs théories et leurs idées. Une bibliographie beaucoup moins importante est consacrée aux intellectuels qui, à la même époque que Carl MENGER, ont voulu participer à une émancipation des idées de la toute jeune Ecole Autrichienne de l'économie politique ("die österreichische Schule der Nationalökonomie »). Un de ces intellectuels fut le philosophe Autrichien Christian von EHRENFELS (18591932). Resté connu dans le monde de la psychologie comme le père de la théorie du Gestalt, le reste de ses ouvrages fit l'objet de peu d'attention. Depuis les années 1980 et 1990, un certain intérêt semble renaître pour ce philosophe et pour son travail, en particulier dans le domaine de la valeur'. Il n'existe cependant aucune analyse de la sorte en français. EHRENFELS publia à la fin du XIX ${ }^{\text {eme }}$ siècle toute une série de travaux sur le phénomène de la valeur ${ }^{2}$. Il développa une théorie générale de la valeur qui à ce jour ne fut analysée par aucun économiste.

Notre travail se consacrera à l'étude de la théorie de la valeur de Christian von EHRENFELS avec une approche économique. Son objectif est double : il s'agira d'une part de situer la théorie du philosophe dans le courant intellectuel de l'Empire Austro-hongrois de la fin du XIX ${ }^{\text {eme }}$ siècle ${ }^{3}$; nous voulons d'autre part montrer que cette théorie reste valable et utilisable en tant qu'alternative dans les sciences économiques modernes.

La valeur est un phénomène aux significations plurielles. En cherchant la définition du mot "valeur» dans un dictionnaire récent, on peut relever le sens premier suivant : la valeur est " ce en quoi une personne est digne d'estime (au regard des qualités que l'on souhaite à l'Homme dans le domaine morale, intellectuel et professionnel) ${ }^{4}$. Le mot valeur est ici employé à l'égard d'une personne, d'un individu.

\footnotetext{
' Barry SMITH et Reinhard FABIAN principalement, consacrèrent quelques livres au philosophe Christian von EHRENFELS.

${ }^{2}$ Ces travaux sont rassemblés dans un ouvrage édité par Reinhard FABIAN : EHRENFELS, Christian von, " Philosophische Schriften - Werttheorie ", 1982.

${ }^{3}$ Par courant intellectuel, nous voulons désigner à la fois les courants philosophiques (sous l'impulsion de Franz BRENTANO) et économiques qui ont marqué cette période.

${ }^{4}$ Le Grand Dictionnaire de la Langue Française, $2^{\text {eme }}$ édition dirigée par Alain REY, Dictionnaire le Robert, Paris 2001.
} 
La notion de valeur peut également être employée lorsqu'il s'agit d'un objet : la valeur peut alors se définir comme étant " le caractère mesurable d'un objet, en tant que susceptible d'être échangé, d'être désiré $)^{5}$.

Cette deuxième définition se rapproche de celle qui est employée par les sciences économiques. A propos de la relation entre le phénomène de la valeur et les sciences économiques, Hans Albrecht HARTMANN écrit que «les économistes sont des gens qui connaissent le prix de tout mais la valeur de rien ${ }^{6}$. De cette manière, il souligne également que le concept de prix est omniprésent dans les sciences économiques alors que celui de la valeur joue un rôle mineur quand il n'est pas assimilé au prix. Cela suppose également que la valeur et le prix sont deux notions distinctes. Une hypothèse que nous posons dans le cadre de ce travail. Il existe de nombreux travaux sur la détermination des prix, mais peu d'études sur la valeur et ses déterminants.

La théorie de Christian von EHRENFELS se concentre sur la détermination de la valeur, ce qui le conduit à s'intéresser aux comportements et aux manières de penser des individus. A l'aide de la psychologie, de la sociologie et de la philosophie, EHRENFELS explique comment la valeur se forme, se modifie et parfois, disparait. Son approche peut être qualifiée d'approche interdisciplinaire d'un point de vue actuel et elle se distingue complètement de celle monodisciplinaire des sciences économiques ${ }^{7}$. Elle constitue en ce sens une alternative à la théorie des choix rationnels qui domine l'approche comportementale en économie. Dire qu'elle constitue une alternative, c'est affirmer que la théorie d'EHRENFELS contient des arguments et des explications qui ne sont pas caduques mais qui sont basés sur des hypothèses différentes ; c'est également affirmer que son utilisation est possible dans les sciences économiques modernes et qu'elle comble le vide en matière d'analyse sur le phénomène de la valeur.

Notre travail sera autant par son contenu que par sa méthode interdisciplinaire. Les arguments et les concepts du philosophe Autrichien appartiennent autant à la philosophie, qu'à la sociologie, à la psychologie et à l'économie. C'est la première difficulté dans l'analyse de son travail: des connaissances durent d'abord être acquises dans les trois premiers domaines cités et pour cela, nous nous sommes

\footnotetext{
${ }^{5}$ Le Grand Dictionnaire de la Langue Française, $2^{\text {ème }}$ édition dirigée par Alain REY, Dictionnaire le Robert, Paris 2001.

${ }^{6}$ HARTMANN Hans Albrecht, „Wirtschaft und Werte - eine menschheitsgeschichtliche Mesalliance“, 1998, p.29.

${ }^{7}$ Remarquons cependant que dans le contexte de la fin du XIX ${ }^{\text {ème }}$ siècle, étant donné les séparations bien moins marquées entre les disciplines qu'à l'heure actuelle, il était certainement plus facile d'adopter ce genre d'approche, en particulier de la part d'un philosophe. Aussi serait-il possible de considérer la théorie d'EHRENFELS dans son contexte comme étant pré-disciplinaire.
} 
appuyés sur des recherches bibliographiques, mais également sur des cours de l'Université de Vienne. Les recherches bibliographiques ont eu lieu à Vienne en Autriche, et à l'Université de l'Alberta à Edmonton au Canada. Nos recherches au Canada nous ont permis de pouvoir consulter des ouvrages anglo-américains dans le domaine de la valeur et ainsi de pouvoir enrichir notre travail. Afin de compléter les connaissances acquises à travers les livres, nous avons assisté au cours du professeur Herlinde PAUER-STUDER, "Einführung in die Ethik ", durant le semestre d'hiver 2004-2005, et à celui du professeur Konrad Paul LIESSMAN, «Von Hegel zu Nietzsche. Geschichte der Philosophie im 19. Jahrhundert » durant le semestre d'hiver 2003-2004 à l'Université de Vienne. Ces cours ont facilité la compréhension et l'analyse de la théorie d'EHRENFELS.

La deuxième difficulté fut celle de la langue : les travaux d'EHRENFELS ne furent édités qu'en langue allemande ; certains ouvrages concernant le philosophe furent certes publiés en anglais, mais à ce jour aucun travail d'analyse de sa théorie de la valeur n'existe en langue française. La lecture de ces travaux durent se faire avec précaution: certains mots ne sont plus usités, voire absents des dictionnaires allemands contemporains. Pour contourner cet obstacle, il est nécessaire d'utiliser d'anciens dictionnaires de traduction allemand-français, contenant encore les définitions et traductions des mots en question ${ }^{8}$. La plupart des concepts mentionnés par EHRENFELS purent être traduits. Certains d'entre eux cependant lui sont propres et leur traduction est soulignée dans notre travail. Ces traductions restent le plus près possible du sens originel.

Pour notre travail, nous avons retenu d'une part les principaux passages de la théorie de la valeur du philosophe Autrichien que nous analysons plus précisément que d'autres passages, moins importants dans le cadre de notre travail. Nous mentionnons la totalité de la théorie, mais son exhaustivité et sa complexité imposèrent une analyse focalisée sur les arguments qui étaient pertinents pour nos propos.

Nous considérons que ce travail présente deux axes de développement principaux : un axe historique et un axe économique.

Au cours de ce travail, nous allons devoir interpréter la théorie d'EHRENFELS. La question qui se pose alors est celle de la rigueur et de l'objectivité de cette interprétation. La rigueur et l'objectivité sont souhaitables et possibles selon l'historien américain Richard EVANS. Pour cela, il reprend lui-même l'historien CARR qui prétend qu'avant d'étudier les faits historiques, on devrait étudier celui

\footnotetext{
${ }^{8}$ Pour notre part, nous utiliserons le « Enzyklopedisches Worterbuch, Langenscheidt, 1933 ».
} 
qui décrit les faits ${ }^{9}$. Dans notre cas, il s'agit de comprendre EHRENFELS avant $\mathrm{d}$ 'analyser son travail et cela sera fait à travers une biographie du philosophe.

Ensuite, toujours selon EVANS, ce qui intéresse les historiens, ce ne n'est pas seulement les faits, mais plutôt les événements liés à ces faits ${ }^{10}$. Autrement dit, on doit s'intéresser au contexte qui a vu l'apparition des faits. Dans le cas d'EHRENFELS, il s'agit de décrire la vie intellectuelle autrichienne de la fin du XIX $^{\text {ème }}$ siècle, qui a certainement influencé son travail et celui de ses amis et connaissances.

Enfin, tout travail contient une thèse, derrière laquelle se cache l'avis de l'auteur, y compris les travaux scientifiques. L'auteur doit pouvoir à ce propos prendre de la distance par rapport à son sujet, développer un certain sens de l'autocritique et enfin, accepter les autres points de vue.

Cette vision des choses se rapproche de celle du courant constructiviste (Karl POPPER) pour lequel tout travail scientifique est une construction humaine puisque réalisée par l'être humain. Notre interprétation de la théorie d'EHRENFELS sera une interprétation du $\mathrm{XXI}^{\mathrm{e}}{ }^{\mathrm{e} e}$ siècle avec néanmoins une prise en compte du contexte de développement de la théorie en question ainsi que des possibilités de son utilisation à l'heure actuelle par les sciences économiques.

D'un point de vue des sciences économiques, notre approche se rapproche de celle de l'Ecole de la Régulation ${ }^{\prime \prime}$. La méthode employée est celle de la perspective interdisciplinaire : les apports de différentes disciplines sont un enrichissement qui permet de mieux cerner un phénomène, par exemple celui de la valeur.

Notre travail se décomposera en trois parties: la première contiendra une biographie de Christian von EHRENFELS ainsi qu'une description de l'environnement intellectuel dans lequel il vécut et dont il s'est inspiré. Nous soulignerons le rôle du courant philosophique mené par Franz BRENTANO ainsi que du courant économiste avec Carl MENGER, Eugen von BÖHM-BAWERK et Friedrich von WIESER. Le but de ce premier chapitre est de présenter le philosophe EHRENFELS et d'amener le lecteur à comprendre les personnes et les théories qui ont inspiré ses travaux sur la valeur.

Notre second chapitre sera consacré exclusivement à l'analyse de sa théorie de la valeur. Avant de s'intéresser à la détermination de la valeur, il faudra d'abord

\footnotetext{
${ }^{9}$ EVANS, Richard : « Fakten und Fiktionen », 1998, p.213.

${ }^{10}$ idem, p.79.

${ }^{11}$ Courant français apparu dans les années 1970 et dont les principaux représentants sont Michel Aglietta et Robert Boyer. Leur méthodologie s'inspire de celle de l'Ecole Historique Allemande et elle est basée sur une analyse interdisciplinaire des phénomènes économiques. Voir AGLIETTA Michel, « Régulation et Crise du Capitalisme », 1997.
} 
énumérer les notions inhérentes; puis nous verrons comment la valeur se construit à travers un processus psychologique influencé par des facteurs internes mais aussi externes. La valeur est pour EHRENFELS un phénomène dynamique et nous analyserons les causes de ses changements.

La théorie de la valeur du philosophe Autrichien contient des arguments qui demeurent pertinents, et dont les fondements psychologiques restent valables. Dans une troisième partie, elle sera comparée aux théories de la valeur d'Adam SMITH, de David RICARDO et de Karl MARX. Leurs points communs et leurs différences seront soulignés. Cette comparaison ne se limitera pas aux théories du passé, nous verrons également dans quelle mesure il serait possible d'utiliser la théorie d'EHRENFELS dans les sciences économiques modernes. Avant cela, nous évoquerons les nouvelles hypothèses émises sur les comportements individuels à la fin du XIX ${ }^{\text {eme }}$ siècle et qui continuent d'imprégner les sciences économiques à l'heure actuelle. Ces hypothèses ont servi à l'élaboration de modèles mathématiques dont l'un d'entre eux fera l'objet d'une attention particulière, le modèle de l'optimum de Pareto. Ce dernier a permis le développement d'une nouvelle branche de l'économie au début du $\mathrm{XX}^{\text {eme }}$ siècle, l'économie du bien être, dont l'objet est de pouvoir fournir des critères d'analyse d'efficacité des politiques économiques sur l'amélioration du bien être. Nous verrons néanmoins que ces nouvelles hypothèses sur les comportements permettent avant tout une abstraction propice à un calcul de maximisation mais que leur réalisme reste controversé. C'est en ce sens que la théorie d'EHRENFELS constitue une alternative intéressante, plus proche de la réalité. Afin de permettre son utilisation, il faut surmonter l'obstacle que constitue l'approche économique du comportement individuel : nous montrerons que les hypothèses sur lesquelles sont basées cette approche sont fragiles et que pour mieux appréhender la réalité et ainsi mieux cerner et expliquer le phénomène de la valeur, la recherche d'une alternative telle que la théorie d'EHRENFELS est nécessaire. 
Youri Tacoun - 978-3-631-75426-9

Downloaded from PubFactory at 01/11/2019 05:04:02AM

via free access 


\section{Chapitre premier}

\section{Christian von EHRENFELS,}

\section{biographie et contexte historique :}

les origines de sa théorie de la valeur 
Youri Tacoun - 978-3-631-75426-9

Downloaded from PubFactory at 01/11/2019 05:04:02AM

via free access 
Le cœur de notre travail portera sur les réflexions de Christian von EHRENFELS sur la valeur. Mais avant de découvrir et d'analyser sa théorie de la valeur proprement dite, il convient dans un premier temps de faire connaissance avec ce philosophe autrichien resté relativement peu connu. L'objet de ce chapitre sera donc d'une part d'établir une biographie du philosophe puis, d'autre part, de découvrir l'environnement intellectuel dans lequel il a développé sa théorie de la valeur.

La biographie que nous nous proposons d'écrire sera élaborée à partir de peu d'ouvrages dans la mesure où, encore une fois, la vie d'EHRENFELS a fait l'objet de peu d'intérêt. L'ouvrage le plus complet est celui de Reinhard FABIAN' ${ }^{1}$. Pour le compléter, nous nous sommes référés aux articles consacrés à la philosophie autrichienne et mentionnant EHRENFELS. Ces articles sont peu nombreux et sont essentiellement ceux qui ont été édités par Barry SMITH. Notre biographie synthétise et complète les rares ouvrages consacrés à la vie du philosophe autrichien, mais elle ne se veut pas exhaustive dans la mesure où l'intérêt de notre travail porte essentiellement sur sa théorie de la valeur.

Afin de mieux comprendre l'œuvre d'EHRENFELS, il est également important de connaitre l'environnement intellectuel dans lequel le philosophe a vécu, à savoir les courants dominants en philosophie et en économie qui ont pu l'influencer. Dans l'Empire austro-hongrois de la fin du XIX ${ }^{\text {eme }}$ siècle, la personnalité du philosophe Franz BRENTANO aura marqué plus d'un académicien et aura contribué au développement d'une perspective s'opposant à celle qui dominait dans l'Empire allemand voisin. Dans le domaine économique, les noms de Carl MENGER, Friedrich von WIESER et Eugen von BÖHMBAWERK auront marqué leur époque. A cause de leurs relations plus ou moins proches avec EHRENFELS et de leur influence sur sa théorie de la valeur, nous allons nous intéresser à leurs vies et à leurs œuvres.

'FABIAN Reinhard (éd.), 1986. 


\section{I- Une biographie de Christian von EHRENFELS}

Maria Christian Julius Leopold Karl Freiherr von EHRENFELS, ou de son nom d'auteur, Christian von EHRENFELS, est né le 20 juin 1859 à Rodaun, petite ville non loin de Vienne, en Basse-Autriche ${ }^{2}$. Christian est le fils aîné d'une famille qui compta au total cinq enfants (Christian, Ferdinand, Lili, Bernhart et Marie). Ferdinand et Marie mourront en 1873 de la diphtérie, le premier était alors âgé de 12 ans et la seconde de 2 ans.

Christian von EHRENFELS est issu d'une famille noble (Freiherr von EHRENFELS est l'équivalent du titre "Baron » dans la noblesse française) dont les aïeux étaient déjà relativement connus, notamment pour leurs travaux et leurs découvertes dans le domaine agricole. Ainsi l'arrière grand-père de Christian, Joseph Michael Freiherr von EHRENFELS, était l'auteur de plusieurs livres agronomiques décrivant par exemple de nouvelles techniques dans le domaine de l'apiculture ou de l'élevage ${ }^{3}$.

Le père de Christian, Leopold von EHRENFELS, est né en 1828 et a épousé en 1856 Klotilde von COITH, laquelle était elle-même issue d'une riche famille noble possédant des usines textiles, et dont la lignée maternelle descend de la noblesse française.

Christian von EHRENFELS passera les 11 premières années de sa vie dans le château de Brunn (près de la ville de Krems en Basse-Autriche) acheté par son arrière grand-père. Durant ces années, son éducation, ainsi que celle de ses frères et sœurs, va se faire au travers de cours privés donnés au château. Par la suite, les enfants EHRENFELS iront à l'école de Krems où les parents ont acheté un appartement. Christian se distinguera dès le début par ses excellentes prestations scolaires et montrera un intérêt particulier pour la littérature et la poésie. Il recueillera ainsi toute l'admiration de sa famille lorsque, durant l'été 1876, après l'examen final de la "Realschule », il écrira trois pièces de théâtre : "Hadmar von Kuenring ", "Brutus », "Richard Löwenherz ». Sa mère et ses sœurs, en particulier, compteront parmi ses premiers et ses plus fidèles admirateurs. Ces compositions montrent l'esprit déjà créatif du jeune EHRENFELS. Et ce n'est sans doute pas un hasard s'il a écrit une pièce consacrée à Hadmar von

\footnotetext{
${ }^{2}$ Rodaun était une commune indépendante jusqu'en 1938, année elle fut rattachée au Land de Vienne. Aujourd'hui, Rodaun est un quartier du $23^{\text {eme }}$ arrondissement de la ville.

${ }^{3}$ Par exemple : EHRENFELS Joseph Michael Freiherr von, « Unterricht fur Bauersleute von den Krankheiten der Pferde des Hornviehs der Schaafe und Schweine. Erdmann Hüfreichs Unterricht für Bauersleute von den Krankheiten der Pferde des Hornviehs der Schaafe und Schweine : Den Lesern des Noth- und Hülfbulchleins gewidmet ", Doll und Schwaiger, Vienne 1790.
} 
KUENRING. En effet, la famille KUENRING possédait de vastes domaines en Basse-Autriche, dont celui de Brunn am Walde jusqu'au XIV ${ }^{\text {eme }}$ siècle, où se trouvait le château familiale ${ }^{4}$.

En tant qu'aîné de la famille, Christian von EHRENFELS est destiné à prendre la succession de son père à la tête du domaine familial. Dans cette perspective il entreprend à partir de 1877 des études à l'Université d'agronomie de Vienne. Mais ce projet n'intéresse pas vraiment le jeune EHRENFELS. Il est en effet plutôt attiré par des études universitaires et en particulier par la philosophie. Il réussit en un an à rattraper et à passer l'équivalent du baccalauréat (soit en allemand le "Gymnasialreifeprüfung "), ce qui lui permet de s'inscrire à l'Université de Vienne dès le semestre 1879-1880, en faculté de philosophie et de droit.

C'est pendant ses années d'études que le jeune EHRENFELS rencontre les professeurs qui sont devenus par la suite ses amis et, dans une certaine mesure, ses maitres à penser. Parmi ceux-ci on retrouve les philosophes Franz BRENTANO et Alexius MEINONG. EHRENFELS et MEINONG sont restés bons amis tout au long de leur vie et leur riche correspondance ${ }^{5}$ donnera lieu à des débats propices pour leur développement intellectuel et pour l'élaboration de leurs théories.

Parallèlement à ses études, Christian von EHRENFELS s'intéréresse beaucoup à la musique et en particulier à celle du compositeur allemand Richard WAGNER, qu'il admire comme bon nombre de ses contemporains. Le compositeur marque en effet les milieux intellectuels de l'espace germanique entre la fin du XIX ${ }^{\text {eme }}$ siècle et le début du $\mathrm{XX}^{\text {ème }}$ siècle $^{6}$. Aujourd'hui encore, il reste l'objet d'un certain nombre d'études. EHRENFELS resta sa vie durant un grand admirateur des œuvres de WAGNER, même si on doit préciser que son admiration était avant tout dirigée vers les talents de compositeur de ce dernier.

\footnotetext{
${ }^{4}$ EHRENFELS Bernhart von, " Geschichte der Schlosser und Güter Brunn am Walde, Lichtenau, Allentsgichwendt, Eppenberg und Raitbach ", 1904, p.14.

${ }^{5}$ Une partie de cette correspondance a été conservée par la famille d'EHRENFELS et, est consultable au château de Lichtenau (Basse-Autriche) ; une autre partie a été laissée par MEINONG et se trouve conservée à la bibliothèque de l'Université de Graz; enfin quelques lettres ont été publiée en 1965 : Christian von EHRENFELS, "Sieben Briefe an MEINONG », p.65-81.

${ }^{6}$ A propos de l'impact de la musique de Richard Wagner dans les milieux intellectuels germaniques à la fin du XIX ${ }^{\text {ème }}$ siècle et au début du XX ${ }^{\text {ème }}$ siècle, voir : KOPPEN Erwin, " Dekadenter Wagnerismus ", 1973 ; SCHÜLER Winfried, « Der Bayreuther Kreis von seiner Entstehung bis zum Ausgang der Wilhelminischen Ära ", 1971 ; ZELINSKY Hartmut, " Richard Wagner - ein deutsches Thema ", 1976; DALHAUS Carl (éd.), " Richard Wagner - Werk und Wirkung », 1971; DÖHRING Sieghart, “ Berlioz, Wagner und die Deutschen ", 2003.
} 
Le premier article qu'EHRENFELS publia sur ce sujet, " Richard Wagner und der Naturalismus $"{ }^{7}$ refléta l'admiration décrite plus haut. Un peu plus tard le philosophe prit ses distances avec la personne de Richard WAGNER ${ }^{8}$, mais continua de vouloir analyser ses œuvres et d'en analyser la forme (en particulier, l'architectonique du drame musical) ${ }^{9}$. A la fin de sa vie, EHRENFELS vit dans les œuvres de WAGNER une inspiration et une justification à ses propres théories sur la religion ${ }^{10}$. Il s'essaya également à plusieurs reprises à l'art de la composition musicale, mais reconnut de lui même n'avoir aucun talent pour cela.

En 1882 Christian von EHRENFELS prit la décision de ne pas accepter la gérance du domaine familiale et, dans une lettre à son père, avoua avoir d'autres centres d'intérêt. Il délégua en conséquence cette responsabilité à son jeune frère, Bernhart.

Après avoir effectué son service militaire (1882-1883), EHRENFELS entreprit de continuer ses études doctorales à l'université de Graz, une décision qui fut prise non sans l'influence de son professeur et ami, Alexius MEINONG, qui fut nommé professeur " extraordinarius " en 1882 dans cette même université. C'est en mars 1885 que Christian von EHRENFELS obtint son titre de Docteur grâce à sa thèse "Über Größenrelationen und Zahlen»" ou "A Propos des Relations de Grandeur et des Nombres ».

C'est également en 1885 que Ehrenfels publia pour la première fois en tant qu'auteur deux tragédies, en prose, intitulées " Die Brüder von Hartenstein ${ }^{12}$ (ou "Les Frères de Hartenstein ») et " Mélusine " ${ }^{13}$. Dans la première, la fascination d'EHRENFELS pour le monde des chevaliers et des êtres fantastiques est manifeste. La seconde décrit une relation romantico-tragique entre une nixe $\mathrm{e}^{14}$ et un chevalier. L'année suivante, il publia les résultats d'une étude à propos de $\mathrm{Du}$ BOIS-REYMOND dans laquelle il traite de la relation de dépendance entre la

\footnotetext{
${ }^{7}$ EHRENFELS Christian Von, « Richard Wagner und der Naturalismus », article paru dans le journal « Freie Bünne für den Entwicklungskampf der Zeit », 1891.

${ }^{8}$ Il critiqua le dogme wagnérien du « Deutschtum » tout comme le théorème de l'allitération ou les origines de la race blanche. Voir EHRENFELS Christian von, " Richard Wagner und seine Apostaten ", 1913, p.21 et WINKLER Gerhard J., "Christian von Ehrenfels als Wagnerianer ", in FABIAN Reinhard (éd.), 1986, p.182-245.

9 EHRENFELS Christian von, "Zur Klärung der Wagner-Controverse », 1896; EHRENFELS Christian von, « Die musikalische Architektonik », 1896.

${ }^{10}$ EHRENFELS Christian von, "Wagner und seine neuen Apostaten », 1914; EHRENFELS Christian von, " Kosmogonie ", 1986.

"Un exemplaire de sa thèse est disponible et consultable à la bibliothèque de la Karl-FranzenUniversităt de Graz.

${ }^{12}$ EHRENFELS Christian von, “ Die Bruder Von Hartenstein », 1885.

${ }^{13}$ EHRENFELS Christian von, " Melusine ", 1887.

${ }^{14}$ Une nixe désigne une nymphe des eaux dans la mythologie germanique.
} 
réalité physique et psychique ${ }^{15}$. Emil Du BOIS-REYMOND (1818-1896), célèbre physiologue et mathématicien allemand, est resté connu comme le fondateur de l'électrophysiologie ${ }^{16}$. Il fut élu membre de l'Académie Royale des Sciences de Berlin dès 1851. Ses recherches sur l'électricité musculaire animale l'occupèrent tout au long de son existence. EHRENFELS présenta son étude sur Du BOISREYMOND, la première du genre qu'il écrivit, à l'Académie des Sciences de Vienne.

En 1887 Ehrenfels partit pour Zurich avec l'intention d'y rester si le lieu saurait éveiller son intérêt. Ce ne fut pas le cas et il revint à Vienne ou il travailla à son habilitation à l'université. Il l'obtint en 1888 grâce à la publication d'une étude psychologique sur les sentiments de sentir et de vouloir ${ }^{17}$. Cette étude montre qu'il s'intéressa très tôt à la psychologie. 1888 fut aussi l'année durant laquelle son père décéda. Il laissa en héritage un ensemble de terres d'une surface totale d'environ 1900 " Joch ", soit l'équivalent de 1083 hectares $^{18}$. Cet ensemble de terres se divisait en fait en deux domaines : celui de Brunn am Walde et celui de Lichtenau ${ }^{19}$. Nous n'avons cependant pas trouvé d'information concernant la surface respective de chacun de ces domaines. Christian von EHRENFELS ayant cédé ses droits d'aînesse à son frère Bernhart, il reçut le second château de la famille, celui de Lichtenau, avec les terres adjacentes, où il installa par la suite sa famille et sa mère.

Bien qu'il fut professeur à l'université de Vienne depuis la réussite de son habilitation, EHRENFELS ne donna aucun cours jusqu'en 1891 et préféra voyager en Europe, en particulier à Berlin, où son attention était attirée par la montée des idées du courant socialiste. A travers le magazine "Freie Bühne", qu'il soutenut financièrement à la manière d'un mécène, il trouva un organe de publication pour ses écrits sur l'art (en particulier la musique), écrits qui ont été publiés entre 1891 et $1893^{20}$. "Freie Bühne" était un magazine littéraire hebdomadaire fondé en 1890 par les membres de l'association théâtrale du même nom, et qui fut rebaptisé "Die Neue Rundschau » en 1894. Il servait de moyen d'expression à de nombreux critiques tels que Otto BRAHM (1856-1912). L'association " Freie Bühne » s'était donnée comme tâche de favoriser la mise en

\footnotetext{
${ }^{15}$ EHRENFELS Christian von, « Metaphysische Ausfuhrung im Anschlusse an Emil Du BoisReymond ", rapport de conférence à l'Académie impériale des Sciences, 1886, p.429 à 503.

${ }^{16}$ L'électrophysiologie est « la partie de la physiologie qui étudie l'activité électrique des cellules des organes et des tissus vivants, notamment des tissus nerveux et musculaires » (Le Petit Larousse 2004).

${ }^{17}$ EHRENFELS Christian von, «Über Fühlen und Wollen », 1888.

${ }^{18}$ Le "Joch" est une ancienne unité de mesure des surfaces. Un Joch équivaut à environ $5700 \mathrm{~m} 2$.

${ }^{19}$ EHRENFELS Bernhart von, 1904, p.10-11.

${ }^{20}$ Voir BRAHM Otto (éd.), "Freie Bühne für modernes Leben ", éditions reliées de 1891, 1892 et 1893.
} 
scène de pièces dramatiques ayant pour point commun leur critique de l'ordre social. Parmi les premières représentations on comptait "Vor Sonnenaufgang " de Gerhart HAUPTMANN (1862-1946) et « Die Gespenster » de Henrik IBSEN (1828-1906).

Mais c'est en 1890, dans la revue "Vierteljahrsschrift für wissenschaftliche Philosophie » que parut un article majeur de Christian von EHRENFELS : « Über Gestaltqualitäten ». Cet article fut en fait une réponse d'EHRENFELS à Ernst $\mathrm{MACH}^{21}(1838-1916)$ et à ses propos sur la perception des choses ${ }^{22}$. Ernst MACH était un physicien et un philosophe qui enseigna à l'Université allemande de Prague entre 1867 et 1895 . Il reste connu pour ses nombreuses découvertes en sciences physiques (l'onde de choc, le nombre de Mach,...) mais aussi pour ses thèses en philosophie des sciences. En tant qu'empiriste, il considérait que tout ce que l'on peut apprendre de la réalité du monde se fait à travers nos sens, ce qui rend le critère de mesurabilité nécessaire à notre compréhension de cette même réalité. Dans cette perspective, les sciences physiques constituent une méthode moyennant laquelle il est possible d'expliquer la réalité quand la psychologie permet, elle, de comprendre nos sensations. Dans son livre « Beiträge zur Analyse der Empfindung " 23 , paru en 1886, MACH affirme que les sensations ont à la fois une dimension physique et une dimension psychologique ${ }^{24}$. Il ne faut pas analyser ces dimensions séparément car elles sont liées, mais essayer de comprendre leur fonctionnement respectif et les relations qui les lient. Par exemple, pour comprendre la perception des notes de musique, il convient d'analyser également leur effet sur la psyché de l'individu ${ }^{25}$.

L'article d'EHRENFELS reprend la problématique de la perception d'une mélodie par un individu sur le plan psychologique. Il pose les bases de la théorie du Gestalt, dont on peut considérer qu'EHRENFELS fut le fondateur, et qui a, aujourd'hui encore, un rôle important à jouer en psychothérapie. Gestalt est un mot allemand que l'on traduit par "forme" dans le sens : aspect qu'une chose peut avoir en apparence ou dans l'imagination. Cependant, l'anglais et le français par exemple, utilisent le même mot pour désigner cette théorie. Le point de départ de l'article d'EHRENFELS est la réponse à la question suivante : qu'est ce qu'une mélodie? Pour EHRENFELS, une mélodie n'est pas simplement la somme des notes de musique jouées qui la compose. Une mélodie est une somme

\footnotetext{
${ }^{21}$ Les archives de la correspondance d'EHRENFELS montrent qu'il échangeait également des lettres avec Ernst MACH entre 1881 et 1903.

22 SMITH Barry, " Foundations of Gestalt Theory ", 1988, p.11.

${ }^{23}$ A partir de sa seconde édition, le livre portera le titre suivant: « Analyse der Empfindungen und das Verhältnis des Physischen zum Psychischen ».

${ }^{24}$ MACH Ernst, « Analyse der Empfindung », 1900, p.1-27.

${ }^{25}$ Idem, p. 169-205.
} 
de notes de musique et chaque note de musique en est un constituant. L'ensemble est d'autant plus stable que les relations entre ses différentes composantes le sont. De même, les relations entre les différentes parties sont d'autant plus stables que la forme ou le Gestalt de l'ensemble est prégnant ${ }^{26}$. En outre, une mélodie est plus que la simple somme des notes qui la composent. Elle est égale à la somme des notes qui la composent plus un élément qu'EHRENFELS nomme une " Gestaltqualität » ou qualité de la forme.

La théorie du Gestalt est une théorie générale qui offre un cadre adéquat pour différentes connaissances psychologiques et leur utilisation. L'être humain y est compris comme un système ouvert, l'homme interagit avec son environnement. Dans le monde contemporain, la théorie du Gestalt est à comprendre dans un sens large et comprend les éléments suivants ${ }^{27}$ :

- La primauté des phénomènes : c'est une position fondamentale de la théorie du Gestalt d'accepter comme seule réalité donnée les expériences vécues de l'être humain et de les prendre au sérieux sans les minimiser par des discussions. L'ampleur féconde de cette position est loin d'être épuisée.

- L'interaction de l'individu et de la situation au sens d'un champ dynamique déterminent l'expérience vécue et le comportement, pas seulement les instincts (psychoanalyse, éthologie) ou les forces extérieures (behaviorisme) ni les traits de caractère immuables (théorie classique de la personnalité).

- Le rapport entre les faits psychiques se fait plus facilement et plus constamment s'il est fondé sur les relations objectives et moins bien par la répétition ou la confirmation.

- Penser et résoudre des problèmes sont marqués par la structuration, la restructuration et la concentration sur les données vis-à-vis de ce qui est demandé dans la situation.

- Dans notre mémoire, les structures se forment et se différencient par des rapprochements associatifs.

- Des perceptions incompatibles d'une personne mènent à une expérience de dissonance et à des processus de perception qui tendent à réduire cette discorde.

- Dans un groupe, compris comme un ensemble interpersonnel, on trouve la tendance vers les rapports excellents par l'échange des forces et des besoins.

\footnotetext{
${ }^{26}$ WALTER Hans Jürgen, " Gestalttheorie und Psychotherapie », 1994, p.27.

${ }^{27}$ Voir sur www.gta.ch, la définition de la théorie du Gestalt.
} 
Carl STUMPF (1848-1936), également professeur à Prague entre 1879 et 1884 et en contact avec EHRENFELS, s'intéressa longtemps à la psychologie de la musique et exerça une influence notable sur les trois fondateurs de l'Ecole de Psychologie expérimentale de Berlin (devenue après 1922, l'Ecole de la Gestaltpsychologie de Berlin) : Max WERTHEIMER (1880-1943), Wolfgang KÖHLER (18871967) et Kurt KOKKFA (1886-1941). Notons que ces deux derniers ont écrit leurs thèses de doctorat sous la direction de Carl STUMPF à Berlin et que Max WERTHEIMER fut un élève d'EHRENFELS durant une partie de ses études de psychologie à Prague ${ }^{28}$. L'article d'EHRENFELS sur les «Gestaltqualitäten » restera une des bases fondamentales de la Gestaltpsychologie développée par Max WERTHEIMER et l'Ecole de Berlin ${ }^{29}$.

Cette volonté d'analyser l'essence de chaque objet est une méthode conforme à l'Ecole Autrichienne, en particulier à Carl MENGER, comme nous le verrons au cours de notre paragraphe concernant l'environnement dans lequel EHRENFELS a vécu. Cette théorie du Gestalt aura en outre des influences sur le développement de sa théorie de la valeur, puisque la valeur en elle-même est un Gestalt, une forme composée d'éléments essentiels qu'EHRENFELS étudia en détail. Il analysa le processus d'apparition de la valeur dans la conscience de l'individu en relation avec son environnement (plus exactement la perception de ce même environnement) et avec les émotions ressenties.

En 1891, EHRENFELS retourna à Vienne et à c'est à partir de ce moment qu'il commença à enseigner à l'université, même si ce ne fut que pendant les semestres d'été (de février à juin). Le reste du temps, il voyageait ou partait se reposer dans son château de Lichtenau. Le premier cours qu'il donne s'intitula "Theorie générale de la valeur avec une considération particulière pour l'éthique ${ }^{30}$. Il fut pour la première fois confronté au problème de la valeur lors de ses études à Graz, et en fit pour les années suivantes un des centres d'intérêt de ses recherches.

C'est ainsi qu'il écrivit à MEINONG, dans une lettre datée du 12 juillet $1891^{31}$, son intention de publier un ouvrage en trois tomes sur les résultats de ses recherches et réflexions sur la valeur, avec une attention particulière aux problèmes de l'éthique et de la morale. En 1893 et 1894 parurent une série d'articles sur la valeur (ce que l'on considérera comme étant le premier tome) et quatre ans plus tard un autre ouvrage "System der Werttheorie » qui constituera

\footnotetext{
${ }^{28}$ Dès la première année de son inscription à l'Université allemande de Prague, WERTHEIMER suivit des cours d'EHRENFELS. L'intérêt qu'il portait au philosophe le conduit à participer à d'autres cours et séminaires durant les années suivantes. Voir KING Brett D. et WERTHEIMER Michael, « Max Wertheimer \& Gestalt Theory », 2005, p.43.

${ }^{29}$ KING Brett D. et WERTHEIMER Michael, 2005, p.96-97.

${ }^{30}$ Allgemeine Werttheorie mit spezieller Berücksichtigung der Ethik.

${ }^{31}$ Christian von EHRENFELS, "Sieben Briefe an MEINONG ", 1965, p.65-81.
} 
le second tome. Il n'y eut pas de troisième tome, qui aurait dû être consacré plus spécialement au problème de la valeur en sciences économiques.

C'est également durant la même période que se développe l'Ecole Autrichienne, laquelle fut une école de pensée consacrée aux sciences économiques en général, et dont certaines théories ainsi que la méthodologie permettant le développement de ces théories, sont restées célèbres. On considère Carl MENGER comme le fondateur et le chef de file de ce courant de pensée, Friedrich von WIESER et Eugen von BÖHM-BAWERK comme ses élèves et parmi les plus importants représentants de l'Ecole Autrichienne.

L'ensemble des articles publiés de 1893 à 1894 ainsi que le livre "System der Werttheorie " ont été rassemblés dans un seul ouvrage édité en 1982 par Reinhard FABIAN $^{32}$. Cet ouvrage sera notre référence pour l'étude de la théorie de la valeur de Christian von EHRENFELS.

En février 1893, à Graz, EHRENFELS fit la connaissance d'Emma von HARTMANN, à l'occasion d'une conférence sur Richard WAGNER donnée par un de leurs amis communs: Houston Stewart CHAMBERLAIN ${ }^{33}$. Emma von HARTMANN était alors veuve depuis peu et mère d'un enfant. Emma et Christian se revirent plusieurs fois par la suite et leur relation devint alors plus intime. Ils se marièrent en octobre 1894. Leur premier enfant, Imma, vint au monde durant l'été 1895 et leur second enfant, Rolf, naquit six ans plus tard. Emma von EHRENFELS est une femme qui a une certaine admiration pour son mari $^{34}$ et qui lui offre son soutien et sa participation dans chacun de ses travaux, scientifiques ou artistiques.

En 1896, alors qu'on lui proposa une place de professeur à l'Université allemande de Prague, EHRENFELS décida définitivement de son sort et aspira à poursuivre une carrière d'enseignant. L'Université allemande de Prague est en fait une des deux entités de l'ancienne Université Charles-Ferdinand de Prague, fondée en 1348 par l'Empeureur du Saint Empire romain germanique, Charles IV, et scindée

\footnotetext{
${ }^{32}$ Christian von EHRENFELS, « Philosophische Schriften. Werttheorie », 1982.

${ }^{33}$ Houston Stewart CHAMBERLAIN (1855-1927) était un grand admirateur de WAGNER qui reflétait selon lui, la " grandeur de la culture germanique ». Influencé par le Comte Arthur De GOBINEAU (Voir « Essai sur l'Inégalité des Races humaines» 1853-1855), il publia par ailleurs plusieurs ouvrages sur la lutte des rasses dans lesquels il affirmait entre autre que la rasse germanique était la plus créative d'un point de vue culturel (" Die Grundlagen des neunzehnten Jahrhunderts » 1899 ; " Arische Weltanschauung » 1905). Adolf HITLER comptait parmi ses fervents lecteurs.

Précisons que Richard WAGNER était la raison des relations entre EHRENFELS et CHAMBERLAIN (FABIAN 1986, p.23) car les notions de lutte des rasses ou de supériorité de la rasse germaine ne sont pas présentes chez le philosophe autrichien.

${ }^{34}$ FABIAN Reinhard (éd.), 1986, p.39-40.
} 
en deux universités (l'une de langue allemande et l'autre de langue tchèque) par la loi du 28 février $1882^{35}$. Cette loi est une des conséquences de la montée du nationalisme tchèque militant en faveur de la reconnaissance culturelle des populations de Bohème et de Moravie ${ }^{36}$. Malgré les tensions engendrées, les universités de Prague se caractérisent par la qualité des enseignements et de ses scientifiques d'une manière générale ${ }^{37}$. EHRENFELS et sa famille emménagèrent à Prague dans une maison dans laquelle vivait une autre famille dont le père est également professeur dans cette même université. Celui-ci n'était autre que Friedrich von WIESER. La promiscuité entre les deux familles les amena à nouer de solides liens d'amitié. Cependant, il semblerait que le fait que Christian von EHRENFELS et Friedrich von WIESER se soient côtoyés n'ait pas influencé leurs travaux respectifs. Autrement dit, Friedrich von WIESER n'aurait pas influencé EHRENFELS dans l'élaboration de sa théorie de la valeur ${ }^{38}$. On verra néanmoins par la suite que EHRENFELS part d'un concept de la valeur qui est aussi celui de von WIESER et que ce dernier est cité à plusieurs reprises dans ses écrits.

En 1898 EHRENFELS trouva de nouveau l'inspiration poétique et se mit à écrire une pièce qui parut en 1899 : "Siegmar und Heliea ", une tragédie en musique. Le message contenu dans la pièce illustre la réflexion d'EHRENFELS sur l'un des nouveaux thèmes sur lequel il travaille : l'éthique sexuelle. EHRENFELS était pour l'introduction d'une "polygamie limitée ", c'est-à-dire d'une polygamie réservée à une certaine élite. Les hommes et femmes considérés comme étant " de valeur ", c'est-à-dire possédant une certaine intelligence ou un certain talent, seraient autorisés à avoir plusieurs partenaires, de manière à obtenir une descendance bénéficiant des qualités de l'un et de l'autre. Les institutions publiques devront alors aider les mères à élever leurs enfants.

\footnotetext{
${ }^{35}$ Selon cette loi, l'Université Charles-Ferdinand de Prague est divisée à partir du semestre universitaire d'hiver 1882-1883 en deux entités conservant toutes les deux le nom CharlesFerdinand, l'une de langue allemande et l'autre de langue tchèque. Cette loi implique également une séparation physique des universités, c'est-à-dire qu'elles ne partageront plus les mêmes espaces, ni la même organisation ou encore la même administration. Un professeur ou un " privatdozent » ne peut appartenir qu'à l'une des deux entités. Enfin, les étudiants ne peuvent s'inscrire que dans une des deux universités, même s'il leur est permis de suivre des cours dans chacune d'entre elles. Voir « Die deutsche Karl-Ferdinands-Universităt in Prag unter der Regierung seiner Majestät des Kaisers Franz-Josef I. ", édité par le Sénat Académique de l'Université Allemande de Prague, 1899, p.27-28.

${ }^{36}$ Voir à ce propos : RAUPACH Hans, „Der Tschechische Frühnationalismus », 1939 ; WIESNER-PIHOFSKY Emmy, “ Die Wiedergeburt der tschechischen Nation im 19. Jahrhundert », 1994.

${ }^{37}$ MADER Melitta, “ 650 Jahre Prager Universităt », 1999, p.33 et CYSARZ Herbert, " Beiträge der Prager deutschen Universität zur Philosophie und Grundlagenforschung in der Zwischenkriegzeit », 1982, p.256-258.

${ }^{38}$ FABIAN Reinhard (éd.), 1986, p.25.
} 
Afin de mieux les comprendre, il convient de replacer les propos d'EHRENFELS dans leur contexte, à savoir la société viennoise à la fin du XIX ${ }^{\text {ème }}$ siècle et au début du XX ${ }^{\text {ème }}$ siècle. Jacques Le RIDER présente cette société comme souffrant d'une crise identitaire ${ }^{39}$. Durant les années 1859 et 1860 , Vienne montrait un certain retard de développement par rapport à Budapest et Berlin. Ce retard fut en partie comblé à partir des années 1890 avec, notamment, la politique de grands travaux de Karl LUEGER (1844-1910), maire de Vienne entre 1897 et 1910. Le retard de Vienne dans les domaines socio-économiques existait également dans le domaine culturel, ce qui conduit les intellectuels à regarder Berlin avec envie ${ }^{40}$. Leur réaction à « la prépondérance allemande, à l'apparente solidité de l'Empire voisin et au prestige de leur culture fut un repli sur soi ${ }^{41}$. Cette crise d'identité est la conséquence d'une modernisation accélérée et elle est liée, selon Hermann $\mathrm{BROCH}$, à une " désintégration des valeurs ", c'est-à-dire à une fragmentation des normes collectives en plusieurs systèmes de valeurs atomisés ${ }^{42}$. Le RIDER distingue deux facteurs principaux à cette crise identitaire : d'une part la crise de l'identité masculine, et d'autre part l'antisémitisme ${ }^{43}$.

L'antisémitisme, présent dans la société viennoise de la fin du XIX ${ }^{\text {ème }}$ siècle, se manifeste entre autre, selon Sander GILMAN, à travers un rejet de leur identité juive par un certain nombre d'intellectuels et la projection des qualités inhérentes à cette identité sur d'autres groupes ${ }^{44}$. Un exemple illustrant cette thèse est celui du célèbre critique d'origine juive, Karl KRAUS (1874-1936). Editeur du journal satirique "Die Fackel " (= Le Flambeau), une bonne partie de ses critiques s'adressait à des écrivains juifs (par exemple, Felix SALTEN 1869-1945), à des médias dits juifs (par exemple, la « Freie Neue Presse ") ou encore à des penseurs juifs (par exemple, Theodor HERZL (1860-1904), dirigeant du mouvement sioniste dans l'Autriche-Hongrie de la fin du XIX ${ }^{\text {eme }}$ siècle) qui avaient pour point commun d'être les soi-disant "représentants d'une véritable contamination du langage, un des arguments antisémites de l'époque ${ }^{45}$. Cette projection de KRAUS lui permettait de rejeter les attaques antisémites, de se prétendre assimilé à la société viennoise et d'agir en tant que critique et non en tant que Juif. Son journal, " Die Fackel », publia également quelques articles de Steward Houston CHAMBERLAIN, avec qui il resta en bons termes jusqu'à la fin de sa vie. KRAUS se convertit par ailleurs en 1911 au catholicisme. Sa vision des choses fut

\footnotetext{
${ }^{39}$ RIDER Jacques Le, "Between Modernism and Postmodernism: The Viennese Identity Crisis ", in TIMMS Edward et ROBERTSON Ritchie (eds), 1990, p.1-11.

${ }^{40}$ Idem, p.l.

${ }^{41}$ Idem, p.1.

${ }^{42}$ BROCH Hermann, „Nachruf auf Robert Musil“", 1975, p.98.

${ }^{43}$ RIDER Jacques Le, 1990, p.7.

${ }^{44}$ GILMAN Sander L., " Karl Kraus's Oscar Wilde: Race, Sex, and Difference », in TIMMS

Edward et ROBERTSON Ritchie (eds), 1990, p.12.

${ }^{45}$ Idem, p. 14.
} 
quelque peu ébranlée par l'accession au pouvoir du parti national-socialiste en Allemagne en 1933 et les prémisses de la fin annoncée d'une époque sont visibles dans son livre "Les Derniers Jours de l'Humanité " (= "Die letzten Tage der Menschheit », 1918).

La crise de l'identité masculine, et plus généralement, la crise de l'identité personnelle trouve son reflet dans le développement considérable que connut la psychologie, plus particulièrement sous l'égide de Sigmund FREUD. La Société de Psychologie de Vienne comptait quelques membres dont les relations illustraient cette crise de l'identité masculine. Edward TIMMS prend l'exemple du " trio érotique » formé par Karl KRAUS, Fritz WITTELS (1880-1950) et Irma KARCZEWSKA (1890-1933), et dans lequel FREUD avait également joué un rôle $^{46}$. Fritz WITTELS est né à Vienne où il fit également ses études de médecine. Il envoya dès 1907 un premier article à Karl KRAUS qui fut publié dans son magazine "Die Fackel ». Son second article publié dans ce même magazine concernait la possibilité d'une légalisation de l'avortement, ce qui provoqua une vague de protestation, notamment de la part des autorités religieuses. Cet article plut non seulement à KRAUS mais aussi à FREUD. WITTELS devint membre du cercle de discussion de KRAUS - qui se réunissait régulièrement le soir au Café Griensteidl, au centre-ville de Vienne - ainsi que de la Société Viennoise de Psychanalyse. Considérant alors FREUD et KRAUS comme ses pères spirituels, les relations entre les trois hommes connurent des bouleversements à partir du moment où KRAUS présenta son amante, Irma KARCZEWSKA, à WITTELS. KRAUS voulut se séparer de KARCZEWSKA et la marier à WITTELS qui, même s'il se sentait attiré par cette belle femme, n'accepta pas la façon de faire du critique viennois. Irma KARCZEWSKA refusa également de se laisser ainsi manipuler tel une femme-objet et voulut garder son indépendance dans ses choix. Cette relation inspira à WITTELS un article, publié en juillet 1907 toujours dans « Die Fackel », et qui s'intitulait « Das Kindweib» ou « La Femme-Enfant ». Il y décrivit la «Femme-Enfant " comme une belle femme, libérée de ses inhibitions sexuelles et ayant la capacité de retirer du plaisir de tous ses amants. Ce concept impliquait la résurrection d'une "religion de la beauté " centrée sur l'idéal grec de l'hétaïre, qui est une femme à la beauté exceptionnelle, une muse pour ses admirateurs masculins ${ }^{47}$. Le culte de l'hétaïre est, selon TIMMS, un des produits les plus extravagants de la subculture érotique au tournant du siècle dernier dans la société autrichienne ${ }^{48}$.

\footnotetext{
${ }^{46}$ TIMMS Edward, «The « Child-Woman »: Kraus, Freud, Wittels and Irma Karczewska », in TIMMS Edward et ROBERTSON Ritchie (eds), 1990, p.87-107.

${ }^{47}$ Idem, p.91.

${ }^{48}$ Idem.
} 
Les relations entre WITTELS, KRAUS et FREUD se détériorèrent dès 1908, en particulier entre les deux premiers. Plus tard, lors de leur dernière rencontre en 1933 à Vienne, FREUD avoua à son fils spirituel sa tristesse de l'avoir "perdu » à cause de l'affaire KARCZEWSKA et de ses suites, à un moment où KRAUS et lui-même en étaient si « proches ".

Pour TIMMS, cette relation montre d'une part les relations particulières que certains intellectuels entretenaient à cette époque, et d'autre part "les énergies libidinales d'attraction et de rivalité qui ont joué un rôle dans cette explosion de créativité dans la culture viennoise [développée] dans les cafés. En ce sens, la subculture érotique n'était pas une dimension isolée ", elle était présente dans les relations de certains intellectuels, avec parfois des tonalités homosexuelles ${ }^{49}$.

Un autre intellectuel au destin tragique, qui refléta cette crise identitaire fut Otto WEININGER (1880-1903). Né à Vienne, WEININGER y mourut également à l'âge de 23 ans, se donnant la mort dans l'ancienne maison de BEETHOVEN, peu après la parution de son seul ouvrage : "Geschlecht und Charakter ». Dans ce livre se côtoient, selon Jacques Le RIDER, "le meilleur et le pire». Il se demande si la "déraison » de "Geschlecht und Charakter » ne reflèterait pas la raison de son temps et si la crise personnelle de WEININGER ne serait pas symptomatique de la crise morale de toute une génération ${ }^{50}$.

Le père d'Otto, Leopold WEININGER, était un homme au caractère dominant, un Juif antisémite, et un admirateur de Richard WAGNER. Otto WEININGER présentait une personnalité troublée, et avait hérité de son père la haine des Juifs et une passion pour la musique de WAGNER. Etudiant en philosophie à l'Université de Vienne, il fréquentait également la Société Viennoise de philosophie où il fit la connaissance, entre autre, de Houston Stewart CHAMBERLAIN. WEININGER rencontra FREUD en 1900 et fut défendu à plusieurs reprises par Karl KRAUS dans sa revue ${ }^{51}$.

"Geschlecht und Charakter » fut d'abord présenté par WEININGER comme son travail de doctorat qu'il présenta sous la direction des philosophes JODL et MÜLLNER en 1902. Une des principales hypothèses de départ, qu'il reprend d'Ernst MACH et de Richard AVENARIUS, est celle de l'unité formée par le corps et l'esprit ${ }^{52}$. Il en ressort que le caractère sexuel est l'expression à la fois des

\footnotetext{
${ }^{49}$ " Libidinal energies of attraction and rivalry also played their part in that explosion of creativity in the coffee-house culture of Vienna. In this sense the erotic subculture was not really a separate dimension. " in TIMMS Edward et ROBERTSON Ritchie (eds), 1990, p.104-105.

${ }^{50}$ RIDER Jacques Le, " Le Cas Otto Weininger », 1982, p.9.

${ }^{51}$ Idem, p.21 et p.33-35.

52 SCHWINGHAMMER Melitta, «Die Diskussion des « Geschlechterproblems » in der Wiener Moderne ", 1995, p.13.
} 
parties physiques et intellectuelles concernées. Parmi les thèmes abordés par WEININGER, on retrouve celui de la bisexualité et celui de l'émancipation de la femme. Concernant la bisexualité, WEININGER affirme que les individus sont tous bisexuels, c'est-à-dire qu'ils ont tous une composante masculine et une composante féminine. L'homme idéal et la femme idéale (qui ne contiennent respectivement que des composantes masculines ou féminines) n'existent pas. Chaque individu est le résultat de l'équation $\mathrm{xM}+\mathrm{yW}$ (où $\mathrm{M}$ est l'homme idéal et $\mathrm{W}$ la femme idéale) ${ }^{53}$. Le principe de la bisexualité est pour WEININGER universel. Cependant les caractéristiques sexuelles de l'individu ne sont pas fixes, elles peuvent varier et se rapprocher de l'homme idéal ou de la femme idéale selon les périodes ${ }^{54}$.

A partir de ces premières thèses, WEININGER voit dans l'émancipation de la femme un mouvement qui est causé en premier lieu par la composante « $M$ » de l'individu. Autrement dit, le besoin et la capacité d'émancipation de la femme sont déclenchés par sa composante masculine et WEININGER ajoute que la femme ne peut finalement être l'égale de l'homme car la composante "W " n'éprouve aucun désir d'émancipation et que celle-ci est dominante chez elle ${ }^{55}$. WEININGER réduit la femme idéale ou " $\mathrm{W}$ » à la sexualité elle-même, il la décrit entre autre comme étant alogique, amorale, sans personnalité et comme vivant soit en tant que mère, soit en tant que prostituée ${ }^{56}$.

Toutes ces thèses sur la supériorité de l'homme sur la femme n'ont pas surpris JODL au moment de commenter la thèse de WEININGER, ce qui est, selon Le RIDER, caractéristique de cette époque ${ }^{57}$, malgré les quelques contre arguments notables du mouvement féministe autrichien ${ }^{58}$. Entre la fin du XIX ${ }^{\text {ème }}$ siècle et le début du XX ${ }^{\text {eme }}$ siècle, Vienne connaît une période de publication de nombreux ouvrages au caractère diffamatoire concernant la femme ${ }^{59}$. C'est aussi une période où les valeurs prônées par les philosophes des Lumières ont perdu toute signification ; une période de crise du système libéral, de « fracture avec le passé d'une génération révoltée contre leurs pères " ${ }^{60}$; une période enfin, où la femme resta sous l'emprise du système patriarcal et où WEININGER prit l'initiative de légitimer scientifiquement et philosophiquement les préjugés de la bourgeoisie.

\footnotetext{
${ }^{53}$ WEININGER Otto, "Geschlecht und Charakter », 1980, p.8-10.

${ }^{54}$ SCHWINGHAMMER Melitta, 1995, p.14.

${ }^{55}$ WEININGER Otto, p.80.

${ }^{56}$ SCHWINGHAMMER Melitta, 1995, p.17.

${ }^{57}$ RIDER Jacques Le, 1982, p.24-25.

${ }^{58}$ Voir à ce propos KEREKES, MILLNER, OROSZ et TELLER, « Mehr oder Weininger », 2005.

${ }^{59}$ SCHWINGHAMMER Melitta, 1995, p.61.

${ }^{60}$ SCHORSKE Carl E., " Wien. Geist und Gesellschaft im Fin de Siècle », 1982, p.IX.
} 
Le philosophe vit dans la menace d'une ère féminisée, le déclin de la " vraie culture ». Dans "Geschlecht und Charakter » il donne des arguments contre la réalisation d'une telle ère et voit en la femme et le Juif, les éléments négatifs d'une société décadente ${ }^{61}$.

Voyant également dans cette période les signes d'un déclin des valeurs morales ${ }^{62}$, EHRENFELS espère, à travers ses propos pour une nouvelle éthique sexuelle, favoriser un renouveau. Pour le philosophe autrichien, la pérennité de l'espèce humaine dépend des capacités à se reproduire des hommes les plus valeureux ( " hochwertige Männer »). On devrait ainsi favoriser la polygamie pour permettre aux hommes les plus talentueux d'assurer une descendance qui permettra à la société d'atteindre un certain " niveau ». La monogamie empêcherait ce processus de remise à niveau. Il est important de noter que dans la théorie d'EHRENFELS, il n'y a pas véritablement de restrictions au niveau des groupes ethniques, c'est-àdire que pour lui, peu importe l'origine ethnique de la personne, seul compte son talent, sa valeur. La seule restriction que l'on peut noter est le fait qu'EHRENFELS ne prenne en compte que les civilisations occidentales, ce qui est compréhensible en ce tournant de siècle, où la plupart des puissances européennes avaient des empires coloniaux, dont les populations autochtones n'étaient pas considérées sur un pied d'égalité. La vision d'EHRENFELS en la matière est donc identique à celle de ses contemporains.

Malgré ses efforts pour faire passer son message, EHRENFELS ne trouve pas l'écho escompté dans le grand public. On peut noter cependant que Sigmund FREUD, avec lequel EHRENFELS est en contact depuis 1903, s'est montré intéressé par certaines de ses idées en matière de comportement sexuel, en particulier en ce qui concerne le mariage ${ }^{63}$. Cette relation permit d'ailleurs à EHRENFELS de donner par deux fois une conférence à la «Wiener psychoanalytische Vereinigung » ou Société Viennoise de Psychanalyse.

Une nouvelle grande période de créativité commença pour le philosophe en 1912. Il s'intéressa à la religion et plus particulièrement à l'existence de Dieu. EHRENFELS eut besoin de quatre ans avant de publier son ouvrage sur la

\footnotetext{
${ }^{61}$ SCHWINGHAMMER Melitta, 1995, p.61-62.

${ }^{62}$ Christian von Ehrenfels, "Sexualethik ", 1907, p.10.

${ }^{63}$ FREUD cite à plusieurs reprises EHRENFELS : une première fois en se reportant à son article "Sexuales Ober- und Unterbewußtsein » paru en 1903 dans lequel FREUD apprécie la vision de la vie de couple d'EHRENFELS (voir FREUD Sigmund, "Psychologische Schriften ", 2000, p.105); une seconde fois en s'appuyant sur l'article "Sexualethik, Grenzfragen des Nerven- und Seelenlebens " paru en 1907 dans lequel FREUD souligne et approuve la distinction effectuée entre la " morale sexuelle naturelle » et la « morale sexuelle culturelle » (voir FREUD Sigmund, «Fragen der Gesellschaft. Ursprunge der Religion », 2000, p.13-14, p.16, p.32).
} 
question, qui s'intitulait "Kosmogonie " ${ }^{64}$. Son livre le plus important après celui sur la théorie de valeur, exposait ses idées métaphysiques, et contenait également un essai sur la question de la création du monde et du cosmos. Le fonctionnement du monde obéirait à deux principes complémentaires : celui d'une "unité de création des formes ${ }^{65}$ " et celui d'un "antagonisme chaotique ${ }^{66}$ ". Le second serait responsable de la destruction de ce qui a été formé par le premier, et apparaîtrait aux personnes comme étant le Mal. Ce qui a été détruit sera par la suite reconstruit par le premier.

L'avènement de la première guerre mondiale interrompit la période de réflexion du philosophe sur les questions métaphysiques. L'assassinat par un extrémiste serbe de l'Archiduc François-Ferdinand de HABSBOURG, héritier du trône, et de sa femme à Sarajevo le 28 juin 1914, a conduit l'Autriche-Hongrie à déclarer la guerre à la Serbie ${ }^{67}$. Avec l'enclenchement des mécanismes d'alliance ${ }^{68}$, l'Europe entière est entrée en guerre ${ }^{69}$. La «Grande Guerre ", comme on l'appelait alors, allait durer quatre ans au bout desquels l'Allemagne et l'Autriche-Hongrie s'avouaient vaincues. L'année 1918 est celle de la dissolution de la double monarchie et du morcellement de son territoire. L'idée de la création d'un Etat regroupant les régions de Bohème, de Moravie, de la Slovaquie et de Transcarpathie surgit dans la tête de Thomas Garrigue MASARYK (1850-1937), intellectuel et philosophe né à Hodonin (Moravie du Sud), professeur de philosophie à l'Université tchèque de Prague depuis 1882, et député de 1900 à 1914 militant en faveur de la souveraineté des peuples slaves ${ }^{70}$. Le 28 octobre 1918, l'Etat de Tchécoslovaquie est fondé et le 14 novembre, la République tchécoslovaque est proclamée, avec à sa présidence Thomas G. MASARYK. Soulignons le fait que MASARYK et EHRENFELS se soient connus à l'Université de Vienne lorsque le premier exerçait en tant que professeur et le second commençait ses études de philosophie. Une véritable amitié s'est nouée entre les deux hommes, une amitié qui s'est renforcée lorsque tous les deux enseignaient la philosophie, l'un à l'Université tchèque, l'autre à l'Université

\footnotetext{
${ }^{64}$ EHRENFELS Christian von, “ Kosmogonie », 1986.

${ }^{65}$ Das schöpferische Gestaltungsprinzip.

${ }^{66}$ Das antagonistische chaotische Prinzip.

${ }^{67}$ L'Autriche-Hongrie adresse un ultimatum de 48 heures à la Serbie dans lequel elle exige de pouvoir mener une enquête sur place, ce à quoi s'opposera la Serbie pour des raisons de souveraineté. L'Autriche-Hongrie déclare alors la guerre à la Serbie le 28 juillet 1914 .

${ }^{68}$ On distingue la Triple Entente (France, Royaume-Uni et Russie) de la Triple-Alliance ou Triplice (Allemagne, Autriche-Hongrie et Italie). La Russie était un pays allié de la Serbie.

${ }^{69}$ Pour une chronologie détaillée des événements précédant la guerre et du déroulement de la guerre, voir par exemple KANN Robert A., " Geschichte des Habsburgerreiches 1526-1918 », 1977, p.367-465.

${ }^{70}$ Pour une biographie détaillée, voir CAPEK Karel, « Gespräche mit Masaryk », 2001.
} 
allemande de Prague ${ }^{71}$. La naissance de la Tchécoslovaquie implique toute une réorganisation à l'intérieur des régions qui la composent, notamment au niveau économique ${ }^{72}$, et cette réorganisation affecte également les campus universitaires allemands et tchèques de Prague où les tensions entre les deux communautés sont explicites ${ }^{73}$. La loi de 1920, dite "Lex Mares ", reconnaît l'Université tchèque de Prague comme étant la seule descendante de l'Université Charles fondée en 1348. L'existence de l'Université allemande est maintenue mais une quelconque parenté avec l'Université Charles lui est refusée. Herbert CYSARZ affirme que MASARYK se montrait ouvert aux discussions concernant l'existence de l'Université allemande de Prague, en particulier avec EHRENFELS et Oskar KRAUS, mais il ne précise pas l'influence que ces derniers auraient pu avoir lors de ces discussions ${ }^{74}$.

La chute de l'Empire d'Autriche-Hongrie et le redécoupage géographique de l'Europe ont affecté le moral d'EHRENFELS. Comme bon nombre d'intellectuels de son époque, il vit à travers la guerre une chance pour les Occidentaux de détruire ce qui est « mauvais » et de reconstruire sur de meilleures bases ${ }^{75}$, faisant ainsi allusion à la fois à ses réflexions sur la religion et à son principe d'éthique sexuelle, il fut déçu par la tournure que prirent finalement les événements. EHRENFELS perdit l'illusion de voir un jour ses idées adoptées. Il sombra peu après dans une profonde dépression, qui l'empêcha d'entreprendre tout travail intellectuel ou artistique. Ayant retrouvé un semblant de moral en 1919, il reprit ses cours à l'Université allemande de Prague et s'intéressa aux nombres primaires. Cet intérêt grandit progressivement, et lorsqu'il sortit définitivement de sa période de dépression l'année suivante, il décida de s'occuper du problème de la construction des nombres primaires avec l'aide de la théorie du Gestalt.

\footnotetext{
${ }^{71}$ CYSARZ Herbert, 1982, p.255.

${ }^{72}$ Voir de la formation de la Tchécoslovaquie et des relations avec l'Autriche, voir KEREKES Lajos, "Von St Germain bis Genf ", 1979, p.77-100 ; concernant la réorganisation économique et ses implications, voir TEICHOVA Alice et MATIS Herbert (eds), "Österreich und die Tschechoslowakei 1918-1938 ", 1996.

${ }^{73}$ Voir WOLMAR Wolfgang Wolfram von, « Prag. Die alteste Universităt des Reiches », 1998.

${ }^{74}$ CYSARZ Herbert, 1982, p.255.

${ }^{75}$ Le phénomène dit de " catharsis ", c'est-à-dire la purgation, le nettoyage de ce qui est mauvais dans la société, et sa présence dans les discours des intellectuels germanophones du début du $\mathrm{XX}^{\mathrm{e}}{ }^{\mathrm{me}}$ siècle est traité en détail dans ERNST Petra, HARING Sabine A., SUPPANZ Werner (eds), "Aggression und Katharsis ", 2004.
} 
C'est ainsi qu'il publia en 1922 le résultat de ses recherches ${ }^{76}$, c'est-à-dire une méthode qui permet de construire une suite de nombres primaires. Il ne s'agit pas d'une théorie mathématique à proprement parler, mais ses résultats sont considérés par le mathématicien Peter SIMONS comme une performance remarquable pour un non-spécialiste de la matière ${ }^{77}$.

Convaincu que l'espoir d'un renouveau de l'Europe, et en particulier de l'Europe centrale, viendrait de l'Europe de l'Est, en particulier des peuples slaves, EHRENFELS crut en une réconciliation entre l'Allemagne et la nouvelle République tchécoslovaque. Tentant d'influencer le cours des choses, il écrivit une nouvelle pièce de théâtre "La Mère du Légionnaire ${ }^{78}$, dont la première représentation eut lieu à Prague en présence du Président Thomas G. MASARYK en 1925. Dans cette pièce, les positions clairement exposées d'EHRENFELS ont reçu un accueil mitigé. Les blessures étaient encore trop récentes entre les deux pays et une réconciliation était encore trop prématurée ${ }^{79}$. Déçu par l'insuccès de sa pièce, y compris auprès des membres de sa famille, EHRENFELS tomba une nouvelle fois dans une phase de dépression, moins profonde néanmoins que la première. Ce fut d'ailleurs la dernière pièce écrite par EHRENFELS.

Ce n'est qu'en 1928 qu'EHRENFELS reprit des forces ainsi que ses activités à l'université. Il travailla alors de nouveau sur la religion et se référa à son ouvrage "Kosmogonie " pour mener à bien son dessein, celui d'élaborer une nouvelle religion dont les bases sont exposées dans son livre "Die Religion der Zukunft» (= la Religion du Futur) paru en 1929. Cette "religion du futur " se distingue du Christianisme par son dualisme, c'est-à-dire par le fait que le monde serait gouverné par deux principes complémentaires et antagonistes, et non par une seule instance, ce qui pourrait expliquer, selon EHRENFELS, un grand nombre de phénomènes malheureux. Et pour mettre en place cette nouvelle religion, le philosophe autrichien vit en Thomas G. MASARYK, la personne idéale; il lui adressa une lettre publique en 1929, dans laquelle il fit l'éloge du président tchécoslovaque en tant que « fondateur » potentiel de cette nouvelle religion.

\footnotetext{
${ }^{76}$ EHRENFELS Christian von, « Das Primzahlengesetz, entwickelt und dargestellt auf Grund der Gestalttheorie ", 1922.

${ }^{77}$ SIMONS Peter, " Mathematik als Wissenschaft der Gestalten ", in Fabian, 1986, p.112.

${ }^{78}$ Il faut savoir que la " Légion tchécoslovaque " était une armée de volontaires, composée de Tchèques et de Slovaques, qui a combattu durant la première Guerre Mondiale aux côtés des pays de l'Entente. Voir à ce propos MUSCHKA Wilhelm, « Der Legionăr », 1995.

${ }^{79}$ Cet accueil mitigé se comprend d'autant mieux lorsque l'on considère notamment le rôle de Masaryk dans la séparation entre la Bohème et l'Autriche-Hongrie ainsi que sur son attitude vis-àvis des Sudètes, voir WOLFRAM von WOLMAR Wolfgang, 1998, p.105-144.
} 
Le fait d'exposer ce genre d'idées peut paraître étrange pour un philosophe qui s'est surtout préoccupé de problèmes concrets comme la valeur, l'esthétique et la psychologie. Mais suite à plusieurs périodes de dépression et dans un contexte de grands bouleversements, il est aussi probable que le philosophe ait cherché pour lui même de nouvelles valeurs et de nouveaux repères.

Ehrenfels continua à donner des cours dans la même université de Prague jusqu'au début de l'année 1932. Il s'arrêta à cause de problèmes de santé, lesquels affectaient sa respiration. La même année, une infection pulmonaire l'affaiblit considérablement et il rentra dans son château de Lichtenau où il mourut le 7 septembre 1932. 


\section{II- Le contexte historique : le monde académique de l'Empire austro-hongrois à la fin du XIXème siècle et ses courants philosophiques et économiques}

Ce monde académique particulier de la seconde moitié du XIX ${ }^{\text {ème }}$ dans l'Empire austro-hongrois était imprégné par un certain aristotélisme, mis en avant dans le courant philosophique alors en plein développement. Ce développement se fit en particulier sous l'impulsion du philosophe Franz BRENTANO, qui fut lui-même un des maîtres à penser d'EHRENFELS. Un autre courant qui fit son apparition et qui connut une grande expansion durant la même période fut le courant économique marginaliste ou plus communément appelé Ecole autrichienne, dont le fondateur Carl MENGER, aura joué un rôle non négligeable dans la réflexion sur la valeur. Considéré comme l'un de ses élèves, von WIESER fut également lié d'amitié avec EHRENFELS.

C'est pourquoi nous allons décrire dans un premier temps ce monde académique ainsi que les idées aristotéliciennes qui l'auront marqué. Puis nous nous intéresserons aux intellectuels que nous avons cités, en évoquant leur vie, leurs ouvrages ainsi que leurs perspectives respectives sur le sujet de la valeur.

\section{1 - Généralités}

A la fin du XIX ${ }^{\text {ème }}$ siècle, Vienne était la capitale de l'Empire d'AutricheHongrie qui s'étendait sur une bonne partie de l'Europe centrale. Elle était également le centre intellectuel de l'Empire avec, entre autres institutions académiques, une grande université. Durant la seconde moitié du XIX ${ }^{\text {eme }}$ siècle cette vie intellectuelle s'est intensifiée et on assista à un développement particulièrement important des courants philosophiques et économiques. L'Empire d'Autriche-Hongrie ne comptait, en dehors de Vienne, qu'un petit nombre de villes universitaires : Prague, Budapest, Graz, Innsbruck et Krakòw. Les universités dans ces villes étaient de taille moyenne, comparées à celle de la capitale. A la fin du XIX ème siècle, le nombre d'universités augmenta avec l'ouverture de celles de Lemberg ${ }^{80}$, Zagreb et Czernowitz. Suite à une série de réformes survenues durant les années 1860 et $1870^{81}$, les universités de l'Empire connurent un développement important. A titre d'exemple, l'Université de Vienne comptait 2444 étudiants en 1850-51, plus de 5000 étudiants en 1884-85 et plus de 10000 en 1910. De même, le nombre d'enseignants dans la Faculté de Philosophie est passé de 17 en 1848 à 109 en 1888.

${ }^{80}$ Les villes de Lemberg ou L'viv et de Czernowitz se trouvent aujourd'hui à l'ouest de l'Ukraine.

${ }^{81}$ ALTENHUBER Hans, "Universitäre Volksbildung in Österreich 1895-1937 », p.27 et p.32. 
A l'image du monde universitaire et de manière conséquente, le monde académique était relativement étroit, si bien que la plupart des intellectuels et scientifiques de l'époque se connaissaient, ou encore, étaient apparentés. A titre d'exemple, les économistes Eugen von BÖHM-BAWERK et Friedrich von WIESER ont effectué leur scolarité dans la même école (le Schottengymnasium de Vienne) puis dans la même université où ils ont entamé les mêmes études de droit. En 1880, Friedrich von WIESER épousa Paulina von BÖHM-BAWERK, la propre sœur de son ami Eugen, faisant de ce dernier son beau-frère ${ }^{82}$. Par ailleurs, von WIESER était le voisin de pallier de Christian von EHRENFELS à Prague entre 1884 et 1903, période durant laquelle les deux intellectuels enseignaient dans l'Université Allemande de Prague. EHRENFELS était un élève du philosophe Alexius MEINONG, lui-même disciple du philosophe Franz BRENTANO, chef de file reconnu du courant de pensée philosophique autrichien durant la seconde moitié du XIX ${ }^{\text {ème }}$ siècle.

On peut ainsi constater les liens étroits qu'entretenaient les scientifiques et intellectuels du monde académique. On devine également que ces relations ont mené dans certains cas à une certaine influence autant dans la manière de penser que dans le contenu de leur travail. Pour preuve citons la correspondance entre Alexius MEINONG et Christian von EHRENFELS ${ }^{83}$, dans laquelle ils discutaient, entre autres choses, de leurs recherches respectives sur les thèmes successivement étudiés. Et on remarque à ce propos que cela a amené EHRENFELS à défendre son point de vue sur la théorie de la valeur par exemple, et MEINONG à réviser le sien sur certains points ${ }^{84}$.

Un monde académique dont les membres se connaissaient tous donc, mais aussi un monde académique dont la manière de penser et de concevoir les sciences était sous l'emprise d'un seul et même paradigme. Il s'agit en fait d'une inspiration commune de la philosophie d'Aristote et des concepts épistémologiques de ce dernier. L'aristotélisme autrichien, comme on pourrait le qualifier, était une caractéristique distinctive de la pensée intellectuelle autrichienne de la fin du $\mathrm{XIX}^{\mathrm{eme}}$. Distinctive car elle voulait se démarquer des courants d'inspiration Kantienne et Hégélienne dominants dans l'Allemagne voisine durant la même époque ${ }^{85}$.

\footnotetext{
${ }^{82}$ Voir à ce propos la biographie de Klaus H. HENNINGS (1997) et celle de Shigeki TOMO (1991), Partie A.

${ }^{83}$ Christian von EHRENFELS, « Sieben Briefe an MEINONG », 1965.

${ }^{84}$ Voir p.71 et p.74-75 de ce travail.

${ }^{85}$ SMITH Barry, 1994, p.3.
} 
Ces mouvements, comme leurs noms l'indiquent, ont été initiés par Immanuel KANT (1724-1804) et Georg Wilhelm Friedrich HEGEL (1770-1831), qui se sont eux-même inspirés des idées et ouvrages de la Révolution Française de la fin du $\mathrm{XVIII}^{\mathrm{e} m e}$ siècle $^{86}$. Or ces idées étaient considérées comme trop libérales par les autorités impériales autrichiennes et donc potentiellement dangereuses, puisque pouvant remettre en cause leur pouvoir. C'est dans ce but que l'on a cherché en Autriche à se protéger de ces idées en influençant le contenu des enseignements et le choix des professeurs à l'université ${ }^{87}$. Par ailleurs, les ouvrages issus de la Révolution Française ne vont pas seulement à l'encontre de la position monarchique, mais aussi à l'encontre de la position et du pouvoir de l'Eglise. Or l'Autriche-Hongrie était un empire catholique, dont le souverain entretenait des relations privilégiées avec le Vatican et qui, enfin, se posait en rempart défenseur du catholicisme contre l'expansion grandissante des orthodoxes (de Russie) et des musulmans (de l'Empire Ottoman) à l'Est. C'est une raison supplémentaire pour laquelle les idées d'Aristote se sont substituées à celles de KANT et de HEGEL.

En résumé, "la philosophie autrichienne est restée largement isolée des influences germaniques durant une grande partie du XIX ${ }^{\text {ème }}$ siècle et son adhésion à des idées particulières a eu un effet notable sur la manière dont les sciences humaines, spécialement l'économie, ont été conçues ${ }^{88}$.

C'est dans ce contexte que naissent et évoluent deux grandes écoles de pensée, l'une philosophique, l'autre économique. Toutes les deux vont subir, dans le temps, une influence réciproque ${ }^{89}$. L'école philosophique fut fondée par Franz BRENTANO (1838-1917) et comptait dans ses rangs de nombreux philosophes qui ont perpétué ses enseignements, parmi lesquels Edmund HUSSERL, Christian von EHRENFELS, Alexius MEINONG, Anton MARTY ou encore Oskar KRAUS.

\footnotetext{
${ }^{86} \mathrm{Si}$ on constate que HEGEL s'est inspiré de la philosophie des Lumières, ses propres concepts s'en distinguent néanmoins, notamment par leur caractère historique (voir RITTER Joachim, " Hegel und die französische Revolution ", 1989). L'influence de HEGEL sur la philosophie au $\mathrm{XIX}^{\mathrm{eme}}$ siècle ne fut pas négligeable (voir HENRICH Dieter, « Hegel im Kontext », 1981). Par ailleurs, il inspira l'école de pensée dite des jeunes hégéliens (à laquelle appartenait Karl MARX) qui portait un regard critique sur la gouvernance prusse de l'Allemagne et son conversatisme (voir ESSBACH Wolfgang, " Die Junghegelianer », 1988). Concernant les idées de KANT, voir par exemple JASPERS Karl, « Kant. Leben, Werk, Wirkung », 1985.

${ }^{87}$ GRASSL et SMITH Barry, 1986, p.12-13.

${ }^{88}$ " Austrian Philosophy remained largely isolated from German formative influences for much of the nineteenth century and its adherence to its own peculiar ideas had a noticeable effect on the way the human sciences, especially economics, evolved ", in OAKLEY Allen, 1997, p.31.

${ }^{89}$ EATON Howard O., 1930, p. 16.
} 
Un des objectifs de cette école était de développer une théorie générale subjective pour toutes les catégories de valeurs existantes, qu'elles soient économiques, esthétiques, morales ou encore sociales. Cela suppose également que les lois régissant leur apparition et leur évolution sont les mêmes, et que les fondements de ces lois sont d'ordre psychologique ${ }^{90}$.

L'histoire de l'école économique commence avec la parution en 1871 d'un des ouvrages principaux de Carl MENGER (1851-1914): «Grundsätze der Volkswirtschaftslehre ». Cette école de pensée comptera, selon la classification de Kurt LEUBE ${ }^{91}$, pas moins de sept générations ${ }^{92}$ : la première étant celle de Carl MENGER, la seconde celle de Eugen von BÖHM-BAWERK (1851-1914) et Friedrich von WIESER (1851-1926), la troisième celle de Ludwig von MISES (1881-1973) et Joseph A. SCHUMPETER (1883-1950). Les générations suivantes furent celles de l'Ecole autrichienne émigrée aux Etats-Unis suite aux événements précédant la seconde guerre mondiale. Dans le cadre de notre travail, nous serons amenés à considérer avant tout les deux premières générations, celles qui ont été actives intellectuellement durant la période où Christian von EHRENFELS a développé sa propre théorie de la valeur.

D'un point de vue méthodologique, les deux écoles partageaient un certain nombre de points communs, parmi lesquels l'attachement aux théories aristotéliciennes. Le paragraphe suivant nous permettra de mieux appréhender ce qui aura été une interprétation autrichienne des idées d'Aristote. Concernant le problème de la valeur, même si on remarque une certaine continuité entre les premières et secondes générations de l'école économique autrichienne, il n'en demeure pas moins que leurs approches respectives font état de différences que nous soulignerons plus tard.

\section{2 - L'Aristotélisme autrichien}

Pour mieux comprendre l'évolution des sciences et la méthodologie employées par les intellectuels autrichiens de la seconde moitié du XIX ${ }^{\text {ème }}$ siècle, il est important de connaître les grands traits de ce que l'on peut nommer l'Aristotélisme autrichien, c'est-à-dire l'interprétation autrichienne d'Aristote ${ }^{93}$. En particulier dans le traitement du problème de la valeur, s'il existe des similitudes, il faut bien noter que chez Aristote, le problème n'est pas posé dans le

\footnotetext{
${ }^{90}$ GRASSL Wolfgang, Avant-Propos, in EHRENFELS, 1982, p.3.

91 Il convient de noter que d'autres classifications ont été faites selon d'autres critères.

${ }^{92}$ LEUBE Kurt (éd.), 1995, p.14-15.

${ }^{93}$ SMITH Barry, 1994, p.19.
} 
même contexte ${ }^{94}$. En effet, la notion de valeur n'apparaît pas en tant que centre d'intérêt, mais plutôt en relation avec d'autres notions telles que la politique, l'éthique ou encore l'esthétique. Aristote n'utilise pas non plus d'unité de mesure pour la valeur, mais une échelle de préférence (ordres supérieurs ou inférieurs de valeurs). Un choix logique puisque celles-ci sont considérées comme subjectives.

Dans le domaine de l'éthique, le point de départ de la philosophie aristotélicienne est celle de " la psychologie de l'aspiration qui est éprouvée envers un bien et qui définit le "bien" comme ce vers quoi tout le monde aspire " "Bien" sont donc ici synonymes. Ce que nous voulons pour lui-même a une valeur plus élevée que ce que nous désirons comme moyen d'atteindre autre chose : même s'il n'emploie pas ces mots, Aristote avait déjà défini les notions de valeur intrinsèque et de valeur extrinsèque. La notion de valeur dérive des buts prédéfinis des actions de l'individu : tout acte, tout art et toute science ont leur dessein immanent. Et parmi ces desseins, il y a ceux qui appartiennent à un ordre supérieur et ceux qui appartiennent à un ordre inférieur. Le but le plus noble est celui de la politique pour Aristote. A noter que pour lui, la politique est un prolongement de l'éthique ou, plus précisément, l'éthique et la politique partagent l'aspiration pour une série de buts ou " biens"

La vie économique et sociale est reconnue par Aristote comme fondement matériel de l'Etat. L'Etat est vu comme une communauté d'individus qui aspirent tous vers le même but, un idéal parfait. L'économie fait partie de la politique, de la vie de l'Etat, qui à côté du droit du mariage et du droit de la famille, consacre le droit du maître sur ses esclaves, une des bases du système économique de la Grèce antique. Dans ce contexte, la richesse consiste en une vie accomplie que ce soit dans le domaine de la gestion de son ménage que dans le domaine de la vie de l'Etat en tant que citoyen. L'acquisition d'argent est un domaine exclu du champ des valeurs.

La vision d'Aristote concernant les valeurs est incluse dans sa vision de l'Etat, de la société et de l'économie, le tout imprégné par l'éthique. Ses propos sont néanmoins ancrés dans un certain contexte, celui de la société grecque antique avec notamment ses esclaves. De plus, le lien entre l'éthique et la politique est aujourd'hui de plus en plus discuté avec même la volonté de revenir à des valeurs plus morales dans ce domaine. On retiendra donc des enseignements d'Aristote, pour ce qui nous concerne, qu'il avait adopté une perspective subjective incluant différentes catégories de valeurs, notamment celles des valeurs intrinsèques et

\footnotetext{
${ }^{94}$ DEMPF Alois, 1989.

${ }^{95}$ Idem, p.11.
} 
extrinsèques. Les valeurs naissent enfin de la relation entre un objet et un individu dans l'action qu'il entreprend pour arriver à son but.

L'interprétation de ces théories par les intellectuels autrichiens a été résumée de manière remarquable par le philosophe américain Barry $\mathrm{SMITH}^{96}$, dont nous reprendrons une grande partie des propos afin d'expliciter les caractéristiques principales de ce courant philosophique de la fin du XIX ${ }^{\text {eme }}$ siècle.

La première des caractéristiques principales concerne la vision du monde : le monde existe indépendamment de nous, c'est-à-dire qu'il pré-existe à l'homme et existe indépendamment des représentations humaines. De ce fait, les théories objectives sur ce monde sont possibles. Les Hommes font partis de ce monde, ils en sont donc une composante.

Ensuite, le monde est constitué d'éléments essentiels ${ }^{97}$ qui sont gouvernés par des lois universelles, valables en tout temps et en tout lieu. Pour MENGER, les éléments simples de la réalité engendrent ensemble une structure et l'étude de leur relation fait partie du procédé d'analyse scientifique ${ }^{98}$. Cela suppose qu'il y ait des lois et des théories générales qui trouvent leur application pour tout objet. EHRENFELS, comme ses prédécesseurs, Franz BRENTANO et Alexius MEINONG ont cru en l'existence d'une théorie de la valeur générale, valable en tout temps et en tout lieu.

La troisième caractéristique concerne la connaissance : celle-ci s'effectue à deux niveaux, à un niveau général et à un niveau individuel. Chaque élément de ce monde est à prendre en considération à un niveau individuel (par l'expérience de nos sens) et à un niveau général (en tenant compte de l'environnement dans lequel se trouve cet élément, environnement dont le fonctionnement répond aux lois générales). Enfin, les éléments constitutifs des différents niveaux ou segments de la réalité constituent un alphabet des parties structurelles. Celles-ci peuvent être combinées de différentes manières, statique ou dynamique, selon leur coexistence ou l'ordre de succession.

Dernière caractéristique principale : il est possible d'avoir une vision objective du monde, c'est-à-dire que la réalité nous est accessible, et il est possible de théoriser cette réalité, même si le risque d'erreur persiste dans ce processus. Percevoir correctement la réalité est possible grâce à notre bon sens ou "common sense " (empirisme) et aux méthodes scientifiques. Le monde se laisse comprendre à travers un individu " en bonne santé psychique " comme à travers des méthodes

\footnotetext{
${ }^{96}$ SMITH Barry, 1994, p.321-324.

${ }^{97}$ "Wesen» selon la terminologie de Franz Brentano.

${ }^{98}$ LITSCHKA Michael, 1999, p.84.
} 
scientifiques. Il peut être expliqué par un observateur scientifique, peu importe son passé et sa culture ${ }^{99}$.

Un scientifique observant n'a pas besoin de faire partie de ce qu'il observe pour en comprendre les éléments. Au contraire, un certain recul favorise son objectivité.

La première caractéristique énoncée est liée en fait au concept de l'apriorisme, lequel implique que certains éléments ou certaines structures du monde réel existent indépendamment de tout. Elles sont données et c'est à partir de ces éléments donnés que nous bâtissons notre vision du monde ${ }^{100}$. Ainsi notre conscience contient un élément a priori qui nous permet d'accéder à la connaissance : l'intuition. L'intuition nous conduit à poser des hypothèses de départ, lesquelles seront par la suite vérifiées empiriquement (raisonnement inductif). C'est exactement ce genre de raisonnement qui est appliqué par EHRENFELS dans le développement de sa théorie de la valeur. Il part d'hypothèses prédéfinies ou encore d'idées reçues sur la question et vérifie leur pertinence le long d'un raisonnement qui comprend des références à la réalité.

Pour les économistes autrichiens, il existe dans l'économie des structures a priori, c'est-à-dire déjà existantes. L'Ecole autrichienne n'essaie cependant pas de remplacer les modèles néo-classiques par des modèles empiriques, mais de fournir une étape supplémentaire dans l'étude économique, celle qui rattache les modèles à la réalité économique, c'est-à-dire d'analyser précisément les structures existantes et de les relier aux études mathématiques.

C'est justement cette compréhension de l'existant que tente EHRENFELS dans son étude sur la théorie de la valeur : il part de la théorie de la valeur développée par l'Ecole autrichienne et essaie ensuite de la relier à la réalité par une analyse approfondie du désir et du lien valeur-désir-objet. EHRENFELS s'inscrit donc complètement dans ce mouvement, cette tendance de l'apriorisme.

Deux autres concepts dominants dans l'Autriche de la fin du XIX ${ }^{\text {eme }}$ siècle, particulièrement chez les économistes, étaient ceux du subjectivisme et de l'individualisme : «[...] les Autrichiens sont subjectivistes et ce subjectivisme se voit dans leur attitude envers les phénomènes sociaux $n^{101}$. Ces deux notions sont amplement à prendre en compte dans la mesure où, là encore, elles ont imprégné le raisonnement du philosophe EHRENFELS.

\footnotetext{
${ }^{99}$ LITSCHKA Michael, 1999, p.83-84.

${ }^{100}$ Voir également SMITH Barry, 1994, p.305-310 et OAKLEY Allen, 1997, p.32-33.

101 « Austrian are subjectivists and that subjectivism is seen in their prevailing attitude to all social phenomena ", OAKLEY citant Alex SHAND in OAKLEY Allen, 1997, p.1.
} 
Le subjectivisme peut se définir comme une « doctrine selon laquelle tout ce qui existe n'a d'autre réalité que celle que lui donne le sujet, la conscience qui le pense ${ }^{102}$. Autrement dit, l'individu est placé au centre d'une réalité qu'il perçoit et celle-ci se définit par rapport à ses perceptions. L'Homme vit dans un environnement composé d'objets. Il va considérer chacun d'entre eux individuellement et les évaluer également un à un, aussi bien de manière qualitative (intérêt de l'objet) que de manière quantitative (dimensions). L'Homme est un être conscient, qui a la capacité d'agir dans son propre intérêt et par rapport à son environnement mais aussi avec la sagesse et la prudence qui s'imposent. Ainsi, les agents économiques seront considérés par les subjectivistes comme des êtres recherchant la satisfaction de leurs besoins, dans un ordre défini, en agissant en réponse à leur environnement ${ }^{103}$. L'être humain est donc au cœur de l'activité économique et l'étude de cette dernière passe, par conséquent, par celle des agissements du premier. C'est pour cette raison que les subjectivistes ont critiqué l'utilisation des méthodes des sciences physiques dans les sciences économiques. Néanmoins, et malgré leur orientation subjectiviste, les économistes autrichiens ont cherché à donner une certaine objectivité à leurs propos, à l'aide de la vérification empirique.

La notion d'individualisme est, de manière logique, directement associée au subjectivisme, puisque l'on considère en premier lieu les agissements et les choix de l'individu. Les phénomènes économiques doivent être étudiés de manière individuelle.

La conception épistémologique des intellectuels et scientifiques autrichiens de la fin du XIX ${ }^{\text {eme }}$ siècle est donc clairement marquée par un attachement aux notions et concepts aristotéliciens. C'est en particulier la conception de l'apriorisme épistémologique qui distingue KANT et Aristote ${ }^{104}$. La notion d'apriori a un sens différent selon les auteurs : chez KANT, les connaissances a priori sont celles qui sont le résultat d'un raisonnement de l'esprit de l'individu; pour Aristote, l'expérience des sens permet de prendre connaissance du monde qui est un élément a priori, c'est-à-dire dont l'existence est donnée. Dans les deux cas, la connaissance est supposée acquise par des moyens non inductifs, mais elle ne provient pas des mêmes processus cognitifs. Pour KANT, la connaissance reflète la structure logique de l'esprit humain, lequel "ordonne" le monde dans des catégories qu'il crée. La connaissance ne provient pas de la réalité elle-même mais de l'esprit du sujet connaissant qui impose ses structures au monde. La catégorisation permet à l'individu de construire un schéma de pensée qui lui

\footnotetext{
${ }^{102}$ Le Petit Larousse 2004, p.969.

${ }^{103}$ OAKLEY Allen, 1997, p.5.

104 BRAMOULLE Gérard, " Apriorisme et faillibilisme : en défense de Rothbard contre Popper », 1995.
} 
permet de comprendre le monde ${ }^{105}$. Comme nous l'avons vu, Aristote considère que la connaissance reflète la structure du monde qui est, en elle-même intelligible. La connaissance résulte du fait que l'esprit humain peut saisir la nature ou l'essence des choses, ces catégories apriori de la réalité, comme si le sujet connaissant et les objets de connaissance étaient "pré-accordés ", selon l'expression de Barry SMITH ${ }^{106}$. Une autre différence importante entre Aristote et KANT, dans le domaine de l'éthique cette fois-ci, est leur conception du bonheur. Pour le premier, chaque individu aspire à un bien suprême qui est le bonheur (" eudaimonismos ") et qui est indépendant des circonstances extérieures. Ce bonheur peut être atteint à travers les activités de l'esprit qui poussent l'individu à agir de manière raisonnable. L'esprit, dans une société où l'ordre est établi par des lois, a pour fonction de reconnaitre les moyens et chemins appropriés qui conduisent au bien suprême pendant que les vertus éthiques fixent les buts des actions. Autrement dit Aristote nous montre la voie qui mène au bonheur ${ }^{107}$. Pour KANT, l'individu est doté de la capacité à établir des lois universelles qui conduisent au bonheur. L'individu est lui-même un être de raison, auteur de lois morales et à la fois, en tant qu'être moral, son propre législateur. Il est le moteur de sa volonté libre dans la mesure où la maxime de son action s'élève du rang d'obligation à celui de législateur ${ }^{108}$. C'est notamment à cause de cette vision des choses, celle de l'individu libre et autonome, auto-législateur, qui s'inspire de la philosophie des Lumières, que KANT n'eut pas les faveurs de la monarchie austro-hongroise.

Pour terminer ce tour d'horizon du contexte dans lequel a vécu EHRENFELS, intéressons nous plus profondément à deux grands personnages qui ont marqué l'histoire de la philosophie et celle de l'économie de l'Europe centrale : Franz BRENTANO et Carl MENGER. L'un et l'autre auront soit connu personnellement EHRENFELS, c'est le cas de Franz BRENTANO qui fut un de ses professeurs à l'université de Vienne et qui influença de cette manière ses idées; soit qui auront marqué une discipline dont se sert EHRENFELS dans l'élaboration de sa théorie de la valeur. C'est le cas de Carl MENGER qui a marqué les sciences économiques en Europe à la fin du XIX ${ }^{\text {ème }}$ siècle, et qui a développé une théorie de la valeur qui a été en partie reprise par EHRENFELS. Nous évoquerons enfin brièvement les théories de Friedrich von WIESER et celle de Eugen von BÖHM-BAWERK.

\footnotetext{
${ }^{105}$ KUNZMANN Peter, BURKARD Franz-Peter et WIEDMANN Franz, « dtv-Atlas Philosophie ", 2001, p.137-139.

${ }^{106}$ SMITH Barry, " Aristoteliarism, Apriorism, Essentialism », 1994, p.36.

${ }^{107}$ SANDVOSS Ernst R., "Immanuel Kant », 1983, p.92.

${ }^{108}$ idem, p.95-96.
} 


\section{3 - Franz BRENTANO}

La philosophie autrichienne de la fin du XIX ${ }^{\text {ème }}$ siècle connaîtra un développement assez important sous l'impulsion du philosophe allemand Franz BRENTANO. Les oeuvres de BRENTANO seront reprises et retravaillées par nombre de ses élèves, parmi lesquels EHRENFELS, et marqueront les sciences et le développement intellectuel de la monarchie danubienne.

Franz BRENTANO est né en 1838 à Marienberg dans le Sud de l'Allemagne. Sa famille comprend déjà un certain nombre d'intellectuels et d'artistes : son père était écrivain, son oncle, Clemens BRENTANO (1778-1842), était poète et son frère, Lujo BRENTANO (1844-1931), économiste. Il fit ses études à Munich, à Würzburg et à Berlin. Son premier livre, "Von den mannigfachen Bedeutung des Seienden nach Aristoteles " montre déjà son intérêt pour le philosophe grec. Se destinant un temps aux ordres, BRENTANO, en proie aux doutes sur sa foi et contestant l'infaillibilité du Pape, se décide finalement pour une carrière d'enseignant. En 1866, il fut nommé «Privatdozent " ${ }^{109}$ à l'université de Würzburg. En 1872, il partit pour Londres où il fit la connaissance d'Herbert SPENCER et de John Stuart MILL avec lequel il entretiendra une longue correspondance. Il fut nommé la même année professeur à l'Université de Vienne, poste qu'il obtint grâce au soutien de son ami Hermann LOTZE. Après son mariage en 1880, il perdit son titre de professeur car l'Empereur François Joseph ne voulut pas reconnaître le mariage d'un ancien prêtre. Il ne retrouvera jamais ce titre, malgré les protestations de ses collègues et amis. Après la mort de sa première femme en 1883 , il resta souvent dans sa résidence italienne à Florence, et se remaria en 1897. Il finira ses jours en Suisse, à Zurich où il décéda en 1917.

BRENTANO était reconnu comme étant un bon professeur et ses cours étaient assez fréquentés, notamment par des étudiants qu'il a inspirés et qui sont devenus plus tard eux-mêmes philosophes. Un témoignage de l'intérêt des étudiants pour ses cours, mais aussi de sa disponibilité pour ses étudiants et de la passion avec laquelle il poursuivait les discussions après les cours, est fourni par un de ses élèves les plus connus : Edmund HUSSERL (1859-1938) dans un de ses écrits paru pour la première fois en $1919^{110}$.

\footnotetext{
${ }^{109}$ Privatdozent était le statut d'une personne enseignant à l'université, sans être payé mais avec tous les privilèges dus au titre.

${ }^{110}$ HUSSERL Edmund, " Erinnerung an Franz Brentano », in FISCHER Kurt Rudolf (éd.), 1995, p.79-89.
} 
Il y décrit son professeur Franz BRENTANO avec beaucoup d'admiration, mais aussi comme celui qui l'a fait quitter le chemin des mathématiques pour celui de la philosophie alors que sa préférence hésitait entre ces deux disciplines :

"A la première impression, je ne restais pas indifférent. Cette forme maigre avec cette grosse tête entourée de cheveux bouclés, ce nez énergique et audacieusement arqué, ces traits du visage expressifs, qui révélaient non seulement ses réflexions mais aussi ses profonds combats de l'esprit ressortaient dans le cadre de la vie commune. Dans chaque geste, dans chaque mouvement habile des yeux pleins de vie vers le haut et vers l'intérieur, dans sa façon de se donner s'exprimait la conscience d'une grande mission. Le langage du cours, parfait dans sa forme, libre de toute tournure artificielle, de tout ornement spirituel, de toute phrase rhétorique, n'était rien de moins qu'un discours scientifique sobre $"$ "

A cet éloge du personnage s'ajoute encore quelques anecdotes sur les nombreuses discussions le soir à la table même de Franz BRENTANO :

"Après la présentation de leurs travaux il avait l'habitude d'inviter les intervenants ainsi que trois ou quatre fervents participants de ses séminaires chez lui, où Madame Ida BRENTANO avait préparé un dîner " 112 .

Le philosophe était donc réputé pour ses cours et son intérêt pour les discussions avec ses étudiants, réputation qui s'étendait dans toute l'Europe centrale, au delà des frontières de l'Empire austro-hongrois. Ses idées se sont propagées dans l'espace mais aussi dans le temps dans la mesure où nombre de ses étudiants sont devenus par la suite d'éminents professeurs de philosophie (MEINONG, von EHRENFELS, STUMPF, NEURATH, HUSSERL, MARTY). Ainsi à Prague, à Lemberg, à Graz, à Czernowitz, à Berlin se sont établis des centres de réflexion brentaniens ou d'inspiration brentanienne ${ }^{113}$. Les élèves de BRENTANO ne se

\footnotetext{
"II « Diese hagere Gestalt mit dem mächtigen, von lockigem Haar umrahmten Haupt, der energischen, kühn geschwungenen Nase, den ausdrucksvollen Gesichtslinien, die nicht nur von Geistesarbeit, sondern von tiefen Seelenkämpfen sprachen, fiel ganz aus dem Rahmen des gemeinen Lebens heraus. In jedem Zug, in jeder Bewegung, in dem aufwarrts- innengewandten Blick der seelenvollen Augen, in der ganzen Art sich zu geben, druckte sich das Bewusstsein einer großen Mission aus. Die Sprache der Vorlesungen, vollendet in der Form, frei von allen künstlichen Wendungen, von allem geistreichen Aufputz, aller rhetorischen Phrase, war doch nichts weniger als die der nüchternen wissenschaftlichen Rede », Edmund HUSSERL in FISCHER Kurt Rudolf (éd.), 1995, p.80.

112 « Nach den Übungen pflegte er den Referenten und noch drei oder vier der eifrigsten

Teilnehmer mit nach Hause zu nehmen, wo Frau Ida Brentano ein Abendessen vorbereitet hatte ", in FISCHER Kurt Rodolf (éd.), 1995, p.81.

${ }^{113}$ SMITH Barry, 1994, p.20-28.
} 
contentèrent pas de répéter les enseignements de leur professeur, ils les développèrent pour créer soit une branche spécifique de la philosophie brentanienne, soit pour créer une nouvelle tendance. Une branche spécifique serait par exemple celle de Graz (Alexius MEINONG, Alois HÖFLER, Ernst MALLY, Franz WEBER pour citer les principaux noms de cette école de Graz). Une nouvelle tendance a été développée par Christian von EHRENFELS qui, tout en s'inspirant de BRENTANO, s'est opposé à son ancien professeur dans bien des domaines, notamment dans celui de la valeur. BRENTANO eut aussi de l'influence en Pologne sous l'impulsion de Kazimierz TWARDOWSKI (18661938), qui orienta ses recherches vers la logique et la psychologie et établit ainsi en Pologne l'Ecole de la Psychologie Exacte. Kevin MULLIGAN souligne par ailleurs l'importance de l'influence de BRENTANO, en particulier en ce qui concerne la méthologie employée en philosophie ${ }^{114}$ : le philosophe considérait cette discipline comme une science qui se devait d'être précise ${ }^{115}$. Cette volonté de précision se retrouve par ailleurs clairement chez EHRENFELS, notamment dans le fait de décrire le phénomène de la valeur de la manière la plus précise possible.

Ces remarques sur le personnage du philosophe allemand et son rayonnement en Europe centrale dans la seconde moitié du XIX ${ }^{\text {eme }}$ siècle ne doivent pas faire oublier l'importance et la diversité de l'œuvre qu'il a laissée. Peu de travaux de BRENTANO furent publiés de son vivant. Aussi « Grundlegung und Aufbau der Ethik ${ }^{116}$, qui rassemble ses idées sur la valeur et l'éthique à travers ses cours entre 1876 et 1894 , a été publié un demi-siècle plus tard.

Il est important en premier lieu de présenter Franz BRENTANO comme celui pour qui la psychologie représente une science à laquelle il est nécessaire de faire appel lorsque l'on veut étudier le comportement humain. Et lorsque BRENTANO parle de psychologie, il entend en fait la $"$ psychologie descriptive $\|^{117}$, laquelle " vaut comme fondement scientifique des sciences philosophiques $" 118$.

Pour le philosophe allemand, les phénomènes psychiques gagnent à être analysés de manière empirique et cette méthode empirique précisément consiste dans le fait de décrire la Psyché, l'âme, la conscience de la personne analysée. De cette méthode descriptive vient en fait le nom de " psychologie descriptive ».

\footnotetext{
${ }^{114}$ MULLIGAN Kevin, « The Expression of Exactness: Ernst Mach, the Brentanists and the Ideal of Clarity ", 1989, p.35.

${ }^{115}$ Idem, p.36.

${ }^{116}$ BRENTANO Franz, " Grundlegung und Aufbau der Ethik », 1952.

117 Deskriptive Psychologie, voir aussi PARK Chan-Young, 1991, p.11-26.

118 PARK Chan-Young, 1991, p.11.
} 
Plus en avant, BRENTANO décrira l'acte psychique comme un acte accidentel durant lequel l'âme se concentre sur un certain nombre d'objets ${ }^{119}$. Les projections de l'âme sur les objets peuvent être classées en trois catégories ${ }^{120}$ :

- celle de l'imagination ou du fait d'imaginer (= "Vorstellen »), car pour pouvoir considérer l'objet, il faut pouvoir se le représenter,

- celle du jugement ou du fait de juger $(=$ «Urteilen ») qui peut prendre la forme concrète de "reconnaître » (= "Anerkennen ») ou "rejeter " (= "Verwerfen »),

- celle du fait d'aimer ou de haïr (= « Lieben oder Hassen »).

Les trois catégories énoncées plus haut ne décrivent pourtant pas des actes psychiques indépendants. En effet, il est possible qu'un acte relève de deux ou de trois de ces catégories à la fois. Par exemple, l'imagination fournit un objet que le jugement pourra évaluer et de ce jugement, la personne pourra soit aimer, soit haïr cet objet. Un acte n'impose pas non plus de réactions en chaîne, c'est-à-dire que toutes les catégories citées n'interviennent pas forcément dans l'acte psychique du sujet pensant.

Le fait d'aimer ou de haïr quelque chose provoque ce que BRENTANO appelle un "mouvement d'humeur " 21 . Il effectue ensuite un autre classement destiné à distinguer les différents types de mouvements d'humeur ${ }^{122}$ selon que l'on aime ou pas l'objet sur lequel on projette notre pensée. Ainsi un objet qui sera aimé pour lui-même sera désigné comme " objet primaire ou intrinsèque " (= " primäres ou intrinsisches Gut ») et celui qui sera aimé pour ce qu'il peut nous apporter sera nommé « objet secondaire ou utile » (= « nützliches oder sekundäres Gut »). Dans le cas contraire, c'est à dire dans le cas où l'objet en lui même est détesté ou non aimé, on le nommera " non-valeur primaire ou intrinsèque " (= " primärer ou intrinsischer Unwert ») et " non-valeur dommageable ou secondaire " (= "schädlicher ou sekundärer Unwert») lorsqu'on le déteste pour ses effets. Cette distinction est très importante à souligner dans la mesure où elle sera reprise plus tard par EHRENFELS dans sa théorie de la valeur.

La valeur est estimée lors du jugement de valeur ou évaluation faite par l'être pensant. Ces évaluations sont en relation directe avec les mouvements d'humeur

\footnotetext{
${ }^{119}$ Idem p.23.

${ }^{120}$ Idem et aussi BRENTANO Franz, « Vom Ursprung sittlicher Erkenntnis ", 1969.

${ }^{121}$ En allemand " Gemütsbewegung " : " das Gemüt " se traduit par " L'âme, le coeur » et " Bewegung " par « mouvement », d'où la traduction par « Mouvement d'humeur » décrivant le changement d'attitude vis-à-vis d'un objet, d'une chose.

${ }^{122}$ BRENTANO Franz, 1982, p. 150.
} 
dans la mesure où une évaluation aura pour objet un mouvement d'humeur ${ }^{123}$. Certains jugements peuvent, selon BRENTANO, être incorrects. Un jugement correct est caractérisé par la connaissance du bon ou du mauvais dans la formule "A est bien » ou "A est mauvais » ou la connaissance « qu'un sentiment positif ou négatif dirigé sur A soit correct ou incorrect $»^{124}$.

Ainsi les jugements de valeur ou estimations seraient, selon BRENTANO, apodictiques, c'est-à-dire qu'il ne pourrait en être autrement.

Après cette brève introduction sur la formation de la valeur chez BRENTANO, nous allons nous intéresser plus précisément à la valeur elle-même, et en présenter les quatre caractéristiques définies par le philosophe dans ses enseignements ${ }^{125}$ :

- D'abord le fait que quelque chose soit «bon" ou "mauvais" ne se rapporte pas directement à la chose en elle-même, mais à la relation émotionnelle entre la personne et la chose. Une chose qui sera qualifiée de "bonne " le sera en prenant en compte que le fait d'aimer cette chose soit correct. Un objet sera évalué ou jugé comme «bon " par une personne qui aura aussi dans le même sens pris en compte le fait que le sentiment positif envers l'objet en question est correct.

- Ensuite la théorie de la valeur de BRENTANO met en avant la différence entre les expériences de la valeur (autrement dit, le fait d'attribuer de la valeur ou non à quelque chose dans sa vie quotidienne) correctes et incorrectes, ce qui lui permet par la même occasion de trouver un compromis entre les différentes théories de la valeur jusqu'alors existantes. Son enseignement de la valeur nous explique comment on passe de nos expériences subjectives de la valeur à une validité générale de nos expériences. L'objectivisme est donc la deuxième caractéristique de sa théorie de la valeur.

- Troisièmement, la valeur chez BRENTANO a un caractère à la fois apodictique et empirique. L'expérience de la valeur autant que le jugement de valeur ou évaluation sont tous deux apodictiques, c'est-à-dire qu'il ne peut en être autrement, ils existent. Empirique puisque l'évaluation se produit lors d'une expérience concrète, on va procéder à des évaluations dans sa vie quotidienne. Nos expériences de la valeur proviennent donc de notre quotidien tout comme les concepts qui en découlent («bien» ou " mal », " correct » ou « incorrect »).

\footnotetext{
${ }^{123}$ PARK Chan-Young, p.70.

124 "Wahre Werturteile sind entweder die Erkenntnis von Gutem und Schlechtem in der Formel " A ist gut ", " A ist schlecht " oder die Erkenntnis " dass eine auf A gerichtete Liebe und ein auf A gerichtete Hass unmöglich richtig (unrichtig) sei " in PARK Chan-Young, p.70.

${ }^{125}$ PARK Chan-Young, p.80-83.
} 
- Enfin BRENTANO considère avant tout les biens dits primaires, c'est à dire ceux qui sont estimés pour eux-mêmes. Il s'agit donc d'un enseignement de la valeur "intrinsèque », puisque l'on s'intéresse en particulier à la valeur intrinsèque des choses.

Pour terminer, il faut retenir encore des enseignements de BRENTANO que les valeurs ne sont pas des caractéristiques, des propriétés des objets évalués, mais des qualificatifs attribués aux objets que l'on est amené à considérer.

Le fait de qualifier un objet de "bon ", et donc le fait de lui donner la valeur "bon " est synonyme du fait de considérer quelque chose mieux qu'autre chose. Etablir une hiérarchie de ce qui est bien et de ce qui est moins bien revient à établir des préférences. Une préférence est un mouvement d'humeur qui suit celui d'aimer. On considère un objet, on l'imagine, on projette un sentiment d'aimer ou de ne pas aimer, on établit une préférence, telle est l'enchaînement qui se produit chez le sujet conscient et qui aboutit sur les sentiments de vouloir ou choisir ${ }^{126}$, qui sont pour BRENTANO synonymes. Chaque nouvelle étape de cet enchaînement est conditionnée par la précédente.

Il ajoute que chaque objet voulu ou choisi est du domaine du possible. On ne peut désirer quelque chose qui a peu de chance de se réaliser ou qui ne se réalisera pas. Cette perspective implique d'une part le fait que l'individu ne désire que ce qui n'existe pas ou pas encore. Cette idée sera reprise par la suite par Alexius MEINONG et par Christian von EHRENFELS, malgré sa perfectibilité. Le fait de croire que l'individu ne désire que ce qui peut devenir réalité se heurte d'autre part aux souhaits émis par les individus dans leurs rêves par exemple.

Enfin, en haut de la pyramide des différents types de biens existants (biens primaires et biens secondaires) se trouve le "plus haut bien réel " (= das höchste praktische Gut). Il peut se définir comme étant le meilleur bien en terme quantitatif comme en terme qualitatif. Il s'agit d'un but lointain, du meilleur que l'on peut atteindre et "seul le monde dans sa totalité ("Weltganze ») serait le meilleur but réel ${ }^{127}$. Autrement dit, le «plus haut bien réel » inclut le plus grand nombre de personnes possible.

Ainsi pourraient se résumer de manière relativement simple les enseignements de BRENTANO sur la valeur. Ceux-ci vont être repris plus tard dans notre travail, parallèlement à l'analyse de la théorie de la valeur d'EHRENFELS qui reprend certains éléments de BRENTANO et s'oppose à d'autres.

\footnotetext{
${ }^{126}$ Wollen et wählen.

${ }^{127}$ BRENTANO, « Vom Ursprung sittlicher Erkenntnis », 1969, p.131.
} 
Après s'être concentré sur le contexte philosophique, intéressons nous maintenant au contexte économique ou plus exactement au développement de l'Ecole autrichienne en cette fin de XIX ${ }^{\text {eme }}$. Lorsqu'on parle d'Ecole autrichienne, on pense immédiatement à celui qui est considéré comme son fondateur ${ }^{128}$ : Carl MENGER.

\section{4 - Carl MENGER}

Né le 28 février 1840 à Neu Sandez ${ }^{129}$, au sud de Cracovie, dans l'actuelle Pologne, Carl MENGER est le descendant d'une vieille famille autrichienne qui a immigré de la Bohème pour s'établir au sud de la Pologne une génération avant sa naissance. Son père, Anton MENGER, était avocat et sa mère, Caroline GERZABEK, était la fille d'une riche famille bohémienne de marchands. Carl avait deux frères: Anton, qui fut plus tard un éminent auteur socialiste et professeur à l'Université de Vienne; et Max qui exerça la profession d'avocat et fut également député libéral au Parlement autrichien.

Après avoir étudié l'économie dans les universités de Prague et de Vienne entre 1853 et 1863 , il travailla comme journaliste durant l'été 1863 . Le jeune Carl MENGER ne tarda pas à se faire remarquer dans la profession en écrivant une série de nouvelles comiques, mais surtout des articles économiques en tant qu'analyste des marchés pour le Wiener Zeitung. Il quitta le journal en 1867 pour préparer son doctorat en droit qu'il obtint en août 1867 de l'Université de Cracovie. Il ne tarda néanmoins pas à se remettre au journalisme et s'intéressa de plus en plus à l'économie. C'est ainsi qu'il remarqua par exemple que la théorie de la formation des prix de l'Ecole Classique ne fonctionnait pas, du moins de son point de vue, et il chercha par la suite à la réformer. En 1870 MENGER obtint un poste dans le département des relations publiques du " Ministerratspräsidium » ${ }^{130}$ qui était alors composé de membres du parti libéral.

C'est en 1871 qu'il publie une de ses œuvres majeures : "Principes d'Economie Politique ${ }^{131}$, œuvre qui rassemble ses vues sur les questions économiques. L'année suivante, il réussit son habilitation à l'Université de Vienne où il

\footnotetext{
${ }^{128}$ SMITH Barry, 1994, p.299.

${ }^{129}$ La ville s'appelle aujourd'hui Nowy Saçz.

${ }^{130}$ Cabinet du « Ministerpräsident », c'est-à-dire du chef du gouvernement durant la monarchie danubienne.

${ }^{131}$ MENGER Carl, " Grundsătze der Volkswirtschaftslehre », première édition 1871 et seconde édition élaborée à partir des notes laissées par Carl Menger 1923.
} 
travaillera un an comme "Privatdozent» puis comme professeur d'université. Bien qu'il ait entretemps abandonné son poste au "Ministerratspräsidium ", MENGER continuera ses activités journalistiques jusqu'en 1875 .

En 1876, MENGER fut appelé à être un des tuteurs du prince héritier Rodolphe de HABSBOURG. Au bout de trois ans et pour le remercier de ses services, le père de Rodolphe, l'Empereur d'Autriche-Hongrie, François-Joseph Ier, le nomma Professeur Ordinarius. A ce titre, l'économiste autrichien occupa donc la chaire de politique économique à la faculté de droit de l'Université de Vienne. Il enseigna jusqu'en 1903.

A la fin des années 1870 Carl MENGER consacra ses recherches aux méthodes employées dans les sciences économiques en particulier et dans les sciences sociales en général. S'opposant au haut degré d'abstraction et de mathématisation initié par l'Ecole Classique, l'économiste autrichien proposa d'étudier les phénomènes économiques d'un point de vue théorique, en tant qu'invariants universaux, et non plus comme étant tous spécifiques car datés historiquement (ce qui interdit toute généralisation théorique). MENGER insista sur la spécificité des phénomènes humains et sociaux qui sont de nature essentiellement subjective, car fondés avant tout sur des opinions, des perceptions, par rapport aux phénomènes naturels qui, eux, sont de nature objective (cette vision est partagée par le monde académique autrichien et plus particulièrement par l'école philosophique, comme on l'a vu précédemment). MENGER récuse de ce fait la possibilité d'importer la méthode des sciences exactes dans le domaine des sciences sociales, et en particulier la possibilité ou l'intérêt d'utiliser les mathématiques en économie.

Ses analyses méthodologiques sont rassemblées dans un ouvrage publié en 1883 sous le titre « Untersuchungen über die Methoden der Sozialwissenschaften und der politischen Ökonomie insbesondere ${ }^{132}$. Cet ouvrage voulait établir de nouvelles bases pour la recherche et la réflexion dans les sciences sociales. La lecture de cet ouvrage par les économistes de la nouvelle l'Ecole Historique allemande ${ }^{133}$ fut ressentie comme une attaque implicite à leurs méthodes.

\footnotetext{
${ }^{132}$ MENGER Carl, « Untersuchung uber die Methoden der Sozialwissenschaften und der politischen Ökonomie insbesondere ", 1883.

${ }^{133}$ L'Ecole Historique Allemande est un courant de pensée économique dominant dans l'Allemagne de la seconde moitié du XIX ${ }^{\text {ème }}$. Ils rejetaient l'individualisme méthodologique, l'hypothèse de l'homo oeconomicus ainsi que la possibilité de lois économiques universelles. Pour cette école de pensée, le développement économique ne peut être analysé grâce à une construction purement logique (en réaction aux courants rationalistes et aux positivistes dominants au début du même siècle). La meilleure méthode est celle de l'histoire et de l'éventuelle recherche des régularités ou des différences ; cela conduit au relativisme et à l'abandon de l'idée d'une science économique formalisable et universelle (" Histoire des Pensées Economiques. Les Fondateurs », deuxième édition, Dalloz, Paris 1993, p.199). On distingue en général deux tendances dans cette
} 
Leur réponse ne tarda d'ailleurs pas et elle fut adressée à l'Ecole autrichienne, c'est à dire à MENGER et ses élèves, Eugen von BÖHM-BAWERK et Friedrich von WIESER. Il est important de souligner que ce fut la première fois que le terme d'Ecole autrichienne fut employé. Cette réponse de la nouvelle l'Ecole Historique marqua le début d'un débat méthodologique intitulé « Methodenstreit » et qui prit parfois des apparences acerbes ${ }^{134}$.

L'influence de MENGER se fit sentir, en particulier dans le monde académique autrichien où ses ouvrages eurent un impact notamment sur la pensée et la politique économiques et ses réflexions furent reprises et développées par ses élèves. A la fin des années 1880 , les idées de MENGER se sont répandues au-delà des frontières autrichiennes et ont atteint la France, la Hollande, les Etats-Unis et la Grande-Bretagne. Durant la même période, MENGER se retira de la " Methodenstreit » et se concentra de nouveau sur des questions d'économie pure et d'économie appliquée. En 1888, il publia un article sur la théorie du Capital ${ }^{135}$ et il fut nommé à la tête d'une commission chargée de réformer le système monétaire autrichien, et dont les résultats parurent en 1892 dans une série d'articles. Par la suite MENGER n'écrivit plus aucun ouvrage majeur.

Dans le domaine des sciences, Carl MENGER adopta une position innovante et développa également une méthodologie adoptée plus tard par les générations suivantes de l'Ecole autrichienne. Ses sources d'inspiration sont nombreuses et diverses, même si pour certains ${ }^{136}$ il était avant tout un aristotélicien. Pour Pierre Le MASNE, si ceci reste globalement vrai, l'économiste autrichien se distingue également d'Aristote dans plusieurs domaines ${ }^{137}$. Parmi les courants qui ont influencé Carl MENGER, on peut citer le courant Romantique ${ }^{138}$ et l'ancienne Ecole Historique allemande. Bien qu'il fût en conflit avec les représentants de la seconde génération de cette école, MENGER appréciait ceux de l'ancienne école,

Ecole : la première, dite ancienne Ecole Historique, avec à sa tête Wilhelm Roscher (1815-1894), et la seconde, la nouvelle Ecole Historique allemande emmenée par Gustav Schmoller (18381917).

${ }^{134}$ A propos du débat méthodologique et de son contenu, voir WENTZEL Bettina, « Der Methodenstreit », 1999.

${ }^{135}$ MENGER Carl, « Zur Theorie des Kapiltals », 1888.

${ }^{136}$ Par exemple Barry Smith (1990), Hutchison (1973), Max Alter (1990), Michael Litschka (1999).

${ }^{137}$ MASNE Pierre Le, « Carl Menger et la séparation entre éthique et économie », p.19.

${ }^{138}$ Le courant Romantique apparut au XVIII ${ }^{\mathrm{eme}}$ siècle, en réaction au rationalisme des Lumières et à la philosophie de Kant. Ce courant se répandit assez largement en Europe occidentale et en particulier en Allemagne. Il était représenté aussi bien par des philosophes (en particulier F.W Schelling (1775-1854) ) que par des poètes (Goethe (1749-1809), Novalis ou F. Von Hardenberg (1772-1829), F. Schlegel (1772-1829) ), et dont les principaux préceptes étaient celui de l'individu placé au centre de la réflexion, celui de l'unité entre la nature et l'esprit et celui de l'art comme la forme d'expression la plus recherchée. 
au point de dédier son ouvrage "Principes d'Economie Politique " à Wilhelm ROSCHER. D'autres sources d'inspirations sont identifiées par Kurt LEUBE à travers Gian Francesco LOTTINI (1512-1572), Bernardo DAVANZATI (15291606), Ferdinando GALIANI (1728-1787) ou encore Hermann Heinrich GOSSEN (1810-1858). Du premier, MENGER retiendra l'idée que les individus valorisent les biens présents plus que les biens futurs, ce qui prépara le terrain à la théorie des préférences variables avec le temps. Du second, il reprit l'aspect subjectif de sa théorie de la valeur. De GALIANI, MENGER retiendra le fait que la relation individu-objet n'est pas suffisante dans l'étude de la valeur et qu'il faut considérer la structure globale moyen-but qui conduit l'individu à effectuer un choix entre différentes possibilités. GOSSEN influença MENGER avec sa méthode "kausal-genetisch » ou causalo-génétique ${ }^{139}$, qui marquera également une partie de l'Ecole économique autrichienne ${ }^{140}$. Ces différentes influences apparaissent clairement dans les écrits de l'économiste autrichien.

Ceci nous amène maintenant à parler de la vision nouvelle de Carl MENGER concernant les sciences et sa méthodologie. Le point de départ de son analyse est qu'il existe deux orientations principales de recherche : l'orientation individuelle et l'orientation générale ${ }^{141}$. La première orientation est historique et vise la connaissance de l'être individuel et des interactions individuelles; la seconde est théorique et vise la connaissance des êtres en général et des relations entre les phénomènes globaux. Ce faisant, il opère donc une première distinction entre domaines théoriques et domaines pratiques. Dans le domaine théorique, le procédé de raisonnement est la déduction, celle qui a pour point de départ les éléments les plus simples de la réalité et comme point d'arrivée des lois exactes $^{142}$. Les sciences exactes analysent donc les éléments les plus simples, hors de leur contexte et de toute influence, et se distinguent par la même de la méthode inductive de l'Ecole Historique allemande, qui analyse avant tout les phénomènes de société dans leur contexte historique.

Le but de toute recherche scientifique est de prendre connaissance ("Erkennen ») d'un phénomène et de le comprendre ("Verstehen»). Dans le même sens, l'économie doit se consacrer à l'étude de l'origine ("die Ursprung») et de l'essence (« das Wesen ») des phénomènes ${ }^{143}$.

\footnotetext{
${ }^{139}$ Méthode selon laquelle l'effet d'un objet est une conséquence liée à son origine dans la relation entre l'origine et l'effet de ce même objet. Par conséquent, il convient de rechercher et de comprendre l'origine des objets afin d'en expliquer les effets. Voir également KRELLE Wilhelm et RECKTENWALD Horst C., " Gossen und seine Gesetze in unserer Zeit », 1987.

${ }^{140}$ LEUBE Kurt (éd.), 1995, p.17.

${ }^{141}$ MENGER Carl, « Untersuchungen », extraits parus dans Kurt LEUBE (éd.), 1995, p.84-85.

${ }^{142}$ Idem, p.89.

${ }^{143}$ ALTER Max, 1990, p.96.
} 
Reconnaître un phénomène est, pour MENGER, le fait de saisir le concept, l'image mentale ou la représentation linguistique du phénomène en question ${ }^{144}$.

Comprendre un phénomène équivaut à saisir son essence, la raison pour laquelle il est, et sa façon d'être. Découvrir l'essence d'un phénomène implique donc sa compréhension, celle de son existence :

" Das Ziel der wissenschaftlichen Forschung ist nicht nur die Erkenntnis sondern auch das Verständnis der Erscheinung “'145

Il fut celui qui introduit la méthode du "Verstehen » dans les sciences sociales, une méthode qui s'est ensuite répandue dans son utilisation à toute l'école autrichienne. Il s'agit d'essayer de saisir le sens que l'individu agissant, donne au but de son action. Nous pouvons affirmer, parce que nous le savons a priori, que toute action humaine a un but, avec l'aspiration rationnelle ou irrationnelle d'atteindre un état meilleur dans le futur que ce qu'il n'est dans le présent. Ceci est en fait la description du principe de maximisation.

Pour comprendre le sens de l'action d'autrui, nous avons besoin d'un autre élément, celui de la détermination du but, c'est-à-dire son fondement ou sa motivation.

Plus en avant concernant les sciences, et cherchant à développer sa distinction entre théorique et pratique, MENGER y ajoute une dimension historique. Cette classification s'applique bien évidemment à l'économie de la manière suivante $^{146}$ :

- Les sciences historiques qui s'intéressent à la nature individuelle des phénomènes économiques et à leurs interactions.

- La science théorique de l'économie (« die theoretische Nationalökonomie ») décrit la nature générale et les interactions générales des phénomènes économiques. Elle comporte une orientation exacte de recherche et une orientation empirique.

- Les sciences pratiques de l'économie recherchent et décrivent les principes d'action adaptés dans le domaine économique, en fonction de la diversité des conditions, ce sont la politique économique («die Volkswirtschaftspolitik») et la gestion des finances («die Finanzwissenschaft »).

\footnotetext{
144 MENGER Carl, 1883, p.6.

${ }^{145}$ MENGER Carl, 1883, p. 14.

${ }^{146}$ MASNE Pierre Le, p.7.
} 
MENGER appelle enfin l'économie politique ("die politische Ökonomie ») le regroupement des sciences théoriques et des sciences pratiques; les sciences historiques ne font pas partie de l'économie politique mais appartiennent néanmoins au domaine de l'économie.

Voulant faire de l'économie une science à part entière, MENGER affirmait en outre que l'on pouvait lui appliquer les méthodes de recherche des sciences exactes, la différenciant ainsi de la philosophie et plus particulièrement de l'éthique, à laquelle elle fut longtemps subordonnée. Ajoutons que par économie, MENGER entend "les activités des individus visant à couvrir leur besoins en biens " et par politique économique, la même activité mais à l'échelle d'une société ${ }^{147}$.

Les éléments simples à l'origine de l'activité économique sont les besoins, les biens et l'aspiration à couvrir le plus grand nombre de besoins possibles. Les recherches théoriques dans ce domaine doivent pouvoir nous apprendre quels sont les facteurs élémentaires de l'économie individuelle qui, en dehors de toute influence extérieure, provoquent le développement de phénomènes économiques complexes. Un exemple nous est proposé par l'économiste autrichien dans ses "Untersuchungen " 148 .

Les recherches théoriques sur le phénomène des prix nous ont amenés à la loi exacte suivante : lorsque le besoin pour un bien marchand croît, alors, et sous certaines conditions, son prix augmentera dans la même proportion. Ces conditions en question sont au nombre de quatre. La première est que l'agent économique doit être conscient de ses envies et de ses intérêts personnels. La seconde condition est celle qu'il ne se trouve pas dans l'erreur que ce soit aussi bien en ce qui concerne le but qu'il poursuit que des moyens employés. Troisièmement, il doit connaître l'état des choses économiques, pour autant que celui-ci ait une influence sur les prix. Enfin, il ne doit pas y avoir de contrainte extérieure s'exerçant sur l'individu. Si ces hypothèses ne sont pas respectées alors la loi énoncée ci-dessus ne se vérifie pas. Et ceci est souvent le cas dans la réalité, avoue MENGER, précisant que les prix réels diffèrent ainsi des prix dits "économiques". Il poursuit en disant que, même si cette loi exacte n'est pas empirique, elle n'en demeure pas moins vraie et d'une importance certaine pour la compréhension théorique du phénomène des prix.

En résumé, la loi exacte affirme que, sous certaines conditions, une augmentation déterminée des besoins entraînera une augmentation proportionnelle des prix. La loi empirique dit, elle, que l'augmentation des besoins entraînera dans la règle une

${ }^{147}$ MENGER Carl, « Untersuchungen », extraits parus dans Kurt LEUBE (éd.), 1995, p. 92.
${ }^{148}$ Idem, p. 102-103. 
augmentation des prix, mais une augmentation dont la proportion avec l'accroissement des besoins ne peut être établie. En conclusion, l'économie politique exacte, de par sa nature, nous apprend les lois de la viabilité économique ("die Wirtschaftlichkeit») alors que les enseignements de la politique économique empirico-réaliste nous éclairent sur les régularités dans la succession et la coexistence des phénomènes de l'économie ${ }^{149}$.

Un concept qui fut également important chez MENGER comme chez les économistes autrichiens en général est celui de l'individualisme méthodologique. Le fait de considérer l'être humain au centre de la réflexion vient du courant Romantique allemand: "l'individu est unique, différent, inégal, complexe et, enfin, indescriptible », autrement dit «Individuum est ineffabile » ${ }^{150}$. Le caractère unique d'un individu est repris par MENGER, qui affirmait par extension que les éléments d'un ensemble doivent être considérés un à un et qu'un phénomène complexe n'est rien d'autre que la somme des éléments le constituant. Ceci se traduit en économie par l'étude des individus et de leurs choix dans un contexte social donné, et non, comme le préconisait l'Ecole Historique allemande, étudier d'abord la société dans son ensemble. L'individualisme méthodologique se remarque également chez Christian von EHRENFELS qui concentre ses réflexions au niveau de l'individu. Il ira cependant plus loin que Carl MENGER dans le fait d'inclure dans son analyse les facteurs extérieurs pouvant influencer les valorisations de l'individu.

Après avoir évoqué les concepts de base de la pensée Mengerienne, nous pouvons à présent énoncer sa réflexion concernant le phénomène de la valeur. Il considère la valeur indépendamment de toute structure sociale ${ }^{151}$, et comme étant une essence ("Wesen ») de l'activité économique. L'analyse de l'individu évaluant est celle d'un seul être à la fois, et l'application à un groupe ou à une société s'effectue par la somme des analyses individuelles.

La théorie de la valeur de MENGER implique un certain nombre d'hypothèses qu'il convient en premier lieu d'énumérer.

D'abord, comme nous l'avons déjà dit, MENGER s'intéressait avant tout aux essences de l'activité économique. La valeur est une de ces essences et elle se distingue ainsi des prix, considérés comme des "phénomènes de surface ".

Une autre essence importante liée à la valeur est celle de la nature du bien : qu'est ce qui fait qu'un objet mérite le qualificatif de bien ? D'une manière générale, un bien est un objet qui a une utilité, celle de pouvoir satisfaire nos besoins ${ }^{152}$.

\footnotetext{
${ }^{149}$ Idem, p. 104.

${ }^{150}$ ALTER Max, 1990, p.32.

${ }^{151}$ MENGER Carl, 1871, p.80.

152 MENGER Carl, « Grundsătze », extraits dans LEUBE Kurt (éd.), 1995, p.27.
} 
De manière plus précise, un bien suppose :

- un besoin humain,

- une propriété de l'objet qui fait qu'il pourrait satisfaire un ou plusieurs besoins d'un individu,

- que l'individu soit conscient de cette relation,

- et le fait de disposer du bien permet à l'individu de satisfaire son besoin.

Les biens sont des moyens qui nous permettent de satisfaire nos besoins, que ce soit de manière directe ou indirecte. Ainsi ceux qui satisfont directement nos besoins lorsqu'on en dispose, sont dénommés "biens de premier ordre " ou "Güter der ersten Ordnung " par MENGER. Les biens de second ordre sont ceux qui permettent la fabrication de biens de premier ordre et peuvent donc satisfaire un besoin indirectement. MENGER évoque également les biens de troisième, quatrième, et $\mathrm{X}^{\mathrm{ieme}}$ ordres, lesquels se distinguent par le fait qu'ils permettent la production de biens d'ordre inférieur et n'entrent en ligne de compte que comme moyen indirect de satisfaire le besoin d'un individu. Concrètement, un pain est un bien de premier ordre car il permet d'atténuer ou de faire disparaitre la faim d'un individu. La farine ou les levures utilisées pour sa fabrication sont des biens de second ordre, et ainsi de suite. De la relation entre l'individu et l'objet, fondée sur la motivation de la satisfaction des besoins encore une fois, naît le phénomène de la valeur. Pour MENGER, les biens non économiques pour lesquels aucun besoin n'existe, n'ont pas de valeur ${ }^{153}$. Nous verrons dans notre chapitre suivant que cette affirmation peut être contrebalancée par la théorie d'EHRENFELS, pour qui tous les objets peuvent se voir attribuer une certaine valeur, car les biens économiques ne sont pas les seuls objets à posséder une utilité capable d'éveiller l'intérêt de l'individu, tout dépend du type d'utilité qui est accordée à l'objet. Sur ce point on peut d'ores et déjà affirmer qu'EHRENFELS développe et complète la théorie de Carl MENGER qu'il prend par ailleurs comme référence.

La deuxième hypothèse posée par MENGER est celle du caractère unique de la valeur à travers l'agent économique : celui-ci attribue de la valeur à un bien et ce n'est que par cet intermédiaire qu'un bien a de la valeur. La valeur n'est donc pas une propriété intrinsèque d'un bien. Et si la valeur est attribuée par l'agent économique, celle-ci ne peut alors être que subjective (MENGER et les économistes autrichiens en général s'opposèrent au concept classique de l'Homo oeconomicus ${ }^{154}$ ). Cette hypothèse sera également reprise puis développée par Christian von EHRENFELS dans ses réflexions sur la valeur.

\footnotetext{
${ }^{153}$ Idem, p.46.

${ }^{154}$ LEUBE Kurt (éd.), 1995, p.20.
} 
Enfin, sa théorie de la valeur contient une dimension psychologique qui nous intéresse d'autant plus que celle-ci est très présente également chez EHRENFELS. Selon Max ALTER, Friedrich von WIESER est communément considéré, à tort, comme celui qui a introduit une dimension psychologique dans la théorie subjective de la valeur ${ }^{155}$. Et pour cause, lorsque l'on faisait référence à la théorie de la valeur de l'Ecole autrichienne, on faisait référence à celle développée par BÖHM-BAWERK et von WIESER. Cependant ses composants étaient déjà présents dans la théorie de la valeur de MENGER.

En effet, ce dernier décrit le fait d'évaluer ou de donner une valeur à travers un processus psychologique : la valeur est le résultat d'un processus de transfert dans lequel l'individu compare la signification de la satisfaction d'un besoin (c'est à dire sa valeur) avec la capacité d'un bien à satisfaire ce besoin ${ }^{156}$ :

« Es ist somit der Werth die Bedeutung, welche concrete Güter oder Güterquantitäten für uns dadurch erlangen, daß wir in der Befriedigung unserer Bedürfnisse von der Verfügung über dieselben abhängig zu sein uns bewußt sind $»^{157}$.

Autrement dit, la valeur est l'importance qu'un bien possède aux yeux d'un individu, et qui est fonction de sa capacité à satisfaire ses besoins.

Cette définition appelle plusieurs remarques :

- D'abord que le concept de valeur chez MENGER n'a rien à voir avec celui microéconomique des écoles Classique et Néoclassique (bien qu'il fut considéré comme faisant partie de cette dernière) qui ont développé un concept de valeur objective qui est assimilée au prix. Le prix d'un bien, en fait d'un bien économique, est déterminé par l'ensemble des coûts dus à l'utilisation des facteurs de production nécessaires à la fabrication du bien en question. MENGER assimile la valeur d'un bien à sa signification c'est-à-dire son importance - pour l'individu. Si un objet est capable de satisfaire un de mes besoins alors sa possession ou sa consommation aura une importance telle qu'elle en déterminera la valeur. Par ailleurs, la valeur d'un bien de consommation finale est obtenue en effectuant la soustraction entre la satisfaction finale procurée par une certaine quantité de ce bien et la satisfaction procurée par une quantité moindre ou nulle de ce bien.

\footnotetext{
${ }^{155}$ ALTER Max, 1990, p.98.

${ }^{156}$ MENGER Carl, 1871, p.81 et p. 126.

${ }^{157}$ Idem, p.78.
} 
- Ensuite on remarque, encore une fois, le cheminement psychologique qui conduit à la valeur : l'individu a conscience de ses besoins, de la rareté des biens disponibles et il est capable, sous les conditions évoquées, d'établir un jugement sur la valeur d'un bien en regard de sa capacité à contribuer à notre bien être.

- Par ailleurs, la valeur contient une dimension quantitative puisque les besoins peuvent être classés en préférences, elles-mêmes ordonnées de la plus grande à la plus petite.

Enfin, pour terminer cet exposé de la théorie de la valeur de Carl MENGER, il convient de présenter un de ses concepts les plus importants, celui de l'utilité marginale. Pour beaucoup d'économistes, c'est Friedrich von WIESER qui formula explicitement le concept susmentionné. Cependant la paternité de cette notion devrait revenir en fait à Carl MENGER (même s'il n'utilisa pas le terme d'utilité marginale) qui s'en servit dans ses "Grundsätze " pour résoudre le problème du paradoxe de la valeur de l'eau et du diamant ${ }^{158}$ :

L'obtention d'un bien permet de satisfaire un besoin et celui-ci diminue à mesure que la satisfaction augmente jusqu'à un point de saturation pour lequel le besoin à satisfaire est nul. Le supplément de satisfaction apporté par l'acquisition ou la consommation d'une unité supplémentaire d'un bien est la donnée essentielle qui permet à l'agent économique de fixer une valeur de ce bien. De même, la signification de l'abandon de la propriété d'une unité d'un bien ou de la renonciation à sa consommation en déterminera sa valeur.

Le nom d'utilité marginale ou "Grenznutzen" par contre a été trouvé par von WIESER et il apparait pour la première dans son ouvrage d'habilitation à l'Université de Vienne, paru en 1884 sous le titre "Über den Ursprung und die Hauptgesetze des wirtschaftlichen Werthes $"{ }^{159}$. Soulignons le fait que ce concept fut repris et utilisé dès 1893 par EHRENFELS dans ses premiers articles traitant du phénomène de la valeur.

Au fur et à mesure que nous avançons dans nos propos, les mêmes noms d'économistes et d'intellectuels autrichiens reviennent et s'enchevêtrent, ce qui montre une relative grande corrélation dans leurs travaux respectifs et l'intérêt d'évoquer le contexte dans lequel EHRENFELS a évolué et développé sa théorie de la valeur.

\footnotetext{
${ }^{158}$ MENGER Carl, « Grundsătze », in LEUBE Kurt (éd.) 1995, p.51-67 et LITSCHKA Michael, 1999, p.21-22.

${ }^{159}$ p. 127-128.
} 
Pour finir notre tour d'horizon de ses principales sources d'influences intellectuelles, nous allons parler des deux principaux économistes de la seconde génération de l'Ecole autrichienne, Friedrich von WIESER et Eugen von BÖHMBAWERK.

2.5 - Deux développements de la théorie de la valeur de Carl MENGER par Friedrich von WIESER (1851-1926) et Eugen von BÖHM-BAWERK (18511914)

Friedrich von WIESER est né en 1851 dans une famille noble autrichienne. La même année naquit Eugen von BÖHM-BAWERK. Les deux enfants effectueront une bonne partie de leur scolarité ensemble, à savoir dans le très réputé "Schottengymnasium » de Vienne, puis à la Faculté de Droit de l'Université de la même ville ${ }^{160}$. La rencontre entre Carl MENGER et les deux étudiants von WIESER et von BÖHM-BAWERK s'effectua de manière indirecte, à travers l'ouvrage du premier " Grundsätze der Volkswirtschaftslehre » que les étudiants devaient lire afin de préparer un de leurs examens de doctorat. En 1875, une opportunité importante s'offrit aux deux doctorants, celle d'une bourse leur permettant de poursuivre leur formation à l'étranger, en l'occurrence, en Allemagne. C'est à cette occasion qu'une rencontre se produisit avec Carl MENGER qui devait choisir les universités où cette formation serait possible, et les professeurs avec lesquels ils allaient devoir travailler. Le choix de Carl MENGER porta sur les universités de Heidelberg, Jena et Leipzig où Eugen et Friedrich allaient rencontrer KNIES, ROSCHER et HILDEBRAND, trois représentants de l'Ecole Historique allemande. Ces rencontres influencèrent von WIESER et von BÖHM-BAWERK.

Dans le domaine de la valeur, leurs réflexions respectives s'appuient certes sur celles de MENGER, mais elles s'en distinguent également dans leurs développements. D'après Erich STREISSLER, Friedrich von WIESER adopte une perspective que l'on pourrait aujourd'hui qualifier de néo-classique, contenant notamment un modèle mathématisable, bien que ne contenant aucune équation $^{161}$. BÖHM-BAWERK s'intéresse, lui, plutôt au phénomène de la valeur dans le cadre de sa théorie du capital et du taux d'intérêt.

\footnotetext{
${ }^{160}$ Pour une biographie plus détaillée, voir par exemple pour Wieser, WILMES Eugen, « Friedrich von Wieser », 1985 et pour Böhm-Bawerk, TOMO Shigeki, « Eugen von Bठhm-Bawerk.Ein großer ठsterreichischer Nationalokonom zwischen Theorie und Praxis », 1991.

${ }^{161}$ STREISSLER Erich, HAX Herbert (eds), « Friedrich von Wiesers wissenschaftliche Grundperspektive ", 1999, p.29-30, et KURZ Heinz D. et STURN Richard « Wiesers " Ursprung " und die Entwicklung der Mikrookkonomie » in Streissler Erich et Hax Herbert (eds), 1999, p.71.
} 
Commençons par nous intéresser à Friedrich von WIESER : ses réflexions sur la valeur apparaissent essentiellement dans deux de ses ouvrages, "Über den Ursprung und die Hauptgesetze des wirtschaftlichen Werthes " paru en 1884 comme thèse de doctorat, et "Der natürliche Werth" paru en 1889. HansHermann HOPPE et Joseph T. SALERNO expliquent que von WIESER eut une influence assez importante dans l'Ecole autrichienne, notamment à cause de sa longévité dans le domaine de l'enseignement ${ }^{162}$ : après presque vingt ans passés dans l'Université allemande de Prague, il reprit la chaire de Carl MENGER à l'Université de Vienne en 1903, où il enseigna jusqu'en 1922. Pendant ce temps, son beau-frère, Eugen von BÖHM-BAWERK, travaillait plutôt pour le gouvernement, notamment en tant que ministre des finances, et ses activités académiques étaient de ce fait limitées. Durant ces presque deux décennies à Vienne, von WIESER put enseigner à un grand nombre d'étudiants parmi lesquels ceux de la troisième génération de l'Ecole autrichienne comme Joseph A. SCHUMPETER (1883-1950), et même ceux de la quatrième génération comme Friedrich August von HAYEK (1899-1992), Gottfried von HABERLER (19001995), Oskar MORGENSTERN (1902-1977) et Paul ROSENSTEIN-RODAN (1902-1985). A noter que Ludwig von MISES (1881-1973), un des principaux représentants de la troisième génération, fut lui un élève de BÖHM-BAWERK.

Les développements de Friedrich von WIESER en matière de théorie de la valeur par rapport à celle de Carl MENGER sont résumés par Erich STREISSLER ${ }^{163}$, dont nous reprenons ici les propos. La théorie de von WIESER est une théorie subjective, contenant également quelques concepts philosophiques, en particuliers ceux de Franz BRENTANO ${ }^{164}$. Dans son programme de recherches, il voit les acteurs (les agents économiques) au cœur d'une philosophie de la valeur causalogénétique $^{165}$ (celle définie par MENGER). Sa théorie de la valeur contient également des composantes psychologiques.

La valeur d'un objet naît de la relation entre un individu et un objet. La valeur n'est donc pas une propriété intrinsèque de l'objet et le fait qu'elle est le produit d'une relation explique les différences de valorisation d'un même objet par différents individus. La valeur est donc un phénomène subjectif. Von WIESER, comme la plupart des économistes de l'Ecole autrichienne, rejette le concept de la

\footnotetext{
${ }^{162}$ HOPPE Hans-Hermann et SALERNO Joseph T., « Friedrich von Wieser und die moderne Österreichische Schule der Nationalökonomie » in Streissler et Herbert (eds) 1999, p.107-113.

${ }^{163}$ STREISSLER Erich et HAX Herbert (eds), « Friedrich von Wiesers wissentschaflitche Grundperspektive ", 1999, p.48-51.

${ }^{164}$ KURZ Heinz et STURN Richard, in Streissler et Herbert 1999, p.67, et FABIAN R. et SIMONS P.M. «The Second Austrian School of Value Theory » in Grassl et Smith (eds), 1986, p.46 et suivantes.

${ }^{165}$ WIESER, Friedrich von, " Der natürliche Werth », 1889, p.10.
} 
valeur objective. De même, le jugement de valeur - dans le sens de l'attribution d'une valeur à un objet - ou préférence de l'agent économique est dépendant du contexte dans lequel cet agent vit, que ce soit sur le long terme ou sur le court terme ${ }^{166}$. Adoptant par ailleurs une perspective d'analyse dynamique, c'est-à-dire dans laquelle il étudie les variations des préférences dans le temps, l'économiste autrichien ne pouvait faire autrement que de considérer les préférences comme une variable endogène de l'économie.

En fin de compte, il s'agit pour von WIESER non pas de rejeter les théories classiques de la valeur (qui est en fait une théorie des coûts) ou celle de l'offre et de la demande, mais "de les réunir sous un même toit, celui d'une théorie générale de la valeur qui nomme l'origine de la valeur et qui considère toute autre théorie comme cas particulier de la théorie générale ${ }^{167}$. Pour trouver une solution au problème de l'origine du phénomène de la valeur, il se rapproche de la philosophie et pose entre autres la question de la dénomination d'un objet en tant que bien. Il arrive à la conclusion que « la valeur est un phénomène homogène et général ${ }^{168}$. L'utilité est un attribut général des biens. Bien que la valeur dans le fond ne puisse être dérivée des coûts (ou du travail), il convient néanmoins de noter qu'elle correspond souvent à ces derniers ${ }^{169}$, du moins dans le cas des biens reproductibles : le principe de la valeur basée sur les coûts ne serait donc pas un principe indépendant qui ne coïnciderait pas avec l'utilité ${ }^{170}$.

Le fait de vouloir diminuer les coûts ne peut s'expliquer avec la théorie des coûts mais avec celle de la valeur d'usage : l'optimisation des coûts est souhaitée car cela permettrait de libérer des forces ou facteurs de production, lesquels pourraient être utilisés ailleurs à bon escient. La notion de coûts renvoie ainsi à celle de valeur d'usage et à celle de "l'estimation subjective de l'utilité selon l'importance de la satisfaction des besoins " ${ }^{171}$. Ainsi, il n'y aurait pas deux lois de la valeur pour deux types de biens, mais une seule loi, celle du désir.

C'est ici un point important de sa théorie de la valeur pour nous puisque celle d'EHRENFELS accorde également une grande importance au désir comme étant à l'origine du phénomène de la valeur.

\footnotetext{
${ }^{166}$ WIESER Friedrich von, « Über den Ursprung und die Hauptgesetze des wirtschaftlichen Werthes", 1884 p.23, 84-89.

167 " Es geht ihm [von Wieser] nicht um die Wiederlegung einer der konkurrierenden Werttheorien [...] sondern um deren Vereinigung unter dem Dach einer allgemeinen Theorie des Werts, die die Ursache des Werts zutreffend benennt und alle anderen Theorien als Spezialfälle enthălt ", KURZ Heinz D. et STURN Richard, in Streissler et Herbert (eds) 1999, p.88.

${ }^{168}$ WIESER Friedrich von, 1884, p. 97.

169 Idem.

${ }^{170}$ Idem, p.99.

171 " [Der Begriff der Kosten wird auf die] subjective Schătzung des Nutzens nach der Wichtigkeit der Bedurfnisbefriedigungen ", in WIESER Friedrich von, 1884, p.103.
} 
Toujours concernant la détermination de la valeur d'un bien, von WIESER ajoute :

« Les fruits de la production ont une valeur bien avant qu'ils ne soient mûrs, avant qu'ils ne soient fabriqués. La valeur prévue du produit est le motif et sa grandeur la mesure de la production. ${ }^{172}$

Von WIESER opère, à l'image de MENGER, une distinction entre produit fini et produit de consommation intermédiaire. Aussi, «la grandeur de la valeur des biens de production sera déterminée par la plus petite utilité marginale qui aura le droit d'être atteinte dans une branche de l'économie $"{ }^{173}$.

Evoquons enfin le concept de "valeur naturelle " ou " natürlicher Werth " crée par von WIESER et exposé dans son ouvrage du même nom. Il nomme valeur naturelle le type de valeur accordée à un bien dans un contexte de concurrence pure et de même revenu pour chacun ${ }^{174}$. Dans ce cas, la valeur naturelle est égale à la valeur marginale du bien produit. Le modèle de la valeur naturelle se réfère à un état idéal dans lequel on a, à la fois, une efficacité de l'allocation des ressources et une distribution égalitaire des revenus. Ce modèle est un modèle fictif, celui d'un équilibre général à la manière de Léon WALRAS, mais avec une composante que l'on pourrait qualifier de "marxiste", celle d'un revenu égal pour chacun. Son concept de valeur a soulevé un certain nombre de critiques, notamment de la part de Ludwig von MISES, un des élèves de BÖHMBAWERK, reprochant à von WIESER, entre autres, de diffuser une perspective plus proche du néoclassicisme que de l'Ecole autrichienne ${ }^{175}$. C'est pourquoi il convient de considérer également les réflexions de BÖHM-BAWERK dans ce domaine.

Le parcours de l'étudiant Eugen von BÖHM-BAWERK fut similaire dans ses grands traits à celui de son collègue von WIESER, et ce jusqu'en 1879. Il présenta cette même année son travail d'habilitation, cinq ans avant son futur beau-frère. Le thème de ce travail portait un titre somme toute assez général : "Reinigung

\footnotetext{
172 « [...] Früchte der Production haben Werth, bevor sie gereift, bevor sie gezeugt sind. Der vorausgesehene Werth der Producte ist das Motiv, seine Grösse ist das Mass der Production ", in WIESER Friedrich von, 1884, p.140.

173 « Die Grösse des Werthes der Productivgüter wird durch den geringsten Grenznutzen bestimmt, der in irgend einem Productionszweige wirthschaftlicher Weise noch erreicht werden darf $[\ldots] »$, in WIESER Friedrich von, 1884, p. 149.

${ }^{174}$ STREISSLER Erich, « Arma virumque cano - Friedrich von Wieser : der Sănger als Ökonom ", in Streissler et Herbert (eds) 1999, p.49.

${ }^{175}$ HOPPE et SALERNO, «F. von Wieser und die moderne Österreichische Schule der Nationalökonomie ", in Streissler et Herbert (eds) 1999, p.117-134.
} 
einiger Grundbegriffe der Volkswirtschaftslehre ». Le président du jury d'habilitation fut Carl MENGER. Nommé ainsi Professeur d'université, BÖHMBAWERK partit enseigner à l'Université d'Innsbruck où il resta d'avril 1880 à septembre 1889. Il publia plusieurs ouvrages dont les plus importants, par leur portée, furent "Geschichte und Kritik der Kapitalzinstheorien » en 1884 et "Positive Theorie des Kapitals " en 1889, respectivement premier et deuxième tome de "Kapital und Kapitalzins ». A partir de 1889 il retourna à Vienne où il travailla pour le gouvernement, notamment en tant que ministre des finances. De 1904 à 1914, il enseigna à l'Université de Vienne et on compta parmi ses élèves Ludwig von MISES ou encore Otto BAUER (1881-1938).

Le but de BÖHM-BAWERK en matière de théorie de la valeur était de poursuivre le développement des théories de MENGER et de von WIESER. Mais il s'en distingua également par le fait d'adopter des points de vue extérieurs, par exemple, en partant d'une perspective microéconomique pour finir par une perspective macroéconomique. Il considérait par ailleurs autant les théories subjectives de la valeur que les théories objectives : une analyse est consacrée à la notion de valeur subjective autant qu'à celle de la valeur objective dans le livre "Grundzüge der Theorie des wirtschaftlichen Güterwerts " ${ }^{176}$. Une des tâches de la politique économique, ajoute l'économiste autrichien, est d'expliquer les phénomènes tels que la valeur qui apparaissent dans la vie économique quotidienne ${ }^{177}$. Il souligne également qu'il s'intéresse avant tout à la valeur dans son sens économique, c'est-à-dire celle des biens économiques ${ }^{178}$.

Il définit la valeur subjective comme étant la signification ou l'importance qu'un bien ou qu'un ensemble de biens possède pour le bien-être d'un individu ${ }^{179}$. Autrement dit, un bien économique a de la valeur pour moi s'il a la capacité d'améliorer mon bien-être ou de m'épargner un certain mal-être. La valeur objective est la force contributrice d'un objet à atteindre un objectif déterminé ${ }^{180}$. Par exemple, la valeur du charbon est sa contribution à la création de chaleur ou encore, la valeur d'un aliment est son apport nutritif c'est-à-dire sa contribution à atteindre l'objectif « renouvellement de la force de travail ».

La valeur d'un bien économique provient de sa capacité à satisfaire les besoins de l'individu et sa grandeur dépend de sa capacité à améliorer le bien-être de

\footnotetext{
${ }^{176}$ Il s'agit en fait du regroupement de deux passages écrits par BÖHM-BAWERK et paru dans le " Jahrbücher für Nationalökonomie ", volume XIII.

${ }^{177}$ BÖHM-BAWERK Eugen von, " Grundzuge der Theorie des wirtschaftlichen Guterwerts ", 1886, p.3.

${ }^{178}$ Idem, p.3-4.

179 "Werth im subjectiven Sinn ist die Bedeutung, die ein Gut oder ein Guterkomplex fur die Wohlfahrtszwecke eines Subjectes besitzt ", in BÖHM-BAWERK Eugen von, 1886, p.4.

${ }^{180}$ " Werth im objectiven Sinn heißt dagegen die Kraft oder Tuchtigkeit eines Gutes zur Herbeifuhrung irgend eines objectiven Erfolges ", idem, p.4.
} 
l'individu. BÖHM-BAWERK considère que tout ce qui peut améliorer le bienêtre d'un individu a de l'utilité. Il reprend de Carl MENGER et Friedrich von WIESER le concept de marginalité et arrive à la même conclusion suivante que la valeur d'un bien dépend de son utilité marginale ${ }^{181}$. Il affirme en outre que la valeur subjective est mesurable à travers l'intensité du sentiment d'envie ou de non envie que l'individu éprouve à l'égard d'un objet. Ses sentiments, ses domaines d'intérêt, ses souhaits, ses buts, tous ces éléments vont se traduire par des émotions d'envie ou de non envie, lesquelles, lors de la considération d'un objet particulier, vont conduire l'individu à lui attribuer une valeur ${ }^{182}$. Nous verrons que la perspective d'EHRENFELS se rapproche également de celle de BÖHM-BAWERK.

Dans le domaine économique, on est amené à parler de valeur objective dans le cas de la valeur d'échange d'un bien économique. Pour l'économiste Eugen von BÖHM-BAWERK, la valeur d'échange objective n'est rien d'autre que la capacité d'un bien à permettre l'acquisition d'autres biens à travers l'échange ${ }^{183}$. En d'autres termes, il s'agit du pouvoir d'achat. L'échange intervient lorsque l'individu y trouve un intérêt c'est-à-dire quand il en retire un gain. BÖHMBAWERK rejette cependant les notions d'offre et de demande comme régissant le prix des biens sur les marchés, dans le sens qui leur est donné par Adam SMITH ou David RICARDO. Il considère que ces mêmes notions devraient inclure d'une part l'intensité du désir ressenti envers l'objet considéré, et d'autre part la demande ne devrait inclure que la partie qui est effectivement en mesure de pouvoir acquérir cet objet ${ }^{184}$.

La théorie de la valeur de BÖHM-BAWERK est donc ancrée dans ce qui était à l'époque la - encore toute jeune - tradition autrichienne de la nature subjective de la valeur. Dans ses ouvrages postérieurs, il s'est intéressé à la valeur d'usage dans le cadre plus vaste de sa théorie du capital et des taux d'intérêt. L'estimation de la valeur d'un bien s'explique par deux types de raison : des raisons psychologiques et des raisons techniques. Les raisons psychologiques trouvent leurs racines dans l'incertitude du futur et dans le fait que les individus cherchent - ou pas - à satisfaire leurs besoins futurs. On pense alors à la fable de LA FONTAINE : « La Cigale et la Fourmi ». Les raisons techniques sont liées aux méthodes de production, dont les plus efficaces sont celles qui ont la possibilité de disposer des biens de consommation intermédiaires et des moyens de production. Disposer de tous les moyens de production suppose en fait de pouvoir disposer du capital

\footnotetext{
${ }^{181}$ BÖHM-BAWERK Eugen von, 1866, p.29.

${ }^{182}$ Idem, p.46-51.

183 «[...] so ist Tauschwerth im objectiven Sinn nichts, als die Faphigkeit eines Gutes, im Austausch anderer Güter zu verschaffen ", in BÖHM-BAWERK Eugen von, 1886, p.477-478.

${ }^{184}$ Idem, p.525-526.
} 
nécessaire permettant de les acquérir. Celui qui en dispose aura un avantage vis-àvis de ses concurrents car il pourra se lancer dans la production de biens qui auront une valeur supérieure à celle des moyens investis. Autrement dit, l'argent investi aujourd'hui aura une valeur supérieure l'année prochaine; celui qui ne l'est pas perdra, en comparaison, de sa valeur. La différence constitue le taux d'intérêt.

Il s'agit là d'une version simplifiée de la théorie du capital de BÖHM-BAWERK mais elle suffit à montrer, encore une fois, que sa théorie de la valeur y est imbriquée et qu'elle ne fait pas non plus l'objet d'une attention particulière comme chez von WIESER par exemple.

Le contexte dans lequel EHRENFELS élabora sa théorie de la valeur fut celui des développements intellectuels dans les domaines économiques et philosophiques à la fin du XIX ${ }^{\text {ème }}$ siècle. Ces développements se sont construits autour du paradigme de l'Aristotélisme autrichien, c'est-à-dire l'interprétation des thèses d'Aristote, prônée en réaction aux changements provoqués par la diffusion des thèses Kantiennes dans l'Allemagne voisine. Parmi les personnalités qui ont eu une influence sur les réflexions d'EHRENFELS, nous avons cité les philosophes BRENTANO et MEINONG, ainsi que les économistes MENGER, von WIESER et, dans une moindre mesure, von BÖHM-BAWERK.

Nous verrons dans notre chapitre suivant que la théorie d'EHRENFELS se situe dans les mêmes courants intellectuels. Elle sous-entend une soumission à l'Aristotélisme autrichien, elle adopte le concept d'une valeur subjective qui est analysée sous l'angle individualiste et avec l'aide de la psychologie. EHRENFELS n'hésita pas non plus à reprendre et à développer à sa manière le concept de marginalité. C'est avant tout dans sa définition même de la valeur qu'il est proche de Carl MENGER en l'assimilant à l'importance d'un objet pour l'individu. 
Youri Tacoun - 978-3-631-75426-9

Downloaded from PubFactory at 01/11/2019 05:04:02AM

via free access 


\section{Chapitre second}

\section{La theorie de la valeur de Christian von EHRENFELS}


Youri Tacoun - 978-3-631-75426-9

Downloaded from PubFactory at 01/11/2019 05:04:02AM

via free access 
La théorie de la valeur du philosophe autrichien Christian von EHRENFELS représente le cœur de notre travail qui va être consacré à la découverte ou plus précisément, à la redécouverte de cette théorie ainsi qu'à son étude.

Les écrits de Christian von EHRENFELS sur la valeur ont été rassemblés dans un livre édité par Reinhard FABIAN en 1982. Ce livre comprend une première série d'articles parue en 1893/1894, puis une deuxième série parue en 1896 et enfin son livre consacré plus spécifiquement à la valeur et à l'éthique, "System der Werttheorie, Grundzüge einer Ethik », paru en 1898. Dans ce chapitre, chaque référence aux écrits d'EHRENFELS se rapportera à l'ouvrage cité précédemment et se fera entre parenthèses (sauf dans le cas des citations), afin d'en faciliter la lecture.

Les premiers articles sur la valeur sont ceux dans lesquels EHRENFELS a commencé à réfléchir sur le problème de la valeur. Comme nous l'avons déjà mentionné, son point de départ est la théorie de la valeur de l'Ecole Autrichienne, c'est-à-dire en fait celle qui fut élaborée par MENGER et développée par von WIESER. EHRENFELS émet des critiques vis-à-vis de cette théorie, soulignant en particulier ses manques. Il propose de pallier à ces derniers par une étude personnelle sur la base de ce qui a déjà été produit. Pour cela, il commence par nous donner quelques définitions qui nous permettent de mieux cerner le concept de la valeur et ses différentes facettes. Ensuite, il se propose d'analyser les causes du changement de la valeur, c'est-à-dire les raisons pour lesquelles les personnes sont amenées à changer la valeur attribuée à un objet. Enfin, il aborde le problème des valeurs éthiques ainsi que de leur évolution.

L'article paru en $1896^{1}$ est en fait une réponse à Alexius MEINONG, et fait suite à un de ses propres articles, paru un an plus tôt ${ }^{2}$. Selon EHRENFELS, MEINONG a, dans cet article, modifié sa définition de la valeur pour se rapprocher de la sienne. EHRENFELS va en conséquence revoir également sa conception de la valeur en montrant la relation entre la valeur et le désir, puis en développant, à partir de la formule de MEINONG, une équation permettant de calculer la grandeur de la valeur. Enfin, le désir et son origine sont évoqués plus en détail à la fin de l'article.

\footnotetext{
' EHRENFELS, 1982, p.167-180.

${ }^{2}$ EHRENFELS, « Über Werthaltung und Wert », 1895, p.325-346.
} 
Le "System der Werttheorie » est l'ouvrage le plus important d'EHRENFELS sur la valeur. Il reprend dans un premier temps les concepts importants autour de la valeur, puis s'intéresse plus précisément aux mouvements de valeur avant d'entrer en détail dans la psychologie du désir. Dans le second volume de son «System der Werttheorie ", le philosophe s'intéresse plus particulièrement aux valeurs éthiques et à la place importante de l'éthique dans le processus de valorisation.

Pour notre part, la présentation de la théorie de la valeur de Christian von EHRENFELS se fera sous la forme d'une synthèse de l'ensemble des articles cités. Le but de cette présentation n'est bien sûr pas de répéter dans le détail la théorie en question mais d'en relever les points les plus intéressants et les plus pertinents pour expliquer la formation de la valeur et son processus d'attribution, tout en accordant une attention toute particulière aux valeurs dans le domaine économique.

Pour ce faire, nous allons commencer par définir la valeur et les concepts qui en dérivent et qui nous aident à mieux la comprendre. La valeur n'est pas immédiatement définie par EHRENFELS, celui-ci préférant développer les concepts autour de la valeur, comme par exemple celui de la satisfaction des besoins ou de l'utilité, avant de s'attaquer à définition de la valeur proprement dite. Il s'attache aussi à distinguer les différents types de valeurs (valeur intrinsèque, valeur d'effet, valeur intermédiaire, valeur non intermédiaire, valeur réelle ou supposée, etc...). EHRENFELS s'intéresse en outre à une facette du concept qui a été développée en philosophie mais qui a été oubliée par l'économie, celle de la non-valeur. Par ailleurs, comme la valeur est ici subjective, elle ne peut logiquement être statique. Au contraire, le philosophe autrichien veut démontrer pourquoi les valeurs apparaissent, évoluent et disparaissent. Les causes de leurs changements sont à trouver non pas du côté de l'objet, mais du côté de l'individu valorisant, et plus précisément au niveau de son état émotionnel. C'est pour cette raison qu'il entreprend une analyse du phénomène du désir. La notion de désir est traitée par EHRENFELS sous l'angle de la psychologie, en tant qu'émotion résultant de la relation établie entre un individu et un objet.

L'éthique et les valeurs sont étroitement liées. En fait, la philosophie et les philosophes, lorsqu'ils ont commencé à s'intéresser au phénomène de la valeur, l'ont reliée à l'éthique. Un problème intemporel qui fut traité déjà du temps de l'Antiquité romaine et grecque. Le point de départ de toute théorie de la valeur était la question de l'existence du "bien supérieur" ou "das höchste Gut». Celui-ci a été remplacé avec le temps par le besoin et la satisfaction du besoin. EHRENFELS dénonce la "surévaluation des enseignements économiques en 
matière de valeur » (p.211), aussi bien chez les Socio-démocrates ${ }^{3}$ que chez les Classiques. Pour lui, les valeurs économiques ne sont en fait qu'un cas particulier de valeur, une catégorie particulière des valeurs humaines. Une place plus importante doit être accordée aux valorisations éthiques, lesquelles ne sont d'ailleurs pas sans rapport avec les valeurs économiques. En effet, dans les deux cas on peut être amené à juger un comportement, par exemple un comportement de consommateur en conformité ou pas avec la morale. EHRENFELS accorde une place particulière à l'éthique et à la morale mais considère encore une fois que les valeurs dérivées ne sont qu'un cas particulier de valeurs et que leur détermination obéit à des mécanismes généraux.

${ }^{3}$ Par « Sociaux-démocrates », EHRENFELS fait probablement référence aux courants socialistes en plein développement dans l'Empire allemand de la fin du XIX ${ }^{\text {eme }}$ siècle. 


\section{I - Approche et définition du phénomène de la valeur}

Le premier aspect de la théorie de la valeur du philosophe autrichien concerne d'une part les hypothèses fondamentales de sa théorie, et d'autre part la définition elle-même de la valeur.

\section{1 - Généralités}

Dans cette première partie, nous allons énoncer les hypothèses et définitions nécessaires à la compréhension de la théorie de la valeur d'EHRENFELS.

En premier lieu, il convient de rappeler que le dessein d'EHRENFELS est de développer une théorie générale - " eine Allgemeine Werttheorie » - c'est à dire une théorie qui peut être utilisée pour expliquer la valeur de n'importe quel objet. Le mot "objet" se réfère ici à tous les éléments matériels ou immatériels qui peuvent être inclus dans le processus d'attribution de la valeur c'est-à-dire dans la valorisation ou jugement de valeur. Un objet peut donc être un bien de consommation, un objet matériel que l'on veut posséder, un sentiment, une relation (amicale ou amoureuse), un talent, une personnalité, un trait de caractère, une règle, une norme ou encore un comportement. Exclus du champ des possibilités les êtres vivants, les Hommes, les animaux et les plantes ne sont pas évoqués en tant que tels par EHRENFELS. Il est possible de valoriser un trait de caractère en particulier mais pas une personne en général. Même lorsqu'on affirme qu'une personne est un être de grande valeur, ce n'est pas la personne elle-même qui est valorisée mais un de ses traits de caractère, un des traits de sa personnalité. Le fait de vouloir développer une théorie générale de la valeur est une ambition partagée par toute une génération de philosophes autrichiens de la fin du XIX ${ }^{\text {eme }}$ siècle $^{4}$. Aussi vouloir expliquer la détermination et l'évolution des valeurs par le biais d'une théorie générale implique que les valeurs économiques et les valeurs éthiques par exemple, ne sont que des cas particuliers de valeur.

La première remarque qu'appelle la théorie du philosophe autrichien concerne la possibilité d'intégrer, voir de fondre les valeurs économiques dans une théorie générale alors que dans la période durant laquelle il a vécu, les économistes se sont plutôt attachés à vouloir faire des sciences économiques une discipline à part entière avec ses propres concepts. Cette séparation qui s'est faite entre l'économie et l'éthique - à laquelle elle était auparavant rattachée - est-elle définitive ?

\footnotetext{
${ }^{4}$ GRASSL Wolfgang, in EHRENFELS 1982, Avant-Propos, p.3.
} 
Lorsque l'on considère le nombre important d'ouvrages récents consacré aux relations existantes entre l'économie et l'éthique, il semble que la réponse à cette question soit plutôt négative ${ }^{5}$. L'étude d'une théorie générale de la valeur n'apparaît donc pas comme caduque dans le contexte actuel, ni même comme déplacée dans le cadre des sciences économiques.

Deuxièmement, la théorie d'EHRENFELS se veut une théorie de la valeur subjective. De ce fait, EHRENFELS reste fidèle à un des principes de l'Ecole Autrichienne en la matière comme on l'a déjà vu dans le chapitre précédent. Il reprend l'idée de Carl MENGER lorsqu'il affirme que les valeurs ne sont pas une propriété intrinsèque d'un objet donné, mais qu'elles sont attribuées par un individu à cet objet. La valeur naît et existe à travers la relation qui s'établit entre un individu et un objet. De cette relation provient également le caractère subjectif de la valeur au sens propre de l'adjectif, c'est-à-dire " qui relève du sujet défini comme être pensant $»^{6}$. EHRENFELS s'oppose ainsi à la conception classique et néoclassique de la valeur objective, fixée par les coûts de production. En effet, même s'il ne le précise pas, on comprend à travers ses propos qu'il ne considère pas l'individu comme un être rationnel privilégiant dans toutes les situations ses intérêts personnels. Cela se voit notamment à travers la place qu'il accorde aux émotions, au regard d'autrui et à la considération sociale dans la détermination de la valeur.

La valeur, comme le rappelle EHRENFELS, est un sujet qui a préoccupé la philosophie et le monde intellectuel dès l'Antiquité. Il souligne néanmoins que la remise en cause des principes métaphysiques et de l'existence de Dieu, principalement durant le siècle des Lumières, a propulsé les courants rationalistes sur les devants de la scène. Le problème de la valeur fut alors résolu par les résultats objectifs d'une démonstration logique, laquelle fait intervenir des connaissances acquises à travers la recherche scientifique. Or ce changement a également entraîné une remise en cause des valeurs établies, ce qui pour EHRENFELS fut un des plus grands problèmes de la société dans laquelle il vécu ${ }^{7}$. Cette société fut marquée par de grands bouleversements en Europe et ceux-ci furent accompagnés de changements sociaux, influençant par la même les valeurs traditionnelles des sociétés ${ }^{8}$. Ces changements ont probablement conduit le philosophe autrichien à penser que les valeurs ne sont pas statiques mais bien dynamiques.

\footnotetext{
${ }^{5}$ Voir également à ce sujet GROENEWEGEN Peter (éd.), „Economics and Ethics“, 1996, Introduction, p.1 et MEIER-SEETHALER Carola, " Gefuhle und Urteilskraft ", 1998, p.361-388.

${ }^{6}$ Le Petit Larousse 2004, p.969.

${ }^{7}$ Voir p.21-22 de ce travail.

${ }^{8}$ Voir par exemple PLETICHA Heinrich (éd.), « Weltgeschichte », Tome 10, 1996, p.201-232.
} 
Troisièmement, EHRENFELS prend comme point de départ de sa réflexion la théorie de la valeur de Carl MENGER et de Friedrich von WIESER. Il les mentionne d'abord comme " eine Gruppe von Nationalökonomen" (p.23) qui se seraient intéressés au phénomène de la valeur et auraient ainsi établi une relation "définitive" ou "wohlendgültig" entre celle-ci et l'utilité d'un objet. En écrivant "définitive" EHRENFELS veut souligner le caractère fixé de cette relation et sous-entend également que les économistes de ce courant n'ont pas envisagé d'autres possibilités (p.23-25). Il est clair pour lui que, s'il existe bien une relation entre valeur et utilité, celle-ci n'est en aucun cas exclusive, au sens où elle serait la seule qui existerait ${ }^{9}$. C'est la première critique qu'il adresse ainsi à la théorie de l'Ecole Autrichienne, celle de ne pas avoir exploré d'autres voies que celle que nous venons d'évoquer, et qui lui permet également de commencer ses réflexions en la matière. Autre reproche effectué à cette école de pensée, est celui d'avoir négligé l'étude de l'évolution des valeurs dans le temps et plus précisément, des lois régissant les changements de valeur. S'inspirant du Darwinisme, qui imprègne en plusieurs points sa théorie, EHRENFELS attribue aux valeurs la faculté d'évoluer et de s'adapter à leur environnement. Ce faisant, il inclut une dimension temporelle et sociologique dans sa théorie.

Par rapport aux théories de MENGER et de von WIESER, il s'agit là d'une innovation puisque ni le premier, ni le second ne considèrent l'évolution de la valeur sous cette perspective. Pour MENGER, il s'agit d'établir la relation entre les objets désirés et la volonté des individus de satisfaire leurs besoins. De cette relation naît la valeur, et plus spécifiquement la valeur d'usage. Dans le cas de von WIESER, le concept de valeur naturelle, que nous avons évoqué dans notre chapitre précédent, est un concept théorique dans un modèle purement théorique. Comme l'explique l'anthropologue Diane AUSTIN-BROOS le marché est devenu un élément intrinsèque des sociétés occidentales, qui produit sa propre culture, la culture du marché, laquelle a des relations conflictuelles, ou pas, avec la culture des individus ${ }^{10}$. Ces relations évoluent avec le temps et l'environnement social. C'est précisément cette dimension qui est contenue dans la théorie d'EHRENFELS et qui constitue donc un apport intéressant en matière d'explication du phénomène de la valeur, qu'elle soit économique ou pas.

\footnotetext{
9 " Die Menger- und Wiesersche Werttheorie, welche ich hier im Auge habe, keineswegs in einwandfreien allgemeinen Begriffsbestimmungen, sondern in der Aufdeckung einer realer Beziehung, des größenverhăltnisses zwischen Wert und Nutzen [...] ihren wesentlichen Beitrag zur Förderung des Wertproblems geliefert hat ", in EHRENFELS, 1982, p.24.

${ }^{10}$ AUSTIN-BROOS Diane, « Morality and the culture of the market », in GROENEWEGEN Peter, 1996, p.173-183.
} 
Enfin remarquons que Christian von EHRENFELS utilise et développe le concept d'utilité marginale ou "Grenznutzen » dans sa théorie. Il montre la volonté de développer ce concept et de l'appliquer à d'autres domaines de la valeur, en particulier à celui des valeurs éthiques.

Dans son exposé, EHRENFELS commence par souligner le fait que la relation valeur-utilité n'est pas simple. Pour illustrer son affirmation, il utilise l'exemple ô combien célèbre du paradoxe de la valeur de l'eau et du diamant d'Adam SMITH: comment expliquer que certains objets comme l'eau, de grande utilité pour nous puisque nous ne pourrions pas vivre sans eux, ne se voient accorder qu'une petite valeur? De la même manière, comment expliquer que les diamants ou l'or, peu utiles, aient une aussi grande valeur? Ce problème qui avait déjà été posé par Adam SMITH ${ }^{11}$ trouve une solution à travers le concept d'utilité marginale de Carl MENGER et de l'Ecole Autrichienne: la dernière unité acquise ou consommée d'un objet est importante dans la détermination de la valeur. Si le stock de cet objet est présent dans la nature en grande quantité alors son accès sera d'autant plus facile et sa consommation aisée. Sa valeur sera en conséquence relativement faible. Cependant, lorsque le stock d'un bien est disponible en quantité limitée, son accès ou sa consommation seront logiquement plus difficiles et la dernière unité acquise de cet objet aura une valeur relativement élevée. Pour reprendre l'exemple d'EHRENFELS, l'air que nous respirons est présent en grande quantité dans notre environnement et il est facilement accessible. Par contre, l'air pur des montagnes n'est pas disponible en aussi grande quantité et est plus difficilement accessible, d'où la valeur plus grande qui lui sera accordée.

EHRENFELS prend ensuite l'exemple d'un père de famille ayant le choix entre un pain et une barrique de poivre pour nourrir sa famille. Notons au passage que le rôle de père de famille est mis en avant, conformément à la vision traditionaliste d'EHRENFELS, et que le poivre était au XIX ${ }^{\mathrm{e} m e}$ une épice bien moins courante et plus chère qu'elle ne l'est aujourd'hui. S'il a le choix entre les deux, lequel va-t-il choisir, ou à quel objet va-t-il accorder la plus grande valeur ? Ce choix se fera en fonction de l'utilité de chacun d'entre eux, mais aussi en fonction des circonstances: si la famille a faim, le père choisira de satisfaire ce besoin primaire, alors que si la faim n'est pas encore présente, il prendra la barrique de poivre et la revendra pour acheter du pain plus tard. Cet exemple illustre une situation de famille réaliste, et cet attachement à la réalité imprègne la plupart des exemples donnés par EHRENFELS. Donc, selon le besoin à satisfaire, l'utilité des objets sera différente et l'acte de valorisation s'effectue toujours en référence aux

\footnotetext{
"Voir à ce propos KOCH Claudia, 1995, p.257-258.
} 
objets valorisés et à l'action qui suit ${ }^{12}$. Ce qui est important dans l'acte de valorisation n'est pas l'utilité totale que nous confère l'objet dont il est question dans cet acte de valorisation, mais plutôt l'utilité inhérente à l'acte qui suit. Plus le but de cet acte correspond à un besoin important (le besoin ici est celui de manger, qui est un besoin vital, et le but de l'action est d'apaiser ce besoin vital), plus la valeur de cet acte sera grande. Cependant, et bien qu'EHRENFELS ne nie pas l'importance du concept d'utilité marginale, il va par la suite démontrer que la détermination de la valeur obéit à des mécanismes plus complexes.

EHRENFELS résume son interprétation de la théorie de la valeur de l'Ecole Autrichienne grâce au raisonnement suivant :

- D'abord les besoins des hommes pour les objets sont limités tout comme la quantité de biens disponibles (hypothèse de rareté des biens).

- A travers la consommation d'un bien déterminé, le besoin satisfait par la consommation d'une première unité de bien est d'importance supérieure à celui qui sera satisfait par la deuxième unité de bien.

- Un même objet peut servir à la satisfaction de plusieurs besoins de différentes importances, par exemple un pain peut servir à assouvir la faim comme à nourrir les pigeons dans un parc.

- Le stock d'un même bien peut présenter différents degrés d'utilité selon le besoin à satisfaire. Par exemple le pain peut être utile pour atténuer la faim ou pour nourrir les oiseaux.

- Lorsqu'un stock de biens de même utilité ne suffit pas à satisfaire tous les besoins, l'individu va satisfaire en premier les besoins les plus urgents. Par exemple, avec un bout de pain on va d'abord chercher à apaiser sa faim avant de nourrir les oiseaux, ce qu'EHRENFELS appelle le "Vernunft im Güterverbrauch» ou encore «la raison dans la consommation de biens $"$.

- Celui qui possède un stock de biens de même utilité et qui en donne une unité, ne perd à travers cet acte qu'une toute petite partie de l'utilité totale que représente le stock entier c'est à dire qu'il perd l'équivalent de l'utilité marginale. De la même manière, s'il ajoute un bien à son stock, il n'augmentera que marginalement l'utilité de son stock.

- Il découle de ces conditions l'habitude d'évaluation des biens dans toutes les actions qui provoquent un flux de biens (production, vente, achat, consommation).

\footnotetext{
${ }^{12}$ « Die Akte der Wertschătzung von Gegenstände werden immer in bezug auf irgendwelche jene Gegenstände und ihr Verhältnis zu uns betreffen Handlungen vollzogen ", in EHRENFELS, 1982, p.25-26.
} 
A ces hypothèses, EHRENFELS ajoute que "ce n'est pas parce qu'on a utilisé beaucoup de travail pour la fabrication d'un bien que celui-ci aura de la valeur. Au contraire, on va consacrer beaucoup de travail à la fabrication d'un bien parce que l'on attend de lui une certaine utilité marginale ${ }^{13}$. Cette vision des choses rejoint celle de Carl MENGER :

"Si les biens d'ordre supérieur sont utilisés pour produire des biens d'ordre inférieur, alors la valeur des biens d'ordre supérieur doit être calculée par rapport à la valeur attendue du bien d'ordre inférieur qu'ils servent à produire. $\|^{14}$

Ayant maintenant exposé les hypothèses de la théorie d'EHRENFELS ainsi que son point de départ, notre prochain point concernera les développements à proprement parler du philosophe. Pour cela, il va dans un premier définir ou redéfinir la valeur en tant que relation entre un sujet valorisant et un objet valorisé, ainsi que les différentes catégories de valeur et les notions qui lui sont associées.

\section{2 - Les origines de la valeur : la relation sujet-objet}

La valeur, comme nous l'avons déjà évoqué, n'est pas une propriété d'un objet, elle n'est pas contenue par l'objet mais provient de la relation établie entre l'individu et l'objet (p.218-219). Cette relation est de nature psychologique et suppose donc un lien émotionnel entre l'individu et l'objet : le fait de considérer un objet éveille chez l'individu un sentiment que l'on qualifiera de désir. Les différentes écoles de pensée en économie partent en général de l'hypothèse que le désir est dicté par la satisfaction des besoins. Autrement dit, nous désirons parce que nous avons des besoins à satisfaire. Cette hypothèse, selon EHRENFELS, est insuffisante pour expliquer réellement l'origine du désir (p.219-220).

Dans un premier temps, nous allons nous intéresser au désir et à la relation de désir chez EHRENFELS, puis à la relation entre l'individu et l'objet. Nous verrons comment il détermine les origines du désir et les facteurs qui favorisent l'apparition d'un désir chez l'individu. Enfin, nous étudierons les limites de l'hypothèse de satisfaction des besoins comme cause du désir

\footnotetext{
${ }^{13}$ " Nicht deswegen ist ein Gut wertvoll, weil wir auf dessen Erzeugung Arbeit verwendet haben sondern umgekehrt : nur deswegen verwenden wir Arbeit auf die Erzeugung eines Gegenstandes, weil wir von ihm « Grenznutzen » erwarten, d.h. weil wir ihm einen präsumtiven Wert zusprechen ", in EHRENFELS, 1982, p.27 (note en bas de page).

14 "If goods of higher order are used to produce goods of lower order, then the value of the goods of higher order has to be imputed from the expected value of the good of lower order they are used to produce ", in ALTER Max, 1990, p.167, citation reprise de MENGER Carl, 1871, p.123-126.
} 
Les sentiments déclenchés par la relation individu-objet décrivent tous les états psychiques qui portent en eux la caractéristique de l'envie ou de la désolation, peu importe leurs qualificatifs :

"Unter Gefühlen verstehen wir alle psychischen Zustände, welche das Merkmal des Lust- oder Leidvollen in sich tragen, gleichgültig, ob sie des näheren als Freude, Annehmlichkeit, Wohlbehagen, Lust, oder als Schmerz, Qual, Pein, Unbehagen, Unlust bezeichnet werden. ${ }^{15}$

Tous les sentiments qui se situent au dessus du point d'indifférence de l'individu (point pour lequel l'individu n'éprouve ni joie, ni peine) entrent dans la catégorie " envie» ou "Lust». Ceux qui par contre se situent en dessous de ce point d'indifférence entrent dans la catégorie " non-envie » ou " Unlust ». Les intensités des différents sentiments de part et d'autre du point d'indifférence peuvent être comparées dans la mesure où les grandeurs des sentiments d'envie sont positives et celles des sentiments de non envie sont négatives. Les grandeurs évoquées ici ne sont bien sûr pas exactes étant donné le caractère subjectif et relatif des choses auquel on est confronté dans le domaine psychologique, d'autant qu'EHRENFELS n'évoque aucune unité de mesure. Mais on peut, encore une fois, les comparer en ce sens où on peut affirmer d'un sentiment d'envie qu'il est supérieur à un sentiment de non envie ${ }^{16}$.

Par désir, EHRENFELS entend « le fait de souhaiter, de vouloir, d'aspirer, etc... tous les actes psychiques donc qui ont comme point commun d'être dirigés sur un but, un dessein précis, celui de l'existence ou de l'apparition d'un objet, celui de la justification d'une manière d'agir (processus), celui de la non-existence ou de l'élimination d'un objet, ou encore celui de l'abandon d'une manière d'agir ${ }^{17}$. EHRENFELS distingue par ailleurs le désir positif - ou désir tout simplement du désir négatif ou rejet.

Une discussion a eu lieu entre EHRENFELS et MEINONG à la fin du XIX ${ }^{\text {ème }}$ concernant le fait que l'individu puisse désirer aussi des objets qui n'existent pas $^{18}$. Pour MEINONG, nous désirons les objets qui n'existent pas ou dont on

\footnotetext{
${ }^{15}$ EHRENFELS, 1982, p.220-221.

${ }^{16}$ Cette classification ordinale est fidèle dans son principe à celle, de la nature des préférences chez Menger.

17 " Unter dem gemeinsamen Begriffe des Begehrens umfassen wir alles Wünschen, Streben und Wollen, - psychische Akte also, welchen es gemeinsam ist, auf ein bestimmtes Ziel oder einen Zweck gerichtet zu sein, nämlich entweder auf die Existenz oder die Entstehung eines Dinges, das Eintreten oder Zutreffen eines Vorganges, oder aber auf die Nichtexistenz oder Vernichtung eines Dinges, das Hintanbleiben oder Aufhören eines Vorganges » in EHRENFELS, 1982, p.221.

${ }^{18}$ Voir article d'EHRENFELS " Vom Wertdefinition zum Motivationsgesetz " paru en 1896 (in EHRENFELS, 1982, p.167-179) en réponse à celui de MEINONG, «Über Werthaltung und
} 
doute de l'existence. EHRENFELS acquiesce le point de vue de MEINONG et affirme en outre qu'un objet, dont on est convaincu de l'existence, ne peut éveiller notre désir. Cette vision est néanmoins perfectible, en particulier dans le domaine des biens économiques: les individus désirent non seulement les biens qui n'existent pas encore (mais dont ils savent que ces biens vont un jour exister), mais également les biens qui existent. Lorsque nous nous trouvons face à un objet réel et que nous le considérons, un sentiment d'envie peut apparaître. Cette affirmation suppose-t-elle que nous ne possédions pas l'objet en question ? Par expérience, on sait que les individus valorisent tout autant ce qu'ils possèdent que ce qu'ils ne possèdent pas. Par contre, ils désirent ce qu'ils ne possèdent pas.

Pour cette raison, nous nous permettrons de modifier l'affirmation d'EHRENFELS de la manière suivante : l'individu désire les objets qui n'existent pas ou n'existent plus, mais dont il est convaincu de l'existence future ou passée, ainsi que les objets qui existent mais qu'il ne possède pas.

Lorsque l'individu perçoit un objet (peu importe que cet objet soit imaginé ou existe réellement), la relation qui s'établit alors découle des effets de l'objet sur l'état psychologique, et plus exactement, sur l'état émotionnel de l'individu. Un sentiment de désir ou d'envie peut naître à l'égard de cet objet. La question que se pose alors EHRENFELS est celle de l'apparition du désir : quels sont les facteurs qui provoquent l'apparition du désir?

Il y répond de la manière suivante : nous allons attribuer de la valeur à un objet réel ou imaginé, selon que la probabilité de sa réalisation - ou de sa possession renforce ou diminue son sentiment de bien être par rapport à la situation où cela ne se produirait pas ${ }^{19}$. Autrement dit, la perception de l'objet doit déclencher le sentiment d'un bien être supérieur (respectivement inférieur) chez l'individu pour qu'il ait envie (ou pas) de cet objet, c'est-à-dire qu'il le désire (ou pas).

Les éléments déclencheurs du désir sont les conceptualisations de l'objet, dont la précision est déterminante dans l'attribution de la valeur. Pour EHRENFELS, la valeur attribuée variera en grandeur selon les capacités de l'individu à s'imaginer l'objet existant et à en avoir une idée précise et concrète. Ce processus psychologique peut survenir alors même que l'objet n'existe pas : l'individu peut simuler l'existence de l'objet et comparer ses états émotionnels dans le cas où l'objet

Wert " paru en 1895. L'idée de ne désirer que des objets qui n'existent pas est reprise de Franz BRENTANO.

${ }_{19}$ "Wert (oder Unwert) werden wir hiernach einem wirklichen oder bloß gedachten Gegenstand insofern zuschreiben, als bei einem bestimmten Subjekte die nach Tunlichkeit anschauliche und lebhafte Vorstellung seiner Verwirklichung gegenuber derjenigen seiner Nichtverwirklichung Glücksförderung (oder Glücksminderung) zu bewirken vermag », in EHRENFELS, 1982, p.176. 
existerait et dans le cas où il n'existerait pas. Le jugement de valeur sur un objet provoque chez l'individu la conceptualisation de celui-ci.

De manière schématique, on peut résumer le processus de la valorisation de la façon suivante :

Perception de l'objet $\rightarrow>$ conceptualisation dans la conscience (plus ou moins précise) -> déclenchement ou non d'un sentiment d'envie ou de non envie lié à une augmentation ou à une diminution du bien être $->$ désir $->$ jugement de valeur $\rightarrow$ attribution de la valeur.

Dans ce processus de détermination de la valeur la notion de satisfaction des besoins n'apparaît pas, et c'est là un des intérêts de cette théorie : elle permet d'expliquer le fait qu'un individu accorde de la valeur à un objet dans toutes les situations possibles. La satisfaction des besoins est traitée comme un cas particulier dans lequel l'individu désire un objet auquel il accorde une valeur. De plus, sa référence constante à la psychologie et à l'état d'esprit de l'individu la rapproche encore plus de la réalité.

Ce processus psychologique est conditionné par certaines émotions (les sentiments d'envie ou de non envie) éprouvées envers l'objet considéré. Or la philosophie du XIX ${ }^{\text {ème }}$ siècle, fortement influencée par les apports d'Immanuel KANT, reconnaît en la raison (" die Vernunft ») une instance supérieure qui aurait le dernier mot en matière de décision de l'individu. Autrement dit, le désir est certes déterminé par les sentiments de l'individu mais sa raison serait plus forte et déciderait en fin de compte de la décision à prendre ou du comportement à adopter selon les situations. EHRENFELS critique cette position: il est impossible que la raison influence la volonté sans l'intermédiaire des émotions. Celui qui ne ressent rien ne peut désirer, le désir implique des émotions et la raison intervient a posteriori. EHRENFELS pense que KANT se trompe lorsqu'il affirme que la raison est l'origine de toute action, de tout agissement. Pour le philosophe autrichien, les sentiments interviennent antérieurement à la raison et peuvent garder le dessus par rapport à cette dernière. Par exemple, la mort provoque en nous un sentiment de non envie, de rejet et c'est ce sentiment de non envie, lié à la raison, qui va nous inciter à agir dans sens de la survie. La raison accompagne donc les dispositions émotionnelles de l'individu.

La raison reste aujourd'hui encore opposée aux émotions et aux sentiments ${ }^{20}$, en particulier dans les sciences économiques. Les sentiments sont associés aux passions et supposent une réaction spontanée, non raisonnée. La raison est, elle,

\footnotetext{
${ }^{20}$ SOLOMON Robert C., « Emotions, Thoughts and Feelings: What is a Cognitive Theory of the Emotions and Does it Neglect Affectivity? ", in Anthony Hatzimoysis (éd.), 2003, p.1 et 3-4.
} 
l'ingrédient essentiel du comportement rationnel. L'individu raisonnable est une des hypothèses fondamentales du comportement des agents économiques. La raison semble donc l'emporter sur l'émotion dans le discours moderne; elle l'a même évincée dans les modèles économiques. Cependant, cette vision des choses est critiquée, voire de plus en plus rejetée ${ }^{21}:$ l'importance des émotions dans la prise de décision ne peut être négligée ${ }^{22}$, de même que les émotions ne conduisent pas toujours l'individu à se conduire de manière irrationnelle. Pour Aaron BENZE'EV, les émotions sont rationnelles si on considère qu'elles ont un mode de fonctionnement différent de celui du raisonnement intellectuel ${ }^{23}$. Pour cela, raison et rationalité doivent voir leur sens évoluer.

La raison et les émotions sont tous deux déterminants dans la réaction d'un individu dans une situation donnée, mais la raison n'a pas toujours le dernier mot, nous dit Patricia GREENSPAN, par exemple dans le cas où une réaction rapide est requise $^{24}$. La peur peut ainsi conduire l'individu à avoir une réaction lui évitant des conséquences négatives. L'argument d'EHRENFELS s'en trouve ainsi conforté.

Du côté des économistes classiques et néoclassiques, la raison se manifeste également a posteriori chez l'agent économique rationnel ou homo oeconomicus. Si cela était effectivement le cas alors la théorie ne pourrait pas expliquer les achats dits "coup de cœur» durant lesquels les individus éprouvent un désir si intense qu'ils ne tiennent compte ni de ses critères financiers, ni de l'utilité réelle de l'objet dans son achat. Les sentiments peuvent donc être plus forts que la raison et conduire en ce sens à une certaine irrationalité de l'individu. Les sentiments ou "die Gefühle» dont parle EHRENFELS pourraient être comparés aux préférences des agents économiques en ce sens où leurs sentiments sont reflétés à travers leurs préférences. Cependant là encore, il convient de différencier les deux notions: les sentiments peuvent dans certaines situations contredire les préférences d'un individu. Par exemple, une personne peut préférer rester à la maison durant le week-end mais par amour pour son ou sa conjoint(e), cette personne l'accompagnera au cinéma.

${ }^{21}$ GREENSPAN Patricia, "Emotions, Rationality, and Mind/Body ", in HATZIMOYSIS Anthony (éd.), 2003, p.113.

${ }^{22}$ HARTMANN Martin, 2005, p. 19.

${ }^{23}$ BEN-ZE'EV Aaron, "The Logic of Emotions ", in Anthony Hatzimoysis (éd.), 2003, p.147154.

${ }^{24}$ GREENSPAN Patricia, in Anthony Hatzimoysis (éd.), 2003, p.118-119 et voir également BENZE'EV Aaron, in Anthony Hatzimoysis (éd.), 2003, p.151, NUSSBAUM Martha, "Upheavals of Thought ", 2001 et LeDOUX Jospeh, « Das Netz der Gefuhle. Wie Emotionen entstehen », 1998. 
Deux points doivent encore être explicités : premièrement, dans le cas où l'individu devrait choisir entre deux objets, comment expliquer que le désir pour l'un des deux soit plus fort et domine ? Deuxièmement, à quel moment apparaît le désir chez l'individu?

A la première question, EHRENFELS répond en s'inspirant, ou plus exactement, en modifiant la loi de motivation de MEINONG ${ }^{25}$. Supposons que l'individu ait à choisir entre deux objets $\mathrm{O} 1$ et $\mathrm{O} 2$.

Soit,

z1 : la situation dans laquelle l'individu s'imagine la matérialisation de $\mathrm{O} 1$ et pas celle de $\mathrm{O} 2$.

$\mathrm{z} 2$ : la situation où il s'imagine la matérialisation de $\mathrm{O} 2$ et pas celle de $\mathrm{O} 1$.

$\mathrm{g}$ : représente l'existence d'un objet ( $\mathrm{g} 1$ pour l'objet $\mathrm{O} 1$ et $\mathrm{g} 2$ pour l'objet $\mathrm{O} 2$ )

$\mathrm{g}$ : représente la non-existence d'un objet (g'1 pour l'objet $\mathrm{O} 1$ et g'2 pour l'objet O2)

On a alors :

$\mathrm{z} 1=\mathrm{g} 1+\mathrm{g}{ }^{\prime} 2$

$\mathrm{z} 2=\mathrm{g} 2+\mathrm{g} 1$

Si B 1 représente le désir pour l'objet 1 et B 2 le désir pour l'objet 2, on peut en déduire :

$\mathrm{B} 1=\mathrm{z} 1-\mathrm{z} 2=\mathrm{g} 1+\mathrm{g}^{\prime} 2-\mathrm{g} 2-\mathrm{g}^{\prime} 1=\left(\mathrm{g} 1-\mathrm{g}^{\prime} 1\right)-\left(\mathrm{g} 2-\mathrm{g}^{\prime} 2\right)$

$\mathrm{B} 2=\mathrm{z} 2-\mathrm{z} 1=-\mathrm{B} 1=\left(\mathrm{g} 2-\mathrm{g}^{\prime} 2\right)-\left(\mathrm{g} 1-\mathrm{g}^{\prime} 1\right)$

$\mathrm{B} 1>0$ quand le désir pour l'objet $\mathrm{O} 1$ est supérieur à celui pour l'objet $\mathrm{O} 2$, ou plus exactement, quand la différence entre l'envie que l'objet $\mathrm{O} 1$ existe et celle qu'il n'existe pas est plus grande que celle entre l'envie que l'objet $\mathrm{O} 2$ existe et l'envie que $\mathrm{O} 2$ n'existe pas. Dans ce cas, plus la différence est grande et positive entre $(\mathrm{g} 1$ - g' 1$)$ et $(\mathrm{g} 2$ - g'2) et plus le désir pour l'objet $\mathrm{O} 1$ est grand. La comparaison entre deux objets permet à l'individu d'une part, de se décider pour l'un ou l'autre objet, et deuxièmement de déterminer la grandeur de son désir pour l'objet préféré. La valeur d'un objet est alors proportionnelle à la somme des intensités des sentiments d'envie et de non envie, EHRENFELS citant ainsi MEINONG. Cependant cette définition n'est pas tout à fait exacte pour lui et nécessite quelques précisions.

D'abord, au lieu de la somme des intensités des sentiments d'envie et de non envie, il convient plutôt d'effectuer la différence entre ces deux mêmes éléments. Ensuite pour la grandeur de la valeur, le sentiment d'existence lié à une conceptualisation la plus concrète et la plus vivante possible de l'objet en

\footnotetext{
${ }^{25}$ MEINONG Alexius, "Über Werthaltung und Wert ", 1895, in " Gesamtausgabe ", Band III 1968, p.247-266.
} 
question, est déterminant. Enfin la référence implicite faite au jugement de valeur est éliminée par EHRENFELS. Sous le terme "sentiment d'existence ", il faut comprendre un sentiment qui s'adapte selon le caractère concret et vivant de la conceptualisation de l'existence ou de la non existence de l'objet. En fait, l'existence de l'objet n'est pas jugée mais imaginée.

La théorie de Christian von EHRENFELS permet donc de déterminer le schéma d'apparition du désir et sa grandeur, mais elle ne fournit pas d'explication quant au moment de l'apparition du désir et à celui de la prise de décision au moment du choix entre deux objets. Et pour cause, selon le philosophe autrichien, "l'expérience montre la plus grande diversité selon les individus " ${ }^{26}$. Il est donc possible d'expliquer le procédé d'apparition du désir et celui de la prise de décision, mais étant donné le caractère émotionnel et compliqué de la psyché humaine, il est difficile d'établir une loi concernant le moment du désir et de la prise de décision, même si les réflexions suivantes du philosophe permettront de mieux comprendre ce phénomène. De plus, ce moment ne peut être théorisé car le désir en lui-même n'est qu'une partie du processus de pensée lié à l'état de bienêtre. En fait, le désir n'est pas une qualité psychique, elle n'en est qu'une composante.

La puissance ou la force du désir n'est pas l'intensité de celui-ci, tel que cela est le cas pour une qualité psychologique comme l'envie ou la non envie, mais la grandeur de la résistance qui provoque sa domination par rapport à d'autres désirs dans la conscience de l'individu. La réflexion dans le cas d'une alternative entre deux ou plusieurs objets, et qui intervient avant l'acte d'envie, n'est pas une précondition mais plutôt le "comportement" normal du processus du désir qui se déroule, selon les cas, plus ou moins rapidement :

" Die Kraft oder Stärke des Begehrens ist daher nicht die Intensität einer bestimmten psychischen Qualität, wie etwas der Lust oder Unlust, sondern das Maß des Widerstandes, welchen der [...] Vorstellungslauf seiner Verdrängung aus der Enge des Bewusstseins durch andere Begehrungen oder überhaupt psychische Kräfte entgegenzusetzen vermag; und das " Erwägen " der Alternativen eines Konfliktsfalles vor dem Zustandekommen des Strebens- oder Willensaktes ist nicht die Vorbindung, sondern der Gebärungszustand des Begehrens, welcher sich je nach dem Walten der [...] Tendenzen (äußere Einwirkung, Assoziation, Ermüdung) rascher oder langsamer, einfacher der wechselvoller abspielt. ${ }^{27}$

${ }^{26}$ "Die Erfahrung zeigt hier die großte Mannigfaltigkeit », in EHRENFELS, 1982, p.179.

${ }^{27}$ EHRENFELS, 1982, p. 180. 
Après cette analyse de la relation entre l'individu et l'objet désiré, et une première analyse des sentiments qui accompagnent cette relation, il s'agit maintenant de considérer un des sentiments déterminants dans cette relation : la satisfaction des besoins. La satisfaction des besoins est pour bon nombre d'écoles de pensée en économie le but ultime de tout désir. Elle est un concept fondamental chez les économistes autrichiens également, et chez Carl MENGER en particulier :

«Sie [die Nationalökonomen] gehen nämlich von der mehr oder minder deutlich ausgesprochenen Voraussetzung aus, daß alles menschliche Begehren seinem Wesen nach überhaupt auf keine anderen Zwecke als auf Bedürfnisbefriedigung gerichtet sein könne. ${ }^{28}$

L'hypothèse de départ des économistes de l'Ecole Autrichienne est que la raison d'être du désir humain trouve sa justification à travers sa projection sur la satisfaction des besoins. Il est alors essentiel pour EHRENFELS de pouvoir donner une définition du « besoin » ou " Bedürfnis », une définition qui est en fait double :

En premier lieu, le besoin peut être défini comme le désir projeté sur une chose qui est l'objet de ce désir. Ou encore, le besoin pour un objet est éveillé par un état de non-satisfaction qui peut cesser grâce à l'acquisition de l'objet désiré (p.28). EHRENFELS souligne par ailleurs que ces deux définitions correspondent à celles que l'on retrouve dans le langage parlé et sont donc empreintes d'un certain empirisme.

La seconde définition du mot " besoin " est celle qui se rapproche le plus de celle des économistes de l'Ecole Autrichienne qui ont focalisé leur attention sur l'aspect pratique de la notion, c'est-à-dire sur son attachement et sa relation avec les théories économiques, et non sur son aspect psychologique. C'est ce manque que le philosophe va essayer de combler à travers sa théorie. Cette seconde définition est selon lui non satisfaisante dans la mesure où elle contient un nonsens : si on admet que le désir est équivalent au besoin alors, d'après cette même définition, le désir n'a pour autre but que la satisfaction du désir.

Mais peut-on assimiler aussi facilement besoin et désir? Dans la dernière définition mentionnée de la satisfaction des besoins, les notions de "désir » et de " besoin » apparaissent comme étant condition l'une pour l'autre : un désir induit automatiquement un besoin et un besoin naît d'un désir. Selon EHRENFELS, le langage courant autorise cette assimilation mais il est nécessaire de redéfinir ces notions et de les préciser pour mieux comprendre le phénomène de la valeur ${ }^{29}$.

\footnotetext{
${ }^{28}$ EHRENFELS, 1982, p.28.

29 "Diese [Definition] ist jedoch in der dargelegten, durch den Sprachgebrauch bedingten Fassung noch zu eng für die Identifizierung von Nutzen mit Făhigkeit zur Bedurfnisbefriedigung. ", in EHRENFELS, 1982, p.28.
} 
D'abord, nous n'attribuons pas de l'utilité seulement aux objets qui suppriment notre frustration mais également à ceux qui éveillent l'envie. En effet nos besoins peuvent être satisfaits par des objets qui suppriment la frustration due à leur nonpossession et/ou par les objets éveillant le sentiment d'envie chez l'individu. L'envie de posséder ou d'acquérir un objet n'est donc pas forcément lié au sentiment de frustration : l'individu peut désirer une chose sans pour autant que la non-possession de cette chose soit pour lui source de frustration. Par exemple un tour de manège éveille certes l'envie, mais ne pas avoir droit à son tour n'est pas automatiquement frustrant. A partir de ces hypothèses, EHRENFELS peut nous livrer sa définition du besoin : le besoin pour un objet désigne chaque état d'un individu qui chasse la frustration ou suggère l'envie, plus généralement, qui est capable d'élever son état de bien être ${ }^{30}$.

Le besoin pour un objet s'apparente donc à un état de bien être ou plus exactement à un état émotionnel de l'individu. L'origine du besoin est donc à chercher ou à rechercher dans l'état émotionnel de l'individu, c'est à dire qu'elle est psychologique, de même que le sentiment de satisfaction ou "Gluckszustand ". EHRENFELS insiste sur l'importance de l'état émotionnel de l'individu et donc de l'implication de la psychologie.

La valeur est donc, pour EHRENFELS, un phénomène qui apparaît dans le cadre d'une relation entre un invididu et un objet. Cette relation est de nature subjective, c'est-à-dire qu'elle implique un jugement de la part d'un individu qui se construit non pas à partir de critères prédéfinis, mais sur la base des émotions ressenties à la vue de l'objet en question. Celui-ci doit éveiller en l'individu un certain intérêt par le fait que sa réalisation ou sa possession conduirait à une amélioration de son bien être. Nous pouvons à présent livrer la définition de la valeur du philosophe autrichien.

\section{3 - Définition générale de la valeur}

Après avoir évoqué les hypothèses de départ d'EHRENFELS dans son travail sur la valeur, et après avoir présenté la valeur comme étant le résultat d'une relation entre un individu et un objet, relation durant laquelle le désir de l'individu est éveillé, et qui le conduit à attribuer une valeur à l'objet ou aux objets qu'il considère, nous pouvons maintenant présenter la définition générale de la valeur de Christian von EHRENFELS.

\footnotetext{
${ }^{30}$ "Wir verstehen somit theoretisch unter Bedurfnis nach einem Gegenstande jede derartige Beschaffenheit eines Individuums, dass jener Gegenstand ihm Unlust zu vertreiben oder Lust zu betreiben - allgemein seinen Gluckszustand zu heben oder zu fordern vermøchte ", in EHRENFELS; 1982, p.29.
} 
Avant d'arriver à sa propre définition de la valeur, le philosophe autrichien reconnaît l'existence de bon nombre de définitions de cette même notion, mais il ajoute également que ces définitions ne lui semblent pas satisfaisantes.

Sa définition doit inclure les hypothèses déjà posées :

- la valeur a un caractère subjectif,

- elle est le résultat d'une relation établie entre un individu et un objet,

- l'individu attribue de la valeur à un objet dont il est persuadé de la nonexistence ou dont il doute de l'existence - ainsi qu'à un objet qui existe mais qu'il ne possède pas,

- et la considération de l'objet doit éveiller chez l'individu un désir positif ou négatif selon l'objet.

La définition de la valeur devra donc tenir compte du désir de l'individu. En fait, la valeur d'un objet est assimilée à sa désirabilité ${ }^{31}$, une thèse reprise plus tard par des auteurs contemporains d'horizons différents ${ }^{32}$. De même, la grandeur de la valeur est déterminée par sa désirabilité, c'est-à-dire que la valeur d'un objet est proportionnelle au désir qui lui est adressé.

Cependant le désir pour un objet n'est pas directement projeté sur l'objet luimême, mais sur son "être ou ne pas être" ou "auf ihr Sein oder Nichtsein ". Désirer un objet équivaut à désirer son existence - ou sa possession - c'est-à-dire en fin de compte, désirer le fait qu'il soit - ou qu'il soit propriété.

On ne désire pas l'objet lui-même mais le fait de pouvoir en disposer et, en même temps, la non existence de tout obstacle qui empêcherait de pouvoir en disposer :

« Ein Ding begehren heißt entweder die Existenz des Dinges begehren, oder seinen Besitz, in welch letzterem Falle das Begehren auch auf ein Sein, jedoch nicht des Dinges selbst, sondern der Verfügungsmöglichkeit über dasselbe, und zugleich auf ein Nichtsein, die Abwesenheit alle jener Verfügungsgewalt behindernden Störungen, gerichtet ist. " ${ }^{33}$

Chaque désir projeté sur l'existence ou le "Sein“ d'un objet établit une valeur positive ; tout désir de "Nichtsein " ou non existence établit une valeur négative ou non-valeur. Cependant, d'autres combinaisons sont possibles, par exemple la persistance d'une douleur peut avoir autant de valeur que son apparition peut

\footnotetext{
${ }^{31}$ " Der Wert eines Dinges ist seine Begehrbarkeit », in EHRENFELS, 1982, p.253.

${ }^{32}$ Les valeurs en tant que conception de la désirabilité d'un objet, ont été évoquées par les anthropologues KLUCKHOHN Klyde dans " Values and Value-orientations in the Theory of Action : an Exploration in Definition and Classification "), 1951, p.395 ; et GRAEBER David, "Toward an Anthropological Theory of Value. The False Coin of our Own Dreams ", 2001, p.3.

${ }^{33}$ EHRENFELS, 1982, p.254.
} 
représenter de non-valeur; de même, la persistance d'une envie peut avoir aussi peu de non-valeur que son apparition aurait de valeur. En outre, on sait que le désir lui-même est déterminé par l'état émotionnel de l'individu. Il faut également en tenir compte dans la définition de la valeur.

EHRENFELS définit la valeur de la manière suivante :

La valeur est une relation entre un sujet et un objet qui exprime soit le désir effectif du sujet pour l'objet, soit son désir éventuel dans le cas où le sujet serait convaincu de la non existence (ou de la non-possession) de l'objet. Autrement dit, le sujet va désirer l'objet lorsque le fait d'imaginer l'existence de l'objet, de manière plus ou moins concrète et vivante, provoque une augmentation du sentiment de son bien être supérieure à celle qui serait provoquée dans le cas où le sujet imaginerait la non existence de l'objet. La grandeur de la valeur est proportionnelle à l'intensité du désir, ce qui correspond à la différence entre les sentiments d'envie et de non envie d'existence de l'objet.

Autrement dit :

"Wert ist eine Beziehung zwischen einem Objekte und einem Subjekte, welche ausdrückt, daß das Subjekt das Objekt entweder tatsächlich begehrt oder doch begehren würde, falls es von dessen Existenz nicht [sic!] überzeugt wäre - oder daß durch die möglichst anschauliche, lebhafte und vollständige Vorstellung von dem Sein des betreffenden Objektes bei dem Subjekt ein auf der Gefühlsskala Unlust-Lust höher gelegener Zustand bedingt wird, als durch die ebenso beschaffene Vorstellung von dem Tatbestand beim Nichtsein des Objektes. Die Größe des Wertes ist proportional der Stärke des Begehrens, sowie dem Abstand zwischen den beiden charakterisierten Gefühlen. " ${ }^{34}$

Le fait de définir ainsi la valeur ne semble pas apporter un élément nouveau ou une perspective nouvelle. Il s'agit d'une définition générale mais qui cerne bien le phénomène dans sa totalité. L'intérêt de sa théorie ne se situe pas tant dans cette définition que dans ce qui suit, c'est-à-dire la classification des types de valeurs ainsi que leurs évolutions. Dans cette deuxième partie, le philosophe sera plus innovant et plus intéressant.

${ }^{34}$ EHRENFELS, 1982, p.261. 


\section{II - Catégorisation du phénomène de la valeur et notions adjacentes}

La valeur est une notion subjective qui revêt divers aspects, qui ont conduit EHRENFELS à distinguer les différents types de valeur.

Les deux premiers types de valeur, et sans aucun doute les plus connus et utilisés en économie, sont les valeurs d'usage et les valeurs d'échange. Elles sont déterminées par l'utilité d'une part et l' " échangeabilité » de l'objet d'autre part. $\mathrm{Si}$ on rencontre ces types de valeur assez souvent, et si leur définition nous permet de mieux comprendre l'apparition de certaines valeurs, elles ne suffisent en aucun cas à cerner le phénomène de la valeur en général, ni même celui de la valeur en économie en particulier. Pour pallier à ce manque, EHRENFELS procède à des catégorisations supplémentaires : il distingue dans un premier temps les valeurs intrinsèques et les valeurs d'effet. Cette distinction n'est pas nouvelle, du moins en philosophie puisqu'il est possible de la retrouver chez Franz BRENTANO par exemple $^{35}$. Néanmoins, les valeurs intrinsèques et les valeurs d'effet recouvrent un plus grand nombre d'évaluations que les valeurs d'échange et les valeurs d'usage, et nous allons voir plus loin dans ce travail, qu'il est tout à fait envisageable de les utiliser en économie. Une distinction plus générale est possible grâce aux valeurs intermédiaires et aux valeurs non-intermédiaires. Enfin, d'autres catégories de valeurs vont être énoncées, des catégories qui, même si elles sont moins importantes, ne doivent pourtant pas être négligées.

\section{1 - La valeur d'usage et la valeur d'échange}

Les notions de valeur d'échange et de valeur d'usage sont celles qui ont le plus souvent été utilisées par les économistes pour définir et expliquer le concept de la valeur. La valeur d'échange fait référence à la valeur qu'un objet peut se voir attribuer au cours d'un échange entre deux individus. La valeur d'usage est liée à l'utilité de l'objet : un objet de grande utilité aura une grande valeur d'usage et vice versa. Autrement dit, la valeur d'usage est proportionnelle à l'utilité d'un objet. EHRENFELS est proche de cette perspective mais il ajoute également une certaine critique quant à la détermination de la valeur d'échange.

Il définit en premier lieu l'échange comme l'acte durant lequel les individus échangent la propriété ou la possession d'un objet pour un autre (p.39). L'échange est possible si l'individu confère plus de valeur à un objet qu'il ne possède pas qu'à l'objet qu'il possède, ou encore, il confère plus de valeur à la possession d'un

\footnotetext{
${ }^{35}$ Voir p.46 de ce travail.
} 
objet qu'il ne possède pas qu'à celle d'un objet qu'il possède déjà. L'échange intervient donc s'il y a un gain pour l'individu. Par ailleurs, l'échange influence notre processus d'attribution de la valeur: si l'échange n'était pas possible, un individu qui a faim accorderait plus de valeur à une livre de pain qu'à une once d'or.

EHRENFELS définit la valeur d'usage comme celle qui est accordée par un individu à la possession d'un objet, abstraction faite de la possibilité de l'échanger. La valeur d'échange est celle qui est attribuée par l'individu à la possession d'un objet en considérant sa potentialité à l'échange. Lorsque la valeur d'échange est supérieure à la valeur d'usage, l'échange s'effectue. De l'acte d'échange se construit le prix d'un bien économique. Le prix est la relation exprimée en argent d'un bien économique dans l'échange. Celui-ci sera déterminé par les individus qualifiés de "Kauflustig » ou "Verkauflustig », c'est-à-dire des individus qui ont envie de vendre ou d'acheter, en considération de leurs valeurs d'usage et d'échange et donc de leurs gains suivant l'acte de l'échange. Sur ce point, l'analyse d'EHRENFELS est très proche de celle de l'économiste autrichien Friedrich von WIESER ${ }^{36}$, notamment par le fait qu'il reprenne les expressions " Kauflustig » et " Verkauflustig ».

Se référant à l'analyse de la formation des prix de BÖHM-BAWERK, EHRENFELS affirme que le prix n'est en fait pas déterminé en fonction des évaluations de la majorité ${ }^{37}$ mais de celles des personnes à fort pouvoir d'achat. Autrement dit, les prix seraient fixés par les personnes ayant des revenus élevés, c'est-à-dire, en cette fin de $\mathrm{XIX}^{\text {ème }}$ siècle, par celles qui possédaient le capital et les moyens de production. Les prix seront donc déterminés en fonction de la valeur d'usage qui sera attribuée par ces individus aux objets qu'ils vendront. Une certaine influence socialiste se fait ici sentir dans le discours d'EHRENFELS. Il semble critiquer ouvertement le système économique dans lequel il vit. Il dénonce même le fait que la valeur attribuée par les personnes à fort pouvoir d'achat soit supérieure à celle qui serait attribuée par les personnes ayant un pouvoir d'achat moins élevé. Il dénonce enfin le fait que cette différence augmente au fur et à mesure que le fossé se creuse entre les classes de revenus et que la richesse soit inégalement répartie (p.40).

\footnotetext{
${ }^{36}$ WIESER Friedrich von, « Der naturliche Werth », 1889, p.48-52.

${ }^{37}$ « Es ist nămlich sofort klar, daß auf die Lage der Grenzpaares einerseits die Zahl, und anderseits die Höhe der Begehrungen bzw. Wertschătzungen, die bei beiden Parteien vorkommen, den entscheidenden Einfluß [auf den Marktpreis] nehmen muss ", in BÖHM BAWERK Eugen von, "Grundzlige der Theorie des wirtschaftlichen Guterwerts », 1886, p.509.
} 
Le fait d'avoir un tel discours n'est sans doute pas étranger à ses nombreux voyages en Allemagne où les courants socialistes étaient de plus en plus forts, et où les réformes sociales étaient de plus en plus nombreuses en cette fin de XIX ${ }^{\text {ème }}$ siècle. On sait que le philosophe autrichien était sensible aux influences socialistes et sa réflexion sur la formation des prix montre la mesure de cette influence.

Si EHRENFELS reconnaît l'existence des valeurs d'échange et des valeurs d'usage, il semble par contre penser qu'elles ne sont pas les seules catégories de valeur existantes. L'utilisation de telles notions semble être associée aux prix du marché : les valeurs d'échange et les valeurs d'usage peuvent découler de la relation entre un individu et un objet, mais elles sont déterminées en fin de compte par le marché sous la forme d'un prix exprimé en unités monétaires. En effet, la notion de valeur d'échange se confond le plus souvent avec le prix de l'objet puisqu'elle apparait sur le marché au moment de l'échange. La valeur d'usage, comme l'affirme EHRENFELS, n'est qu'un type particulier de valeur qui est fonction de l'utilité d'un objet. Autrement dit, elle n'intervient que dans les situations où les individus désirent l'objet pour son utilité. Ceci n'est pas toujours le cas, y compris en économie. Pour pallier à ce manque EHRENFELS tente une approche différente en considérant la valeur comme l'importance qu'un individu accorde à un objet, les notions sus mentionnées étant par ailleurs insuffisantes dans le cadre d'une théorie générale. C'est pourquoi il présente d'autres catégories, dont la plus importante est celle des valeurs intrinsèques et des valeurs d'effet.

\section{2 - La valeur intrinsèque et la valeur d'effet}

Dans ses premiers articles sur la valeur en 1893/1894, EHRENFELS ne parle que des valeurs intrinsèques et des valeurs d'effet, alors que quelques années plus tard, dans son livre "System der Werttheorie", il subordonne ces deux catégories à deux autres encore plus générales : celle des valeurs intermédiaires ou " vermittelte Werte», et celles des valeurs non-intermédiaires ou " unvermittelte Werte » (p.268-270).

Les valeurs non-intermédiaires désignent les valeurs qui sont accordées aux objets qui sont désirés pour eux-mêmes. Les valeurs intermédiaires décrivent un type de valeur qui est accordé à un élément inhérent à l'existence de l'objet. Parmi les valeurs intermédiaires, il faut distinguer trois sous-catégories :

1) Les valeurs intermédiaires dites purement constitutives, qui sont attribuées lorsque l'individu valorise un élément constitutif de l'objet désiré. Par exemple, un minerai qui serait valorisé pour son contenu en fer. 
2) Les valeurs intermédiaires dites purement causales, qui sont attribuées lorsque l'individu valorise l'objet en tant que cause provoquant un effet attendu. Par exemple, le cachet d'aspirine qui sera désiré pour ses effets.

3) Les valeurs intermédiaires causales et constitutives, attribuées à un objet à la fois pour un de ses éléments constitutifs, mais aussi pour ses effets attendus. Par exemple, le minerai qui sera valorisé pour son contenu en fer, mais aussi pour l'utilisation possible de ce fer.

En fait, dans le cas des valeurs non-intermédiaires et des valeurs intermédiaires constitutives, l'objet sera désiré pour lui-même ou pour une partie de lui-même. Dans les autres cas, il le sera pour les effets qu'il provoque.

Dans le cas où on désire une chose pour elle-même, EHRENFELS parle également de "Eigenwert " ou valeur intrinsèque. Dans le cas où la chose est considérée comme un moyen d'atteindre un but ou plus exactement, lorsque l'individu désire un objet pour les effets directs ou indirects induits par l'existence ou la possession de celui-ci, EHRENFELS parle de "Wirkungswert " ou valeur d'effet. Les qualificatifs utilisés par le philosophe lui sont propres, mais leurs définitions laissent supposer qu'elles lui ont été inspirées par son ancien professeur Franz BRENTANO ${ }^{38}$ : ses notions de biens primaires et secondaires correspondent à celles de valeur intrinsèque et valeur d'effet chez EHRENFELS. Il est possible de trouver d'autres sources d'inspiration ainsi que d'autres correspondances chez d'autres philosophes et économistes, mais nous nous intéressons pour l'instant plus précisément aux notions elles-mêmes. Précisons enfin qu'il est possible de trouver une autre traduction de "Wirkungswert » dans la littérature française : le philosophe Pierre LIVET traduit "Wirkungswert " par valeur fonctionnelle ${ }^{39}$. Bien que les deux termes soient synonymes et puissent être utilisés comme tels, nous lui préférons notre traduction plus explicite quant à la signification de la "Wirkungswert». En effet, le mot allemand "Wirkung " se traduit en français par " effet, influence, résultat». Par conséquent, en utilisant le mot " effet", nous restons le plus proche possible du sens originel. En outre la nuance de sens entre " effet » et «fonction » d'un objet peut être clarifiée à l'aide d'un exemple. Si l'on considère le cachet d'aspirine, il a pour fonction d'atténuer ou de soulager du mal de tête. Mais ce que l'on va en fait désirer en lui, ce n'est pas tant sa fonction que son effet. En ce sens l'effet est le résultat de l'action de l'aspirine, c'est-à-dire les effets de sa fonction.

\footnotetext{
${ }^{38}$ PARK Chan-Young, 1991, p.66-67.

${ }^{39}$ LIVET Pierre, « Emotions et valeurs chez Meinong et Ehrenfels », 2001.
} 
Pour illustrer maintenant les deux notions de valeur intrinsèque et d'effet, prenons l'exemple d'une pomme : celle-ci sera désirée par un individu ayant faim et dont le but sera, à travers la consommation de cette pomme, d'apaiser sa faim. Le but ultime, l'apaisement de la faim se verra attribuer une valeur intrinsèque, laquelle sera plus ou moins grande selon l'urgence du besoin. La pomme, elle, est le moyen de satisfaire ce besoin, ou plus exactement, l'effet de la pomme se concrétisera sous la forme d'une diminution de la faim. La valeur d'effet ici attribuée sera plus ou moins élevée selon la capacité de la pomme à apaiser la faim.

La question qui se pose à la suite de ces définitions est la suivante : est-ce qu'un bien économique, c'est-à-dire un bien issu de la production industrielle et qui se vend sur un marché, peut se voir attribuer de la valeur intrinsèque?

Pour les économistes de l'Ecole Autrichienne, l'individu va désirer et attribuer de la valeur à un objet qu'il veut posséder, et il veut le posséder avec l'intention de satisfaire ses besoins. Le bien en question n'est donc qu'un moyen pour atteindre un but final et de ce fait, et même s'ils ne le précisent pas, ils sous-entendent au sens d'EHRENFELS, que les biens économiques ne se voient attribuer qu'une valeur d'effet. EHRENFELS ne semble pas contredire les économistes en question et laisse également entendre que les biens économiques n'ont qu'une valeur d'effet et ne sont en fait que des instruments servant d'autres desseins. Il convient de noter par ailleurs que pour les économistes autrichiens, l'objet qui se voit attribuer de la valeur est un objet réel, concret. Il doit exister pour être l'objet d'une évaluation par l'individu. Pour EHRENFELS, il suffit que l'individu l'imagine pour éveiller son désir pour l'objet et donc pour lui attribuer une valeur.

Le processus d'attribution de la valeur est pour EHRENFELS un processus individuel, dans la mesure où il considère la valeur comme étant subjective et donc relative à chaque individu. Il s'accorde de la sorte avec la perspective de l'individualisme méthodologique développée par MENGER et adoptée par l'Ecole Autrichienne.

La mesure de la valeur d'effet s'inspire cependant des enseignements de la valeur des économistes autrichiens, de l'aveu même d'EHRENFELS (p.270). Pour arriver à mesurer une valeur d'effet, il faut tout d'abord tenir compte du contexte ou de la situation dans laquelle elle a été attribuée, puis il convient de définir le type de valeur d'effet dont il s'agit.

Prenons l'exemple d'un bateau en pleine mer. La réserve en eau douce à son bord a une grande valeur, qui est en fait égale à celle de la vie de l'équipage, puisque celui-ci ne pourrait pas survivre sans eau douce. Par contre lorsque le bateau est à quai, la valeur de la même réserve n'est plus aussi grande, puisqu'en cas de 
pénurie il suffira d'aller se ravitailler sur la terre ferme. Il faut dans cet exemple distinguer les différents types de valeur en présence : d'abord la vie de l'équipage a une valeur intrinsèque dont l'existence dépend de celle de la réserve d'eau douce à bord lorsque le bateau est en haute mer. Autrement dit, l'eau a une valeur d'effet. La survie ou plus exactement le bon état de santé de l'équipage a aussi une valeur d'effet qu'EHRENFELS dénomme "valeur d'origine" ou "Stammwert ». La valeur d'effet de l'eau est aussi élevée que la valeur d'origine qui est attribuée à la survie de l'équipage dans le cas où le bateau est en haute mer. En revanche, lorsque le navire reste à quai, la valeur d'effet de l'eau est plus faible, en fait beaucoup plus faible que celle de la survie de l'équipage. Cette différence est due, selon EHRENFELS, à la substituabilité ou non de l'eau. Lorsqu'il est dans le port, l'eau à bord du bateau peut être remplacée sans difficulté, de telle manière que la vie des marins n'est jamais en danger :

"Unersetzbare Wirkungswerte (d.h. also die Wirkungswerte unersetzbarer Objekte) sind [...] schlechthin ihren Stammwerten gleich; ersetzbare Wirkungswerte können an Größe ihre Stammwerte niemals übertreffen, wohl aber erheblich hinter ihnen zurückbleiben. $»^{40}$

On peut donc affirmer que la grandeur d'une valeur d'effet ne peut en aucun cas dépasser celle de la valeur d'origine qui en dépend. Elle lui est égale lorsque l'objet est irremplaçable, et elle lui est inférieure lorsque l'objet est remplaçable.

Considérons l'exemple d'un bateau en haute mer transportant 40 sacs de biscottes rangés par ensembles de 10 sacs. Des 10 derniers sacs dépend la vie de l'équipage; des 10 avant-derniers dépend leur bonne santé; des 10 suivants dépend le bien-être du propriétaire des sacs et des 10 premiers dépend le bien-être des animaux de compagnie de celui-ci. Dans ce cas, les dix premiers sacs vaudront 100 unités monétaires, les 10 suivants 2000, les 10 avant-derniers 300 000 et les dix derniers sont irremplaçables. Notons que l'exemple des sacs de biscottes disponibles à bord d'un bateau en haute mer s'inspire fortement d'un exemple donné par Carl MENGER dans son explication de la détermination de la valeur d'un bien, une explication menant directement au concept de valeur marginale ${ }^{41}$. Les conclusions que l'un et l'autre en tirent ne sont donc pas si éloignées que cela. Pour EHRENFELS cet exemple montre que la valeur d'effet d'une quantité quelconque d'objets substituables est déterminée par son utilité marginale. La différence entre valeur d'effet et valeur d'échange est que la première peut être attribuée à un bien non économique et/ou à un bien non substituable.

\footnotetext{
${ }^{40}$ EHRENFELS, 1982, p.271.

${ }^{41}$ MENGER Carl, « Grundsătze der Volkswirtschaftslehre », 1871, extraits dans LEUBE Kurt, 1995, p.63.
} 
Dans ce paragraphe nous avons évoqué les quatre grands types de valeurs. Les deux premiers, les valeurs intermédiaires et non intermédiaires, ont été rajoutés plus tard par le philosophe. Mais on pourrait se demander quel est l'intérêt d'une telle catégorisation de la part d'EHRENFELS. Elles sont théoriquement correctes en ce sens où il est possible d'opérer une telle distinction et elles apportent une précision supplémentaire, même si les deux autres catégories, celles des valeurs intermédiaires et des valeurs d'effet, semblent présenter un plus grand intérêt. On sait qu'EHRENFELS tient à être le plus précis et le plus complet possible et c'est pour cela qu'il a développé autant de types différents de valeurs.

Voyons à présent comment il envisage la notion d'utilité.

\section{3 - La relation valeur-utilité et valeur-avantage}

Après avoir précisé la notion de valeur et développé celles de la valeur intrinsèque et de la valeur d'effet, nous allons nous intéresser aux relations entre la valeur et l'utilité. Pour ce faire, nous allons commencer par considérer de plus près le lien entre le désir et la valeur, avant de s'intéresser à la relation qui lie la valeur à l'utilité.

Concernant la valeur intrinsèque ou "Eigenwert", chaque objet dont la représentation dans l'imagination entraine une intensification relative du sentiment de bien-être, sera désiré et possède ainsi pour l'individu une valeur intrinsèque (p.34). On parlera d'ailleurs dans ce cas de valorisation de l'objet, en ce sens où l'individu lui donne de la valeur. Tout ce qui peut être imaginé ou plus précisément, tout ce qui peut se concrétiser dans l'imagination, peut se voir attribuer de la valeur intrinsèque, $y$ compris certains sentiments tels que la douleur par exemple. Même s'il ne les mentionne pas explicitement, EHRENFELS n'exclut pas les comportements dans lesquels les individus éprouvent du désir lorsqu'ils s'imaginent la douleur créée par un acte. Il s'agit bien évidemment de comportements sadomasochistes, durant lesquels la douleur est le but ultime que désire atteindre l'individu. Elle se voit donc attribuer une certaine valeur intrinsèque par les mêmes individus. La grandeur de la valeur intrinsèque dépend de l'intensité du désir correspondant, lequel est déterminé par l'importance de l'augmentation du bien-être relatif.

En ce qui concerne la valeur d'effet, EHRENFELS trouve son origine grâce à une extension de la théorie sur l'utilité marginale. Dans cette théorie, l'augmentation du bien-être est le but ultime qui est visé par chaque individu et les biens acquis sont les moyens qui permettent d'atteindre ce but. 
On attribue au bien être une valeur intrinsèque, alors que les biens acquis ont une valeur d'effet. L'effet attendu de ces biens est l'augmentation du bien-être et de cette augmentation dépend la grandeur de la valeur d'effet.

Dans les deux cas, la valeur d'un objet sera proportionnelle à l'augmentation du bien-être engendrée par la possession de l'objet en question. Dans le premier cas, l'augmentation du bien-être est liée à l'objet lui-même et dans le second cas, elle est liée aux effets attendus de l'objet.

La relation liant l'objet et la valeur comprend un autre élément qui est souvent évoqué dans les théories de la valeur : l'utilité. L'utilité d'un objet se comprend, d'une manière générale, par l'usage qu'un individu retire d'un objet. L'usage de l'objet en question permet à l'individu de satisfaire un besoin.

En économie, l'utilité joue un rôle important dans la détermination de la valeur : pour les économistes autrichiens, et en particulier pour Carl MENGER, la valeur d'un objet dépend de son utilité et avant tout de son utilité marginale. Mais avant d'aller plus loin sur la relation utilité-valeur, il nous faut d'abord mentionner la définition d'EHRENFELS. Il définit l'utilité d'un objet comme "sa capacité à provoquer une accélération, une augmentation de la joie ou du bonheur d'un individu ${ }^{42}$.

L'utilité est analysée ensuite plus finement par le philosophe autrichien qui différencie celle qui est en cause dans la détermination de la valeur d'effet, de celle qui est en cause dans la valeur intrinsèque. Cette distinction impose le choix d'un mot supplémentaire et pour cela, le philosophe autrichien va utiliser un ancien mot allemand qui a aujourd'hui disparu des dictionnaires: "Das Frommen ». Le choix de ce mot trouve son origine dans la vieille expression populaire : "Es gereicht mir zu Nutz und Frommen ». Dans cette expression, les deux mots sont synonymes et peuvent se traduire par utilité. Néanmoins, une traduction plus précise de " Frommen » nous permet d'apprécier la nuance de sens existante : cet ancien mot allemand signifie plus exactement « au profit de, à l'avantage de ${ }^{43}$. Le «Frommen » est donc l'avantage ou le profit apporté par un objet ou une chose :

«Wir [...] nennen das « Frommen » der Objekte ihre Mitwirkung bei der Hervorbringung von Eigenwerten im allgemein, ebenso wie der «Nutzen » der Gegenstände uns ihre Mitwirkung bei der Hervorbringung speziell der Glücksförderung bedeutet. » ${ }^{44}$

\footnotetext{
${ }^{42}$ « Einfacher jedoch [...] ist die Definition der Nutzlichkeit eines Gegenstandes als seiner Făhigkeit, Glucksforderung zu bewirken ", in EHRENFELS, 1982, p.29.

${ }^{43}$ Enzyklopådisches Worterbuch, Langenscheidt, 1933.

${ }^{44}$ EHRENFELS, 1982, p.36.
} 
Le « Frommen » ou avantage d'un objet désigne sa contribution à la production de valeur intrinsèque en général ; l'utilité d'un objet est sa capacité à contribuer à l'amélioration du bien être en particulier (p.36). Autrement dit, dans les cas où l'individu vise une amélioration de son bien être comme but ultime, les objets pouvant y contribuer auront une utilité. Dans les cas où l'individu vise un but, autre qu'une amélioration du bien être, ayant une valeur intrinsèque, on parlera de l'avantage des objets qui peuvent y contribuer. Les biens ayant une valeur d'effet peuvent présenter une utilité ou un avantage, selon le but de l'individu. Dans le domaine économique le but des individus, selon les enseignements de l'Ecole Autrichienne, est de satisfaire ses besoins (ce qui provoque une amélioration de son bien être) au moyen des biens économiques. Ces biens auront une utilité et se verront attribuer une valeur d'effet selon EHRENFELS.

Le but de cette catégorisation, selon Wolfgang GRASSL, serait de rendre sa théorie de la valeur utilisable pour les biens n'étant pas des biens économiques au sens propre, mais dont le désir n'est pas non plus explicable d'un point de vue hédoniste $^{45}$. EHRENFELS suppose donc qu'il existe des biens désirés par les individus non pas pour leur utilité dans la perspective de satisfaire leurs besoins, mais pour leur avantage qui est lié à un but autre que l'augmentation du bien être individuel. Concrètement, des parents peuvent vouloir envoyer leurs enfants dans une école particulière dans le but de leur offrir la meilleure éducation possible. L'école en question éveille leur désir par rapport à l'avantage qu'elle procure pour atteindre leur but. Les stylos qu'ils vont acheter à leurs enfants auront eux, une utilité et se verront attribuer une valeur d'effet (ils sont un moyen).

La grandeur de l'utilité d'un objet est déterminée par celle de l'amélioration du bien être qu'il provoque. De la même manière, la grandeur de l'avantage d'un objet définit celle de la valeur intrinsèque qui lui est accordée.

Enfin, de la même manière qu'il existe une utilité marginale, il existe également un avantage marginal. Le principe de marginalité est commun aux deux notions : l'utilité marginale est le bien-être crée par l'acquisition d'une unité supplémentaire d'objet donné ; l'avantage marginal ou "Grenzfrommen » est l'effet causé par l'acquisition ou la possession d'une unité supplémentaire d'un objet. La grandeur de la valeur d'effet d'un objet est proportionnelle à l'avantage marginal qui lui est attribué. Si le but de l'individu est la satisfaction de ses besoins, alors la grandeur de la valeur d'effet dépendra de son utilité marginale ${ }^{46}$.

\footnotetext{
${ }^{45}$ GRASSL Wolfgang, Avant-Propos in EHRENFELS, 1982, p.8.

${ }^{46}$ "Wir gewinnen [...] den allgemeinen Satz, daß die Größe des Wirkungswertes des Objektes proportional ist dem ihnen zugeschriebenen Grenzfrommen », in EHRENFELS, 1982, p.37.
} 
Maintenant que la relation valeur-utilité et valeur-avantage a été précisée, EHRENFELS va analyser les différents aspects de la notion de valeur elle-même en commençant par ceux de la valeur véritable et de la valeur imaginée.

\section{4 - Autres types de valeurs}

$\mathrm{Si}$ on peut considérer que les valeurs intrinsèques et les valeurs d'effet sont les deux grands types de valeurs, il est nécessaire de préciser que ce ne sont pas les seuls. EHRENFELS effectue un classement secondaire des valeurs, c'est-àdire qu'une fois qu'elles ont été rentrées dans une des deux premières grandes catégories, il affine leur classement en développant d'autres catégories. Ceci nous permet de caractériser de manière plus précise chaque valeur mais aussi de mieux en comprendre leur apparition et leur évolution. Parmi les types secondaires de valeurs, on retrouve les valeurs véritables qu'il convient de distinguer des valeurs imaginées ou encore des valeurs supposées. Ensuite on évoquera les valeurs temporaires, normales et normatives avant d'aborder la distinction entre les valeurs générales et les valeurs individuelles ${ }^{47}$.

Selon EHRENFELS, on entend souvent parler de valeurs véritables ou " wahre Werte ", de même que de valeurs imaginées ou " eingebildete Werte ". Notons que cette distinction est également proposée par Carl MENGER ${ }^{48}$. Les valeurs véritables se réfèrent, dans son discours qui se veut en accord avec la réalité du langage, aux valeurs qui sont considérées comme étant objectivement vraies. Les valeurs imaginées sont celles qui découlent d'un jugement de valeur erroné. Autrement dit, l'individu va attribuer de la valeur à un objet qui, en fait, n'en a pas. Or, selon EHRENFELS, la valeur n'est pas une propriété intrinsèque de l'objet, elle est attribuée par les individus le désirant, c'est à dire que la valeur est subjective. Il n'existe pas de critères qui permettent d'affirmer qu'une valeur est véritable ou pas. De plus, un jugement de valeur se rapporte souvent à une valeur d'effet, car celui-ci se destine à évaluer l'objet c'est-à-dire à lui attribuer une valeur, ce qui dans la majorité des cas se fait pour l'effet causé par l'objet, selon le philosophe autrichien. La distinction entre valeur véritable et valeur imaginée n'intervient que rarement dans le domaine des valeurs intrinsèques. Et dans les rares cas où cette distinction se produit, l'individu s'imagine accorder de la valeur à un objet pour lui-même alors qu'en réalité ce sont les avantages procurés par cet objet qui sont au centre de son désir. Autrement dit, l'individu se trompe en accordant à un objet de la valeur intrinsèque qui est en fait une valeur d'effet.

\footnotetext{
${ }^{47}$ Pour les valeurs réelles et imaginées, voir EHRENFELS, 1982, p.38-39 ; pour les autres types de valeurs voir EHRENFELS, 1982, p.263-264.

${ }^{48}$ MENGER Carl, "Grundsătze », extraits parus dans LEUBE Kurt, 1995, p.49.
} 
Ceci se produit dans trois cas :

1) Lorsque l'objet désiré est une partie d'un tout. Par exemple, on dit aimer et désirer son séjour dans une ville pour la ville elle-même alors qu'en réalité ce sont les souvenirs d'enfance associés à la ville que l'on apprécie.

2) Lorsque l'on croit désirer une chose pour elle-même alors qu'elle n'est en fait que le moyen d'atteindre un but final dont on n'a pas conscience.

3) Lorsque l'on croit désirer quelque chose comme moyen d'atteindre un but alors qu'en fait le moyen lui-même est plus désirable à nos yeux. C'est le cas de l'alpiniste qui va gravir la montagne avec pour but avoué le point de vue au sommet de celle-ci. Cependant il éprouvera un plus grand intérêt pour l'ascension de la montagne, son plaisir et son désir ne seront pas tant dirigés sur la vue au sommet que sur l'effort fourni pour y arriver.

En fin de compte, nous différencions la valeur intrinsèque véritable de celle qui est imaginée selon que celle-ci soit effectivement désirée ou pas.

EHRENFELS parle également de l'existence des valeurs supposées. Les valeurs supposées sont des valeurs d'effet, et elles se réfèrent à l'effet ou aux effets d'un objet qu'un individu désire, mais qu'il n'est pas en mesure d'apprécier réellement. Dans le cas d'un médicament par exemple, l'individu désire l'objet pour son effet mais il ne peut pas apprécier réellement a priori l'effet réel qu'aura ce médicament, il ne peut que le supposer. La valeur supposée s'oppose donc à la valeur réelle, celle qui sera attribuée en connaissance de cause. On peut néanmoins affirmer que l'individu, dans un très grand nombre de cas - ceux où il n'est pas a priori en mesure d'évaluer les effets de l'objet - ne pourra attribuer que des valeurs supposées.

Outre les valeurs supposées, réelles ou imaginées, EHRENFELS affirme l'existence des valeurs temporaires, normales et normatives. La valorisation d'un individu, qui dépend directement de son état émotionnel, varie, comme ce dernier, avec le temps et selon les circonstances. Par exemple, l'enfant qui aime les sucreries va leur accorder une grande valeur d'une manière générale. Par contre, lorsqu'il en aura mangé jusqu'à ne plus en pouvoir, la valeur qu'il leur accordera sera plus petite (comme on l'a déjà vu, plus le besoin est satisfait et plus le désir pour un objet et donc sa valeur temporaire, auront tendance à diminuer). Cependant, cette valeur, accordée dans un état de saturation certain, ne sera donc en ce sens qu'une valeur temporaire, alors que la valeur accordée " en moyenne " aux sucreries, dans l'exemple précédent, aura une valeur dite normale. On peut également citer le cas où l'individu valorise un objet qu'il sait pourtant ne pas être bon pour lui. Par exemple, lorsqu'il fait chaud, on aime prendre un bain froid, sachant que cela n'est pas toujours bon pour notre organisme. 
On valorise bien sûr sa propre santé plutôt que le contraire, sauf dans certaines situations, comme celle que l'on vient d'énoncer. Dans ce cas, EHRENFELS parle de valeur normative, encore une fois à différencier des valeurs normales, et qui désignent les valeurs accordées selon certaines normes.

Enfin, avant de clore ce paragraphe, évoquons encore les valeurs générales et les valeurs individuelles. La valeur générale désigne une valeur commune, qui est accordée par un ensemble d'individu à un objet donné. Un ensemble d'individus peut signifier un groupe de personnes, une classe sociale, un groupe social, une classe d'âge, un genre ou encore une nation. Les valeurs individuelles sont les valeurs accordées spécifiquement par un individu et qui, la plupart du temps, lui sont propres.

Tous les différents types de valeurs que nous venons d'évoquer sont ceux que l'on est amené à rencontrer le plus souvent dans la vie de tous les jours. On pourrait évoquer et développer bien d'autres catégories de valeurs. Intéressons nous à présent à une catégorie qui n'a pas été traitée par les économistes, mais dont l'importance n'est pas à négliger : celle des valeurs négatives ou de la non-valeur.

\section{5 - La notion de la non-valeur}

S'il existe des objets que l'individu désire, il y en a d'autres pour lesquels il éprouvera un désir négatif, c'est-à-dire qu'il désirera leur non-existence. Les objets désirés se voient attribuer une certaine valeur qualifiée de valeur positive ; ceux dont on désire par contre la non-existence se verront également attribuer une valeur, mais on parlera dans ce cas d'une valeur négative ou d'une non-valeur (" der Unwert "). Le problème de la non-valeur a été traité par la philosophie en général et par certains philosophes en particulier comme Franz BRENTANO ${ }^{49}$. EHRENFELS, en tant que philosophe, ne peut passer sous silence cet aspect du problème. Le fait de reprendre sa réflexion sur la non-valeur dans un travail qui se veut avant tout économique se justifie par le fait que cette notion n'est pas seulement théorique et que des études empiriques ont montré son existence et ont explicité son principe ${ }^{50}$.

\footnotetext{
${ }^{49}$ Voir p.39 de ce travail.

${ }^{50}$ Le phénomène de la non-valeur a été traité dans des études empiriques et anthropologiques contemporaines comme celles de Nancy MUNN (" The Frame of Gawa : A Symbolic Study of Value Transformation in a Massim Society », 1986), Maurice BLOCH (" Death, Women and Power ", 1982, p.211-230, David GRAEBER (" Dancing with Corpses Reconsidered : an Interpretation of Famadihana ", in American Ethnologist nr 22, 1995, p.258-278). A noter que ces études se réfêrent certes à des sociétés non-industrielles, mais que la notion de non-valeur, dans son principe, peut également être appliquée à des sociétés industrielles.
} 
L'individu attribue donc de la non-valeur à un objet ou à une chose dont il désire la non-existence, la disparition ou l'élimination. De la même manière que pour la valeur, c'est l'intensité du désir, ici d'un désir négatif ( « negatives Begehren ») ou plus exactement d'un rejet ("Verabscheuen »), qui détermine la grandeur de la non-valeur. De même qu'il différencie la valeur intrinsèque de la valeur d'effet, EHRENFELS distingue de manière similaire la non-valeur intrinsèque de la nonvaleur d'effet. Dans le premier cas, l'objet ou la chose est rejeté ou détesté pour lui-même. Dans le cas de la non-valeur d'effet, on désire l'élimination de l'effet de l'objet qui est ici un effet négatif.

Un objet qui présente un avantage et qui est désiré par un individu se verra attribuer une valeur d'effet; et s'il présente une utilité, il se verra attribuer une valeur intrinsèque. De la même manière, la valeur négative sera attribuée à un objet qui présente soit une utilité négative, soit un avantage négatif. Afin de caractériser plus précisément les notions d'utilité négative, EHRENFELS aura recours aux notions de "Schaden » et de « Schädlichkeit » qui peuvent se traduire par dommage et nuisance (p.40). En effet, pour exprimer le contraire de " Nutzen» ou utilité, on choisit généralement le mot "Schaden» ou dommage. Mais pour distinguer le dommage, ou " Schaden ", du dommage correspondant à un avantage négatif, EHRENFELS utilise pour sa part le mot "Schädlichkeit », lequel peut se traduire en français par le terme "nuisance ». La nuance de sens entre " dommage » et " nuisance " est certes plus importante que celle qui existe entre "Schaden " et "Schädlichkeit » dans le contexte posé par EHRENFELS, mais les deux mots restent synonymes et sont facilement compréhensibles dans le problème qui nous préoccupe.

Définir un objet comme nuisible signifie qu'il a une certaine tendance à produire de la non-valeur intrinsèque. Par dommage, EHRENFELS entend la mesure de la non-valeur intrinsèque que l'objet produit effectivement. Pour illustrer ces notions, il prend l'exemple d'un domaine viticole menacé par une invasion de sauterelles (p.42-43). Si les sauterelles arrivent jusqu'aux vignes, elles vont les détruire et la perte sera grande pour le viticulteur. Cependant, celui-ci refusera, selon EHRENFELS, de payer pour leur élimination car, si l'essaim est important, elle ne pourra être que partielle et la perte d'une partie du vignoble ne pourra être évitée. Il affirme en outre que « la nuisance tout comme le dommage réel présumé sont très grands, par contre le dommage dépendant de l'existence de l'objet [les sauterelles] - qui sert à établir la non-valeur d'effet - sera égale à zéro ${ }^{51}$.

EHRENFELS veut créer un parallèle avec la valeur quasi-nulle de l'eau et de l'air : étant donné que ces éléments sont présents en grande quantité dans notre

\footnotetext{
${ }^{51}$ « Hier ist also die Schädlichkeit sowie der präsumtive tatsăchliche Schaden sehr groß, der « von der Existenz des Objektes [...] als abhängig erachtete Schaden » aber - die Größe, nach welcher der Wirkungsunwert bestimmt wird - gleich Null ", in EHRENFELS, 1982, p.43.
} 
environnement (du moins dans certaines régions du monde, pourrait-on ajouter), une unité supplémentaire ou une unité de moins de ces éléments n'affecterait pas notre bien être de manière significative. Dans l'exemple ci-dessus, la quantité de sauterelles est telle qu'une élimination partielle de celles-ci ne réduirait pas le dommage réel dû à leur existence d'où la non-valeur d'effet nulle. Il faut admettre que la formulation de ses idées dans ce passage est quelque peu confuse.

Il est difficile de s'imaginer un viticulteur n'accordant qu'une non-valeur d'effet insignifiante à un essaim de sauterelles menaçant ses vignes. Encore une fois, la non-valeur d'effet est établie par rapport au dommage dépendant de l'existence de l'objet et le dommage est à la valeur négative ce que l'utilité est à la valeur positive. L'utilité est liée à la notion de bien être, on peut alors en déduire que le dommage est lié à la notion de non bien être ou mal être. Dans l'exemple d'EHRENFELS, le mal être du viticulteur ne diminuerait pas même s'il arriverait à éliminer une partie des sauterelles car leur nombre fait qu'il en restera suffisamment pour détruire en partie son domaine. En ce sens, le dommage lié à l'existence des sauterelles par rapport auquel la non valeur d'effet s'établit, serait quasi-nul.

Autant les notions de non valeur intrinsèque et de non valeur d'effet sont relativement faciles à comprendre et à utiliser, autant celles de dommage et de nuisance sont plus difficiles à saisir selon la définition d'EHRENFELS.

La notion de non-valeur n'est donc pas négligée par EHRENFELS, au contraire elle trouve ici une place dans sa théorie générale de la valeur. La non-valeur est le pendant naturel de la valeur ou valeur positive. On a vu également que ce type de valeur fut surtout étudié en philosophie, beaucoup plus qu'en économie. Néanmoins, il serait tout à fait possible d'en trouver quelque usage dans le quotidien, également dans le quotidien économique. Par exemple dans le cas d'une personne ayant pour objectif ou but ultime d'atteindre un poids qu'elle considère comme idéal. Les aliments contenant du sucre se verront accorder une non valeur d'effet par cette personne : leur existence crée un dommage affectant de manière négative son bien être et l'éloigne de son objectif. Au contraire, cette même personne va accorder une grande valeur d'effet à des aliments lui permettant d'atteindre son objectif. Ainsi peut-on comprendre que cet objectif poursuivi par un nombre de personnes de plus en plus grand, a conduit l'industrie à produire de plus en plus de ces produits.

Les différents types de valeur présentés par EHRENFELS nous permettent en fait d'expliquer quasiment toutes les situations quotidiennes dans lesquelles on trouve des évaluations. Et même si les notions de valeur intrinsèque, d'effet ou encore de non valeur, ne sont pas véritablement nouvelles, leur définition par le philosophe autrichien les rend par contre utilisables dans beaucoup de contextes, notamment 
dans le contexte économique. C'est là que se situe l'intérêt principal de cette théorie. Ceci sous-entend de notre part que les explications des sciences économiques en la matière sont insuffisantes et nous verrons dans notre troisième partie comment, à l'aide de la présente théorie, nous pourrions les compléter. Mais avant cela, étudions un autre aspect innovateur de la théorie d'EHRENFELS, celui du mouvement des valeurs. 


\section{III - Les mouvements de valeur et leurs déterminants}

Christian von EHRENFELS considère la valeur comme étant un phénomène non statique. Par "mouvement de valeur", qui est la traduction de "Wertbewegung ", nous voulons désigner en fait les changements que subissent les valeurs dans le temps. Un changement de la valeur attribuée est la conséquence logique d'un changement dans la valorisation effectuée par l'individu, c'est-à-dire que ce dernier aura révisé son jugement sur l'objet désiré. Cette modification intervient, rappelons-le, dans le cadre de la relation qui s'est établie entre un individu désirant et un objet désiré. Autre hypothèse établie, cette relation est de nature subjective, elle est donc soumise aux variations de l'état émotionnel de l'individu ou plus exactement à celles de ses dispositions émotionnelles. Après avoir défini plus précisément cette notion, nous allons nous intéresser aux facteurs qui peuvent influencer ces dispositions et qui sont donc à l'origine d'un changement de la valeur attribuée. Outre les dispositions émotionnelles, un autre élément peut entraîner un mouvement de valeur : le but poursuivi par l'individu. En effet, lorsqu'un individu désire un objet, il va agir dans le but de le posséder. L'action qu'il va mettre en œuvre aura des conséquences sur sa valorisation de l'objet. En nous intéressant plus en détail à cette action, nous mettrons en valeur les facteurs susceptibles d'induire une modification du but poursuivi et donc de la valeur accordée.

\section{1 - Les dispositions émotionnelles}

A la question, existe-t-il des valeurs qui ne changent pas, EHRENFELS répond logiquement par la négative. La valeur est accordée à un objet en fonction du désir projeté sur cet objet. Le désir est un sentiment, un état d'esprit durant lequel l'individu est capable de désirer. Il s'agit plus exactement d'une disposition d'esprit de l'individu, laquelle est dominée par des émotions comme l'envie. Ainsi, le désir est déterminé par les dispositions émotionnelles de l'individu. Cette affirmation en implique deux autres : d'abord que le processus d'attribution de la valeur est un processus individuel. Ensuite que les dispositions émotionnelles ont un caractère relatif au temps et à l'environnement de l'individu. Par définition, une disposition désigne le moment durant lequel un certain état d'être, au sens d'état psychologique de l'individu, apparait, ce qui se répercute par la suite sur le comportement de l'individu. Cet état d'être peut être inné ou acquis et dépend dans tous les cas de l'environnement de l'individu ${ }^{52}$.

52 ARNOLD Wilhelm, EYSENCK Hans Jürgen et MEILI Richard (eds), “ Lexikon der Psychologie ", 1996, p.381. 
Par ailleurs, le désir, en tant que désir pour une chose, peut se référer à une chose ou à un objet existant comme à une chose ou à un objet qui n'existe pas dans les faits mais qui est imaginé ou pensé par l'individu. Par exemple, j'accorde de la valeur à mon bonheur futur dans ma vie, sans pour autant y penser dans le présent. Désirer son propre bonheur dans sa vie future est du domaine du possible, mais ce désir se concrétise dès que son objet est évoqué : EHRENFELS parle de dispositions du désir ou plus exactement de "Begehrensdisposition». Les "Begehrensdispositionen " désignent les dispositions ou l'état psychique dans lequel se trouve l'individu qui désire quelque chose.

Encore une fois, le processus d'attribution de la valeur contient des composantes psychologiques qui vont influencer la valeur elle-même. Plus précisément, ces composantes psychologiques sont des sentiments, des émotions qu'EHRENFELS regroupe sous le terme générique de "Gefühlsdispositionen " ou dispositions émotionnelles. Les dispositions émotionnelles ou "Gefühlsdispositionen 》 se définissent d'une manière générale par "l'art et la manière de réagir à des représentations [d'objets ou de choses au sens large du terme] et des jugements avec envie ou non ${ }^{53}$. La notion de "Gefühlsdispositionen " peut se traduire en français par le mot "disposition", dans le sens "état d'esprit à un moment donné $"$ " , auquel on peut ajouter l'adjectif " émotionnelle " pour insister sur le caractère possiblement variable de cette disposition ainsi que sur le fait qu'elle soit liée à un sentiment. Ce sentiment peut être le désir pour un objet ou une chose et EHRENFELS redéfinit les dispositions émotionnelles en fonction du désir de la manière suivante: elles représentent les capacités de lier certains sentiments d'envie et de non-envie avec certains phénomènes psychiques ${ }^{55}$. Nous allons nous intéresser dans un deuxième temps aux phénomènes psychiques qui sont liés au sentiment d'envie ou de non-envie. Pour l'instant, voyons plus précisément la relation entre l'envie, les dispositions émotionnelles et les valeurs, et essayons de répondre à la question de la détermination des dispositions émotionnelles.

Les dispositions émotionnelles sont associées à des circonstances, à des événements. Quel genre d'événement ou de circonstance favorise les dispositions du désir? Etant donné encore une fois l'hypothèse de la subjectivité de l'individu, il n'est malheureusement pas possible d'établir de loi concernant les émotions selon EHRENFELS. Mais il est par contre possible d'affirmer que d'une manière générale, les événements qui sont liés directement ou indirectement à la survie de

\footnotetext{
${ }^{53}$ " [...] die Art und Weise, wie er auf Vorstellungen und Urteile mit Lust oder Unlust reagiert ", in EHRENFELS, 1982, p.45.

${ }_{54}$ Dictionnaire Le Petit Larousse Compact 2004.

55 «Als Gefühlsdisposition bezeichen wir die Fähigkeiten, mit bestimmten psychischen Phänomenen bestimmte Gefuihle der Lust und der Unlust zu verbinden ", in EHRENFELS, 1982, p.51.
} 
l'individu ("Selbsterhaltung ») et de l'espèce ("Arterhaltung ») favorisent une disposition émotionnelle qui provoque un sentiment d'envie. De plus, l'individu va généralement rechercher ce qui est agréable (" das Lustvolle ») et éviter ce qui est douloureux ("das Unlustvolle "). Tout ce qui est utile à notre survie va provoquer un sentiment d'envie, et on va par conséquent accorder de la valeur intrinsèque à la chose qui a éveillé cette envie, et modifié par la même nos dispositions émotionnelles en ce sens. Par exemple, le fait de désirer des enfants est lié à la survie de l'espèce. Celui de vouloir une alimentation équilibrée est lié à notre propre survie. L'instinct de survie crée donc une disposition d'esprit qui nous met dans une position d'être désirant ou ayant des envies pour des objets ou des choses et nous permet d'atteindre un but ultime en rapport à notre propre survie.

Cependant, cette règle comporte quelques exceptions: certains individus accordent de la valeur à ce qui est mauvais ou néfaste à leur survie. Par exemple, les alcooliques, les drogués ou encore les amateurs de sports extrêmes. Ces personnes éprouvent du désir vis-à-vis d'objets qui semblent entrer en contradiction avec l'instinct de survie.

Enfin, étant donné que la valeur est subjective et que sa détermination se fait par l'individu désirant, on pourrait être amené à penser qu'elle est relativement instable. EHRENFELS ne nie pas une certaine instabilité de la valeur d'effet, mais il affirme également que la valeur intrinsèque est relativement stable (p.50). Ainsi, l'effet provoqué par la possession d'un objet varie en fonction des circonstances dans lesquelles se trouve ou agit l'être possédant : l'effet de la consommation d'un morceau de pain peut être plus important lorsque la faim est présente que lorsqu'elle ne l'est pas $^{56}$. Par contre, la valeur de l'objet elle-même, sa valeur intrinsèque, ne varie que peu ou pas selon les circonstances : la valeur d'un objet que l'on désire pour lui-même est peu influencée par le contexte. Par exemple, la valeur accordée à la dégustation d'un bon vin reste la même, peu importe le temps, l'âge de la personne ou son état de santé. Selon EHRENFELS, on ne va parler d'un changement de la valeur intrinsèque que dans le cas d'un changement durable des dispositions émotionnelles.

Avant de s'intéresser aux facteurs pouvant causer une modification des dispositions émotionnelles, signalons que les théories modernes concernant les dispositions de l'individu en psychologie, voient en elles des " traits de caractère dans lesquelles se reflètent des tendances du comportement innées ou acquises $"$ ". Un type de théorie du nom de "Wechselwirkungstheorien » ou

\footnotetext{
${ }^{56}$ Voir à ce propos les notions de valeurs temporaires et de valeurs normales.

${ }^{57}$ FROHLICH Werner D. (éd.), « Worterbuch der Psychologie », 1998 et ALLPORT Gordon W., " Personlichkeit », 1960.
} 
théories des interactions, suit un chemin cognitif et décrit la personnalité de l'individu comme un système d'espérances par rapport aux conséquences de ses agissements et qui lui permettent de planifier une action ${ }^{58}$. Cette voie, qui fut développée par FREUD, fut également employée par EHRENFELS. La perspective de ce dernier se trouve donc confortée par ses contemporains en psychologie et par le fait que les dispositions émotionnelles sont influencées autant par des facteurs internes que par des facteurs externes.

\section{2 - Les facteurs influençant les dispositions émotionnelles}

Comme facteurs d'influence des dispositions émotionnelles, le philosophe autrichien distingue ceux qui sont d'ordre psychologique de ceux qui sont d'ordre physiologique. Pour chacun de ces deux types de facteurs, il y en a qui sont connus et d'autres qui sont moins ou pas du tout. EHRENFELS classe les facteurs influençant les dispositions émotionnelles en deux catégories :

- la catégorie des causes psychologiques connues,

- la catégorie des causes psychologiques inconnues qui comprend également les causes physiologiques connues et inconnues.

Enfin, il énonce les facteurs extérieurs d'influence, c'est-à-dire les situations dans lesquelles un individu, de par son comportement, va influencer un autre individu et modifier par la même ses dispositions émotionnelles.

\subsection{1 - Les facteurs psychologiques inconnus influençant les dispositions émotionnelles}

Parmi les facteurs psychologiques inconnus qui influencent les dispositions émotionnelles, on peut distinguer trois sous-catégories :

- Les dispositions émotionnelles provoquées par des dispositions innées, autrement dit les causes innées des dispositions émotionnelles. Certaines dispositions peuvent en effet être héritées des parents, sous la forme de prédisposition dans le cas par exemple de "la dégustation d'alcool, l'amour de la musique, du sexe,... »(p.52). Ces prédispositions n'apparaissent pas forcément dès la naissance mais se manifestent dans la plupart des cas avec le temps.

- Le développement organique ou «Ursachen des organischen Entwicklungsganges ». Au cours des différents âges de la vie apparaissent ou

\footnotetext{
${ }^{58}$ HERMANN et LANTERMANN (eds), « Persठnlichkeitspsychologie : Ein Handbuch in Schlüsselbegriffen ", 1985.
} 
disparaissent certaines dispositions émotionnelles pendant que d'autres sont modifiées. EHRENFELS donne l'exemple du désir sexuel qui est une disposition émotionnelle qui apparaît à l'adolescence.

- Les causes totalement obscures ou "vollkommen dunkle Veränderungsursache " qui sont les causes qui ne sont ni héritées, ni liées à l'âge de la personne. Autrement dit, ce sont les causes spontanées, celles qui, on ne sait pourquoi, provoquent une disposition émotionnelle donnée (elle peut être une manifestation de l'inconscient de l'individu).

Ces causes peu connues, du moins par EHRENFELS, de l'apparition, de l'épanouissement, du développement, du rajeunissement, de la disparition des dispositions émotionnelles, constituent néanmoins les forces fondamentales du changement. Le développement organique conduit, chacun peut le constater, à des changements des dispositions individuelles vis-à-vis d'objets particuliers. Les causes de ces changements peuvent s'expliquer aujourd'hui. Par exemple dans le cas du désir sexuel, on sait que les hormones jouent un rôle incontournable. Certaines représentations de la conscience et la mémoire sont par ailleurs des facteurs que l'on peut qualifier d'innés et qui influencent les dispositions émotionnelles, dans la mesure où les individus sont dotés dès leur naissance de la capacité de représentation comme de celle de la mémoire ${ }^{59}$. Quant aux causes totalement obscures, il nous est difficile de les commenter car cela demanderait une connaissance approfondie des mécanismes de l'inconscience, un domaine qui se situe en dehors de nos compétences.

A côté de celles-ci, ils existent également des facteurs psychologiques explicables et descriptibles.

\subsection{2 - Les facteurs psychologiques connus influençant les dispositions émotionnelles}

Outre les causes psychologiques inconnues, les dispositions émotionnelles peuvent être également influencées par des causes qu'il est possible de préciser. Parmi celles-ci, on trouve l'habitude, la " déshabitude ${ }^{60}$, l'association, le transfert émotionnel, l'illusion et le désir.

Le premier facteur identifiable est l'habitude. Elle apparaît à travers la répétition d'une action. Cette habitude peut provoquer un désir croissant ou décroissant. Par exemple, le désir décroît dans le cas du repas : on a l'habitude de manger à un certain moment de la journée et l'heure du repas déclenche l'envie de manger à

\footnotetext{
${ }^{59}$ ZIMBARDO Philip G., « Psychologie », 1995, p.444.

${ }^{60}$ Nous nous permettons ce néologisme afin de retranscrire au mieux le mot allemand originel "Entwöhnung".
} 
travers le phénomène de l'habitude. Cependant cette envie décroît à mesure que le besoin est satisfait. Dans le cas du fumeur, l'habitude provoque un désir croissant de fumer: l'envie de fumer ne disparaît pas après une cigarette, elle est au contraire entretenue par le fait de fumer. Dans ce cas, comme dans celui de l'alcool par exemple, l'habitude crée une disposition favorable à leur consommation, c'est à dire un désir de consommer ces deux produits. En définitive, l'habitude finit dans ce genre de cas par créer un besoin. Dans la psychologie moderne, l'habitude est considérée chez Clark HULL (1884-1952) par exemple, comme une réaction répétée à un stimulus identique. L'individu apprend à agir de manière identique lorsqu'il rencontre une situation qu'il connaît ${ }^{61}$.

Après l'habitude, une autre cause identifiable est celle de la « déshabitude » ou la perte d'habitude: un besoin qui reste longtemps insatisfait renforce dans un premier temps l'envie, le désir éprouvé envers l'objet permettant de satisfaire ce besoin. Cependant, après un lapse de temps relativement long, selon les individus, cette envie ou ce désir disparait à travers une certaine lassitude. Une personne qui désire longtemps un objet sans jamais pouvoir se le procurer va finir par abandonner l'idée de vouloir se l'approprier.

L'association est la troisième cause identifiable qui induit un changement dans les dispositions émotionnelles. Lorsqu'on associe un sentiment à un souvenir, ce dernier va influencer le sentiment au point de pouvoir provoquer une envie ou, son contraire, le rejet d'un objet. Par exemple, son envie d'aller dans la ville où l'on a fait ses études est liée souvent au sentiment de bien être éprouvé lors du temps des études et ce sentiment est associé à cette ville. Il faut cependant faire attention aux risques de confusion entre l'association et l'habitude. L'habitude peut provoquer un désir, par exemple l'habitude de manger un certain plat peut finir par en provoquer le désir, alors que l'association ne crée pas de désir, celui-ci est déjà présent. L'association est un concept qui est également présent chez Sigmund FREUD sous la dénomination "libre association » et qui désigne une méthode d'analyse du patient par le psychanalyste ${ }^{62}$. Dans cette méthode, le psychanalyste demande à son patient de lui révéler ce qu'il associe à des mots ou des objets précis.

Un changement des dispositions d'un individu peut en outre être provoqué par un transfert émotionnel. Par transfert émotionnel, EHRENFELS entend le transfert du sentiment d'envie ou de non-envie de la conséquence d'une action à sa cause, c'est-à-dire qu'au lieu de valoriser le but de l'action, notre attention va plutôt se

\footnotetext{
${ }^{61}$ Voir à ce propos HULL Clark L., « Principles of Behaviour », 1966.

${ }^{62}$ Voir à ce propos FREUD Sigmund, « Vorlesungen zur Einführung in die Psychoanalyse », volume 1, 2000, p.454-457.
} 
porter sur le moyen utilisé pour arriver au but. Dans ce cas, c'est donc le moyen de l'action, et non sa conséquence, qui va induire un changement de nos dispositions émotionnelles. La notion de transfert émotionnel est également utilisée par Sigmund FREUD, cependant dans un sens différent. FREUD définit le transfert émotionnel ${ }^{63}$ comme les souhaits inconscients de l'enfance du patient qui refont surface et qui sont projetés sur le thérapeute. Les modèles infantiles sont donc répétés et vécus comme étant actuels.

L'illusion ou "Einbildung " est également évoquée comme facteur d'influence. L'illusion est le fait d'être convaincu de pouvoir simuler des capacités psychiques que l'on possèderait. Concrètement, l'individu s'imagine pouvoir résister au désir pour un objet ou encore, simule le dégoût vis-à-vis d'un objet, ce qui change en fait sa relation avec celui-ci et donc son évaluation de l'objet. La définition donnée par EHRENFELS se rapproche de la définition générale de cette notion en psychologie où l'illusion désigne un élément imaginé qui ne correspond pas à la réalité. Cette capacité à imaginer les choses est également vue comme la source d'une certaine créativité de l'esprit ${ }^{64}$.

Enfin le désir lui-même est une des raisons du changement des dispositions émotionnelles. Le désir pour un état émotionnel peut provoquer un changement des dispositions émotionnelles. Par exemple, la narration d'un conte de fées peut provoquer chez l'individu qui écoute l'envie d'être le héros et influencer de cette manière son comportement et donc ses dispositions émotionnelles. La pratique d'une religion peut vouloir signifier pour la personne croyante un désir d'atteindre un comportement déterminé, notamment un comportement émotionnel déterminé, ce qui va influencer ses dispositions émotionnelles.

Ainsi a-t-on décrit les causes des changements des dispositions émotionnels et donc des changements des valeurs et non-valeurs intrinsèques. Dans la vie quotidienne, les facteurs évoqués ne se manifestent pas de manière isolée mais plutôt sous forme de combinaison de plusieurs d'entre elles et les possibilités de combinaison sont nombreuses.

\footnotetext{
${ }^{63}$ SCHUSTER Peter et SPRINGER-KREMSER Marianne, " Anwendungen der Psychoanalyse ", 1998, p. 128; FREUD Sigmund, « Vorlesungen zur Einfuhrung in die Psychoanalyse », volume 1, 2000, p.415-430 et « Schriften zur Behandlungstechnik », 2000, p.159-168.

${ }^{64}$ ARNOLD Wilhelm, EYSENCK Hans Jürgen et MEILI Richard (eds), 1996, p.428-429.
} 


\subsection{3 - Les influences extérieures}

Outre les changements des dispositions émotionnelles intervenant au niveau de l'individu lui-même, il existe des formes d'influence extérieure, c'est-à-dire des formes d'influence d'un individu sur autrui ou vice-versa, qui vont provoquer une modification des dispositions émotionnelles de l'individu sous influence. Autrement dit, le comportement d'autrui peut influencer ses propres dispositions et donc ses désirs personnels. Cette influence peut prendre la forme de la contrainte, de l'effet à travers l'exemple, de la suggestion ou de l'influence à travers l'ordre.

Une contrainte régulière influe l'état des dispositions émotionnelles d'autrui à travers l'habitude et la "déshabitude ». Par exemple le fait d'aller à l'école est une contrainte régulière qui prend la forme d'une habitude avec le temps. La contrainte peut également être associée à un transfert émotionnel. Dans ce cas, EHRENFELS différencie l'influence de ce phénomène sur les individus dotés d'une capacité intellectuelle supérieure aux autres ${ }^{65}$ : les moins bien dotés vont, selon lui, transférer leur sentiment de non-envie de la punition sur la cause de cette punition c'est à dire sur l'acte appelant la punition. Pour les personnes mieux dotées, la non-envie se transfert sur le punissant lui-même, le "Strafende », c'est à dire sur le fait d'être puni. La contrainte s'applique comme on l'a vu, en relation avec une punition, mais également avec une récompense. Par exemple, la contrainte d'étude pour un examen peut se solder par la récompense de se voir reçu à ce même examen. La contrainte enfin, possède l'effet le plus visible et le plus efficace sur l'individu. La contrainte comprend en psychologie deux aspects distincts : elle peut être externe, comme dans la définition d'EHRENFELS, ou interne. Les contraintes internes ou névroses ont fait l'objet d'une analyse approfondie par Sigmund FREUD ${ }^{66}$ et continuent d'intéresser la psychologie ${ }^{67}$.

L'influence d'autrui sur nos dispositions émotionnelles peut aussi se faire à travers l'effet de l'exemple. L'influence, dans ce cas précis, s'effectue autant sur les jeunes que sur les adultes et se manifeste sous la forme d'une imitation ou d'une identification. Lorsque la personne admirée ou considérée comme digne d'admiration effectue un geste de dégoût devant une situation donnée, l'enfant par

\footnotetext{
${ }^{65}$ " Versuche, mittelst Zwanges nach dem Gesetz der Gefühlsübertragung zu wirken, sind meist nur bei tiefstehenden Intellekten von Erfolg begleitet. [...] Bei etwas ausgebildeteren Intellekten jedoch und wo nicht große Liebe und Hingebung fur den Strafenden vorhanden, uberträgt sich der Unwille statt auf die gestrafte Tat vielmehr auf den anderen Teil der Ursache - den Strafenden selbst, welcher statt der Tat nun um der Strafe willen verhaßt wird " in EHRENFELS, 1982, p.55.

${ }^{66}$ FREUD Sigmund, "Vorlesungen zur Einfuhrung in die Psychoanalyse », 2000, p.245-447.

${ }^{67}$ Voir par exemple TOMAN Walter, « An Introduction to Psychoanalytic Theory of Motivation », 1960 ; FENICHEL Otto, " Psychoanalytische Neurosenlehre », Volume 3, 1977.
} 
exemple aura tendance à imiter ce geste dans les mêmes circonstances. L'enfant va donc associer une situation à un geste, un sentiment qu'il aura imité. Chez les adultes, le cas se produit lorsqu'une personne au caractère affirmé influence celle à la personnalité plus faible ${ }^{68}$. D'une manière générale dans le monde contemporain, les individus cherchent à imiter les personnalités médiatiques, en particulier les célébrités du monde du spectacle et du sport. L'imitation peut se faire de manière consciente ou de manière inconsciente, en particulier chez les enfants.

La suggestion est le troisième type d'influence qu'il convient maintenant d'évoquer. La suggestion est l'acte par lequel un individu arrive à convaincre un autre individu que ce dernier possèderait des capacités physiques ou morales déterminées. Un exemple donné par EHRENFELS est celui de l'hypnose ou encore celui des personnes se laissant convaincre par une autre à la forte personnalité. La suggestion est utilisée en psychothérapie pour traiter les patients. Cette méthode consiste à transmettre au patient une idée positive qui induit un changement de son état de santé ${ }^{69}$. La suggestion influence donc les dispositions émotionnelles de l'individu et par conséquent sa valorisation d'objets déterminés.

Enfin, le dernier type d'influence est celui de l'influence à travers l'ordre. L'ordre s'exprime le plus souvent sous la forme d'une menace explicite, d'une contrainte : "Fais-le sinon... ", ou " si tu ne le fais pas alors... »

Il éveille chez l'individu qui le reçoit la disposition émotionnelle appropriée, c'est à dire qu'il va se soumettre à l'ordre en question. Si un ordre est donné souvent ou régulièrement, il va créer une disposition émotionnelle durable (par exemple dans le cas du conditionnement militaire). L'ordre peut également prendre la forme d'une suggestion et fonctionne alors à la manière de l'exemple par l'association. A travers l'exemple, on suggère un comportement, une manière d'être qui peut être en réalité un ordre. Il ne faut cependant pas confondre l'influence de l'ordre par l'exemple et l'influence de la suggestion: dans le cas de la suggestion, celui qui veut influencer autrui n'est pas sûr du résultat. Dans le cas de l'ordre, autrui aura plutôt tendance à obéir, c'est-à-dire que l'influence y sera plus certaine et plus explicite.

Nous avons ainsi évoqué à travers cette liste de facteurs les différents types d'influences extérieures que peuvent subir les dispositions émotionnelles. Cellesci vont alors varier en fonction de leur environnement et engendrer en conséquence un changement dans les valorisations effectuées par l'individu.

\footnotetext{
${ }^{68}$ Voir également BANDURA Albert, "Lernen am Modell », 1976 et ZUMKLEY-MÜNKEL Cordula, "Imitationslernen. Theorien und empirische Befunde ", 1976.

${ }^{69}$ Voir KATZENSTEIN Alfred, "Suggestion und Hypnose in der psychotherapeutischen Praxis ", 1978.
} 
Ce paragraphe concernant les facteurs d'influence des dispositions du désir se révèle intéressant pour plusieurs raisons. D'abord, il montre que le fait de désirer n'est en aucun cas lié exclusivement à la satisfaction des besoins. Les éléments qui font que l'individu est en mesure de désirer ou pas sont, par ailleurs, de deux natures: psychologiques et physiologiques. Une partie de ces facteurs est identifiée ici par EHRENFELS et cela nous permet de mieux comprendre le phénomène de la valeur dans son ensemble. Il attire enfin notre attention sur le fait que le processus de valorisation de l'individu peut être le résultat de l'influence d'autrui, une information négligée dans les théories de la valeur en économie. Le troisième groupe de facteurs susceptibles d'influencer les dispositions du désir de l'individu est celui qui est lié au dessein de l'individu.

\section{3 - Les mouvements de valeur en fonction du dessein de l'individu}

Après avoir présenté les mouvements de valeurs en fonction des influences psychologiques, nous allons nous intéresser à une seconde catégorie de mouvements de valeur définie par EHRENFELS comme celle des mouvements en fonction des buts poursuivis ou « Wertbewegung in der Zielfolge » (p.60-62).

A chaque fois que nous agissons, nous avons un but en tête. Le but de notre action va être un facteur déterminant dans l'établissement des valeurs. Chaque élément d'une action (cause, moyen, but ou effets directs) peut être valorisé selon la nature de l'action et son but. Ainsi le moyen sera valorisé comme élément permettant d'atteindre le but ; le but lui-même sera valorisé en tant que tel; enfin les effets directs de l'action seront valorisés comme éléments dérivant de l'action quand son but a été atteint.

Le processus d'attribution de la valeur lui-même peut aussi faire l'objet d'une estimation. Autrement dit, je peux donner de la valeur au fait d'accorder de la valeur à un objet donné. Et dans ce cas, toutes les combinaisons sont possibles entre les types de valeurs accordées à la valorisation elle-même et à l'objet valorisé. Par exemple, l'alcoolique qui accorde de la valeur propre à la consommation d'alcool, peut en revanche ne pas supporter le fait qu'il accorde de la valeur à la consommation d'alcool. C'est-à-dire, le fait d'accorder de la valeur ou d'estimer la consommation d'alcool a pour lui une non-valeur intrinsèque alors que l'alcool lui-même a une valeur intrinsèque, voire dans certains cas, une valeur d'effet (pour celui qui cherche à oublier ses problèmes par exemple). L'alcoolisme est considéré de nos jours comme une maladie où le patient est dans une relation de dépendance par rapport à l'alcool. Il aime l'alcool pour ses effets, 
c'est-à-dire pour le fait qu'il l'aide à oublier les raisons de son mal être ${ }^{70}$. Cependant, le patient n'aime pas forcément l'état dans lequel il se trouve.

En considérant le ou les buts d'une action dans la détermination de la valeur, on peut mieux comprendre les mouvements de valeur en fonction du dessein de l'individu. Lorsque le but d'une action change, c'est-à-dire lorsqu'il y a un mouvement du but d'une action, la valeur accordée à cette action ou à l'un de ses éléments va également changer. Prenons notre exemple de la cuisine : si le but de l'action (cuisiner) est de se nourrir ou si le but de cette action est de nourrir une certaine clientèle dans le cadre d'un restaurant, l'action ne se verra pas accorder la même valeur par l'individu. L'action est la même dans les deux cas, mais son but est différent et sa valeur va par conséquent elle aussi changer : si l'individu aime plus cuisiner pour les autres que pour lui-même, il va accorder deux valeurs différentes aux deux actions. On peut considérer le problème de manière différente : selon que l'individu cuisine des plats dans le but de flatter le palet des personnes qui vont les déguster ou qu'il cuisine dans le but de faire une cuisine qui plait au plus grand nombre, la valeur du premier sera plus élevée que celle du second. Dans l'exemple énoncé ci-dessus, l'action (cuisiner) est le moyen similaire d'atteindre des buts différents aux valeurs différentes.

Des mouvements de valeur peuvent se produire lorsque le but de l'action change mais également lorsque le but de l'action n'est plus l'élément qui est valorisé. Lorsque l'objet de valorisation n'est plus le but mais le moyen, EHRENFELS parle de " mouvement de valeur vers le bas "; lorsque ce n'est plus le but mais les effets produits par les conséquences de l'action, EHRENFELS parle de " mouvement de valeur vers le haut»; les mouvements de valeurs peuvent aussi se produire vers le côté ou vers l'intérieur. Nous allons reprendre chacun de ces cas en détail.

Ce qui au départ vaut comme moyen d'atteindre un but, peut lui-même devenir le but de l'action. Par exemple celui qui travaille, le fait dans un premier temps dans le but de pouvoir se nourrir avec le produit de son travail (soit en cultivant son jardin, soit en achetant du pain comme nous le dit EHRENFELS), mais dans un deuxième temps ce travail peut prévaloir sur le fait de pouvoir se nourrir avec le salaire perçu. Autrement dit, le travail lui-même et son produit, le salaire perçu, se voient attribuer une valeur intrinsèque. La preuve en est faite au moment de la retraite où l'absence de travail se fait sentir: la perte est alors grande pour l'individu. La valeur créée par le processus de mouvement vers le bas est appelée "valeur dérivée " ou " abgeleiteter Wert" (p.63), car elle dérive d'une valeur

\footnotetext{
${ }^{70}$ GERGER Silvia, « Chronischer Alkoholismus - ein Beitrag zur Geschichte der Pflege ", 2004, p.60-61. Voir également DICKHAUT Hans, « Der Alkoholkranke Patient », 1992.
} 
intrinsèque première. La valeur dérivée apparaît progressivement, elle est une valeur propre de l'objet qui garde cependant une valeur d'effet (le fait de travailler permet de se nourrir) qui reste supérieure à la valeur intrinsèque (celle accordée au travail dans notre exemple). La valeur dérivée peut être induite par d'autres individus à travers la suggestion, la contrainte ou encore l'exemple. Elle peut également se transmettre de génération en génération, à l'image des traditions. EHRENFELS parle alors de "valeurs dérivées transmises " ou "sukzessorisch abgeleitete Werte", à la différence des "valeurs dérivée individuelles" ou " individuell abgeleitete Werte » qui désignent donc les valeurs dérivées produites au niveau individuel comme dans l'exemple mentionné plus haut. La valeur dérivée ne peut par contre en aucun cas devenir une valeur intrinsèque, elle reste avant tout une valeur d'effet ${ }^{71}$.

La valeur dérivée aurait selon EHRENFELS une certaine importance en matière économique et cette importance serait décrite par Friedrich von WIESER dans ses publications. En effet, ce dernier affirme que les biens économiques sont évalués par rapport à leur utilité, et plus précisément par rapport à l'utilité de leurs effets ${ }^{72}$. Dans un exemple, il précise que celui qui se retrouve dans le désert va désirer l'eau et lui attribuer une très grande valeur d'effet dans la mesure où l'effet en question est le maintien en vie de l'individu (qui possède lui, une valeur intrinsèque $)^{73}$.

Les mouvements de valeur vers le haut désignent un changement de la valeur intrinsèque qui se dirige non plus sur le but de l'action mais sur les effets consécutifs à cette action. L'instinct de survie constitue un des facteurs qui provoque ce changement de valeur. Par exemple, le bébé accorde de la valeur à la faim et va agir en ce sens, alors que, selon EHRENFELS, l'adulte, lui, va accorder de la valeur à ce qui apaise sa faim c'est-à-dire à la nourriture. Pour le bébé ou l'enfant, le but de son action sera d'apaiser sa faim et manger aura par conséquent une grande valeur intrinsèque. Il n'accordera par contre pas d'importance à la qualité de ce qu'il va manger, alors que l'adulte saura différencier ce qui est bon pour lui de ce qui ne l'est pas. Il attribuera de la valeur à la nourriture qui servira à apaiser sa faim, mais qui lui permettra aussi de mieux vivre. Le but de l'action (ici, manger) est certes d'apaiser sa faim, mais l'individu va en fait, à travers cette action, valoriser sa survie puisque l'effet attendu du but de cette action est justement le maintien en vie de la personne qui a mangé. On parlera donc dans ce cas d'un mouvement de valeur vers le haut et les valeurs nées de ce mouvement seront nommées « valeurs supérieures » ou « übergeordnete Werte » (p.67).

\footnotetext{
${ }^{71}$ EHRENFELS, 1982, p.64, note 19.

${ }^{72}$ WIESER, Friedrich von, « Über den Ursprung und die Hauptgesetze des wirtschaftlichen Werthes ", 1884, p.79.

${ }^{73}$ Idem, p.81.
} 
Ce processus peut également, à l'image des valeurs dérivées, se produire au niveau de l'individu ou alors se transmettre entre les générations. Ceci nous amène à parler de « valeurs supérieures individuelles » et de " valeurs supérieures transmises ».

EHRENFELS évoque également le cas des valeurs supérieures qui sont transmises par des individus à forte personnalité, à travers l'exemple et la suggestion. Il parle alors d'une "valorisation hyperindividuelle" ou "überindividuelle Wertung» (p.67) qui se produit lorsque les individus en présence attendent de la personne qui les leur transmet une telle valorisation. On pense par exemple aux professeurs des écoles pour lesquels la transmission de valeurs faisant référence à un certain comportement en conformité avec la morale, et considérées ainsi comme supérieures, fait partie de la formation qu'ils doivent apporter à leurs élèves. Autrement dit, la transmission de ce genre de valeurs est partie intégrante de leur profession.

Les mouvements de valeurs vers le haut, c'est-à-dire le déplacement de l'objet de la valorisation du but de l'action aux suites attendues de celle-ci, peuvent être assimilés aux mouvements de valeur vers l'extérieur ou «Wertbewegung nach seitwärts ». Mais il s'agit cette fois-ci d'effets indirects, c'est-à-dire d'effets attendus et désirés mais non supérieurs au but de l'action, en ce sens où il ne concerne pas des valeurs telles que la survie de l'individu ou de l'espèce, qui sont pour EHRENFELS des valeurs supérieures. Ainsi on peut affirmer que les mouvements de valeurs vers haut ne sont qu'un cas particulier des mouvements décalés, lesquels créent des "valeurs adjacentes" ou " nebengeordnete Werte " (p.69).

Lorsque le but de l'action produit des effets abstraits, d'ordre émotionnel, sur l'individu, EHRENFELS parle de mouvement de valeur vers l'intérieur. Ces mouvements sont liés aux émotions ressenties par la personne agissant. Celles-ci ont un caractère abstrait et sont propres à chaque individu, de telle sorte que lorsqu'il agit et que son action atteint son but, les effets ressentis seront d'ordre émotionnel. Ces émotions auront une valeur dite «intrasèque " ou " eingeordneter Wert » (p.69).

Un changement dans la manière d'agir, ou plus précisément dans le but de l'action, peut entraîner un changement dans le processus de valorisation. Ce changement est décrit de manière précise par EHRENFELS qui envisage quasiment tous les cas de figure pour lesquels il trouve à chaque fois une nouvelle dénomination de la valeur. 
Ceci conduit à une certaine complexité de sa théorie que nous essaierons de pallier en partie grâce au tableau suivant :

\begin{tabular}{|l|c|c|c|}
\hline $\begin{array}{l}\text { Changement dans le } \\
\text { processus d'action }\end{array}$ & Type de valeur crée & Valeur globale & Valeur individuelle \\
\hline $\begin{array}{l}\text { Cas général : } \\
\text { changement dans le } \\
\text { processus de } \\
\text { valorisation de } \\
\text { l'action }\end{array}$ & Valeur dérivée & $\begin{array}{c}\text { Valeur dérivée } \\
\text { transmise }\end{array}$ & $\begin{array}{c}\text { Valeur dérivée } \\
\text { individuelle }\end{array}$ \\
\hline $\begin{array}{l}\text { Mouvement de valeur } \\
\text { vers haut : valeur } \\
\text { intrinsèque accordée } \\
\text { non plus au but mais } \\
\text { aux effets consécutifs } \\
\text { à l'action }\end{array}$ & Valeur supérieure & $\begin{array}{c}\text { Valeur supérieure } \\
\text { transmise }\end{array}$ & $\begin{array}{c}\text { Valeur supérieure } \\
\text { individuelle }\end{array}$ \\
\hline $\begin{array}{l}\text { Mouvement de valeur } \\
\text { vers le haut sous } \\
\text { l'influence d'autrui }\end{array}$ & hyperindividuelle & - & - \\
\hline $\begin{array}{l}\text { Mouvement de valeur } \\
\text { du but aux effets } \\
\text { secondaires ou } \\
\text { adjacents à l'action }\end{array}$ & Valeur adjacente \\
\hline $\begin{array}{l}\text { Mouvement de valeur } \\
\text { vers l'intérieur: } \\
\text { valorisation des } \\
\text { émotions produites } \\
\text { par le but de l'action }\end{array}$ & Valeur intrasèque & - & - \\
\hline
\end{tabular}

D'une manière générale, lorsqu'un changement intervient dans le processus de valorisation, c'est-à-dire qu'un autre élément de l'action attire plus particulièrement l'attention de l'individu, un nouveau type de valeur sera accordé : celui de la valeur dérivée qui peut soit être transmise entre les individus, soit être propre à l'individu.

Une valeur peut en remplacer une autre dans ce processus de changement et EHRENFELS va même plus loin en affirmant qu'il existe un "combat pour l'existence entre les différents types de valeur ${ }^{74}$. Il ajoute que chaque combat pour l'existence est en fait un combat pour les conditions d'existence. On devine dans ces affirmations une référence explicite au Darwinisme, cette référence devenant évidente lorsqu'EHRENFELS écrit que «toutes les écoles de physiologie s'accordent sur le fait que l'existence des êtres vivants peut être

${ }^{74}$ " [Es] soll nun dem bereits vorausgesetzten Tatbestand eines Kampfes ums Dasein zwischen den einzelnen Wertungen ein năheres Augenmerk zugewandt werden ", in EHRENFELS, 1982, p.70. 
assimilée à un processus d'adaptation et que dans cette perspective tout organisme se voit attribuer une capacité d'adaptation ${ }^{75}$. Comme les espèces vivantes, les valeurs doivent s'adapter et se battre pour survivre.

EHRENFELS effectue de manière originale un parallèle entre le combat pour la survie des espèces et celui des valeurs, également dans l'optique de leur propre survie. De même que la force de survie ou "Lebenskraft " est limitée, celle qui est dévolue à l'attribution des valeurs n'est pas infinie. Le fait de donner une valeur à un objet consomme une partie de l'énergie de l'individu, énergie qui encore une fois n'est présente qu'en quantité limitée. Le fait de procéder à une valorisation limite les possibilités d'une seconde valorisation simultanée et donc constitue un obstacle à l'apparition d'autres valeurs. En conséquence, chaque processus de valorisation possèderait selon EHRENFELS une propension à se transmettre à travers l'exemple, c'est-à-dire à se reproduire chez autrui et ainsi à survivre. Néanmoins ce combat entre différents types de valeur n'empêche pas de manière définitive l'apparition de plusieurs valeurs. Certaines valeurs étant interdépendantes, l'existence de l'une conditionnant celle de l'autre. Par exemple, l'individu ne peut valoriser la connaissance et l'envie d'apprendre que s'il valorise d'abord son existence (le premier n'est évidemment pas possible sans le second).

Par ailleurs, il convient de différencier le processus de dérivation de la valeur de celui de la dévalorisation. Dans le premier cas une valeur sera dérivée d'une autre qui l'aura précédée, ce qui se produit par exemple lors de grands bouleversements dans une société. Dans le second cas, la valeur disparaît au fur et à mesure, par exemple dans le cas d'un changement radical, et sur le long terme, dans l'environnement de l'individu. Pour EHRENFELS, les changements dans le mode d'alimentation des hommes ont provoqué la disparition de certaines valeurs liées aux modes plus anciens tels que la chasse par exemple, qui a disparu au profit de l'élevage. Pour chaque type des valeurs évoqués ci-dessus, le philosophe autrichien propose un terme précis afin de les qualifier : lorsque l'existence d'une valeur intrinsèque dépend de son existence concomitante comme valeur d'effet, il parle de " valeur intrinsèque dépendante " ou " abhängiger Eigenwert "; lorsque au contraire les valeurs intrinsèques perdent leur propriété comme valeur d'effet, il parle de " perte d'avantage » ou " Entfrommung » (p.75).

Le processus de perte d'avantage ne se produit pas toujours au niveau d'un individu, il peut se dérouler sur plusieurs générations. On parlera de valeur individuelle dépendante lorsque sa perte d'avantage intervient au niveau d'un seul individu et de valeur dépendante transmise lorsque sa perte d'avantage se fait au

\footnotetext{
75 " Darüber sind nämlich alle physiologischen Schulen einig, dass der organische Lebensprozess wesentlich als ein Assimilationsprozess aufzufassen sei, und dass mithin dem Organismus Fähigkeit zur Assimilation zukommen musse ", in EHRENFELS, 1982, p.70.
} 
fil des générations. De plus, il convient de souligner que les valeurs dérivées sont dans leur grande majorité des valeurs dépendantes dans la mesure où leur existence en tant que valeur intrinsèque (le moyen devient l'objet de la valorisation) dépend de la leur en tant que valeur d'effet (le but de l'action possède une valeur d'effet, sans laquelle le but ultime, le moyen, ne pourrait exister).

Par opposition aux valeurs dépendantes, les valeurs indépendantes ne sont que des valeurs intrinsèques dont l'existence n'est conditionnée par aucune autre valeur. Les valeurs indépendantes ont une existence qui ne peut être menacée, ni par un individu seul, ni par un ensemble d'individus sur plusieurs générations. C'est le cas des valeurs telles que la survie de l'individu ou celle de l'espèce qui ne peuvent être remises en question, et qui constituent à ce titre des valeurs indépendantes.

Dans les paragraphes précédents, les changements de valeurs concernaient avant tout ceux des valeurs intrinsèques, auxquels Christian von EHRENFELS accorde la primeur de sa présentation. Mais il n'oublie pas pour autant les valeurs d'effet. Des changements affectent également les valeurs d'effet à travers des processus parallèles à ceux des valeurs intrinsèques. Néanmoins les causes de ces changements sont quelque peu différentes : "des modifications surviennent à cause des changements de conviction de l'individu, de nouvelles capacités acquises, mais aussi à cause de ses relations différentes avec son environnement $"{ }^{76}$. Des précisions ne sont cependant pas apportées par EHRENFELS qui reconnaît par la même un manque dans sa théorie (p.83). Un changement de la valeur se produit également de manière plus fréquente et les changements de l'environnement sont beaucoup trop nombreux et complexes pour être synthétisés dans une seule théorie de la valeur. Néanmoins, il est facile de s'imaginer des situations dans lesquelles les valeurs d'effet ne sont plus attribuées au but de l'action proprement dit, mais au but de l'action en tant que moyen ou encore aux suites de cette action. Prenons l'exemple de l'achat d'une voiture de sport : l'individu qui s'offre une telle automobile le fait dans un premier temps dans le but d'affirmer son statut social. Le but de l'achat est donc de montrer sa réussite, qui se voit donc attribuer une valeur d'effet puisque la réussite sociale se mesure par rapport aux regards d'autrui et par comparaison à la position d'autrui. Dans un deuxième temps, la valorisation peut se focaliser non plus sur le but de l'action en tant que tel mais en tant que moyen, c'est-à-dire la réussite en tant que moyen de se faire accepter par une certaine catégorie de personnes par exemple.

\footnotetext{
${ }^{76}$ «[...] Modifikationen [treten] durch den Wandel menschlicher Überzeugungen und Kunstfertigkeiten, sowie durch die Veränderung der Verhăltnisse in der Außenwelt ", in EHRENFELS, 1982, p.83.
} 
Ce changement de valeur peut également s'effectuer de la manière suivante : l'individu va valoriser les effets consécutifs à son action, lorsque son but a été atteint. C'est le cas quand sa réussite entraîne, par exemple, la reconnaissance de ses pairs. Cette reconnaissance peut elle aussi avoir une valeur d'effet.

Dans ce paragraphe, les causes et les conditions de changements des valeurs ont été éclaircies à travers la théorie d'EHRENFELS. L'attribution d'une valeur est liée au désir qui est projeté sur un objet déterminé. Ce désir est lui-même lié aux dispositions émotionnelles de l'individu, c'est-à-dire son état psychique à un moment donné. Ses dispositions émotionnelles permettent à l'individu d'éprouver du désir pour une chose ou un objet. Les mouvements de valeurs sont entraînés par une variation des dispositions émotionnelles qui modifient à leur tour le désir pour l'objet ou plus exactement, le type de désir projeté sur l'objet. La question des mouvements de valeur est par conséquent celle des causes de la variation des dispositions émotionnelles. Ces causes sont multiples et peuvent être psychologiques comme physiologiques. Parmi les influences psychologiques connues, EHRENFELS énumère l'habitude, la "deshabitude ", l'association, le transfert émotionnel, l'illusion et le désir. Une disposition émotionnelle peut également être adoptée par un individu sous l'influence d'autrui, à travers les phénomènes de contrainte, de l'effet par l'exemple, de la suggestion et de l'ordre. La variation d'une valeur peut enfin être le résultat du changement de but dans une action. Le but d'une action est aussi l'objectif établi par l'individu qui désire et qui a les dispositions émotionnelles correspondantes. Un changement des dispositions émotionnelles peut entraîner un changement de l'objectif d'une action ainsi que de la valeur qui sera attribuée à travers cette action. Enfin EHRENFELS énonce les différentes possibilités de changements dans le processus de valorisation. Ces changements entrainent l'apparition de nouveaux types de valeurs lesquels contribuent à la grande précision de cette théorie.

Les deux aspects qu'il convient de souligner dans ce paragraphe sont les suivants : d'abord, la théorie d'EHRENFELS montre que les valeurs, quelque soit le domaine considéré, ne sont pas fixes. Au contraire, elles évoluent dans le temps. Ensuite, l'évolution des valeurs a des causes aussi bien internes (à l'individu) qu'externes (influence d'autrui). Cette vision des choses achève d'ancrer sa théorie dans la réalité. Notre paragraphe suivant concernera un autre élément important dans le phénomène de la valeur, celui du désir. 


\section{IV - Analyse du désir}

Dans l'attribution de la valeur, un élément déterminant est le désir. Concernant la notion de désir, remarquons que le philosophe autrichien utilise le mot "das Begehren" qui est, aujourd'hui, très peu utilisé et remplacé par "motif» ou "but de l'action " ${ }^{77}$. Le désir peut être assimilé à une émotion accompagnant la considération de l'objet. La valorisation de l'objet est fonction de l'importance du sentiment d'envie que l'individu éprouve à son égard. Ce sentiment étant lui-même lié au désir de l'individu, la valorisation dépend donc indirectement du désir. Il est aussi vrai que l'analyse du désir en tant que telle appartiendrait plutôt au domaine de la psychologie et c'est d'ailleurs une analyse de cette nature que nous propose EHRENFELS. La question que l'on pourrait se poser est celle de la pertinence d'une telle analyse en économie. Comme nous l'avons dit, le désir est avant tout une émotion et, en tant que telle, il revêt un aspect psychologique indéniable. Dans le courant néoclassique dominant actuellement les sciences économiques, le désir de l'agent économique s'exprime à travers ses préférences qui sont fonction de la satisfaction de ses besoins. Nous verrons dans notre troisième chapitre que ces axiomes sont très perfectibles, et qu'il serait plus réaliste de remplacer ceux-ci par une approche totalement différente, à l'image de ce que propose Amartya SEN, incluant le fait que l'individu ne désire pas seulement ce qui lui est utile ou encore ce que peut satisfaire ses besoins ${ }^{78}$. Le désir est un phénomène complexe, qui est avant tout de nature psychologique, d'où l'angle d'approche de Christian von EHRENFELS. Son analyse permet d'une part de comprendre comment le désir se rattache à l'imagination de l'individu, et d'autre part, de voir qu'il s'agit non pas d'une simple émotion, mais d'un phénomène rassemblant et déclenchant toute une série de réactions et d'émotions psychologiques. En d'autres termes, le désir serait plutôt un état psychologique dans lequel serait un individu qui, en présence d'un objet, éprouverait un sentiment d'envie ou de non envie à l'égard de ce dernier.

Le désir est le résultat d'un processus que nous allons reprendre, en commençant par la relation entre l'individu et l'objet menant à une représentation de ce dernier dans sa conscience; puis nous réitérons l'exemple d'EHRENFELS qui décrit les différents moments psychologiques du désir. A chaque fois, nous essaierons de comparer les propos du philosophe autrichien avec ceux de la psychologie contemporaine afin de vérifier s'ils sont encore valables.

\footnotetext{
77 « Heute nur noch selten gebrauchte Bezeichnung für einen Wunsch, der sich auf ein klar bewußtes bzw. erkennbares Objekt bezieht. Synonym: Motiv, Handlungsziel » in FRÖHLICH Werner D. (éd.), 1998, p.86.

${ }^{78}$ SEN Amartya, " On Ethics and Economics », 1987, p.15-22 et p.55.
} 
4.1 - Le fonctionnement des mécanismes de représentation de la conscience

L'individu qui est en présence d'un objet va établir avec celui-ci une relation, d'où peut émaner un sentiment d'envie ou de non-envie à l'égard de ce même objet. Le sentiment d'envie ou de non-envie est lui-même la résultante de plusieurs mécanismes psychologiques, en particulier ceux de la représentation.

Lorsqu'un individu considère un objet, il va le faire à travers ses sens. Autrement dit, la perception d'un objet, quelque soit sa nature, se fera à travers les sens de l'individu. Cette perception sera renvoyée sous forme d'interprétation imagée au cerveau, ou plus exactement à la conscience, laquelle se chargera d'offrir à l'individu une représentation de l'objet en question.

Sur ce point, et certainement sans le savoir, EHRENFELS décrit un schéma qui a été expliqué plus tard par la psychobiologie, à savoir la relation entre le cerveau et le comportement d'un point de vue biologique ${ }^{79}$. Le cerveau humain réagit à un événement extérieur par l'intermédiaire de ses sens qui apportent les informations collectées au cerveau. C'est à partir de ces informations qu'il construit une représentation de la réalité. Ce passage de la réalité physique à la dimension psychologigue est décrit par la partie de la psychologie dite psychologie de la perception $^{80}$.

La relation entre le cerveau et le comportement a été également analysée par le philosophe Karl POPPER (1902-1994) et le physiologue australien John Carew ECCLES (1903-1997) dans leur livre " The Self and Its Brain - An Argument for Interactionism " paru en 1977. Ils décrivent l'interaction de l'individu avec son environnement à travers la théorie dite des trois mondes. Le premier monde est le monde physique, celui des objets matériels; le deuxième monde est celui des états psychiques qui comprend les états de la conscience, les dispositions psychiques et les états insconscients; le troisième monde inclut le contenu de la pensée et les produits de l'esprit humain ${ }^{81}$. Les théories scientifiques se retrouvent dans le troisième monde. Il existe une interaction entre le premier et le deuxième monde, également entre le deuxième et le troisième monde par l'intermédiaire du premier $^{82}$. Entre la valeur, produit de l'esprit humain nous dit EHRENFELS, et les dispositions psychiques de l'individu, il y aurait une interaction par l'intermédiaire de la perception de l'objet matériel, d'après la théorie de POPPER et ECCLES. En outre, une des thèses principales de ces derniers est celle que les objets immatériels, produits par l'esprit humain et appartenant donc au troisième monde, peuvent être réels, indépendamment de leur possible matérialisation dans

\footnotetext{
${ }^{79}$ BIRBAUMER Niels et SCHMIDT Robert F. (eds), "Biologische Psychologie ", 2003, p.3.

${ }^{80}$ BENESCH Hellmuth (éd.), « dtv-Atlas zur Psychologie. Tafeln und Texte », 1994, p.90 à 123.

${ }^{81}$ POPPER Karl et ECCLES John, « Das Ich und sein Gehirn », 1997, p.63.

82 Idem, p.433.
} 
le premier monde ${ }^{83}$. Nous pouvons établir un parallèle avec la théorie d'EHRENFELS qui définit la valeur comme le produit de la relation entre un individu et un objet, y compris dans le cas où l'individu est convaincu de la non existence de cet objet. Autrement dit, l'individu peut désirer un objet qui n'est réel que dans sa conscience et dont la matérialisation peut être la conséquence de son agissement en ce sens. On peut affirmer que les développements contemporains de la psychologie ont confirmé, du moins dans le domaine de la perception et de la conceptualisation, les propos d'EHRENFELS.

La représentation de la réalité est une image fabriquée par la conscience de l'individu nous dit ensuite EHRENFELS. Leur diversité et leur nombre ne sont pas quantifiables, mais leur nombre à un instant donné est lui par contre limité (p.338-341). Une représentation peut être le résultat d'une perception concrète ou empirique, mais elle peut également être le simple fait de l'imagination de l'individu, qui par exemple, conceptualise un objet dans le but de déterminer si le supplément de bien être apporté par cet objet serait positif ou négatif. Une représentation n'est donc pas uniquement associée à des perceptions physiologiques. Cependant, précise EHRENFELS, il n'est pas possible de s'imaginer tous les objets: ce procédé ne fonctionnerait pas dans le cas des couleurs ou des notes de musique par exemple. On peut douter de cette affirmation dans la mesure où pour beaucoup d'individus travaillant dans les domaines de la musique ou de l'art, l'habitude de manipuler les notes ou les couleurs peut développer en eux la capacité d'imaginer une note supplémentaire par exemple ou une nuance de couleur plus foncée. Les compositeurs doivent pouvoir se représenter une mélodie avant même qu'elle ne soit écrite, comme un écrivain se représente l'histoire qu'il va conter.

Les représentations sont en effet des conceptualisations qui sont produites par un processus d'association (une sensation $\rightarrow$ une image) qui peut être simple ou complexe avec une réaction en chaîne (sensation $\rightarrow>$ première image qui en appelle une seconde, elle-même appelant une troisième et ainsi de suite). Le processus d'association est lié aux habitudes de l'individu : le fait de percevoir un objet qui nous est familier va conduire l'individu à en avoir une représentation associée à d'autres images, par habitude ${ }^{84}$. Néanmoins chaque reproduction de l'image d'un objet dans la conscience de l'individu ne se fait pas toujours à l'identique : en répétant une chanson par exemple, une personne peut, avec le temps, en oublier un mot ou une strophe.

\footnotetext{
${ }^{83}$ Idem, p.64.

${ }^{84}$ Les refléxions d'Ehrenfels sur l'habitude lui sont été inspirées par les travaux de Franz BRENTANO sur le sujet. Pour plus de précisions, voir BRENTANO Franz, « Grundzüge der Ästhetik », 1959, p.88-119.
} 
D'autres représentations seront reproduites de manière plus ou moins identique et leur maintien dans la conscience de l'individu, ainsi que le réalisme et la vivacité de cette représentation, seront également liés à l'habitude. Mais pas seulement. La lassitude ou "Ermüdung " joue également un rôle non négligeable dans le maintien avec le temps d'une représentation dans la conscience: lorsque je regarde une peinture par exemple, je serai las de cette contemplation au bout d'un moment et détournerai alors mon attention de cet objet. De la même manière, au bout d'un certain temps, la conscience se débarrassera d'une représentation, par lassitude. Il n'est pas possible d'établir une grandeur concernant ce lapse de temps car il varie d'une personne à l'autre et, pour une même personne, il varie dans le temps (p.345-346)

Enfin, dernier facteur qui influencera la durée et le degré de vivacité de la représentation d'un objet dans la conscience : les sentiments qui accompagnent cette représentation. Chaque image est accompagnée d'un sentiment qui, selon sa nature (favorable ou défavorable) fera que l'objet imaginé restera, ou pas, dans sa conscience. De plus, ce sentiment aura quelque chose à voir avec le bien être de l'individu : si une représentation est accompagnée d'un sentiment favorable, c'est que l'objet en question est susceptible d'augmenter le bien être de l'individu. Etant donnée cette relation, EHRENFELS nommera " loi de soutien relative au bien être " ou " Gesetz der relativen Glücksförderung " le processus d'influence des sentiments sur les représentations dans la conscience.

Le point d'analyse suivant concerne l'origine physiologique du bien-être. Autrement dit, quel genre d'expérience sensorielle sera accompagné par une émotion positive?

La loi du soutien relatif au bien être détermine le maintien de l'image et sa durée dans la conscience de l'individu. Elle ne détermine par contre pas son apparition, sauf dans les cas de répétition où l'imagination d'un objet, lorsqu'elle est accompagnée d'une émotion positive, déclenchera un bien-être, lequel à son tour favorisera la réapparition de l'objet dans la conscience. La raison de ce processus est simple : l'individu recherche, dans la plupart des cas, tout ce qui lui est agréable et qui lui procure donc un certain bien-être.

Tous les objets provoquant un bien être supplémentaire chez l'individu, resteront plus longtemps dans sa conscience. Par la suite, à chaque fois que l'individu se retrouvera en face du même objet ou d'un objet du même genre, la même image apparaîtra dans sa conscience, par habitude ou par association. Ou encore, parce qu'un objet déterminé provoque un sentiment positif chez l'individu, il va s'imaginer cet objet de manière répétée, recherchant le bien être lié à cette image. 
La loi du soutien relatif au bien être détermine donc l'apparition des images dans la conscience dans le cadre des répétitions ou habitudes liées à un sentiment de bien être.

Dans les autres cas, l'apparition de représentations est liée à des expériences sensorielles: lorsqu'un des sens de l'individu est stimulé, un message, qui est transmis par le système nerveux, arrive dans la conscience et celle-ci se concrétise par une représentation accompagnée d'un sentiment ou émotion, positive ou négative (p.350). Ce processus est appelé processus de dissimilation par EHRENFELS. Le mot est, de nos jours, utilisé dans un autre sens, et désigne la suppression des liens qui ont été établis lors du processus d'assimilation. Autrement dit, il s'agit du contraire de l'assimilation. L'assimilation est le processus d'intériorisation des différents aspects de l'environnement de l'individu et peut également décrire l'intériorisation des aspects culturels d'un environnement ${ }^{85}$. Ce qu'EHRENFELS décrit dans ses propos se rapproche donc plus du processus d'assimilation, au sens contemporain du terme.

Ses remarques sur la relation entre les expériences sensorielles et le bien être de l'individu s'inspirent des travaux d'Alfred LEHMANN $(1858-1921)^{86}$. La principale thèse de LEHMANN sur le sujet est que l'envie est le sentiment déclenché par un organe durant son temps de travail lorsqu'il consomme moins d'énergie qu'un repas ne peut lui fournir. Au contraire, lorsque l'organe consomme plus d'énergie qu'il ne lui est fourni au cours d'un repas, l'individu va éprouver un sentiment de non-envie ou de rejet; de même, lorsque la quantité d'énergie fournie est de loin supérieure à l'énergie dépensée pour cause de non travail de l'organe. A noter qu'EHRENFELS laisse à ses lecteurs l'appréciation de cette théorie (p.352). Pour sa part, il conclut que la réalité, tout comme la déduction, nous montrent que l'agréable serait, dans la règle, ce qui est supportable, et le désagréable serait ce qui est insupportable. Mais il ajoute que personne ne nous dit que cette règle est sans exception. En effet, si on considère, pour reprendre un précédent exemple, les personnes qui éprouvent du plaisir quand on leur inflige une douleur, elles constituent une exception à la règle que nous venons d'évoquer.

\footnotetext{
${ }^{85}$ ARNOLD Wilhelm, EYSENCK Hans Jürgen et MEILI Richard (eds), « Lexikon der Psychologie ", 1996, p.155 et p.382.

${ }^{86}$ Alfred LEHMANN est connu pour avoir été un des plus célèbres élèves du philosophe Wilhelm WUNDT (1832-1920), fondateur du « Institut für experimentelle Psychologie » en 1879 à l'Université de Leipzig. Son principal ouvrage, sur lequel EHRENFELS se base s'intitule : « die Hauptgesetze des menschlichen Gefuhlens », 1892, p.155.
} 
Un autre élément intervenant dans le processus du désir est le jugement existentiel ou « Existenzialurteil » qui peut être défini comme le jugement d'un individu sur l'existence ou l'absence d'un objet. Selon la définition d'EHRENFELS de la valeur, un individu va désirer quelque chose dont il est persuadé de la nonexistence ou dont il doute de l'existence. Le jugement existentiel est donc important dans le processus de désir de l'individu. Or, EHRENFELS semble ici se contredire quelque peu dans ses propos, puisqu'après nous avoir dit que l'individu ne peut désirer que ce qui n'existe pas ou les objets dont il doute de l'existence, il affirme ici que ce que nous croyons être vrai et exister va obtenir une place privilégiée dans notre conscience (p.354). Par exemple, les personnes qui ont une grande imagination et qui créent un autre monde par besoin, comme les écrivains. Cette nouvelle vision des choses est plus proche de la réalité et corrobore tout à fait notre remarque sur le fait qu'un individu va désirer avant tout les objets qu'il ne possède pas mais qui existent ou peuvent aussi ne pas exister dans le temps présent $^{87}$.

L'important pour l'individu est donc de savoir si l'objet qu'il considère existe ou non, ou plus exactement, il est important qu'il perçoive l'objet comme existant pour pouvoir le désirer. La question est alors, comment différencier quelque chose qui existe vraiment de quelque chose que l'on croit exister?

Il y a deux façons de se représenter un objet, selon EHRENFELS : soit en le considérant comme existant, soit en le considérant comme non existant. Il y a également la possibilité de ne pas penser à la question de l'existence de l'objet, ce que la plupart des personnes font probablement. Considérer qu'un objet existe, c'est avouer l'existence de la relation causale ("kausale Verbindung ») qui relie l'individu à cet objet. Une relation de nature causale, car si l'individu accepte l'existence d'un objet, donc d'un élément extérieur à lui-même, alors il accepte par la même sa propre existence ou ce qu'EHRENFELS appelle son " je présent " ou " gegenwärtiges Ich ». Le " je présent " est défini comme la somme de toutes les perceptions intérieures d'un l'individu, tout comme la représentation de son corps en tant qu'élément dans une relation causale liant deux objets ${ }^{88}$, objet au sens large du terme. L'individu reconnaît donc l'existence de l'objet à travers la sienne, et donc pour qu'un objet existe à ses yeux, il faut donc qu'il le considère comme tel.

\footnotetext{
${ }^{87}$ Voir paragraphe 1.2 du premier chapitre de ce travail.

${ }^{88}$ " Unter dem gegenwärtigen Ich verstehen wir die Summe aller gegenwărtigen inneren Wahrnehmungen des betreffenden Individuums, sowie auch die Vorstellung seines eigenen Leibes; unter kausaler Verbindung aber in diesem speziellen Fall ein derartiges Verhaltnis zweier Objekte (gleichgultig ob Dinge, Vorgănge oder Zustände), vermøge welches sie als gemeinsame Wirkungen einer vorhergegangenen Ursache, oder als mögliche gemeinsame Ursachen einer kunftigen Wirkung gedacht werden ", in EHRENFELS, 1982, p.356.
} 
On remarque, en outre, que l'habitude encore une fois joue un rôle dans la relation sujet-objet : la répétition dans le temps d'une relation entre un objet et un sujet va conduire l'individu à lui accorder une place particulière dans sa conscience.

Schématiquement : le sujet perçoit une pomme qu'il considère comme vraie. Cette perception se répétant, elle finit par obtenir la première place dans sa conscience et la représentation de celle-ci sera alors accompagnée par un sentiment favorable ou défavorable, lequel poussera l'individu à désirer la pomme ou pas, selon que celle-ci lui procure un sentiment de bien être ou pas.

La situation dans laquelle le sujet et l'objet ont une relation causale est dénommée "réalité subjective" par EHRENFELS : l'individu perçoit l'objet à travers une représentation subjective de celui-ci. Subjective car elle est également accompagnée d'émotions.

A noter que l'acte de jugement n'aurait pas d'influence sur la durée, la vivacité et la précision des représentations. Selon EHRENFELS, l'acte de jugement jouerait plutôt un rôle dans le fait que l'entrée dans la réalité subjective se fasse sans difficultés. Autrement dit, l'acte de jugement accompagnerait, ou plus exactement, introduirait l'individu, son "je présent», dans la relation sujet-objet décrivant la dite réalité subjective.

En résumé, l'individu qui perçoit un objet, va le faire à travers une expérience sensorielle qui va elle-même conduire à une représentation de l'objet dans la conscience de l'individu. Cette représentation persistera ou non, selon qu'elle soit accompagnée d'une émotion favorable ou défavorable, ou encore qu'elle relève de l'habitude, ou qu'elle appelle une certaine lassitude au bout d'un certain temps, une lassitude variable selon les individus. La relation entre le sujet et l'objet sera dénommée " réalité subjective ", car cette relation sera une interprétation abstraite de l'objet par l'individu. L'élément le plus déterminant dans le processus de représentation est le supplément de bien être que l'objet est susceptible d'apporter à l'individu, ce qui va conditionner son évaluation et son désir vis-à-vis de cet objet.

\section{2 - Le phénomène du désir}

Le fonctionnement du processus de représentation étant présenté, il convient de s'intéresser au désir proprement dit. Les questions que se pose EHRENFELS, concernent le moment de l'apparition du désir, son évolution dans la conscience et, dans quelle mesure celui-ci influence le processus de valorisation. 
Avant de présenter les réflexions d'EHRENFELS sur le désir, nous allons reprendre l'exemple qu'il a lui-même donné et dont il se sert comme point de départ et de référence au fur et à mesure de ses propos (p.363-365). Cet exemple est celui d'un étudiant en droit, qui, par une belle après-midi d'été, est assis chez lui à son bureau pour réviser en prévision d'un examen proche. La chaleur l'accable et sa concentration est perturbée par les bruits extérieurs ainsi que par l'idée d'aller se promener en montagne. Réflexion faite sur les inconvénients d'une telle sortie - perte de temps pour les révisions, prix du billet de train, etc... - l'étudiant se remet dans un premier temps au travail. Mais rien n'y fait, la pensée d'une sortie en montagne pour prendre l'air frais le gagne encore une fois, et il finit par céder.

Pour EHRENFELS, cet exemple est riche des enseignements suivants :

d'abord, l'idée de partir pour la montagne apparaît dans un premier temps dans la conscience de l'étudiant, mais elle est évincée rapidement par celle de l'examen proche et du prix du billet de train. Cette idée revient une seconde fois et finit par se transformer en désir que l'étudiant voudra réaliser. L'idée d'une promenade est accompagnée d'un sentiment favorable, qui fait miroiter un accroissement du bien être, un accroissement qui finit par être supérieur à celui procuré par le fait d'avoir révisé. Ceci entraîne une modification des dispositions émotionnelles qui vont induire un désir de l'individu pour cet objet, la promenade en montagne, et l'inciter à agir en ce sens.

Ensuite, l'objet du désir sera imaginé comme possiblement réalisable et réel. Il sera inscrit dans une relation causale avec l'individu, c'est-à-dire dans le cadre d'une réalité subjective. Autrement dit, le fait de considérer l'objet «aller faire une promenade en montagne ", et sa représentation dans la conscience de l'individu, vont établir une relation entre le sujet et l'objet, laquelle sera inscrite dans la dite réalité.

Lorsque la représentation de l'objet s'accompagne d'une émotion positive (envie) et que ceux-ci amènent l'individu à désirer, alors l'objet restera longtemps ancré dans sa conscience. Au contraire, les images désagréables ou accompagnées d'un sentiment défavorable (non-envie), comme par exemple aller chez le dentiste pour certaines personnes, seront remplacées au plus vite par d'autres représentations dans la conscience de l'individu. Ce fonctionnement décrit en fait le processus d'entrée, respectivement de sortie, dans et de la réalité subjective. 
Dans l'exemple énoncé par EHRENFELS, le souhait d'aller faire une promenade en montagne s'est ensuite transformé, après réflexion, en aspiration ou "Streben " puis en volonté. On se doit donc de distinguer plusieurs étapes dans le désiir ${ }^{89}$ : d'abord le moment du souhait de l'individu de voir l'objet avec lequel il est en relation, se réaliser; ensuite le moment de l'aspiration ou du "Streben », qui décrit l'état psychologique dans lequel se trouve l'étudiant qui, cédant à son souhait, va agir en ce sens; enfin, le moment de la volonté, qui décrit l'état psychologique dans lequel se trouve l'étudiant qui bannit les derniers doutes et les dernières objections de sa conscience, et qui finalise son action. Dans ce cas, aucun désir ne peut s'opposer à celui qui domine la conscience de l'individu à un moment donné.

Les moments de la volonté et de l'aspiration se distinguent de celui du souhait par le fait que ce dernier n'est qu'une image abstraite dans la conscience de l'individu, alors que les deux autres sont accompagnés d'actes concrets. Le moment du "Streben » s'accompagne d'une mise en action physique (l'étudiant se lève) et le moment de la volonté est en fait un certain jugement ou, on pourrait dire, un dernier mot de la conscience concernant le fait d'accomplir l'acte visant à satisfaire son désir. De plus, la volonté suppose que l'individu est conscient de son désir et qu'il est déterminé à le réaliser. Ainsi le moment de volonté, tout comme celui de l'aspiration, sont orientés vers le futur proche de l'individu, alors que celui du souhait concerne le moment présent ou le passé. C'est d'ailleurs pour cette raison que nous parlons de "moment du souhait» et non de souhait seulement : le moment où l'on émet un souhait est un moment présent ou passé, mais il est difficile de parler de moment de souhait dans le futur.

Enfin, ces trois moments du désir n'évoluent pas indépendamment les uns des autres, mais ils sont souvent associés.

La remarque suivante concerne plus particulièrement le moment du "Streben » : celui-ci n'est pas continuellement présent à l'esprit de l'individu. Rappelons que le moment de l'aspiration est la partie du désir qui va inciter l'individu à agir pour atteindre le but désiré. Or, lorsque l'individu agit, l'action mise en œuvre peut s'étaler dans le temps et ce moment n'est alors pas présent constamment à l'esprit de l'individu. De plus, il faut distinguer le "Streben intérieur», du "Streben extérieur »: le premier est dirigé vers le but de l'action (dans notre exemple, arriver à la montagne); le second est plutôt dirigé vers le moyen (en général, physique) mis en œuvre pour atteindre le but (ici, marcher, puis prendre le train, puis marcher de nouveau).

\footnotetext{
${ }^{89}$ EHRENFELS s'appuie pour ce point sur les écrits de Johann Friedrich HERBART, dans « Sămtliche Werke », Band VI, 1850, p.361.
} 
Concernant les moyens d'atteindre son but, ceux qui éveilleront un sentiment neutre ou positif chez l'individu, seront qualifiés de "bon» ou "bien » et valorisés comme tels. L'individu désirera également ces objets comme moyen d'atteindre son but et rejettera tout moyen lui procurant un sentiment négatif. Dans notre exemple, le train et la marche sont de "bons " moyens d'atteindre le but que l'individu s'est fixé : aller se promener à la montagne. En outre, l'objet, d'abord désiré comme moyen d'atteindre un but, peut par la suite devenir but et être désiré comme tel par l'individu (il s'agit, comme on l'a vu, d'un mouvement de valeur vers le bas, c'est-à-dire ici que le fait de sortir de la ville peut devenir le but). En définitive, le moyen et le but de l'action sont inséparables.

Voyons maintenant la distinction qu'EHRENFELS effectue entre désir extérieur et désir intérieur: le désir intérieur est le désir pour un objet de nature psychologique, par exemple, la détente dans le cas de notre étudiant. Le désir extérieur est le désir pour un objet de nature physique, par exemple la promenade en montagne. A noter qu'un désir peut être également intérieur et extérieur à la fois.

Le nombre de représentations dans la conscience de l'individu est limité : il ne peut se représenter une infinité d'objets à la fois, dans la mesure où les sens ne peuvent lui transmettre autant de messages à un même moment. De plus, et d'après la loi du soutien relatif au bien être, une représentation qui s'accompagne d'un sentiment favorable et qui est susceptible d'augmenter le bien être de l'individu aura tendance à rester un certain temps dans la conscience de celui-ci. Si cette image venait à disparaître, elle serait, du fait de la motivation de l'individu, relayée par une image semblable par procédé d'association. Mais d'une manière générale EHRENFELS affirme qu'il est possible de parler d'une compétition ou d'un combat entre les désirs qui demandent à se réaliser. Derrière cette compétition se cache la motivation de chaque souhait. Plus cette motivation est grande et plus le souhait a des chances de se voir réaliser. La représentation des mouvements nécessaires à la réalisation du désir, c'est-à-dire le moyen de l'action et l'action d'une manière générale, me procure le plus souvent un certain bien être et renforce par la même occasion ma motivation. Lorsque la représentation du moyen de l'action provoque un sentiment de bien être (luimême résultat d'une comparaison entre soutien au bien être ou " Glücksförderung » et dommage au bien être ou « Glücksschädigung »), le désir de l'individu sera renforcé par une motivation supplémentaire et conduira alors à la réalisation de celui-ci (moments du Streben et de la volonté). Dans le cas contraire, lorsque l'imagination du moyen de l'action est accompagnée d'un sentiment défavorable, alors le désir ne sera pas renforcé par une motivation supplémentaire et la représentation disparaîtra de la conscience de l'individu. 
L'arbitrage entre soutien au bien être et nuisance au bien être sera déterminé dans le processus du désir: si le moyen d'arriver à ses fins ne suscite aucune motivation, alors le souhait ne sera pas réalisé.

La motivation, la combativité sont des phénomènes psychiques qui n'apparaissent pas seulement dans le cadre du désir mais aussi en dehors. En effet, il y a des motivations qui restent en tête sans pour autant que la motivation de les réaliser n'incite à agir. Il faut impérativement que la composante « augmentation du bien être » soit présente pour pousser l'individu à agir. Le désir est donc une conséquence directe du soutien relatif au bien être.

La motivation, elle, agit par rapport à des paramètres physiques et psychiques. Voici ses principales formes :

1) L'action sous contrainte ou "Zwanghandlung » : il existe des cas où l'individu va effectuer des gestes qu'il n'a pas désiré ou qu'il sait ne pas être désirables. Par exemple, celui qui va commettre un acte répréhensible est conscient de faire quelque chose de mal et les raisons qui le poussent à agir ainsi sont nombreuses. EHRENFELS lui-même ne s'intéresse pas vraiment à la question, mais des réponses seront apportées par la psychologie criminelle initiée notamment par FREUD en 1915, lorsqu'il s'intéresse aux caractères exceptionnels c'est-à-dire qui montrent une certaine agressivité envers la vie et autrui ${ }^{90}$. Ce dernier considère qu'une des causes des comportements criminelles provient d'un sentiment de frustration et donc de mal être, qui pousse l'individu à agir ainsi. Un cas particulier est l'hypnose.

2) Les actions mécaniques ou "mechanische Handlungen »: il s'agit de gestes habituels, dont la répétition maintes fois (favorisée par l'aspiration et la volonté) conduit à une certaine habitude.

3) La détermination ou " Entschluß » : il s'agit d'un acte de volonté qui débouche directement sur une action, malgré la conscience d'obstacles présents lors de l'action. Par exemple, lorsque je suis déterminé à gravir une montagne, malgré les dangers existants (chute, avalanche ou glissement de terrain). La détermination apparait dans la plupart des cas comme un court moment avant la prise de décision finale. Dans le cas d'actions quotidiennes motivées par une détermination de l'individu, il n'y a pas réellement de combat, ni de "Streben » ou encore de moment de volonté. Ces éléments n'apparaissent que dans le cas où l'individu est conscient de prendre une décision.

\footnotetext{
${ }^{90}$ Sur la psychologie criminelle et les causes identifiées des actes de cette nature, voir RATTNER Josef, « Basiswissen Tiefenpsychologie. Die wichtigsten Neurosenarten », 1999, p.327-373.
} 
Pour la suite de notre propos, il serait intéressant d'analyser plus précisément le terme de motif ou "Motiv » en allemand. Il est, selon EHRENFELS, employé dans des sens différents en philosophie (p.383-384): il peut désigner premièrement, le dessein d'un individu, l'intention finale de son désir. Par exemple, une personne qui agirait pour l'argent, son motif serait dans ce cas celui de gagner le plus d'argent possible. Le motif, deuxièmement, peut désigner un acte de volonté dont les prérequis sont déjà présents : j'ai envie de me promener, par exemple, mais il ne fait pas beau. L'apparition du soleil sera donc ici un motif pour aller faire ma promenade. Troisièmement, le terme motif est la plupart du temps employé pour désigner la disposition émotionnelle qui est déterminante dans la concrétisation d'un acte du désir. Le philosophe choisit, pour la suite de ses propos, d'utiliser le mot " Motivenkampf » ou combat des motifs pour décrire ce qui se passe dans la conscience de l'individu, à savoir, un combat des différents motifs incitant l'individu à agir dans un sens ou dans l'autre. Dans le langage psychologique actuel, le motif ou " Motiv » désigne le facteur qui incite l'individu à agir dans un sens déterminé. Par facteur, on entend en fait un stimulus, qu'il soit extérieur (par exemple la perception d'un objet particulier) ou intérieur (par exemple la faim). Pour plus de précision, ajoutons que la motivation décrit l'état de l'individu qui adopte un comportement dans un but précis et dont on peut mesurer l'intensité, la durée et le sens ${ }^{91}$. La dernière définition donnée par EHRENFELS, et qui selon lui fut la plus souvent utilisée, garde donc toute sa validité de nos jours.

La motivation est pour le philosophe autrichien, ce qui permet à un souhait de se réaliser, ce qui se traduit aussi par le passage aux moments du "Streben » et de la volonté. Cependant, il convient de noter l'existence de situations dans lesquelles l'individu n'éprouve aucun désir, mais où il a une aspiration et une volonté qui déclenchent un geste. Par exemple, lorsque je manque d'air parce que je fais du sport, je vais devoir respirer plus vite. Pour autant le fait de vouloir respirer plus vite n'appelle pas un souhait de la part de l'individu, le geste est effectué automatiquement lorsque les moments énoncés ci-dessus se produisent. Et ce geste va provoquer une augmentation incontestable du bien être de l'individu.

Ces gestes, associés directement ou indirectement à la survie de l'individu, sont des gestes dits instinctifs. Les mouvements instinctifs n'impliquent pas un désir a priori, mais ils sont favorables à la survie de l'individu et donc à son bien être ${ }^{92}$. Ceci nous amène également à souligner le fait que l'augmentation du bien être n'est pas forcément liée à un désir non plus.

\footnotetext{
${ }^{91}$ ARNOLD Wilhelm, EYSENCK Hans Jurgen et MEILI Richard (eds), 1996, Tome 2, p. 1399.

92 Voir HÖFLER Alois, « Grundlehren der Psychologie. Lehrtext und Übungen fur den Unterricht an Gymnasien ", 1897, sur lequel EHRENFELS s'appuie concernant les gestes instinctifs.
} 
Le phénomène de la motivation fut également l'objet de nombreuses études dans la psychologie contemporaine ${ }^{93}$. D'une manière générale, on constate que ses origines sont biologiques : l'individu perçoit - à l'aide de ses sens - des éléments dans son environnement qui le poussent à réagir. Ces éléments sont appelés stimuli et déclenchent une réaction au niveau du cerveau de l'individu, ce qui entrainera tout un processus émotionnel qui peut comprendre la motivation de réagir par rapport à la perception ${ }^{94}$. A noter que les individus recherchent toujours dans leur environnement les situations qui pourraient améliorer leur bilan affectif et ils évitent celles qui pourraient l'influencer négativement.

La réaction ou la manière de réagir d'un individu par rapport à un stimulus, est le fruit à la fois de ses dispositions génétiques et des expériences plus ou moins nombreuses qu'il aura pu faire au cours de son existence. Les dispositions génétiques sont elles mêmes le résultat du processus d'évolution de l'humanité. Les motifs, les raisons pour lesquelles on agit, sont de ce fait des propositions de résolution des problèmes et des tâches, accumulées au fil du temps. La survie de l'espèce par exemple, fait partie des buts établis par des motifs bio-génétiques.

La faim, la soif, la sexualité, les relations à autrui et le pouvoir sont les thèmes qui jouent un rôle plus ou moins important dans la vie des individus, en fonction de leur personnalité. On part du principe que l'intensité du motif sera déterminée par les expériences vécues. Par exemple, ceux qui ont des contacts satisfaisants avec autrui, auront une raison d'entretenir une certaine intimité avec ceux-ci, alors que les personnes qui ont des contacts froids, voire conflictuels avec autrui n'auront pas de telle raison ${ }^{95}$. Cet aspect du désir n'est pas évoqué explicitement et de cette manière par EHRENFELS, qui semble s'en tenir aux causes psychologiques. Cependant, on verra dans le paragraphe suivant qu'il assimile le désir à une disposition émotionnelle et, dans les sous-parties précédentes, nous avons vu les différents déterminants des dispositions émotionnelles, parmi lesquels certains étaient de nature physique. Ceci nous amène à penser que le philosophe avait identifié les stimuli de l'environnement comme un élément pouvant influencer l'individu. De plus, dans l'exemple qu'il donne, la chaleur qui accable l'étudiant en droit est un composant qui influence son désir de vouloir partir en montagne.

Autant les dispositions biologiques donc que les leçons quotidiennes construisent et influencent les réactions des individus et les motifs qui les poussent à agir.

\footnotetext{
${ }^{93}$ Pour une perspective générale, voir MÜSSELER Jochen et PRINZ Wolfgang (eds), "Allgemeine Psychologie », 2002.

${ }^{94}$ DAVIDSON Richard J., " The functional neuroanatomy of affective style ", 2000, p.371-388.

${ }^{95}$ McCLELLAND David C., « Human Motivation », 1985 ; McADAMS Dan P., "Intimacy: the need to be closed », 1989.
} 
Les individus aspirent en outre à la réalisation de buts dont ils sont, selon BRUNSTEIN, conscients et qu'ils peuvent communiquer. BRUNSTEIN et MAIER voient dans l'établissement d'un but, une anticipation selon laquelle le comportement s'organise et les actions qui s'en suivent se retrouvent alors justifiées ${ }^{96}$. Cette perspective contemporaine fut également celle qui marqua le discours d'EHRENFELS en la matière. En effet, nous avons vu que pour lui, le fait pour l'individu de considérer un objet le place dans une relation avec ce dernier qui entraine une conceptualisation du but de son action dans sa conscience. Dans l'exemple que le philosophe donne, en réaction à la chaleur accablante, l'étudiant conceptualise dans sa conscience le fait de faire une escapade à la montagne. Son but est de se rafraichir et de se changer les idées. L'anticipation du but permet d'ajuster son comportement afin de le réaliser.

L'importance accordée à la poursuite d'un but en fonction notamment de sa difficulté, est appelée " commitment " dans le langage psychologique moderne. A ce propos, BRUNSTEIN affirme que le commitment, ou engagement, se distingue par la relation entre un but et le « je » de l'individu ${ }^{97}$. Cette relation est également identifiée par EHRENFELS, mais en des termes différents, puisqu'il parle de la relation entre l'objet et le "je présent» de l'individu. Par exemple, pour ceux qui aspirent à des carrières de dirigeants, poursuit BRUNSTEIN, ils ne le font pas que pour l'argent ou pour une autre récompense matérielle, mais aussi à cause de l'identité acquise une fois que le but sera atteint. Pour reprendre EHRENFELS, dans cet exemple, l'individu pose comme but de sa vie professionnelle le fait de devenir dirigeant, et le fait de visualiser dans sa conscience cette perspective conduit au déclenchement d'une certaine motivation ainsi qu'à la réflexion sur les différents moyens pour y parvenir. Le but en lui-même se voit conférer une valeur intrinsèque d'autant plus grande que le désir de le réaliser - à savoir la motivation, l'aspiration et la volonté de le réaliser - sera grand. Les moyens mis en œuvre se verront eux attribuer une valeur d'effet.

\footnotetext{
${ }^{96}$ BRUNSTEIN Joachim et MAIER Gunther, « Persőnliche Ziele: ein Überblick zum Stand der Forschung ", 1996, p.146-160.

${ }^{97}$ BRUNSTEIN J.C., LAUTENSCHLAGER U., NAWROTH B., PÖHLMANN K. et SCHULTHEISS O., " Persönliche Anliegen, soziale Motive und emotionales Wohlbefinden ", 1995, p.1-10.
} 
La théorie de l'établissement des buts (ou «Zielsetzungstheorie ») de LOCKE et LATHAM $^{98}$ montre qu'en dehors du " commitment » ou engagement, il existe d'autres critères qui déclenchent la motivation chez l'individu, à savoir la difficulté et la spécificité du but posé. Ces critères sont bien sûr variables selon les individus. Ainsi, certaines personnes seront d'autant plus motivées que le parcours pour atteindre le but est difficile ou encore, lorsque celui-ci est spécifié, dans le sens identifié, l'individu saura d'autant mieux dans quelle direction ou de quelle manière il devra agir, ce qui contribuera à sa motivation.

Nous pouvons également citer BANDURA qui montre, entre autre, que les buts influencent la motivation et les actions des individus non pas directement, mais par l'intermédiaire des processus de «Selbstbewertung » ou d'auto-évaluation" Quand l'individu se fixe un but, il y aura une divergence entre l'état présent (ce qui est) et l'état qui est visé (ce qui devra être). La motivation sera le résultat de la volonté de réduire cette divergence en question. L'individu devra évaluer les actions à mettre en œuvre afin d'atteindre le but fixé. A cela, il faut ajouter qu'un but peut être fixé par l'individu lui-même ou par autrui (sous la forme d'un ordre ou d'une suggestion par exemple). Cependant, la poursuite d'un but fixé par autrui ne soulève aucune émotion chez l'individu, tandis que la réalisation de ses propres buts va l'affecter positivement ${ }^{100}$. On pense alors aux employés, soucieux d'exécuter les directives du chef, sans pour autant se sentir émotionnellement impliqués. Cette situation sert également à illustrer les propos d'EHRENFELS concernant l'action sous contrainte ou "Zwangshandlung». D'une manière générale, la poursuite d'un but sera d'autant plus fructueuse qu'une série de processus appuyant la poursuite en question, aura été déclenchée ${ }^{101}$. Si cet appui manque, alors on retombera dans le schéma mentionné ci-dessus.

De ce bref exposé sur quelques résultats des recherches contemporaines sur la motivation, il en ressort que sur ce point, ainsi que concernant le désir dans son ensemble, la théorie du philosophe autrichien offre une perspective qui reste encore valable, et qui se voit même corroborée par les avancées récentes.

\footnotetext{
${ }^{98}$ LOCKE Edwin A. et LATHAM Gary P., " A Theory of Goal setting and Task performance ", 1990.

99 BANDURA Albert, « Self-regulation of Motivation and action through internal standards and goal systems "), 1989, p.19-85.

${ }^{100}$ SHELDON Ken M. et ELLIOT Alison J., " Goal Striving need satisfaction, and longitudinal well-being. The self-concordance model ", 1999, p.482-497 et SHELDON Ken M. et KASSER Tim, "Pursuing personal goals: Skills enable progress, but not all progress is beneficial ", 1998, p.1319-1331.

${ }^{101}$ SOKOLOWSKI Kurt, « Wille und Bewußtheit », 1996, p.485-530.
} 
De plus, les origines biologiques de la motivation sont reconnues par l'économiste Robert ROWTHORN, même s'il ajoute qu'elles sont d'importance secondaire dans les sciences économiques ${ }^{102}$.

Pour finir, EHRENFELS arrive à la conclusion que le désir n'est pas un élément psychique fondamental. Ce que nous nommons désir n'est en fait que la représentation d'une relation subjective entre un sujet, en particulier son «je présent ", et un objet, une relation qui favoriserait une augmentation du bien être :

"Ein besonderes psychisches Grundelement "Begehren» (Wünschen, Streben oder Wollen) gibt es nicht. Was wir Begehren nennen, ist nichts anderen, als die - eine relative Glücksförderung begründende - Vorstellung von der Ein- oder Ausschaltung irgendeines Objektes in das oder aus dem Kausalgewebe [die subjektive Realität] um das Zentrum der gegenwärtigen konkreten Ichvorstellung. ${ }^{103}$

Le désir n'est donc pas une émotion, c'est un état psychologique ou une disposition émotionnelle dans laquelle se trouve l'individu au moment où il souhaite voir l'objet avec lequel il est en relation, se réaliser. Cet état s'accompagne d'émotions particulières (les moments du souhait, du Streben et de la volonté) qui sont favorables à la réalisation de l'objet et qui, dans cette perspective, supposent une augmentation du bien être. EHRENFELS voit donc le désir comme la conséquence d'une relation entre un sujet et en objet. Il s'agit d'un processus dans la conscience de l'individu qui peut mener à la réalisation de son désir par une action précise. Cette action présente elle aussi des composantes appelant le désir : le moyen comme le résultat de l'action doit être souhaité par l'individu. L'accomplissement de l'action lui procure alors un certain bien être. Cependant, pour EHRENFELS, le bien être n'est pas ce pour quoi nous désirons forcément : un individu peut aussi éprouver du bien être à la suite d'une action qu'il n'aura pas désiré (c'est le cas des actions instinctives).

EHRENFELS considère donc que le désir est un phénomène psychologique qui est lié à l'imagination de l'individu, et que l'on peut d'autre part décomposer en trois moments (ceux de la motivation, de l'aspiration et de la volonté). Lorsque l'individu considère un objet dans son environnement, il le fait à travers ses sens. A partir de ce moment s'installe une relation entre les deux, et l'individu est plongé dans ce que le philosophe appelle la réalité subjective. Celle-ci décrit l'état de celui qui, en considérant un objet, le conceptualise dans son imagination et enclenche ainsi tout un processus émotionnel. L'individu se retrouve alors dans une disposition émotionnelle particulière qui est celle du désir. Il se fixe un but

\footnotetext{
${ }^{102}$ ROWTHORN Robert, " Ethics and Economics: an Economist's View », in GROENEWEGEN Peter (éd.), 1996, p.17.

${ }^{103}$ EHRENFELS, 1982, p.386.
} 
par rapport à un objet, et réfléchit aux moyens de l'atteindre. L'objet se voit conférer une valeur intrinsèque, alors que les moyens de l'atteindre obtiennent une valeur intrinsèque.

Les différents moments composant le désir et décrits par EHRENFELS sont la motivation, l'aspiration et la volonté. Ces moments peuvent se succéder les uns après les autres, selon l'intensité du désir de l'individu d'atteindre le but. Nous avons également vu que les théories actuelles de la motivation en psychologie n'entrent pas en contradiction avec les affirmations d'EHRENFELS, bien au contraire, elles tendraient plutôt à les confirmer. Même s'il est discutable que le désir puisse être décomposé en plusieurs moments, il est important de souligner encore une fois les apports du philosophe autrichien en la matière. D'abord, le désir est un phénomène qui n'est pas exclusivement dirigé par les préférences de l'individu. Deuxièmement, l'individu ne désire pas uniquement la satisfaction de ses besoins ou, en d'autres termes, lorsqu'il agit, il ne le fait pas uniquement dans le but de satisfaire un besoin. Enfin, le rôle de l'environnement est particulièrement important dans la fixation et la poursuite d'un but.

Dans la dernière partie de notre chapitre, nous allons nous intéresser à un aspect important de la théorie d'EHRENFELS, celui concernant les valeurs éthiques et de leur place dans la théorie de la valeur. Il s'agit là encore d'un point qui garde toute sa validité dans le discours actuel, même si les discussions à ce propos sont nombreuses. 


\section{V - Valeurs et éthique : étude psychologique des valorisations conformes aux bonnes mœurs.}

La question de l'éthique et de son rôle dans le processus de valorisation occupe une place importante dans l'analyse d'EHRENFELS : une bonne partie de son étude porte sur l'étude de l'origine des valeurs éthiques et du comportement humain, en particulier du désir, qui est associé à ce processus. Cette importance s'explique entre autre par la perception de l'éthique par EHRENFELS, mais aussi par son appartenance idéologique, dont nous avons déjà parlé en introduction de ce chapitre ${ }^{104}$. Nous nous proposons de reprendre le chemin emprunté par EHRENFELS dans ses réflexions : d'abord, il commence par analyser l'éthique en tant que discipline traitant entre autre le problème de la valeur et les différentes possibilités ou méthodes d'analyse des faits éthiques, parmi lesquelles l'analyse réelle qui sera celle choisie par EHRENFELS. Cette dernière le mènera à considérer les origines de la valorisation éthique ainsi que leur nature et le désir qui est lié. Il s'agira par ailleurs de se familiariser avec la notion de "Wohl der Gesamtheit » ou bien être de tous.

Dans un second point, nous évoquerons le fait que les valeurs éthiques et les valeurs morales, tout comme les autres types de valeurs, subissent une évolution dont nous identifierons également les causes. Les valeurs éthiques et l'éthique sont liées par ailleurs au droit, à la coutume et aux maximes qui sont décrits comme des régulateurs du comportement. L'individu vit dans une collectivité ou dans une société qui établit des valeurs et qui vont donc l'influencer dans ses actions. D'autres instances régulatrices telles que la conscience morale et la sanction éthique sont aussi identifiées par EHRENFELS. Enfin, nous aborderons le problème, souvent posé, de l'existence des valeurs absolues.

\section{1 - L'éthique en tant que science}

EHRENFELS affirme qu'il existe deux courants dominants dans l'éthique : le premier qui voit l'éthique comme une discipline pratique, dont la tâche principale est d'éditer des impératifs catégoriques, ce qu'il assimile à un " tu dois » absolu. Cependant, il ajoute en note de bas de page (p.413) que les impératifs catégoriques ne se réfèrent pas exclusivement à la conception kantienne

\footnotetext{
${ }^{104}$ Appartenant au courant philosophique autrichien de la fin du XIX ${ }^{\text {tme }}$ siècle, ses réflexions se caractérisent notamment par leur opposition aux thèses de Kant. Par ailleurs, le fait de vouloir développer une théorie de la valeur pour un philosophe implique son attachement à évoquer le problème des valeurs éthiques, une approche qui se différencie de celle des économistes qui s'attachent exclusivement à expliquer les valeurs des biens marchands.
} 
de cette notion. L'éthique, toujours selon ce courant, se doit de désigner les objets qui se distinguent par le fait que leur appropriation constitue en soi un acte louable et estimable (p.414). Le second courant évoqué par EHRENFELS est qualifié de historico-relativiste et perçoit l'éthique comme étant une discipline normative et dont la tâche principale est de décrire les faits éthiques et leurs histoires dans les différentes cultures. Enfin, EHRENFELS évoque la conception kantienne de l'éthique (p.415) qu'il interprète comme étant celle qui posa l'impératif catégorique en postulat de la raison pure («reine Vernunft») et qui, par conséquent, reconnaît les actes découlant de cette raison comme les seuls pouvant être appréciables. Pour le philosophe autrichien, ce postulat intervient sans considération pour les penchants émotionnels. Il critique ainsi indirectement la vision kantienne et propose une perspective alternative à travers son analyse psychologique des faits ou des actes éthiques.

La conception kantienne de l'éthique est liée à celle de la liberté et de la société $^{105}$ : s'inspirant de HOBBES et de ROUSSEAU, il reprend l'idée de contrat qui lie les institutions aux individus qui composent la société. Les institutions créent le droit qui, à la fois, garantit aux individus leur liberté et constitue une limite à cette liberté. Cette limite est en fait la liberté d'autrui. La question est de savoir comment l'individu peut se soumettre à la volonté de la majorité et en même temps garder sa liberté d'agir et de penser. Pour KANT, c'est la raison pratique $^{106}$ qui fait en sorte que l'individu crée sa propre liberté en s'imposant une règle de conduite susceptible d'avoir une validité universelle, c'est-à-dire d'être reconnue et acceptée en tant que telle par tous. La raison pratique influence la volonté qui est un élément essentiel en éthique pour le philosophe allemand. Un manque de volonté ou son contraire, un excès de volonté ne peuvent conduire à un comportement éthiquement acceptable. La volonté comprend la volonté d'agir selon un but prédéfini et celle de suivre une règle pour atteindre ce but. La raison nous conduit à fixer une règle de conduite qui devient un devoir que nous nous donnons volontairement. Le contraire d'agir par devoir est d'agir par envie ou selon ses penchants. Un exemple d'action par devoir serait pour KANT, un individu qui aiderait un autre, non pas par compassion pour autrui et pour sa souffrance, mais parce qu'il s'est donnée comme devoir d'agir de la sorte (aider les gens en difficulté).

\footnotetext{
${ }^{105}$ ANDERSEN Svend, « Einfuhrung in die Ethik », 2000, p. 143-144.

${ }^{106}$ Kant distingue la raison pratique de la raison théorique (une différenciation qu'il a repris d'Aristote). La raison théorique est celle qui produit les idées ; lorsque la raison donne des directives à la volonté alors elle devient pratique.
} 
Le devoir a alors un caractère d'impératif dont KANT distingue plusieurs types $^{107}$ :

- L'impératif hypothétique : l'individu agit selon la formule "si...alors... ». Par exemple, «si tu veux atteindre ton but, alors il faudra agir de la sorte ».

- L'impératif pragmatique : agir avec précaution, sachant que tous les individus aspirent au bonheur (dans le sens de la satisfaction des besoins).

- L'impératif catégorique : agir en fonction de la maxime que la raison a défini comme étant susceptible de devenir une loi universelle.

C'est grâce à la notion d'impératif catégorique que KANT définit la manière de se comporter de manière éthique. Il ajoute que l'individu ne peut agir de manière éthique que s'il est libre de le faire, c'est-à-dire si la raison est libre d'influencer la volonté d'agir ${ }^{108}$.

EHRENFELS critique le fait de considérer la raison comme le seul élément qui pousse l'individu à agir de manière éthique. Son analyse évoque le rôle joué par les dispositions émotionnelles et la religion. Le fait de ne pas négliger le rôle des émotions dans le processus de décision des individus - notamment en ce qui concerne le caractère éthique de leur comportement - est un argument repris et développé par la philosophe et psychologue Carola MEIER-SEETHALER dans son ouvrage "Gefühl und Urteilskraft ». Elle argumente en faveur de la prise en compte des émotions comme élément complémentaire à la raison dans le processus de prise de décision et de valorisation de l'individu ${ }^{109}$.

Le philosophe autrichien conçoit l'éthique comme une science. Pour EHRENFELS, les sciences sont partagées entre les disciplines théoriques et les disciplines pratiques (p.584). Il précise que ces catégories ne doivent pas être isolées l'une de l'autre. Cette catégorisation ne s'est pas effectuée selon la manière d'arriver à la connaissance, ni selon la nature de la valeur de la connaissance (valeur théorique ou pratique). Les connaissances elles-mêmes ne sont ni théoriques, ni pratiques, mais elles peuvent être partagées ou rassemblées selon la nature du besoin. La classification peut donc se faire selon l'utilisation ou l'utilité de la connaissance. Par exemple, les connaissances permettant de fonder les impératifs éthiques par exemple ont une utilité pratique. Il existe également des connaissances dont l'utilisation peut être à la fois pratique et théorique. Cette classification que nous propose EHRENFELS est en fait reprise d'Alexius MEINONG (p.584) et sert à fonder la nature de l'éthique en tant que discipline.

\footnotetext{
${ }^{107}$ ANDERSEN Svend, 2000, p. 147-148.

${ }^{108}$ Idem, p. 148.

${ }^{109}$ MEIER-SEETHALER Carola, " Gefuhl und Urteilskraft », 1998, p.249-251.
} 
Tous les désirs supérieurs décrits par les impératifs éthiques et qui suivent des buts pratiques, s'appuient sur des connaissances des deux types. Aussi la psychologie des valeurs éthiques réelles est une discipline théorique et pratique. Une sous-discipline, théorique celle-ci, est celle de l'éthique générale qui traite des problèmes généraux et théoriques.

EHRENFELS énonce également d'autres catégories spécialisées de l'éthique (p.586-588) :

- L'éthique théorique, qui dans sa partie descriptive raconte l'histoire des faits éthiques depuis ses débuts jusqu'à nos jours; dans sa partie génétique ("genetischer Teil»), elle traite plutôt de l'histoire philosophique des faits éthiques, c'est-à-dire des lois régissant leur évolution. Enfin, l'éthique théorique se préoccupe également de la vitesse de développement de l'éthique dans le futur et ce faisant, empiète sur le domaine de l'éthique pratique.

- La tâche de l'éthique pratique est en fait l'étude des facteurs de régulation des comportements et de leur contribution dans la réalisation du but initial ou "Zielmäßigkeit », ainsi que de la conception de nouveaux facteurs à la place des facteurs survivants ou contraires à l'évolution de l'éthique même. Pour EHRENFELS, la conception de ces nouveaux facteurs de régulation est une tâche immense et qui concerne tous les domaines de la vie. Elle peut se réaliser avant tout à travers des essais et moins à travers des connaissances anticipées ou théoriques, et c'est pour cela que les hommes d'Etat et les poètes ont des concepts aux effets plus grands que les scientifiques. Tout ceci constitue simplement le fondement d'un système éthique, un apport à la discipline.

L'objet d'analyse de l'éthique pratique est la contribution dans la réalisation du but des valorisations éthiques et des maximes morales. Les deux éléments sont cependant liés au droit et aux coutumes et il est nécessaire de considérer l'ensemble afin de se construire un avis. Ainsi, l'éthique pratique s'applique à la politique, à l'économie nationale et sociale. Cependant les domaines cités ne sont en aucun cas des branches spéciales de l'éthique pratique. En politique par exemple, le désir du législateur de tenir compte de la morale ou des maximes morales est le fait de sa volonté ou de ses dispositions émotionnelles. En fait, on peut distinguer deux catégories de disciplines pratiques: celles, comme l'économie ou la politique, où il s'agit de lister les buts et les moyens de les atteindre (l'éthique devra approuver ou non ces moyens et ces buts) et celles où les moyens et les buts sont éthiquement neutres et n'appellent donc pas de jugement. 
BIRNBACHER définit l'éthique en tant que discipline comme étant la théorie philosophique de la morale ${ }^{110}$. L'éthique revêt par conséquent un aspect théorique alors que la morale (définie comme le système complexe des règles, des normes et des échelles de valeur qui constitue l'objet d'analyse de l'éthique ${ }^{111}$ ) analyse plutôt les situations pratiques du comportement et de la décision. En ce sens, les disciplines telles que la psychologie, la sociologie, l'ethnologie ou encore l'histoire qui s'attachent à comprendre la morale, le font d'un point de vue empirique, ce qui n'est pas le cas de l'éthique. L'éthique se préoccupe de l'analyse théorique de la morale, c'est-à-dire des phénomènes moraux, du contenu de la morale et de son évolution, de la construction et de l'évolution des normes ainsi que de la connaissance de la morale ${ }^{112}$.

A titre comparatif nous allons énoncer l'approche de l'économiste Rick SZOSTAK en la matière ${ }^{113}$. Reprenant HALDANE, SZOSTAK décrit trois niveaux d'analyse de l'éthique ${ }^{114}$ :

- D'abord celui des règles de conduite, comme par exemple l'honnêteté. Ces règles de conduite peuvent être combinées ou pas, avec des systèmes éthiques tels que l'éthique chrétienne.

- Ensuite celui des grands principes tels que la règle d'or, par exemple, qui dérive de la morale chrétienne et qui dit : traite les autres comme tu voudrais qu'ils te traitent. Les grands principes justifient un certain nombre de règles, sachant qu'un même principe peut justifier plusieurs règles à la fois.

- Le troisième niveau d'analyse est celui de la méta-éthique, c'est-à-dire l'étude de la signification des termes moraux, de la relation logique entre les jugements moraux et d'autres formes de jugements, du statut épistémologique des jugements moraux, de leur justification et du statut métaphysique des propriétés morales, abstraction faite de toute conception éthique particulière ${ }^{115}$.

L'approche de SZOSTAK se situera plutôt au premier niveau, nous le dit-il lui même ${ }^{116}$, sans pour autant se faire de manière isolée par rapport aux autres niveaux. Ceci nous amène à faire une première remarque sur le fait que Christian von EHRENFELS également n'eut pas une approche isolationniste, bien au

\footnotetext{
${ }^{110}$ BIRNBACHER Dieter, " Analytische Einfuhrung in die Ethik », 2003, p.2.

111 Idem.

112 Idem, p.58-59.

${ }^{113}$ Cette approche est contenue dans un ouvrage intitulé « Unifying Human Ethics ", qui est paru au mois d'octobre 2005 mais dont nous avons obtenue une copie du manuscrit datant de 2002 . Les références que nous donnons sont celles des pages de ce manuscrit, le livre n'étant pas encore disponible au moment de l'écriture de ce travail.

114 SZOSTAK Rick, " Unifying Ethics », 2002, p.26.

${ }^{115}$ Définition du Dictionnaire d'éthique et de Philosophie morale, p.960.

${ }^{116}$ SZOSTAK Rick, 2002, p.29.
} 
contraire. Il s'intéressa aussi bien aux règles elles-mêmes, et à leur valeur pour les individus, qu'aux grands principes ayant pour lui une valeur intrinsèque.

Une autre distinction effectuée par SZOSTAK, et qui est le résultat de ses nombreuses lectures, est la distinction de cinq grands types de théories ou courants éthiques. Il évoque en premier lieu trois types de théories dites " formelles " (ou « formal ethical theory ») :

1) Celui qui juge l'action en fonction de ses conséquences (si elles sont désirables ou pas). C'est le type de théorie prônée par les utilitaristes, pour qui les conséquences s'assimilent à la joie retirée de l'action.

2) Les théories qui se concentrent sur les vertus humaines : certaines qualités telles que l'honnêteté ou le courage sont bonnes en soi, peu importe leurs conséquences.

3) Le troisième type de théorie est celui qui s'intéresse aux règles éthiques guidant notre comportement comme les lois par exemple. L'action de l'individu sera jugée en fonction de ses conséquences sur autrui.

Ces trois premiers types de théorie sont basés sur la raison.

Viennent ensuite les théories basées sur l'intuition et l'existence de Dieu. Ce dernier aurait crée les individus de manière à ce que ceux-ci agissent instinctivement en fonction des règles éthiques.

Enfin, il y a les théories se basant sur les traditions. Beaucoup d'individus obéissent à des règles sociales en pensant qu'elles sont bonnes parce qu'elles existent depuis longtemps. Néanmoins, les sciences et les philosophes s'accordent à dire, selon SZOSTAK, que les traditions évoluent avec le temps.

Pour l'économiste canadien, ces cinq approches sont complémentaires et les individus les utilisent simultanément ou indépendamment lors de leur prise de décision ${ }^{117}$. Il ajoute que dans le passé, de nombreux philosophes ont eu une approche incluant ces cinq possibilités. C'est d'ailleurs le cas chez EHRENFELS, pour qui les conséquences des actions, les règles, l'intuition, les vertus ou encore les traditions offrent une série de repères éthiques guidant les individus dans leur comportement. Cette approche a l'avantage d'être exhaustive, ou en tout cas, de l'être plus que l'approche utilisée en économie. Pour ce qui est du phénomène de la valeur, EHRENFELS explique sa détermination pour un objet en fonction des cinq paramètres évoqués. Tous jouent un rôle incontestable dans la plupart des situations quotidiennes.

${ }^{117}$ Idem, p.33. 
Avant de commencer à parler des valorisations éthiques, EHRENFELS s'intéresse d'abord aux notions associées à l'éthique en tant que discipline. Il justifie ce procédé par sa conception personnelle des méthodes d'analyse scientifique : les notions et leurs définitions sont des outils essentiels de la recherche car sans une formulation verbale, donc avec des mots, les nouvelles connaissances ainsi acquises ne sont pas transmissibles. Cependant la formulation d'une définition peut mener à une certaine confusion puisqu'un objet réel peut être caractérisé de trois manières différentes : la première est celle qui est établie par rapport au contenu de l'objet; la seconde est établie par rapport à l'environnement de l'objet ; la troisième manière de définir un objet est le sens qui lui est attribué, la définition qui est considérée comme la bonne de l'objet réel en question $^{118}$. C'est pour éviter cette confusion dans son analyse qu'EHRENFELS décide de reprendre les notions importantes de l'éthique. Il lui faut un point de départ qui sera ici le point commun, s'il existe, entre les faits qualifiés de conformes à la morale et ceux qui ne le sont pas (p.420). Il s'agit, précise t-il, d'une analyse psychologique et empirique. Empirique car il veut rester ancré à la réalité en considérant les réflexions populaires sur l'éthique ou encore les jugements quotidiens dans ce même domaine; psychologique car le philosophe s'intéresse aux réflexions qui se cachent derrière un jugement éthique : quelles composantes de la conscience entrent en jeu au moment du processus de valorisation et quels facteurs influencent ce processus?

Il qualifie cette façon de faire de "Realanalyse », une méthodologie inspirée, de son propre aveu, de celle de David HUME et, dans une moindre mesure, de celle d'Alexius MEINONG (p.421, note en bas de page).

Certaines notions associées à l'éthique sont ensuite redéfinies par EHRENFELS. En premier lieu, les faits éthiques sociaux ou "sozial-ethische Tatbestände " décrivent des événements de la société qui émanent de l'action des hommes. Ces événements ou faits seront qualifiés d'éthiques lorsqu'ils sont conformes aux règles éthiques en vigueur dans une société donnée. Lorsque l'action conforme à l'éthique est le fait d'un individu en particulier, cette action sera qualifiée de fait éthique individuel ou « individuell-ethischer Tatbestand » (p.87).

\footnotetext{
118 « In Bezug auf jeden [...] Begriff für reale Objekte kann zunăchst zweierlei unterschieden werden: die Bedeutung mit dem zugehörigen Inhalt des Begriffes einer-, und der Kreis jener realen Objekte andererseits, auf welche der Begriff von den ihn Gebrauchenden richtig oder vielleicht auch fälschlich angewandt wird. Außer diesen beiden Bezeichnungsstucken aber zeigt sich oft noch ein drittes; neben der tatsăchlichen nåmlich auch oft noch eine dafurgehaltene, resp. vermeintliche Bedeutung des Begriffes » In EHRENFELS, p.419.
} 
Les notions d'approbation et de désapprobation sont associées aux actes qu'elles sont censées qualifier. Approuver un acte est la conséquence d'un jugement éthique, découlant lui-même d'une valorisation éthique préalable. La valorisation éthique désigne l'acte par lequel l'individu confère de la valeur à un attribut d'un objet, par rapport à la morale en vigueur dans une société. L'attribut est une partie, un caractère ou une propriété de l'objet en question et il constitue à lui seul l'objet de la valorisation éthique :

«Wir verlangen von der ethischen Wertung, daß sie ein beliebiges Attribut eines als beseelt gedachten Wesens zum Objekt habe, welches Attribut eine ursächliche Beziehung $\mathrm{zu}$ irgendwelchen Handlungen involviert. " ${ }^{119}$

Jusqu'à présent les objets pouvaient être désirés pour eux-mêmes ou pour leur effet et se voyaient attribuer en conséquence une valeur intrinsèque dans le premier cas ou une valeur d'effet dans le second. Dans le cadre des valeurs éthiques, l'individu va désirer l'objet pour son attribut et va lui conférer une valeur éthique qui sera en fait une valeur éthique intrinsèque ou en fait une valeur constitutive, puisque l'attribut est une partie de l'objet. Par ailleurs, le même objet peut également se voir attribuer une valeur d'effet. La compréhension de la valorisation éthique comme telle, nous donne des éclaircissements aussi bien sur l'objet de la valorisation que sur le sujet valorisant, les sujets observant et les références des individus (par rapport à leur manière habituelle de qualifier un objet de « bon » ou de « mauvais ») (p.422).

La caractéristique commune des valorisations éthiques est le fait de conduire les individus à approuver ou à désapprouver un comportement. Il convient cependant de poser une première limite, celle de la culture de la personne. Ainsi, pour reprendre l'exemple d'EHRENFELS, la notion de patrie est associée à la France pour un Français ou à l'Allemagne pour un Allemand. Un même objet peut donc apparaitre de manière différente selon le point de vue adopté. C'est pour cette raison que le philosophe autrichien va, là encore, limiter ses investigations aux objets des valorisations éthiques de sa société à l'époque à laquelle il vivait. Que ce soit à l'époque d'EHRENFELS que de nos jours, il existe des formes d'éthique différentes selon les sociétés ou les cultures ${ }^{120}$. Pour comprendre une valorisation éthique, il convient de tenir compte du monde culturel dans lequel vit la personne qui émet ce jugement ainsi que son environnement social, c'est-à-dire l'appartenance à un certain groupe social ou une certaine classe sociale à travers son métier, ses activités, tout comme son sexe ou son appartenance ethnique. BIRNBACHER définit la morale de groupe comme étant celle qui est reconnue par un ensemble de personnes dont les valorisations des comportements

\footnotetext{
${ }^{119}$ EHRENFELS, 1982, p.88.

${ }^{120}$ BIRNBACHER Dieter, 2003, p.36.
} 
dépendent de l'appartenance de l'individu au groupe ou pas ${ }^{121}$. En fin de compte, il faut situer la personne dans son contexte afin de mieux comprendre leurs dispositions émotionnelles et celles du désir :

" Angesichts der letzterwähnten Beziehung ist es notwendig, sich eines Unterschiedes mit Bestimmtheit bewusst zu werden: Wir können von Geschlechts-, Standes- oder Berufstugenden und -untugenden in zweierlei Sinne sprechen, zunächst in dem eben dargelegten, nach welchem hierunter diejenigen Gefühldispositionen zu verstehen sind, welche von allen oder doch nahezu allen ethisch wertenden Angehörigen eines Kulturgebietes an den Angehörigen eines bestimmten Geschlechtes, Standes oder Berufes positiv resp. negativ gewertet werden. » (p.451)

L'objet par excellence de la valorisation éthique qui appelle une approbation ou une désapprobation éthique est l'acte humain (p.92). L'acte humain découle de l'aspiration ou de la volonté de l'individu, et induit des conséquences intentionnelles ou recherchées ${ }^{122}$. Dans le processus qui conduit à l'action, il faut distinguer l'intention de l'individu ("die Absicht») de son but (" der Zweck ») et des conséquences attendues de son acte (" die erwarteten Wirkungen »). Il s'agit de trouver parmi les composantes de l'action, depuis le désir jusqu'aux conséquences attendues, celle qui est pertinente pour le jugement éthique. EHRENFELS arrive à la conclusion que ce dernier ne concerne pas une de ces composantes en particulier, mais que chacune d'entre-elle donne des indications sur un autre élément important dans le processus de valorisation éthique : les dispositions du désir. Toujours selon le philosophe, on peut constater à travers notre expérience que l'approbation d'un acte ou son contraire se fait par rapport au désir intentionnel de la personne qui agit (p.92 et p.426). Il nomme l'intention originelle de l'action, "die Urheberschaft der Handlung " ou origine de l'acte. L'intention est donc déterminante dans l'engagement de l'action et le but, les moyens et les conséquences attendues en sont le reflet. L'intention et le désir sont liés puisque le second déclenche le premier. Par désir, EHRENFELS entend en fait les dispositions du désir qui sont en définitive l'élément qui sera éthiquement approuvé ou désapprouvé.

« [Es kann] nicht nur das Vorhandensein von Begehrensdispositionen bestimmter Art, sondern auch deren Mangel ethisch - und zwar abfällig - gewertet werden [...] ( (p.95)

"Man sieht: überall gelten Zweck, Mittel und vorausgesehene Folgewirkungen nur als Anzeichen für das Vorhandensein von Begehrensdispositionen, oder furr deren Mangel in der nötigen Intensität. " (p.96)

${ }^{121}$ Idem, p.36.37.

122 « Die menschliche Handlung ist ein Akt des Strebens oder Wollens, durch welchen beabsichtigte Wirkungen hervorgerufen werden. " in EHRENFELS, 1982, p.423. 
Concrètement l'individu qui n'est pas poli ou qui est peu disposé à l'être (absence ou manque de dispositions éthiques) sera désapprouvé par son environnement. Enfin, les dispositions du désir étant déterminées à travers les dispositions émotionnelles, alors le jugement éthique de l'acte individuel ou collectif s'effectuera en fin de compte par rapport à ses dispositions émotionnelles ou par rapport à leur manque (p.427).

L'acte humain n'est pas le seul objet des valorisations éthiques où on considère les dispositions émotionnelles. Il en est de même pour la retenue d'une personne ou le fait de ne pas agir ("Unterlassung »): là aussi, l'approbation ou la désapprobation découlera d'un jugement éthique des dispositions émotionnelles ou de leur absence.

Maintenant que les notions d'approbation ou de désapprobation éthiques sont clarifiées et que les notions adjacentes ont été soulignées, il convient dans un second temps de trouver leur point commun. Pour ce faire, EHRENFELS va tout d'abord énoncer dans l'ordre décroissant les dispositions émotionnelles c'est-àdire celles du désir qui sont les mieux évaluées éthiquement et ensuite, celles qui sont les plus désapprouvées. Il précise encore une fois que cette liste est valable dans son monde culturel (p.428).

La disposition émotionnelle considérée comme la plus noble et la mieux évaluée est celle qui est énoncée par la morale chrétienne : "Aime Dieu plus que tout et ton prochain comme toi-même ". Il ajoute cependant que l'amour de Dieu est en recul dans la société dans laquelle il vit, et on peut sans problème affirmer que ce phénomène n'a fait que s'accentuer depuis. Dans notre société contemporaine, les sentiments religieux affichent un net recul tout comme ceux liés à autrui. Les sociétés occidentales individualistes valorisent avant tout l'individu tandis que les relations avec autrui sont passées au second plan. Pour certains intellectuels, ce phénomène est lié à l'assimilation des valeurs économiques et des préceptes inhérents, notamment celui de l'individualisme méthodologique. ${ }^{123}$

Svend ANDERSEN distingue deux caractéristiques principales des sociétés occidentales actuelles $^{124}$ :

- Elles sont sécularisées : le Christianisme et la religion n'y ont plus la même place que durant les siècles passés, de même que leur conception de la vie qui déterminait l'attitude à adopter face aux questions éthiques.

${ }^{123}$ PETERSON V. Spike, « A Critical Rewriting of Global Political Economy. Integrating reproductive, productive and virtual Economies ", 2003, p.150-151.

${ }^{124}$ ANDERSEN Svend, 2000, p.4. 
- Elles sont pluralistes : le Christianisme est remplacé par un certain nombre de conceptions différentes de la vie, comme par exemple l'athéisme. Ce phénomène conduit à avoir plusieurs opinions de ce qui est «bien » et de ce qui est " mal " dans une même collectivité et à d'éventuels conflits.

Pour EHRENFELS, l'amour du prochain reste une des dispositions émotionnelles les mieux valorisées d'un point de vue éthique. Cette notion appelle néanmoins quelques éclaircissements car elle est très générale et peut être utilisée dans des cas très différents. Le prochain sous-entend certes autrui mais aussi les autres êtres vivants de la planète autre que les humains, autres que ceux de l'espèce humaine, c'est-à-dire les animaux, nous dit EHRENFELS (p.428).

Une disposition émotionnelle sera déterminée par un certain nombre de facteurs psychologiques, dont l'apparition déclenchera un sentiment d'envie ou de non envie chez l'individu. En ce sens, la compassion ou " Mitleid ", c'est-à-dire le fait d'être affecté soi-même par la souffrance d'autrui, peut aussi compter parmi les situations où l'on parle d'amour du prochain. L'amour de son prochain est défini par EHRENFELS comme le partage des joies et des souffrances d'autrui :

" [...] daß doch mit demselben Recht wie das Mitleid auch die Mitfreunde als Bekundung der Menschenliebe angesehen werden müsse. ${ }^{125}$

Mais comment apparaît et comment se développe ce sentiment de compassion pour autrui ?

Selon EHRENFELS, la prise de conscience du malheur ou du bonheur d'autrui affecte nos dispositions émotionnelles ainsi que la capacité de l'imagination à construire des représentations de cette joie ou de ce bonheur. Les dispositions émotionnelles sont certes un facteur important mais elles ne sont pas le seul facteur considéré dans le jugement éthique. Il y a aussi l'imagination. Le fait d'imaginer un individu qui rie ou qui pleure peut également influencer nos dispositions émotionnelles. De nos jours, l'imagination a tendance à être remplacée par la force des images, en particulier celle des images télévisuelles. Celles-ci suggèrent à l'individu une joie ou une peine qui va affecter ses dispositions émotionnelles.

Pour Lawrence BLUM, la compassion n'est pas un sentiment impulsif et par conséquent, il est tout à fait fiable dans des situations qui exigent une attitude morale. Ainsi, celui qui agit par compassion, le fait en mettant de côté ses propres

${ }^{125}$ EHRENFELS, 1982, p. 100. 
tentations et envies pour venir en aide à son prochain ${ }^{126}$. Néanmoins, dans le cas d'une relation amicale ou amoureuse, les frontières entre altruisme et amourpropre s'estompent, et Patricia GREENSPAN parle alors de sentiments d'identification ("identificatory love ») dans lesquels les impulsions altruistes et égocentristes auraient tendance à se renforcer mutuellement ${ }^{12 \dagger}$.

EHRENFELS évoque également des situations dans lesquelles le sentiment de partage des peines empêche l'individu d'aider son prochain. Par exemple, pour les sœurs qui durant les guerres aidaient les blessés, une trop grande compassion les aurait empêché d'effectuer correctement leur travail. Un certain recul est nécessaire afin d'éviter une trop grande implication émotionnelle. L'amour d'autrui, lui, se manifeste dans le fait qu'elles apaisent leur peine en les soignant (p.104). D'une manière générale, la notion de l'amour du prochain est impliquée dans la valorisation d'objets que l'on pourrait regrouper dans une catégorie "participation au bonheur et au malheur d'autrui » (ou "Teilnahme für fremdes Wohl und Wehe "). Ceci apporte un peu de précision à la notion en question, même si le philosophe autrichien avoue que l'on pourrait aller plus loin dans l'analyse psychologique. Cependant, pour ses propos, une approche générale suffit $^{128}$.

Après les dispositions émotionnelles en faveur de l'amour de son prochain, on retrouve en deuxième position les dispositions en faveur d'un cercle de personnes plus restreint (p.431). Ce cercle plus restreint peut comprendre les membres d'une nation, d'un Etat, la famille, les enfants ou encore les amis. Viennent ensuite un certain nombre de qualités qui appellent elles aussi une considération éthique très positive. Dans cette liste énoncée par EHRENFELS, on retrouve notamment la justice, la loyauté, l'honnêteté, le sens du devoir, la vérité et le tact. Il souligne en outre le fait que certaines dispositions émotionnelles sont différemment valorisées par les hommes ou par les femmes. La valorisation éthique peut également être différente selon la classe sociale. Les individus d'une même classe sociale ont conscience d'appartenir à celle-ci en fonction notamment des "valeurs " communes, dont les valeurs éthiques font partie. Ces valeurs seront attribuées à

\footnotetext{
${ }^{126}$ BLUM Lawrence, "Compassion », 1980, p.512. Voir également BLUM Lawrence, "Friendship, Altruism and Morality », 1980.

${ }^{127}$ GREENSPAN Patricia, " Emotions and Reasons ", 1988, p.11 et p.37.

128 « Hiermit wäre wohl der vage Begriff der Menschenliebe in einen etwas bestimmteren Rahmen gefasst, zu seiner psychologischen Charakteristik aber [...] keinerlei Beitrag geliefert - eine Aufgabe, welche überhaupt nicht mit einem Schlag, sondern nur durch eine [...] psychologische Analyse gelöst werden könnte. Hierzu liegt jedoch für uns keine Nötigung vor. Uns genügt es, zum Zwecke der Abstraktion des Gemeinsamen einen summarischen Überblick zu gewinnen. In diesem Sinne haben wir die der allgemeinen Menschenliebe zugrunde liegenden Gefuhlsdisposition an erster Stelle unter den von unserer Kulturwelt ethisch hochgeschätzten anzuführen ", in EHRENFELS, 1982, p.431.
} 
des objets déterminés et il est évident que la règle s'applique également d'un point de vue éthique : un certain comportement par exemple peut être valorisé dans une certaine classe sociale et pas dans une autre.

Il établit également une autre liste, celle des dispositions émotionnelles moralement condamnées ou pour le moins, négativement valorisées. On y retrouve, entre autre, l'indifférence, l'égoïsme, "l'ipsissimismus » (celui qui se satisfait de l'amour qu'il se donne à lui-même et qui décrit un manque d'amour pour autrui), l'absence d'honneur, la méchanceté, la vengeance, etc...

De toutes les dispositions émotionnelles citées, qu'elles soient positives ou négatives, il convient de rechercher leur point commun et ceci à travers ce que EHRENFELS nomme le " Gemeinnützlichkeit » ou le " Gemeinschädlichkeit ", c'est-à-dire l'utilité commune ou la nuisance commune. Concrètement, toutes les dispositions émotionnelles présentent dans leurs effets, c'est-à-dire dans l'acte de l'individu, et dans la grande majorité des cas, une utilité commune ; au contraire, toutes les dispositions émotionnelles désapprouvées ou la valorisation négative du manque de dispositions émotionnelles présentent dans leurs effets une nuisance commune. Cette perspective, nous dit-il ensuite, s'appuie sur celle de l'utilitarisme pour laquelle, nous évaluons une disposition selon son caractère utile ou nuisible.

Cependant, EHRENFELS critique cette vision des choses, car pour lui certaines catégories de dispositions sont certes utiles mais ne se voient pas attribuer de valeur éthique élevée, par exemple le fait de se battre pour sa survie. Cette disposition est d'une utilité incontestable car sans elle, l'espèce n'aurait peut être pas survécue (p.435-436). Par contre sa valeur éthique n'est pas très élevée puisque ce genre de dispositions est présent chez tous les individus. Comparativement, l'amour du prochain a une utilité moindre, mais sa valeur éthique est plus élevée. Ceci s'explique par le fait que cette disposition n'est pas présente dans la même mesure chez les individus. EHRENFELS effectue ici un parallèle avec les valeurs économiques pour lesquelles une augmentation du nombre d'objets, si ceux-ci sont originellement présents en quantité limitée, sera d'une grande utilité pour les individus. Ce parallèle montre que pour le philosophe, le raisonnement qui vaut dans le domaine de l'économie est également applicable dans celui de l'éthique. Une façon de faire logique puisqu'il est dans la perspective d'une théorie générale de la valeur. L'utilité commune des dispositions ne peut donc pas être un critère déterminant, ou en tout cas, pas le seul critère dans le jugement éthique. De plus, l'individu n'est pas toujours conscient de l'utilité ou de la nuisance d'une disposition émotionnelle, ce qui pousse EHRENFELS à aller plus loin. 
Empiriquement, on va qualifier un objet de précieux pour le bien de tous et on va lui attribuer par conséquent une valeur d'effet si une augmentation de la quantité de cet objet serait favorable au bien de tous. Alors que dans le domaine de l'acte immoral, une désapprobation éthique sera déterminée par le critère de nuisance commune. En d'autres termes, l'approbation éthique d'une disposition et sa valeur d'effet dépendra de son effet sur le bien être de tous; la valorisation éthique négative sera, elle, fonction de la nuisance commune engendrée par les objets évalués (p.438).

La notion du bien être de tous ou "Wohl der Gesamtheit " est définie de la manière suivante par EHRENFELS: il s'agit de la plus grande dominance possible de l'envie sur la non envie ${ }^{129}$. Cette notion apparaît de prime abord assez peu claire et le philosophe tente de quantifier le rapport entre les deux grandeurs, avant d'affirmer qu'une formalisation n'apporterait finalement que peu d'éclaircissements ${ }^{130}$. De plus, la recherche du plus grand bien être pour tous conduit à se poser la question de sa valeur intrinsèque : si le bien être de tous a une telle valeur alors sa recherche appelle des moyens dont la valeur éthique est aussi à déterminer, notamment sur le plan politique (par exemple, quel régime étatique est le plus à même de mener au bien être pour tous ?). Mais voyant qu'il s'éloigne de son propos originel, EHRENFELS laisse la discussion de côté et poursuit dans la direction de départ (p.441). La notion de bien être pour tous est une notion complexe. Elle est composée en fait d'un grand nombre d'éléments tels que la santé ou la paix intérieure par exemple. Si tous ces éléments sont réunis alors l'individu éprouvera un grand bien être. Au fur et à mesure que chacun de ces éléments se rajoute favorablement, le bien être individuel croit. Il convient donc de ne négliger aucun de ces facteurs. En ce sens, la notion d'utilité commune, insatisfaisante pour EHRENFELS, est remplacée, ou plutôt rangée sous celle d'élément commun favorisant ou "Gemeinförderlich » qui regroupe tous les objets dont l'effet va dans le sens du bien être pour tous (p.445).

En fin de compte, le point commun entre les objets approuvés éthiquement est leur capacité à favoriser - ou pas - le bien être de tous. Plus un objet - de manière générale, un comportement - favorise le bien être de tous, et plus il sera positivement valorisé. Cette relation admet cependant une limite. En effet, un acte

\footnotetext{
${ }^{129}$ «Wenn man dem Terminus des allgemeinen Wohles einen präzisen Sinn unterzulegen sich bemüht, so geschieht dies zu allermeist in der Weise, daß man unter dem Begriff [...] das höchstmögliche Überwiegen der Lust gegenubber der Unlust, resp. das kleinstmögliche Überwiegen der Unlust gegenüber der Lust versteht » in EHRENFELS, 1982, p.439.

${ }^{130}$ Il pose la question suivante : est-ce que l'existence de $\mathrm{n}$ personnes avec un surplus d'envie individuel u est à préférer à l'existence de $\mathrm{mn}$ personnes avec un surplus d'envie individuel $\mathrm{u} / \mathrm{m}$ ? Dans les deux cas, le surplus d'envie total est le même, ce qui apporte peu d'éclaircissements. De plus, $2 u$ n'est pas égal à $2 u / 2$ pour chaque personne puisque le surplus d'envie est partagé de manière inégale entre les individus. Voir EHRENFELS, 1982, p.440.
} 
a une conséquence sur le bien être de tous, et à l'origine de cet acte on retrouve des dispositions émotionnelles. En fonction de l'effet de ces dernières, une valeur correspondante sera attribuée. Pour EHRENFELS, il serait erroné de vouloir conférer un attribut moral aux seules dispositions émotionnelles qu'il serait souhaitable que tous les individus possèdent, de la même manière qu'il serait erroné de vouloir arriver, à partir d'une valorisation éthique, à l'opinion que cela conduirait tout le monde à la piété si chacun possédait cette disposition émotionnelle. Ces affirmations supposent l'existence d'un caractère moral idéal, une hypothèse qu'EHRENFELS réfute car elle ne serait pas compatible avec les valorisations éthiques empiriques (p.566). A travers cet argument, il nous montre encore une fois qu'il ne partage pas le même point de vue que KANT concernant l'éthique : si les individus élevaient la retenue du désir sexuel (ascétisme sexuel) au rang d'impératif universel et si cet impératif était appliqué par tous, cela conduirait selon EHRENFELS à la disparition de l'espèce humaine. De plus, il affirme que la disposition émotionnelle de la reproduction est présente en quantité telle chez tous les individus, que l'application d'un tel impératif ne serait pas possible (p.565). En outre, rien ne dit que la propagation de cet impératif ne conduirait à un bien être de tous plus élevé. En d'autres termes, EHRENFELS critique les thèses universalistes de KANT.

Reste enfin la question de la relation entre l'objet éthique et la grandeur de la valeur qui lui sera accordée. Dans le cas de l'approbation éthique, deux facteurs sont déterminants dans la valorisation de l'objet : le type et l'intensité relative des dispositions émotionnelles (p.446). Pour illustrer son argument, le philosophe autrichien nous donne deux exemples. Le premier est celui de deux personnes qui doivent volontairement sauver la vie d'un troisième individu. Pour cela, le premier doit mettre sa propre vie en danger alors que le second ne sacrifie que quelques heures de son temps. Dans les deux cas, la disposition de l'amour du prochain est présente de manière incontestable et elle sera chez les deux personnes positivement valorisée. Cependant, le premier en montre plus que le second car il est prêt à sacrifier sa vie pour son prochain et la valeur éthique de son acte sera donc plus grande.

Le second exemple est celui de deux personnes qui sacrifient plusieurs semaines de leur temps, l'une à travailler pour le bien être de son supérieur hiérarchique, l'autre pour la vie de ce même supérieur hiérarchique. L'action est la même, mais dans le second cas, la grandeur des dispositions émotionnelles est plus élevée et en conséquence, elles se verront attribuer la plus grande valeur éthique. D'une manière générale, concernant le type de disposition émotionnelle, l'amour du prochain en général est considéré, selon EHRENFELS, comme supérieur à celui pour un proche en particulier car le second type de disposition est limité à une personne ou un petit groupe de personnes. L'autre facteur important, que nous 
avons déjà évoqué, est l'intensité de la disposition émotionnelle ainsi que l'effet adjacent qui est déterminant pour la grandeur de la valeur d'effet.

Une précision quantitative de ces deux notions est cependant difficile sous une forme mathématique, ajoute EHRENFELS sans plus de précisions (p.447). On peut néanmoins l'interpréter en affirmant que l'intensité d'une disposition émotionnelle ne se laisse que difficilement mesurer et que l'évaluation d'un acte est donc difficilement combinable avec le souhait de formalisation.

Dans le cas de la désapprobation éthique, la relation entre disposition émotionnelle et objet valorisé est la même que dans le cas de l'approbation éthique, à savoir, la non valeur éthique qui est accordée dépendra de l'existence d'un certain type de dispositions émotionnelles (la méchanceté par exemple). Par contre, lorsque les dispositions sont présentes en quantité limitée ou lorsqu'elles ne sont pas présentes du tout, la relation se complique quelque peu. Ainsi la nonvaleur accordée sera fonction de la différence entre le niveau de disposition de l'individu et la moyenne des individus d'une société, plus cette différence est grande et plus grande sera la non valeur.

Toujours dans son analyse empirique de la valorisation éthique, EHRENFELS aborde le problème de la nature de la valeur accordée à l'objet subissant un jugement éthique, s'agit-il d'une valeur intrinsèque, d'une valeur d'effet ou des deux en même temps? (p.448-458)

Ceux pour qui le bien être de tous représente une valeur intrinsèque et qui ont un certain aperçu des relations sociales, ceux-ci vont accorder aux objets de la valorisation éthique une valeur d'effet ou une non valeur d'effet, en combinaison donc avec la première valeur citée. Le bien être de tous peut aussi, on l'a déjà vu, se restreindre à un petit groupe de personnes, mais il se voit également dans ce cas accorder une valeur intrinsèque, laquelle encore une fois se combinera aux valeurs d'effet accordée aux qualités morales telles que la justice, la vérité ou encore la loyauté.

En résumé, quand on considère les relations sociales les plus importantes, basées sur les dispositions du désir, les objets de la valorisation éthique auront une valeur d'effet ou une non valeur d'effet plus ou moins élevée, selon que ces objets prennent part au bien être d'un cercle de personnes plus ou moins important (p.449).

Considérer l'environnement social de l'individu ne suffit pourtant pas à comprendre de manière exhaustive la valorisation éthique. Pour compléter notre analyse, il faut retourner encore une fois aux origines de la valorisation éthique, c'est-à-dire au niveau des dispositions émotionnelles. Pour considérer un acte 
comme éthique, il faut considérer, comme on l'a vu, non seulement le dessein de cet acte mais également les intentions préalables à l'acte et les moyens utilisés pour atteindre l'objectif. Ne considérer qu'un élément ne suffit pas toujours. Prenons l'exemple de deux hommes ayant comme objectif commun celui de s'enrichir. Le premier va, pour se faire, monter un commerce au travers duquel il espère faire des profits, alors que le second décide de cambrioler pour parvenir à ses fins. Dans cet exemple, l'acte de la seconde personne est en lui-même répréhensible alors que l'objectif reste acceptable. Dans le cadre de la valorisation éthique, ce qui compte pour EHRENFELS est donc le but ou le dessein en accord avec le moyen ou "der Zweck in Vereinigung mit den Mitteln». Il ne faut pas non plus négliger les effets attendus de l'acte ou « die Gesamtheit der erwarteten Wirkungen der Handlungen » (p.94).

Dans le cas où l'individu désapprouve un comportement, cette disposition émotionnelle est accompagnée par un complexe d'images (ou " Begleiterscheinungen ") dans sa conscience. Celles-ci peuvent être par exemple l'écœurement ou le rejet qui éveilleront par ailleurs un sentiment de non envie. Ces images sont la réflexion de la "vie intérieure " ou "Innenleben » de l'individu qui relève de l'activité de sa conscience. Cette "Innenleben » est en fait une conscience morale ou « Gewissen » de l'individu qui l'influence par l'intermédiaire des dispositions émotionnelles.

La conscience est liée au sentiment de culpabilité et il est, selon Erika FISCHER, l'expression de l'expérience de l'individu dans des situations vécues ${ }^{131}$. On distingue la bonne conscience (celle que ressent l'individu lorsqu'il a évité de faire une faute de comportement par exemple) de la mauvaise conscience (celle qui apparaît lorsque l'individu a transgressé une norme ou une règle). Le sentiment de culpabilité est ressenti dans le second cas de figure. Pour le psychologue Lawrence KOHLBERG, un critère permettant de reconnaître l'internalisation des règles morales est la présence du sentiment de culpabilité chez l'individu ${ }^{132}$. Les dispositions émotionnelles et la conscience individuelle vont donc bien jouer un rôle important dans le comportement moral de l'individu, comme l'a souligné EHRENFELS.

Un facteur extérieur qui va aussi influencer ses dispositions émotionnelles ainsi que sa conscience morale est la crainte de la sanction éthique. La sanction éthique est décrite par EHRENFELS comme étant "le caractère sacré de certaines dispositions émotionnelles ou de certaines dispositions du désir qui conduisent à

\footnotetext{
${ }^{131}$ FISCHER Erika, “ Moralische Einstellungsmuster und ihre Verănderbarkeit », 1989, p.17.

132 KOHLBERG Lawrence, « Die Psychologie der Moralentwicklung », 1995, p.8.
} 
une "guérison " interne, gage de paix et de joie intérieures ${ }^{133}$. Cette expression nous apparait peu claire, en particulier concernant la nature exacte de ces dispositions émotionnelles ou du désir «sacrés». Mais le philosophe ne nous donnera pas plus d'explications à ce propos. On peut simplement supposer que ce genre de dispositions a à voir avec la morale religieuse. Ce que confirme ses propos concernant les effets de la sanction éthique : ceux-ci sont liés en partie aux convictions métaphysiques de l'individu. En effet, si la crainte d'une sanction est présente dans sa conscience, l'instance morale influencera ses dispositions de manière à éviter ce genre de sanctions. Il reconnaît également que leur effet est en recul à cause des nouvelles perspectives matérialistes offertes par la société industrielle, ce qu'il déplore personnellement (p.454). Par ailleurs, les sanctions conduisent l'individu à attribuer des valeurs dites valeurs d'effet éthiques et individuelles que le philosophe définit comme celles qui sont attribuées à des dispositions psychiques par rapport à leur contribution à la paix intérieure (dans l'hypothèse où celles-ci ont une valeur intrinsèque pour l'individu). Il ajoute enfin que ces valeurs d'effet éthiques et individuelles coïncident en général avec les valeurs d'effet accordées à des objets pour leur contribution au bien être de tous (p.453) car les humains recherchent la plupart du temps la paix intérieure laquelle leur procure un sentiment de bien être favorable à la recherche de la paix sociale, celle avec autrui, menant ainsi à un bien être général. La sanction éthique peut être effectivement celle qui vient de l'individu lui-même, c'est-à-dire de sa conscience. Elle peut être également extérieure, sous la forme d'une sanction judiciaire dans la mesure où le droit sanctionne un comportement non-conforme à l'éthique ${ }^{134}$.

EHRENFELS a attiré notre attention sur trois éléments importants : d'abord, la valorisation éthique s'effectue par rapport aux dispositions émotionnelles de l'individu, c'est à dire ses intentions avant d'agir. Ensuite, la valorisation éthique se fait en fonction de la contribution de l'action de l'individu au bien être général et le bien être général, il faut bien le souligner, ne désigne pas ici celui d'un état de satisfaction des besoins mais celui d'une satisfaction générale. Ce faisant, le philosophe critique les théories utilitaristes et l'insuffisance de l'argument d'une augmentation du bien être seulement par l'intermédiaire de celle de la satisfaction des besoins. Enfin, comme dans les autres domaines, la notion de non-valeur peut également être utilisée dans celui de l'éthique.

De la même façon, nous verrons dans le paragraphe suivant que les valeurs éthiques connaissent également des évolutions.

${ }^{133}$ " Unter der ethischen Sanktionen verstehen wir die [...] « Heiligkeit » gewisser Gefuhls- und Begehrungsdispositionen, welche diese zu einem inneren « Heiltum », zu einer Bürgschaft inneren Frieden und Gluckes macht "), in EHRENFELS, 1982, p. 453.

${ }^{134}$ BIRNBACHER Dieter, 2003, p.54. 
L'éthique est un élément inhérent à la vie quotidienne selon EHRENFELS, et en tant que tel, elle subit des changements liés notamment à ceux affectant ce même quotidien. Concrètement, les changements dans les habitudes comportementales peuvent influencer l'éthique et ses valeurs. Mais également, dans le sens contraire, des changements au niveau des normes, des coutumes ou les lois peuvent amener à des changements dans le comportement des individus. Les facteurs favorisant l'évolution des valeurs éthiques seraient cependant différents selon les époques et les lieux. EHRENFELS prend en exemple les sociétés primitives de chasse et de pêche où le bien être de tous concernait en fait qu'un cercle restreint de personnes. De plus, ce bien être dépendait d'autres facteurs que ceux des sociétés plus avancées. Pour le philosophe autrichien, une société développée suppose la présence d'éléments particuliers tels que la capacité d'abstraction de ses membres ou encore leurs connaissances en psychologie. Ceci leur permet d'être conscients de la notion de dispositions émotionnelles et de la causalité des phénomènes ( $\mathrm{p}$.455). Par conséquent, le jugement éthique des civilisations dites moins évoluées est différent de celui des sociétés dites évoluées ${ }^{135}$.

EHRENFELS établit une relation entre les changements sociaux et ceux qui interviennent dans l'éthique, ce qui, après ce qui a été dit précédemment, semble tout à fait logique. Evoquant le contexte social, il fait explicitement allusion aux développements de l'Ecole Historique Allemande sur les évolutions entre les différentes périodes de l'histoire. Il ajoute que, si ces évolutions sont expliquées par les sciences, celle de l'éthique devrait faire l'objet du même traitement et il serait alors intéressant de dégager les lois de son développement (voir " die ethischen Entwicklungsgesetze », p.134 à 148 et p.474 à 487).

Pour cela, il faudra dans un premier temps préciser les notions de valorisation morale ou immorale. Le philosophe autrichien opère une distinction entre les valorisations morales et les valorisations éthiques. Les premières désignent toutes les évaluations qui sont susceptibles de faire elles-mêmes l'objet d'un jugement éthique. Concrètement, dire la vérité possède en soi une valeur morale et l'évaluation de la disposition émotionnelle "aimer dire la vérité " est une évaluation éthique. Par voie de conséquence, l'évolution des valorisations éthiques est appelée "ethische Wertbewegung" ou mouvement des valeurs éthiques ; celle des valeurs morales sera quant à elle qualifiée de "moralische Wertbewegung » ou mouvement des valeurs morales.

\footnotetext{
${ }^{135}$ Remarquons que sur ce point, le philosophe autrichien est d'accord sur le fond avec l'économiste Amartya SEN, ce que nous verrons plus en détail dans notre troisième chapitre.
} 
EHRENFELS veut ensuite découvrir les lois gouvernant ces mouvements respectifs, pour autant qu'elles existent et qu'on puisse les définir.

Pour comprendre les variations des valeurs éthiques, il est important de considérer les effets sociaux de la valorisation éthique. Les jugements éthiques jouent également un rôle important dans la considération sociale de l'individu : celui qui aura un comportement conforme à l'éthique en vigueur, aura une place privilégiée dans la société dans laquelle il vit. Ceux qui par contre auront un comportement opposé, ne bénéficieront que de peu ou d'aucune considération de la part de leur semblable. Un second effet de la valorisation éthique est sa tendance naturelle à favoriser l'émergence des objets conformes à l'éthique et à freiner celle des objets éthiquement désapprouvés. Autrement dit, tout objet ou toute chose, quelque soit sa nature, qui est éthiquement correct, aura une existence sociale favorisée. Le jugement éthique freine donc l'individu dans ses attitudes. La peur de se faire rejeter aiguise en quelque sorte le désir de l'individu à agir conformément à l'éthique. Un autre facteur qui favorise la retenue de l'individu dans ses attitudes est celui des institutions, en particulier à travers le processus de socialisation où l'individu intègre les normes et les règles de la société dans laquelle il vit, ce qui va par ailleurs influencer ses dispositions émotionnelles ${ }^{136}$. Les institutions influencent en outre l'individu par l'intermédiaire de la sanction, de nature juridique cette fois.

Lawrence KOHLBERG, psychologue spécialiste du développement de la morale, en particulier chez l'enfant, souligne le fait que les individus internalisent les règles et valeurs morales à travers leur expérience de la société ${ }^{137}$. Autrement dit, le développement moral de l'individu s'effectue à travers son contact avec le monde. Mais cette relation est également réciproque : les individus influencent la moralité de la société qu'ils composent. Ainsi le développement moral et le développement social sont liés. L'apprentissage de la moralité se déroule parallèlement au processus de socialisation. L'individu apprend à se comporter conformément aux normes en vigueur dans la société dans laquelle il vit, une qualité qui est révélée par sa capacité à adopter des jugements en fonction des règles apprises et à se justifier par rapport à soi-même et aux autres ${ }^{138}$. KOHLBERG voit dans la société le facteur principal dans les variations des valorisations éthiques et morales ${ }^{139}$. Plus précisément, ce sont les parents (héritage culturel), l'école (et autres institutions similaires) et l'environnement de l'individu

\footnotetext{
${ }^{136}$ Voir le paragraphe 3.2.3 « Les influences extérieures » dans ce chapitre, en particulier sur l'exemple et la suggestion.

${ }^{137}$ KOHLBERG Lawrence, 1995, p.7.

${ }^{138}$ Cet argument est au coeur de la théorie du développement moral chez PIAGET. Voir PIAGET Jean, « Le Jugement moral chez l'enfant », 1932.

${ }^{139}$ KOHLBERG Lawrence, 1995, p.17.
} 
(famille, groupe d'amis) qui vont influencer ses valeurs. Les changements dans les valorisations éthiques et morales font partie intégrante de la société et de la vie des individus ${ }^{140}$.

Dans le jugement éthique, EHRENFELS souligne l'importance de la croyance religieuse : celle-ci servirait de repère et fixe des limites du comportement de l'individu. La principale fonction de la religion selon le philosophe, est de déterminer la sanction éthique de l'individu :

" [...] die wichtigste Funktion der religiösen Überzeugung, [ist] nämlich die Bestimmung der ethischen Sanktion des Einzelindividuums [...]» (p.114)

Tous ces arguments incitent donc l'individu à avoir un comportement éthiquement approuvable. Cette aspiration passe par une éducation morale de l'individu par autrui ou d'autrui par l'individu, ou encore par une auto-éducation. Cette dernière se produit, on peut le deviner, à travers l'expérience de l'individu avec son environnement. Le mouvement de valorisation éthique, au niveau individuel, s'effectue grâce à la motivation de l'individu déclenché par son désir d'acceptation dans la société dans laquelle il vit.

Outre ces facteurs incitant l'individu à agir de manière conforme à la morale, on peut distinguer trois principaux facteurs du mouvement des valeurs éthiques :

D'abord l'apparition de nouvelles qualités humaines. Celles-ci ont lieu lors de l'évolution et du développement des sociétés. Ces nouvelles qualités peuvent être positivement ou négativement évaluées. Par exemple, la société industrielle a vu se développer de nouveaux comportements plus entrepreneurs. Les bouleversements sociaux entrainent donc également des bouleversements au niveau des valeurs, et en particulier des valeurs éthiques. EHRENFELS parle alors de la "sympathie" d'autrui face à ces changements (p.474), permettant aux individus de les accepter et de les intégrer. Il ne le dit pas explicitement, mais il est clair que cette mention fait référence au concept du même nom d'Adam SMITH. Christian von EHRENFELS reste d'ailleurs assez peu précis à ce propos, et parle simplement de la sympathie comme d'une disposition :

"Von hervorragender Bedeutung ist hier jedenfalls die Tatsache des menschlichen Zusammenlebens als solche und die Vermehrung und Verengerung der gegenseitigen Abhängigkeitsbeziehungen, wie sie durch die Ausbildung des staatlichen und wirtschaftlichen Lebens gegeben erscheint - welche wohl vermöge der natürlichen psychologischen Wachstumstendenzen Dispositionen der gegenseitigen Sympathie zeitigt. » (p.474)

${ }^{140}$ BERGER Peter L. et BERGER Brigitte, „Wir und die Gesellschaft“, 1976, p.221-238. 
Cette disposition de la sympathie est liée à la volonté ou au désir des individus de vivre en harmonie et d'être apprécié d'autrui. Dans ce passage, la référence à la notion de sympathie est intéressante puisque EHRENFELS montre ainsi ses connaissances dans le domaine économique. Ceci ouvre la voie à un rapprochement entre EHRENFELS et Adam SMITH dans les domaines du désir et du comportement humain. En effet, la notion est traitée par les deux intellectuels comme une qualité psychologique de l'individu qui influence son comportement et donc ses valorisations.

Le deuxième facteur pouvant induire un mouvement de valeur, est celui des connaissances en psychologie concernant le fonctionnement humain dans les rapports sociaux. Les progrès dans ce domaine permettent une meilleure compréhension de ces rapports ainsi qu'une révision éventuelle du jugement à propos d'une qualité humaine. On pense notamment ici aux maladies psychologiques qui bénéficient d'une certaines indulgence lorsqu'elles sont mieux comprises.

Le troisième facteur enfin, concerne les changements des rapports sociaux et de leurs formes. En fait, EHRENFELS fait ici allusion aux changements sociaux structurels : il évoque les rapports sociaux dans les sociétés monarchiques où les valeurs religieuses ont une place prépondérante en comparaison des sociétés industrielles par exemple. Un cas particulier de ce genre de changement est celui de l'évolution du rapport entre ceux qu'EHRENFELS nomme les "aktiven Wertbildner " et ceux qu'il nomme les "passiven Wertbildner ». "Aktive" et "passive Wertbildner» désignent ceux qui influencent activement et respectivement ceux qui subissent passivement l'évolution des valeurs. La traduction de ces expressions allemandes étant difficiles, nous nous proposons d'utiliser les mots d'EHRENFELS dans la suite de notre travail. Si les " passiven Wertbildner " se voient conférer ou acquièrent un pouvoir plus important, leurs valorisations vont être plus prépondérantes dans la société qui les reconnait comme tels. Au contraire, les « aktiven Wertbildner » peuvent également voir leur influence restreinte ou supprimée (perte de privilège de la noblesse par exemple). Un autre cas particulier est celui de l'agrandissement ou de la diminution du territoire de validité de la morale : la conquête d'un nouveau territoire implique dans de nombreux cas la transmission de nouvelles valeurs, notamment de nouvelles valeurs éthiques. Ce fut le cas lors des colonisations occidentales ${ }^{141}$, et pour certains l'influence culturelle, sous toutes ses formes possibles, reste d'actualité $^{142}$. En extension, EHRENFELS affirme qu'une intensification des

\footnotetext{
${ }^{141}$ FERRO Marc (sous la direction de), « Le Livre Noir du colonialisme, $\mathrm{XVI}^{\text {ème }}-\mathrm{XXI}^{\text {ème }}$ siècle : de l'extermination à la repentance », 2003.

${ }^{142}$ WOOD Ellen Meiksins, « Empire of Capital ", 2003 ; RAMEDHAN Erwin, " La Société Indonésienne face à la pénétration du modèle occidental », 1981 ; LARDELLIER Pascal, « Le
} 
échanges entre les sociétés conduit à une assimilation non seulement des perspectives et des convictions, mais aussi des valeurs éthiques. Cet argument est non seulement pertinent, mais aussi actuel puisque, dans un monde où les échanges prennent quotidiennement une importance grandissante, les questions de la standardisation et des influences culturelles, voir de la domination culturelle, se posent de plus en plus ${ }^{143}$. L'idéologie politique est un élément susceptible de faire évoluer les valeurs accordées par les individus ${ }^{144}$. Les personnalités politiques ayant la possibilité de diffuser leur idéologie sont également des exemples de " aktive Wertbildner ».

On a vu dans les parties précédentes qu'il existe une relation étroite entre les valeurs intrinsèques et les valeurs d'effet. Les premières dérivent bien souvent des secondes dans les situations de report du désir de l'effet d'un objet aux origines de celui-ci, c'est-à-dire en fait à l'objet lui-même. En d'autres termes, après avoir désiré un objet pour ses effets, l'individu va finir par le désirer pour lui-même et celui-ci se verra donc par la même occasion accorder non plus une valeur d'effet mais une valeur intrinsèque. Dans le domaine éthique, le processus de dérivation d'une valeur de l'autre se produit certes de la même manière mais pas en même temps, ni de la même façon chez tous les individus. Et ceci à cause de l'existence des " aktiven Wertbildner ", dont le domaine d'influence et la créativité atteignent également les valeurs éthiques. Sachant que les dispositions émotionnelles d'autrui n'ont pas la même valeur pour chaque individu, dépendant de sa place sociale, celles des "aktiven Wertbildner" auront tendance à être plus prises en considération que celles des autres individus. Ces personnes possèdent incontestablement une position sociale et un pouvoir social importants. En effet, si un "aktiver Wertbildner" transforme ses valeurs d'effet accordées à des dispositions émotionnelles en valeurs intrinsèques et s'il arrive à les transmettre à ses congénères, il pourrait alors influencer leur profil moral en entier. L'assimilation de ces valorisations éthiques par autrui pourrait ainsi déboucher sur un mouvement de valeur.

Si nous reprenons l'argument du désir de gagner en considération sociale, on comprend alors que les individus chercheront à imiter les " aktiven Wertbildner ». Si ce dernier arrive à étendre son domaine d'influence à toute une société alors il provoquera, indirectement, un mouvement des valeurs.

Steak caché des Fast-Foods ", 2003 ; CASSEN Bernard, " Un Monde Polyglotte pour échapper à la dictacture de l'anglais ", 2005.

${ }_{143}$ STIGLITZ Jospeh E., " La Grande Désillusion », 2002, p.67-98.

${ }^{144}$ BRZEZINSKI Zbigniew, " Macht und Moral », 1994, p.34-48. 
EHRENFELS va même jusqu'à établir un parallèle entre les fortes personnalités dans le domaine de l'éthique et dans celui de l'économie : comme le prix d'un bien établit la norme pour la production de ce bien, la formation des caractères humains sera influencée essentiellement par les valorisations éthiques. Si les estimations de ceux qui dominent l'économie - les riches - se reflètent dans les prix, celles des fortes personnalités se reflètent dans les valorisations éthiques d'une culture (p.466) :

«Wie der Güterpreis die Norm für die Güterproduktion abgibt, so wird die Ausbildung menschlicher Charaktere wesentlich durch die ethischen Wertungen beeinflußt. [...] Spiegelt sich in dem Preise die Wertschätzung der wirtschaftlichen Mächtigen, d.h. die Reichen, so in den ethischen Wertungen - in der spezifischen "Moral " eines Wertungsgebietes - die Wertschätzung der « Starken im Geiste ». » ${ }^{145}$

Les racines des relations éthiques sont cependant plus profondes que celles des relations économiques dans la mesure où les premières ne sont pas liées à un droit de propriété sur un objet mais ancrées dans la nature humaine.

Le parallèle énoncé par EHRENFELS montre d'une part sa conception de la société et de son fonctionnement: les hommes puissants ont une influence considérable sur les autres personnes. Cette vision de la société divisée entre ceux qui dirigent ou qui se distinguent, se retrouve chez Max WEBER ${ }^{146}$, Vilfredo PARETO $^{147}$ ou encore Karl MARX ${ }^{148}$. Plus proche de nous, Eric ZBINDEN montre que le pouvoir influence les valeurs collectives ${ }^{149}$. EHRENFELS établit également un parallèle entre économie et éthique en ce sens où dans l'un comme dans l'autre, des normes sont établis par certains individus, et en extrapolant quelque peu ses propos, on pourrait ajouter que ces individus semblent être similaires dans les deux cas, à savoir ceux qui dominent leurs domaines respectifs. En particulier, l'argument du prix comme étant le reflet des estimations des puissants semble rencontrer une certaine approbation contemporaine ${ }^{150}$.

\footnotetext{
${ }^{145}$ EHRENFELS, 1982, p.119.

${ }^{146}$ Max WEBER distingue les leaders ou meneurs de ceux qui les suivent. Dans toute société, groupe ou collectivité, il y a un leader qui sert de référence aux autres. Voir WEBER Max, "Wirtschaft und Gesellschaft ", 1972, chapitre 1.

${ }^{147}$ Pour PARETO, il y a dans toute société des élites qui se divisent elles-mêmes entre les loups (membres plutôt conservateurs qui s'imposent par la violence) et les renards (membres plus ouverts et qui s'imposent par la ruse). Voir PARETO Vilfredo, "Allgemeine Soziologie ", 1955. ${ }^{148}$ MARX distingue ceux qui possèdent les moyens de production et qui possèdent également de ce fait un pouvoir, celui de disposer et d'offrir des biens, de ceux qui ne possèdent que leur force de travail. Ce pouvoir s'étend au niveau de la société toute entière puisque les rapports de production déterminent les rapports sociaux. Voir dans notre chapitre 3 le paragraphe II.

${ }_{49}$ ZBINDEN Eric, "Werte und Macht in sozietalen Systemen », 1984, p.108-115.

${ }^{150}$ Idem, p.45.
} 
EHRENFELS essaiera en outre d'identifier les raisons biologiques pour lesquelles il y a des "aktiven Wertbildner", sans pour autant y parvenir véritablement, affirmant que le niveau de développement de la psychologie de l'époque ne le permettait pas (p.477). La seule cause qui est identifiée est celle de la "disposition naturelle": les "aktiven Wertbildner " possèderait une tendance innée qui les pousserait à influencer les dispositions émotionnelles d'autrui.

Concernant enfin la conscience du type de valeur accordée par l'individu, EHRENFELS affirme que les individus ne le savent pas nécessairement. Ils ne sont pas non plus conscients de la corrélation entre une valeur d'effet et une valeur intrinsèque qui en dériverait par exemple. Mais cette distinction est-elle véritablement pertinente pour l'individu? On peut en douter. Par contre son intérêt est incontestable pour l'analyse des valeurs et de leur origine. EHRENFELS cite en exemple le comportement de certaines populations, sans préciser lesquelles, qui considèrent la virginité comme une valeur éthique intrinsèque, à laquelle ils associent une valeur d'effet par rapport à la descendance, censée être ainsi plus résistante et en meilleure santé. Pour le philosophe, ce genre de valorisations est associé avant tout à des coutumes et traditions, moins à des connaissances fondées. Nous pouvons citer un exemple de population pour laquelle la virginité, en plus de sa valeur intrinsèque éthique, possède une autre valeur d'effet, celle de garantir l'honneur de la famille et donc sa place dans la société. L'auteure norvégienne Åsne SEIERSTAD décrit dans son livre "Le Libraire de Kaboul» la place des femmes et notamment leur soumission physique et psychique aux hommes dans une famille de Kaboul et dans leur entourage ${ }^{|5|}$. Elle montre l'importance liée à la pureté de la femme avant son mariage pour toute sa famille. Dans cet exemple, la valeur intrinsèque dérive de la valeur d'effet de l'objet mentionné. Cependant, les individus ne sont pas conscients de la nature des valeurs accordées dans les deux cas. La question qui vient alors à l'esprit est la suivante : est-il important de savoir si la valeur qui est accordée est une valeur intrinsèque ou une valeur d'effet et quelle est la relation qui les lie?

Du point de vue de l'individu, il est évident qu'il ne se pose pas véritablement la question et que la réponse ne lui apparaîtrait pas d'une grande importance. Son attention portera surtout sur la valeur elle-même, c'est-à-dire son aspect quantitatif (objet de grande ou de petite valeur). Il s'agit d'un des aspects de la théorie d'EHRENFELS qui nous parait perfectible et dont nous discuterons à la fin de ce chapitre.

${ }^{151}$ SEIERSTAD Åsne, " Le Libraire de Kaboul », 2003. 
L'attribution des valeurs éthiques, c'est-à-dire leur apparition, se fait en relation avec l'objet valorisé et par rapport au contexte socio-culturel. La durée de vie d'une valeur éthique par contre, obéit à d'autres mécanismes. Les valeurs éthiques intrinsèques qui découlent des valeurs éthiques d'effet suivent, dans leur évolution, ces dernières : lorsque l'effet d'une valeur éthique disparait, la valeur associée disparaît également, emportant dans son sillage la valeur intrinsèque qui en dérive. Ainsi les valeurs d'effet éthiques peuvent être qualifiées de valeurs dépendantes. L'effet en question ou plus exactement l'avantage qui fonde la valeur d'effet, peut être par exemple l'approbation par autrui d'un comportement en particulier. Si cette approbation disparait, les valeurs éthiques associées n'ont alors plus de raison d'être. Ce processus de disparition de l'avantage est qualifié de "Entfrommung" par EHRENFELS, un terme que l'on pourrait traduire simplement par perte d'avantage ou perte d'intérêt. Ce processus se produit de manière plus ou moins rapide, selon l'évolution de la société, mais il s'étale en général sur plusieurs générations de l'avis du philosophe. Il ajoute cependant que toutes les valeurs éthiques intrinsèques ne sont pas dérivées de valeurs d'effet de même nature, tout comme toutes les valeurs éthiques intrinsèques ne disparaissent pas après la perte d'avantage des valeurs d'effet dont elles dérivent (p.468). De même, le processus de perte d'avantage ne peut avoir de conséquence que pour les valeurs éthiques d'effet: si celui-ci s'apparente à la perte de l'approbation d'autrui, il n'empêche que la valeur éthique intrinsèque qui en dérive peut perdurer dans la mesure où l'attribut de l'objet valorisé n'est pas automatiquement remis en cause et son aspect éthique peut rester intact.

L'évolution des valorisations des objets dans le domaine éthique va se répercuter sur la morale de la société, mais avec un certain temps de retard. Le temps d'intégration des nouvelles valeurs dans la morale peut être plus ou moins long, selon le processus d'intégration social qui intervient parallèlement dans cette situation. Il y a donc toujours un décalage entre la morale en vigueur dans une société et les nouvelles valeurs éthiques: cette différence peut expliquer notamment le conflit des générations, une extrapolation non évoquée par EHRENFELS mais qui semble être une interprétation logique de son argumentation. Ce processus est un cycle et pendant que la morale prend en compte les nouvelles valeurs éthiques, d'autres valorisations apparaissent et vont plus tard s'établir dans la société.

Le rôle des "aktiven Wertbildner » apparaît alors comme très important dans ce développement puisqu'ils sont à l'origine des innovations en matière de valorisation.

En conséquence des arguments précédents, EHRENFELS distingue trois catégories de valeurs éthiques. En premier lieu, celle dont les valeurs ont perdu leur avantage ou sont en train de subir cette perte et qui est qualifiée de catégorie 
des valeurs survivantes ; ensuite, celle dont les objets des valorisations ont toujours leurs valeurs d'effet et qui est qualifiée de catégorie des valeurs normales; enfin celle dont les valorisations sont en devenir (p.469). Il serait également possible d'ajouter d'autres catégories, par exemple celle des valeurs éthiques dérivées des valeurs d'effet, lesquelles sont accordées par rapport à une erreur de jugement sur la relation entre valeur d'effet et valeur intrinsèque. Cette erreur de jugement, selon EHRENFELS serait des plus rares et il n'est donc pas pertinent de vouloir construire une telle catégorie. En fait, les trois premières catégories évoquées rassemblent déjà les principaux types de valeurs éthiques, et les autres catégories que l'on pourrait encore dessiner, ne contiendrait alors plus que des cas de valeurs exceptionnels ou rares. A ces catégories, il convient d'ajouter des formes apparentes des valorisations éthiques qui ne peuvent être classées (p.564) : la règle qui veut que les tendances comportementales soient valorisées plus ou moins positivement selon leur contribution au bien être de tous admet une exception dans le cas des valeurs éthiques survivantes qui caractérisent un comportement appartenant au passé, et dont la contribution au bien être de tous n'est plus d'actualité. Les valeurs éthiques en devenir, de par leur définition, dérivent de valorisations dont la réalisation est le fait, dans un premier temps, d'un petit cercle de personnes (p.564-565). Son extension et son acceptation par un nombre plus grand d'individus s'effectueront dans un futur qui est plus ou moins proche selon la rapidité de cette acceptation. Il nous faut également signaler un risque de non acceptation - par certaines tranches de la population - de certaines valeurs en devenir, une hypothèse non traitée par le philosophe autrichien.

La question de la classification des valeurs devient quelque peu complexe : comment distinguer une valeur en devenir d'une valeur survivante ? Une réponse facile, selon EHRENFELS, serait de classer les valeurs selon leur ressemblance respective. Si une valeur ressemble à une valeur éthique survivante, alors on pourrait la mettre dans la catégorie correspondante. Mais ce procédé apparaît peu satisfaisant pour le philosophe (p.569). Il lui préfère une méthode proche de celle des sciences naturelles où les espèces sont classées par rapport à leurs caractéristiques génétiques. Ainsi deux animaux peuvent, en apparence, n'avoir aucun point commun, mais appartenir malgré tout à la même famille (par exemple, la tortue et le crocodile appartenant tous les deux à la famille des reptiles) ${ }^{152}$. Ce même procédé devrait être appliqué aux valeurs éthiques afin de pouvoir les classer (il faut chercher leurs caractéristiques fondamentales communes). Cette façon de faire qui se veut rigoureuse, nous semble un peu confuse pour permettre une telle utilisation. D'abord EHRENFELS ne donne pas

\footnotetext{
152 Ehrenfels donne l'exemple des « Megacarpaea » et " Tetrapoma " qui appartiennent à la famille des Cruciferes, un exemple qu'il a repris des études de ses collègues, le Dr. B. Hatscheck, professeur de Zoologie à l'Université de Vienne et le Dr. R. v. Wettstein, professeur de botanique à l'Université allemande de Prague. EHRENFELS, p.569.
} 
plus de précision sur l'application d'une telle méthode dans le domaine des valeurs éthiques : comment reconnaître le point commun génétique des valeurs ? Il serait cependant possible de classer les valeurs selon leur degré d'utilisation ou de reconnaissance dans une société donnée. Le type de valorisation qui se rencontre le plus fréquemment est celui qui est le plus actuel car le plus répandu. Les valorisations minoritaires sont celles qui concernent soit les valeurs en devenir, soit les valeurs survivantes. Si la valeur attribuée est déjà connue depuis longtemps, alors il s'agit d'une valeur survivante. Si cette valeur est peu connue dans la société alors il s'agit d'une valeur en devenir (exemple, la valorisation des comportements homosexuels).

Si on s'intéresse de plus près à la perspective du philosophe autrichien, on note que son explication s'apparente encore une fois à un cycle dans lequel les valeurs éthiques naissent, se développent et meurent ou tombent dans l'oubli. Les valeurs sont donc, en quelque sorte, des éléments "vivants» ou, du moins, dont la dynamique de développement se rapproche d'un cycle de vie.

Toujours dans le cadre de l'évolution des valeurs éthiques, EHRENFELS effectue un parallèle entre la relation valeur-utilité en économie et celle entre valeur et avantage dans le domaine de l'éthique (p.470). Ainsi, l'eau et l'air, qui ont une grande utilité, n'ont pas une valeur élevée car ils sont présents en grande quantité dans la nature et leur acquisition ne pose aucun problème. Dans le domaine de l'éthique, la survie de l'individu et celle de l'espèce humaine en général, bien qu'ayant un avantage ou " Frommen » élevé, n'ont pas une grande valeur d'effet car ils sont également présents en grande quantité chez les individus.

Le doute n'est pas permis sur le fait que la survie d'un individu ou de l'espèce n'aient ni une valeur intrinsèque, ni une valeur d'effet élévées: selon EHRENFELS la nature a doté tous les individus d'une grande envie de survivre de telle manière qu'ils ne pensent même pas à la remettre en question. Il est d'ailleurs familier de parler "d'instinct de survie », faisant alors de la survie un objet inné, présent dès le départ chez les êtres humains. De plus, la possession de l'objet « survie » ne présenterait pas un avantage particulier dans la mesure où la quasi-totalité de la population le possède déjà.

L'envie de survivre, contrairement à ce que le philosophe autrichien semble croire, n'est pas toujours présente en grande quantité chez tous les individus. On distingue en particulier ceux qui ont des tendances suicidaires. A l'époque d'EHRENFELS, bon nombre d'ouvrages ont également tenté d'analyser le phénomène du suicide ${ }^{153}$.

${ }^{153} \mathrm{~A}$ la fin du XIX ${ }^{\text {ème }}$ siècle, le phénomène du suicide a suscité l'intérêt d'un grand nombre d'intellectuels et de scientifiques. Son analyse fut entreprise en psychologie (voir par exemple FREUD Sigmund, "Trauer und Melancholie ", 1916) et en sociologie (voir par exemple DURKHEIM Emile, « Le Suicide », 1897, CHYLE Franz, « Der Selbstmord als Spekulation des 
Outre ce parallèle avec le domaine économique, EHRENFELS veut appliquer le concept de marginalité aux valeurs éthiques (p.470). Il commence cependant par énoncer une restriction : la libre utilisation des réserves de biens marchands n'est pas possible dans le domaine de l'éthique où on ne peut pas disposer librement des dispositions émotionnelles d'autrui pour les augmenter ou les diminuer. Autrement dit, dans le cas des biens marchands, il est possible de déterminer leur quantité à travers leur production alors qu'il n'est pas possible de pré-définir la production de dispositions émotionnelles. Tout au plus peut on noter une tendance de la valorisation éthique à augmenter le stock d'objets éthiques. Le problème est alors de pouvoir définir une quantité de dispositions émotionnelles. Il s'agit avant tout d'une notion qualitative, mais EHRENFELS tente également une approche quantitative (p.471). Les dispositions émotionnelles évoluent en quantité comme en intensité. Leur quantité augmente lorsqu'un plus grand nombre éprouve une même disposition émotionnelle. Leur intensité augmente lorsque cette disposition est émotionnellement plus forte dans chaque individu. L'augmentation de la quantité de dispositions émotionnelles identiques s'effectue à travers l'influence d'un individu sur autrui. EHRENFELS donne l'exemple d'une société dans laquelle la moitié des prêtres serait instruite et l'autre moitié ne le serait pas (p.124). Une première moitié de la population sera alors influencée par la première moitié instruite des prêtres et l'autre moitié de la population le sera par la seconde moitié des prêtres non instruits. Cette évolution quantitative n'en reste pas moins très théorique et il est empiriquement difficile de prédire l'importance d'une influence sur autrui. En effet, si on suppose qu'une moitié des prêtres prêchant dans une société est instruite, il est relativement peu probable que leur sermon soit entendu par la moitié de la population. De plus, le degré d'influence d'un prêtre sur autrui varie d'un individu à l'autre. L'exemple donné par EHRENFELS s'avère donc peu convaincant.

S'il est difficile de déterminer les stocks de dispositions émotionnelles, il est par contre plus facile de distinguer les causes de leurs mouvements: leur augmentation ou leur diminution se fait à travers l'exemple et/ou la suggestion. On pense ainsi aux institutions publiques telles que l'école ou encore aux personnes qui ont une forte personnalité pour illustrer cet argument. On peut donc expliquer la variation de la quantité de dispositions émotionnelles dans les individus, mais on ne peut pas en mesurer la quantité totale dans une population ou une société. L'approche quantitative est par conséquent limitée.

modernen Verbrechertums ", 1908, ou MASARYK Thomas G., " Der Selbstmord als sociale Massenerscheinung der modernen Civilisation ", 1881). On s'intéressa en outre à sa présence dans la littérature (voir par exemple ROST Hans, " Bibliographie des Selbstmordes ", Regensburg 1927) ou encore au théâtre (FERRARI Paolo, " Ein Selbstmord », pièce en 5 actes, 1881 ou HALBE Max, " Mutter Erde », 1898). 
Pour EHRENFELS, les dispositions émotionnelles vont inciter l'individu à agir dans le sens du "Wohl der Gesamtheit » ou bien de tous. En d'autres termes, elles feront en sorte que l'individu se décide pour l'objet ayant la plus grande valeur intrinsèque éthique. Il en découlera une augmentation du "stock " de telles dispositions dans l'individu, mais, ajoute le philosophe, une augmentation décroissante de ce stock. Plus la disposition est présente chez un grand nombre d'individus et moins la croissance de ce stock sera forte : il s'agit clairement ici d'une tentative d'application du concept de marginalité aux valeurs éthiques. Lorsque le stock de comportement ayant une valeur morale augmente, son avantage lui, n'augmentera pas, au contraire, il aura plutôt tendance à diminuer. En effet, si un comportement, dit conforme à l'éthique, est largement répandu dans une société donnée, le fait pour un individu supplémentaire d'avoir ce comportement ne représentera qu'un avantage limité. L'augmentation décroissante du stock de dispositions émotionnelles éthiques chez l'individu s'explique par la perte progressive de l'avantage de l'objet valorisé. Plus un objet est possédé par un grand nombre d'individus, plus l'avantage lié à sa possession diminue, tout comme l'augmentation des dispositions émotionnelles inhérente.

L'application du concept de marginalité ne peut par contre se faire concernant le dommage (Schaden) puisque une augmentation des dispositions immorales entraîne une augmentation plus que proportionnelle du "stock" de telles dispositions. Concrètement, une multiplication par dix du nombre de meurtriers dans une société causerait une multiplication du dommage crée par plus de dix. Une nouvelle fois l'argument du philosophe autrichien s'avère très théorique. Dans son livre paru en 1994 et s'intitulant «Punir les Pauvres : le nouveau gouvernement de l'insécurité sociale ", Loïc WACQUANT, professeur de sociologie à l'Université de Californie à Berkeley, montre qu'il n'y a pas de corrélation entre l'augmentation ou la baisse de la criminalité et sa perception par les individus. En effet, il affirme que malgré une baisse statistique des homicides en France depuis 1995, la population aurait plutôt une impression contraire à cause de l'impact des médias ${ }^{134}$. L'influence des dispositions émotionnelles des individus ne se base donc pas toujours sur des faits réels mais sur la perception qu'ils ont de ces faits.

Après le thème du mouvement des valeurs éthiques, intéressons nous à présent aux mouvements de valeurs morales ainsi que de la recherche du bien être pour tous, un objet dont la valeur éthique est des plus élevée.

${ }^{154}$ WACQUANT Loïc, „Fermons les Prisons !“, in Le Monde Diplomatique, septembre 2004, p.67. 
Les valorisations morales sont liées aux valorisations éthiques dans la mesure où ces dernières sont celles qui attribuent une valeur aux qualités de la personne. De par ce lien, les mouvements de valorisation morale sont un produit des valorisations éthiques et leur influence se limite à la tendance qu'elles donnent aux secondes (p.478).

Les valorisations morales se font donc à l'égard des comportements des individus, eux-mêmes dictés par les dispositions émotionnelles. Les dispositions émotionnelles se voient donc attribuer une valeur d'effet puisqu'elles causent le désir d'agir. Dans le domaine moral, la recherche du comportement ou plus exactement de la disposition ayant la plus grande valeur d'effet, est la règle. On assiste alors à un mouvement de valeur vers le "Erhaltungsglied " c'est à dire vers l'élément coordinateur (élément qui fait que la chaine de l'action tienne), ce qui revient à parler d'un changement des valorisations vers le haut ou vers l'intérieur. Concrètement, le but de la valorisation ne concerne plus l'effet de l'objet mais l'objet lui-même. Ce changement s'opère logiquement selon EHRENFELS puisque le bien être de tous, comme objet, ne pourrait pas avoir la valeur d'effet la plus élevée. En effet, si cela était le cas, l'énergie de l'individu se concentrerait sur la manière d'agir la plus appropriée au lieu d'agir proprement dit (p.479), ce qui serait nuisible dans les situations où une action rapide est nécessaire. Cet argument est à mettre en rapport avec les réflexions d'EHRENFELS sur l'utilisation de la "Lebenskraft » ou force vitale : celle-ci n'existe pas de manière infinie et elle doit être partagée entre les différentes activités de l'individu. Ainsi la conscience de l'individu va plutôt orienter son attention sur l'objet, permettant de fixer un but conduisant l'individu à agir. L'énergie de l'individu sera donc dans un second temps utilisée pour atteindre ce but et ne restera pas fixée sur le moyen de l'atteindre. Parallèlement, un changement de valeur s'effectue de la façon précédemment expliquée.

L'objet moral " bien être de tous » doit être considéré plutôt comme un but à long terme. Dans sa vie quotidienne, l'individu se donne pour but d'autres objets qui sont conformes à la morale en vigueur. Ainsi s'opère une distinction entre le " moralische Bestrebung " ou l'aspiration morale, et le "moralischer Trieb " ou pulsion morale. Le premier désigne les efforts de l'individu dans un but moral, un but qui se veut de moyen ou de long terme. L'agissement moral désigne le fait d'agir dans le sens de la morale au quotidien. Cependant, il peut parfois être plus fort que l'effort moral (p.481) en ce sens où l'individu va privilégier sa morale quotidienne et considérer au second plan des buts de long terme. Le doute est par ailleurs permis sur le fait que les dispositions émotionnelles possèderaient une tendance à influencer les valeurs dans le sens du bien être de tous, c'est-à-dire vers un but général et de long terme. Le sens du concept de "bien être pour tous" reste ici à préciser : s'agit-il d'une notion au sens fixe ou dont la signification 
évolue avec le temps ? Le but moral de long terme demande en effet, un agissement sur le long terme, à l'échelle d'une vie donc. Pour atteindre ce but, l'individu doit acquérir des dispositions émotionnelles qui le mèneraient à son but et qui détermineraient par conséquent sa manière d'agir au long de sa vie. On voit ici apparaitre le sens religieux de l'argument du philosophe autrichien : l'effort moral est en fin de compte une référence qui conduit l'individu vers une vie qui sera très positivement valorisée et cet effort moral est celui qui est contenu dans les écrits religieux.

Le bien de tous qui est alors commandité, se définit-il par rapport à un état dynamique ou statique ? Il semble que pour EHRENFELS la perspective d'un développement soit la bonne réponse à cette question (p.482). En effet, on sait que les valorisations morales dérivent des valorisations éthiques et que ces dernières subissent une évolution qui est à mettre en rapport avec celle de la société. Un changement chez l'une provoquera un changement chez l'autre : les valorisations morales sont donc à considérer dans une perspective de développement. Ainsi l'aspiration au développement pour tous aura l'avantage ou le "Frommen " le plus élevé et se verra donc attribuer la plus grande valeur d'effet possible. On parlera alors de "morale qui s'élève" ou " aufstrebende Moral» (p.482). Le développement moral et celui de la société sont deux phénomènes parallèles et corrélés.

D'autres facteurs n'ayant pas de rapport direct avec l'éthique peuvent par ailleurs influencer le mouvement des valeurs morales. Les actions dans le but d'acquérir ou de posséder des objets ne se produisent pas tant en conséquence du désir dirigé sur ces derniers qu'en conséquence du désir d'agir pour les acquérir ou les posséder. En d'autres termes, c'est l'action qui est privilégiée dans le désir et non l'objet lui-même. Pour illustrer ses propos, EHRENFELS nous donne l'exemple des joueurs de billards ou des jeux d'adresse en général. Celui qui est mieux prédisposé à jouer n'est pas celui qui désire le gain, mais celui qui désire avant tout l'action qui le mène au gain, c'est à dire jouer. Tous ceux qui se sont enrichis, n'ont pas tant désiré la richesse que le combat et le travail qui mènent à cette richesse. Le désir se dirige avant tout sur l'action, mais il ne néglige pour autant pas le but ultime. Le jugement moral se construit par rapport aux dispositions de l'individu agissant mais aussi par rapport à son but. Ainsi, le fait de vouloir la richesse n'était, par exemple, pas positivement valorisé dans les sociétés catholiques d'antan, contrairement au labeur du travailleur. De même, le joueur qui agit par plaisir pour le jeu lui-même, ne sera pas désapprouvé par autrui, contrairement à celui qui ne vise que le gain. Aussi les personnes privilégiant l'action auront l'esprit tranquille et s'attireront une certaine sympathie, au sens premier du terme, de la part d'autrui (p.484). 
En outre, la personne qui considère l'action plus que le but ultime, va également déterminer le type d'action en favorisant au maximum la réalisation de l'objet. En d'autres termes, l'individu va privilégier le moyen ayant la plus grande probabilité de réalisation possible du but initialement fixé. EHRENFELS, pour montrer la véracité de son argument, nous propose aussi une démonstration mathématique : la volonté d'atteindre un but 10 qui a une probabilité de réalisation de $1 / 3$ l'emportera sur celle d'atteindre un but 4 avec une probabilité de réalisation de $2 / 3$ car 10/3 est supérieur à $8 / 3$. Il suppose aussi que, de par la loi des grands nombres, plus la probabilité de réalisation est faible et plus le nombre d'essais doit être grand. Ainsi celui qui vise un gain de 100 avec une probabilité de réalisation de $1 / 30$, va privilégier l'action qui le mènera au gain 4 avec une probabilité de réalisation de $2 / 3$, malgré le fait que $100 / 30$ soit supérieur à $8 / 3$, car la première possibilité demanderait une répétition de l'action un trop grand nombre de fois avant de voir le but atteint. Il nous parait réaliste de rajouter à l'argument du philosophe autrichien que la grande majorité des personnes ne connaissent pas la probabilité de réalisation exacte du but d'un acte. Si c'est la première fois qu'elles agissent de la sorte, elles ne peuvent qu'estimer cette probabilité notamment grâce aux expériences d'autrui. Si elles agissent par habitude alors la probabilité de réalisation estimée sera fonction de leurs expériences passées.

EHRENFELS affirme par ailleurs que le fait de valoriser l'action plus que le but favorise sa probabilité de réalisation (le joueur de billard qui s'intéresse plus au jeu qu'aux gains subira moins de stress que celui qui privilégie la victoire, et sa probabilité de réussite sera ainsi plus élevée). On parlera ici d'un mouvement de valeur du but vers le moyen (ou "Wertbewegung nach abwärts") ou encore d'un mouvement de valeur en faveur de l'action.

EHRENFELS voit donc également les valeurs éthiques et les valeurs morales comme n'étant pas figées dans le temps. Leur évolution est la conséquence des changements survenus dans l'éthique d'une société donnée. L'éthique n'est pas la seule composante d'une société pouvant influencer la valorisation des individus. Ceux-ci vont aussi tenir compte des maximes, des coutumes et du droit.

\section{4 - Maximes morales, coutumes et droit}

Dans la plupart des collectivités ou des sociétés, on retrouve des valorisations éthiques régulant le comportement, c'est-à-dire des règles et des consensus autour desquels la population s'accorde. Les comportements des membres de la collectivité seront, en référence aux consensus évoqués, valorisés sous l'angle éthique. Un comportement auquel on accorde une valeur éthique négative sera désapprouvé par autrui et peut être également puni à travers une 
sanction. S'il y a une sanction alors il y a un interdit correspondant. L'interdit suppose l'existence de son contraire : un comportement ne peut être interdit que si son contraire est approuvé. L'opposé de l'interdit est incarné par l'impératif éthique et le commandement (le second au sens religieux du terme puisque le mot employé par EHRENFELS est celui de "Gebot» en allemand qui se traduit par commandement religieux en français). L'application des impératifs et des commandements devrait conduire, selon le philosophe, au bien être, un objectif qui se voit accorder la valeur intrinsèque la plus élevée.

Commençons par nous intéresser à la notion de la maxime. Pour EHRENFELS, les maximes morales sont toutes les règles du comportement ayant pour but la poursuite du bien être pour tous (p.489). Elles concernent tous les domaines de la vie et elles impliquent une certaine habitude la part des individus qui la suivent. En outre, la poursuite des maximes constitue un moyen d'agir pour celui dont les dispositions émotionnelles seront dirigées vers le bien être pour tous. Elles auront par conséquent une grande valeur d'effet. L'habitude prise de suivre les impératifs moraux est induite, soit par l'obligation que l'individu s'impose d'adopter de tels comportements ; soit par son éducation, ou encore par les deux à la fois (p.491).

Le philosophe autrichien ajoute que les cas d'individus n'agissant que par rapport aux maximes, tout comme ceux des individus qui n'agiraient que par rapport à leurs envies, sont des cas extrêmes qui ne sont pas souvent observables (p.490). De plus, un individu respectant les maximes morales sans avoir pour but ultime le bien être de tous, bénéficiera d'une valorisation positive de son comportement.

Il suppose par ailleurs que le système de maximes est en situation de quasimonopole ou, du moins, en situation dominante dans une société le reconnaissant.

Par conséquent, la personne dont le comportement se dirige plutôt vers un autre système (par exemple un système privilégiant plus la famille ou les amis), même si ses dispositions morales se verront positivement valorisées, on pourra lui reprocher un manque de dispositions en faveur des cercles de personnes plus larges.

Une liste des maximes morales, par ordre d'importance, est également fournie par EHRENFELS (p.491-492). La première place revient au bien être pour tous. Viennent ensuite le bien être des concitoyens ("Volksgenossen »), de la famille, l'éveil des jugements vrais chez autrui, la préservation de la virginité, etc... qui peuvent être qualifiés de maximes de second ou de troisième rang. Ce classement, qui est établi par EHRENFELS lui-même, est influencé par ses propres valeurs conservatrices et religieuses. De plus, si on devait établir un tel classement pour 
nos sociétés occidentales actuelles, il serait certainement différent, mais ceci est également envisagé par la théorie d'EHRENFELS qui parle, nous l'avons vu, de l'évolution de la morale et des valeurs adjacentes.

Ayant ainsi établi l'importance des maximes morales, EHRENFELS va s'intéresser plus précisément à leur fondement et à leur validité. Les maximes morales ont pour lui une connotation religieuse indéniable (p.504). Les religions proposent des commandements réglant, d'une manière générale, les comportements des croyants. Aller à l'encontre d'un commandement sera éthiquement sanctionné. Les maximes du comportement, tout comme certains commandements religieux trouvent leurs racines dans la conscience morale générale. Dans ce cas, on parlera de la validité réelle d'une maxime morale (" die tatsächliche Geltung einer moralischen Maxime »). Sa portée peut varier d'un individu (qui s'impose des règles de conduite) à une communauté toute entière. Le fait de ne pas respecter une maxime morale sera négativement valorisé car la maxime morale est favorable au bien être de tous. Ne pas la respecter, c'est manquer de disposition du désir pour le bien être de tous et c'est en fin de compte ce manque qui est négativement valorisé. Cette valorisation négative n'est cependant pas reliée automatiquement à une sanction, celle-ci intervient plutôt à travers les coutumes et les habitudes inhérentes à leur transgression. De plus, pour être sanctionnées, ces transgressions doivent être visibles ou extérieures, c'est-àdire que l'on doit pouvoir les prouver.

Evoquons à présent la relation qu'EHRENFELS établit entre les normes, les maximes morales et le droit (p.493). L'existence ou la raison d'être du droit se conçoit à travers l'image d'une collectivité sociale dans laquelle toutes les conditions fondamentales du droit sont réunies ${ }^{155}$. Dans cette collectivité, les personnes transgressant une maxime morale à travers leur comportement, jugé alors comme nuisible pour autrui et n'allant donc pas dans le sens du bien être de tous, serait sanctionné. Ce processus serait la conséquence d'un consensus collectif et favoriserait, implicitement, l'harmonie entre les membres de cette collectivité. Cette vision se rapproche de celle de Thomas HOBBES qui voit dans l'Etat et ses institutions l'incarnation de la volonté d'une collectivité d'individus ayant pour objectif l'harmonie et la paix. Ces buts seront garantis grâce aux lois auxquelles la collectivité accepte de se soumettre ${ }^{156}$.

Si chacun poursuit ses intérêts personnels, la société aura une base instable défavorable en fin de compte à l'intérêt général. Aussi les individus retireront un

\footnotetext{
155 « Das Wesen des Rechtes kann man am besten erfassen, indem man das Idealbild einer sozialen Genossenschaft konstruiert, in welcher alle bei der Rechtsbegrundung konkurrierenden

Bedingungen gegeben sind ", in EHRENFELS, p.493.

${ }^{156}$ HOBBES Thomas, "Leviathan », 1984, p.134-135 et p.139.
} 
avantage à se soumettre à un ensemble de règles qui favorisent un état social stable $^{157}$. La présence de l'Etat ou d'une instance régulatrice, n'est pas explicitement évoquée par EHRENFELS, mais ses propos ne laissent aucun doute quand à ce sujet.

Les notions de droit et de collectivité sont associées à celle de "Rechtsgenossenschaft » ou droit collectif, c'est-à-dire d'un droit valable pour tous les membres d'une même communauté (p.494). Ceci implique concrètement une entente quant aux attitudes réprouvées ainsi que sur la sanction appliquée dans ce cas. Autant le consensus que la sanction seront écrits pour éviter par exemple les situations de vengeance personnelle, néfaste au bien être de tous.

Concernant encore une fois les condamnations, EHRENFELS dénonce ceux qui en useraient comme d'un pouvoir arbitraire (p.495). En d'autres termes, il évoque les avantages d'une décision consensuelle et de la démocratie en matière de justice, non seulement pour ce qui est de l'écriture des sanctions, mais aussi de leur disparition dans le cas où les attitudes correspondantes ne seraient plus condamnées par la majorité de la population. Cependant, même dans une démocratie, il convient de faire la différence entre les membres actifs et les membres passifs : aussi bien en matière de valeurs éthiques que du droit, les premiers joueront un rôle plus important que les seconds. Enfin, dernier avantage énoncé du droit écrit est le fait que tout le monde n'a pas la même disposition à sanctionner : un individu pourrait être plus clément qu'un autre pour une même erreur effectuée. Ainsi pour éviter les inégalités dans la sanction, un droit écrit s'avère nécessaire, autant que les institutions chargées de les faire appliquer.

EHRENFELS semble cependant affirmer les limites de l'appareil judiciaire : si on cherche à établir des sanctions pour les cas rares de transgression des maximes, alors on doit s'attendre à une perte de considération de cet appareil. Il reste néanmoins relativement imprécis sur les raisons précises de cette perte de considération. Pour le philosophe, certaines maximes morales doivent rester en dehors du domaine juridique dans la mesure où, dans le cas contraire, cela pourrait être nuisible au bien de tous (p.550). Il ne précise encore une fois pas les maximes en question, mais on peut supposer qu'il redoute en fait un pouvoir trop étendu du législatif. Il évoque ainsi les cas de droit positif qui sont institués par ceux qui sont au pouvoir afin justement d'asseoir leur pouvoir.

Il établit en outre une relation entre le niveau moral d'une société et son système juridique : plus le niveau moral est élevé et plus le système judiciaire sera étendu (p.506). Le contraire est aussi valable puisqu'un système de droit et de mœurs est

\footnotetext{
${ }^{157}$ NIDA-RÜMELIN Julian, « Rationality and Morality », in Pauer-Studer Herlinde (éd.), 1994, p.222-223.
} 
une condition fondamentale à un développement moral. Si chacun est incité, d'une manière ou d'une autre, à respecter des règles de conduite élaborées en référence aux maximes morales, alors le niveau général de la morale évoluera positivement. Un changement dans l'évaluation des dispositions émotionnelles peut conduire à une modification de la morale, laquelle peut ensuite mener à une modification de la législation ou à la création d'une législation concernant ces dispositions. La nouvelle valorisation ne tarde pas à se généraliser dans les mentalités des personnes vivant dans une même société. Par contre, le fait de faire évoluer le droit fait évoluer plus ou moins rapidement les mentalités (par exemple l'abolition de l'esclavage a pris du temps pour être intégrée par les individus ${ }^{158}$ ).

EHRENFELS n'ira pas plus loin dans son développement, considérant que des précisions appartiendraient au domaine de l'enseignement du droit. La pertinence de ce paragraphe se situe pour nous dans la corrélation entre le droit et l'éthique, ainsi que dans les influences mutuelles qui s'en suivent sur les valeurs. Le droit et l'éthique restent éminemment liés, surtout dans les débats actuels autour de l'évolution de la médecine ${ }^{159}$. De plus, pour Wibren van der BURG, le droit et l'éthique ne peuvent être considérés séparément car leur raison d'être repose notamment sur l'appréciation du comportement des individus, un objet commun qui rend leur présence respective obligatoire dans les débats de société ${ }^{160}$.

EHRENFELS lie le droit et les valeurs morales par l'intermédiaire des maximes morales en ce sens où ces dernières influencent en partie l'élaboration du droit. Il n'est effectivement pas possible de montrer une stricte séparation entre le droit et la morale ${ }^{161}$. La présence des valeurs morales dans le système juridique est donc incontestable, même si sa place varie d'une société à l'autre et si les changements dans un domaine peuvent favoriser des changements dans l'autre domaine. EHRENFELS s'intéresse avant tout à la relation entre droit et valeurs morales dans une direction qui va du second au premier, mais il n'évoque pas les influences possibles qui vont dans le sens contraire. On pourra simplement rajouter que le droit peut aussi avoir une influence sur les valeurs morales.

\footnotetext{
158 Voir par exemple FEDERINI Fabienne, « L'abolition de l'esclavage de 1848 », 1998.

${ }^{159}$ BONDOLFI Alberto, "Ethik und Gesetzgebung ", in BONDOLFI Alberto et GROTEFELD Stefan (eds), "Ethik und Gesetzgebung », 2000, p.13-14.

${ }^{160}$ BURG Wibren van der, "Zwei Modelle von Recht und Moral », in BONDOLFI Alberto et GROTEFELD Stefan (eds), " Ethik und Gesetzgebung ", 2000, p.23-41.

${ }^{161}$ LA TORRE Massimo, "Law and Morality: a modest Assessment ", in PAUER-STUDER Herlinde, 1994, p.115-127.
} 
Par exemple le droit peut prendre le pas sur les valeurs morales en ce sens où l'individualité peut être privilégiée par rapport au communautarisme plus proche des valeurs morales dans les sociétés catholiques européennes en plein bouleversement à la fin du XVIII ${ }^{\text {eme }}$ siècle (la Loi Le Chapelier en vigueur à partir de 1791, interdisait le corporatisme en France).

Après avoir discuté la relation entre les valeurs éthiques et le droit à travers les maximes morales, voyons à présent celle qui unit ces mêmes valeurs aux coutumes.

Les coutumes sont des règles qui ne sont ni codifiées, ni écrites. Elles ont cours entre un ensemble de personnes dans leurs vies quotidiennes respectives. Des changements dans les coutumes interviennent de manière non officielle, incontrôlée et non intentionnelle, selon EHRENFELS (p.501). Celui qui ne respecte pas les coutumes en vigueur dans la société dans laquelle il vit, sera sanctionné socialement, c'est-à-dire que son attitude appellera la désapprobation et le rejet d'autrui. Cette sanction n'est pas semblable à celle qui intervient dans le domaine du droit. C'est une sanction purement sociale qui se produit indépendamment de la sanction judiciaire (par exemple lorsque celle-ci n'existe pas) ou en complément de celle-ci. Concrètement certaines attitudes ne sont codifiées que par les coutumes. On pense par exemple au fait de porter une robe blanche pour la mariée, qui est une tradition dans certains pays, sans pour autant être codifié par la loi.

Le philosophe distingue par ailleurs différentes catégories de coutumes. La première d'entre-elle est celle des mœurs positives, par opposition à la seconde qui comprend les mœurs morales : la différence est la même qu'entre droit positif et le droit moral, nous dit EHRENFELS (p.502). Cette différence n'est cependant pas la seule que l'on peut noter. En effet, le droit positif est un droit écrit, alors que les coutumes d'une manière générale, par définition, ne le sont pas. Les coutumes ou mœurs morales désignent donc les exigences subjectives dont la réalisation par autrui représente pour lui-même l'accomplissement de l'exigence d'une maxime morale.

Enfin, avant de passer au problème de conscience morale, notons l'affirmation d'EHRENFELS concernant le fait que les maximes, comme le droit ou les coutumes, sont soumises à un processus d'évolution (p.507-508). Dans une société en développement, le comportement de l'individu change, ce qui se répercute par ailleurs sur les rapports sociaux. Ce changement va mener à une évolution de la maxime correspondante, laquelle aura plus tard des conséquences sur le droit et la coutume. Aussi voit-on se dessiner la relation entre l'évolution des comportements et celle des maximes, mais aussi entre les dispositions 
émotionnelles et la valorisation éthique. Il y a un décalage entre la maxime morale en vigueur et les relations sociales : ces dernières évoluent plus rapidement que les premières. Ainsi on pourra parler de maximes normales en vigueur, de maximes survivantes ou de maximes en devenir ; de même on parlera des coutumes et du droit en vigueur, survivants ou en devenir. Que ce soit les maximes, les coutumes, le droit ou encore les valorisations éthiques, tous subissent un processus de changement assimilable au cycle de vie. Cependant, si certaines lois sont en parfait accord avec les maximes morales en vigueur, d'autres entrent par contre en contradiction avec ces dernières. De ce fait, l'histoire de l'une ne peut se comparer à celle de l'autre.

\section{5 - Conscience morale et sanction éthique}

Deux notions importantes qui sont traitées par EHRENFELS sont celles de la conscience morale et de la sanction éthique. La première comme la seconde jouent un rôle non négligeable dans le processus de valorisation éthique. C'est pour cela que nous allons nous y attarder dans ce paragraphe.

La langue allemande différencie la conscience, " das Bewusstsein ", c'est-à-dire le fait de réaliser les choses, de la conscience ou "das Gewissen », c'est-à-dire la conscience morale de l'individu. La conscience ou "Gewissen" est donc une instance morale qui permet par ailleurs à l'individu de se mettre dans un état psychique donné et de réaliser par la même qu'il possède cette capacité.

Chaque valorisation positive ou négative, émanant d'un jugement de la conscience ou "Gewissen » et concernant un état psychique, un vécu, une expérience ou une action est appelée sanction éthique par EHRENFELS. La notion de "sanction " est donc à comprendre ici en tant que synonyme de "jugement». En fait, le philosophe voudrait réunir sous le même toit du fait éthique les notions de jugement éthique et de sanction éthique. En effet, lorsqu'un individu attribue une valeur éthique, il le fait à travers un jugement de valeur, c'est-à-dire une sanction éthique positive ou négative. Chaque individu possède une conscience qui émet un jugement sur un état psychique, une expérience ou un agissement. Ce jugement peut concerner aussi bien autrui que soi-même. Cette conception psychologique d'instance d'auto-censure à travers la conscience morale, se retrouve également chez FREUD sous la forme du « Über-Ich » ou « Sur-Moi » ${ }^{162}$.

${ }^{162}$ FREUD Sigmund, " Vorlesung zur Einfuhrung in die Psychoanalyse und neue Folge ", Volume 1, 2000, p.413, p.504-516. 
Selon EHRENFELS, on peut constater empiriquement que celui qui dit avoir " une prédisposition au désir et aux sentiments moraux ", sera conscient par la même occasion de posséder une certaine tranquillité d'esprit, notamment face à sa propre disparition. Cette tranquillité est liée à certaines perspectives métaphysiques (p.509). L'individu ayant des désirs et des sentiments en rapport au bien de tous, ou en tous cas, respectueux de la morale en vigueur, gagnera donc par conséquent une certaine sérénité, en particulier par rapport à la mort. Derrière l'expression utilisée par EHRENFELS de "perspectives métaphysiques » ou " metaphysische Ausblicke ", on devine qu'il fait allusion au jugement dernier, celui auquel est confronté l'individu après son décès dans la religion catholique comme dans d'autres religions. La sérénité de l'individu naît donc de la perspective de se voir juger positivement par rapport aux actes accomplis durant sa vie. Ainsi celui qui agit en respectant les coutumes et le droit peut avoir l'esprit tranquille et serein, même si parfois ses agissements ne sont pas conformes aux maximes morales (p.511). La motivation profonde guidant l'individu dans ses actions est donc à chercher du côté de ses croyances. L'explication du philosophe est rattachée à la prépondérance de la religion dans la vie de l'individu et de celle du jugement dernier. Si la croyance des personnes en un jugement dernier est effective, leur motivation à agir correctement influencera certainement leurs désirs et leurs sentiments. Cependant, comme nous le fait remarquer EHRENFELS, la motivation quotidienne est influencée par d'autres facteurs. De plus, il ne s'intéresse semble-t-il pas aux individus sans croyances, ce qui pourrait s'expliquer encore une fois par sa propre éducation et par son intérêt évident pour la religion. Or, dans nos sociétés occidentales actuelles, la religion et donc ses maximes adjacentes ainsi que les perspectives qu'elle offre, ont une place moindre dans l'esprit des individus. Peut-on alors penser que leur motivation est influencée exclusivement par des facteurs de court terme ? Beaucoup de personnes ont un projet ou un but situé dans un futur plus ou moins lointain et pour atteindre ce but ou pour réaliser ce projet, auxquels elles accordent une grande valeur, elles vont utiliser des moyens ayant des effets de court terme comme de long terme. Ainsi, même si la religion a une place moindre dans nos sociétés, les personnes n'en ont pas moins des objectifs de long terme avec des valeurs correspondantes.

Le phénomène de la conscience morale (" das Gewissen ») est également soumis à des variations. Celles-ci sont liées à des mouvements d'envie (ou de non envie) qui apparaissent comme une conséquence des tendances ou des prédispositions morales. Ces mouvements se manifestent après les agissements d'un individu qui seraient conformes ou non-conformes aux maximes morales. On note également des mouvements de la conscience dans le cas du regret qui pousse l'individu à agir en ayant en tête les conséquences attendues de ses actes. Il cherchera à éviter des gestes qui pourraient par la suite décevoir autrui et produire un sentiment de regret chez celui qui a agi. Le regret peut dans certains cas apparaître bien après 
l'action. Le philosophe autrichien nous donne l'exemple du meurtrier qui regrette son acte en constatant, bien après, la tristesse des parents de la victime. Bien qu'il ne le précise pas, on peut néanmoins penser que ce genre de regret ne se produit pas chez tous les individus.

Par contre, en ce qui concerne les mouvements de la conscience en direction du bien être de tous, EHRENFELS affirme qu'ils ne sont pas présents chez tous les individus de la même manière. Ainsi une conscience morale " sensible " (p.525), c'est-à-dire réagissant conformément à la morale, est considérée comme des plus nobles et sera donc très positivement valorisée. Mais il existe aussi des consciences moins sensibles et donc moins positivement valorisées.

EHRENFELS plaide pour la diffusion de la religion et de ses maximes afin d'éduquer les individus et les inciter à agir conformément à la morale (p.525-529). La conscience de l'individu sera influencée par celui qui agit conformément aux maximes morales et qui bénéficie par conséquent d'une valorisation positive de la part de la société. Cet individu voudra également connaître ces maximes et entrera alors dans un processus d'auto-éducation.

Enfin, la valorisation éthique peut être affectée par l'apparition de conflits. Par conflit éthique EHRENFELS entend les cas où le désir est incertain ou partagé et ceux où le désirant met en doute le ou les moyens utilisés pour atteindre son but. On parle ici des conflits de la conscience et non des conflits entre deux personnes. Concrètement, une personne peut être tentée par la vengeance tout en rejetant la notion de vengeance même. Il y a donc un conflit moral quand l'individu est confronté à la classification des choix qui lui sont offerts pour l'action qu'il veut réaliser. Les conflits éthiques sont un cas particulier des conflits moraux, dans lesquels l'être désirant veut à la fois faire le meilleur choix éthique possible et effectuer un classement des manières d'agir possibles.

Il n'est pas vrai que celui qui agit en fonction des mœurs évite la sanction éthique car celle-ci se réfère non seulement aux dispositions du désir précédant l'agissement, mais aussi à leur manque. Un manque de disposition du désir peut inciter l'individu à penser qu'il agit en fonction des mœurs, bien qu'en fait son manque de disposition soit éthiquement désapprouvé. Parmi les dispositions du désir morales, les dispositions éthiques ne sont pas les plus nobles. Enfin, celui qui agit en fonction du bien de tous n'est pas à l'abri d'un jugement éthique négatif (justement à cause du fait qu'il puisse être jugé par rapport à un manque de dispositions).

Dans ce paragraphe nous avons résumé les perspectives de Christian von EHRENFELS concernant le rôle régulateur de la conscience morale et de la sanction éthique. La conscience morale va inciter l'individu à agir de manière 
conforme aux maximes morales en agissant au niveau de son imagination et de ses dispositions émotionnelles. Celles-ci vont conduire l'individu à désirer et à valoriser des objets favorables au bien être de tous. La sanction éthique est une instance extérieure à l'individu et qui influence ses dispositions émotionnelles par rapport à un but de long terme - lié le plus souvent à des convictions métaphysiques - qu'il valorisera positivement. Sa conscience morale comme la sanction éthique se développent dans le temps grâce au processus de socialisation. Elles ne sont donc pas fixes mais subissent un processus d'évolution lié à l'évolution des maximes, des coutumes, du droit et des valeurs correspondantes. Et comme ces derniers, la conscience morale et la sanction éthique sont des éléments régulateurs du comportement par leur influence sur les valorisations des individus. Enfin, dans certaines situations où les valeurs éthiques apparaissent, l'individu peut être confronté à un conflit interne. L'issue de ce conflit est difficile à déterminer puisqu'il dépend en partie de l'individu concerné, mais il se laisse également influencer par la conscience morale et les règles intériorisées.

Le dernier point qu'il nous faut encore évoquer concernant les valeurs éthiques est celui des valeurs absolues.

\section{6 - Le concept de valeur absolue}

EHRENFELS considère que l'éthique, en tant que discipline, s'est préoccupée dès le départ de justifier et de fonder l'existence des valeurs absolues sans pour autant y être parvenue. Il s'agit d'une des questions les plus importantes de la discipline (p.539). En effet, la qualification de «bien " ou de " mal " peutelle être une qualification absolue, c'est-à-dire, est-ce que le fait de qualifier un objet de «bien » vaut pour tous, partout et en tout temps ?

La réponse à cette question n'a trouvé aucun compromis parmi ceux qui ont tenté d'y réponse, ajoute-t-il (p.539).

En se remémorant la théorie de la valeur d'EHRENFELS, on peut deviner d'avance qu'il va rejeter l'hypothèse d'existence des valeurs absolues. En effet, l'individu étant subjectif et les valorisations étant propres à chacun, il est difficile sous ces conditions d'imaginer qu'il puisse exister des objets dont la valeur est universelle et intemporelle.

Il cherche pourtant à en démontrer l'existence (p.539-562), et finit, sans surprise à la conclusion qu'elles n'existent pas ( $p .563$ ). Le rejet de l'hypothèse des valeurs absolues exclut également la conception de l'éthique comme une discipline absolue et normative avec des impératifs universels (p.587). Pour EHRENFELS, les impératifs dans l'éthique se différencient des autres impératifs, de disciplines 
telles que la pédagogie ou la thérapeutique, par le fait qu'ils ont un effet réel. D'une manière générale, l'impératif est lié à l'existence d'un désir. Dans le cas de l'éthique, il existe également des désirs supérieurs qui sont présents de manière variable chez tous les individus et c'est ce fait qui contribue à la différencier des autres disciplines. Il peut ainsi affirmer que les impératifs éthiques et leurs désirs supérieurs sont " empiriquement " ou " physiquement " absolus (p.588). C'est-àdire que leurs valeurs ont une force absolue dans la réalité présente de l'individu qui émet un jugement éthique, ou encore dans la réalité de la société où les impératifs trouvent une valeur absolue dans leur application.

Pour KANT, la raison nous conduit à déterminer une loi du comportement (une maxime) qui pourrait être érigée en loi universelle. Ce principe d'universalisation des maximes reste un sujet d'étude des philosophes actuels et certains, comme Hans KÜNG, ont essayé de formuler des valeurs dites universelles sur la base des valeurs religieuses ${ }^{163}$. D'autres, comme Carola MEIER-SEETHALER, préfèrent trouver à travers les langues et la littérature des expressions communes en référence à des attitudes et des valeurs morales ${ }^{164}$. Enfin, mentionnons la voie ouverte par Erich FROMM ${ }^{165}$, Agnes HELLER ${ }^{166}$, Hans JONAS ${ }^{167}$ et Carol GILLIGAN $^{168}$, qui consiste à définir des besoins psychiques fondamentaux communs à tous et qui appellent des jugements moraux similaires aux réactions engendrées par ces besoins ${ }^{169}$. Un exemple est le besoin de se consacrer aux autres et de leur venir en aide : les réactions engendrées et positivement évaluées sont celles de la compassion, de la solidarité ou du sentiment de responsabilité ; au contraire, l'indifférence, l'abandon, l'absence de responsabilité se verraient attribuer des valeurs morales négatives.

En conclusion, la valorisation éthique est à mettre en relation avec les impératifs, les mœurs et le droit. Les impératifs, les mœurs et le droit sont à classer dans la catégorie des régulateurs sociaux du comportement. Les sous-catégories qui en dérivent se construisent par rapport aux types d'objets sur lesquels portent les valorisations ainsi que sur les "Begleiterscheinung" ou les images les accompagnant. Le droit et les mœurs permettent la valorisation des comportements humains (aussi bien les actions que les abstentions) sans véritable référence aux dispositions du désir de l'individu. Ces jugements s'accompagnent

\footnotetext{
${ }^{163}$ Voir KÜNG Hans, « Projekt Weltethos », 1990.

${ }^{164}$ MEIER-SEETHALER Carola, 1998, p.274.

${ }^{165}$ Voir FROMM Erich, " Psychoanalyse und Ethik »,

${ }^{166}$ Voir HELLER Agnes, " Philosophie des linken Radikalismus », 1984 et HELLER Agnes, "General Ethics ", 1988.

${ }^{167}$ Voir JONAS Hans, « Das Prinzip Verantwortung », 1979.

${ }^{168}$ Voir GILLIGAN Carol, « Moralische Orientierung und moralische Entwicklung », 1991.

${ }^{169}$ MEIER-SEETHALER Carola, 1998, p.270.
} 
d'habitudes de sanction par l'environnement (sanction judiciaire formalisée ou sanction non formelle sous la forme d'une non approbation social dans le cas des mœurs) (p.563). Le "contraire" de cette forme de jugement est le fait des valorisations éthiques, lesquelles se produisent par rapport aux tendances comportementales, et plus spécifiquement par rapport aux dispositions du désir de l'individu (p.564). Entre les deux catégories se place celle des impératifs liés aux maximes morales qui permettent un jugement en fonction de règles de conduite pré-établies. EHRENFELS précise cependant que les frontières entre ces catégories doivent rester flexibles car certains jugements se font par rapport au droit et aux valeurs éthiques par exemple. Les régulateurs du comportement influencent donc de plusieurs manières les jugements comportementaux.

Le développement social et intellectuel conduit à une prise d'importance croissante des régulateurs du comportement. Autrement dit, la recherche du développement passe par le développement d'instances de régulation du comportement, lesquels sont eux-mêmes liés à l'éthique, à la morale et aux valeurs correspondantes. La façon dont le droit est introduit et appliqué va également influencer les valeurs éthiques qui en dérivent ( $\mathrm{p} .580)$. 


\section{Résumé et mise au point}

La théorie de la valeur du philosophe autrichien Christian von EHRENFELS est une théorie générale c'est-à-dire qu'elle peut s'appliquer à tout type de valeur. Elle se doit par conséquent d'être précise et exhaustive. Nous venons, dans ce chapitre, d'en exposer les grandes lignes mais il est probablement difficile pour le lecteur d'en retenir les points principaux étant donné justement son degré d'exhaustivité. C'est pourquoi nous proposons d'en faire un résumé et d'en dégager les points qui nous semblent les plus pertinents dans le cadre de ce travail, à savoir ceux qui intéressent ou sont susceptibles d'intéresser les sciences économiques ainsi que ceux qui mériteraient de ne pas être oubliés. Notons qu'il serait possible d'en souligner d'autres points, selon le cadre d'analyse choisi. Ce résumé sert par ailleurs de transition avec le chapitre suivant et permettra une comparaison directe avec d'autres théories de la valeur et la mention des développements actuels en la matière.

La théorie de la valeur de Christian von EHRENFELS prend pour base ce que l'on pourrait appeler la théorie de l'Ecole Autrichienne de la fin du XIX ${ }^{\text {eme }}$, et qui est en fait une synthèse entre la théorie de Carl MENGER et celle de Friedrich von WIESER. Le philosophe reprend un certain nombre de résultats mais aussi de méthodes d'analyse. Sa théorie de la valeur est une théorie générale et subjective qui implique que la valeur naisse de la relation établie entre un individu et un objet pour lequel il éprouve un certain désir. La perspective adoptée est celle de l'individu en particulier, même si pour certaines notions EHRENFELS évoque l'influence de la société ou de l'environnement social de l'individu. Mais en fin de compte, ses réflexions nous ramènent toujours au point de vue individuel.

Il est intéressant de constater que le philosophe utilise la notion de marginalité, encore récente au moment où il écrit son premier article sur le sujet, et qu'il cherche même à en étendre les domaines d'application.

Il reproche aux économistes de l'Ecole Autrichienne de ne baser le développement de la valeur que sur le fait que les individus ne désirent en fin de compte que la satisfaction de leurs besoins. Pour EHRENFELS, il existe d'autres raisons pour lesquelles les individus procèdent à l'évaluation d'un objet qu'ils considèrent. Précisons encore une fois que la valeur n'est pas le prix d'un objet mais l'importance que l'individu accorde à cet objet. Cette définition s'inspire nettement de celle de Carl MENGER.

La valeur nait donc de la relation établie entre un individu et un objet en ce sens où elle est attribuée par ce dernier et ne provient en aucun cas de l'objet lui-même. 
Pour que cette relation s'établisse, il faut que l'individu réagisse à la vue de l'objet, c'est-à-dire qu'il y ait une réaction émotionnelle. Celle-ci peut se traduire concrètement par un sentiment d'envie ou de non-envie. L'envie ou la non-envie vont déclencher à leur tour un désir positif ou un désir négatif de l'individu pour l'objet. La grandeur de la valeur de l'objet est fixée en fonction de l'intensité du désir ou du non-désir. Jusqu'ici rien ne distingue véritablement la théorie d'EHRENFELS d'une autre théorie de la valeur et encore moins de celle de l'Ecole Autrichienne des première et seconde générations. Son innovation se situe ailleurs, au niveau de l'intérêt qu'il accorde à la psychologie et aux émotions dans la détermination de la valeur. Aussi, même s'il ne le dit pas explicitement, on comprend qu'il ne croit pas à l'hypothèse de la rationalité de l'individu, d'autant qu'il rejette la raison pure comme ayant le dernier mot avant l'acte de l'être pensant. Il ne nie pas qu'elle puisse jouer un rôle et influencer le processus de valorisation, mais il existe de nombreuses situations où les émotions prennent le dessus sur la raison. Tout le monde peut donner un exemple personnel où il a pris possession d'un objet uniquement parce que celui-ci était plaisant mais qu'il ne satisfaisait aucun besoin particulier. Le fait d'admettre que la satisfaction des besoins n'est pas la seule circonstance dans laquelle l'individu procède à une évaluation, permet de créer une base plus large pour une analyse plus réaliste pouvant inclure un plus grand nombre de cas possibles, y compris dans le domaine économique. Il faut ajouter que dans les deux cas, que le désir soit lié à la satisfaction d'un besoin ou non, l'augmentation du bien être de l'individu est une des causes pour lesquelles il va considérer et valoriser un objet. Le bien être n'est par contre pas le seul but de l'individu que ce soit dans sa vie en général que dans le domaine économique. Là aussi la théorie d'EHRENFELS soulève un point intéressant qui lui permet de rester plus proche de la réalité.

La définition de la valeur par EHRENFELS est la suivante :

La valeur est une relation entre un objet et un sujet, exprimant le désir, actuel ou éventuel, du sujet pour l'objet, dans le cas où il serait convaincu de la non existence (ou de la non-possession) de ce dernier. Autrement dit, le sujet va désirer l'objet lorsque le fait d'imaginer son existence, de manière plus ou moins concrète et vivante, provoque une augmentation du sentiment de son bien être supérieure à celle qui serait provoquée dans le cas où le sujet imaginerait la non existence de l'objet. La grandeur de la valeur est proportionnelle à l'intensité du désir, ce qui correspond à la différence entre les sentiments d'envie et de non envie d'existence de l'objet.

La notion de la valeur prend une toute autre dimension lorsque le philosophe autrichien commence à explorer toutes ses facettes. En effet, la valeur qui est accordée à un objet peut avoir plusieurs natures différentes, selon ce qui est valorisé par l'individu. 
D'une manière générale, EHRENFELS distingue la valeur intrinsèque de la valeur d'effet, deux notions dont il n'a pas la paternité et qui sont plutôt utilisées en philosophie. Une valeur intrinsèque est accordée à un objet lorsque l'individu le désire pour lui-même. Lorsqu'il le désire pour un de ses effets, c'est-à-dire comme moyen d'atteindre un but initialement fixé, alors l'objet se verra accorder une valeur d'effet. Dans le domaine économique, la plupart des objets se voient accorder une valeur d'effet car ils ne sont que des moyens d'atteindre un but (par exemple, celui de satisfaire un besoin).

La grandeur de la valeur d'effet et celle de la valeur intrinsèque sont déterminées en fonction de l'utilité ou de l'avantage procuré par l'objet. La notion d'utilité employée par EHRENFELS n'est pas uniquement liée à la satisfaction des besoins, mais plutôt à celle du bien être. Un objet est utile et il éveille le désir de l'individu s'il est capable d'influencer positivement son bien être. Ajoutons que le philosophe autrichien ne réduit pas le bien être à la satisfaction des besoins. En outre, la notion d'avantage lui est propre. Le concept de marginalité peut aussi s'appliquer à la notion d'avantage comme il s'applique à la notion d'utilité : l'avantage marginal désigne l'avantage ou l'effet causé par l'acquisition ou la possession d'une unité supplémentaire d'un objet.

Outre les valeurs intrinsèques et les valeurs d'effet, EHRENFELS développe d'autres types de valeurs telles que les valeurs imaginées et les valeurs véritables (une différenciation qui se retrouve également chez Carl MENGER), les valeurs supposées, les valeurs réelles ou encore les valeurs temporaires, normales et normatives. Toutes ces facettes du phénomène de la valeur se réfèrent à des situations concrètes et contribuent à la précision et à l'exhaustivité de la théorie du philosophe autrichien. Dans le troisième chapitre de ce travail, nous allons nous concentrer sur les deux premiers types de valeur (valeur intrinsèque et valeur d'effet).

EHRENFELS a consacré une partie de son analyse au thème de la non-valeur. Il s'agit d'un concept utilisé et traité avant tout en philosophie et qui se réfère au désir négatif éprouvé par un individu envers un objet. Ce désir négatif est relié au rejet de l'objet ou au sentiment de non-envie de son existence ou de la non-envie de l'effet de son existence. Par conséquent, il se verra attribuer soit une non-valeur intrinsèque, soit une non-valeur d'effet. La grandeur de ces valeurs est liée au dommage ou à la nuisance créée par l'objet considéré. Lorsque l'objet en luimême affecte de manière négative le bien être de l'individu alors il présentera un dommage. Dans le cas où l'objet affecte autre chose que le bien être de l'individu (par exemple un autre objet qu'il possède et auquel il attache une grande importance) alors on parlera de nuisance. La non-valeur est une notion qui n'est pas utilisée en économie, mais qui pourtant pourrait y trouver une place, 
notamment en matière d'environnement, et plus particulièrement de pollution. $\mathrm{Si}$ par exemple un tanker s'échoue sur une côte et provoque une marée noire, les conséquences se feront sentir non seulement sur le bien être des individus vivant à proximité, mais cette marée noire créera également une nuisance puisqu'elle aura souillé la plage et les eaux dont ils tirent leurs revenus soit à travers le tourisme, soit à travers la pêche. On accordera alors à cette catastrophe une non-valeur d'effet plus ou moins grande selon la nuisance créée.

Un des points les plus intéressants de la théorie de Christian von EHRENFELS est celui qui concerne le mouvement des valeurs. Plus exactement, il considère que les valeurs ne sont pas un phénomène fixe mais qu'elles évoluent avec le temps. Très marqué par le Darwinisme, le philosophe attribue aux valeurs la faculté de s'adapter à leur environnement. Les valeurs naissent au sein d'une société ou d'une collectivité, elles se propagent et parfois se transforment, puis finissent par tomber dans l'oubli ou par disparaitre. La naissance et la propagation des valeurs s'effectue par l'intermédiaire des "aktive Wertbildner » c'est-à-dire par des personnes ayant une personnalité assez forte et une réputation telle que leurs nouvelles valorisations serviront de référence pour le reste de la société ou de la collectivité. Ceux qui adoptent les valeurs nouvellement créées sont dénommés " passive Wertbildner » par EHRENFELS. Il distingue donc deux courants dans une société ou une collectivité, dont l'un peut être assimilé à une élite. Le processus de valorisation individuel peut donc se laisser influencer par autrui ou par l'environnement dans lequel le sujet valorisant vit.

EHRENFELS identifie les dispositions émotionnelles comme l'élément déterminant dans le processus de valorisation qui subit en outre des influences extérieures et intérieures. Le fait de considérer un objet déclenche une réaction de la part de l'individu qui peut être positive ou négative. Cette réaction est de nature émotionnelle avant tout puisque l'individu peut développer un sentiment d'envie vis-à-vis de cet objet. Ces sentiments le disposent à désirer ou pas l'objet en question. Les dispositions du désir ne sont à leur tour qu'un cas particulier de dispositions émotionnelles, lesquelles sont donc l'instance originelle dans la prise de décision de l'individu, que ce soit dans sa valorisation que dans l'action qui fait suite à l'attribution d'une valeur à l'objet.

Parmi les facteurs pouvant influencer les dispositions émotionnelles, EHRENFELS distingue ceux dont l'origine est connue et ceux dont l'origine ne l'est pas. Les facteurs psychologiques connus influençant les dispositions émotionnelles sont l'habitude, la "déshabitude " ou perte d'habitude, l'association, le transfert émotionnel, l'illusion et le désir. Ces facteurs sont d'ordre internes, mais il existe aussi des facteurs externes : la contrainte, l'effet par l'exemple, la suggestion et l'ordre exercés par autrui peuvent modifier les dispositions émotionnelles de 
l'individu. Cette énumération nous montre que le processus de valorisation individuel n'est pas un phénomène simple et que nombreux sont les éléments qui peuvent l'influencer dans un sens ou dans un autre. Les facteurs que l'on vient d'évoquer sont présents dans notre vie quotidienne et sont couramment utilisés. Par exemple la publicité se définit, selon KROEBER-RIEL, comme la suggestion du comportement à travers l'utilisation d'informations et de moyens d'influence de la motivation ' (d'achat dans la plupart des cas). La publicité, qui est un facteur externe, agit au niveau de la perception de l'individu et cherche ainsi à changer ses dispositions émotionnelles vis-à-vis d'un objet ${ }^{2}$. Il n'est encore une fois pas question de prix, mais du désir et de la valeur accordée par l'individu à l'objet pour son effet ou son effet supposé.

Voulant aller plus loin dans son analyse, le philosophe autrichien se propose d'étudier les mouvements de valeur en fonction du but de l'individu. Dans le cas où le but venait à changer, la valeur accordée à celui-ci tout comme au moyen utilisé pour l'atteindre pourraient subir une évolution. De même, dans certaines situations, le moyen utilisé pour arriver au but peut devenir l'objet de la valorisation à la place du but lui-même. En d'autres termes, l'individu peut attribuer plus d'importance au moyen de son action qu'à son but. Il est en outre possible que les personnes valorisent plus les effets consécutifs à l'action plutôt que son but ou les effets adjacents à la réalisation d'un but d'une action. Dans chacune des situations, un nouveau type de valeur est crée.

Même si le désir de précision d'EHRENFELS est louable, sa description comprenant autant de nouvelles dénominations mène à une certaine confusion. Nous en retiendrons que le processus de valorisation de l'individu peut être influencé autant par des facteurs externes (évolution de son environnement social ou influence par le contact avec autrui sous une forme ou une autre) que par des facteurs internes et que ces mêmes facteurs peuvent conduire l'individu à modifier sa valorisation première que ce soit au niveau du but fixé, du moyen choisi ou des deux à la fois.

Le deuxième point d'analyse de la théorie de la valeur concerne le désir. Habituellement en économie le désir de l'individu est lié automatiquement à la satisfaction de ses besoins : il ne désire que ce qui peut satisfaire ses besoins. EHRENFELS montre que le phénomène du désir est plus complexe et qu'il est lié à l'imagination des individus. En effet, la perception d'un objet ou d'une chose déclenche la conceptualisation de son image dans la conscience de l'individu.

\footnotetext{
'MARZEN Walter, "Marketing der Handelsbetriebe ", 1986, p.127. Voir également: KROEBERRIEL Werner, "Werbung - Steuerung des Konsumentenverhaltens », 1982, p.26, 27-29.

${ }^{2}$ SCHOLL Alice, «Einfluß der Marketinginstrumente auf die Kaufentscheidung und das damit verbundene Kaufverhalten », 1989, p.90-93.
} 
Cette conceptualisation s'accompagne, ou pas, d'autres images, et cet ensemble va à son tour déclencher un certain nombre d'émotions. D'une manière générale, les images qui sont associées à un sentiment agréable sont celles qui restent le plus longtemps dans sa conscience. La relation émotionnelle créée entre un individu et l'objet qu'il considère, est appelée réalité subjective par EHRENFELS.

EHRENFELS explique le phénomène du désir comme étant le rassemblement de trois moments : celui du souhait, celui de l'aspiration et celui de la volonté. Le souhait décrit le moment où l'individu se fixe un but qu'il espère atteindre. Pour qu'un souhait soit réalisé, il faut qu'il y ait une motivation qui pousse l'individu dans ce sens, c'est le moment de l'aspiration. Durant cette phase du processus, l'individu réfléchit aux différents moyens possibles d'atteindre son but et valorise chacun de ces moyens. Il se décidera pour celui qui obtiendra la valeur positive la plus élevée. A partir de ce point, le moment de la volonté entre en jeu.

A la lumière de ce schéma, on comprend pourquoi le philosophe autrichien considère que le désir n'est pas simplement une émotion mais une disposition émotionnelle dans laquelle se trouve un individu et qui peut le conduire à agir dans un but précis. Les valeurs attribuées, le sont au cours de ce processus et le fait de désirer ne peut pas être confondu avec la volonté de satisfaire des besoins. Le premier peut conduire au second, mais cette relation n'est pas exclusive.

Enfin, comme nous l'avons vu, l'éthique occupe une place importante dans la théorie de la valeur de Christian von EHRENFELS. Il commence tout d'abord par définir l'éthique en tant que discipline et distingue ensuite les différentes branches de cette discipline, de son propre avis. Il distingue l'éthique théorique (histoire des faits éthiques) de l'éthique pratique (analyse des facteurs de régulation des comportements). Ses réflexions sur la valeur se situent plutôt du côté de l'éthique pratique. Il évoque le fait que parmi toutes les disciplines existantes, il y a celles qui sont éthiquement neutres et celles qui ne le sont pas, en ce sens où l'éthique devra approuver ou non les moyens utilisés et les buts fixés. Les sciences économiques par exemple présentent l'individu comme un être cherchant à maximiser son bien être. Ce but ayant une valeur éthique, les moyens utilisés pour l'atteindre peuvent, par voie de conséquence, se voir également attribuer une telle valeur. Pour EHRENFELS, les sciences économiques ne sont donc pas éthiquement neutres.

A travers un jugement éthique, l'individu attribue à un objet soit une valeur intrinsèque éthique, soit une valeur d'effet éthique. L'objet le plus courant du processus de valorisation dans ce cas est le comportement ou l'agissement des individus. Une valeur d'effet éthique est accordée en fonction de la capacité de l'objet à favoriser la réalisation d'un but ayant la plus grande valeur intrinsèque 
éthique. Parmi ces buts, on retrouve en première position le bien être de tous puis le bien être d'un cercle de personnes plus restreint. Pour pouvoir juger un comportement, un individu va se référer aux dispositions émotionnelles qui l'ont poussé à agir.

Dans un second temps, le philosophe autrichien s'attache à découvrir les lois de l'évolution des valeurs éthiques et morales. Dans le cas de l'évolution du jugement éthique, les facteurs identifiés seront la volonté de bénéficier d'une considération sociale positive, la croyance religieuse et le processus de socialisation par les institutions en charge de cette tâche. De la même manière, l'apparition de nouvelles qualités humaines, liée le plus souvent à des changements dans la société, le progrès des connaissances en psychologie concernant le fonctionnement des individus dans les rapports sociaux, et les changements dans les rapports sociaux et plus particulièrement dans leur forme, peuvent constituer également des facteurs pouvant faire évoluer les jugements éthiques.

Les valeurs morales se construisent par rapport aux comportements des individus, et leur évolution est liée à celle des valeurs éthiques, mais avec un certain décalage : un changement dans les valeurs morales s'opère après un changement de la valeur éthique correspondante.

Les valeurs éthiques sont présentes dans la société à travers les instances régulatrices du comportement telles que le droit, les coutumes et les maximes morales. Elles guident les individus dans leurs agissements et fixent des limites. Ces limites, si elles ne sont pas respectées, peuvent faire l'objet d'une sanction éthique qui peut être écrite (droit) ou pas (coutumes). La sanction éthique peut également se faire au niveau des croyances métaphysiques de l'individu: la perspective du jugement dernier peut l'amener à repenser et réguler ses actes dans le quotidien. L'individu serait, selon EHRENFELS, doté d'une instance autorégulatrice qui corrigerait ses erreurs ou l'empêcherait d'en commettre. Cette fonction se construit avec le temps, soit grâce à des apports extérieurs, soit grâce à une auto-éducation, également en rapport avec l'environnement de l'individu dans lequel il cherche à adapter son comportement.

Le fait de considérer que les valeurs éthiques, comme les autres types de valeur, ne sont pas fixes, que les valeurs sont attribuées par des individus se laissant influencer par leur environnement et que les normes écrites ou orales jouent un rôle dans la détermination des valeurs éthiques, toutes ces hypothèses conduisent EHRENFELS à la conclusion logique que les valeurs absolues n'existent pas. Dire qu'une valeur est absolue signifierait que le désir situé en amont est toujours le même dans une situation donnée. Ceci impliquerait que le processus émotionnel 
que décrit le désir, serait le même ou encore que l'influence de l'environnement ou d'autrui serait telle que la conscience de l'individu ne puisse attribuer qu'une valeur dite universelle. Or, nous avons vu que le processus du désir est complexe et qu'il peut changer à tout moment. De plus l'environnement de l'individu n'est pas stable non plus et les évolutions se reflètent en partie dans celles des coutumes, du droit et des maximes. Nous ajouterons à ceci que d'un point de vue anthropologique, il n'est pas possible de considérer qu'une valeur puisse être universelle dans la mesure où la signification d'un objet pour un individu, pour reprendre des termes « mengeriens », est différente selon sa culture.

$\mathrm{Du}$ résumé ci-dessus, nous aimerions faire ressortir les concepts les plus importants de la théorie de la valeur de Christian von EHRENFELS dans le cadre de ce travail :

- La valeur est une notion subjective et elle est déterminée par l'individu.

- La valeur n'est pas le prix d'un objet, elle est l'importance qu'un individu lui accorde.

- La valeur est liée au désir de l'individu et ce désir est un phénomène psychologique complexe. Les dispositions émotionnelles de l'individu sont déterminantes dans l'apparition du désir et dans le fait de motiver l'individu à valoriser un objet et à agir en conséquence.

- La valeur est un phénomène dynamique qui évolue dans le temps et selon les lieux, en fonction de facteurs aussi bien internes qu'externes.

- Les valeurs éthiques et morales sont des cas particuliers de valeur. Elles se réfèrent le plus souvent à un comportement dont la valeur est fonction des dispositions émotionnelles initiales (ou intentions initiales) de l'individu. Les valeurs éthiques et morales sont également dynamiques et leur détermination est le résultat de l'influence de facteurs externes et internes.

EHRENFELS considère que les valeurs économiques sont un cas particulier de valeur. Leur détermination obéit cependant aux mêmes lois générales. Ces lois sont différentes chez les économistes, et une comparaison avec les théories de la valeur d'Adam SMITH, de David RICARDO et de Karl MARX le montrera. De cette comparaison émergeront également quelques points de rapprochement. Les théories de la valeur en sciences économiques sont basées sur des hypothèses comportementales très éloignées de celles posées par EHRENFELS. Nous verrons que ces hypothèses sont perfectibles et que la théorie d'EHRENFELS constitue en ce sens une alternative intéressante pour expliquer le phénomène de la valeur. 


\section{Chapitre troisième}

\section{Discussions sur la valeur avec la théorie de Christian von EHRENFELS}


Youri Tacoun - 978-3-631-75426-9

Downloaded from PubFactory at 01/11/2019 05:04:02AM

via free access 
La théorie de la valeur du philosophe autrichien Christian von EHRENFELS n'aura trouvé que peu d'intérêt de la part des philosophes contemporains et elle n'a encore jamais été utilisée dans le domaine des sciences économiques. Dans l'Empire austro-hongrois de la fin du $\mathrm{XIX}^{\mathrm{e} m e}$ siècle, on distinguait deux grandes approches du phénomène de la valeur: d'une part celle de l'école économique dont le but était de développer une théorie de la valeur spécifique au domaine économique; d'autre part, l'école philosophique qui voulait développer une théorie générale de la valeur'. De ces deux approches, c'est la première qui fut le plus souvent reprise et qui eut la plus grande influence. Le fait de vouloir intégrer la théorie d'EHRENFELS aux sciences économiques constitue l'originalité principale de notre travail.

Nous allons dans un premier temps, comparer la théorie d'EHRENFELS à d'autres théories économiques. Nous avons dû procéder à un choix parmi ces théories et celui-ci s'est porté sur celles d'Adam SMITH (1723-1790), de David RICARDO (1772-1823) et de Karl MARX (1818-1883). La théorie d'Adam SMITH est incontournable dans la mesure où le philosophe écossais est considéré comme le père fondateur des sciences économiques en tant que discipline ${ }^{2}$, mais aussi parce que sa théorie présente un certain nombre de points communs avec celle d'EHRENFELS. Notre approche se distingue de l'interprétation usuelle et vise à montrer les corrélations entre l'économie et la philosophie qui transparaissent dans les différents arguments d'Adam SMITH dans "Théorie des Sentiments moraux ". Les théories de la valeur des deux philosophes contiennent une dimension éthique qui a été négligée chez SMITH.

David RICARDO est considéré par plus d'un comme le successeur de SMITH dont il a repris et développé la théorie de la valeur notamment. Nous verrons que RICARDO marque un pas décisif dans l'étude de la valeur : ses développements ouvrent la voie à une théorie de la valeur exclusivement économique. Ses conclusions s'éloignent ainsi de celles d'EHRENFELS et achèvent ainsi d'affirmer la position des sciences économiques en la matière.

Une autre théorie de la valeur qui a marqué le $\mathrm{XIX}^{\text {ème }}$ siècle, est celle du philosophe allemand Karl MARX. L'idée d'un rapprochement avec Christian von EHRENFELS est venue d'abord du fait que ce dernier montre une certaine

\footnotetext{
${ }^{\prime}$ Cette distinction est évoquée par Reinhard FABIAN dans son Introduction au livre de EHRENFELS, " Die Werttheorie ", 1982, p.3. Pour plus de précision, voir également EATON H.O., "The Austrian Theory of Values ", 1930, p.16, et FABIAN Reinhard et SIMONS P.M., "The Second Austrian School of Value Theory ", in GRASSL et SMITH (éd.), 1983.

${ }^{2}$ Par exemple, " La Richesse des Nations » est considérée par Max LERNER comme étant " sans doute l'ouvrage fondateur de la pensée économique moderne ". Il l'affirme dans son Introduction à "The Wealth of Nations », Edition Cannan, The Modern Library, New York 1937, p.V.
} 
adhésion aux courants socialistes allemands. Un autre point qui favorise le rapprochement entre les deux philosophes est celui du fait d'avoir vécu les bouleversements qui ont marqué les sociétés européennes à la fin du XIX ${ }^{\text {ème }}$ siècle. Après une biographie de Karl MARX, nous exposerons ses thèses sur la valeur, avant d'aborder la question du rapprochement avec les idées d'EHRENFELS. Nous avons pu constater qu'EHRENFELS était influencé par les thèses socialistes et certains de ses propos laissent penser qu'il connaissait également les théories d'Adam SMITH, mais rien ne nous permet d'affirmer qu'il avait bien lu les ouvrages de leurs auteurs comme ceux de RICARDO.

Dans une troisième partie, nous allons nous intéresser au changement survenu à la fin du XIX ${ }^{\text {eme }}$ siècle dans les sciences économiques et concernant les hypothèses sur le comportement humain. Sous l'impulsion de Léon WALRAS et de Vilfredo PARETO notamment, la discipline se dote de concepts fondamentaux qui permettent l'utilisation de méthodes similaires à celles utilisées par les sciences naturelles et qui garantissent un haut degré d'objectivité. Parmi ces concepts, celui de l'individu rationnel cherchant à maximiser son intérêt personnel et celui de l'optimum de Pareto font leur apparition. Le second va marquer le début du développement d'un domaine d'analyse, celui de l'économie du bien être ou "welfare economics». Dans cette perspective, les valeurs émises par les individus sont éliminées tandis que l'économie du bien être fournit ce qui sont en réalité des jugements de valeur sur l'efficacité des politiques économiques à atteindre un niveau de bien être plus élevé pour l'ensemble de la population.

Enfin dans la dernière partie, nous verrons quel intérêt la théorie d'EHRENFELS peut présenter pour les sciences économiques actuelles qui assimilent la valeur au prix d'une marchandise et qui décrivent l'individu comme un être rationnel, égoïste et recherchant exclusivement la maximisation de son bien être par l'intermédiaire de la satisfaction de ses besoins. Ces hypothèses restrictives s'avèrent insuffisantes pour expliquer le phénomène de la valeur. L'utilisation de la théorie de Christian von EHRENFELS, plus proche de la réalité, permettrait une meilleure compréhension du processus de valorisation. Par ailleurs, la place qu'elle accorde à l'éthique correspondrait à l'attente de certains économistes qui plaident pour une approche interdisciplinaire incluant une composante éthique. 


\section{I - Adam SMITH et David RICARDO, la théorie de la valeur de l'Ecole Classique}

Adam SMITH est considéré, aujourd'hui encore, comme le précurseur d'une nouvelle vision de l'économie, fondatrice des sciences économiques. L'ouvrage qui lui a valu une telle considération est "La Richesse des Nations " (1776) qui contient ses réflexions sur les fondements et le fonctionnement du système économique. Il évoque notamment le problème de la valeur qu'il voit comme un phénomène objectif dont la détermination s'effectue par l'intermédiaire des coûts de production. Autrement dit, la valeur est assimilée au prix d'un objet en fait d'un objet marchand. Nous verrons que l'interprétation de la théorie d'Adam SMITH s'est focalisée sur les agents économiques rationnels recherchant, à travers l'activité économique, à satisfaire leur intérêt personnel.

En nous intéressant de plus près au philosophe qu'était Adam SMITH, et à ses travaux sur la valeur et sur le comportement individuel, non seulement dans le cadre de l'ouvrage sus cité, mais aussi dans "Théorie des Sentiments moraux " (1759), nous nous sommes aperçus que ses propos concernant la valeur étaient plus proches de ceux d'EHRENFELS que ce que l'on pourrait supposer. En effet, sa théorie de la valeur ne se limite pas à celle de l'individu égoïste cherchant à maximiser son utilité, elle va bien au-delà et elle intègre également une composante éthique. La théorie d'EHRENFELS va cependant plus loin et pourrait sur certains points, compléter celle de SMITH.

La séparation entre économie et éthique, que l'on pensait opérée par SMITH, n'apparaît pas tant dans ses propos que dans ceux de David RICARDO. Ce dernier reprit la théorie de celui qui fut son maitre à penser, et développa les passages qu'il jugeait insuffisants. Il se basa néanmoins sur les théories de SMITH émanant de "La Richesse des Nations ", et acheva d'élaborer dans le domaine de la valeur une théorie propre aux sciences économiques. En définitive, sa théorie de la valeur s'éloigne de celle d'EHRENFELS et s'ancre dans une volonté de recherche d'objectivité et de précision.

\section{1 - Approche du problème de la valeur par Adam SMITH}

Adam SMITH est né le 5 juin 1723 à Kirkcaldy en Ecosse, d'un père exerçant les fonctions d'officier public et d'une mère issue de la petite noblesse écossaise. Au cours de sa formation scolaire et universitaire à Glasgow puis à Oxford, il se montra brillant élève et suivit, notamment, les enseignements de Francis HUTCHESON, professeur de philosophie morale. A la fin de ses études 
en 1748, il donna des cours libres à l'université d'Edimbourg jusqu'en 1751, date à laquelle il fut nommé professeur de logique à l'université de Glasgow. En 1752, il reprit la chaire de philosophie morale de ce même établissement; ses cours étaient divisés en quatre parties, la première traitait la théologie, la seconde l'éthique au sens strict du terme, la troisième partie était consacrée à la partie de la morale liée à la justice, et enfin, en quatrième partie, il se proposait d'examiner les politiques mises en place en Angleterre afin d'augmenter la richesse et la prospérité du pays ${ }^{3}$.

Il rencontra d'autres grands intellectuels de l'époque tels que David HUME, avec lequel il resta lié toute sa vie, ainsi que le célèbre inventeur James WATT. En 1759, SMITH publia "The Theory of Moral Sentiments» ou "Théorie des Sentiments moraux ", ouvrage qui présentait ses vues en matière de comportement humain et d'éthique. En 1763, il démissionna de son poste à l'université pour devenir le précepteur du jeune Henry SCOTT, troisième Duc de Buccleuch, qu'il accompagna notamment dans un voyage en France et en Suisse de 1764 à 1766 . C'est au cours de ce voyage qu'il rencontra des intellectuels français tels que Jean Le ROND dit D'ALEMBERT (1717-1783), Anne Robert TURGOT (1727-1781), François QUESNAY (1694-1774) ou encore VOLTAIRE (1694-1778), qui eurent sur lui une grande influence. De retour en GrandeBretagne, SMITH se consacra à la rédaction de son œuvre principale : " Recherches sur la Nature et les Causes de la Richesse des Nations », paru pour la première fois en 1776. Ce livre connut un grand succès et eut une certaine influence sur la politique économique anglaise en cette fin de XVIII ${ }^{\text {ème }}$ siècle. SMITH fut nommé Commissaire des douanes en 1777. Son état de santé se dégrada à partir de 1786, à la suite d'une attaque de paralysie. Il fut élu recteur de Glasgow en 1787, mais ne pu prononcer son discours d'intronisation en raison de sa maladie. Il mourut en 1790 à l'âge de 67 ans.

Dans notre analyse, nous allons concentrer notre attention sur deux thèmes majeurs chez Adam SMITH : d'une part sur sa théorie de la valeur et d'autre part sur ses réflexions concernant l'éthique et le comportement humain. Pour ce faire il convient, comme pour EHRENFELS, de replacer l'auteur dans son contexte et de voir quelles furent ses sources d'inspiration extérieures. Puis, à travers une présentation des écrits du philosophe et économiste écossais, nous essaierons de proposer une nouvelle perspective qui mettra en valeur les points communs et les différences entre SMITH et EHRENFELS.

\footnotetext{
${ }^{3}$ STEWART Dugald, " Account of the Life and Writtings of Adam Smith ", chap. 1, paragraphe 18, cité dans SKINNER Andrew S., 1996, p.1.
} 
Commençons par évoquer les courants intellectuels qui ont influencé Adam SMITH. Il fut influencé en premier lieu par les Physiocrates, en particulier par François QUESNAY et Richard CANTILLON (1680-1734). Leurs théories deviennent familières à SMITH lors de son séjour en France et en Suisse entre 1764 et $1766^{4}$. Il en retient la notion de liberté économique (le "Laissez faire "), le fait que le capital soit une avance dans le temps pour une production à venir, c'est-à-dire un moyen permettant une production. Le capital avancé sera par la suite remboursé et permettra à son possesseur une accumulation de celui-ci. Par ailleurs, même s'il ne garde pas l'idée de l'agriculture comme étant le seul secteur produisant de la richesse, SMITH concède le fait que les agriculteurs produisent deux surplus : le premier leur permettant de renouveler leur force de travail et le second leur permettant de payer le capital prêté pour la production. Enfin, il adhère à l'idée d'une auto-régulation de l'économie, laquelle se traduit par un schéma de flux interdépendants chez QUESNAY et par l'établissement des marchés chez SMITH avec la rencontre entre une offre et une demande ${ }^{5}$.

Lorsque Adam SMITH écrit "La Richesse des Nations", il le fait dans une Angleterre qui se prépare à la révolution industrielle et qui est marquée par l'adoption d'un certain nombre de lois protectionnistes accompagnant une montée des prix du blé ${ }^{6}$. Le livre d'Adam SMITH constitue en partie une réponse à ces lois et propose des réformes économiques concrètes. La théorie de la valeur d'Adam SMITH est fondée sur un certain nombre d'hypothèses qu'il convient d'énoncer. D'abord, les sociétés développées adoptent la division du travail qui le rend plus efficace. Ainsi, au lieu de produire tout par soi-même, l'individu va produire un certain nombre de biens dont il échangera le surplus non-utilisé contre d'autres biens, lesquels lui permettront de satisfaire ses autres besoins. L'échange est donc une nécessité dans une telle société. Ces échanges vont avoir lieu sur un marché où l'offre et la demande vont se rencontrer. Les biens échangés le seront en fonction de leur valeur, dite valeur d'échange, en distinction de leur valeur d'usage.

La valeur d'usage d'un objet est celle qui est fonction de l'utilité de celui-ci utilité dans le sens usage que l'individu peut faire de l'objet: plus un bien a d'usage pour un individu et plus il lui est utile. Au contraire, l'individu cherchera

\footnotetext{
${ }^{4}$ DOBB Maurice, "Theories of Value and Distribution since Adam Smith ", 1973, p.41.

${ }_{5}^{5}$ Pour plus de précisions, voir SKINNER Andrew S., 1996, p.121-140, DOBB Maurice, 1973, p.41-43 et, CANNAN Edwin, "Inroduction de l'Editeur ", in SMITH Adam, "The Wealth of Nations ", The Modern Library, New York 1937, p.xxxix à xlii.

${ }^{6}$ Voulant profiter de la montée des prix du blé, les capitalistes mettent en culture un grand nombre de terres jusqu'alors inexploitées. Un certain nombre de lois empêchent alors l'importation de blé meilleur marché. Voir BIAUJEAUD Huguette, « Essai sur la Théorie Ricardienne de la valeur ", 1988 (première édition 1933), p.9-10.
} 
à échanger des objets qu'il possède et qui ont peu d'utilité contre d'autres lui procurant un usage plus grand. La valeur d'usage est donc le motif qui incite l'individu à échanger. La valeur d'échange est la valeur relative d'un objet par rapport à un autre et elle est fixée sur le marché où se rencontrent l'offre et la demande. Enfin, la mesure de cette valeur peut se faire grâce au travail.

Le travail est, pour Adam SMITH, la référence en matière de mesure de la valeur, car c'est le seul élément commun et universel à tous les biens produits ${ }^{7}$ :

« Equal quantities of labour, at all times and places, may be said to be of equal value to the labourer. In his ordinary state of health, strength and spirits; in the ordinary degree of his skill and dexterity, he must always lay down the same portion of his ease, liberty and his happiness [...] Labour alone, therefore, never varying in its own value, is alone the ultimate and real standard by the best value of all commodities can at all times and places be estimated and compared. It is their real price; money is their nominal price only. ${ }^{8}$

Le travail peut être comparé dans le monde entier, peu importe sa dureté ou l'habileté requise. En effet, SMITH distingue une composante commune dans tout type de travail : le sacrifice qu'il implique. Ainsi, plus un travail est pénible, plus il demande de sacrifice à l'individu, par exemple lorsque ce travail demande une formation particulière. Et plus ce sacrifice est grand, plus la valeur du travail mis en œuvre dans la fabrication du bien le sera également. SMITH identifie donc un élément commun permettant de déterminer la valeur d'un bien. La question est de savoir, de quelle manière le "sacrifice " ou effort, va influencer la valeur. Il faut alors noter que la position adoptée par SMITH à ce propos a quelque peu évoluée, un changement souvent occulté par bon nombre d'analystes ${ }^{9}$. En effet, SMITH décrit d'abord la valeur d'un bien comme étant la quantité de travail qu'un individu qui le possède peut commander par ailleurs, ou la quantité de travail qu'il peut acheter ou s'offrir ${ }^{10}$. Il parle ici en termes de travail commandé, une perspective qu'il abandonne par la suite pour celle du travail incorporé dans un bien. Cette évolution est illustrée par l'exemple suivant : lorsque la fabrication d'un bien $A$ a demandé une heure de travail et celle d'un bien $B$ une demi-heure, alors le bien A s'échangera contre deux biens $\mathrm{B}^{11}$.

Dans l'affirmation ci-dessus, il convient également de souligner le fait que le travail incorporé dans un bien permet d'établir une valeur qui est en fait le prix réel de ce bien. Le prix en unités monétaires n'en est que le prix nominal.

\footnotetext{
${ }^{7}$ DOBB Maurice, 1973, p.48.

${ }^{8}$ SMITH Adam, " An Inquiry into the Nature and Causes of the Wealth of Nations ", édition Cannan, 1937, p.33.

${ }^{9}$ O'DONNEL Rory, « Adam Smith's Theory of Value and Distribution », 1990, p.65.

${ }^{10}$ SMITH Adam, 1937, p.30.

${ }^{11}$ Idem, p.47.
} 
Ceci nous amène à écrire qu'Adam SMITH assimile la valeur réelle d'un objet à son prix réel, ce qui dénote une approche exclusivement économique de la valeur, celle qui concerne les biens marchands. Il poursuit son analyse avec le lien entre le prix réel, c'est-à-dire donc la valeur du travail incorporé dans le bien, et le prix du blé. Pour le philosophe et économiste écossais, la valeur du travail est déterminée par celle du blé, car, une fois son énergie dépensée à cet ouvrage, l'individu va chercher à la renouveler. Donc l'effort ou le sacrifice consenti devra être compensé par le prix du bien. Or, à l'époque de SMITH, le blé est la céréale de base dans l'alimentation des individus. Mais si la valeur du travail, ou plutôt celle de l'effort ou du sacrifice, peut être jugée comparable et donc fixe, peut-il en être autant de la valeur du blé ?

SMITH doit alors poser une hypothèse supplémentaire, celle que le prix réel du travail, exprimé par rapport à la ration de blé nécessaire à la subsistance de l'individu, a toujours la même valeur sur le long terme. Ceci suppose de nouveau que le blé est produit à un coût sensiblement identique sur le long terme également ${ }^{12}$, mais aussi qu'il est considéré comme une valeur standard permettant d'évaluer également le prix du travail.

Le travail est le principal facteur de production, mais il n'est pas le seul. En effet, SMITH distingue dans son analyse, les sociétés "avancées" des sociétés " primitives $" ~{ }^{13}$. Dans ces dernières, le travail est le seul facteur de production et la valeur des objets échangés dépend donc complètement de la quantité de travail dépensé. Dans les sociétés dites avancées, qui sont en fait les sociétés où l'accumulation du capital fait son apparition, à l'image de l'Angleterre de la fin du XVIII ${ }^{\text {eme }}$, le travail n'est plus le seul facteur de production. L'ensemble des facteurs de production devra être pris en compte afin de déterminer ce que SMITH dénomme le " prix naturel» du bien. En des termes plus modernes, le prix naturel désignerait le coût de production du bien. Les composants du prix naturel sont identifiés par SMITH comme étant le salaire et la rente. Le salaire étant le prix du travail incorporé dans le bien, et la rente désigne la somme d'argent avancée afin de couvrir les coûts de fabrication du bien, à savoir les matériaux bruts et les salaires. La rente est remboursée une fois le bien vendu sur un marché avec un taux d'intérêt qui s'apparente au bénéfice tiré de l'emprunt.

\footnotetext{
12 "First, he [Adam SMITH] assumed that the corn wage of common labour is constant accross long periods of time. Second, he assumed that corn was produced at near constant cost. This assumption not only allowed him to use the price of corn as a standard value, as a proxy for the price of labour, but also provided a rational foundation for the use of a labour measure in the first place " in O'DONNEL Rory, 1990, p.67.

${ }^{13}$ SMITH Adam, Chapitre VIII « Of the Wages of Labour », 1937, p.64-86.
} 
Lorsqu'un bien est produit il faut ensuite l'échanger, ou en des termes actuels, le commercialiser sur un marché. La personne qui se chargera de le faire se verra rémunérée en récupérant une partie du prix de vente du bien. Autrement dit, elle tirera un profit de la vente du bien sur le marché. Le profit est, pour Adam SMITH, "juste un autre nom pour le salaire d'une sorte particulière de travail, celui de l'inspection et de la direction ${ }^{14}$. De plus, le niveau du profit n'est pas proportionnel à la dureté, ou à l'ingénuité de ce supposé travail d'inspection et de direction :

"They [the profits of stock] are regulated altogether by the value of the stock employed, and are greater or smaller in proportion to the extent of this stock. $\|^{15}$

Le prix auquel le bien est cédé sur un marché est nommé "prix du marché » ou " valeur d'échange " par SMITH, et il est égal au prix naturel auquel on ajoute le profit. Le prix du marché varie selon l'offre et la demande sur ce même marché. Le mécanisme offre-demande est décrit de la manière suivante par SMITH : lorsque la quantité amenée sur le marché est légèrement inférieure à la demande effective, le prix du marché dépasse le prix naturel du bien; lorsque la quantité proposée est supérieure à la demande effective, alors le prix du marché chute au dessous du prix naturel; enfin lorsque les quantités offertes sont égales à la demande effective, le prix du marché et le prix naturel coïncident ${ }^{16}$. Ce mécanisme est resté celui qui est utilisé pour décrire le fonctionnement des marchés en sciences économiques.

La théorie de la valeur d'Adam SMITH est donc une théorie basée d'une part sur la valeur du travail incorporé dans le bien fabriqué, et d'autre part sur les coûts de production comme élément déterminant de la valeur d'échange du bien. Cependant, cette théorie présente à nos yeux quelques limites évidentes. En premier lieu, elle s'intéresse en priorité à la valeur d'échange des biens, et par conséquent, elle semble négliger la valeur d'usage de ces mêmes biens. Ensuite, SMITH considère, sans vraiment y faire allusion, uniquement les biens marchands c'est-à-dire ceux qui sont susceptibles d'être échangés sur un marché, ce qui parait logique puisque c'est le sujet de son ouvrage et de ses réflexions. Néanmoins, il convient de le considérer comme une limite dans la mesure où les biens marchands ne sont pas les seuls à faire l'objet d'un processus de valorisation : les individus accordent également une valeur aux biens non marchands. Ces derniers ne se situent pas systématiquement en dehors de la sphère économique. Prenons l'exemple de l'environnement : les réserves halieutiques d'un pays ont une valeur

\footnotetext{
${ }^{14}$ " The profit of stock [...] are only a different name for the wages of a particular sort of labour, the labour of inspection and direction ", in SMITH Adam, 1937, p.48.

${ }^{15}$ SMITH Adam, 1937, p.48.

${ }^{16}$ SMITH Adam, 1937, p.56-57.
} 
(d'effet) pour ses pêcheurs, mais pas de prix ${ }^{17}$. Une diminution des ces mêmes réserves a des conséquences économiques pour cette classe de travailleurs. L'environnement et ses composantes appartiennent à la sphère économique, même s'il est difficile d'en fixer le prix de manière objectif comme le suggère Adam SMITH. Par contre, il est tout à fait concevable d'en établir une valeur pour un pays ou une population concernée.

On pourrait alors penser que la valeur dans ce cas peut être le prix naturel de l'objet, c'est-à-dire son coût de production. S'il n'est pas difficile d'établir le coût de production de la santé d'un patient en tenant compte du coût des traitements nécessaires à sa guérison, il faut avouer que dans le cas de l'environnement, le calcul est plus complexe. En outre, la valeur de la santé d'une personne - comme celle de l'environnement - comprend, en plus de leur composante économique, une composante éthique. Une des obligations du médecin traitant consiste à guérir son patient en utilisant tous les moyens mis à sa disposition. Cette obligation est une obligation éthique, influençant sans aucun doute la valeur dont il est question dans cet exemple. Enfin, le fait de développer une théorie de la valeur d'échange sur les coûts de production laisse penser que celle-ci peut être déterminée de manière objective. Cet argument se retrouve appuyé par le fait que l'individu recherche son intérêt personnel. La valeur d'échange étant objective, il pourra alors agir de manière rationnelle afin de satisfaire ses besoins. Considérer l'objectivité de la valeur comme une limite peut être sujet à une longue discussion, et ce n'est pas là notre propos. En réalité, la limite n'est pas tant l'aspect objectif de la valeur que l'interprétation de la théorie d'Adam SMITH comme étant une théorie purement objective, ainsi que sa réflexion sur la soidisant rationalité de l'individu à travers la poursuite de son intérêt personnel.

En matière de comportement humain, comme en matière de valeur, s'il l'on s'en tient aux seules réflexions contenues dans la "Richesse des Nations ", comme l'ont fait un certain nombre d'interprètes de $\mathrm{SMITH}^{18}$, alors ses perspectives sur la valeur se limitent à celles que nous venons d'évoquer.

\footnotetext{
${ }^{17} \mathrm{~L}$ 'évaluation de l'environnement se fait habituellement en économie par l'intermédiaire d'une analyse utilité-coûts. Cette méthode avoue ses limites, notamment d'un point de vue éthique et certains environnementalistes demandent de nouvelles méthodes d'évaluation interdisciplinaires. Voir à ce propos MARGGRAF Rainer et STREB Sabine, "Ökonomische Bewertung der naturlichen Umwelt ", 1997 ; FOSTER John (ed.), "Valuing Nature ", 1997, p. 1-17.

${ }^{18}$ Par exemple, DOBB Maurice, 1973; HOLLANDER Samuel, « Classical Economics ", 1992; HOLLANDER Samuel, « Economics of Adam Smith », 1973, SCHUMPETER Joseph A., « History of Economic Analysis », 1954; MEEK R.L., 1956, p.62 et suivantes.
} 
Si, par contre, nous y ajoutons ses développements contenus dans « La Théorie des Sentiments Moraux", alors nous verrons que les limites que nous avons mentionnées ci-dessus, trouvent en partie une réponse dans les propos de SMITH, ce qui en outre le rapproche quelque peu de la position d'EHRENFELS.

Comme nous l'avons déjà évoqué, une partie des cours d'Adam SMITH, occupant alors la chaire de philosophie de Glasgow, était consacrée à l'éthique et à la morale. Une autre partie était consacrée à la théologie. Et bien qu'il l'enseignât, on ne peut pas nier par ailleurs l'adhésion de SMITH aux idées de NEWTON ${ }^{19}$. Il considérait le monde comme obéissant à d'autres règles que les règles divines et effectuait une analogie entre le fonctionnement du monde et celui d'une machine, décrivant l'univers comme " une formidable machine dans laquelle on peut observer des instruments ajustés de la plus agréable manière afin de produire les finalités auxquelles ils sont destinés. ${ }^{20}$

Le monde est donc régi par un certain nombre de lois et de règles, de la même manière que le comportement humain. Pour mieux appréhender cet aspect du problème, SMITH pose en plus certaines hypothèses psychologiques : «il affirme que l'individu est doté de certaines facultés telles que la raison, la réflexion et l'imagination, et de certains penchants le motivant à désirer les sources de plaisir et à éviter les sources de peine $»^{21}$.

Dès lors un parallèle avec EHRENFELS est inévitable puisque ce dernier, avant d'exposer sa théorie, pose également des hypothèses psychologiques sur la nature des comportements humains. Comme nous l'avons vu, la réflexion et l'imagination jouent un rôle important dans la motivation de l'individu et dans sa relation avec l'objet valorisé. De plus, comme SMITH, EHRENFELS affirme que l'individu recherche avant tout son plaisir et que le sentiment d'envie qui lui est associé obtiendra une place privilégiée dans sa conscience, alors que le sentiment contraire, la non-envie, domine la conscience lorsque l'individu est face à un objet qui pourrait lui apporter du déplaisir. EHRENFELS semble pourtant aller plus loin, considérant même les comportements des individus trouvant du plaisir psychologique dans l'effort, voir dans la peine physique. On peut alors penser aux sportifs motivés par le fait de dépasser leurs limites, ce qui implique un grand effort physique. Le philosophe autrichien complète ses propos en tenant compte,

\footnotetext{
${ }^{19}$ SKINNER Andrew S., 1996, p.1.

${ }^{20}$ " [the universe is] a great machine wherein we may observe means adjusted with the nicest artifice to the ends which they are intended to produce " in SMITH Adam, "The Theory of Moral Sentiments ", Section II, Partie II, Chapitre 3, § 5, 1963, p. 147.

${ }^{21}$ « He [Adam Smith] assumed that a man is endowed with certain faculties and propensities such as reason, reflection, and imagination, and that he is motivated by a desire to acquire or to avoid the source of pleasure or pain » SKINNER Andrew S., 1996, p.28.
} 
non seulement du but de la personne (acquérir la source de plaisir ou éviter celle procurant de la peine), mais aussi du moyen utilisé pour atteindre ce but. Car le moyen également peut être source de plaisir (ou peut permettre d'éviter la peine) et contribuera à la motivation totale de l'individu et aussi à son désir.

On peut également noter chez SMITH l'idée que le plaisir proviendrait de la contemplation d'une relation, d'une similarité ou d'un ordre d'idées. Il affirme en effet que l'individu a du plaisir à connaître la relation entre les choses, et qu'il lui parait plus agréable de voir un objet ou un ensemble d'objets structurés que le contraire $^{22}$. Il exprime cette idée d'abord dans un contexte différent, puisque l'on retrouve ce passage dans un autre de ses ouvrages, dédié à l'histoire de l'astronomie. Mais ceci n'est pas surprenant puisque, comme nous l'avons vu, il effectue un parallèle entre le fonctionnement mécanique de l'univers et celui du comportement humain : tous deux répondent à des lois précises ${ }^{23}$. Aussi il serait tout à fait envisageable d'interpréter l'exemple suivant, apparu dans son « Histoire de l'Astronomie ", comme étant celui de deux individus dont le rapprochement débouche sur une influence mutuelle et, par la suite, sur une association ou partage d'idées :

"When two objects, however unlike, have often been observed to follow each other, and have constantly presented themselves to the senses in that order, they come to be so connected together in the fancy, that the idea of the one seems of its own accord, to call up and introduce that of the other. If the objects are still observed to succeed each other as before, this connection, or, as it has been called, this association of ideas, become stricter and stricter, and the habit of the imagination to pass from the concept of the one to that of the other, grows more and more revetted and confirmed. ${ }^{24}$

Autrement dit, SMITH veut nous montrer que l'individu est intrigué par un objet ou un certain nombre d'objets qui ne rentrent pas dans une structure dont il a l'habitude ou qu'il connaît. Il va alors chercher à découvrir et à replacer l'objet dans son cadre ${ }^{25}$. Ainsi l'individu montre une certaine curiosité naturelle et, en définitive, un désir certain pour la connaissance. Encore une fois, nous trouvons un point d'accord, certes non explicite mais réel entre SMITH et EHRENFELS, pour qui la connaissance fait parti des objets ayant la valeur intrinsèque la plus élevée pour les individus en général, et qui donc éveille un désir certain chez eux. Ajoutons à ceci que dans le cas de la connaissance, en tant qu'objet du désir d'un individu, EHRENFELS inclut l'attribution d'une valeur, ce que SMITH ne semble

\footnotetext{
${ }^{22}$ SMITH Adam, "The Theory of Moral Sentiments ", Partie v, chapitre 1, § 9, 1963, p.342-345.

${ }^{23}$ SKINNER Andrews S., 1996, p.28-29.

${ }^{24}$ SMITH Adam, " The History of Astronomy ", section II, paragraphe 6, cité dans SKINNER Andrew S., 1996, p.29.

${ }^{25}$ SKINNER Andrews S., 1996, p.29.
} 
pas faire. Il se contente d'affirmer que l'individu a un désir pour la connaissance, mais ne précise pas si cet individu va pour autant la valoriser. Pour Pierre GRAVOT, Adam SMITH est "le père fondateur de l'économie de l'éducation " et il a été « un des premiers, sinon le premier à s'interroger sur la notion de capital humain (même s'il n'utilise pas le terme) $»^{26}$.

Cependant, si on considère que la connaissance est utile à l'individu, elle aura donc une valeur d'usage, au sens même de SMITH. La connaissance a bien une valeur, la question est de savoir quelle est la nature de cette valeur. La connaissance est un excellent exemple pour en discuter puisqu'elle est présente dans les deux domaines : la connaissance est un objet du désir aussi bien en philosophie qu'en économie où, dans les théories modernes, elle est incluse dans le capital humain ${ }^{27}$. Quelle valeur peut-on alors attribuer à la connaissance ? Il est incontestable qu'elle ait une utilité pour l'individu, lié à un désir naturel, nous dit Adam SMITH. Elle aurait donc une valeur d'usage, mais pas seulement. Si on suit la théorie d'EHRENFELS, l'individu est également à la recherche de sa reconnaissance sociale. Cet argument rejoint celui de la "position sociale " de Raymond BOUDON qui montre que l'origine sociale, et donc les valeurs acquises (influençant chez EHRENFELS les dispositions émotionnelles de l'individu) sont déterminantes dans la prise de décision de l'individu en ce qui concerne les buts qu'il se fixe et les moyens qu'il emploie pour les atteindre ${ }^{28}$. Pour le philosophe autrichien, la connaissance est un moyen de parvenir à la reconnaissance sociale puisque d'une part l'individu instruit est mieux vu que celui qui ne l'est pas ; d'autre part, la connaissance permet la progression de l'individu dans l'échelle sociale, et lui attire donc des regards bienveillants. La connaissance a donc une valeur d'effet comme une valeur intrinsèque dérivée de sa valeur d'effet. Autrement dit, elle peut devenir un but en soi : l'individu désire acquérir un certain savoir pour lui-même, en plus des conséquences sociales inhérentes.

Enfin, en considération de la théorie du capital humain moderne, on peut affirmer que la connaissance a une valeur d'échange qui se traduit généralement par son prix sur le marché du travail : l'individu va se former avec la perspective d'avoir un emploi qui lui fournit des revenus tels que ces derniers soient supérieurs aux coûts de formation ${ }^{29}$. L'individu fait valoir ses connaissances auprès des

\footnotetext{
${ }^{26}$ GRAVOT Pierre, "Economie de l'Education », 1993, p.VI (Introduction).

${ }^{27}$ Les deux determinants du capital humain selon BECKER sont la formation scolaire et universitaire et la formation continue durant la vie active (BECKER Gary S., " Human Capital, A Theoretical and Empirical Analysis with Special Reference to Education », 1993, p.17). SCHULTZ complète ces deux catégories par la santé de l'individu, les formations privées individuelles et la propension à voyager ou à émigrer pour le travail (SCHULTZ Theodore W., "Education and Economic Growth », 1961, p.1).

${ }^{28}$ BOUDON Raymond, «L'Inégalité des Chances: La Mobilité Sociale dans les Sociétés Industrielles ", 1978.

${ }_{29}$ Voir BECKER Gary S., 1993 ; BLAUG Mark, « The Empirical Status of Human Capital Theory: A Slightly Jaundiced Survey ", in BLAUG Mark (éd.), 1992, p.3-31 ; MINCER Jacob,
} 
entreprises prêtes à l'embaucher, et leur prix constitue également le revenu attendu. La valeur qui précède ce prix est une valeur d'effet puisque les connaissances permettent d'atteindre un but, ici gagner un certain revenu ou avoir une certaine position sociale.

Outre son intérêt pour le comportement humain, SMITH semble vouloir également tenir compte du contexte dans lequel l'individu évolue, son environnement social. Ainsi, bon nombre d'analystes reconnaissent que, si dans les premiers paragraphes du chapitre V de la Richesse des Nations ("Of the real and nominal price of commodities, or of their price in labour, and their price in money "), il est question en fait d'une société pré-capitaliste, dans les paragraphes suivants, le philosophe et économiste écossais fait allusion à une société capitaliste $^{30}$. Et cette prise en compte a évidemment des conséquences sur les valeurs des biens marchands: SYLOS-LABINI montre que SMITH fait une distinction, non seulement entre les différents stades de développement de la société, mais également entre le long terme et le court terme ${ }^{31}$. Durant un stade de développement, les méthodes de production sont susceptibles de changer, changeant par la même occasion les prix naturels. Et c'est en passant d'un stade de développement à un autre que les salaires, les profits et la rente vont changer. Les prix des métaux précieux, eux, vont fluctuer sur le court terme, alors que le prix du travail restera stable sur la même période, d'où l'intérêt de le considérer comme étalon de mesure de la valeur. SMITH précise également que le prix du blé, lui, est relativement stable sur le long terme, car le coût de sa production, sous des conditions climatiques et de fertilité des sols identiques, restera plus ou moins constant. Il ajoute également que l'amélioration de la productivité, qui pourrait faire baisser les prix, est en général contre-balancée par le coût croissant des instruments de travail ${ }^{32}$. Les valeurs d'échange, autres que celles du blé et du travail, évoluent avec le temps et le contexte social. L'évolution des valeurs d'échange en l'occurrence - est donc également influencée par le système dont elles font partie et qui les génère. Ici aussi, on peut établir un parallèle entre SMITH et EHRENFELS : pour ce dernier, les valeurs ne sont pas statiques mais dynamiques et elles évoluent avec le temps et avec le contexte social. Les valeurs sont imbriquées dans un système qui, en évoluant lui-même, fait aussi évoluer ses

" Human Capital and the Labor Market: A Review of Current Research ", in BLAUG Mark (éd.), 1992, p.186-193.

${ }^{30}$ Voir BLAUG Mark, " Economic Theory in Retrospect », 1978, p.52; HOLLANDER Samuel, 1973, p.128; SKINNER Andrew S., " Editor's Introduction " in SMITH Adam, " The Wealth of Nations ", Penguin, Harmondsworth 1970, p.50 ; ROGIN Leo, "The Meaning and Validity of Economic Theory », 1956, p.79; MEEK Ronald L., "Studies in the Labour Theory of Value ", p.62 et suivantes.

${ }^{31}$ SYLOS-LABINI, “ Competition: the Product Markets ", in WILSON et SKINNER (éd.), 1976, p.200-232.

32 O'DONNEL Rory, “ Adam Smith's Theory of Value and Distribution », 1990, p.68. 
composantes. Pour les deux philosophes, le contexte social fait référence à la structure de la société, c'est-à-dire à son niveau de développement et aux rapports sociaux qui la caractérisent. Le parallèle s'arrête également à ce niveau, car SMITH parle des valeurs d'échange spécifiquement et EHRENFELS considère les valeurs en général, c'est-à-dire celles qui sont attribuées aussi bien aux biens économiques qu'aux autres objets désirés par l'individu. De plus, les rapports sociaux désignent les rapports dans la division du travail pour l'un, et les rapports d'un individu avec autrui pour le second. EHRENFELS aborde les rapports sociaux sous un angle économique, mais sa vision se concentre sur la division entre les classes dominantes (celle des "aktive Wertbilder") et les classes dominées ("passive Wertbildner»), et même s'il évoque les liens avec l'économie et le mode de production, sa vision s'approche plus de celle de MARX que de celle de SMITH.

Dernier point de notre analyse des réflexions d'Adam SMITH sur la valeur : le lien entre valeur et éthique ainsi que l'importance et l'influence de l'éthique sur le comportement de l'individu ${ }^{33}$.

Une caractéristique essentielle de l'individu, nous dit Adam SMITH, est son amour-propre. Tous les individus vont chercher à en prendre soin, c'est-à-dire qu'ils vont privilégier leur propre intérêt mais aussi leur propre plaisir dans la plupart de leurs actions ${ }^{34}$. Une application majeure de cette thèse se manifeste dans "La Richesse des Nations", sur le plan économique donc. Le fait que l'individu privilégie avant tout sa propre satisfaction est interprété par nombre d'analystes, comme étant un argument de sa rationalité ${ }^{35}$. Or cette interprétation peut être remise en question. En effet, pour Amartya SEN, la poursuite de l'intérêt personnel comme critère de rationalité est difficilement défendable, et le fait d'en attribuer la paternité à SMITH est une erreur ${ }^{36}$. SMITH, selon ce dernier, a voulu décrire et expliquer les échanges entre les individus lorsqu'il écrit le fameux exemple du boucher, du boulanger et du brasseur, ce qui constitue d'ailleurs l'objet du chapitre dans lequel on peut le trouver ${ }^{37}$.

\footnotetext{
${ }^{33}$ SKINNER Andrew S., "Moral Philosophy and Civil Society : Ethics and Self-Love », in " A System of Social Science », 1996, p.51-75.

${ }^{34}$ SMITH Adam, "Theory of Moral Sentiments ", Section VII, Chapitre 3, § 12, 1963, p.536.

${ }^{35}$ D'après Amartya SEN, les deux manières de définir la rationalité du comportant dans les courants dominants en économie sont, d'une part, la constance interne d'un choix, et d'autre part, le fait d'identifier la rationalité avec la maximisation de son propre intérêt. Voir SEN, 1987, p.12. ${ }^{36}$ SEN Amartya, 1987, p.14 et PUTNAM Hilary, 2002, p.50.

${ }^{37} \mathrm{~L}$ 'exemple des échanges entre un boucher et un brassier est considéré par bon nombres d'analystes comme étant la preuve écrite qu'Adam SMITH croyait en un individu poursuivant exclusivement la satisfaction de ses intérêts personnels. Voir à ce propos PUTNAM, p.14 et l'exemple dans SMITH, 1937, Livre Premier, Chapitre II, p.14.
} 
Citons de nouveau ce passage sur la recherche de l'intérêt personnel :

" It is not from the benevolence of the butcher, the brewer, or the baker, that we expect our dinner, but from their regard to their own interest. We address ourselves, not to their humanity but to their self-love, and never talk to them of our own necessities but of their avantages. $)^{38}$

SEN interprète ce passage de la manière suivante : ce que SMITH fait ici, est d'indiquer pourquoi et comment la division du travail opère, ce qui est d'ailleurs le sujet du chapitre duquel ce passage est repris. Le fait que SMITH souligne qu'un commerce aux avantages réciproques est très commun ne veut pas dire que l'amour-propre seulement est adéquat pour une bonne société. En fait, il maintient précisément le contraire : le salut de l'économie ne repose pas sur une motivation unique. SMITH appelle, comme on l'a dit, plutôt à la prudence et à la sympathie. Il défend la recherche de l'intérêt personnel dans des situations particulières liées à des restrictions conjoncturelles qui rendent le commerce et la production difficiles (c'est d'ailleurs à ce propos qu'il se réfêre, dans les différentes éditions de la Richesse des Nations, au " present state of things »).

En fait, si on regarde plus attentivement les propos de SMITH concernant le comportement humain, on s'aperçoit qu'il ne fait pas abstraction de toute subjectivité dans le processus de valorisation et que, par ailleurs, la recherche de l'intérêt propre ne se fait pas sans tenir compte du jugement d'autrui. Autrement dit, Adam SMITH ne considère pas les individus en dehors de tout cadre social, bien au contraire.

La subjectivité partielle de l'individu est mise en évidence dans un autre exemple célèbre de SMITH, celui du paradoxe de la valeur de l'eau et du diamant ${ }^{39}:$ l'eau, qui a une grande utilité, a également une petite valeur d'échange, alors que le diamant, dont l'utilité est faible pour l'individu, a une grande valeur d'échange. Si le diamant a une petite utilité pour l'individu, comment expliquer qu'il le désire ? Pour SMITH, la demande pour la pierre précieuse est dûe à sa beauté. Les diamants n'ont pas d'utilité, ils ne sont que des ornements et leur beauté est d'autant plus attirante qu'elle est rare.

Ce n'est donc pas la rareté seule de l'objet qui lui procure une grande valeur d'échange, c'est avant tout sa beauté. Or, comme nous l'avons déjà dit, pour SMITH, l'individu désire tout ce qui lui inspire un plaisir dans son observation de l'objet. La beauté d'un objet lui procurant du plaisir, il va désirer cet objet, même

\footnotetext{
${ }^{38}$ SMITH Adam, 1937, p.26-27.

${ }^{39}$ SMITH Adam, 1937, p.28.
} 
si celui-ci ne lui est pas utile. En fait, l'objet de son désir ne va pas satisfaire un besoin, mais un goût :

«These qualities, which are the ground of preference and which give occasion to pleasure and pain, are the cause of many insignificant demands which we by no means stand in need of. The whole industry of human life is employed, not in procuring the supply of our three humble necessities, food, cloaths, and lodging, but in procuring the convenience of it according to the nicety and delicacy of our taste. To improve and multiply the materials which are the principal objects of our necessities, gives occasion to all the variety of the arts. ${ }^{40}$

Les goûts des individus jouent un rôle non-négligeable dans leurs désirs puisque, lorsqu'un individu acquiert un objet, il satisfait d'abord un goût et parfois aussi le besoin qui lui est associé. Cet argument est repris par SMITH dans le premier chapitre de la quatrième partie de "The Theory of Moral Sentiments", qui s'intitule : "Of the Beauty which the Appearance of Utility bestows upon all the Productions of Art, and of the Extensive Influence of this Species of Beauty ${ }^{41}$. Dans ce chapitre, le philosophe écossais donne l'argument suivant, qui est en fait repris de David HUME : "l'utilité d'un objet quelconque plait au maitre en lui suggérant en permanence le plaisir ou la commodité qu'il est apte à promouvoir » et « le spectateur adhère aux sentiments du maître par sympathie et considère alors nécessairement l'objet du même point de vue agréable. ${ }^{42}$

Cette citation est intéressante à plus d'un titre. L'utilité d'un objet est assimilée au plaisir qu'il procure ou à son côté pratique. L'individu est ici dans une relation avec l'objet, dans laquelle il est question des effets de l'utilité sur ses sentiments. L'utilité se révèle à l'individu à travers la commodité ou le plaisir (esthétique) que lui procure la vue de cet objet. La beauté d'un objet est donc également un critère pour sa valorisation. Pour EHRENFELS, l'individu peut valoriser l'esthétique au point de lui accorder une valeur intrinsèque élevée. L'objet ayant cette qualité va se voir attribuer une valeur d'effet, à cause précisément de son utilité esthétique.

\footnotetext{
${ }^{40}$ SMITH Adam, « Lectures on Jurisprudence », article daté de 1766, cité dans SKINNER Andrew, 1996, p.62-63.

${ }^{41}$ SMITH, "The Theory of Moral Sentiments ", partie IV, chap. 1, 1963, p.308-334.

${ }^{42}$ « The utility of any object [...] pleases the master by perpetually suggesting to him the pleasure or conveniency which it is fitted to promote [...] The spectactor enters by sympathy into the sentiments of the master, and necessarily views the object under the same agreeable aspect ", SMITH Adam, 1963, p.309.
} 
Dans la deuxième partie de la citation, il est question d'un spectateur éprouvant un sentiment de sympathie pour le maître, ce qui l'amène à adopter la même perspective sur l'objet. Ceci n'est pas sans rappeler le chapitre d'EHRENFELS sur les mouvements de valeur. Chez le philosophe autrichien, le maître est l'"aktiver Wertbildner» et le spectateur le "passiver Wertbildner". Le changement de valorisation s'opère sous l'effet de l'imitation ou de la suggestion.

Il pourrait nous être reproché de citer les ouvrages d'Adam SMITH antérieurs à "La Richesse des Nations » et que donc les propos qui en dérivent ne reflètent plus l'opinion de l'auteur lorsqu'il écrit ce dernier. A cela nous répondons que la "Théorie des Sentiments Moraux " a connu six éditions, dont la dernière est parue peu avant la mort du philosophe écossais, ce qui nous amène à dire que ses réflexions en matière de comportement humain n'ont pas changé. En fait, à notre sens, et nous l'avons montré, il ne semble pas y avoir de contradiction entre les deux livres, du moins dans le domaine de la valeur.

Evoquons à présent l'influence de l'environnement social sur le comportement humain chez Adam SMITH. Selon Andrew S. SKINNER, le point central dans l'argumentation de SMITH sur ce sujet est son insistance sur le fait que le motif principal qui nous conduit à améliorer notre condition d'existence est enraciné dans le désir d'être approuvé, ou en fait, d'être admiré par autrui ${ }^{43}$. Autrement dit, l'individu désire la reconnaissance d'autrui et c'est ce qui le pousse le plus souvent à agir. Même lorsqu'il agit dans son propre intérêt, il tient compte des opinions et du regard d'autrui :

" [If the individual] would act so that the impartial spectator may enter into the principles of his conduct, which is what of all things he has the greatest desire to do, he must, upon this, as upon other occasions, humble the arrogance of his self-love, and bring it down to something which other men can go along with. They will indulge it so far as to allow him to be more anxious about, and to pursue with more earnest assiduity, his own happiness than that of any other person. Thus far, whenever they place themselves in his situation, they will readily go along with him. In the race for wealth, and honours, and preferments, he may run as hard as he can, and strain every nerve and every muscle, in order to outstrip all his competitors. But if he should justle, or throw down any of them, the indulgence of the spectators is entirely at an end. It is a violation of fair play, which they cannot admit of. $1^{4}$

\footnotetext{
${ }^{43}$ SKINNER Andrew S., 1996, p.65.

${ }^{44}$ SMITH Adam, " The Theory of Moral Sentiments ", Partie II, section 2, chapitre 2, § 1, 1963, p.139-141.
} 
Dans ce passage, SMITH décrit la compétition pour la richesse et la gloire comme étant approuvées par les "spectateurs " tant que celle-ci ne transgresse pas certaines règles et n'éveille pas la réprobation de ces mêmes spectateurs. Les règles en question peuvent être interprétées comme étant les règles de la loi et celles de la morale. Les spectateurs étant eux, les personnes constituant l'environnement de l'individu ou des individus en compétition. Et on voit déjà se dessiner le lien entre l'éthique et l'économie.

Pour éviter le regard désapprobateur de ces congénères, l'individu devra donc être en mesure de juger de son propre comportement, ce qui implique qu'il devra le faire tout en restant impartial, et ceci n'est pas souvent le cas, selon SMITH :

"There are two different occasions upon which we examine our own conduct and endeavour to view it in the light in which the impartial spectator would view it; first, when we are about to act; and secondly, after we have acted. Our views are apt to be very partial in both cases; but they are apt to be more partial when it is of most importance that they should be otherwise. ${ }^{45}$

La partialité de l'individu s'explique par le fait que, d'une part, lorsque nous agissons, nous le faisons parfois avec passion, ce qui nous empêche de rester objectif. D'autre part, nous dit SMITH, dans certains cas, nous ne voulons pas considérer les conséquences de nos actions sachant par avance qu'elles seront négatives, et nous ne nous imposons pas les restrictions qui seraient appropriées ${ }^{46}$. Il s'agit là en fait d'un problème de conscience morale : l'individu agit parfois sans tenir compte de cette dernière et s'attire ainsi les réprimandes de ses semblables.

Il ajoute que la Nature ne nous a pas laissé seuls avec notre amour de soi. Elle nous a doté de la capacité d'observation et de celle de l'induction. La première nous permet de connaître les comportements d'autrui, ce qui nous conduit à établir des règles générales de conduite grâce à la seconde. Ainsi ces règles définissent ce qui est bien et ce qui ne l'est pas, autrement dit, l'observation et la dérivation de règles mènent à l'élaboration d'une morale ${ }^{47}$. Cette morale fonctionne comme un point de référence dont on se sert pour juger du comportement d'un individu. Les règles morales et juridiques sont nécessaires au bon fonctionnement d'une société, puisque sans elles l'ordre ne serait pas possible ${ }^{48}$. Enfin, pour SMITH, si les individus agissent en ayant toujours en tête les règles de morale, et que leur comportement est marqué par la considération d'autrui (tous deux parfaitement

\footnotetext{
${ }^{45}$ Idem, Partie III, Chapitre 4, § 2, 1963, p.266-267.

${ }^{46}$ SKINNER Andrew S., 1996, p.66.

${ }^{47}$ Idem, p.67.

${ }^{48}$ SMITH Adam, "The Theory of Moral Sentiments ", Partie VII, chapitre 4, 1963, p.542-557.
} 
compatibles avec la poursuite de leurs intérêts propres), alors la société pourrait $s^{\prime}$ 'épanouir ${ }^{49}$. Le lien entre éthique et économie est ici évident : l'individu agit dans le but de satisfaire ses besoins et ses goûts, mais ses agissements doivent respecter les règles morales. Dans ces conditions, la société pourra se développer et l'économie prospérer. Ainsi la distribution des richesses par exemple, fait partie des comportements à adopter :

"The homely and vulgar proverb, that the eye is larger than the belly, never was more fully verified than with regard to him [the landlord]. The capacity of his stomach bears no proportion to the immensity of his desires, and will receive no more than that of the meanest peasant. The rest he is obliged to distribute among those, who prepare, in the nicest manner, that little which he himself makes use of [...]. They [the rich] consume little more than the poor, and in spite of their natural selfishness and rapacity, though they mean only their own conveniency, though the sole end which they propose from the labour of all the thousands whom they employ, be the gratification of their own vain and insatiable desires, they divide with the poor the produce of all their improvements. They are led by an invisible hand to make nearly the same distribution of the necessaries of life, which would have been made, had the earth been divided into equal portions among all its inhabitants, and thus without intending it, without knowing it, advance the interest of the society, and afford means to the multiplication of the species. ${ }^{50}$

Ce passage de la « Théorie des Sentiments moraux » contient une évocation de la fameuse "Main Invisible ", mais dans un contexte quelque peu différent. Cette approche n'a cependant rien de contradictoire avec celle de "La Richesse des Nations ". Dans les deux cas, la main invisible semble guider les individus vers un état meilleur.

Le fait de reconsidérer Adam SMITH sous un angle différent, c'est-à-dire en tenant compte de ses réflexions philosophiques, nous offre une vision nouvelle du phénomène de la valeur par ce même personnage. La vision que nous proposons n'est certes pas nouvelle, et elle n'est pas tout à fait en accord avec l'interprétation de la majeure partie des économistes, mais elle est néanmoins fondée et appuyée par des intellectuels ayant lus SMITH dans sa globalité et dans son contexte. En poste à l'université et occupant la chaire de philosophie morale, SMITH a développé une théorie de la valeur basée sur les coûts de production. Mais cette première approche n'explique en fait pas le phénomène de la valeur en luimême ${ }^{51}$, c'est-à-dire qu'elle n'offre qu'une vue partielle de la détermination de la valeur. Une vue partielle car la valeur comme étant la somme des coûts de production d'un bien, est une explication qui n'est pas complète: elle ne s'applique qu'aux biens marchands qui sont échangés sur un marché.

\footnotetext{
${ }^{49}$ SKINNER Andrew S., 1996, p.68.

${ }^{50}$ SMITH Adam, « The Theory of Moral Sentiments », Partie IV, chapitre 1, § 10, 1963, p.318.

${ }^{51}$ O’DONNEL Rory, 1990, p.56.
} 
Or, d'autres objets peuvent aussi avoir de la valeur et être valorisés par un individu. SMITH nous donne cependant quelques pistes pouvant nous mener à une réponse : en plus de sa valeur d'échange, un bien a une valeur d'usage. Et cette valeur d'usage est déterminée de manière subjective, puisqu'elle est basée sur l'utilité qu'un bien a pour un individu, mais aussi sur son goût pour cet objet. L'individu est subjectif, même dans ses actions en tant qu'acteur économique car il agit autant en fonction de ses goûts que du regard d'autrui. Conscient qu'il est " jugé » par ses semblables, l'individu devra ajuster son comportement, y compris lorsqu'il est en compétition, car il désire non seulement sa satisfaction personnelle au moyen de l'acquisition de biens marchands, mais il désire également la reconnaissance d'autrui. Voila un bon exemple d'un objet désiré par un individu et qui a une valeur d'usage pour lui, mais qui ne peut avoir une valeur d'échange. La reconnaissance d'autrui implique, selon les propres propos d'Adam SMITH, une composante éthique : pour que son comportement soit approuvé, l'individu devra respecter un certain nombre de règles morales. Le philosophe et économiste écossais tient également compte des valeurs éthiques, utiles à l'individu pour obtenir une certaine reconnaissance.

La reconnaissance sociale est aussi une donnée importante chez EHRENFELS pour qui l'individu agit en fonction du regard d'autrui sur ses agissements. Mais plus encore que l'acte lui-même, ce sont les intentions ou dispositions de l'individu qui seront jugées d'un point de vue éthique. Le comportement devra être conforme aux maximes morales en vigueur dans une société donnée pour être approuvé éthiquement. EHRENFELS ne le précise pas véritablement, mais puisqu'il parle de comportement de manière générale, sans émettre de restriction, on peut en déduire qu'il inclut donc les "agents économiques ". Ici aussi, son comportement n'est pas simplement le fait de ses intérêts personnels, il est aussi fonction du système morale de la société dans laquelle vit l'individu. Or, le système moral ou encore la valorisation des comportements impliquent tous deux des valeurs morales. Dans ce domaine, EHRENFELS se montre plus précis que SMITH, en partie parce que son but est justement une théorie générale de la valeur, et que donc les valeurs morales obéissent à des lois de fonctionnement au même titre que les valeurs économiques.

En adoptant une nouvelle approche de SMITH, on arrive à dégager des points communs avec EHRENFELS dans leur traitement respectif du problème de la valeur. D'abord, tous deux considèrent l'individu comme étant un être subjectif, accordant à certains objets une valeur d'usage chez SMITH, une valeur intrinsèque chez EHRENFELS. Ensuite, SMITH comme EHRENFELS identifient l'influence de la société sur l'individu et donc sur son comportement. Si cette relation est établie, alors la société influence les valorisations d'autrui. Cependant EHRENFELS donne plus d'explications que SMITH sur la nature de cette 
influence. Enfin, le rôle de l'éthique dans les agissements des individus n'est pas négligé chez les deux philosophes, y compris dans le cadre économique.

Si on peut distinguer quelques points communs entre les deux philosophes, il faut également garder à l'esprit les différences existantes.

En premier lieu, "La Richesse des Nations" se veut un ouvrage explicatif et instructeur sur le fonctionnement de l'économie dans une Angleterre qui aborde une période protectionniste. Son but n'est pas l'élaboration d'une théorie générale de la valeur, mais une théorie concernant les biens marchands exclusivement. SMITH effectue la distinction entre deux types de valeur : la valeur d'usage et la valeur d'échange. Cette distinction restera une caractéristique des études économiques sur le sujet, alors que la philosophie cherchera à développer la voie d'une distinction entre la valeur intrinsèque et la valeur d'effet. Les deux approches de la valeur sont donc différentes par le fait que la première se consacre à l'étude de la valeur en économie spécifiquement et la seconde par celle de la valeur en général. Ceci n'empêche cependant pas les rapprochements tels que ceux que nous avons effectués. De plus, et nous le verrons un peu plus tard dans ce travail, il est possible d'établir un parallèle entre les valeurs d'usage et valeurs d'échange ainsi qu'entre les valeurs intrinsèques et les valeurs d'effet. En effet, la valeur d'usage se réfère à l'utilité que l'objet procure à l'individu. Or, l'utilité d'un objet peut aussi être qualifiée de caractéristique de ce même objet. La valeur intrinsèque est attribuée par rapport à l'objet désiré pour lui-même ou encore pour l'une de ses propriétés. La valeur intrinsèque n'est donc pas si éloignée que cela de la valeur d'usage. Mais il convient de noter que cette dernière est contenue dans l'objet ou encore qu'elle provient de l'objet, alors que pour EHRENFELS, la valeur est attribuée par l'individu et n'est donc pas inhérente à l'objet. D'où la nature subjective de la valeur pour ce dernier. On tendrait à penser que pour SMITH la valeur serait une notion objective basée sur la valeur du travail contenu dans l'objet. Mais nous avons vu les limites de cette interprétation par le fait que SMITH admet que la production d'un bien se fait pour satisfaire un besoin de l'individu mais aussi pour satisfaire ses goûts. Cette proposition se vérifie facilement par l'exemple du choix des couleurs proposées pour un grand nombre d'objets : l'individu choisit d'acquérir l'objet pour répondre à un de ses besoins mais il choisit également sa couleur pour satisfaire ses goûts. Ajoutons enfin qu'EHRENFELS ira plus loin dans son analyse de la valeur en développant un certain nombre d'autres catégories et de sous-catégories de valeurs.

Le deuxième grand point de différence entre les deux philosophes concerne l'approche psychologique d'EHRENFELS. Cette perspective est la conséquence d'une de ses hypothèses, celle de la valeur comme étant attribuée par l'individu et non comme étant une propriété de l'objet chez SMITH. Le philosophe autrichien se propose de découvrir l'origine de la valeur et de sa détermination à travers une 
analyse qui se veut en partie axée sur celle du désir et des émotions. Les réflexions de SMITH sur la valeur se veulent plutôt économiques, empreintes de quelques apports de la sociologie, notamment en ce qui concerne la considération d'autrui et le fait de chercher à plaire. Mais l'essentiel de sa théorie se concentre sur le travail.

La troisième différence, et non des moindres, est celle qui concerne la relation entre la valeur et les prix. Pour SMITH, les prix sont la traduction en unités monétaires de la valeur d'un objet. Mais ce faisant, il considère en fait les biens marchands, c'est-à-dire les objets qui ont un prix, qui sont achetables et/ou vendables. De son côté, EHRENFELS, toujours dans le but de concevoir une théorie générale de la valeur, ne peut faire abstraction des autres types de biens. Il tient compte de tous les types d'objets pouvant faire l'objet d'une valorisation de la part d'un individu. Il considère par ailleurs les biens marchands comme un type particulier de bien et les valeurs qui en découlent, comme un type particulier de valeur.

En définitive, c'est en considérant les propos d'Adam SMITH sur le comportement des individus en tant qu'agent économique et sur la valeur d'usage, qu'il est possible de le rapprocher de Christian von EHRENFELS. Des différences, en partie dues à l'objectif initial de chacun des deux auteurs, persistent cependant. Et elles vont s'accentuer avec la théorie de la valeur de David RICARDO.

\section{2 - Les apports de David RICARDO à la théorie de la valeur d'Adam SMITH}

David RICARDO est né en Angleterre à Londres le 18 avril 1772. Il bénéficie d'une éducation non conventionnelle en ce sens où elle se fait à la fois en Angleterre et en Hollande ${ }^{52}$. Voulant que son fils apprenne suffisamment pour lui succéder plus tard dans ses entreprises ${ }^{53}$, le père de David RICARDO l'envoie passer deux ans, à Amsterdam de 1783 à 1785. RICARDO, seul, en sera assez malheureux ${ }^{54}$. Dès son retour dans son pays natal, à 14 ans, son père l'associe à la gestion de ses affaires, une expérience empirique qui influencera plus tard le jeune

\footnotetext{
52 La famille de David RICARDO avait immigré à Londres juste avant sa naissance.

${ }^{53}$ MAHESHWARI P.D. (éd.), "Towards the Theory of Value. Focus on David Ricardo ", p. 14.

${ }^{54}$ Dans une lettre de Maria Edgeworth datée du 14 novembre 1821 , elle répète les confidences de Ricardo à propos de son expérience à Amsterdam (in "Letters from England ", édité par Christina Calvin, Oxford 1971, p.266): « My father gave me but little education. He tought reading, writing and arithmetic sufficient because he doomed me to be nothing but a man of business, he sent me at eleven to Amsterdam to learn Dutch, French and Spanish. But I was so unhappy at being separated from my brothers, sisters and family, that I learned nothing in two years but Dutch which I could not help learning ".
} 
RICARDO ${ }^{55}$. Il grandit par ailleurs dans un pays subissant de grands changements liés à la Révolution Industrielle naissante en cette fin de XVIII ${ }^{\text {eme }}$ siècle.

C'est en 1799 que RICARDO lit pour la première fois "La Richesse des Nations ", ouvrage qui constituera la base de ses réflexions ultérieures, notamment dans le domaine de la valeur. Le livre de SMITH occupait les devants de la scène en économie dans l'Angleterre de ces années, et ce malgré la parution d'autres ouvrages dans ce domaine ${ }^{56}$.

Au début du XIX ${ }^{\text {ème }}$ siècle, et particulièrement entre 1809 et 1811 , l'intérêt de RICARDO dans le domaine économique concernait le débat sur la gestion de la masse monétaire par la Banque d'Angleterre ainsi que le prix du blé. Dans ses premiers ouvrages traitant de ces questions ${ }^{57}$ RICARDO se montra très critique vis-à-vis de la politique de la Banque d'Angleterre, lui reprochant notamment de ne pas contrôler l'émission des billets de banque c'est-à-dire l'augmentation de la masse monétaire, ce qui, toujours selon RICARDO, achevait d'affaiblir la Livre Sterling par rapport aux autres monnaies d'Europe continentale ${ }^{58}$. Dans un premier temps, il ne traita que brièvement la question de la valeur et sa théorie fut véritablement exposée dans "Principles of Political Economy and Taxation " ou « Des Principes de l'Economie politique et de l'Impôt » édité en $1817^{59}$.

La théorie de la valeur de RICARDO est soumise à plusieurs hypothèses. La première d'entre elles est que l'or et l'argent doivent être considérés comme de simples marchandises dont les prix varient et dont l'extraction nécessite du travail et du capital. La valeur de l'un ou l'autre métal précieux est donc définie de la même manière que le serait celle de tout bien marchand, c'est-à-dire en fonction de ses coûts de production. La question de l'or ou de l'argent en tant qu'étalon de référence pour mesurer la valeur sera considérée plus tard par RICARDO.

Ensuite, sa théorie de la valeur s'applique avant tout aux marchandises produites dans un régime de libre concurrence et dont la reproduction est permise par la nature. Il distingue par la même occasion les biens reproductibles des biens nonreproductibles (ceux dont la reproduction n'est pas permise par la nature) et décide que la valeur de ces dernières ne dépend que de leur rareté ${ }^{60}$.

\footnotetext{
${ }^{55}$ BIAUJEAUD Huguette, 1988, p.3.

${ }^{56}$ Notamment "Essay on Population " de Robert Malthus en 1798 et " Commerce Defended " de James Mill en 1808. Voir à ce propos DOBB Maurice, 1973, p.66.

57 "Le Haut Prix des Lingots est une Preuve de la Dépréciation des billets de Banque " en 1810, " Essai sur l'Influence du bas Prix du Blé sur les Profits du Capital » en 1815 et " Projets pour une Circulation Monétaire Economique et sûre " en 1816.

${ }_{58}$ DOBB Maurice, 1973, p.67.

${ }^{59}$ BIAUJEAUD Huguette, 1988, p.33-39.

${ }^{60}$ RICARDO David, "Essay on the influence of a low price of corn on the profits of stocks ", 1996, p.375-377.
} 
Puis, considérant que ces objets ne forment qu'une très petite partie des marchandises échangées quotidiennement, il en néglige l'étude ${ }^{61}$. Il précise par ailleurs que cette rareté a un caractère absolu, physique et non subjectif. Son caractère est objectif mais pas sa valeur absolue. Celle-ci « dépend uniquement de la fortune, du goût et du caprice de ceux qui ont envie de posséder de tels objets $"{ }^{62}$. A travers cette affirmation il est possible de discerner le caractère subjectif de la valeur des biens non-reproductibles : les goûts et les caprices des individus leur sont propres. Ceci dit, il est intéressant de noter que RICARDO aussi fait allusion aux goûts des individus tout comme SMITH, mais dans un contexte différent puisque ce dernier parle de l'influence des goûts dans le cas de la valeur d'usage. La valeur des biens reproductibles reste objective, dépendant de leur coût de production.

Troisième hypothèse : le travail est l'élément principal qui donne sa valeur à l'objet pour RICARDO et sa théorie est de ce fait une théorie de la valeur basée sur le travail. Il rejette la notion de "travail commandé » de SMITH, pour s'intéresser au "travail contenu" dans un bien marchand. Plus un tel bien en contient et plus sa valeur d'échange sera élevée. Cependant RICARDO, comme on l'a vu, ne nie pas la présence d'autres coûts de production mais il affirmera par la suite que leurs valeurs peuvent être converties en une seule et même unité, celle du travail contenu, puisqu'il faut également du travail pour les produire ${ }^{63}$.

Enfin, l'économiste anglais accorde dès le départ de l'importance à la variation de la valeur et au capital comme facteur de détermination de la valeur :

"Quelques marchandises augmentent de valeur par l'effet de l'impôt, ou de la rareté des matières premières dont elles sont faites ou de toute autre chose qui accroît la difficulté de production. D'autres baissent avec le perfectionnement des machines, une meilleure division du travail et une plus grande habileté de l'ouvrier, une plus grande abondance de matière première et généralement avec une facilité plus grande de production ${ }^{64}$.

Ainsi, juste avant la parution de son ouvrage majeur, "Des Principes de l'Economie Politique et de l'Impôt " en 1817, RICARDO a déjà une conception de la valeur comme étant une notion objective dont la détermination peut se faire grâce aux coûts de production et par rapport au travail contenu. Jusqu'en 1823, il y aura trois éditions des "Principes », dans lesquelles il est possible de voir une évolution de la conception de RICARDO concernant le problème de la valeur, en

\footnotetext{
${ }^{61}$ BIAUJEAUD, p.35.

${ }^{62}$ RICARDO David, "Principes », 1819, chapitre 1, p.2 et 3.

${ }^{63}$ HOLLANDER Samuel, 1992, p.87.

${ }^{64}$ RICARDO David, « Propositions tendant à l'établissement d'une circulation monétaire et économique et sûre ", cité dans BIAUJEAUD, 1988, p.38-39.
} 
particulier dans la dernière édition datant de $1821^{65}$. Durant la période antérieure aux "Principes", l'économiste anglais n'était pas tant intéressé par le problème de la valeur que par ceux du blé et de la masse monétaire, et c'est en parti pour cela qu'il ne posa que quelques hypothèses avant d'entreprendre un développement plus conséquent. L'évolution de sa perspective se fit également par rapport aux critiques, en particulier celles de Thomas MALTHUS, autre intellectuel s'intéressant à l'économie durant la même période ${ }^{66}$. Pour notre part, nous allons effectuer une synthèse de l'analyse Ricardienne de la valeur en montrant tout d'abord les points d'évolution par rapport à SMITH, puis en soulignant leurs différences et enfin en dessinant un parallèle avec la théorie d'EHRENFELS. Notre but étant de s'intéresser avant tout à la notion de la valeur, à sa détermination et à sa mesure, nous n'avons pas pour prétention de présenter tous les aspects de la théorie de RICARDO sur le sujet, mais simplement l'essentiel de sa théorie permettant une comparaison avec celle d'EHRENFELS.

La théorie de la valeur de David RICARDO se base sur celle de SMITH, comme il le reconnaît lui-même à travers ses écrits ${ }^{67}$. Plus exactement, il s'en inspire pour élaborer sa propre théorie, comblant au passage les soi-disant manques de la théorie de $\mathrm{SMITH}^{68}$. Pour exposer les réflexions de RICARDO sur le sujet, nous avons choisi de suivre ses principales critiques de son maître à penser à partir desquelles il développa sa théorie.

Le premier reproche que l'on relèvera et que RICARDO fait à SMITH concerne la confusion faite par ce dernier en ce qui concerne le travail commandé et le travail dépensé dans une marchandise ${ }^{69}$ : pour RICARDO le travail contenu est le seul facteur déterminant dans l'établissement de la valeur d'échange ${ }^{70}$. En effet, la quantité de travail fixée dans un objet est invariable alors que celle qu'il commandera est variable car la quantité dépensée à la production d'une chose ne commandera pas une quantité de travail égale à elle-même. Rappelons que RICARDO s'intéresse à la valeur relative des choses et que donc pour lui, le travail commandé ne peut être une référence à cause justement de sa variabilité. Par contre le travail dépensé, lui, est stable, permettant par la même occasion de le considérer pour établir cette valeur.

\footnotetext{
${ }^{65}$ BIAUJEAUD, dans la deuxième partie de son livre, elle montre justement cette évolution dans les réflexions de David Ricardo.

${ }^{66}$ BIAUJEAUD, 1988, p.98-99.

${ }^{67}$ O'DONNEL Rory, 1990, p.136-137.

${ }^{68}$ HOLLANDER Samuel, 1992, p.86.

${ }^{69}$ DOBB Maurice, 1973, p.77.

${ }^{70}$ HOLLANDER, 1992, p.87.
} 
Dans la première édition de ses "Principes ", l'économiste anglais arrive à cette conclusion par élimination, c'est-à-dire qu'il a envisagé les deux possibilités données par SMITH et qu'il a montré que seulement l'une d'entre elle est possible pour mesurer la valeur. Ce n'est que dans la troisième édition de ce même livre qu'il effectuera une démonstration par induction, à la suite notamment de critiques formulées par MALTHUS :

"C'est par l'établissement d'une table de degrés des variations de la valeur d'une marchandise et de la quantité de travail nécessaire à sa production qu'il [Ricardo] est conduit à formuler l'hypothèse d'une relation causale entre ces deux variations, puis une expérimentation vient vérifier son hypothèse et lui permet enfin de la transformer en loi » ${ }^{71}$

A la suite de cette remarque, Huguette BIAUJEAUD ne manque pas d'ajouter que ces "tables de degrés" ne sont pas des statistiques réelles, mais des tableaux élaborés par RICARDO lui-même sur des bases empiriques. La base de sa démonstration est donc fragile.

Rappelons qu'EHRENFELS procéda également par induction, mais il ne manipule à aucun moment les valeurs relatives. RICARDO érige le travail dépensé en tant qu'élément donnant sa valeur à une marchandise en postulat. Suivant SMITH, il considère que la valeur d'un bien peut, en outre, être calculée à partir des coûts de production. Il reconnaît par la même occasion que le travail n'est pas seul facteur de production et qu'il faut compter aussi avec le capital.

Contrairement à SMITH, et c'est un second reproche qu'il lui fait, RICARDO affirme que, quelque soit le stade de développement de la société, un produit ou une marchandise est toujours le fruit du travail assisté par du capital sous toutes les formes que ce soit. Ainsi le chasseur a besoin pour pouvoir emprisonner et tuer le gibier d'un instrument de chasse. De la société la plus primitive à la société la plus civilisée donc, le capital intervient dans la production et influence la valeur en fonction de la portion de travail fixée en lui, laquelle se transmet à la marchandise envisagée et s'ajoute à la quantité de travail immédiatement dépensée. C'est donc toujours le travail et le travail seul qui règle la valeur relative. Concrètement, et si on reprend l'exemple du chasseur que nous venons d'évoquer, le travail dépensé dans la fabrication du ou des instruments de chasse s'ajoutera à celui dépensé pour attraper le gibier pour former la valeur d'échange de ce dernier, s'il venait à être échangé.

${ }^{71}$ BIAUJEAUD, 1988, p.98-103. 
RICARDO introduit dans sa troisième édition des ses "Principes" le facteur " duré de vie du capital », qui influence également la valeur du bien :

"Supposons que la même quantité de travail soit nécessaire pour faire les deux armes mais qu'elles soient de durées très inégales; une petite portion de la valeur de l'instrument durable serait seulement transférée à la marchandise, une portion beaucoup plus grande de la valeur de l'instrument le moins durable serait réalisée dans la marchandise qu'il a contribué à produire ${ }^{72}$.

Cette remarque fait suite à la distinction effectuée entre capital fixe et capital circulant. Les machines par exemple appartiennent à la deuxième catégorie car leur durée de vie est limitée dans le temps. L'expression durée du capital prend alors un sens nouveau: la durée du capital est maintenant estimée d'après la longueur du processus de fabrication de la marchandise dans lequel il est impliqué. C'est au prix de cette transformation seulement que RICARDO peut expliquer par la même cause la valeur différente de marchandises produites par la même quantité de travail, mais dont la vente est plus ou moins éloignée du moment de leur achèvement ${ }^{73}$. De nos jours, ce temps de mise sur le marché pourrait être assimilé au temps de transport et de distribution.

Le temps va jouer un rôle certain dans la détermination de la valeur d'une marchandise puisque sa valeur initiale n'est pas immuable, et suivant le temps plus ou moins long qui s'écoule entre le moment où la marchandise est produite et celui où elle est vendue, elle subit un accroissement de valeur proportionnel à cet espace de temps, à la valeur du capital et au taux de profit. En fait cet accroissement est même plus que proportionnel nous dit RICARDO, car les profits se capitalisent. Il en est de même pour les marchandises produites par du travail seulement : leur valeur augmentera en fonction du temps nécessaire pour que le capital circulant qui a rémunéré ce travail, revienne par la vente des marchandises au capitaliste. Cette augmentation de la valeur serait donc due à des intérêts sur le capital avancé, pour employer des termes contemporains. Ceci nous fait penser à la rente chez SMITH, laquelle est constituée par le capital emprunté sous forme d'avance pour payer les coûts de production et qui est ensuite remboursée avec des intérêts.

\footnotetext{
${ }^{72}$ RICARDO David, « Principes " (3 ${ }^{\text {ème }}$ édition 1821), 1993, p. 16.

${ }^{73}$ BIAUJEAUD Huguette, 1988, p. 123.
} 
Le capital est donc présent quelque soit le stade d'avancement de la société, mais le travail reste en définitive le dénominateur commun permettant de déterminer la valeur d'un bien marchand. En d'autres termes, la valeur relative des marchandises est égale à la somme des différents travaux nécessaires à leur production, c'est-à-dire la fabrication des instruments qui assistent le travail (=capital sous toutes ses formes) et la production directe du bien lui-même (=effort physique que l'on retrouve également chez SMITH).

Si le travail est l'élément déterminant, la question qui vient ensuite à l'esprit est celle de la détermination de la valeur du travail lui-même. Pour RICARDO, comme pour SMITH, le prix du travail ou salaire est lié à la subsistance de l'individu, c'est-à-dire au renouvellement de sa force de travail. Le prix du travail est donc associé à celui du blé, base de l'alimentation à cette époque. Le prix du blé, quant à lui, dépend des conditions de sa production. En effet, la valeur du blé produit dépend d'après la théorie Ricardienne, de son coût de production. Or, celui-ci inclut la rémunération du travail, celle du capital, la rente et le profit.

Pour l'économiste anglais, les terres mises en exploitation n'auront pas le même rendement; les premières à être cultivées seront les plus fertiles, puis, avec l'augmentation de la population et donc celle de la demande de nourriture ${ }^{74}$, d'autres terres, moins fertiles, vont être mises en exploitation. Etant donné qu'elles seront moins productives, c'est-à-dire qu'à quantité de travail égale elles donneront moins de blé, leur coût d'exploitation sera plus élevé. Le prix du blé suivant les coûts de production, il augmentera lors de la mise en culture des terres moins fertiles. Cette différence apparaissant alors entre le coût de production relativement moins élevé sur les terres les plus fertiles - et le prix du blé - fixé encore une fois en fonction des coûts de production sur les terres les moins fertiles - sera consacrée au paiement de la rente pour les terres les plus productives. La rente étant cette portion du produit de la terre que les fermiers doivent abandonner aux propriétaires, en rémunération du droit qu'ils leur accordent d'exploiter les facultés productives et impérissables du sol. Elle est le résultat de l'appropriation du sol, des différences de fertilité et de l'insuffisance des terres fertiles pour produire toute la nourriture nécessaire à la population ${ }^{75}$.

Autrement dit, le prix du blé dépendra de son coût de production dans les conditions les plus défavorables, c'est-à-dire en fait son coût de production sur la

\footnotetext{
${ }^{74}$ En fait, les prémisses de sa théorie de la rente étaient déjà exposées dans son essai de 1815 ("Essai sur l'influence du bas prix du blé sur les profits du capital »). Il s'était d'ailleurs pour cela inspiré de celui de Malthus, "An Inquiry into Rent ", paru trois semaines plus tôt. Ricardo reconnaissait par ailleurs la théorie sur l'augmentation de la population de son compatriote. Voir BIAUJEAUD, 1988, p.66 et DOBB, 1973, p.68.

${ }^{75}$ Voir à ce propos RICARDO, « Essais sur l'Influence du Bas Prix du blé » et « Principes », Chapitre II: " De la Rente ".
} 
dernière terre mise en culture. A la lumière de cet argument, il serait possible de penser que RICARDO avait anticipé la théorie de la valeur marginale. Le rapprochement semble d'ailleurs cohérent, même s'il ne faut pas négliger le contexte dans lequel l'économiste anglais a développé sa théorie.

Ses réflexions sur le phénomène de la valeur sont marquées par un taux de profit unique, une variation des salaires à la hausse et celle du taux de profit à la baisse $^{76}$. Cette perspective s'explique par le fait que le début du XIX ${ }^{\text {ème }}$ siècle en Angleterre fut caractérisé, non seulement par une montée des salaires et une baisse des prix, mais aussi par une contestation grandissante envers les lois protectionnistes sur le blé, les fameuses "Corn Laws». RICARDO, pour expliquer les variations des salaires et des prix, suppose d'une part un taux de profit unique et une mobilité parfaite du capital. Il suppose par ailleurs que l'individu est rationnel et que la libre concurrence est la règle. Dans ce cas, les individus vont chercher à placer leurs capitaux là où le profit est le plus élevé. Ainsi les activités les moins profitables disparaîtront au profit des plus profitables. Le taux de profit commun est déterminé par celui des fermiers ${ }^{77}$, c'est pourquoi lorsque celui-ci baisse, les taux de profits dans les autres secteurs vont aussi baisser. Or, le taux de profit dans l'agriculture va accuser une baisse en raison de la productivité moindre des dernières terres mises en culture, ce qui va provoquer une hausse des prix du blé et par conséquent une augmentation des salaires de subsistance. Rappelons que pour RICARDO une augmentation du taux de salaire ne va pas se traduire par une hausse des prix mais par une baisse des profits.

C'est dans ce contexte qu'il milite pour une abolition des "Corn Laws". Une suppression de ces dernières aurait favorisé, selon lui, une augmentation des importations de blé moins cher, provoquant par la même occasion une baisse des salaires de subsistance et une augmentation des profits dans l'agriculture, une augmentation qui se répercuterait sur les autres secteurs de l'économie. Le prix du blé est donc une donnée importante dans la détermination du salaire de subsistance, lequel sert de référence pour la mesure de la valeur d'une marchandise puisque son coût de production peut être exprimé en quantité de travail dépensé. Cependant, il est important d'insister sur le fait que pour RICARDO, une augmentation du prix du blé peut aussi traduire une variation de la valeur du métal précieux. Si celui-ci voit sa valeur baisser, cela va provoquer mécaniquement une augmentation du prix du blé, ce dernier ayant toujours une valeur inchangée. Cette augmentation se répercutera également sur le prix des biens marchands. La valeur des biens marchands, exprimée par rapport à celle du blé sera inchangée, tandis que leurs valeurs respectives, exprimées en unités

\footnotetext{
${ }^{76}$ BIAUJEAUD, 1988, p.63-74.

${ }^{77}$ DOBB Maurice, 1973, p.70-71.
} 
monétaires, seront plus élevées ${ }^{78}$. La valeur réelle d'un bien dépend donc bien de la quantité de travail dépensée à sa fabrication.

Enfin, il convient d'aborder le problème de la mesure de la valeur chez RICARDO. Au départ, il avait adopté deux mesures de la valeur: le travail dépensé et l'or. Il faut dire que les réflexions de RICARDO sur la question se sont étalées dans le temps et qu'en fin de compte, il n'a jamais vraiment trouvé de solution unique $^{79}$. Il essaya d'abord de chercher dans le travail dépensé un étalon de mesure invariable de la valeur : étant donné qu'un certain effort exigerait toujours un même sacrifice de repos, le travail dépensé pouvait être considéré comme tel. Et puisque sa valeur se reflète à travers le salaire, alors il pourrait être considéré comme étalon de mesure. Mais étant donné que le salaire de subsistance varie avec l'état de civilisation ou d'avancement du pays, il n'est alors plus une mesure invariable.

RICARDO va alors chercher dans l'or et l'argent une référence stable, avec laquelle on pourra évaluer la valeur des marchandises. En réalité, l'intention première de RICARDO, nous dit Huguette BIAUJEAUD, n'était pas tant de trouver un étalon que de répondre à la politique de la Banque d'Angleterre à son époque :

" Afin de prouver que le prix des lingots tenait à la surabondance et à la dépréciation des billets de banque, RICARDO dans son premier pamphlet, avait évoqué l'invariabilité ou plutôt la stabilité de la valeur des métaux précieux, et montré qu'il fallait, pour apprécier l'altération de la monnaie de papier, posséder une étalon invariable auquel la comparer $n^{80}$

Il établit alors l'argent (or) comme un étalon monétaire et un étalon général de valeur :

« D'aucuns pensent que cette mesure du commerce (l'argent) est, comme toutes les autres mesures, arbitraire et peut être modifiée à volonté. Mais il n'en est pas ainsi de l'argent : c'est la chose que l'on marchande aussi bien que la mesure du marché ; dans le commerce

\footnotetext{
${ }^{78}$ Ce point est abordé par Ricardo en note en bas de page ( $"$ Works and Correspondence of David Ricardo ", édité par Sraffa, volume IV, p.21) : "It has been thought that the price of corn regulates the prices of all other things. This appears to me to be a mistake. If the price of corn is affected by the rise or fall of the precious metals themselves, then indeed will the price of commodities be also affected, but they vary because the value of money varies, not because the value of corn is altered. Commodities I think cannot materially rise or fall, whilst money and commodities continue in the same proportions, or rather whilst the cost of production of both estimated in corn continues the same." "

${ }^{79}$ DOBB Maurice, 1973, p.83

${ }^{80}$ BIAUJEAUD, 1988 , p.79.
} 
il passe de l'acheteur au vendeur: ainsi il ne mesure pas seulement la valeur de la marchandise à laquelle on l'applique, mais on le donne en échange de celle-ci, comme étant de valeur égale. On dit, je pense, avec raison qu'une marchandise monte, quand la même quantité s'échangera pour une plus grande quantité d'une autre chose, mais plus particulièrement de cette chose qui est la mesure du commerce de ce pays... $)^{81}$

RICARDO a, en fait, adopté deux étalons de mesure de la valeur mais il les emploie à des fins différentes. Pour déterminer la valeur échangeable de deux marchandises, il a recourt de préférence à l'étalon théorique, les quantités relatives de travail, et pour étudier les variations de la valeur, ce qu'il fait par l'intermédiaire de la valeur absolue, il choisit plutôt de la mesurer par rapport à l'or ou l'argent.

En définitive, bien qu'il considère la valeur comme étant objective, RICARDO n'arriva pas à en déterminer la mesure.

En outre, l'apport du capital dans sa propre théorie de la valeur rendait par la même occasion le principe d'universalité de la mesure par le travail questionnable. La valeur relative des marchandises est le rapport de leurs valeurs réelles, et elle est en même temps le rapport de leurs coûts de production respectifs, puisque la valeur réelle est un synonyme du coût de production. Mais le travail étant l'essentiel de celui-ci, il s'établit en fonction des salaires qui, finalement, règlent le rapport d'échange.

Après de nombreuses tentatives pour trouver un étalon de mesure de la valeur, et s'étant finalement décidé pour l'or, RICARDO avoua cependant quelques doutes et son insatisfaction, lesquels sont exprimées explicitement dans sa correspondance avec son ami et partisan de ses doctrines économiques, John Ramsey MAC CULLOCH, datée du 25 janvier 1821 :

" Je ne suis pas satisfait, comme je vous l'ai souvent dit, de l'exposé que j'ai donné de la valeur, parce que je ne sais pas exactement où fixer mon étalon. Je suis entièrement persuadé qu'en le fixant sur la quantité de travail réalisée dans les marchandises comme règle qui gouverne leur valeur relative, nous sommes sur la bonne voie, mais, quand je désire fixer un étalon de valeur absolue, je suis indéterminé quand à choisir le travail d'un an, d'un mois, d'une semaine, d'un jour. ${ }^{82}$

Le facteur temps fait donc hésiter RICARDO sur la question de la détermination d'un étalon de mesure unique et universel de la valeur. La réponse de

\footnotetext{
${ }^{81}$ RICARDO David, 1932, p.3.

${ }^{82}$ La lettre entière est reproduite en appendice du livre de Huguette BIAUJEAUD, 1988, p.207209.
} 
l'économiste au problème de la valeur ne sera en fait pas complète, même s'il s'est consacré jusqu'à la fin de sa vie à sa résolution.

En résumé, pour RICARDO la valeur d'un bien marchand dérive de son coût de production, lequel est lui-même déterminé par la quantité de travail incorporée à travers la fabrication de ce bien. Deux autres facteurs sont aussi importants : la rareté (plus un bien est rare et plus sa valeur sera grande) et le capital (la production d'un bien nécessite, quelque soit le stade de développement de la société, l'apport de capital). Ce dernier facteur de production peut être en fait converti en travail puisque, pour la production du capital il faut de nouveau du travail, lequel en détermine donc la valeur. Il ajoute ensuite que, selon son type, le capital aura une durée de vie variable. L'introduction du facteur temps pour expliquer les différences de valeur entre deux biens utilisant la même quantité de capital, ainsi que la prise en compte du temps de mise sur le marché, ne satisfera en fin de compte pas totalement l'économiste ${ }^{83}$.

RICARDO s'intéresse avant tout à la valeur relative des biens marchands, c'est-àdire à leur valeur par rapport à un autre bien. Ceci suppose bien sûr que les biens sont censés être échangés, autrement dit, le but de l'établissement de la valeur est l'échange.

Il en ressort que le traitement du problème de la valeur s'est focalisé chez RICARDO sur celui de la valeur des biens marchands susceptibles d'être échangés sur un marché. Une vision tout à fait logique puisqu'il était lui-même homme d'affaires et avait donc à faire avec des produits manufacturés. Aussi RICARDO est-il considéré comme celui qui a confirmé la rupture entre l'économie, l'éthique et la politique gouvernementale ${ }^{84}$. Avec lui, le traitement du problème de la valeur, pourrait-on dire, s'est restreint au champ des biens échangeables. Ceci nous amène à lui reprocher la négligence des autres types de biens, qu'il évoque certes courtement, mais sans y attacher trop d'importance.

L'intérêt de la théorie générale de la valeur d'EHRENFELS par rapport à celle de RICARDO est justement qu'elle ne néglige aucun type d'objet valorisé, mais en plus qu'elle considère les valeurs économiques comme cas particulier de valeur. Les différences entre les approches des deux intellectuels est grande : d'un côté une théorie économique qui se veut objective et qui est basée sur les coûts de production, avec le travail pour étalon de mesure de la valeur ; de l'autre côté, une théorie générale de la valeur, considérant tout processus de valorisation et intégrant l'individu valorisant dans un contexte social plus vaste que le seul environnement économique.

\footnotetext{
${ }^{83}$ MEHRA U.K., " Review of Ricardian Economics », in MAHESHWARI P.D., 1978, p.101.

${ }^{84}$ Idem, p.99.
} 
La question d'un éventuel rapprochement entre ces deux théories se pose alors : est-il pertinent de vouloir établir un parallèle entre celles-ci ?

Il est difficile de trouver des points communs aux deux approches, et pour cause, leurs fondements tout comme les objectifs initiaux de leurs auteurs sont très différents. Pour autant l'utilisation de leurs différences ne doit pas forcément mener à leur opposition radicale. En fait, l'évocation de la théorie de David RICARDO se justifie plus par la place de ce dernier dans l'histoire du développement de la pensée économique, moins pour son rapprochement avec la théorie d'EHRENFELS. Les arguments de RICARDO marquent la séparation entre la philosophie et l'économie en focalisant sa théorie notamment sur la valeur relative des biens marchands. Une opposition des deux théories déboucherait sur une longue discussion qui, à nos yeux, serait moins prolifique que d'essayer de les voir plutôt de manière complémentaire. En effet, si on considère l'éventail des biens susceptibles d'être échangés sur un marché, la théorie des coûts de production de l'économiste anglais apporte des éclaircissements sur la détermination de la valeur en rapport à ces coûts. Même si on accepte l'idée que les prix soient l'expression de la valeur de ces biens, on ne peut pas s'arrêter au fait que le prix d'un objet soit exclusivement déterminé par son coût de production ajouté au profit. D'autres facteurs, moins objectifs, entrent également en jeu. Pour EHRENFELS, le désir d'acquérir d'un objet est fonction non seulement de la valeur intrinsèque qui lui est attribuée, mais aussi de sa valeur d'effet. Or, il ajoute que la plupart des biens valorisés dans le domaine économique se voient attribuer une valeur d'effet, celle-ci étant relative à leurs avantages. Plus l'avantage d'un objet est grand, plus celui-ci gagne en valeur auprès de l'individu et plus celui-ci aura tendance à demander l'objet, c'est-à-dire à payer plus cher pour l'acquérir.

Plus proche d'EHRENFELS, et ce à plus d'un titre, le philosophe Karl MARX chercha à comprendre le phénomène de la valeur dans un système de production donné. Il développa une théorie objective de la valeur dont l'étude constituera l'objet du paragraphe suivant. 


\section{II - La théorie de la valeur ajoutée de Karl Marx}

Après les théories de la valeur Smithienne et Ricardienne, nous avons choisi de nous intéresser à celle de Karl MARX. Le choix de Karl MARX dans un travail consacré pour l'essentiel à EHRENFELS se justifie notamment par le fait que ce dernier fut influencé par le premier. Karl MARX fut un adepte des révolutions dans une période marquée par de nombreux changements; EHRENFELS, plutôt conservateur, n'en sera pas moins intéressé et influencé par ces mêmes changements. De plus, et à plusieurs reprises dans les chapitres précédents, nous avons signalé les passages où EHRENFELS était proche de la position de MARX, en particulier dans sa vision de l'organisation sociale avec ses membres dominants (les "aktiven Wertbildner») et ses membres dominés (les " passiven Wertbildner ").

Outre ses réflexions sur la construction de la société et en particulier sa perspective historique sur l'évolution des modes de production, la première chose qui rapproche MARX et EHRENFELS est le fait qu'ils furent tous les deux philosophes, de par leurs parcours universitaires respectifs. Et tous deux se sont par la suite intéressés à l'économie, bien que pour des raisons différentes et de manière plus marquée chez MARX.

La théorie de la valeur de Karl MARX offre une perspective toute à la fois différente, puisqu'elle se base sur le travail contenu dans l'objet pour expliquer sa valeur, et proche de celle d'EHRENFELS dans la mesure où l'une comme l'autre sont à comprendre dans un environnement social déterminé (la première théorie suppose une société divisée en deux classes opposées, la seconde trouve sa validité dans le monde culturel occidental). Cependant, nous verrons en fin de paragraphe que la théorie de MARX reste plutôt éloignée de celle du philosophe autrichien, et plus proche des théories purement économiques à la manière de David RICARDO, par exemple.

Nous allons, comme pour chaque auteur que nous avons évoqué dans ce travail, commencer par une biographie du philosophe allemand. Puis nous présenterons sa théorie de la valeur, que nous allons par la suite comparer avec celle de Christian von EHRENFELS.

\section{1 - L'essentiel de la vie de Karl MARX}

Karl MARX est né le 5 mai 1818 à Trèves, dans une famille bourgeoise, dont le père était avocat. Trèves était à cette époque une ville de taille moyenne, redevenue allemande à la suite du Congrès de Vienne en 1815. Avant cela, la région, au même titre que la Moselle voisine, était rattachée à la France. Ceci 
explique le fait que Hirschel MARX (1777-1838), le père de Karl, eut longtemps la nationalité française ainsi que l'occasion de s'intéresser à la philosophie des Lumières. Issu d'une famille de confession juive, Hirschel se convertit à la religion protestante et se fit rebaptiser Heinrich MARX, afin d'éviter les problèmes que cela aurait pu lui causer lorsque la région redevint possession de l'Empire allemand, dominé alors par les Prussiens ${ }^{85}$. Heinrich MARX était un homme ouvert et cultivé, n'attachant pas une importance particulière à la religion, comme le prouve sa conversion. D'un point de vue politique, il appartenait à une tendance libérale, mais sans pour autant être révolutionnaire. Sa femme, Henriette MARX (1787-1863), descendait d'une famille d'origine hollandaise dont les fils étaient de grands rabbins, comme le fut par exemple son père. Elle était peu cultivée et savait à peine lire et écrire. Son intérêt portait avant tout sur sa famille et la gestion quotidienne du ménage. Les relations entre Karl et sa mère étaient relativement froides et il en était beaucoup moins proche que de son père ${ }^{86}$.

Karl MARX était l'aîné d'une famille nombreuse. Il fut un brillant élève à l'école et en 1835 son père l'envoya étudier le droit à l'Université de Bonn. Dès le premier semestre, il consacra beaucoup de son temps à lire, parfois durant des nuits entières, ce qui au bout d'un an affecta sensiblement son état de santé, au point de le faire réformer du service militaire. Mais si son comportement pendant les cours était exemplaire, il n'en était pas de même en dehors des salles d'enseignement. Il reçut en effet quelques amendes pour le bruit causé par l'état d'ébriété qui était le sien lors des soirées étudiantes. Il fit également parti, dès ses premiers mois à l'université, d'un club de poètes («Dichterklub ») officiellement interdit, et dont les thèmes de discussion concernaient moins la rhétorique et la poésie que la contestation de la politique impériale prussienne. En 1836, Karl MARX partit continuer ses études à l'Université de Berlin, avec l'approbation de son père. A partir de ce moment, il s'intéressa de manière croissante à la philosophie de HEGEL (1770-1831), en phase avec une mouvance menée notamment par le philosophe Ludwig FEUERBACH (1804-1872) et qui était qualifiée de "hégélianisme de gauche ». Il fit parti du cercle des Hegéliens de gauche qui cherchaient à tirer de la philosophie de HEGEL des conclusions athées et révolutionnaires. Il semblait ainsi se consacrer de moins en moins au droit, et de plus en plus à la philosophie. En novembre 1837, MARX écrivit à son père une longue lettre dans laquelle il lui annonça son intention de réorienter ses études en faveur de la philosophie, ce qui ne rassura pas ce dernier sur l'avenir de son fils ${ }^{87}$. Karl MARX termina ses études en 1841 avec le titre de docteur en philosophie, qu'il obtint à l'université d'léna grâce à un travail sur Démocrite et Epicure.

\footnotetext{
${ }^{85}$ Les juifs de Trèves étaient soumis à un édit prussien de 1812 qui les excluait de l'administration publique autant que de l'enseignement. Voir Francis WHEEN, 2001, p.21.

${ }^{86}$ WHEEN Francis, 2001, p. 18.

${ }^{87}$ Idem, p.41.
} 
MARX renonça bien vite à une carrière de professeur à l'université, car dans un Empire prussien peu enclin aux idées révolutionnaires, ses déboires d'étudiant lui avaient déjà construit une réputation qui n'était pas favorable à une telle position. Il s'orienta plutôt vers le journalisme et commença à travailler pour le "Rheinische Zeitung", un quotidien aux tendances libérales et dont il devint rédacteur en chef en octobre 1842 . Ses articles se distinguaient par leurs attaques contre le régime dirigeant mais aussi comme un miroir reflétant les disputes de MARX avec d'autres personnalités de son entourage. On peut citer par exemple sa dispute avec Georg JUNG (1821-1888), un des actionnaires du journal, qui le voyait déjà comme un révolutionnaire dangereux. La censure ne tarda d'ailleurs pas à se manifester, et malgré les avertissements MARX continua d'écrire dans le même style, ce qui conduisit à une interdiction de parution en mars 1843.

En juin de cette même année, il se maria avec Johanna Bertha Julia Jenny von WESTPHALEN (1814-1881), dite "Jenny ", une amie d'enfance qui était trois ans plus âgée que lui. Jenny était une fille de famille noble, mais dont le père entretenait de bonnes relations avec Heinrich MARX et qui était également ouvert d'esprit et cultivé. Elle joua un rôle important dans la vie de Karl, notamment lorsqu'elle lui servit de secrétaire pour la rédaction et la traduction de ses écrits.

Karl et Jenny MARX eurent six enfants (Jenny, Laura, Edgar, Heinrich Guido, Franziska et Eleanor) dont la plupart ne vécurent pas longtemps et moururent souvent à la suite de maladies peu ou mal soignées par manque d'argent.

Attiré par Paris, une ville dont les révolutions de 1789 et 1839 avaient laissé une odeur attirante pour un contestataire tel que MARX, il décida de s'y installer à partir de l'automne 1843. A Paris habitaient également d'autres intellectuels socialistes, parmi lesquels Pierre Joseph PROUDHON (1809-1865), un anarchiste, que son livre "Qu'est ce que la propriété ? », paru en 1840, avait rendu célèbre. Avec, entre autre, la collaboration d'Arnold RUGE (1802-1880), un hégélien de gauche, il entreprit la rédaction d'un journal encore plus radical que ne le fut le " Rheinische Zeitung ", sous le titre de "Deutsch-Französische Jahrbücher ». L'édition s'arrêta dès la première parution après que MARX se fût disputé de façon irréversible avec RUGE. A partir de l'été 1844, MARX se consacra à l'étude des principaux représentants de la théorie économique anglaise : Adam SMITH, David RICARDO et John Stuart MILL. C'est également cette année que commença véritablement son amitié avec Friedrich ENGELS (18201895). Les deux personnes s'étaient certes déjà rencontrées en 1842, lorsqu'ENGELS lui rendit visite à la rédaction du « Rheinische Zeitung ", mais ce premier contact était resté assez froid. En 1844, ENGELS écrivit une série d'articles pour le "Deutsch-Französische Jahrbücher » qui avaient immédiatement éveillé l'attention de MARX, notamment parce qu'ils décrivaient la réalité 
du prolétariat en Angleterre ${ }^{88}$, une réalité dont MARX avait alors peu conscience puisqu'il s'était jusqu'alors penché essentiellement sur les idées sousjacentes aux mouvements de contestation grandissants en Europe continentale. Friedrich ENGELS joua également un rôle important dans la vie de Karl MARX puisqu'il fut un des seuls proches, avec Jenny, à être resté en bons termes avec lui jusqu'à la fin de sa vie. Selon Francis WHEEN, il n'y avait aucun secret ni tabous entre les deux hommes, et ENGELS remplaçait quelque part la mère affectueuse et attentionnée que MARX n'avait jamais eu. Ainsi, il lui donnait de l'argent, se souciait de sa santé et le rappelait de ne pas négliger ses études ${ }^{89}$. ENGELS participa à l'écriture de nombreux ouvrages et n'hésita pas à écrire à la place de son ami, par exemple dans les années 1860, lorsque MARX était payé par le journal américain « New York Daily Tribune " pour les articles qu'il envoyait.

En 1844 encore, le Roi Prussien Friedrich Wilhelm IV (1795-1861) échappa de justesse à un attentat sur lequel MARX ironisa, ce qui lui valut le fait d'être expulsé de France l'année suivante, par l'intermédiaire d'une lettre du Roi prussien au Roi de France. Il fuit alors en Belgique, où il organisa et dirigea un réseau de groupes révolutionnaires dispersés à travers l'Europe et connus sous le nom de Comités de correspondance communistes. Il joua un rôle décisif dans la consolidation de ces comités qui, en 1847, prirent le nom de Ligue communiste ("Bund der Kommunisten »). MARX et ENGELS furent chargés de rédiger le programme de cette première organisation ouvrière internationale. Le texte qu'ils soumirent alors, connu sous le titre de "Manifeste du Parti communiste ", fut le premier écrit systématique de la doctrine socialiste moderne et fut rédigé par Karl MARX, en partie d'après des brouillons d'ENGELS. Paru en février 1848, juste avant les mouvements révolutionnaires qui déferlèrent par la suite en Europe continentale, son contenu reflétait les visions de ses auteurs concernant l'histoire des développements sociaux sous la forme d'un antagonisme de classes ${ }^{90}$. En effet, les sociétés civilisées se caractérisaient par leurs deux classes sociales, celle des dominants et celle des dominés, quelque soit l'époque (durant le Moyen-Âge avec les seigneurs et leurs paysans, puis durant la Monarchie avec les nobles et les paysans et enfin dans les sociétés industrielles avec les bourgeois et les prolétaires).

\footnotetext{
${ }^{88}$ Le père de Friedrich ENGELS voulait qu'il lui succède à la tête de son entreprise et c'est avec l'intention de l'y préparer qu'il envoya se former dans une filiale implantée à Manchester. Ceci explique les connaissances de ENGELS quant à la condition ouvrière dans les fabriques de textiles.

${ }^{89}$ WHEEN Francis, 2001, p.105-106.

90 BURCHARDT Michael, " Marxistische Wirtschaftstheorie ", 1997, p.223.
} 
Suite aux évènements survenus en 1848 en France (chute de la monarchie et avènement de la seconde République), et aux grondements grandissants en Allemagne, le roi de Belgique, Leopold Ier, prenant peur des possibles soulèvements dans son pays, en particulier avec un personnage tel que Karl MARX sur son sol, signa le document ordonnant à la famille MARX de quitter le pays sous 24 heures. Après un bref passage par Paris, les MARX s'installèrent à Cologne, et Karl entreprit, avec l'aide d'ENGELS, de publier un nouveau journal, le "neue Rheinische Zeitung ». Mais dès les premiers éditoriaux, les autorités prusses se sentirent attaquées et au bout d'un an, l'entreprise dut s'arrêter. En 1849, la famille MARX se retrouva à Londres où Karl et Jenny vécurent jusqu'à la fin de leurs jours.

Les années 50 furent difficiles pour la famille qui vécut dans des conditions très miséreuses, malgré le soutien généreux d'ENGELS, qui, entre-temps s'était remis à travailler dans l'entreprise familiale à Manchester, afin de gagner de l'argent pour subvenir à ses propres besoins ainsi qu'à ceux de son meilleur ami ${ }^{91}$.

De 1853 à 1860, MARX fournit des articles au journal «New-York Daily Tribune »; en 1855 il travailla également pour le "Neuen Oder-Zeitung ", ou encore en 1858 pour le "New American Cyclopaedia ». Ses articles lui assurèrent une source de revenu non négligeable, même si selon Francis WHEEN, ENGELS en écrivit une certaine partie ${ }^{92}$. Malgré cet argent, MARX avait toujours besoin de l'aide financière de son ami. Ceci nous amène à dire qu'il n'a jamais vraiment su gérer ses dépenses par rapport à ses revenus, souhaitant également conserver un certain niveau de vie, quelque soit les circonstances, et peut être aussi pour sa femme aux origines nobles mais qu'il n'avait pas réussi à satisfaire matériellement. C'est ainsi qu'il s'était permis un secrétaire particulier répondant au nom de Wilhelm PIEPER. Il offrit également à sa famille des vacances à la mer, des leçons de piano et de langues aux enfants, pour ses filles des robes de bals ainsi que des cours de danse ou encore un "séminaire pour les dames" durant leur adolescence. Bref, MARX avait un style de vie qui, s'il correspondait à celui de son enfance et à son éducation, ainsi qu'à celui de sa femme, n'avait aucun rapport avec ses revenus ${ }^{93}$.

D'un autre côté, le fait de ne pas avoir un travail fixe lui permettait de s'asseoir de longues heures dans les bibliothèques afin d'enrichir ses connaissances. Après quinze ans de lecture des ouvrages économiques, il décida au tournant de l'année 1857-1858, d'écrire une critique de la politique économique. Ce travail n'aboutit

\footnotetext{
91 HAUTMANN Hans, « Karl Marx - Friedrich Engels : ein Vedemekum uber ihr Leben und Werk ", 2001, p.182-183.

${ }^{92}$ WHEEN Francis, 2001, p. 257-309.

${ }^{93}$ Idem, p.221-222.
} 
qu'après un certain nombre d'années malgré les incitations et encouragements d'ENGELS. MARX publia un premier ouvrage en 1859, connu sous le nom de "Zur Kritik der Politischen Ökonomie. Erstes Heft». Ce premier livre reçut un accueil mitigé, surtout de la part de ses amis ${ }^{94}$. Plusieurs intermèdes (intérêts pour d'autres sujets ou encore maladie) allaient retarder la parution de l'ouvrage qui devait en fin compte contenir l'essentiel de ses thèses économiques. Cependant, pour Hans HAUTMANN, cette période fut probablement la plus productive dans la vie de $\mathrm{MARX}^{95}$. Parmi les intermèdes qui détournèrent son attention, citons par exemple la rivalité avec Ferdinand LASALLE (1825-1864), dont les idées eurent plus d'influence que celles de MARX au début des années 1860. En particulier, il se distingua par son discours de 1862 devant de nombreux travailleurs, et qui était resté connu sous le nom de "Arbeiterprogramm ». Il s'agissait, toujours selon HAUTMANN $^{96}$, d'une version quelque peu transformée et moins radicale du Manifeste du Parti Communiste, avec également une adaptation à la réalité légale dans la Prusse d'alors. Ce discours prônait donc l'organisation des travailleurs au sein d'une structure reconnue et se présentait, depuis la défaite des révolutions et mouvements de 1848, comme une nouvelle espérance pour ces derniers. En 1863, il prit la tête du "Allgemeiner deutsche Verein", fondé au mois de mai de la même année. Les relations entre MARX et LASALLE ne furent jamais sans conflit, mais elles empirèrent après les voyages de MARX en Allemagne en 1861 et celui de LASALLE à Londres en 1862. Francis WHEEN décrit d'ailleurs l'attitude de plus en plus distante du premier vis-à-vis du second en Angleterre, MARX trouvant LASALLE de plus en plus insupportable dans son attitude, mais surtout par ses idées. Les deux personnages n'étaient pas d'accord sur les questions fondamentales telles que la politique économique, la tactique politique à adopter ainsi que leur distance avec l'Etat prussien ${ }^{97}$. MARX soutint plus tard la création d'un parti révolutionnaire allemand opposé à celui de LASALLE, un projet qui aboutit en 1869, grâce à Wilhelm LIEBKNECHT (1826-1900), considéré comme un de ses disciples. On sait également que ce parti fusionna dix ans plus tard avec le parti des radicaux nationalistes de LASALLE pour former le parti démocrate socialiste d'Allemagne.

Le 28 septembre 1864 dans le Hall Saint Martin à Londres, l'Association internationale des travailleurs fut fondée comme celle du rassemblement des travailleurs de différents pays européens et dont le congrès général se déroulait dans la capitale anglaise. MARX se vit remettre, grâce à son insistance, la tâche d'en écrire les statuts ${ }^{98}$.

\footnotetext{
${ }^{94}$ Idem, p. 284.

${ }^{95}$ HAUTMANN Hans, 2001, p. 177.

${ }^{96}$ Idem, p. 177.

${ }^{97}$ Voir également HAUTMANN Hans, 2001, p.178-180.

${ }^{98}$ Le fonctionnement de cette association est décrit par HAUTMANN Hans, 2001, p.188-189.
} 
En 1867 parut « Das Kapital. Kritik der politischen Ökonomie. Erster Band, Buch I : Der Produktionsprozeß des Kapitals ». Le premier tirage fut de 1000 exemplaires, ce qui ne fut pas peu pour un tel ouvrage à cette époque. ENGELS réussit à publier sous différents pseudonymes des articles dans des journaux qui contribuèrent à la popularité du "Capital ${ }^{99}$. La première traduction dans une langue étrangère fut celle Nikolai DANIELSON et German LOPATIN : "Le Capital » parut ainsi en russe à partir de 1872. Le succès, bien qu'inattendu, fut au rendez-vous. La traduction française fut entreprise par Joseph ROY, mais celle-ci ne plut pas à MARX qui la trouvait trop littérale. Il y apporta ses propres corrections, modifiant même par endroits la structure du texte. Aussi l'édition française, qui parut pour la première fois en 1875, était considérée par le philosophe allemand comme ayant "une valeur scientifique indépendante de l'œuvre originelle ${ }^{100}$. Le livre était structuré de manière originale par rapport aux autres ouvrages de politique économique. MARX innova en imprégnant son texte de sa vision historique des événements et d'une dialectique hégélienne : le système de production et ses éléments adjacents étaient le résultat d'un développement historique et son étude passait par un raisonnement logique permettant d'en comprendre tous les engrenages. Les trois volumes du "Capital » reconstituèrent, en l'expliquant, le système de production capitaliste en commençant par l'origine du capital, sa circulation dans le circuit économique et enfin le processus global de production capitaliste.

De 1867 à 1873, MARX se consacra à ses fonctions au sein de l'Association internationale des travailleurs et publia quelques prises de positions et commentaires sur les événements tels que la guerre entre la Prusse et l'Autriche, ainsi que sur celle entre la France et la Prusse, puis sur la Commune de Paris en 1871. Dans le premier cas, MARX et ENGELS avaient parié sur une victoire de l'Autriche, ce qui ne se vérifia pas dans les faits ; dans le second cas, ils avaient souhaités une victoire de la République Française fondée en septembre 1870, puis celle des travailleurs sur la bourgeoisie dominant les gouvernements durant les barricades à Paris. En 1873, MARX et ENGELS proposèrent de transférer le siège de l'association de Londres à New York, ce qui signifiait aussi que MARX ne siègerait plus au conseil d'administration. En fait, cette décision marquera le début de la fin du mouvement, puisque l'Association internationale des Travailleurs fut, après une existence dans l'ombre sur le territoire états-unien, dissolue en $1876 \mathrm{au}$ Congrès annuel de l'association à Philadelphie.

\footnotetext{
${ }^{99}$ HAUTMANN Hans, 2001, p.197.

${ }^{100}$ Idem, p. 198.
} 
Les dix dernières années de la vie de Karl MARX furent marquées par de nombreuses heures de lecture et l'intérêt pour de nouveaux thèmes. Ceux-ci allaient de l'économie (surtout le développement économique aux USA et en Russie), aux mathématiques, en passant par la chimie, la biologie,...

Son état de santé était affaibli par de nombreuses années de manque et par les maladies passées et peu soignées. MARX passa une grande partie de son temps à travailler sur le second volume du "Capital ». Sa femme, Jenny, décéda le 2 décembre 1882, ce qui fut un coup dur pour lui. En janvier 1883, sa fille Eleonor mourut à son tour à la suite d'une maladie, une mort qu'il ne supporta pas. Karl MARX décéda deux mois plus tard, le 14 mars 1883.

\section{2 - La théorie de la valeur de Karl MARX}

La théorie de la valeur de Karl MARX est une théorie basée sur le travail. Comme nous l'avons déjà évoqué, avant de se lancer dans une analyse de la sorte, il a étudié les principaux ouvrages d'économie de l'époque, en s'intéressant en particulier aux auteurs anglais. Ses propos sur la valeur sont présents dans son œuvre la plus connue : "Le Capital ", dont le premier des trois volumes est paru en 1867. Les deux suivants ont en fait été édités par ENGELS après la mort de MARX. "Le Capital " est une analyse des rapports de production dans la société et il souligne la domination du système capitaliste. La compréhension de son fonctionnement suppose celui de la notion de valeur ${ }^{101}$. Un autre ouvrage important et qui contient ses idées en matière de théorie de la valeur et d'économie est celui qui est paru en 1898 en anglais sous le titre "Value, Price and Profit ". Il reprend en fait le discours que MARX fit en 1865 en réaction aux propos du Oweniste John WESTON qui avait déclaré que les syndicats étaient néfastes et qu'une augmentation des salaires ne servirait à rien ${ }^{102}$. L'objectif du philosophe allemand à travers ses ouvrages, était d'identifier les lois du système capitaliste et ses effets négatifs ${ }^{103}$. Sa théorie de la valeur fut une contribution à cette dénonciation.

\footnotetext{
${ }^{101}$ GRÜNDBICHLER Alois, " Werte und Preise in der Theorie von Karl Marx ", 1986, p.49.

${ }^{102}$ HAUTMANN Hans, 2001, p. 193.

${ }^{103}$ HOFER Wolfgang, "Vergleichende Darstellung der Wirtschaftskreislauftheorien von Quesnay, Marx und Keynes unter Berucksichtigung ihrer Bedingtheit aus der Entwicklung der kapitalistischen Witschaftsweise ", 1987, p.189.
} 
Le point de départ de son analyse est l'étude des marchandises :

« Der Reichtum der Gesellschaften, in welchen kapitalistische Produktionsweise herrscht, erscheint als " ungeheure Warensammlung ", die einzelne Ware als seine Elementarform. Unsere Untersuchung beginnt daher mit der Analyse der Ware. ${ }^{104}$

La marchandise est un des éléments dits essentiels dans la société capitaliste, et MARX s'intéresse en particulier à sa valeur. La problématique qu'il pose est celle de la raison, de la façon et des critères selon lesquels la marchandise est échangée. Une marchandise peut être soit consommée, soit utilisée, soit échangée contre une autre marchandise. Mais avant tout, la marchandise est un objet au caractère double : elle est une chose utile, capable de satisfaire un désir humain ${ }^{105}$. Et c'est grâce à ses deux propriétés que la marchandise acquiert à la fois une valeur d'usage (en fonction de son utilité donc) et une valeur d'échange (si elle peut satisfaire un besoin).

MARX utilise, comme bon nombre d'économistes, les termes de valeur d'usage et valeur d'échange. La valeur d'usage dérive de sa capacité à satisfaire les besoins humains, ce qui induit donc une relation sujet-objet. D'après LIESSMAN, interprétant MARX, la valeur d'usage ne serait établie que dans le cadre de la consommation et non lors de l'échange puisque l'individu peut également revendre la marchandise sans la consommer ${ }^{106}$. Il nous semble que pour MARX, la marchandise se voit accorder une valeur d'usage par rapport à sa capacité à satisfaire un besoin. La définition qu'il en donne ne diffère pas de celle des économistes Classiques. En outre, une marchandise, en tant que bien de consommation intermédiaire, peut également avoir une utilité, celle de servir à la production d'un autre bien et d'être consommée à cet effet. Ceci ne l'empêche pas d'avoir une valeur d'échange. La valeur d'échange justement, s'établit dans une relation objet-objet, dans laquelle un objet est échangé contre un autre, si cet autre possède une utilité telle qu'elle soit en mesure de satisfaire le besoin de l'échangeur.

MARX se concentre néanmoins plus sur la relation sujet-objet dans le cas de la valeur d'usage que sur la relation objet-objet de la valeur d'échange. Dans le premier cas, la valeur de la marchandise n'est pas exclusivement déterminée par les préférences des agents économiques. Son analyse souligne le fait que le phénomène de la valeur est lié à des catégorisations sociales, c'est-à-dire à toutes les tâches qui précèdent la fabrication de la marchandise et qui induisent une

\footnotetext{
${ }^{104}$ MARX Karl, « Das Kapital. Erster Produktionsprozeß des Kapitals », in Marx-Engels-Werke Vol. 23, 1962, p.49.

${ }^{105}$ GRÜNDBICHLER Alois, 1986, p.5 et LIESSMAN Konrad P., « Karl Marx 1818-1989. Man stirbt nur zweimal », Sonderzahl, 1992, p.92.

${ }^{106}$ LIESSMAN Konrad P., 1992, p.93.
} 
relation sujet-sujet. LIESSMAN dit à ce propos, et pour illustrer la perspective de MARX, que « nous vivons à travers l'acte d'échange et si nous vivons, alors nous échangeons ${ }^{107}$. En d'autres termes, les relations entres individus sont toujours impliquées dans la détermination des valeurs, car ces mêmes relations existent dans le processus de fabrication ou parce qu'elles influencent les individus dans leurs choix.

La détermination de la valeur dans le cadre de l'échange doit se faire par l'intermédiaire d'un dénominateur commun qui sert de référence ou d'étalon aux échangeurs. Cette référence ne peut être la valeur d'échange elle-même puisqu'elle diffère d'objet en objet. L'élément commun doit être une propriété commune qui lie les marchandises entres-elles et qui sert de référence à l'échange. Cet élément est identifié par MARX comme étant le temps de travail qui donne à une marchandise sa valeur. Il distingue le travail - l'activité - du temps de travail qui est le temps qui est dépensé pour accomplir une tâche particulière. Un objet à l'état brut (un arbre par exemple) n'a pas de valeur d'échange s'il n'est pas mis en valeur par le travail humain qui fait de lui une marchandise :

«Ein Gebrauchswert oder Gut hat also nur einen Wert, weil abstrakt menschliche Arbeit in ihm vergegenständlicht oder materialisiert ist. $)^{108}$

Ceci implique que la valeur n'est pas une propriété intrinsèque de l'objet, c'est la société, dans le cadre de son mode de production, qui va décider de la mise en valeur d'un objet en le transformant à l'aide du travail humain. La valeur se construit donc également dans le cadre de la société, une hypothèse qui exclut la possibilité de valeurs individuelles. MARX repère par ailleurs l'existence de biens ayant une valeur d'usage, mais qui sont utilisables et qui satisfont un besoin sans l'intermédiaire du travail humain. Par exemple l'air ou le bois ne sont pas des marchandises, mais ils ont une utilité. A partir du moment où du temps de travail humain devient nécessaire afin que ces éléments puissent être utilisés pour satisfaire un certains nombre de besoins, alors ces mêmes éléments deviennent des marchandises. Il s'agit d'une évolution que l'on a pu constater pour l'eau par exemple, dont la consommation suppose un traitement préalable. Plus en avant, on peut y voir une anticipation de l'extension du marché avec un nombre de plus en plus important d'objets devenus des marchandises avec le temps.

Le problème de prendre le travail comme étalon de la valeur pose le problème de sa qualité, c'est-à-dire que pour la fabrication d'un même objet, le temps de travail peut être différent selon que la personne soit motivée ou pas. Ce à quoi MARX rétorque par le fait que la grandeur de la valeur d'une marchandise est toujours le

\footnotetext{
${ }^{107}$ Idem.

${ }^{108}$ MARX Karl, « Das Kapital », 1962, p.55.
} 
"temps de travail social nécessaire" (ou "die gesellschaftlich notwendige Arbeitszeit ») mesuré en moyenne. Le temps de travail social nécessaire est défini comme le temps indispensable pour produire une marchandise dans les conditions de production données dans la société où la marchandise en question est produite, et avec le niveau moyen d'adresse et d'intensité du travail dans cette même société $^{109}$. La moyenne qui est proposé par MARX prend pour unité de référence le niveau de qualification le plus bas, celui décrivant la force de travail la plus simple, que possèdent tous les individus sans formation particulière ${ }^{110}$. Les travaux plus compliqués ou demandant plus de qualifications seront comptabilisés comme étant le résultat de la multiplication du travail le moins qualifié par un coefficient déterminé. Les spécialistes ont reçu la plupart du temps une formation qui a elle-même demandé des heures de travail. La difficulté est de trouver le bon coefficient multiplicateur avec lequel on peut comparer (soit en multipliant, soit en divisant) le travail simple et le travail plus complexe. Le travail complexe peut lui-même être décomposé en une somme de travaux simples. Par exemple, le travail qualifié d'un médecin est la somme des années d'études durant lesquelles il aura lui-même contribué à son savoir, mais aussi durant lesquelles il aura eu des professeurs qualifiés qui lui auront enseigné la discipline. La valeur des connaissances acquises dépendra également des moyens qui y auront contribué, à savoir l'ensemble des éléments matériels disponibles qui permettent à un étudiant d'effectuer ses études dans de bonnes conditions.

En résumé, la grandeur de la valeur est déterminée à travers la quantité de travail dépensé à sa production. Il faut encore ajouter que ce temps de travail devient nécessaire pour la société lorsque son résultat est une marchandise capable de satisfaire des besoins. Cette nécessité correspond également au caractère double du bien : il doit être désiré pour être marchandise et sa valeur doit être produite et reconnue sur le marché. Notons que le temps de travail social nécessaire est une abstraction dont les travailleurs ne font jamais l'expérience concrète ${ }^{111}$.

Dans les sociétés capitalistes de la fin du XIX ${ }^{\text {ème }}$ siècle, les conditions de production impliquent la présence de moyens de production tels que les machines. Participent-elles à la formation de la valeur d'un objet, ou le travail humain est-il le seul élément capable de créer de la valeur?

Pour MARX, les moyens de production sont également des marchandises qui ont une valeur. Ils ont une valeur parce qu'il a bien fallu du travail pour les fabriquer et c'est à partir du travail dépensé à leur fabrication que va s'établir leur valeur.

\footnotetext{
${ }^{109}$ MARX Karl, 1962, p.53, GRÜNDBICHLER Alois, 1986, p.6 et HERF Markus, « Wert- und Preismodell als Koordinationsmechanismen bei Arbeitsteilung : das Konzept von Karl Marx ", 1988, p.23-24.

${ }^{110}$ MARX, 1962, p.59.

"' LIESSMAN Konrad P., 1992, p.96.
} 
Concrètement, un pêcheur qui attrape son poisson à l'aide d'une canne à pêche, lorsqu'il va le revendre, devra tenir compte non seulement de l'effort effectué mais aussi de la valeur participative de la canne à pêche, qui sera elle-même proportionnelle au temps de fabrication divisé par la durée d'utilisation.

Les hypothèses de MARX jusqu'à présent, permettent d'affirmer que pour lui la valeur d'une marchandise n'est ni une propriété intrinsèque de cette marchandise, ni une grandeur fixe. La détermination d'une valeur dépend de facteurs extérieurs tels que les conditions de production ou l'habileté des travailleurs. Or ces facteurs diffèrent selon les sociétés et évoluent avec le temps.

En ce qui concerne la question de la forme d'apparition de la valeur, c'est-à-dire sa forme concrète, MARX se réfère encore une fois au niveau de développement de la société et aux conditions de production qu'il implique. Ainsi, dans les sociétés de troque, les marchandises vont s'échanger l'une contre l'autre, et la valeur d'échange d'un objet sera donc établie en nombre d'unités de l'autre objet qu'il est possible d'acquérir avec le premier. Il est possible de cette manière de construire une liste des objets que l'on peut obtenir avec une certaine quantité d'une marchandise déterminée. Lorsqu'une marchandise est reconnue et acceptée par tous les membres d'une société comme unité de référence servant d'intermédiaire dans les échanges, alors celle-ci obtient des propriétés similaires à la monnaie. Du temps de MARX, cette marchandise était l'or. La quantité de cette marchandise de référence nécessaire à l'acquisition d'un bien est alors son prix. Le prix donne la mesure de la valeur d'échange d'une marchandise et permet d'être ainsi la forme générale d'équivalence puisque toutes les marchandises échangées se voient conférer un prix. Ce faisant MARX sous entend que l'argent sert d'intermédiaire et que les individus calculent en prix et non en valeur. Etant donné le caractère abstrait de la formation de la valeur, il ne serait pas possible d'effectuer des calculs en termes de valeur ${ }^{112}$.

Evoquions à présent une notion importante dans la théorie de Karl MARX, celle de la plus value ou "Mehrwert». La plus value est la réponse à la question de l'origine de la richesse dans le système capitaliste, ou formulé autrement, comment la valeur peut être accumulée dans une société d'échange où seuls des équivalents sont échangés ${ }^{113}$ ?

Son existence est liée à celle du système de production capitaliste qui se caractérise pour l'intellectuel allemand par la division de la société en deux classes : celle qui possède les moyens de production (les bourgeois) et celle qui vend sa force de travail (les prolétaires). L'appartenance à une classe ou une autre est déterminée par le type de revenu perçu par l'individu (les propriétaires des

\footnotetext{
112 Idem, p. 19.

${ }^{113}$ Idem, p. 115.
} 
moyens de production perçoivent des rentes ou des dividendes alors que les prolétaires reçoivent un salaire). Les prolétaires vendent donc leur force de travail comme une marchandise et le revenu qu'ils en tirent ne provient pas de la vente de la marchandise fabriquée mais du salaire qui leur est versé en échange de leur travail. La production de biens sert, selon MARX, uniquement le profit et non plus la satisfaction des besoins personnels ou la création de valeur d'usage. La motivation sous-jacente à tout acte de production est l'augmentation de la valeur d'échange, ce qui peut par ailleurs se schématiser sous la forme suivante :

$\mathrm{G}->\mathrm{W}->\mathrm{G}$ ', où $\mathrm{G}$ désigne la monnaie, W la marchandise et $G$ ' la monnaie $G$ augmentée d'un supplément.

Cette formule caractérise le paradoxe de la société décrite par MARX : elle est une société d'échange où s'échangent uniquement des marchandises équivalentes par leurs valeurs, mais où en même temps, "l'échange pur d'équivalent y est fade $»^{114}$. A travers l'échange, il s'agit non pas d'échanger de l'argent contre la même quantité d'argent, mais, par l'intermédiaire de la circulation des biens, de gagner de l'argent ${ }^{115}$.

MARX attribue au mot "capital " un sens nouveau, celui d'une réserve de biens dans le but de faire des profits ${ }^{116}$. Cette réserve de biens peut prendre la forme de monnaie, de moyens de production, de matières premières, de produits finis ou encore d'un potentiel en force de travail. Le propriétaire de cette réserve est qualifié de capitaliste.

C'est dans ce contexte que le philosophe allemand introduit sa notion de plus value. Les prolétaires ne possèdent globalement qu'un seul bien : leur force de travail. C'est contre une certaine rémunération qu'ils vont accepter de la vendre au propriétaire du capital, qui s'en servira pour la production de biens. Mais comme nous l'avons déjà mentionné, cette production, pour qu'elle soit intéressante pour le capitaliste, doit être source de revenus. Or, selon les thèses Marxistes, l'échange n'est possible qu'entre deux marchandises de même valeur ou entre une marchandise et une certaine somme de monnaie de valeur équivalente. Le salaire versé au prolétaire devra permettre, selon MARX, la reproduction de sa force de travail. En d'autres termes, la somme qui lui sera versée devra être égale à la valeur d'un panier de consommation permettant ce processus de reproduction ${ }^{117}$. Cependant, il ne s'agit pas d'un salaire de subsistance, c'est-à-dire d'un salaire minimum permettant au travailleur de survivre, mais d'un salaire suffisant pour pouvoir manger, se loger, se vêtir, et permettre à la personne de se cultiver,

\footnotetext{
114 Idem, p.116.

${ }^{115}$ MARX Karl, 1962, p. 168.

${ }^{116}$ BURCHARDT Michael, 1997, p.107.

${ }^{117}$ GRÜNDBICHLER Alois, 1986, p.12 et HOFER Wolfgang, 1987, p.95.
} 
d'apprendre sur l'histoire de son pays et sur sa culture. On pourrait à ce propos, tel que le fait BURCHARDT ${ }^{118}$, reprocher à MARX que la notion de revenu permettant la reproduction de la force de travail soit difficile à déterminer, d'une part parce que le travail considéré est, d'après les hypothèses de MARX, celui qui est le plus simple. Qu'en est-il du salaire pour un travail aux exigences physiques intensives? Peut-on trouver un coefficient multiplicateur? La tâche parait difficile. D'autre part, MARX ne donne pas suffisamment de précisions sur les critères permettant de déterminer un panier de consommation idéal, si du moins il existe.

Dans le capitalisme donc, un salaire est versé au travailleur en échange de sa force de travail et ce salaire lui permet de renouveler cette force. Comme on l'a vu, pour le capitaliste l'intérêt de produire réside dans le fait de pouvoir en tirer des profits et ceci sera possible à l'intérieur du processus de production. Etant donné que ce qui donne la valeur au produit est la quantité de travail qui est dépensée pour le fabriquer, le profit ne pourra donc se faire qu'à ce niveau. Lorsqu'un ouvrier travaille six heures dans une fabrique, la valeur de son travail à la fin de ce temps sera égale à la quantité de travail dépensée, c'est-à-dire six heures. Dans le cas d'une journée de travail réglementée à dix heures (c'est-à-dire que le capitaliste achète dix heures de force de travail à l'ouvrier), si le temps de travail nécessaire à la reproduction de la force de travail est de six heures, et si durant ces six heures le travailleur crée une valeur équivalente, alors le reste de la valeur créée durant les heures de travail restantes, sera celle que MARX nomme la plus value ou "Mehrwert». Cette séparation qui est ici schématisée, n'est que purement théorique. Les employeurs ne calculent pas en temps de travail, c'est-à-dire qu'ils ne s'intéressent pas véritablement à la valeur de la marchandise produite, mais à son prix ${ }^{119}$. Dans la réalité, nous dit MARX, ce processus se produit simultanément $^{120}$. La plus value n'apparaît d'abord pas sous forme d'argent, mais sous la forme d'un produit ou d'un stock de produits ayant une valeur d'échange intégrant celle-ci.

Il est important de souligner encore une fois que l'échange entre travailleur et employé ne donne à aucune des parties un avantage excessif : le premier échange sa force de travail contre un salaire lui permettant de la renouveler, le second tire une plus value du surplus de valeur créée par le travailleur. Pourquoi le capitaliste devrait-il payer la marchandise «force de travail » plus chère que sa valeur, alors qu'il ne le fait pas pour les autres marchandises? De ce fait, il n'est possible de parler d'un avantage d'une classe sur une autre dans le processus d'échange.

${ }^{118}$ BURCHARDT Michael, 1986, p.114-115 ; voir également LIESSMAN Konrad P., 1992, p.124.

${ }_{19}$ HERF Markus, 1988, p.17.

${ }^{120}$ BURCHARDT Michael, 1997, p.116. 
L'avantage de la classe bourgeoise est de posséder du capital productif dont ils peuvent tirer un profit, alors que les prolétaires ne possèdent que leur propre force de travail qu'ils ne peuvent qu'offrir sur le marché du travail. Sur le marché du travail se rencontrent des travailleurs et des entrepreneurs libres et égaux. Les premiers possèdent une force de travail et les seconds de l'argent. En tant que propriétaires d'un élément susceptible d'être échangé, les deux catégories de personnes sont égales. Non sans cynisme MARX écrit que "sur le marché la liberté, l'égalité, la propriété dominent » : la liberté car les travailleurs sont libres d'offrir leur force de travail; égalité car les deux parties échangent deux marchandises aux valeurs équivalentes; propriété car chacun possède quelque chose. Il ajoute :

" [Arbeitskraft und Geldbesitzer] begegnen sich auf dem Markt und treten in Verhältnis zueinander als ebenbürtige Warenbesitzer, nur dadurch unterschieden, daß der eine ein Käufer, der andere Verkäufer, beide also juristisch gleiche Personen sind. Die Fortdauer dieses Verhältnis erheischt, daß der Eigentümer der Arbeitskraft sie stets nur für bestimmte Zeit verkaufe, denn verkauft er sich in Bausch und Bogen, ein für allermal, so verkauft er sich selbst, verwandelt sich aus einem Freien in einen Sklaven, aus einem Warenbesitzer in eine Ware. Er als Person muss sich beständig zu seiner Arbeitskraft als seinem Eigentum verhalten. ${ }^{121}$

Lorsqu'on lit ces lignes, on peut se demander ce qu'il en est vraiment de l'affirmation d'exploitation des travailleurs par les entrepreneurs. Pour MARX, le problème se situe dans le fait que, premièrement, la plus value n'est pas le produit du travail des entrepreneurs mais de celui de leurs employés, qu'ils se sont appropriés. La plus value n'est possible que parce que la force de travail est rémunérée comme une marchandise et non comme la participation à la création de valeur. En tant que marchandise, la force de travail est soumise aux mêmes règles de marché que les autres marchandises. Le problème sur ce marché est que les producteurs bénéficient d'un monopole, du moins du temps de MARX, sur la détermination du temps de travail journalier et que cela leur permet d'augmenter la plus value à leur guise ${ }^{122}$.

Le secret de la société capitaliste bourgeoise réside dans la séparation entre la sphère de l'échange et celle de l'utilisation ${ }^{123}$ : la force de travail sera échangée, en tant que marchandise, contre de l'argent, pour une valeur équivalente; l'entrepreneur l'utilise et vend ensuite la marchandise que le travailleur aura fabriquée ou dont il aura contribué à la fabrication (on est dans la sphère de l'utilisation). Cette marchandise sera vendue sur le marché, contre une somme

\footnotetext{
${ }^{121}$ MARX Karl, 1962, p. 182.

${ }^{122}$ BURCHARDT Michael, 1997, p.116-117.

${ }^{123}$ LIESSMAN Konrad P., 1992, p. 125.
} 
d'argent correspondant à sa valeur d'échange (on se situe de nouveau dans la sphère de l'échange) qui inclut un surplus allant directement à l'entrepreneur. Vu de cette manière, personne n'est trompé ou encore considéré de manière injuste. MARX montre ainsi avec la plus value que l'exploitation des travailleurs n'est pas un élément abominable, méchant ou mauvais de ce type de société. Il s'agit en réalité de son principe de fonctionnement, d'un pilier fondamental qui inclut en outre la liberté, l'égalité et la propriété. Si on veut arrêter avec cette exploitation, il faut donc changer le système. En fin de compte, la plus value apparait car le salaire que reçoit le travailleur reflète la valeur de sa force de travail et non du produit de son travail ${ }^{124}$.

Enfin, pour terminer notre tour d'horizon autour de la question de la valeur chez MARX, nous allons considérer la différence qu'il effectue entre la valeur et le prix d'une marchandise. Nous avons vu que la valeur de celle-ci dépend de la quantité de travail qui a été dépensée pour sa production, alors que le prix, qui est fixé sur le marché, se situe autour de cette valeur, et inclut également la plus value. Il est intéressant de noter que pour MARX, le prix d'une marchandise n'est pas égal à sa valeur, mais il en dérive systématiquement. Le prix, qui est donc l'expression de la valeur en unités monétaires, peut correspondre à cette dernière si la quantité demandée sur le marché coïncide avec la quantité offerte. Si la quantité demandée surpasse la quantité offerte ou si elle lui est inférieure, alors le prix sera respectivement plus élevé ou moins élevé que la valeur de la marchandise. Cependant, il ajoute que les capitalistes ne s'intéressent qu'aux prix et non à la valeur qui en constitue la base. Le problème du passage de la valeur au prix est qualifié de problème de la transformation dans la littérature spécialisée ${ }^{125}$.

Pour MARX, le salaire oscille toujours autour de la valeur de la force de travail et la plus value constitue la base du profit. La question est de savoir comment on passe des grandeurs constitutives de la valeur à celles qui composent le prix d'une marchandise.

Le profit est la forme sociale sous laquelle apparaît la plus value ou encore la plus value exprimée sous forme de prix. Cependant, le profit n'est pas obligatoirement égal à la plus value. Ainsi le taux de plus value est égal à la plus value divisée par le capital variable utilisé dans la production, alors que le taux de profit est lui égal à la plus value divisée par la quantité totale de capital utilisée dans la production (c'est-à-dire capital fixe plus capital variable) $^{126}$. La plus value se base donc uniquement sur le capital variable - les ouvriers ou plus exactement leur force de travail - alors que le profit dérive du capital total engagé dans la production. Lorsque l'entrepreneur veut produire une marchandise, il devra pour cela acheter

\footnotetext{
${ }^{124}$ Idem, p. 126.

${ }^{125}$ BURCHARDT Michael, 1997, p.155.

${ }^{126}$ Idem, p. 156.
} 
sur le marché du capital fixe et du capital variable, s'il n'en dispose pas préalablement. Acheter signifie qu'ils ont un prix et la différence entre ce prix (que MARX nomme le « Kostpreis » ou prix-coût de l'objet) et le prix de vente du produit fini constituera le profit. On comprend ainsi pourquoi ce dernier et la plus value ne sont pas égaux ${ }^{127}$. Le capitaliste ne serait intéressé que par le profit, et non pas par son origine ou son lien avec la valeur. Pour MARX, le prix et la valeur d'un objet ne sont pas égaux ou, le cas échéant, il pourrait s'agir que d'un pur hasard. Enfin, le travail doit être compris comme la substance de la valeur, c'est lui qui donne à un objet sa valeur.

Le prix d'une marchandise sera égal à son prix-coût (= capital fixe et capital variable exprimés sous forme de prix) ajouté au profit. Il serait possible d'affiner l'analyse de ce processus de transformation surtout en ce qui concerne la détermination des prix à la production, mais dans le cadre de ce travail, nous nous en tiendrons là, concentrant notre attention sur le problème de l'origine de la valeur et son lien avec le prix ${ }^{128}$.

La théorie de Karl MARX se distingue de celles des autres économistes par le fait qu'il considère la valeur comme un phénomène social, c'est-à-dire appartenant ou faisant partie de la société. Il en est de même pour les prix et l'argent. Le développement de la monnaie est lié à celui de la production des biens. Dans une société de production de masse, telle que celle du XX ${ }^{\text {ème }}$ dans le monde occidental, l'argent est devenu un moyen d'échange et de mesure de valeur incontournable qui est très répandu. Dans une société où la production est limitée aux cultures maraîchères ou vivrières par exemple, l'argent n'est pas aussi développé puisque l'échange se fait essentiellement entre marchandises. Pour MARX, le développement de l'échange conduit à l'apparition d'un étalon de la valeur qui, s'il est accepté et reconnu comme tel par tous, devient alors monnaie d'échange. Dans ce cas, la monnaie reste un moyen d'échange, puisque le but ultime des individus est d'acquérir des marchandises susceptibles de satisfaire leurs besoins, ce qui correspond au schéma marchandise - monnaie marchandise.

Un changement dans la manière de produire et le développement de la circulation des marchandises vont conduire à l'apparition du capital. Dans ce schéma de pensée, la marchandise devient le moyen et l'argent le but et on a alors le processus suivant : monnaie - marchandise - monnaie. Cependant, il ne s'agit pas comme dans le premier schéma d'un échange de valeurs équivalentes sinon

\footnotetext{
${ }^{127}$ Sur ce point voir également GRÜNDBICHLER Alois, 1986, p.22-26.

${ }^{128}$ Pour ceux qui veulent aller plus loin : HERF Markus, 1988, p.33-46. Le problème dit de la transformation (comment passer de la valeur d'une marchandise à son prix) est traité par GRÜNDBICHLER Alois, 1986, p.34-43.
} 
l'opération ne se révèlerait pas intéressante. Ainsi, la somme qui est retournée au propriétaire du capital, se fait avec un supplément. On devrait donc écrire : monnaie - marchandise - monnaie + supplément. La question est de savoir comment se forme ce supplément. Pour MARX, il ne peut provenir que dans la phase "monnaie - marchandise" c'est-à-dire lors de l'achat de moyens permettant de produire la marchandise en question. Or, dans l'acte d'achat, l'échange ne peut concerner que des marchandises de valeur équivalentes. Parmi les moyens de production acquis, il y a la force de travail, qui est le seul élément capable de créer de la valeur. En d'autres termes, la force de travail est la marchandise à l'origine du surplus de valeur créée et qui constitue plus tard le supplément dont profite le propriétaire du capital ${ }^{129}$.

Ces schémas de production et de circulation ne sont possibles que dans le cadre d'une structure particulière de la société, celle d'une société divisée en deux classes : celle des capitalistes ou propriétaires de capitaux, et celle des ouvriers qui vendent leur force de travail.

Dans la théorie de la valeur de Karl MARX, la valeur est le symbole des relations sociales entre les individus. Elle existe par et à travers ces relations à l'intérieur, ou, entre plusieurs sociétés. Le but de MARX n'est pas de montrer qu'il existe un lien entre valeur et prix ${ }^{130}$, mais de discuter l'essence et la détermination de la valeur. Il établit le fait qu'il existe des nécessités vitales («Lebensnotwendigkeiten») qui exigent la dépense de travail, tout comme un partage de ce travail. Les sciences se doivent ainsi de trouver les formes sous lesquelles les nécessités vitales apparaissent concrètement. Un lieu où le travail dépensé se manifeste est le marché. Les échanges qui s'y produisent apparaissent en surface comme ceux d'objets entre eux. En fait, selon MARX, il s'agit d'échanges entre individus : les premiers vendent des marchandises ayant une valeur d'usage pour les seconds qui vont chercher à les acquérir. A travers ce mécanisme le travail dépensé se disperse à travers la société. La valeur est alors l'enveloppe qui sert d'intermédiaire et de référence et qui permet d'échanger une même quantité de travail dépensé. La forme apparente du marché fait que les individus ne sont pas conscients des éléments évoqués ${ }^{131}$.

La théorie de la valeur de Karl MARX propose donc une approche élargie dans la mesure où elle s'intègre dans une réflexion plus large sur la société capitaliste, c'est-à-dire sur le mode de production en vigueur. Le travail est un processus de confrontation avec la nature, et c'est ce qui, d'après le philosophe allemand,

\footnotetext{
${ }^{129}$ GRÜNDBICHLER Alois, 1986, p.11.

${ }^{130}$ Idem, p.45.

${ }^{131}$ Idem, p.46-47.
} 
constitue la base de l'économie ${ }^{132}$. L'individu doit pouvoir accorder ses besoins avec ses capacités de travail de manière à continuer à vivre dans de bonnes conditions. Grâce aux moyens de production, il peut travailler la matière première pour en faire un objet utile. Une fois que la marchandise est consommée, elle perd son caractère de produit et devient moyen de reproduction, celui de la force de travail ou encore, moyen de production pour la fabrication d'autres marchandises. Outre ce processus de transformation, MARX décrit le contexte social dans lequel il se produit, comprenant le mode de production en vigueur. Dans le système capitaliste, le propriétaire des capitaux est celui qui combine les facteurs de production et planifie ainsi le processus de production. Il possède les moyens de production et, pour une certaine durée journalière, une certaine quantité de force de travail. Le résultat de ce processus lui revient de droit.

Comme nous l'avons vu, le point de départ de la théorie marxiste de la valeur se situe au niveau de la marchandise produite. De ce point de vue, MARX n'innove pas véritablement par rapport aux auteurs anglais Classiques ou même par rapport à leurs confrères de l'Ecole Autrichienne. Un autre point commun avec les théories de SMITH et RICARDO, est qu'il voit dans le travail l'origine de la valeur. MARX est d'ailleurs considéré par certains comme un des derniers grands représentants de l'Ecole Classique ${ }^{133}$. Il faut cependant ajouter que la problématique qu'il propose tout comme les solutions sont éloignés des autres représentants de cette école.

Une marchandise se voit conférer son utilité par le biais du travail qui sert à la mettre en valeur, au sens propre comme au sens figuré. Et c'est l'ouvrier qui va donner à la matière brute sa valeur, et même encore plus puisque pour MARX, le système de production capitaliste fait que ce dernier produit une plus value pour laquelle il n'est pas rémunérée. Le produit fini sera ensuite offert sur le marché où le prix sert d'étalon pour la mesure de la valeur et donc pour l'échange. Le fait de passer de la valeur au prix pour un bien apparaît comme une sorte de confirmation de la valeur pour MARX. La valeur est un élément abstrait qui ne peut prendre que la forme concrète d'un prix (la valeur devient alors valeur d'usage). Etant prêt à payer un certain prix pour une marchandise, une personne lui reconnait par la même une valeur et c'est de cette manière que se produit le processus de confirmation $^{134}$. Cependant, le prix n'est pas équivalent à la valeur. Une correspondance entre les deux ne serait que pur hasard selon le philosophe allemand. Il s'agit là d'une différence avec les auteurs anglais, mais d'un point commun avec Christian von EHRENFELS, pour qui le prix reflète certes la valeur d'un objet, mais ne lui est pas égal, autant quantitativement que qualitativement.

\footnotetext{
${ }^{132}$ HERF Markus, 1988, p.57.

${ }^{133}$ RIEDER Karl, 2003, p.8.

${ }^{134}$ HERF Markus, 1988, p.30.
} 
Nous pouvons encore mentionner le fait que leurs théories respectives soient quelques peu tombées dans l'oubli. En particulier celle de Karl MARX n'apparaît plus dans les théories économiques contemporaines ${ }^{135}$. Anthony BREWER y ajoutera même que sa théorie "n'apporta pratiquement rien d'utile à l'héritage de l'Ecole Classique ${ }^{136}$.

La distinction entre la valeur et le prix d'un objet est un premier point de rapprochement entre les deux philosophes. Pour autant nous verrons que les similitudes ne sont pas si nombreuses que cela, ce qui s'explique notamment par le fait que leurs intentions respectives n'étaient pas les mêmes au départ. Une discussion sur ce thème sera d'ailleurs l'objet de notre prochain paragraphe

\section{3 - Un rapprochement entre EHRENFELS et MARX est-il possible ?}

L'idée de vouloir effectuer une comparaison entre les deux théories de la valeur semble a priori présenter un certain intérêt, mais elle semble également se heurter à un obstacle non négligeable : les intentions originelles des deux auteurs ne furent pas identiques. En effet, MARX avait pour objectif, à travers son travail, l'élaboration d'une critique des théories économiques dominantes et d'un ouvrage plus ou moins exhaustif sur sa vision des choses en la matière, dont la valeur n'en serait qu'un constituant. EHRENFELS, lui, voulait également écrire un ouvrage relativement complet mais centré sur la problématique de la valeur dans tous les domaines possibles. Sa théorie, au risque de nous répéter, est une théorie générale de la valeur, et elle en analyse l'origine et la détermination pour tous les objets possibles, qu'ils soient matériels ou immatériels. MARX limite son analyse aux objets matériels qui méritent le qualificatif de marchandise. Sa perspective se limite donc à un type d'objet alors que la théorie d'EHRENFELS permet d'analyser la détermination de la valeur de tout type d'objet désiré. Dans le domaine économique, la plupart des marchandises échangées sur un marché (matérielles ou non) contiennent une certaine quantité de travail. Dans cette optique la théorie de Karl MARX nous propose une explication de la valeur de l'objet marchand. Par contre, d'autres objets comme la qualité de vie ont une valeur dont l'appréciation est possible à travers la théorie d'EHRENFELS.

\footnotetext{
135 Idem, p.9.

${ }^{136}$ " An examination of the main elements of the Marx's economics shows [...] that what was new was not helpful and that what was usable was simply a restatement of well-known ideas in new terms. [...] The point is that Marx added little or nothing useful to the classical heritage ", in BREWER Anthony, " A Minor Post-Ricardian? Marx as an Economist », 1995, p.113.
} 
Il est difficile d'apprécier la qualité de vie de manière objective en tenant compte de la quantité de travail nécessaire à sa réalisation. Ses facteurs sont nombreux et varient d'un individu à l'autre, et dans ce cas la théorie du philosophe autrichien nous permet de mieux comprendre la détermination de sa valeur.

Un point commun entre les deux théories est le fait de considérer que la valeur n'est pas une propriété intrinsèque de l'objet, mais quelque chose qui lui est extérieur, qui lui est attribué. Pour EHRENFELS comme pour MARX, la valeur ne peut être attribuée que par un individu. La similitude entre les deux réflexions s'arrête cependant là. Pour le philosophe autrichien, la valeur est subjective car propre à chaque individu, même si certaines valeurs peuvent être communes à un groupe de personnes ou encore si celles-ci peuvent subir des influences extérieures. La valeur ne peut se déterminer par des critères objectifs. Pour MARX, le travail est le dénominateur commun qui permet de déterminer la valeur de toute marchandise. En mesurant la quantité de travail dépensé dans la fabrication d'un objet, on peut en déterminer la valeur finale. Cependant, dans un cas comme dans l'autre, il subsiste une certaine imprécision quant à une détermination quantitative exacte. Elle reste volontaire chez EHRENFELS, qui dit à plusieurs reprises qu'une précision mathématique est difficile voire impossible. Elle est un problème irrésolu dans la théorie de MARX, surtout en ce qui concerne la mesure de la valeur d'un travail plus complexe par rapport à un travail simple ou encore la détermination de la valeur apportée à un produit fini par un facteur de production tel qu'une machine. MARX se limite au travail comme élément créateur de valeur. Ce point de vue ne peut être objecté, car c'est bien le bûcheron qui fait de l'arbre une marchandise susceptible d'éveiller l'intérêt d'autrui. De par son effort, il transforme un objet a priori sans utilité en une marchandise pouvant être échangée. Pour EHRENFELS, l'arbre a lui-même une valeur, et plus précisément une valeur d'effet parce qu'il est écologiquement utile par exemple, ou encore parce qu'en été il nous fait de l'ombre dont on peut profiter. En ce sens, l'arbre, sans travail, c'est-à-dire sans intervention humaine, a un intérêt pour les individus. Un objet peut donc avoir de la valeur sans être une marchandise, et c'est en ce sens que nous pouvons affirmer que la théorie de Karl MARX présente des limites dans son approche. Et on parle bien de la valeur et non du prix de l'objet, qui pour les deux intellectuels ne sont pas la même chose. On pourrait nous rétorquer le fait que tout objet, dont la valeur n'est pas convertie en prix sur un marché, ne doit pas obligatoirement être inclus dans une théorie de la valeur dans le domaine économique. Il ne faut pas oublier que le marché connaît une extension progressive et que de plus en plus de biens ou de services font l'objet d'un échange. De plus, et pour revenir à notre exemple précédent, l'arbre n'est pas amené à être échangé sur un marché, mais cela ne veut pas dire pour autant que sa valeur ne sera jamais convertie en prix. Ainsi, dans la situation où cet arbre serait dans une ville où la pollution en viendrait à bout, si les habitants de la ville lui 
accordent une certaine valeur, alors il fera l'objet d'un remplacement. Ce remplacement a un coût, et celui-ci s'exprime sous la forme d'un prix. Le domaine considéré par MARX est aujourd'hui un peu plus vaste et complexe, une complexité qu'il est possible de traiter avec la théorie d'EHRENFELS.

Un troisième élément de discussion concerne la plus value et, plus généralement, le contexte dans lequel elle apparaît. Que ce soit chez MARX ou chez EHRENFELS, le contexte social joue un rôle important. Pour le philosophe allemand, le système de production détermine la nature des relations sociales à l'intérieur d'une société. Sa théorie suppose une société capitaliste caractérisée par deux classes sociales antagonistes, celle des capitalistes et celle des travailleurs. La plus value ne peut apparaitre que dans ce contexte d'exploitation des seconds par les premiers. Elle est plus exactement le supplément de valeur, non rémunéré, que le travailleur confère à une marchandise. Cette dimension du problème de la valeur n'est pas prise en compte chez EHRENFELS. Chez lui aussi la valeur est un phénomène qui ne peut $s$ 'étudier en dehors du contexte social, car celui-ci l'influence forcément. La notion de plus value n'apparaît tout simplement pas dans son discours. Il vaut mieux en rester au contexte social. Pour le philosophe autrichien, sa théorie est valable dans le monde culturel occidental, c'est-à-dire dans un type de société identifié. Là où il se rapproche de MARX, c'est dans le fait de voir dans sa composition deux entités : celle des dominants et celle des dominés ou, pour EHRENFELS, des passifs. Il nomme les personnes dominantes les « aktiven Wertbildner » qui construisent activement les valeurs. Il écrivit à ce propos, que ces gens sont ceux qui déterminent les prix sur les marchés, avouant par la même son influence marxiste ${ }^{137}$. Faut-il pour autant trouver une similitude entre les " aktiven Wertbildner " et les bourgeois ? Dans les deux cas, il s'agit de personnes ayant une influence telle dans la société qu'elles sont en mesure d'en déterminer un certain nombre de paramètres. Les bourgeois possèdent le capital nécessaire à la mise en production, et qui leur rapporte un bénéfice. Ils sont actifs en ce sens où ils décident de ce qui peut être produit et de la manière de le produire. Ils décident également de la longueur de journée de travail, nous dit MARX, c'est-à-dire qu'ils ont le pouvoir législatif.

Les bourgeois sont-ils des "aktiven Wertbildner", ou encore, les "aktiven Wertbildner " sont-ils des bourgeois ? Il nous est difficile de répondre à une telle question en ce qui concerne la société dans laquelle vivaient les deux philosophes. Il est peut être aussi plus pertinent de vouloir en donner une réponse pour nos sociétés occidentales contemporaines. Il s'agit d'abord d'identifier les " aktiven Wertbildner » dans ces sociétés. Ce sont ceux qui établissent de nouvelles valeurs, au sens d'EHRENFELS, ceux qui donnent de l'importance à un objet ou

${ }^{137}$ EHRENFELS, 1982, p.119. 
modifient l'importance d'autres objets, qu'ils soient matériels ou immatériels d'ailleurs. Prenons l'exemple d'une tendance actuelle de plus en plus décriée, mais aussi de plus en plus suivie, celle de soigner son apparence. Qui sont ceux qui nous influencent sur ce point ? Quelles personnes contribuent activement à un renforcement de l'importance de l'apparence physique ? D'une manière générale, il s'agit de celles qui apparaissent souvent dans les médias. Ces personnes fontelles partie de la classe bourgeoise ? Au sens de MARX, oui. Même si cette classe a des contours un peu différents aujourd'hui. EHRENFELS parlerait là d'un mouvement de valeur causé par la suggestion. Mais tous les bourgeois suggèrent-ils? Parmi ceux-ci, il y a ceux que l'on voit et qui suggèrent par leur image, et ceux que l'on ne voit pas, mais qui ont des capitaux impliqués dans un processus de production d'une marchandise. Etant donné que, selon MARX, leur objectif est de faire fructifier leurs capitaux, ces derniers vont chercher à faire vendre leurs produits, et les méthodes marketing utilisées utilisent également la suggestion. Indirectement, ces personnes influencent aussi les valeurs. Enfin, les bourgeois sont-ils toujours au pouvoir? Dans les sociétés occidentales, il est difficile de trouver un exemple montrant le contraire. On peut même, dans certains cas comme la France ou les Etats-Unis, parler d'une succession au pouvoir au sein d'une même classe ${ }^{138}$. Les bourgeois sont donc le plus souvent des "aktiven Wertbildner", que ce soit directement ou indirectement, mais tous les "aktiven Wertbildner" ne sont pas des bourgeois. En utilisant ce terme, EHRENFELS désigne également les personnes ayant une grande sagesse et obéissant aux principes moraux. A travers ses propos, on devine qu'il veut parler par exemple de la personne du Pape. Les dignitaires religieux sont également des " aktiven Wertbildner », dont l'influence est toujours présente de nos jours.

Il existe donc bien une corrélation entre les "aktiven Wertbildner" d'EHRENFELS et les Bourgeois de chez MARX. Cependant, les premiers ne désignent pas une catégorie sociale et ne se retrouvent pas uniquement dans une seule catégorie sociale.

En définitive, la théorie de la valeur de Karl MARX est certes une réflexion incontournable sur le phénomène qui nous préoccupe et elle comporte des points qu'il convient de ne pas négliger. Un parallèle avec la théorie du philosophe autrichien Christian von EHRENFELS montre qu'un rapprochement n'est pas possible, malgré les quelques points communs que l'on a soulignés. Les deux approches sont assez différentes et les objectifs dans les deux cas ne sont pas les mêmes. La théorie d'EHRENFELS semble cependant pouvoir nous donner plus

\footnotetext{
${ }^{138}$ Voir à ce propos GARRIGOU Alain, « Les Elites contre la République : Sciences Po et l'ENA ", 2001 ; BOURDIEU Pierre, "La Noblesse d'Etat : grandes écoles et esprit de corps ", 1989 ; FANTASIA Rick, " Délits d'initiés sur le Marché Universitaire Américain », Novembre 2004, p.4-5.
} 
de possibilité de cerner le phénomène de la valeur. Elle est aussi celle qui s'adapterait plus facilement à une application contemporaine. La théorie de MARX se veut une réponse à celles des auteurs anglais SMITH, RICARDO et MILL. Comme dans le cas de RICARDO, leurs analyses respectives s'inscrivent dans une tendance du XIX ${ }^{\text {ème }}$ siècle qui a voulu faire de l'économie une science à part entière avec ses propres théories. L'isolement de l'économie de son contexte, que certains pourraient d'ailleurs qualifier d'un isolement de la réalité, ne lui permet pas de trouver une solution satisfaisante au problème de la détermination de la valeur. 


\section{III - Welfare Economics : fondements, théorie du choix et bien être des individus en sciences économiques}

A la fin du XIX ${ }^{\text {ème }}$ siècle s'ouvre un nouveau chapitre de l'histoire de la pensée économique : on remarque la volonté chez certains économistes tels que Léon WALRAS (1834-1910) ou encore Vilfredo PARETO (1848-1910), de faire des sciences économiques une discipline scientifique à part entière. Ce dessein les conduit à redéfinir certaines hypothèses, en particulier celles concernant le comportement des individus. La théorie du choix ou "choice theory" de l'économiste français Léon WALRAS est basée sur le principe d'un individu rationnel qui décide en fonction de ses préférences. Cette nouvelle perspective est une abstraction de la réalité qui permet notamment sa formalisation mathématique. Les thèses de WALRAS participeront à la naissance du courant néo-classique, à l'introduction des mathématiques en sciences économiques et à l'élaboration d'une théorie des choix orientée vers le bien être individuel par la satisfaction des besoins.

Les hypothèses comportementales émises par WALRAS seront reprises et développées par Vilfredo PARETO, ingénieur italien qui s'est intéressé aux sciences économiques et à la sociologie à partir des années 1890. Un des apports de PARETO qui nous intéresse dans le cadre de ce travail est la notion de la " Pareto optimalité » qui constitue un critère de jugement pour évaluer l'efficacité de politiques économiques destinées à améliorer le bien être des individus. L'économie du bien être ou "welfare economics" est devenue une branche des sciences économiques dont l'objet implique en fait un jugement de valeur. Le processus de valorisation intervient à deux niveaux : d'une part au niveau de la détermination des préférences des individus, dont la formalisation sert le développement d'un modèle de maximisation; et d'autre part, dans l'évaluation des politiques économiques et de leurs effets sur le bien être des individus. Les concepts que nous mentionnerons dans ce paragraphe continuent par ailleurs d'imprégner les sciences économiques actuelles et se distinguent sensiblement de ceux développés par Christian von EHRENFELS dans le domaine des valeurs et de la valorisation.

3.1 - Léon WALRAS et l'adoption de nouvelles hypothèses comportementales en sciences économiques

Léon WALRAS naquit le 16 décembre 1834 en France, à Evreux. Il était le fils d'Antoine Auguste WALRAS (1801-1866), qui montrait un intérêt particulier pour l'économie, et de Louise Aline de SAINT-BEUVE (1811-1892), fille de notaire. Après un baccalauréat littéraire en 1851 et un second baccalauréat 
scientifique en 1853, Léon WALRAS essaya par deux fois d'entrer à l'Ecole Polytechnique sans y arriver. Reçu à l'Ecole des Mines de Paris en 1854, il abandonna ses études peu de temps après à cause du peu d'intérêt que la formation dispensée éveillait en lui. Il entreprit alors d'autres études pour approfondir ses connaissances en philosophie, en histoire, en critique de la littérature et de l'art, en économie politique et en sciences sociales. WALRAS publia une nouvelle en 1858, «Francis Sauveur », qui n'eut aucun succès ${ }^{139}$. En accord avec son père, il décida de se consacrer aux sciences économiques ${ }^{140}$. Entre 1859 et 1862, il écrivit en tant que journaliste des articles pour le « Journal des Economistes " et "La Presse ». Dans ces articles, il argumenta contre les thèses de Pierre Joseph PROUDHON et en 1860, il publia son premier ouvrage économique: "Examen critique et réfutation des doctrines économiques de M.P.J. Proudhon précédés d'une introduction à l'étude de la question sociale ». Il participa la même année au Congrès International de l'Impôt réuni à Lausanne et y fit une très bonne impression. Entre 1860 et 1870, Léon WALRAS exerça différentes professions ${ }^{141}$ avant d'être appelé à passer un concours pour obtenir la chaire d'économie politique nouvellement créée à l'Université de Lausanne. Il réussit ce concours et enseigna douze ans, jusqu'en 1892, dans cette même université. Sur le plan personnel, Léon WALRAS vécut en concubinage avec Céline Aline FERBACH (1834-1879), qu'il connut à la fin des années 1850 et avec qui il eut deux enfants, dont l'un décéda très jeune. Ils se marièrent en 1869. Cécile Aline ayant une santé fragile, Léon WALRAS, dont les revenus ne suffisaient pas à couvrir les frais médicaux, travaillait, en plus de l'université, à la Gazette de Lausanne, à la bibliothèque de la ville, et en tant qu'expert pour la compagnie d'assurance " La Suisse ". Après le décès de Céline Aline, il se remaria avec Léonide Désirée MAILLY (1826-1900). Donald WALKER décrit WALRAS comme "un enseignant très consciencieux, mais un lecteur peu inspirant et dont les étudiants visaient une carrière dans le droit, pas en économie, et il ne réussit pas, par conséquent, à trouver de potentiels disciples parmi eux ${ }^{142}$. Son état de santé, en particulier sur le plan psychologique, se détériorait et WALRAS dut arrêter l'enseignement en 1892. Il continua cependant ses activités de recherches et d'écriture. Vilfredo PARETO lui succéda et reprit la chaire d'économie politique. Léon WALRAS mourut le 5 janvier 1910 à Montreux.

\footnotetext{
${ }^{139}$ Remarquons que comme un grand nombre d'intellectuels de cette époque, parmi lesquels Christian von EHRENFELS, WALRAS s'essaya à la littérature. Et comme le philosophe autrichien, il n'eut que peu de succès.

${ }^{140}$ WALKER Donald A., „Walras's market models“, 1996, p.1.

${ }^{141}$ Voir WALKER Donald A., 1996, p.1-2.

142 WALKER Donald A., 1996, p.5.
} 
Parmi ses ouvrages importants, nous mentionnerons les trois principaux :

- "Eléments d'économie pure ", dont la première partie, "Théorie de l'échange ", parut en 1874 et la seconde, "Théorie de la production", parut en 1877.

- «Etudes d'économie sociale. Théorie de la répartition de la richesse sociale » parut en 1896.

- " Etudes d'économie politique appliquée », publié en 1898.

Ce qui distingue WALRAS des autres économistes de son temps, c'est sa manière de voir les sciences économiques. Pour Michael G. KRAFT, l'économiste français représente une rupture dans l'histoire des sciences économiques ${ }^{143}$. Pour comprendre cette affirmation, il nous faut expliciter la façon dont WALRAS voyait les sciences. Il distingue en premier lieu la science de l'art. En science, l'individu observe, décrit et explique ; l'art conseille, prescrit et guide. L'art est une forme sous laquelle se manifeste l'imagination et la créativité des individus $^{144}$. WALRAS, inspiré par les philosophes des Lumières, considère l'individu comme libre et raisonnable, capable d'imposer sa volonté à la nature ${ }^{145}$. Il détermine donc les structures sociales dans lesquelles il vit. WALRAS définit par ailleurs tout chose utile et rare comme étant appropriable, et la somme de toutes ces choses appropriables constitue la richesse sociale ${ }^{146}$. Chaque individu va chercher à acquérir ou à échanger une partie de cette richesse sociale. L'échange constitue le cœur du système économique. L'analyse de l'échange et de la production, en tant que phénomènes économiques, est l'objet de l'économie politique pure. L'économie politique appliquée analyse la production de la richesse sociale, c'est-à-dire de l'organisation de l'industrie dans le cadre de la division du travail ${ }^{147}$. La production et l'échange s'effectuent par rapport à des considérations d'intérêt : les individus ne produisent ou n'échangent que ce qui présente de l'intérêt pour eux. La distribution de la richesse sociale et les politiques inhérentes (taxation, justice sociale) sont des domaines réservés à l'économie sociale. L'économie sociale s'oriente par rapport à des considérations de justice. Chacun de ses ouvrages principaux cités ci-dessus, est consacré à une partie composant les sciences économiques.

WALRAS commence donc par redéfinir la discipline avant de commencer un travail d'analyse. Le phénomène de la valeur, qu'il considère comme subjectif, appartient à l'économie sociale où l'individu est amené à émettre un jugement sur

\footnotetext{
${ }^{143}$ KRAFT Michael G., "Ökonomie zwischen Wissenschaft und Ethik », 2005, p.25.

${ }^{144}$ Idem, p.46-47 ; voir également WALRAS, « Element of pure economics », 1954, p.58-60.

${ }^{145}$ KRAFT Michael G., 2005, p.40-41.

${ }^{146}$ Idem, p.49 et WALRAS Léon, "Eléments d'économie pure ", 1952, § 40, p.43.

147 WALRAS Léon, 1952, § 34, p.35 ; SINGH Harcharan K.M., " Marie Esprit Léon Walras ", in John Cunningham Wood, 1993, p.56.
} 
la façon dont les richesses sont réparties. L'économie pure est une analyse dite objective et cette objectivité est garantie par son instrument d'analyse, la modélisation mathématique.

Notre objectif, en exposant le point de vue de Léon WALRAS, n'est pas de faire part au lecteur de sa théorie économique dans son ensemble ${ }^{148}$, ni de son modèle d'équilibre général en particulier ${ }^{149}$. Nous nous intéressons à sa conception des sciences économiques, et avant tout aux hypothèses liées au comportement individuel.

La manière dont WALRAS aborde les sciences économiques lui est inspirée d'abord par son père, Antoine Auguste WALRAS, ainsi que par William Stanley JEVONS (1835-1882) et par le mathématicien français Antoine Augustin COURNOT (1801-1877) ${ }^{150}$. SINGH précise cependant que WALRAS est un des rares économistes dont les thèses dérivent aussi peu d'autres économistes ${ }^{151}$.

Dans son premier ouvrage, "Eléments d'économie pure ", l'économiste français innove en voulant faire de l'économie une science exacte au même titre que les sciences physiques ${ }^{152}$. Milton FRIEDMAN considère que ce livre " est un excellent travail qui marque un pas important en direction d'une science économique en tant que science, et qui joue toujours un rôle important dans la pensée économique [actuelle] $)^{153}$.

$\mathrm{Au}$ centre de sa théorie économique se trouve le phénomène de l'échange. Les individus sont dotés chacun d'une richesse sociale dont ils cherchent à échanger une partie dans le but d'augmenter leur bien être ${ }^{154}$. Cette hypothèse implique que

\footnotetext{
${ }^{148}$ A propos des théories économiques de Walras, voir par exemple MORISHIMA Michio, "Walras'economics ", 1981 ; WOOD John C. (éd.), " Léon Walras ", vol. 2 : "Walrasian Economics », 1993.

${ }^{149}$ Pour une analyse détaillée de l'équilibre général, voir par exemple HILDENBRAND Werner, "Equilibrium Analysis ", 1988 ; DAAL Jan van et JOLINK Albert, " The Equilibrium economics of Léon Walras ", 1993 ; WALKER Donald A., 1996, p.21-426.

${ }^{150}$ Voir SINGH Harcharan K.M., in John Cunningham Wood, 1993, p.54 ; PLANTZ Don V., "Cournot's « Recherches " : Some Insights on its Influence upon the Development of Economics Thought ", in John Cunningham Wood, 1993, p.143-158 et HICKS John R., "Léon Walras ", in John Cunningham Wood, 1993, p.4-7.

151 " [...] he [Walras] is one of those very few economists who derived so little from others, and one who relied so confidently on himself ", SINGH Harcharan K.M., in John Cunningham Wood, 1993, p.54-55.

${ }^{152}$ Le principe d'équilibre général en particulier est emprunté à la mécanique. Voir KRAFT Michael G., 2005, p.27.

153 " [...] the " Elements " is a great work which marked an important step foward in the development of economics as a science, and which still plays an important role in economic thinking ", FRIEDMAN Milton, "Léon Walras and His Economic System ", in John Cunningham Wood, 1993, p.19.

154 JOLINK Albert, „The Evolutionist Economics of Léon Walras“, 1996, p.74.
} 
chaque objet composant la richesse sociale de l'individu ait une valeur. L'individu attribue donc une valeur à l'objet quelque soit sa position (celui qui offre comme celui qui demande). Cette valeur est une valeur d'échange, et selon WALRAS, elle doit être considérée comme un phénomène naturel en ce sens où elle est déterminée par les forces de la nature. L'essence de chaque objet susceptible d'être échangé est déterminée par la nature, de même que ses caractéristiques telles que sa rareté ${ }^{155}$. De ce point de vue, l'individu est subordonné aux forces de la nature. La caractéristique principale de l'objet qui fait qu'il peut être échangé, est son utilité valorisée par l'individu. Par conséquent, la nature de l'objet est indépendante de la volonté des personnes alors que son utilité dépend directement de sa valorisation.

WALRAS analyse l'utilité au niveau individuel (microéconomique) ${ }^{156}$. Il distingue par ailleurs deux types d'utilité :

- L'utilité virtuelle qui représente les désirs virtuels de l'individu, ceux qui sont liés à son imagination.

- L'utilité effective qui est la manifestation réelle des désirs individuels.

L'utilité virtuelle comporte elle-même deux dimensions: une dimension dite extensive (c'est-à-dire la magnitude des désirs) et une dimension dite intensive (qui est fonction de l'urgence des désirs) ${ }^{157}$. Les deux dimensions de l'utilité virtuelle sont déterminées par l'individu, elles sont donc subjectives. WALRAS ajoute que lorsque l'utilité extensive croît, l'utilité intensive décroît. En même temps que William Stanley JEVONS et Carl MENGER (mais indépendamment les uns des autres), il formula ainsi la loi de l'utilité marginale.

L'individu est libre de décider de ce qui a de l'utilité pour lui et de ce qui en a suffisamment pour justifier un échange ${ }^{158}$. S'inspirant du philosophe Jean-Jacques ROUSSEAU (1712-1778) et des thèses utilitaristes, WALRAS attribue aux individus le fait de toujours rechercher leur intérêt personnel ${ }^{159}$, et ce, quelque soit sa place dans l'économie : les consommateurs vont chercher à maximiser leur utilité à travers l'acquisition de biens, l'entrepreneur tout comme le capitaliste vont chercher à maximiser leurs profits, les travailleurs leurs salaires et les propriétaires terriens la rente de la terre.

\footnotetext{
${ }^{155}$ Idem.

${ }^{156}$ Idem, p.85.

${ }^{157}$ WALRAS Léon, $1952, \S 72-75$, p.73-76.

${ }^{158}$ KRAFT Michael G., 2005, p.53.

159 Idem, p.66.
} 
Autrement dit, la maximisation de l'utilité est pour WALRAS la motivation qui se cache derrière tout comportement économique. De plus, chaque individu étant rationnel, du moins dans les domaines de l'échange et de la production, ses préférences vont le guider vers un état de satisfaction maximale.

Sous l'hypothèse de concurrence pure et parfaite ${ }^{160}$, les individus seront libres de rechercher leur bien être et d'agir en conséquence. L'analyse du comportement des personnes en économie sera réduite à une série d'équations dans un modèle mathématique. WALRAS développe un modèle complet décrivant la situation entre l'offre et la demande sur tous les marchés et la résolution des équations lui permet d'obtenir un point d'équilibre dit général, c'est-à-dire sur tous les marchés. Il ajoute également que l'équilibre général correspond au point où tous les agents ont maximisé leurs intérêts personnels et qu'il peut être considéré comme un optimum non seulement du point de vue du bien être personnel, mais aussi du bien être collectif. En d'autres termes la recherche de ce point d'équilibre devient équivalent à celle du bien être pour tous et constitue en ce sens un objectif moral. Le comportement de l'homo œconomicus devient une norme éthique qui trouve ainsi sa justification ${ }^{161}$.

La perspective de Léon WALRAS fut, en son temps innovante, et il est intéressant de noter que c'est cette perspective qui va dominer dans les sciences économiques par la suite ${ }^{162}$. Il faut également souligner que la volonté d'analyser le comportement individuel du point de vue de la "politique économique pure ", c'est-à-dire en le formalisant et en l'intégrant dans un modèle d'équilibre, peut amener quelques critiques.

WALRAS est conscient que son modèle d'équilibre général est un modèle théorique et que les conditions de concurrence pure et parfaite notamment, n'ont pas de rapport direct avec la réalité ${ }^{163}$. On peut cependant lui reprocher de réduire, sans l'analyser véritablement, le comportement individuel à celui d'un être

\footnotetext{
${ }^{160}$ La concurrence parfaite est un modèle décrivant une structure de marché hypothétique dans laquelle aucun producteur ni consommateur ne dispose d'un pouvoir discrétionnaire sur la fixation des prix ou sur les décisions des autres acteurs, et que tous les acteurs ont accès à la même information, ce qui suppose une égalité des positions à l'origine de la relation marchande. La situation de concurrence pure et parfaite est associée à cinq hypothèses : l'atomicité du marché, l'homogénéité des produits, la transparence de l'information, la libre entrée sur le marché et la libre circulation des facteurs de production.

${ }^{161}$ KRAFT Michael G., 2005 , p.66.

162 WALKER Donald A., 1996, p.23.

${ }^{163}$ WALRAS Léon, « Mathematische Theorie der Preisbestimmung der wirtschaftlichen Güter ", 1972, p.62.
} 
maximisant ${ }^{164}$. Nous verrons également dans notre paragraphe suivant que la réalité de la nature humaine est plus complexe et que cet aspect réducteur des choses peut amener à des conclusions théoriques ayant peu de rapport avec la réalité.

Concernant le phénomène de la valeur, WALRAS l'identifie à l'utilité de l'objet pour l'individu, qu'il assimile aussi à l'importance qu'un objet a pour ce même individu. Son point de vue rejoint celui d'EHRENFELS lorsqu'il considère que cette importance est subjective et qu'elle dépend des désirs de l'individu. Celle-ci revêt en outre pour WALRAS une dimension qualitative et une dimension quantitative, qui sont exclusivement liées à l'utilité de l'objet. Pour le philosophe autrichien, l'utilité est certes un facteur de valorisation de l'objet, mais il n'est pas le seul. L'utilité peut être associée par ailleurs à différents types de valeurs (valeur intrinsèque ou valeur d'effet). Cependant, l'importance globale de l'objet considéré est le résultat d'un processus psychologique qui ne peut être réduit à la simple évocation de ses préférences personnelles en fonction de la seule utilité.

Les thèses économiques de Léon WALRAS ne peuvent être réduites à celles qui sont émises dans son premier ouvrage principal. En effet, lorsqu'on considère son livre «Etudes d'économie sociale. Théorie de la répartition de la richesse sociale », on s'aperçoit que l'économiste français s'est également intéressé aux politiques économiques favorisant une répartition au plus juste de la richesse sociale. Il conseille par exemple une restriction du " laissez faire, laissez passer » dans le cas d'un monopole ou encore l'intervention de l'Etat afin de favoriser une redistribution des richesses ${ }^{165}$.

La voie ouverte par WALRAS, qui distingue plusieurs domaines d'étude dans les sciences économiques, sera prise également par celui qui lui succèdera à la chaire d'économie politique de l'Université de Lausanne: Vilfredo PARETO. Comme WALRAS, il n'est pas venu tout de suite aux sciences économiques et comme WALRAS, il s'inspire fortement des sciences physiques pour redéfinir les principes fondamentaux en économie.

\footnotetext{
${ }^{164}$ Voir à ce propos POLANYI Karl, «Ökonomie und Gesellschaft », 1979, p.131.

165 JOLINK Albert, 1996, p.110-122.
} 
Vilfredo PARETO, de son vrai nom Fritz Wilfried PARETO ${ }^{166}$, naquit le 15 juillet 1848 à Paris d'un père italien noble, Raffaele PARETO Marchese di Parigi (1812-1882), et d'une mère française, Marie MÉTÉNIER (1816-1889). La famille PARETO vivait en exil à Paris depuis $1815^{167}$ et Vilfredo grandit jusqu'en 1854 dans la capitale française en apprenant à la fois le français et l'italien. La famille regagna ensuite Gênes, avant de déménager à Casale Monferrato sur les bords du Pô où Raffaele PARETO avait obtenu un poste de professeur. Il obtint en 1862 un poste de fonctionnaire au ministère de l'agriculture à Turin et c'est également dans cette ville que son fils, Vilfredo, effectua ses études d'ingénieur, après avoir obtenu une "Licenza in scienze matematiche e fisiche" (licence en mathématiques et en sciences physiques) à l'Université de Turin. Il termina ses études d'ingénieur en 1870 avec une thèse consacrée aux principes de l'élasticité des corps solides, et intitulée en conséquence « Principi fondamentali della teoria della elasticità dei corpi solidi ». Ses bonnes aptitudes en mathématiques lui viendraient de son père ${ }^{168}$. PARETO s'intéressait également à d'autres domaines et notamment à l'économie ${ }^{169}$. Après ses études, il travailla dans le secteur des transports durant presque vingt ans, avant de se consacrer totalement à l'économie, puis à la sociologie. Signalons qu'il consacra du temps, durant cette période, à promouvoir un certain libéralisme autant sur le plan politique que sur le plan économique. Membre de la « Accademia Economico-Agraria dei Georgofili di Firenze » (Académie économico-agricole des agriculteurs de Florence), il y fit plusieurs lectures concernant des questions d'actualité (intervention de l'Etat dans l'économie, l'introduction d'un salaire minimum par exemple). Ceci lui donnait également l'occasion de connaître de nombreux intellectuels italiens avec qui il put échanger ses idées ${ }^{170}$. Il se maria avec Allessandra BAKOUNINE (18601940) en 1889. C'est aussi à la fin des années 1880 qu'il rencontra celui qui deviendra un de ses amis les plus proches, l'économiste Maffeo PANTALEONI $^{171}$ (1857-1927), qui fut un des premiers à adhérer aux théories

\footnotetext{
${ }^{166}$ Gottfried EISERMANN écrit qu'il n'existe aucun document indiquant la raison pour laquelle un prénom allemand fut attribué à PARETO. Voir EISERMANN Gottfried, 1987, p.1. La lecture d'autres biographies de Vilfredo PARETO ne nous a pas permis non plus d'apporter une réponse à cette question qui reste posée.

${ }^{167}$ Le grand-père de Vilfredo, Giovanni Benedetto PARETO, a été annobli par Napoléon lorsqu'il aida ce dernier à la création de la République Subalpine. La famille prit la fuite après la Restauration du royaume de Savoie en 1814. Voir EISERMANN Gottfried, "Vilfredo Pareto ", 1987, p.1.

${ }^{168}$ EISERMANN Gottfried, 1987, p.6.

169 Idem.

${ }^{170}$ Idem, p.9.

${ }^{171}$ BRUNI Luigino, " Vilfredo Pareto and the Birth of Modern Microeconomics ", 2002. p.7.
} 
marginalistes ${ }^{172}$. C'est par l'intermédiaire de ce dernier que PARETO obtint un rendez-vous avec Léon WALRAS à Lausanne. Et avec le soutien de WALRAS, PARETO fut appelé en tant que professeur, à enseigner à partir de 1893 à l'Université de Lausanne. Son ouvrage principal en économie, "Cours d'Economie politique ", parut en 1896-97. A partir de 1897, il commença à s'intéresser également à la sociologie, qu'il fut prié d'enseigner. Le décès de son oncle Domenico PARETO, dont il hérita de la fortune, permit à PARETO de se retirer de l'enseignement pour pouvoir se consacrer aux recherches, en particulier en sociologie. Il publia en 1916 "Trattato di sociologia generale ", divisé en deux volumes et résumant ses principales idées en la matière. PARETO mourut le 19 août 1923 à Céligny, en Suisse.

C'est en lisant "Principi di Economia Pura (1889) de PANTALEONI que PARETO prend connaissance de la théorie de l'équilibre général de WALRAS et qu'il comprend que l'économie pure était un territoire encore vierge qu'il décide d'explorer ${ }^{173}$. Les équations décrivant les comportements individuels et les marchés et dont la résolution mène à l'équilibre général, ne sont pas sans lui rappeler les équations de l'équilibre mécanique. La méthode - l'utilisation des mathématiques comme outil d'analyse - lui est familière.

Dans un premier temps, nous aimerions exposer la méthodologie employée par Vilfredo PARETO, ainsi que sa conception des sciences économiques. Pour Luigino BRUNI, PARETO était un positiviste ${ }^{174}$. L'observation doit être par conséquent objective c'est-à-dire que l'observateur doit regarder les choses avec recul, sans être impliqué dans ce qu'il voit. Cette approche expérimentale le pousse à adopter progressivement une méthode inductive, que ce soit en économie ou en sociologie. Le problème restant celui de savoir comment l'observateur peut rester objectif en interprétant des faits. PARETO y répond en affirmant qu'il faut distinguer les éléments objectifs des éléments subjectifs. En effet, la description d'un fait contient une certaine interprétation, c'est-à-dire un certain jugement de valeur, de la part de l'observateur. Plus le fait est décrit de manière exacte et plus il constitue un fondement solide pour un travail scientifique. Cette définition implique que la dimension historique des faits n'est pas exclue. Cependant, à cause de l'imperfection de l'esprit humain et des sentiments de l'observateur, il est impossible d'atteindre l'objectivité absolue en sciences. C'est pourquoi, il préconise le démantèlement des phénomènes complexes en composants simples qu'il est possible d'analyser avant de faire une synthèse de l'ensemble ${ }^{175}$. L'analyse des composants simples passe par leur abstraction, qui est, toujours

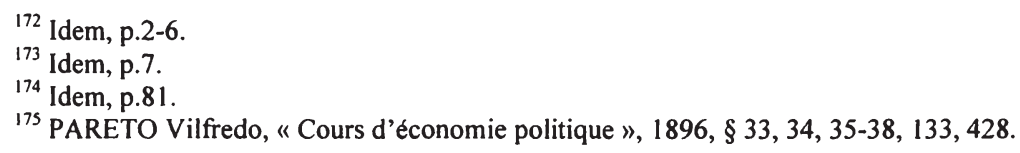


selon PARETO, la première étape en sciences ${ }^{176}$. BRUNI précise cependant que l'économiste et sociologue italien n'a pas complètement abandonné la déduction dans son raisonnement ${ }^{177}$. PARSONS affirme qu'à partir de 1897, PARETO adopte "l'induction analytique " ${ }^{178}$ à travers ce que ce dernier définit lui-même comme étant la " metodo logico-sperimentale » ou méthode logico-expérimentale, c'est-à-dire basée sur des lois statistiques qu'il est possible de généraliser. PARETO écrit à ce propos :

" Dans le future, le progrès de l'économie politique dépendra en grande partie de la recherche de lois empiriques à l'aide de statistiques, et qui seront comparées avec les lois théoriques connues. $1{ }^{179}$

Et une des abstractions faites par l'économiste et sociologue italien, est celle du comportement humain à travers l'homo œconomicus.

PARETO considérait en outre la psychologie comme une science expérimentale qui comporte une certaine incertitude car elle est soumise à l'interprétation, c'està-dire à la subjectivité de l'analyste. Celui-ci décrit l'état d'esprit de la personne observée. Pour cette raison, PARETO fait le choix de baser les sciences économiques sur une approche dite objective, avec un individu cherchant à maximiser ses besoins en faisant des choix rationnels ${ }^{180}$. L'approche psychologique est isolée de l'approche économique, ce qui à cette époque, distinguait PARETO de la plupart des autres économistes ${ }^{181}$.

Les aspects méthodologiques étant exposés, nous pouvons à présent discuter des thèses de PARETO en matière de comportement individuel.

Comme Léon WALRAS, Vilfredo PARETO voulait faire de la politique économique pure une science comme la physique ou la mécanique. Ses positions changent au début du XX ${ }^{\text {eme }}$ siècle : il considère alors que l'économie constitue une partie de la sociologie, c'est-à-dire de l'analyse des sociétés humaines ${ }^{182}$. Economie et sociologie sont cependant soumises aux mêmes méthodes

\footnotetext{
${ }^{176}$ Voir PARETO Vilfredo, « Manuel d'économie politique », 1963, tome 1, chap. 1, § 20-26.

${ }^{177}$ BRUNI Luigino, 2002, p.83.

${ }^{178}$ PARSONS Talcott, « The Structure of Social Action », Free Press, New York 1968.

179 PARETO Vilfredo, "L'interpolazione per la ricerca delle leggi economiche " in Giornale degli Economisti, Mai 1907, p.377-385. Voir PARETO Vilfredo, "CEuvres Complètes ", vol. 26, 1982, p.586-587.

${ }_{80}^{80}$ Voir PARETO Vilfredo, « Sunto di alcuni capitoli di un nuovo trattato di economia politica del Prof. Pareto ", in Giornale degli Economisti, Mars 1900, p.216-235. Voir PARETO Vilfredo, "CEuvres Complètes ", vol. 26, 1982, p.365-384.

${ }^{181}$ BRUNI Luigino, 2002, p.87.

${ }^{182}$ EISERMANN Gottfried, 1987, p.56 et p.75.
} 
scientifiques. Dans la perspective qu'il adopte, PARETO voit l'individu comme un être qui cherche à obtenir l'utilité maximale. Il distingue cependant l'utilité de ce qu'il appelle l'ophélimité. Le premier est un concept objectif alors que le second est plutôt subjectif (cette distinction s'apparente à celle que Léon WALRAS fait entre l'utilité extensive et l'utilité intensive). Le mot ophélimité vient en fait du grec "ophelimos", qui signifie "utile, avantageux, qui peut rendre service ». En réalité, le mot était à l'origine un synonyme d'utilité. Avec PARETO, il prend un sens différent : l'ophélimité est ce qui dans l'objet procure du plaisir à l'individu et qui est bon pour lui (par opposition à ce qui peut être utile dans certaines situations, mais nuire à l'individu) ${ }^{183}$.

Dans son livre "Cours d'économie politique ", PARETO définit deux types d'action individuelle :

- L'action dont le but est simplement de procurer une sensation plaisante (recherche de l'ophélimité).

- Les actions visant à procurer aux individus certaines conditions de santé, de développement du corps et de l'esprit (associées avec l'utilité).

En économie, l'individu établit des choix en fonction de son utilité, c'est-à-dire en fonction d'un critère objectif. En développant le concept d'ophélimité, PARETO veut libérer l'économie de toute trace de subjectivité. L'ophélimité est une notion plutôt hédoniste, qui se rapporte à la notion de plaisir. Or le plaisir est un sentiment dont l'analyse appartient au domaine de la psychologie.

Lorsque PARETO commence à s'intéresser à la sociologie, il redécouvre les actions dites non rationnelles. Dans son "Trattato di Sociologia Generale ", il établit une distinction claire entre l'économie qui analyse les actions dites logiques (résultats d'un raisonnement rationnel) et la sociologie, qui s'occupe d'expliquer les actions dites non logiques (qui découlent des états psychologiques des individus, en particulier de leurs sentiments) ${ }^{184}$. La rationalité est donc pour PARETO un instrument d'analyse, ce qui se retrouve dans les théories des choix modernes.

Un point sur lequel PARETO a innové, est celui de la mesure de l'utilité. Les préférences individuelles sont établies en fonction de l'utilité que l'individu attribue à chaque objet qu'il considère. Pour PARETO, il est possible de mesurer les préférences en leur donnant une valeur. Il reprend le concept de courbes d'indifférence développé par EDGEWORTH (1845-1926) en les basant non plus sur les points pour lesquels les individus n'éprouvent pas de préférence entre deux

${ }^{183}$ EISERMANN Gottfried, 1987, p.78.

${ }^{184}$ PIETRI-TONELLI Alfonso de, et BOUSQUET Georges H., « Vilfredo Pareto », 1994, p.xii. 
biens, mais sur l'acte de choix. Autrement dit, PARETO ne mesure pas l'utilité, il ne l'additionne pas non plus, il élabore un nouveau concept. L'acte de choix décrit la demande effective pour certains biens. La demande effective doit se distinguer des préférences des individus. L'acte de choix se compose de deux éléments :

- le "gusti », c'est-à-dire les souhaits, les aspirations et les goûts des individus,

- l' " ostacoli » qui désigne les obstacles directs ou indirects à la réalisation des " gusti » (par exemple, les goûts d'autrui, la non disponibilité du bien désiré, ou encore le fait de devoir partager le bien acquis).

L'équilibre sur les marchés est atteint lorsqu'une balance est trouvée entre les "gusti » et les "ostacoli " ${ }^{185}$. Le point d'équilibre est le prix d'équilibre, qu'il assimile à la valeur d'échange. Il se distingue sur ce point de l'analyse de l'équilibre économique de Léon WALRAS.

Enfin, à propos de cet état d'équilibre général, PARETO affirme :

« Nous dirons que les membres d'une collectivité jouissent, dans une certaine position, du maximum d'ophélimité, quand il est impossible de trouver un moyen de s'éloigner très peu de cette position, de telle sorte que l'ophélimité dont jouit chacun des individus de cette collectivité augmente ou diminue. C'est-à-dire que tout petit déplacement à partir de cette position a nécessairement pour effet d'augmenter l'ophélimité dont jouissent certains individus, et de diminuer celle dont jouissent d'autres : d'être agréable aux uns, désagréable aux autres. ${ }^{186}$

C'est cet état d'optimum décrit par PARETO qui est aujourd'hui connu sous le nom d'optimum de PARETO, et qui constitue un des fondements de l'économie du bien être ou « welfare economics ».

Vilfredo PARETO marqua une certaine rupture dans l'histoire des sciences économiques : sa méthode d'analyse des phénomènes économiques s'inspire de celle de Léon WALRAS. Il continue sur la même voie que ce dernier en redéfinissant les sciences économiques avec une composante dite pure, c'est-à-dire se voulant objective et employant les mathématiques comme instrument d'analyse. Un autre instrument employé par PARETO fut l'abstraction du comportement individuel : l'individu est un être rationnel dont le but est de maximiser son utilité en minimisant l'effort. Ses choix sont guidés par ses préférences qui sont le reflet de son raisonnement rationnel. Cette manière de voir les choses exclut la psychologie comme source d'explication des comportements.

${ }^{185}$ EISERMANN Gottfried, 1987, p.83-85.

${ }^{186}$ PARETO, « Manuel d'économie politique ", 1963, $2^{\text {eme }}$ tome, chap. VI, § 33. 
Un développement ultérieur des thèses de PARETO fut celui de la théorie des choix, impliquée dans une autre branche, celle du " welfare economics".

3.3 - Le concept de Pareto optimalité comme base de développement de l'économie du bien être ou "welfare economics »

Un des problèmes qui a longtemps préoccupé et qui préoccupe toujours les économistes et les philosophes, est celui de la valorisation de la répartition des revenus $^{187}$. Le choix d'une politique économique adéquate est nécessaire pour garantir une répartition des revenus telle que chaque individu puisse, avec son revenu, satisfaire ses besoins et ainsi améliorer son bien être. Une branche de l'économie qui s'est développée au début du $\mathrm{XX}^{\mathrm{eme}}$ siècle $^{188}$ et dont la tâche principale est de trouver les politiques adéquates pour atteindre le but précédemment évoqué, est l'économie du bien être ou "welfare economics " ${ }^{189}$. L'économie du bien être permet d'offrir des critères de jugement de l'efficacité d'une mesure allant dans le sens d'une amélioration de la satisfaction des individus ${ }^{190}$.

Un des concepts fondamentaux de l'économie du bien être est celui de la Pareto optimalité. Ce concept implique des hypothèses de comportement, notamment sur la valorisation d'un objet par les individus, qui continuent d'imprégner les sciences économiques actuelles et qui se distinguent nettement de celles proposées par EHRENFELS. L'optimum de Pareto est en réalité un point théorique qui a fait l'objet de développements censés éliminer les points faibles de la théorie sur laquelle il se base. Une des alternatives les plus connues est celle de l'analyse coût-profit ou " cost-benefit analysis ». Notre propos dans ce paragraphe n'est pas d'exposer de manière exhaustive les théories et les discussions ayant cours en économie du bien être. Ne voulant pas nous écarter de notre sujet principal, le phénomène de la valeur et le processus de valorisation en particulier dans les sciences économiques, nous nous en tiendrons aux hypothèses inhérentes au comportement individuel et qui sont susceptibles d'influencer la valorisation des objets. L'autre aspect intéressant pour notre travail est la valorisation des politiques économiques favorisant le bien être des individus.

\footnotetext{
${ }^{187}$ EBERT Udo, « Beitrăge zur Wohlfahrtsőkonomie », 1987, p.82.

${ }^{188}$ Selon Yew-Kwang NG, Arthur PIGOU fut le premier à présenter l'économie du bien être en tant que domaine d'étude distinct. Voir NG Yew-Kwang, "Welfare Economics ", 2004, p.1.

189 JOHANSSON Per-Olov, "An Introduction to modern welfare economics ", 1991, p.1. Pour plus de précisions concernant les domaines d'étude de l'économie du bien être, voir DICK Eugen, " Untersuchungen einiger Grundprobleme der Wohlfahrtsökonomie », 1993, p.12-13. Voir également concernant les origines de l'économie du bien être HILLINGER Claude, "Applied Cardinal Welfare Economics : Conceptual Fondation, Empirical Measurement, and Aggregation ", 1994, p.16-34.

${ }^{190}$ NG Yew-Kwang, 2004, p.2.
} 
Le concept de Pareto optimalité s'appuie sur plusieurs hypothèses, parmi lesquelles certaines concernent le comportement des individus. On suppose en effet, que les individus, en tant qu'agents économiques, sont des êtres rationnels qui cherchent à maximiser leur satisfaction personnelle sous la contrainte du budget disponible. Chaque individu recherche sur les marchés, les biens et les services susceptibles de satisfaire ses besoins. Il émet face aux biens et services disponibles des préférences. On suppose que ses préférences sont soumises également à l'hypothèse de rationalité, c'est-à-dire qu'il va préférer les objets qui ont la plus grande utilité pour lui, c'est-à-dire qui vont maximiser sa satisfaction. Ceci nous amène à évoquer un premier débat dans le monde de l'économie du bien être : celui qui a lieu entre les partisans d'une conception cardinale des préférences et ceux qui lui préfèrent une conception ordinale. L'hypothèse de préférences ordinale se retrouve chez des économistes tels que JEVONS ${ }^{191}$, MENGER $^{192}$, EDGEWORTH ${ }^{193}$ et PARETO $^{194}$. Ils estiment alors que les individus ne sont pas en mesure de donner une valeur cardinale à l'utilité d'un objet, mais qu'ils peuvent sans problème ordonner une liste d'objets selon la satisfaction qu'ils procurent. Avec le développement de l'économétrie, la conception cardinale des préférences prit de l'ampleur ${ }^{195}$. HILLINGER souligne que la première approche permet de mesurer la satisfaction des individus, mais elle ne permet pas d'apprécier la distribution des revenus, contrairement à la seconde approche ${ }^{196}$. L'idéal serait de trouver un compromis qui autorise la mesure à la fois du bien être des individus et la répartition leurs revenus. Il propose une perspective monétaire, celle de la "money metric utility " en partant du principe que dans une économie monétaire (dominée par les prix), l'individu préfère avoir plus d'argent que moins d'argent ${ }^{197}$. De notre point de vue, cette solution n'est pas des plus satisfaisantes car elle suppose que l'argent ait une valeur prépondérante dans la société analysée et que l'argent soit la seule cause du bien être.

\footnotetext{
191 Voir JEVONS William Stanley, " The Theory of Political Economy ", 1871.

${ }^{192}$ Voir MENGER Carl, « Grundsătze der Volkswirtschaftslehre », 1871.

${ }^{193}$ Voir EDGEWORTH Francis Ysidro, " Mathematical Psychics », 1881.

${ }^{194}$ Voir PARETO Vilfredo, "Cours d'économie politique », 1896-1897.

${ }^{195}$ HILLINGER Claude, 1994, p.24.

${ }^{196}$ Idem, p.4.

${ }^{197}$ Idem, p.1, p.6 et p.7-16.
} 
Revenons au modèle proposé par PARETO, dans lequel les préférences sont formalisées sous la forme de courbes d'indifférence qui sont soumises aux hypothèses suivantes :

- La complétude : les consommateurs connaissent leurs préférences individuelles. Ils peuvent choisir entre consommer le couple $\mathrm{X}$ et $\mathrm{Y}$. Ils savent si $\mathrm{X}$ est préféré à $\mathrm{Y}$, $\mathrm{Y}$ est préféré à $\mathrm{X}$, ou s'ils sont indifférents entre consommer $\mathrm{X}$ ou $\mathrm{Y}$.

- La transitivité : si un consommateur préfère le couple $X$ au couple $Y$, et préfère le couple $\mathrm{Z}$ au couple $\mathrm{Y}$, alors il préfèrera le couple $\mathrm{X}$ au couple $\mathrm{Z}$.

- La continuité : cela signifie que l'individu peut choisir de consommer n'importe quelle quantité d'un bien. Il peut boire par exemple $11 \mathrm{ml}$ ou $132 \mathrm{ml}$ de soda.

- La non satiété : le fait que la consommation d'un bien ne conduise jamais à un état de saturation.

- La convexité : la valeur marginale qu'un individu retire de la consommation de chaque bien diminue. Dans un monde à deux biens, si un consommateur a relativement plus d'un bien, il sera plus heureux avec relativement moins de ce bien et un peu plus d'un autre bien.

La comparaison entre les courbes d'indifférence permet de trouver un point pour lequel il n'est plus possible d'améliorer la satisfaction d'un ou de plusieurs individus sans détériorer celle d'un ou de plusieurs autres individus. Ce point constitue donc un optimum dans une situation donnée.

Dans cette situation où l'allocation des ressources est optimale, tous les individus ont atteint la satisfaction maximale possible dans les conditions de marché données. Puisque leurs demandes auront été satisfaites, on suppose que leur bien être a augmenté et qu'il sera également maximum. Toutes les politiques économiques allant dans le sens de l'amélioration du bien être de tous les individus seront positivement valorisées. Ce modèle suppose par ailleurs que les valorisations individuelles, c'est-à-dire les préférences exprimées par les individus, ne dépendent que de la satisfaction de leurs besoins et que les individus préfèrent les objets qui ont l'utilité la plus élevée. Ainsi l'optimum de Pareto n'est pas un optimum au sens éthique puisque l'équilibre de marché peut être injuste, mais la meilleure situation possible dans des conditions de marché déterminées ${ }^{198}$.

Le concept de Pareto optimalité présente quelques points faibles que les économistes ont essayé par la suite de pallier. Pour JOHANSSON, le concept de Pareto optimalité est inutile en tant que critère pour les choix sociaux dans la

198 JOHANNSSON Per-Olov, 1991, p.21. 
plupart des situations réelles ${ }^{199}$. Deux théories alternatives ont été développées : celle du principe de compensation et celle de la fonction du bien être social.

Dans la réalité, la majorité des mesures économiques prises produisent des gagnants et des perdants. Dans ces situations, il n'est pas possible d'utiliser le critère de Pareto. Le principe de compensation a été proposé par HICKS ${ }^{200}$ et $\mathrm{KALDOR}^{201}$ à la fin des années 1930 comme alternative. Selon ledit critère de KALDOR, un projet est désirable si, avec ce projet, il est hypothétiquement possible de redistribuer les revenus de telle manière que tous pourraient s'en sortir mieux que sans le projet. Ceux qui tirent profit du projet seraient en mesure de payer des compensations aux perdants, mais le critère de KALDOR ne requiert pas l'application de la redistribution. Le critère de HICKS, quant à lui, dit que le projet de passer d'un état $A$ à un état $B$ est désirable si dans l'état $A$, il est impossible de redistribuer les revenus. La possibilité de le faire dans l'état B, fournit une meilleure perspective aux individus. On suppose que les gagnants dans l'état B n'ont pas la capacité de corrompre les perdants et de les dissuader de passer de l'état A à l'état $\mathrm{B}$. Dans les deux cas, le principe de compensation est lié à une potentielle redistribution des revenus et non à une redistribution effective. $\mathrm{Si}$ une compensation était requise, il n'y aurait alors pas de différence avec le critère de Pareto.

L'analyse coûts-profits (ou "cost-benefit analysis") est la méthode la plus communément employée pour l'évaluation des projets du secteur public (par exemple, des investissements dans des infrastructures publiques), des programmes de réforme du marché du travail (par exemple, une mesure de dérégulation), de (dé-)régulation du secteur privé ou encore des programmes environnementaux ${ }^{202}$. L'idée fondamentale de cette méthode d'analyse est de mesurer, en unités monétaires, les conséquences d'un projet déterminé sur le bien être des individus. Si on considère par exemple la construction d'un pont, la comparaison entre les coûts engendrés (construction, impact sur l'environnement, impact financier en cas d'instauration d'un péage à l'entrée du pont, etc...) et les bénéfices d'un tel ouvrage (gain de temps au niveau de la circulation par exemple) sera déterminante dans la décision de réalisation de ce projet.

\footnotetext{
${ }^{199}$ Idem, p.22.

${ }^{200}$ Voir HICKS John R., "The foundations of welfare economics ", in Economic Journal, nr 49, 1939, p.696-712.

${ }^{201}$ Voir KALDOR Nicholas, « Welfare propositions of economics and personal intercomparisons of utility ", in Economic Journal, nr 49, 1939, p.549-552.

202 JOHANSSON Per-Olov, 1991, p.112.
} 
Une autre alternative au théorème de Pareto est celle de la fonction du choix social (" social choice fonction ») ${ }^{203}$. Les individus expriment leurs préférences par rapport à divers choix sociaux possibles et on recherche, par un processus de maximisation mathématique, l'état social optimal, celui pour lequel il ne serait pas possible d'améliorer le bien être social de certains individus sans détériorer le bien être social d'autres individus. Le concept de bien être social est plus vaste que celui de bien être au sens de Pareto qui se limite en fait à un état de satisfaction matérielle. ARROW affirme que dans le choix d'un état social, on devrait pouvoir considérer toutes les valeurs imprégnant une collectivité, une société, y compris les valeurs éthiques. Cependant, les mécanismes de marchés ne retiennent que les préférences matérielles ${ }^{204}$. Il montre par ailleurs qu'il est impossible de dériver (de l'ordonnancement des préférences des individus) un ordre social qui serait consistant sous des conditions raisonnables ${ }^{205}$. Autrement dit, en respectant la règle de la majorité absolue, il n'est pas possible de dériver des fonctions de bien être social, un ordre social issu d'un choix rationnel des individus. Yew-Kwang NG montre que cette impossibilité résulte du fait que pour dériver une nouvelle fonction de bien être social d'un nouvel ensemble de préférences, ARROW utilise la même règle que pour dériver l'ancienne fonction de bien être social. Cette règle peut être par exemple le vote à la majoritéé ${ }^{206}$. NG doute du bien fondé de la remise en cause d'une telle règle pour atteindre un état optimale (le changement de la règle peut se faire au détriment de la majorité de la population). Selon NG, il n'existe pas de règle raisonnable qui permette de dériver un ordre social basé seulement sur les préférences individuelles.

Un autre reproche émis à l'encontre de la théorie de la Pareto optimalité est celui concernant la prise en compte des préférences et celle de l'agrégation des courbes d'indifférences dans le modèle de maximisation. Les modèles mathématiques proposés dès la fin $\mathrm{du} \mathrm{XIX}^{\mathrm{ème}}$ siècle considèrent les préférences des individus à un moment précis ${ }^{207}$, comme le reflet de leurs choix rationnels et dont le critère de détermination est la recherche de l'intérêt personnel. Pour un certain nombre de scientifiques, ce concept de préférence est insuffisamment réaliste. En effet, les individus ont également des tendances altruistes ${ }^{208}$, et leurs préférences sont le résultat à la fois d'un héritage culturel et génétique ${ }^{209}$. Une vision des choses qui

\footnotetext{
${ }^{203}$ NG Yew-Kwang, 2004, p.92-126.

${ }^{204}$ ARROW Kenneth J., "Social Choice and Individual Values », 1966, p.18.

${ }^{205}$ Idem.

${ }^{206}$ NG Yew-Kwang, 2004, p.98-99.

${ }^{207}$ Des modèles dynamiques ont également été développés. Voir AUER Ludwig von, « Dynamic Preferences, Choices Mechanisms, and Welfare ", 1998.

${ }^{208}$ Voir par exemple HOFFMAN Martin L., « Is altruism part of human nature? », in Journal of Personality and Social Psychology, nr 40, 1981, p.121-137.

${ }^{209}$ Voir BOYD Robert et RICHERSON Peter J., "Culture and the Evolutionnary Process ", 1985 ; SOBER Elliot et WILSON David S., « Unto Others : The Evolution and Psychology of Unselfish
} 
inclut en outre, un aspect psychologique et qui rejoint celle de Christian von EHRENFELS. Autre difficulté posée par le concept des préférences est celle de sa mesurabilité : le débat concernant la possibilité de mesurer les préférences des individus est un vieux débat dans les sciences économiques. Enfin, la question dite de la comparaison interpersonnelle des préférences reste également posée. Est-il réaliste de vouloir comparer les préférences de deux individus qui vivent dans des situations sociales et matérielles différentes? Cette problématique en implique une autre, celle de la subjectivité des préférences. Si on considère que les préférences individuelles sont subjectives, il est alors difficile d'envisager leur comparaison entre les individus et encore moins, leur agrégation ${ }^{210}$. Si au contraire, les préférences sont considérées comme objectives car établies par rapport aux mêmes critères de rationalité et de recherche de l'intérêt personnel, une comparaison interpersonnelle ne se heurte à aucun obstacle. Nous discuterons plus amplement dans le chapitre suivant, de ce problème toujours actuel dans les sciences économiques ${ }^{211}$.

La volonté de faire des sciences économiques une discipline objective à l'image des sciences physiques, a conduit Léon WALRAS et Vilfredo PARETO à développer de nouveaux concepts d'analyse théorique. Ils soulignent également le fait que ce que WALRAS appelle l'économie pure, ne constitue qu'une partie des sciences économiques et que l'abstraction mathématique ne constitue qu'un instrument d'analyse. Léon WALRAS modélise le processus de choix individuel comme étant celui de l'expression de préférences individuelles rationnelles allant dans le sens d'une maximisation de l'utilité. L'utilité est assimilée à la satisfaction personnelle, elle-même confondue avec le bien être. Cette perspective est reprise et développée par PARETO, et elle constitue aujourd'hui le cœur des méthodes d'analyse de la microéconomie. Le concept de Pareto optimalité est lui, utilisé comme critère d'analyse de l'efficacité des politiques économiques sur le plan du bien être. On utilise également la méthode comparative coûts-profits pour décider de la réalisation d'un projet qui aura un impact sur le bien être des individus.

\footnotetext{
Behavior ", 1998 ; BOWLES Samuel, « Individual Interactions, Group Conflicts and the Evolution of Preferences ", in DURLAUF Steven et YOUNG Peyton (eds), " Social Dynamics ", 2001.

${ }^{210}$ SEN Amartya, " Interpersonal Comparison of Welfare ", in BOSKIN Michael J. (éd.), "Economics and Human Welfare ", 1979, p.183-202.

${ }^{211}$ Voir paragraphe 4.1.3 de ce travail.
} 
L'abstraction du comportement des individus est critiquée pour son manque de réalisme. Elle s'oppose également aux hypothèses fournies par EHRENFELS dans sa théorie de la valeur. Dans notre dernière partie, nous verrons les limites de la conception abstraite des comportements en sciences économiques et la nécessité de trouver une alternative et de s'inspirer pour cela de la théorie du philosophe autrichien. 


\section{IV - Réflexions sur les limites de la théorie économique actuelle et sur la pertinence de la théorie d'EHRENFELS comme alternative dans le domaine de la valeur.}

La théorie de la valeur de Christian von EHRENFELS se distingue sensiblement de celles d'économistes tels que RICARDO ou MARX. Son utilisation par les sciences économiques actuelles semble a priori difficile. En effet, la théorie du philosophe autrichien n'est pas utilisable sous forme mathématique, elle est basée sur des hypothèses comportementales différentes et elle contient une approche interdisciplinaire avec notamment des apports issus de la psychologie. Ce qui la distingue en premier lieu est la définition qu'elle donne à la valeur. La valeur, nous l'avons vu, n'est pas le prix d'un objet, mais son importance aux yeux d'un individu. Elle est donc subjective et sa détermination dépend en partie des émotions consécutives à la considération de l'objet, ainsi que des expériences et influences de son environnement. La théorie d'EHRENFELS n'est pas seulement une théorie de la valeur, elle est aussi une théorie des comportements, notamment au niveau psychologique. La réaction qui précède le jugement de valeur, conditionne en fait la détermination de la valeur par la suite accordée. Son étude est donc nécessaire pour mieux comprendre le phénomène de la valeur.

Les sciences économiques offrent leur propre vision du comportement individuel qui précède par ailleurs le processus de valorisation. L'individu est schématisé à travers le concept de "l'homo œconomicus". L'origine de cette notion est incertaine, mais elle constitue la figure de proue des sciences économiques depuis les travaux de RICARDO et de MALTHUS, c'est-à-dire depuis le XIX ${ }^{\text {ème }}$ $\operatorname{siècle~}^{212}$. L'homo œconomicus est un être rationnel recherchant la maximisation de son bien être à travers la satisfaction de ses besoins. Cette conception de l'individu s'oppose à celle d'EHRENFELS pour qui les êtres humains recherchent certes leur bien être, mais pas uniquement à travers la satisfaction des besoins. De plus, il ne le précise pas, mais l'individu qu'il présente est plus émotionnel que rationnel. Et la place des émotions dans la prise de décision (allocation d'une valeur à un objet) conduit à une notion de la valeur subjective.

Nous allons montrer que cette différence de point de vue ne constitue pas un obstacle à l'utilisation de la théorie d'EHRENFELS par les sciences économiques. Les hypothèses sur lesquelles s'appuie leur concept de l'individu sont critiquées pour leur manque de réalisme. Nous discuterons dans un premier temps de l'individualisme méthodologique et de la rationalité des agents économiques.

${ }^{212}$ SABOOGLU Mufit, " Rationalité et Déterminisme », 1994, p.2. 
Ensuite, nous nous concentrerons sur la notion d'utilité et sur la dichotomie valeur/fait présente dans les sciences économiques. Nous verrons à chaque fois que les hypothèses sur lesquelles se base la perspective économique de l'individu, ne permettent pas d'expliquer de manière satisfaisante le phénomène de la valeur. La recherche d'une alternative devient nécessaire et c'est à travers la théorie du philosophe autrichien que nous devrions nous orienter.

\section{1 - La perfectibilité des hypothèses sur les comportements humains}

La théorie de la valeur qui domine actuellement le discours économique et qui est présente dans les manuels de sciences économiques considère l'individu comme un agent rationnel qui va chercher à maximiser son bien être. Pour cela il va se poser en demandeur sur le marché lorsqu'un objet - un bien économique en l'occurrence - sera susceptible de satisfaire ses besoins.

Cette perspective est fondée sur les hypothèses suivantes ${ }^{213}$ :

- La nature humaine est telle que les individus recherchent leur intérêt personnel et qu'ils sont rationnels dans leurs choix (ce qui les conduit à faire des choix en faveur de la maximisation de leur bien être personnel).

- Le but de la vie de l'individu est de chercher à être heureux selon leur définition du bonheur. Pour cela, il est important qu'ils soient libres de pouvoir le faire.

- Le monde social idéal est un rassemblement d'individus libres qui se font concurrence dans leur intérêt personnel mais également parce qu'un certain nombre de biens sont rares. Dans le monde social, il existe des forces qui conduisent les individus vers des positions d'équilibre (hypothèse dérivée de celle de la main invisible).

On dit d'un individu qu'il a un comportement rationnel lorsqu'il a utilisé complètement l'information disponible et qu'il a eu un raisonnement solide et rigoureux ${ }^{214}$. La rationalité revêt plusieurs aspects. On distingue ainsi la rationalité subjective (rationalité basée sur l'information à la disposition de l'agent économique), de la rationalité objective (rationalité basée sur l'information maximale disponible à un moment donné de l'histoire).

\footnotetext{
${ }^{213}$ Nous reprenons ici les propos de Charles K. WILBER, « Ethics and Economics ", in WHALEN Charles J. (éd.), 1996, p.47-48.

${ }^{214}$ SABOOGLU Mufit, 1994, p.3.
} 
Dans la théorie néo-classique, l'agent économique utilise toutes les informations à sa disposition et la raison devient un instrument qu'il utilise afin de découvrir l'action le menant à la maximisation de son bien être. Ce type de rationalité objective est dit instrumental ${ }^{215}$.

L'individu heureux est celui qui satisfait tous ses besoins. Ainsi les sciences économiques assimilent la satisfaction des besoins avec le bonheur.

Reprenons une à une chacune des caractéristiques principales de l'homo œconomicus (l'individualisme, la rationalité et la maximisation du bien être).

\subsection{1 - L'individualisme méthodologique}

L'hypothèse du comportement orienté vers la satisfaction de l'intérêt personnel serait plus complexe qu'il n'y paraît selon Amartya SEN, et contiendrait trois composantes caractéristiques ${ }^{216}$ : celle du "self-centred welfare" ou bien être autocentré, celle du " self-welfare goals » ou les buts visant le bien être personnel et troisièmement celle du " self-goal choice " ou choix du but personnel.

Le bien être autocentré décrit le fait que le bien être d'une personne dépende de sa consommation personnelle. Autrement dit, le bien être est réduit ou assimilé à la consommation individuelle et il n'implique à aucun moment les relations avec autrui. Deuxièmement, le but de toute personne est de maximiser son bien être personnel et, considérant une part d'incertitude, de maximiser la valeur attendue de ce bien être. Les sciences économiques admettent que l'individu ne dispose pas de toutes les informations concernant le gain d'utilité fournie par l'acquisition d'un objet. Par conséquent, l'individu va estimer ce gain par l'intermédiaire des probabilités. Cette proposition est celle du théorème de l'utilité espérée, élaborée à l'origine par CRAMER et BERNOULLI, puis perfectionnée dans les années 1940 par von NEUMANN, MORGENSTERN et SAVAGE ${ }^{217}$. Encore une fois, les buts d'une personne sont fixés en fonction de son intérêt personnel, de manière isolée par rapport à autrui. Notons cependant qu'une valeur est accordée au bien être et que, à travers ses actions, l'individu cherche à maximiser cette valeur.

Enfin, le "self-goal choice " implique le fait que chaque choix effectué par un individu est guidé par la poursuite de son intérêt personnel. Ces trois éléments sont contenus simultanément dans les théories économiques du courant dominant nous dit SEN, mais il serait possible de les considérer un à un ${ }^{218}$.

\footnotetext{
${ }^{215}$ Idem, p.5.

${ }^{216}$ SEN Amartya, 1987, p.80.

${ }^{217}$ SABOOGLU Mufit, 1994, p.31-45.

218 Idem.
} 
D'une manière générale, l'individualisme méthodologique ne décrit pas de manière satisfaisante le comportement des individus, même dans le domaine économique. Un exemple dans lequel l'individu s'intéresse à autrui est celui des parents s'occupant de leurs enfants. Ainsi, les parents sont prêts à investir dans l'éducation de leurs enfants, non pas en fonction de leur bien être personnel, mais en fonction de celui de leurs enfants. On pourrait également penser que le bien être des parents dépend de celui des enfants et c'est pour cela qu'ils favorisent une bonne éducation scolaire : en fin de compte, leur but ultime reste leur bien être personnel. Même si cela était vraiment le cas, ce serait la preuve que le bien être d'une personne dépend de celui d'une autre, ce qui revient en fait à confirmer notre affirmation selon laquelle les individus n'agissent pas toujours de manière égocentrique mais parfois aussi de manière allocentrique et que leur bien être n'est pas indépendant de celui des autres.

Pour l'économiste Robert ROWTHORN ${ }^{219}$, l'altruisme, d'un point de vue biologique, est un caractère qui est présent autant chez l'homme que chez l'animal et il a donc des origines génétiques. Il ajoute que la plupart des économistes modernes ignorent l'altruisme ou cherchent à l'expliquer comme une manifestation de l'intérêt personnel de long terme. Or, plusieurs expériences ont montré que la majorité des individus plaçaient l'intérêt d'autrui au-dessus du leur ${ }^{220}$. Il est donc erroné de penser que l'individu n'est altruiste que par intérêt personnel ou encore qu'il ne l'est pas du tout.

Les comportements égoïstes, ajoutés à la soi-disant rationalité individuelle, passent pour être également efficaces puisqu'ils conduisent l'individu à faire les meilleurs choix possibles. Pour Friedhelm HENGSBACH, la coopération et le consensus sont considérés comme trop chers alors que la concurrence est vue comme le signe inévitable d'une économie et d'une société mondialisées ${ }^{221}$. Andreas SUCHANEK nous explique que les comportements coopératifs sont souvent plus bénéfiques et qu'en réalité, si l'individu est véritablement rationnel, il fera le choix de coopérer ${ }^{222}$. Pour arriver à cette conclusion il passe par l'exemple du dilemme des prisonniers dans la théorie des jeux : deux personnes sont arrêtées pour avoir commis un délit. Chacune d'entre-elle est placée dans une pièce séparée afin de procéder aux interrogatoires, sachant qu'elles ne peuvent à

\footnotetext{
${ }^{219}$ ROWTHORN Robert, " Ethics and Economics: an Economist's View », in GROENEWEGEN Peter, 1996, p.17-18.

${ }^{220}$ Voir à ce propos ETZIONI Amitai, "The Moral Dimension : Towards a New Economics ", 1988 ; FRANK Robert H., " Passions within Reason : The Strategic Role of the Emotions ", 1988 ; WILSON James Q., « The Moral Sense », 1993.

${ }^{221}$ HENGSBACH Friedhelm, « Marktkonkurrenz auf der Grundlage gesellschaftlicher Kooperation » in BRIESKORN Nobert et WALLACHER Johannes (eds), 1998, p.47.

${ }^{222}$ SUCHANEK Andreas, "Ökonomische Ethik », 2001, p.62-67.
} 
aucun moment se parler entres-elles. Elles ont le choix entre " coopérer " et " ne pas coopérer ». Le tableau suivant reprend les valorisations des deux personnes selon leur mode d'agissement :

\begin{tabular}{|l|c|c|}
\hline & B coopère & B ne coopère pas \\
\hline A coopère & $I(2 ; 2)$ & II $(0 ; 3)$ \\
\hline A ne coopère pas & III $(3 ; 0)$ & IV $(1,1)$ \\
\hline
\end{tabular}

Dans chaque quadrant, le premier chiffre correspond à la valorisation de la situation par A, le second chiffre correspond à la valorisation par B. Dans ce tableau, la situation IV dans laquelle les deux personnes ne coopèrent pas, n'est pas bien évaluée, contrairement à la situation I où elles coopèrent toutes les deux. Dans les situations intermédiaires II et III, une des deux personnes a un comportement égoïste et profite ainsi du fait que l'autre coopère.

Il en ressort que si les deux personnes s'accordent, elles en retireront un bénéfice total plus élevé que si elles ne coopèrent pas du tout ou que si elles agissent de manière égoïste. Nous voyons ainsi que l'individualisme n'est pas un comportement efficace, bien au contraire. Dans les économies de marché, la coopération ne s'oppose pas au principe de la concurrence, au contraire, elle le complète : c'est en travaillant ensemble que l'on atteint de meilleurs résultats et être ainsi plus concurrentiel ${ }^{223}$. L'individualisme, selon ce schéma, ne conduit pas à l'intérêt général. SUCHANEK ajoute que les individus doivent parfois être incités vers la coopération et que ceci peut se faire par l'intermédiaire de règles établies par les institutions ${ }^{224}$. La coopération peut également être favorisée par l'habitude et les convictions. Elle reste en tous les cas liée à la morale : si chacun ne se sent pas moralement obligé de respecter les termes du contrat de coopération, alors cette dernière est vaine et les résultats obtenus sont encore moins efficaces ${ }^{225}$.

Concrètement, un travail en groupe sur un projet, malgré les divergences éventuelles, peut conduire à un résultat positivement valorisé, non seulement par les travailleurs, mais aussi par autrui. La politesse entre les membres du groupe facilite la coopération et permet en outre, de prévoir les réactions d'autrui.

\footnotetext{
${ }^{223}$ HENGSBACH Friedhelm, 1998, p.54.

${ }^{224}$ SUCHANEK Andreas, 2001, p.50-58.

${ }^{225}$ HENGSBACH Friedhelm, 1998, p.58-59.
} 
On peut en déduire que les évaluations individualistes ${ }^{226}$ ne sont pas les plus efficaces non plus. EHRENFELS évoque lui-même les évaluations individuelles, tout comme les valeurs collectives, mais sa théorie laisse penser que les valeurs individualistes sont fortement influencées par les évaluations collectives. Par ailleurs, l'individualisme, d'une manière générale, n'est pas un comportement positivement valorisé, en particulier au sein d'une collectivité ou d'un groupe restreint comme la famille. Celui qui agira de cette manière, se verra reprocher son comportement par autrui. Or, parmi les buts des membres d'une société, il y a le fait de se faire reconnaitre et de se faire accepter par les autres. Autrement dit, la réputation d'un individu dans la société dans laquelle il vit, joue un rôle dans sa manière d'agir. Un individu rationnel fera attention à sa réputation puisque celleci lui fournira un avantage lors de négociations, mais aussi pour le plaisir de se sentir apprécié. On peut craindre cependant qu'il ne saisisse toutes les occasions d'agir de manière opportuniste ${ }^{227}$. L'individu qui a des valeurs morales et des buts de long terme en ce qui concerne sa place dans la société, évitera d'agir de la sorte, même si cela est dans son intérêt de court terme ${ }^{228}$. Se faire accepter par la société et y avoir une place c'est aussi adhérer à ses valeurs, notamment ses valeurs morales. En n'agissant que par rapport à ses intérêts, l'individu prend le risque de se voir mal considéré par autrui, ou encore de ne pas pouvoir participer aux contrats de coopération qui lui seront proposés. Ne pas coopérer peut conduire à un résultat qui ne sera pas le meilleur possible.

Pour EHRENFELS, l'individu valorise avant tout des buts de long terme. Le fait d'attribuer des valeurs morales conformément aux règles en vigueur dans une société, aura pour autrui une grande valeur éthique. Le regard d'autrui lui procurera alors une satisfaction personnelle contribuant à son bien être. Un jugement de valeur négatif ne l'y aidera pas, au contraire. De plus, si on reprend l'exemple de la famille, les parents n'auront pas intérêt à valoriser l'individualisme, pouvant à long terme en être victime de la part de leurs propres enfants, s'ils venaient à avoir besoin d'aide ou de soins dans la dernière partie de leur vie. Cependant, le nombre croissant de personnes âgées restant dans les maisons de retraite montre que ces dernières années, le comportement de leurs enfants tend vers un plus grand individualisme.

\footnotetext{
${ }^{226}$ On distinguera les évaluations individuelles, celles émises par les individus en particulier, des évaluations individualistes, celles qui sont émises par l'individu visant uniquement la satisfaction de son intérêt personnel.

${ }^{227}$ KLIEMT Hartmut, " Selbstbindung und Selbstverwirklichung », in RAMB Bernd T. et TIETZEL Manfred (eds), 1993, p.301.

${ }^{228}$ Idem, p.302.
} 
La perspective de l'individualisme méthodologique proposée et propagée par les sciences économiques semble avoir une influence sur les comportements réels :

«Learning economics, it seems, may make people more selfish ${ }^{229}$

Des études du comportement des économistes ont en effet montré que ces derniers avaient une tendance à adopter une attitude égoïste dans certaines circonstances, et que cette tendance se renforçait au fur et à mesure des études ${ }^{230}$. Ces résultats vont encore plus loin : la vie économique implique la plupart du temps le travail en équipe et d'autres formes de coopérations où l'altruisme est présent. Non seulement les économistes coopèrent mal, mais en plus ils ont tendance à sousestimer la proportion d'altruistes dans la population. Leur attitude se reflète donc dans leurs réflexions et dans leurs travaux.

La tendance à l'individualisme ne concerne en fait pas ou plus seulement les économistes. On a donc une valorisation duale du comportement individualiste, et il semble que ce soit le résultat d'une évolution. Selon les études que l'on vient d'évoquer, ce type de comportement est dû en partie à l'influence du savoir acquis. Ce savoir est, on le sait, dominé par des paradigmes néo-classiques. Ceuxci sont intégrés, puis transmis par les économistes qui sont, pour certains, conseillers d'hommes politiques. L'introduction de ces paradigmes va se faire dans le cadre institutionnel, introduisant un certain nombre de règles. Ces mêmes règles vont influencer les valorisations individuelles (mouvement de valeur provoqué par la contrainte ou par l'exemple).

La question est de savoir s'il est moral de vouloir favoriser l'individualisme méthodologique, sachant par ailleurs qu'il ne s'agit pas du comportement le plus efficace, y compris pour les individus rationnels. Nous verrons plus loin que cette évolution est liée à des changements globaux, concernant en particulier l'avancée du marché dans les sociétés occidentales.

\footnotetext{
${ }^{229}$ HAUSMAN Daniel M. et McPHERSON Michael S., « Taking Ethics seriously : Economics and Contemporary Moral Philosophy ", 1993, p.674.

${ }^{230}$ ROWTHORN Robert, "Ethics and Economics. An Economist's View », in GROENEWEGEN Peter, 1996, p.19-20 ; KERSTING Wolfgang, " Diskussion: Moral Based Rational Man ", in BRIESKORN Nobert et WALLACHER Johannes (eds), 1998, p.34.
} 


\subsection{2 - L'hypothèse de rationalité}

La deuxième hypothèse fondamentale qui nous préoccupe et qui concerne les comportements humains, est celle de l'individu rationnel et cherchant à maximiser son bien être. Une hypothèse dont on attribue la paternité à Adam SMITH. L'individu va agir de manière égoïste et chercher à satisfaire ses besoins personnels. Et c'est cette recherche qui le mènera à produire ou à consommer. Selon SEN, cette interprétation, reprise par la grande majorité des économistes et la plupart des manuels de sciences économiques, est erronée ${ }^{231}$. On prête au philosophe écossais des propos sans tenir compte du contexte dans lequel ils ont été énoncés, et on oublie souvent les thèses d'Adam SMITH sur les comportements. En effet, il met en avant entre autre, les sentiments de sympathie et d'autodiscipline. L'individu fait parti d'un tout et il est prêt à sacrifier son propre intérêt pour autrui ${ }^{232}$. Ce genre de réflexions a eu tendance à être oublié en faveur de celui concernant la recherche de l'intérêt personnel. Certes, selon SEN toujours, SMITH a su voir que beaucoup de nos actions étaient en fait guidées par notre intérêt. Il n'accorde à aucun moment un rôle particulier à l'intérêt personnel et il ne dit pas non plus qu'il s'agit de la seule motivation de l'individu agissant ${ }^{233}$.

Le caractère rationnel des personnes n'est en aucun cas un caractère absolu. Pour BRATMAN et GIBBARD, les êtres humains sont des animaux planifiant, et dans leur vie sociale, par l'intermédiaire du langage, ils expriment leur besoin de planifier leurs actions ${ }^{234}$. Du point de vue d'un économiste, les raisons d'agir sont déterminées par les préférences d'une personne, plus que par les résultats attendus de son action. Pour David GAUTHIER, il y a du vrai comme du faux dans cet argument $^{235}$. La planification est un instrument nous permettant d'atteindre nos objectifs et non un objectif en lui-même. On effectue une planification en fonction de nos préférences, et ce faisant, nous prévoyons nos actes de manière à ce qu'ils apportent le meilleur résultat possible, celui que nous préférons. Il convient cependant de ne pas sous-estimer le rôle du résultat. Par exemple, je prévois d'être au bureau demain matin et d'aller faire les courses demain après-midi. J'apprends ensuite qu'une collègue, avec qui je dois discuter de quelque chose, n'est pas là

\footnotetext{
${ }^{231}$ SEN Amartya, 1987, p.22-28.

232 «Man, according to the Stoics, ought to regard himself, not as something separated and detached, but as a citizen of the world, a member of the vast commonwealth of nature [...] to the interest of this great communauty, he ought at all times to be willing that his own little interest should be sacrificed ", in SMITH Adam, " The Theory of Moral Sentiments ", 1790, p.140.

${ }^{233}$ Voir p.206-207 de ce travail.

${ }^{234}$ BRATMAN Michael, "Intentions, Plans, and Practical Reason ", 1987, p.2-3 ; GIBBARD Allan, "Wise choices, Apt Feelings: A Theory of Normative Judgement ", 1990, p.57.

${ }^{235}$ GAUTHIER David, "Commitment and Choice: An Essay on the Rationality of Plan », in FARINA Francesco, HAHN Frank et VANNUCCI Stefano (eds), 1996, p.218-244.
} 
demain après-midi. Je vais alors réajuster mes plans et venir au bureau l'aprèsmidi au lieu du matin.

Dans cet exemple, mes préférences vont à la première variante. Mais étant donné mon objectif - pouvoir parler avec ma collègue - je vais me décider pour la seconde variante. Le contexte dans lequel $\mathrm{j}$ 'agis va influencer mes décisions mais pas mes préférences. La personne peut ici, de manière rationnelle, choisir de changer ses plans, mais pour autant elle ne va pas maximiser sa satisfaction.

Selon la théorie d'EHRENFELS, dans l'action tous ces éléments se voient attribuer une certaine valeur, que ce soit le moyen, le but, ou encore les conséquences attendues de l'action. Dans l'exemple précédent, l'individu a deux buts : le premier est d'aller faire les courses, et le second est de parler avec sa collègue de travail. Sa préférence va certes vers le fait de faire les courses l'aprèsmidi, en ce sens où c'est ce qui lui procurerait le plus de plaisir et qu'il va donc valoriser positivement. Mais le fait de pouvoir discuter avec sa collègue semble avoir une plus grande importance à ses yeux, puisque pour atteindre ce but, il est prêt à changer l'ordre de préférence du déroulement de sa journée. Les personnes n'agissent donc pas seulement par rapport à leurs préférences, mais avant tout par rapport à l'ordre d'importance qu'elles accordent aux choses, c'est-à-dire à la valeur accordée à leurs buts.

La question que pose GAUTHIER par ailleurs est celle des limites de l'engagement d'un agent par rapport à son plan ${ }^{236}$. Pour lui, un agent peut avoir une raison basée sur un plan même s'il sait que d'autres raisons basées sur ses préférences favorisent une reconsidération de son plan. Cette thèse est celle qui est défendue par l'auteur et qui s'oppose également à l'orthodoxie économique. Parmi les raisons d'agir, il convient de distinguer deux perspectives :

- La perspective subjective de l'agent, qui reflète ses croyances concernant les situations, les actions et résultats possibles, ainsi que leurs probabilités respectives en fonction de l'action.

- La perspective idéale, objective, qui reflète la situation actuelle de l'agent, les actions possibles et leurs résultats. Ceci suppose que l'individu soit parfaitement informé sur ces éléments.

Dans le processus de planification, il y a deux éléments qu'il faut distinguer : les raisons d'adopter un plan et les raisons de s'y tenir. On peut à ce propos, distinguer les agents dits myopes des agents dits sophistiqués. Les premiers agissent uniquement en fonction de leurs préférences présentes et, si elles changent en cours d'application de son plan, alors ils les reconsidéreront en

${ }^{236}$ Idem. 
fonction de leurs nouvelles préférences. Les seconds s'attacheront à respecter l'objectif qu'ils se seront fixés dans le plan adopté car le fait d'atteindre leur objectif aura une plus grande importance pour eux. MAC CLENNEN souligne le fait que l'individu myope n'a pas un comportement constant et que le plan qu'il adopte n'est peut être pas celui qu'il exécute, alors que l'individu sophistiqué s'en tient lui à son plan originel ${ }^{237}$. De plus, ne pas reconsidérer son plan se produit fréquemment dans les situations qui requièrent une coordination interpersonnelle. MAC CLENNEN ne suppose pas qu'un choix résolu est toujours faisable et il reconnaît qu'il est impossible pour une personne de l'être si elle n'a rien à gagner de cette constance. La raison de s'en tenir à son plan doit donc être avantageuse.

Si on reprend l'exemple précédent de GAUTHIER, la personne qui change son emploi du temps va aussi très probablement s'y tenir, car le fait de pouvoir parler avec sa collègue sera une raison plus importante que le fait de pouvoir faire les courses. Ceci montre encore une fois la possibilité pour un individu de ne pas agir en fonction de ses préférences mais de choisir malgré tout une solution optimale (même si celle-ci n'est pas forcément contraire à ses préférences). Le fait d'être constant pour un individu signifie également que son comportement est prévisible, c'est-à-dire qu'il permet à autrui d'ajuster le sien de manière optimale.

Les comportements que nous venons de décrire dépendent en définitive de la valeur qui est accordée par l'individu aux buts qu'il s'est fixé. Certes le chemin qui mène jusqu'à eux peut subir l'influence des préférences. Néanmoins, l'individu n'agit pas uniquement en fonction de ses préférences, mais aussi en fonction de son environnement, et le fait qu'il soit rationnel ne veut pas dire qu'il va choisir la préférence qui lui est la plus avantageuse. De plus, l'individu peut agir en fonction de ses préférences, sans pour autant être rationnel. Par exemple, il peut absolument vouloir acquérir un objet, et lui accorder donc une grande valeur d'effet, sans que cette acquisition soit justifiée, voire qu'elle ait des conséquences (financières) négatives. Le fait qu'il accorde une telle valeur à cet objet peut être le fait de ses préférences personnelles (dans le cas d'un collectionneur par exemple) ou le résultat d'une influence extérieure (suggestion, comme l'effet de mode par exemple).

GAUTHIER ajoute enfin qu'une raison d'agir d'une certaine manière pour un individu peut être une raison morale $\mathrm{e}^{238}$. Des préférences morales sont par exemple la résolution, l'engagement et l'obligation, et suffisent à justifier l'attitude d'une personne. Une attitude d'autant plus appréciée qu'elle permet à autrui de

\footnotetext{
${ }^{237}$ MCCLENNEN Edward F., " Rationality and Dynamic Choice: Foundational Explorations ", 1990.

${ }^{238}$ GAUTHIER David, in FARINA Francesco, HAHN Frank et VANNUCCI Stefano (eds), 1996, p.242.
} 
s'adapter, ce qui peut conduire à une optimalité interpersonnelle. En effet, toujours en citant GAUTHIER, chacun d'entre-nous doit pouvoir coordonner ses propres activités entres-elles, et coordonner ses propres activités avec celles des autres. Planifier est un bon moyen d'agir, et être résolu dans son action nous permet d'agir efficacement. On comprend ainsi l'importance des conventions qui facilitent la coordination interpersonnelle. Le respect mutuel de ces conventions prend alors une signification morale. Encore une fois on remarque le rôle joué par la morale dans la perspective d'un comportement menant aux meilleurs résultats possibles.

Concernant précisément la coordination, Robert SUGDEN se propose de traiter la question de savoir si elle est un sous-produit d'un comportement irrationnel ou alors le résultat d'un processus de raisonnement ${ }^{239}$. Il suggère en fait que la coordination est le produit du raisonnement mais d'un type de raisonnement non compatible avec la conception conventionnelle de la rationalité utilisée en économie et dans la théorie de la décision.

Pour arriver à cette conclusion, il distingue d'abord deux types de raisonnement :

- Le raisonnement dit "primary salience", c'est-à-dire celui qui intervient lorsqu'un individu répond à une situation de manière spontanée. Par exemple, une personne qui doit choisir entre deux enveloppes, prendra l'une d'entres-elles de manière spontanée. Ou encore, lorsqu'on lui demande de choisir une couleur, une personne peut répondre en nommant la première couleur qui lui vient à l'esprit.

- Le raisonnement dit « secondary salience » qui est le produit d'une réflexion. Par exemple, si je dois trouver la ville de naissance du plus grand nombre de personnes en Angleterre, je vais répondre Londres par déduction, car il s'agit de la plus grande ville en nombre d'habitants.

Une expérience de SCHELLING montre comment les individus sont incités à adopter des raisonnements dits de " secondary salience " dans certaines situations impliquant une coopération ${ }^{240}$. Dans cette expérience, deux groupes de plusieurs individus doivent nommer un nombre. Le premier groupe est motivé par le fait que les membres sont rémunérés si leur réponse est coordonnée. Le deuxième groupe n'est pas motivé, ses membres sont rémunérés seulement s'ils donnent une réponse, peu importe laquelle. Le résultat montre que le premier groupe manifeste une coordination bien supérieure à celle du deuxième. Ses membres se sont en fait focalisés sur le chiffre 1, lequel sort de l'ordinaire. Il faut noter que les individus n'ont alors pas agi comme des êtres maximisant. Chacun a ainsi considéré la situation à la manière d'un chef d'équipe, cherchant la combinaison d'action donnant le meilleur résultat. Ce mode de raisonnement est nommé « maximisation

${ }^{239}$ SUGDEN Robert, " Rational Co-ordination », in FARINA Francesco, HAHN Frank et VANNUCCl Stefano (eds), 1996, p.244-261.

${ }^{240}$ SCHELLING Thomas C., " The Strategy of Conflict ", 1997, p.89-99. 
conjointe " par SUGDEN, par opposition aux individus maximisant des théories économiques. Le problème est de savoir si cette maximisation conjointe est rationnelle. Pour répondre à cette question, il faut se mettre encore une fois dans la situation d'un chef d'équipe qui a pour mission d'amener ses joueurs à choisir une solution coordonnée. Il lui faut utiliser un moyen de persuasion efficace. On peut dire du chef d'équipe qu'il agit en être maximisant, puisqu'il est de son intérêt que l'équipe agisse de manière coordonnée. Il lui faut par contre justifier l'adoption d'un processus coordonné si les joueurs sont indifférents aux solutions. Certains d'auteurs ont souligné le fait que le principe de coordination ne peut être dérivé des théories conventionnelles du choix rationnel ${ }^{241}$. On ne peut produire un travail ou un comportement coordonné uniquement par préférence, ni même en tant que choix maximisant, reflet d'une quelconque rationalité.

Le fait de choisir d'agir de manière coordonnée ne peut non plus se faire contre la volonté de l'individu, c'est-à-dire donc que s'il accepte de le faire, c'est qu'il y a intérêt, mais ce dernier ne correspond pas forcément à ses préférences. Par exemple, un travailleur peut accepter de travailler en équipe afin d'améliorer son image dans son entreprise, tout en sachant que sa préférence irait plutôt vers le fait de travailler seul. Son but ultime est-il sa satisfaction personnelle? Ou ne serait-il pas plus exact de dire qu'il s'agirait ici de la réussite sociale ? La réussite sociale apporte un certain degré de satisfaction à l'individu, mais pas forcément du bien être (l'individu serait probablement plus à son aise s'il travaillait moins et passerait plus de temps avec sa famille). Il est difficile dans un exemple de généraliser un comportement car tout le monde est différent et chacun a ses propres buts avec une valorisation individuelle de ces derniers, comme nous le dirait EHRENFELS. En tous les cas, le fait d'accepter un comportement coordonné est le résultat d'un raisonnement, et il oblige aussi l'individu à un certain engagement et donc à des obligations, sans lesquelles la coordination ne serait pas possible. On le voit une fois de plus, le rôle de la morale ne peut être négligé en matière d'étude des comportements.

Pour ajouter encore un argument à l'encontre de l'hypothèse de rationalité, exposons à présent la thèse de l'économiste grec VAROUFAKIS. Grâce à un exemple révélateur, il montre que la rationalité ne peut servir à justifier les comportements individuels quotidiens, en particulier ceux qui sont répréhensibles $^{242}$.

\footnotetext{
${ }^{241}$ Voir par exemple HODGSON David.H., « Consequences of Utilitarism », 1967.

${ }^{242}$ VAROUFAKIS Yanis, "O Tempora, O mores! Economics as the Ethos of our Times », in GROENEWEGEN Peter, 1996, p.157-171.
} 
Il considère la situation où une mère, professeur d'économie, essaie d'expliquer à son fils de 6 ans, pourquoi il n'aurait pas dû frapper un plus petit que lui pour lui prendre son jouet. La première raison qu'elle lui donne : que dirais-tu si un plus vieux que toi faisait la même chose ? Imaginons que l'enfant lui réponde qu'il ne l'apprécierait pas, et alors?

Parmi les théories possibles, on peut distinguer celles qui prennent appui sur les raisons internes et celles qui prennent appui sur les raisons externes. Dans les théories économiques, ce qui est raisonnable (rationnel) est basé sur des raisons internes, puisque les agents ne sont pas censés subir les influences extérieures. Une raison interne possible est celle de la réputation : l'individu cherche à avoir une bonne réputation auprès de ceux avec qui on sera amené à vivre. Cet argument fut fourni à l'origine par HOBBES ${ }^{243}$. A cela, le fils peut lui répondre qu'il est probable qu'il ne soit pas amené à vivre avec celui à qui il a pris le jouet, donc il n'a pas de raison de ne pas le lui prendre. Une deuxième raison interne, basée sur les thèses de HUME, est celle de la sympathie pour autrui et de la part d'autrui : de par son comportement, l'individu peut recevoir ou accumuler la sympathie d'autrui ${ }^{244}$. L'enfant peut alors répondre que sa préférence va plus vers le jouet qu'une éventuelle sympathie. Il en retirerait d'ailleurs plus de satisfaction. Ne pouvant argumenter contre le geste de son fils, la mère est obligée de se référer à des raisons externes. Elle pourrait invoquer l'argument de la coopération : comme nous l'avons énoncé auparavant, les comportements coopératifs produisent des bénéfices mutuels. Coopérer implique un engagement vis-à-vis d'autrui, mais qu'est-ce qui incite l'individu à respecter ses engagements ? Est-ce le gain qu'il peut en retirer?

Pour KANT, le gain ne peut être la cause d'un comportement raisonnable (ce qui implique l'abandon du paradigme de HUME, selon lequel la raison est l'esclave des passions). Etre raisonnable implique une capacité à reconnaître ses devoirs envers les autres. A travers son interprétation du procédé d'application de l'impératif catégorique, John RAWLS nous livre la justification kantienne de ce comportement ${ }^{245}$. Lorsqu'une personne rationnelle, dans des circonstances déterminées, a besoin d'argent et sait qu'elle ne pourra pas le rembourser, cette personne ne pourra pas faire de promesse qu'elle ne tiendra pas. En effet, si chacun agissait de la même manière, c'est-à-dire si chacun pouvait emprunter de

\footnotetext{
${ }^{243}$ Thomas HOBBES décrit l'individu comme individualiste. Sa vision de la morale est celle d'une morale psychologique subjective dans laquelle tous les sensations et les attitudes sont à reconsidérer sous un angle égocentrique. Ainsi l'impudeur n'est pas pour lui un comportement moralement répréhensible mais une conduite non intelligente de celui qui ne fait pas attention à sa réputation. Voir à ce propos : KERSTING Wolfgang, "Thomas Hobbes zur Einfuhrung ", 2002, p.75-77 et note 40 p.212-213.

${ }^{444}$ RYSAVY Karin, " Ethik, Utilitarismus, Politik », 2002, p.29.

${ }^{245}$ RAWLS John, " Geschichte der Moralphilosophie ", 2002, p.230-236.
} 
l'argent en sachant qu'il ne pourrait pas le rendre, alors la promesse n'en serait plus une et on ne leur prêterait rien. Le but de la personne rationnelle est d'obtenir cette somme et cela ne lui est possible que si elle s'engage à la rembourser, c'està-dire si elle tient ses engagements. Son comportement est alors raisonnable et suit implicitement un impératif catégorique (tenir ses promesses). Mais pourquoi voudrais-je être raisonnable de cette manière ? Est-ce à dire que je valoriserais le raisonnable pour lui-même? Le fait d'être raisonnable, nous disent HEGEL et HABERMAS, conduit à avoir une bonne image reflétée par autrui ${ }^{246}$. Mais là encore, l'enfant peut répondre que son image auprès d'autrui l'intéresse moins que le jouet qu'il a pris par la force. Jeremy SHEARMUR pose la question qui est la porte d'entrée menant au néolibéralisme et qui est une réponse à HUME : si le bien commun est seulement imaginable comme un produit du marché, mais que le marché ne réussit pas à la produire, alors pourquoi les bureaucrates s'évertueraient-ils à vouloir imposer quelque chose qui ne sera jamais produit? Pour David HUME et ses disciples, toujours selon VAROUFAKIS, la moralité est une illusion fonctionnelle servant les intérêts mesquins des individus. Pour HUME, quand les passions minent l'action, alors la raison regarde ailleurs, à la recherche d'une référence. Il voit les conventions comme des produits non rationnels et instables d'un processus de sélection sociale qui élimine les conventions instables. Comme en biologie, les conventions qui s'imposent, sont celles qui arrivent à avoir le plus d'approbation au sein de la population. Leur survie dépend de l'adhésion non seulement des partisans mais aussi des opposants. MARX voit aussi la morale comme une construction, qui est spécifique à chaque classe sociale. Les classes dominantes donnent cependant le «la ».

Le modèle de HUME sert de référence aux théories économiques actuelles et BROOME distingue deux tendances, l'une modérée et l'autre extrême, dans les courants Huméens ${ }^{247}$. Si la morale est une construction sociale, et si elle nécessite des explications endogènes, il n'en est pas de même pour l'intérêt personnel. HUME a montré que les idées du bien, de la justice et du devoir sont des créations liées à l'intérêt personnel ${ }^{248}$. MARX ajouta que notre morale et nos desseins sont adjacents à l'histoire des relations sociales.

Pour EHRENFELS la morale semble être en partie une construction, mais qui repose sur des maximes ayant elles-mêmes une provenance religieuse. Il précise que les valeurs éthiques et les valeurs morales sont attribuées par le plus grand

\footnotetext{
${ }^{246}$ RAWLS John, " Geschichte der Moralphilosophie », 2002, p. 450-454 ; HEGEL Georg Wilhelm Friedrich, " Reason in History », 1953 ; HABERMAS Jürgen, « Erkenntnis und Interesse », 1991, p.244-245.

${ }^{247}$ BROOME John, « Ethics out of Economics », 1999, p.68-87.

${ }^{248}$ ROSEN Frederick, « Classical Utilitarism from Hume to Mill », 2003, p.33-47.
} 
nombre lorsqu'elles font l'objet d'un consensus au niveau de la société. L'individu agit dans le cadre des règles imposées par la société (règles morales, lois, coutumes, traditions,...), mais son comportement est également influencé par ses passions. Le processus de valorisation dépend des dispositions émotionnelles des personnes. Or, dans le cas de l'enfant dans l'exemple précédent, ses dispositions émotionnelles sont orientées par le désir de posséder le jouet de l'autre. Pour y parvenir, il choisit de le prendre par la force. Il valorise donc l'objet de telle manière qu'il en justifie son action. Il agit certes par rapport à ses préférences et le résultat est pour lui satisfaisant. Cependant, le moyen utilisé pour y parvenir est moralement inadmissible. EHRENFELS ne pourrait justifier l'action de cet enfant par l'argument de la rationalité. Par ailleurs, il est difficile de montrer que cette action va à l'encontre de sa satisfaction ou de ses préférences. En définitive, il faut faire appel à la morale et aux valeurs inhérentes pour expliquer à l'enfant que son geste n'est pas acceptable. Dans la vie économique également, les différents acteurs ne doivent pas pouvoir non plus utiliser n'importe quel moyen pour arriver à leurs fins, c'est-à-dire leur satisfaction personnelle, en particulier si cela se fait aux dépens d'autrui.

Si on laissait les individus agir de la sorte sous le prétexte qu'ils le font rationnellement, le monde serait celui où, comme dans l'exemple de VAROUFAKIS, les plus forts profiteraient de leur position par rapport aux plus faibles. Au final, l'intérêt général ne serait pas garanti, tout comme la maximisation du bien être de la population. La tension entre les deux groupes serait telle qu'elle créerait une instabilité sociale néfaste au bien être de tous.

Ainsi le fait de considérer les individus comme des êtres rationnels et intéressés uniquement par leur intérêt personnel présente des limites, avant tout par rapport à la diversité des comportements réels. Les individus ne sont pas toujours rationnels et lorsqu'ils le sont, leur comportement ne mène pas automatiquement à la maximisation de leur satisfaction. Nous poursuivrons sur cette voie en réfléchissant à la question concernant le lien entre les préférences et le bien être de l'individu : suivre ses préférences mène-t-il automatiquement à une augmentation de son bien être?

\subsection{3 - La recherche du bien être}

Dans les théories économiques on suppose que l'individu ne recherche que sa satisfaction personnelle supposée par voie de conséquence provoquer une augmentation de son bien-être. La notion de satisfaction est souvent assimilée, voire confondue avec celle de bien-être et, lorsque l'individu émet des préférences, elles se référent automatiquement à la satisfaction de ses besoins. 
De manière synthétique, une chose $\mathrm{A}$ est meilleure pour une personne qu'une autre chose B si et seulement si la personne préfère A à B. "Etre meilleur que " est équivalent à dire "est plus dans son intérêt " ${ }^{249}$. Ceci implique que l'on préfère toujours ce qui est meilleur pour nous, et on va choisir par conséquent la meilleure voie possible susceptible de satisfaire nos besoins. Préférer un objet $\mathrm{A}$ à un objet B signifie dans la littérature économique, selon BROOME, que cette personne choisirait A plutôt que B si elle devait faire un choix entre ces deux objets $^{250}$.

Une thèse concernant les préférences qui est largement admise par les économistes, est celle de la stabilité de l'ordre des préférences de Gary BECKER $^{251}$. BECKER considère que les sciences économiques proposent avant tout une explication du comportement de l'agent économique, c'est-à-dire de l'individu en économie ${ }^{252}$. L'individu cherche à maximiser son bien être et agira pour cela en fonction de ses préférences. Pour BECKER, le schéma des préférences individuelles est le même pour tous :

" [...] all human behavior can be viewed as involving participants who maximize their utility from a stable set of preferences and accumulate an optimal amount of information and other inputs in a variety of markets. $»^{253}$

Il relativise cependant ce point de vue en évoquant l'existence de facteurs non économiques pouvant influencer les comportements :

« Even those believing that the economic approach is applicable to all human behavior recognize that many noneconomic variables also significantly affect human behavior. Obviously, the laws of mathematics, chemistry, physics, and biology have a tremendous influence on behavior through their influence on preferences and production possibilities. ${ }^{254}$

En définitive pour BECKER, malgré ses manques, la théorie économique du comportement individuel offre un cadre adéquat pour la compréhension de tous les comportements humains ${ }^{255}$. Il semble que pour lui cette théorie soit satisfaisante dans la mesure où elle permet d'acquérir des connaissances en sciences économiques et qu'elle remplisse ainsi son rôle ${ }^{256}$. Il s'appuie pour cela

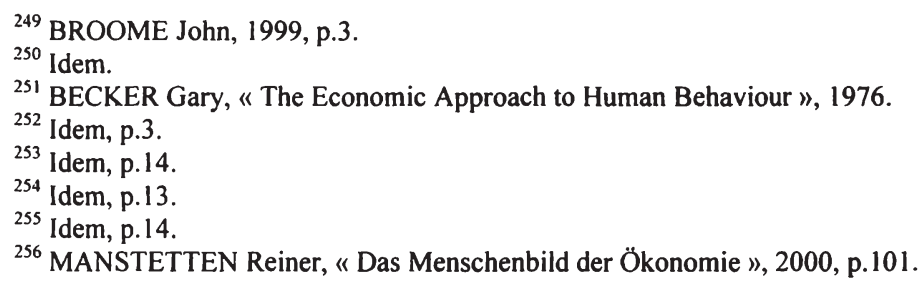


sur un argument de Milton FRIEDMAN pour qui il est possible dans certains cas d'émettre des hypothèses non pertinentes au niveau microéconomique, si par la suite au niveau macroéconomique, des explications exactes peuvent être trouvées pour expliquer des phénomènes observables ${ }^{257}$. L'intérêt du concept d'homo œconomicus ne réside pas dans sa validité empirique mais en tant que "schéma pré-empirique » qui permet par la suite d'explorer et d'expliquer la réalitée ${ }^{258}$.

Reiner MANSTETTEN critique le point de vue de FRIEDMAN, et par conséquent, celui de BECKER : la méthodologie proposée par le premier pourrait conduire à l'invulnérabilité des théories économiques contre la critique et au danger que n'importe quelle hypothèse puisse être utilisée du moment que le résultat s'avère exact ${ }^{259}$.

En ce qui concerne la proposition de stabilité des préférences des individus, l'universalisme implicite dans ce schéma fait abstraction des différences culturelles, y compris au niveau des comportements en économie. Les préférences individuelles dérivent de l'utilité des objets pour les individus, mais l'utilité n'est pas une notion objective. Elle est perçue différemment selon les individus, et un même objet n'apportera pas la même satisfaction à un individu qui n'a pas la même considération de l'objet qu'un autre.

Pour EHRENFELS, les valeurs, c'est-à-dire l'importance accordée à un objet, sont fonction non seulement de l'individu mais aussi du temps et de l'espace, au sens de l'espace culturel dans lequel l'individu se trouve. En outre, vouloir attribuer à tous les individus le même schéma de préférence est perçu par certains comme une approche impérialiste des sciences économiques ${ }^{260}$. Une approche qui est moralement critiquable.

Revenons à présent à BROOME pour qui les économistes sont "typiquement" libéraux et que, "typiquement ", ils croient que les gens devraient être libres de gérer leurs vies. Il s'agit certes d'une bonne idée, mais elle ne conduit cependant pas à la théorie préférences-satisfaction. Pour illustrer sa thèse, il prend l'exemple suivant : soit une vallée dans laquelle vivent des fermiers et au milieu de laquelle coule une rivière. Une fois par an, la rivière sort de son lit et vient inonder les terres de ces fermiers. Ils ont construit des digues, mais il faudrait en construire une encore plus résistante en prévision d'une crue exceptionnelle. La question est de savoir si les fermiers vont vouloir accepter la construction de cette nouvelle digue et payer plus d'impôts en contrepartie, ou s'ils acceptent de prendre le risque d'attendre la crue exceptionnelle et espérer qu'une série de digue, du côté

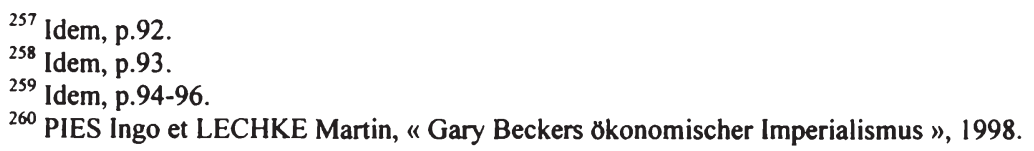


droit ou du côté gauche, ne cède. Dans le cas du second scénario, si l'eau inonde la partie droite des champs, on suppose que la partie gauche sera épargnée et donc une partie seulement des agriculteurs pourra tirer profit de ses exploitations alors que l'autre partie sera ruinée. Si les agriculteurs estiment que le risque que leurs terres soient détruites par les eaux est faible, ils vont préférer que l'on ne construise pas une nouvelle digue. Cette décision, comme on l'a dit, peut aboutir à une grande inégalité dans le cas où l'eau n'inonderait qu'une partie des terres. Autrement dit, la préférence des agriculteurs, fondée sur des informations non parfaites, ne constitue pas toujours la meilleure solution pour eux.

Dans cet exemple, les agriculteurs accordent une non-valeur intrinsèque à une inondation exceptionnelle, due à la nuisance qu'elle représente. Cependant, cette non-valeur intrinsèque ne doit pas être élevée s'ils n'acceptent pas la construction d'une digue plus importante. Si pour les agriculteurs, le risque d'un tel événement est faible, alors ils n'accorderont pas une grande importance à une protection supplémentaire. Rien ne dit cependant que leur décision est la meilleure. BROOME semble penser que la décision des agriculteurs est imprudente. Mais si ces personnes adoptent le même comportement, il ne paraîtra pas imprudent de ne pas vouloir construire une nouvelle digue. Tout dépend du but des cultivateurs : s'ils veulent une prospérité de long terme et sûre alors leur décision pourra être qualifiée d'imprudente. Si par contre, leur objectif est de prospérer le plus possible sur le court terme, tant que les terres ne sont pas inondées, alors leur réaction par rapport à la digue ne pourra être qualifiée d'imprudente.

Dans la pratique, les préférences des personnes vont différer selon leur probabilité de réalisation. EHRENFELS dirait plutôt que leurs préférences dépendent d'un grand nombre de paramètres, lesquelles influencent les dispositions émotionnelles qui conduisent l'individu à accorder un certain type de valeur. Nous avons d'ailleurs évoqué les nombreux facteurs pouvant influencer les dispositions émotionnelles dans notre second chapitre. La probabilité de réalisation d'un but est comptée parmi ces facteurs.

La relation supposée entre les préférences d'un individu et sa satisfaction ne se vérifie pas plus que sa rationalité et son égocentrisme. Le fait d'agir selon ses préférences ne constitue pas toujours un choix rationnel et il ne mène pas toujours à une augmentation du bien être. Le bien être et la satisfaction des besoins ne doivent pas être confondus dans la mesure où le premier est le résultat de plusieurs circonstances parmi lesquelles on retrouve le second.

La théorie économique a voulu schématiser le comportement humain dans les situations propres au domaine de la production et de la consommation. Une schématisation qui a permis sa formalisation mathématique. Or, celle-ci a conduit 
à une réduction ou à une abstraction qui est assez éloignée de la réalité. Une théorie telle que celle d'EHRENFELS permet de mieux cerner le phénomène de la valeur car elle a une approche comportementale généraliste, c'est-à-dire qu'elle ne pose aucune hypothèse limitant le choix des possibilités à un seul type de comportement.

4.2 - De la philosophie (morale) à l'économie : pour un retour à une approche philosophique, aux valeurs et à l'éthique dans les sciences économiques

Les sciences économiques sont une discipline qui durant l'Antiquité était adossée à philosophie et à la morale. L'influence de la seconde sur la première s'est produite durant de nombreuses années, et se manifeste encore à travers l'adoption des théories utilitaristes par exemple. La présence des théories utilitaristes en économie sera le sujet d'une discussion dans ce paragraphe.

$\mathrm{Au} \mathrm{XIX}^{\mathrm{ème}}$ siècle, les sciences économiques se sont affirmées comme une discipline à part entière, dont les principes fondamentaux renforçaient sa spécificité. Sous l'influence de Max WEBER au début du XX ${ }^{\text {ème }}$ siècle, les sciences économiques se sont affirmées objectives et vides de toute valeur (une valeur étant considérée comme un élément subjectif). Cette séparation entre les valeurs et les faits est cependant perfectible et nous verrons que l'un n'est pas possible sans l'autre. Les valeurs sont présentes autant dans la sphère économique que dans les sciences économiques.

\subsection{1 - Les théories utilitaristes et la notion d'utilité dans les sciences économiques}

Dans la théorie économique, on suppose que l'intérêt qu'une personne porte à un bien dépend de son utilité. L'utilité d'un objet peut être définie comme la capacité de cet objet à satisfaire un besoin. Plus cette capacité est élevée et plus grande sera, par conséquent, son utilité. La préférence de l'homo œconomicus va à la chose ayant la plus grande utilité, c'est-à-dire ayant la plus grande capacité à satisfaire un besoin.

Dire qu'un individu ne considère une chose que par rapport à son utilité est une perspective assez réductrice, y compris dans le domaine économique. Les biens acquis ne le sont pas uniquement par rapport à leur capacité à satisfaire un besoin, ils peuvent éveiller l'intérêt d'une personne par rapport à une autre ou à plusieurs autres de ses caractéristiques. Il convient de revenir d'abord sur les origines de la théorie utilitariste, avant d'en montrer les points faibles et la nécessité d'une alternative. 
L'utilitarisme est une théorie qui apparaît d'abord dans le domaine de la philosophie morale, dans le monde anglophone où il a gardé une place importante jusqu'à nos jours. D'après Otfried HÖFFE, on retrouve les éléments essentiels de l'utilitarisme déjà chez l'évêque CUMBERLAND (1631-1718), puis chez David HUME (1711-1776) ${ }^{261}$, mais le premier à avoir parlé explicitement d'utilitarisme et à en avoir exposé les principes, fut Jeremy BENTHAM (1748-1832) à travers son livre "An Introduction to the Principles of Morals and Legislation" en $1789^{262}$. L'utilitarisme arrive sur les devants de la scène dans un contexte où la religion est de plus en plus contestée, en particulier en matière de justification morale. L'utilité d'un objet se voit alors attribuer une signification particulière par BENTHAM. Il la définit comme la capacité d'un objet à produire un certain nombre d'effets positifs ${ }^{263}$. La théorie utilitariste se base sur quatre grands principes fondamentaux :

- La justesse ou le bien-fondé d'une action est déterminé(e) par rapport à ses conséquences.

- Les conséquences seront appréciées en fonction de leur utilité.

- L'utilité est accordée à ce qui est bon en soi. En fait ce qui est bon en soi est l'objet d'une théorie de la valeur. Pour BENTHAM et John Stuart MILL par exemple, la satisfaction des besoins et l'intérêt de l'individu sont bons en soi.

- L'utilitarisme oblige l'action humaine au bien-être et cherche à justifier cette obligation autant que possible par les sciences.

En résumé, il s'agit pour les individus d'agir de telle manière que les conséquences de leurs actions affectent de manière positive le bien être d'autrui. Le fait de juger une conduite par rapport à ses conséquences, fait de l'utilitarisme une théorie conséquentialiste ou téléologique qui considère l'action comme un moyen d'atteindre un but. L'action ne peut donc avoir de valeur intrinsèque. Le critère de sélection d'une action est le bien être qu'elle procure. Il s'agit d'une vision hédoniste des choses. Dans le texte de BENTHAM, le bien être ne concerne pas seulement la personne mais également ses semblables. Il essaie par ailleurs, par l'intermédiaire d'une argumentation sans métaphysique, de montrer que le but ultime de l'action étatique est la plus grande joie du plus grand nombre ${ }^{264}$. Cet état est le résultat de la somme de toutes les joies et de toutes les peines des personnes concernées. Cette vision change par l'intermédiaire d'Adam SMITH, pour qui la recherche de l'intérêt personnel à court terme conduit à une amélioration du bien être sur le long terme.

\footnotetext{
${ }^{261}$ ROSEN Frederik, 2003, p.29-57.

${ }^{262}$ HÖFFE Otfried (éd.), " Einfuhrung in die utilitaristische Ethik », 1994, p.13.

${ }^{263}$ BROOME John, 1999, p.19.

${ }^{264}$ RYSAVY Karin, 2002, p.25.
} 
Le sens différent que l'utilité acquit dans les sciences économiques commença à la fin du XIX ${ }^{\text {ème }}$ siècle, notamment chez Alfred MARSHALL (1842-1924) et Francis Ysidro EDGEWORTH $(1845-1926)^{265}$.

Le premier ouvrage qui se consacre exclusivement à l'utilitarisme est celui de John Stuart MILL (1806-1873) sous le titre révélateur "Utilitarism » en 1863. L'objectif de MILL était de reconduire l'utilitarisme sur le chemin des sciences. C'est sous sa plume que l'utilitarisme devint le principe de l'utilité ou encore, le principe de la plus grande joie. Ses propos differrent ainsi de ceux de BENTHAM, par exemple lorsqu'il argumente en faveur d'un hédonisme qualitatif ${ }^{266}$. Il est important de considérer l'aspect qualitatif du plaisir (physique ou intellectuel) et affirme à ce propos :

"Es wäre unsinnig anzunehmen, daß der Wert einer Freude ausschließlich von der Quantität abhängen sollte, wo doch in der Wertbestimmung aller anderen Dinge neben der Quantität auch die Qualität Berücksichtigung findet. ${ }^{267}$

Il considère par ailleurs que les plaisirs intellectuels sont plus recherchés car leur intensité procure à l'individu plus d'utilité dans la durée. L'individu décide par ailleurs de la catégorie dans laquelle il classe le plaisir qu'il ressent (physique ou intellectuel). Il représente enfin un courant hédoniste pour lequel les individus émettent des préférences en connaissance de cause, et non pas des vœux pour la satisfaction d'une volonté momentanée.

MILL définit les normes morales comme "l'ensemble des règles et des recommandations de conduite dont la poursuite offre une vie autant que possible libre de toute non envie et pleine d'envies, qu'elles soient quantitatives ou qualitatives. Cet idéal est accessible à toute l'humanité ${ }^{268}$.

A la question de savoir pourquoi une personne se sentirait obligée de favoriser le bien être de tous, le philosophe anglais répond que, d'une part, les sanctions (qui représentent la motivation fondamentale de l'utilitarisme) ne sont pas simplement extérieures mais également intérieures. Les sentiments moraux, comme la sanction intérieure, peuvent gagner en conviction grâce à la religion, mais n'en n'ont pas nécessairement besoin car ils sont présents déjà de manière innée chez les individus.

\footnotetext{
${ }^{265}$ BROOME John, 1999, p.20.

${ }^{266}$ PAZOS Manuel García, « Die Moralphilosophie John Stuart Mills », 2001, p.97-104.

${ }^{267}$ MILL John Stuart, « Utilitarismus », 2000, p.15.

${ }^{268}$ Idem, p. 21.
} 
MILL essaya en outre de montrer que le principe utilitariste est le but le plus haut que l'individu peut chercher à atteindre. Sa démonstration se base sur deux arguments : d'abord, le bonheur est la seule chose à laquelle les individus aspirent directement. Ceci implique qu'il n'y ait rien qui soit bon ou digne d'être souhaité, qui ne soit aussi désiré. Concrètement, l'amour pour l'argent ne représenterait qu'un moyen pour arriver au but ultime qui est le bonheur. Ensuite, de l'affirmation précédente, il en déduit que le bonheur est la seule chose qui soit digne d'aspiration. Il ne peut être que moral de vouloir le bonheur de tous.

Il faut noter enfin que l'utilitarisme de MILL se défend d'être égoïste ${ }^{269}$. L'individu ne doit certes pas toujours avoir un œil sur le bien être de son voisin, mais les lois étatiques tout comme l'avis général doivent exercer la pression nécessaire, à travers le système de sanction, afin qu'aucun dommage ne soit fait à autrui ou que personne ne contrarie le bonheur d'autrui. L'être humain est pour le philosophe anglais un être social et la vie en société n'est possible que sur la base de la considération de l'intérêt de tous ${ }^{270}$.

Un autre auteur marquant pour sa contribution dans l'histoire de l'utilitarisme fut Henry SIDGWICK (1838-1900), en particulier par son ouvrage « The Methods of Ethics " dont la première édition parut en 1874. Pour lui, il y a deux grands principes d'action pour les etres humains: le principe moral et le principe rationnel de l'intérêt propre. Le seul moyen d'accorder ces deux principes, a priori rivaux, est de supposer l'existence d'un Dieu qui récompense tous ceux qui suivent les principes moraux, et qui sanctionnent tous ceux qui s'en écartent. Ainsi, suivre les principes moraux est dans l'intérêt de tout individu ${ }^{271}$. Par ailleurs, pour SIDGWICK, la joie n'est pas le seul but de l'action humaine. Aux obligations morales des individus appartiennent non seulement le bien être de tous, mais aussi un partage équitable de ce bien être.

L'utilitarisme sera très critiqué dans la première moitié du $\mathrm{XX}^{\text {ème }}$ siècle. Dans les années 50 , on veut réformer ses principes, ce qui conduit à un grand débat mais aussi à une division. Deux courants apparaissent: l'utilitarisme d'action ( Handlungsutilitarismus» ou "act utilitarism») et l'utilitarisme de règle («Regelutilitarismus » ou « rule utilitarism »). Les deux courants s'accordent sur le fait que le but de toute moralité est de servir l'intérêt commun d'une société en maximisant l'utilité sociale attendue ${ }^{272}$. D'après le premier courant, toute action

\footnotetext{
${ }^{269}$ PAZOS Manuel García, 2001, p.107-109.

${ }^{270}$ Idem, p. 109 et MILL John Stuart, 2000, p.56-57.

${ }^{271}$ SIDGWICK Henry, " The Method of Ethics », 1996, p.337-361 et HÖFFE Otfried (éd.), 1992, p.26.

${ }^{272}$ HARSANYI John C., " Morality and Incentives ", in FARINA Francesco, HAHN Frank et VANNUCCI Stefano (eds), 1996, p.25.
} 
peut être qualifiée de morale si ses conséquences conduisent à un bien être général maximum, c'est-à-dire une action morale serait une action qui maximiserait l'utilité sociale dans une situation donnée ; pour le second courant, une action est morale si elle est conforme aux règles qui vont dans le sens du bien être pour tous. Autrement dit, il faut premièrement définir une règle morale sur laquelle tout le monde s'accorde, par exemple la maximisation de l'utilité sociale. Ensuite, il faut définir l'action appropriée qui correspond à cette règle morale ${ }^{273}$.

BROOME dénonce le fait que les philosophes aient tendance de nos jours, à adopter le mot utilité au sens économique du terme, ce qui est pour lui un non sens et qui mène à une certaine confusion ${ }^{274}$. Dans l'idée de BENTHAM ou de MILL, les individus doivent agir dans le sens d'une amélioration du bien être de tous. Aussi, sous cette forme, l'utilitarisme ne pourrait justifier un développement économique qui désavantagerait une partie des personnes impliquées. Par exemple, dans les échanges internationaux, les pays en développement sont désavantagés par les termes de l'échange et le bien être de leurs populations en subit les conséquences. Si l'échange est utile pour les uns, il affecte négativement dans ses effets le bien être des autres. Ceci ne serait pas compatible avec les idées de BENTHAM ou de MILL. La notion d'agir pour l'amélioration du bien être de tous se rapproche de celle du "Wohl der Gesamtheit» de Christian von EHRENFELS. Pour ce dernier, le bien être de tous possède une valeur éthique intrinsèque élevée pour tous les individus et leurs décisions ne devraient pas aller à son encontre. Si dans son sens originel le bien être de tous avait une composante éthique, il semblerait par contre qu'elle l'ait perdue dans l'utilitarisme moderne qui privilégie l'individu à la collectivité.

Pour la branche moderne de l'utilitarisme d'action, il peut être correct de tuer un individu si le résultat de cette action est l'amélioration du bien être de la majorité ${ }^{275}$. Cependant, dans le cadre des échanges internationaux, c'est la majorité (en termes de population) qui se retrouve défavorisée.

Une autre critique qui peut être adressée à l'utilitarisme est son approche quantitative. En économie, l'utilité de chaque objet désiré peut être exactement quantifiée de manière à permettre une formalisation du raisonnement. Cette approche met en évidence l'objet ayant la plus grande utilité et permet de calculer en outre le gain de satisfaction à la suite d'un échange. Cette forme mathématisée permet enfin la comparaison entre différents états de satisfaction pour une même personne, de même qu'une comparaison dite interpersonnelle, c'est-à-dire entre

${ }^{273}$ HÖFFER Otfried (éd.), 1992, p.31 ; HARSANYI John C., in FARINA Francesco, HAHN Frank, et VANNUCCI Stefano (eds), 1996, p.25.

${ }^{274}$ BROOME John, 1999, p.21.

${ }^{275}$ HÖFFER Otfried (éd.), 1992 p.41. 
plusieurs personnes ${ }^{276}$. L'individu maximisant dans les sciences économiques va choisir l'objet ayant l'utilité la plus élevée puisque c'est celui qui est censé lui apporter la plus grande satisfaction possible. Afin de représenter l'évolution de l'utilité d'un individu en fonction de son bien être, les sciences économiques font appel à une fonction mathématique. L'utilité de chaque objet est celle qui est procurée à l'individu lorsque son but est atteint (par exemple, lorsqu'il a consommé cet objet).

Il n'est pourtant pas exact de vouloir assimiler systématiquement l'utilité avec le bien être d'une personne : en maximisant le premier, l'individu ne maximise pas automatiquement, par voie de conséquence, le second. Nous l'avons vu, le meilleur choix pour l'individu n'est pas toujours celui qui correspond à ses préférences; tout comme le fait de suivre ses préférences ne conduit pas automatiquement à faire le meilleur choix. Prenons l'exemple d'une personne qui a pour but de satisfaire sa faim. Admettons qu'elle se trouve en face d'une pomme et d'un morceau de gâteau : lequel de ces deux objets va-t-elle prendre pour satisfaire son besoin?

Pour les utilitaristes, l'individu va effectuer ce choix en fonction de l'utilité de chaque objet. Le morceau de gâteau est censé avoir la plus grande utilité puisqu'il va provoquer une plus grande augmentation du bien être. Manger un bout de gâteau provoque certainement (mais non sans exception) une plus grande joie que la dégustation d'une pomme. Cependant, l'utilité, c'est-à-dire la capacité de l'un comme de l'autre à satisfaire la faim de cette personne, est sensiblement la même.

Pour EHRENFELS, les choses sont plus complexes : dans cet exemple, le but de l'action possède une valeur intrinsèque élevée. L'individu devra se décider pour le moyen censé lui faire atteindre ce but et il choisira le moyen ayant la plus grande valeur d'effet. Cette même valeur sera fonction de son désir pour l'objet mais aussi des influences extérieures (par exemple la suggestion ou l'ordre). Le désir de l'individu s'orientera dans un premier temps vers la part de gâteau, mais son éducation alimentaire ou encore les prescriptions de son médecin pourront l'en dissuader. Si le but de l'individu se limite à la satisfaction de ses besoins et de ses goûts, il accordera une plus grande valeur d'effet au gâteau; si par contre le fait d'être en bonne santé (qui est un des facteurs de son bien être) ou encore le fait de vouloir garder la ligne ou tout simplement par ses habitudes alimentaires, la pomme pourra être considérée comme le moyen le plus approprié de satisfaire son but.

${ }^{276}$ Idem, p.42. 
Pour BROOME également une confusion persiste entre la maximisation du bien être et celle de l'utilité. Il prend pour exemple à ce propos, un article de BERNOULLI daté de 1954, dans lequel ce dernier écrit que la personne rationnelle cherche à maximiser la probabilité de se faire du bien, ce qui a été repris et interprété comme étant le fait que la personne cherche à maximiser son utilitét ${ }^{277}$.

Frank HAHN reproche à la vision utilitariste de ne pas tenir compte de l'influence des normes sociales et éthiques sur les comportements individuels ${ }^{278}$. Les personnes ne décident pas seulement en fonction de l'utilité d'un objet, elles peuvent aussi réagir par rapport à leurs valeurs religieuses. Si on propose à une personne de dénoncer son ami contre de l'argent, et qu'elle trouve plus d'utilité à la récompense pécuniaire qu'à l'amitié en question, va-t-elle pour autant agir de la sorte? Pour HAHN, il faut considérer les valeurs morales et les normes sociales que la personne aura intégrées (par exemple qu'il n'est pas bien de dénoncer son ami). De plus, il n'y a pas de justification utilitariste pour le fait d'ignorer l'utilité d'autrui : par exemple, si l'Etat décide d'augmenter ses dépenses pour financer de nouveaux biens publics, cela peut se faire au dépend de l'aide extérieure, c'est-àdire aux dépends d'autres pays qui trouvent une grande utilité à recevoir ce genre d'aide ${ }^{279}$.

Dans le même ordre d'idée, SEN prend lui l'exemple de l'optimum de PARETO selon lequel une situation d'échange (argent contre biens ou services) est optimale lorsqu'on ne peut plus améliorer la satisfaction d'un individu sans détériorer celle de l'autre. Ceci implique que la comparaison interpersonnelle des utilités est possible. Mais cette perspective est contestable. En effet, la satisfaction ou le bien être d'une personne ne peuvent se mesurer uniquement à travers la maximisation de son utilité ${ }^{280}$. Le critère de Pareto-optimalité ne tient pas compte du contexte social dans lequel évolue la personne. Sa satisfaction en dépend, selon SEN, d'autant que ce contexte va fausser l'échelle de mesure de l'utilité, certaines personnes se contenteront de petits plaisirs pendant que d'autres voudront toujours plus ${ }^{281}$. Une personne peut être vue sous l'angle de ses activités ou "agencies ", c'est-à-dire sa capacité à établir des buts, des engagements, des valeurs, etc...ou sous l'angle du bien être. Si on fait abstraction de l'hypothèse de l'individu uniquement motivé par son propre intérêt, on peut s'apercevoir qu'il agit

\footnotetext{
${ }^{277}$ Idem, p.25-26.

${ }^{278}$ HAHN Frank, "Some Economical Reflections on Ethics », in FARINA Francesco, HAHN Frank et VANNUCCI Stefano (eds), 1996, p.13-21.

${ }^{279}$ Idem, p. 16.

${ }^{280}$ SEN Amartya, " On the Foundations of Welfare Economics: Utility, Capability, and Practical Reason ", in FARINA Francesco, HAHN Frank et VANNUCCI Stefano (eds), 1996, p.40-41.

${ }^{281}$ Idem, p.52.
} 
également de manière différente, là où l'utilité, peu importe sa définition, n'intervient pas. C'est aussi sur ce point que la théorie d'EHRENFELS garde sa pertinence : la motivation qui précède une action est un phénomène complexe, dont les facteurs sont nombreux. On le voit nettement dans l'exemple où l'étudiant hésite entre rester dans sa chambre à étudier ou partir à la montagne prendre l'air ${ }^{282}$. S'il était rationnel et s'il ne prenait ses décisions qu'en fonction de son utilité, alors il choisirait de rester étudier. Or, ce n'est pas ce qu'il fait car l'utilité ne fait pas le poids face à la perspective d'être en montagne. La valeur qu'il donne à une promenade à l'extérieur est plus élevée que celle qu'il accorde à rester étudier : le comportement individuel est ici mieux cerné.

Enfin, BROOME propose de donner à l'utilité deux définitions : c'est d'une part ce qui représente les préférences d'une personne; ou d'autre part, ce qui est bon pour une personne $e^{283}$. Dans les sciences économiques, on utilise surtout la première définition, se référant donc systématiquement aux préférences des personnes. Pour SEN également, il ne faut pas confondre un changement avantageux pour la société avec un changement qui lui est utile ${ }^{284}$. Il ajoute que les préférences d'une personne rationnelle et égoïste peuvent être représentées par une fonction d'utilité. Dans ce cas elle va préférer, entre deux alternatives possibles, celle qui est la meilleure pour elle. La fonction d'utilité peut donc représenter ce qui est bon pour les individus. Encore faut-il qu'ils soient rationnels et qu'ils recherchent leur intérêt personnel, ce qui, on l'a vu, n'est pas toujours le cas. BROOME affirme que l'utilité, en tant qu'élément représentant ce qui est bon pour l'individu, est implicite dans la fonction d'utilité. Cela peut alors créer une certaine confusion à laquelle il convient de pallier en appelant les choses par leur nom, c'est-à-dire en employant le mot «bien » et non utilité pour préciser ce dont on parle.

Nous concédons un certain désaccord avec BROOME sur ce dernier point. La fonction d'utilité représente un choix trop restrictif et trop abstrait pour nous permettre d'expliquer la détermination des valeurs chez les individus. SEN reproche le fait de juger seulement en fonction du critère d'utilité assimilée à la joie qui en est retirée $e^{285}$. Les agents économiques désirent un objet pour lui-même ou pour ses effets, mais ce n'est pas toujours l'effet de son utilité qui est le plus valorisé par l'individu. C'est aussi pour cette raison que la théorie d'EHRENFELS constitue une alternative intéressante.

\footnotetext{
${ }^{282}$ Voir Paragraphe 4.2 du second chapitre de ce travail.

${ }^{283}$ BROOME John, 1999, p.28.

${ }^{284}$ SEN Amartya, 1987, p.38.

${ }^{285}$ Idem, p.45.
} 
Un autre aspect critiquable de l'utilitarisme selon SEN, concerne la valorisation de l'aspect utile d'un objet. Dire qu'une chose ou qu'une marchandise est utile, c'est émettre un jugement de valeur à son égard. Or, selon l'économiste, cette valorisation est relative à l'état d'esprit de la personne qui juge : si elle est dans un état de déprime par exemple, elle aura tendance à survaloriser ce qui pourrait lui apporter un peu de joie. C'est ce que EHRENFELS nomme une valorisation temporaire, une valeur qu'un individu attribue dans un état émotionnel (ou dans une disposition émotionnelle) particulier(e). Par extension, il affirme que le bien être est la qualification d'un état découlant d'un processus de valorisation ${ }^{286}$. Le problème que soulève SEN ici est complexe, et sous-tend par ailleurs une bonne partie des bases des théories économiques : dire que l'on se sent bien, c'est juger de son propre état en le qualifiant de "bien». Ceci ne veut pour autant pas dire que l'on est satisfait, ni que l'on n'ait plus de besoins à satisfaire. Le bien être ne peut donc être assimilé à l'état de satisfaction. Certes, la corrélation est grande, mais on peut par exemple se sentir bien car en bonne santé, sans pour autant se sentir satisfait, au sens d'avoir satisfait un ou plusieurs besoins. SEN milite donc pour une séparation entre la maximisation de l'utilité et celle du bien être ${ }^{287}$.

Pour EHRENFELS, il s'agit là d'un jugement de valeur lié à une disposition émotionnelle déterminée. Le bien être possède une valeur intrinsèque élevée, et une bonne santé est un des moyens d'atteindre cet objectif. Il possède par conséquent une valeur d'effet. La valeur intrinsèque du bien être est en fait une valeur dépendante de la valeur d'effet dont elle est dérivée. Dans le cas du bien être, sa valeur dérive de plusieurs valeurs d'effet (santé, aisance matérielle, amour, famille, vie professionnelle, amis, statut sociale, etc...). En outre, le bien être d'une personne peut être un jugement extérieur, en ce sens où on peut affirmer qu'une personne se sent bien en la voyant ou en émettant un jugement sur son état émotionnel ou matériel (bien que ne tenir compte que de l'état matériel ne serait que superficiel). La théorie d'EHRENFELS prévoit à ce propos l'influence d'autrui ou encore l'effet d'une valeur reprise d'un "aktiver Wertbildner " (ce dernier peut imposer une norme de bien être qui sert de référence à un jugement de valeur dans ce domaine). Son approche rejoint celle des "capabilities" de SEN, dans laquelle le bien être de l'individu est mesuré en fonction de ses capacités à réaliser un certain nombre d'éléments y contribuant comme par exemple la santé, l'éducation, une habitation ou encore la satisfaction des besoins alimentaires vitaux. Il nomme ces éléments "functionings" parmi lesquels il distingue ceux qui sont élémentaires (comme ceux que l'on a cité

\footnotetext{
${ }^{286}$ Idem, p.46.

${ }^{287}$ SEN Amartya, in FARINA Francesco, HAHN Frank et VANNUCCI Stefano (eds), 1996, p.56.
} 
précédemment) ${ }^{288}$. Il ajoute que chaque «functioning " a une valeur différente selon les individus, affirmant une fois de plus son accord avec EHRENFELS.

\subsection{2 - A propos de la dichotomie valeur/fait dans les sciences économiques}

Dans sa théorie EHRENFELS étudie la détermination de la valeur accordée par l'individu en fonction de ses dispositions émotionnelles qui sont elles-mêmes influencées par des éléments extérieurs. L'individu se place alors dans une réalité subjective, c'est-à-dire qu'il établit une relation avec l'objet à travers ses perceptions sensorielles. La valeur qui est accordée est donc aussi le résultat de la perception individuelle de l'objet, des émotions provoquées par cette perception ainsi que de l'environnement. La valeur et le fait ne sont pas isolés l'un de l'autre, au contraire, chez EHRENFELS les deux éléments sont omniprésents. Les sciences économiques modernes sont censées être dépourvues de jugements de valeur : voulant se rapprocher des sciences naturelles, et fortement influencées par le courant positiviste, elles se donnent pour tâche de décrire de manière objective les comportements des agents économiques et d'en expliquer les raisons. Une séparation s'opère donc entre la valeur et le fait, une séparation qualifiée de dichotomie par Hilary PUTNAM qui en critique les fondements dans son ouvrage "The Collapse of the Fact/Value Dichotomy " ${ }^{289}$.

Cette séparation entre le fait et la valeur a été initiée, selon PUTNAM, par David HUME et Immanuel KANT ${ }^{290}$. Selon Martin HARTMANN, beaucoup de philosophes contemporains pensent que c'est DESCARTES qui a introduit une dévaluation des émotions, une dévaluation dite normative ou épistémique ${ }^{291}$. Cette dévaluation s'est poursuivie au cours des XVII ${ }^{\text {eme }}$ et $\mathrm{XVIII}^{\text {ème }}$ siècles, notamment avec HUME et KANT.

Pour HUME, un fait est quelque chose qui est perceptible à travers les sens humains ${ }^{292}$. Avec les progrès de la science, cette réflexion n'est plus fondée puisqu'on a pu établir l'existence des microbes qui ne sont pas observables à l'œil nu par exemple. Pour le philosophe anglais, le fait doit être différencié de l'idée. En effet, l'idée ne peut qu'interpréter ou représenter le fait. Les idées sont associées aux sentiments, c'est-à-dire aux émotions et, c'est par cette voie que l'individu est conduit à émettre un jugement. KANT, quant à lui, distingue

${ }^{288}$ Idem, p.57 et p.59-60 concernant les « Capabilities »; SEN Amartya, «Ökonomie für den Menschen ", 2000, p.110-138.

289 PUTNAM Hilary, "The Collapse of the Fact/Value Dichotomy and Other Essays », 2002.

${ }^{290}$ Idem, p.7-27.

${ }^{291}$ HARTMANN Martin, 2005, p.15.

${ }^{292}$ RAWLS John, " Geschichte der Moralphilosophie », 2002, p.55-59. 
l'analytique du synthétique, sachant que le premier se réfère à ce qui est vrai, simplement en vertu de leur sens. Par exemple, si on affirme que tous les diplômés sont célibataires, il s'agit d'un propos analytique. Le synthétique est ce qui est construit par la pensée, il est le produit du raisonnement et de la raison.

Beaucoup de philosophes de la morale, par leur interprétation de KANT, sont arrivés à la conclusion que ce dernier conférait aux jugements de valeur le caractère d'impératif ${ }^{293}$. Aussi, dire « tuer est mal » c'est dire « ne tue pas » ce qui n'est pas la description d'un fait.

Puisque les faits doivent être traités de manière séparée des valeurs, il doit en être de même pour l'éthique et l'économie. L'éthique analyse les comportements et les jugements de valeur émis à leur égard; l'économie analyse le comportement dit objectif des agents économiques dans des situations observables, donc également objectives :

"It does not seem logically possible to associate the two studies [ethics and economics] in any form but mere juxtaposition. Economics deals with ascertainable facts; ethics with valuation and obligations. ${ }^{294}$

L'économie semble donc être une discipline objective, traitant et analysant des faits. Pour Max WEBER, les valeurs n'y ont pas leur place. Max WEBER était positiviste et considérait que les individus étaient des êtres tous dotés d'une raison $^{295}$. Cette dernière permet aux individus de comprendre les faits et d'élaborer des théories vides de jugements de valeur, c'est-à-dire décrivant et analysant les faits de manière objective. Le jugement sous-entend une critique, qui n'est pas objective si elle n'est pas justifiée scientifiquement. Cependant, pour Dieter HENRICH, les théories de Max WEBER ne sont pas complètement exemptes de jugements de valeur: il part d'un certain nombre d'hypothèses anthropologiques qui correspondent à une certaine éthique, par exemple celle de l'honnêteté intellectuelle ${ }^{296}$. En fin de compte, la méthodologie proposée par WEBER conduit à une perspective limitée dans l'étude de la prise de décision en éliminant les émotions ainsi que les normes susceptibles d'influencer cette dernière.

${ }^{293}$ PUTNAM Hilary, 2002, p.16.

${ }^{294}$ ROBBINS Lionel, "On the Nature and Significance of Economic Science ", 1932, p.134.

${ }^{295}$ THEUER Rolf, 1986, p.7-13.

${ }^{296}$ HENRICH Dieter, « Die Einheit der Wissenschaft Max Webers », 1952, p.111. 
Non seulement au niveau des méthodes, mais également au niveau des modèles utilisés on constate la présence de valeurs. PUTNAM prend l'exemple du critère de Pareto-optimalité 297 $^{29}$ et affirme :

"If the reason for favouring Pareto optimality as a criterion is that one approves of the underlying value judgment that every agent's right to maximize his or her utility is as important as every other's, then it would seem that Pareto optimality isn't a value neutral criterion of "optimality" at all. How could there be a value neutral criterion of optimality, anyway? » ${ }^{298}$

Un critère d'optimalité ne peut être neutre puisque cette optimalité implique la satisfaction des individus et que, comme on l'a déjà vu, cette satisfaction n'est pas objectivement constatable - surtout pas à travers la perspective utilitariste réduisant la satisfaction à celle des besoins matériels et l'assimilant au bien être. De plus, dire de soi qu'on est satisfait est déjà un jugement de valeur par rapport à son état à un moment donné.

$\mathrm{Au} \mathrm{XX} \mathrm{X}^{\mathrm{eme}}$ siècle, cette séparation s'est accentuée jusqu'au point de penser que l'éthique n'est pas un fait constatable. Un représentant de cet avis, cité par PUTNAM, est Rudolf CARNAP. Pour lui, l'éthique ne fait pas partie du domaine du rationnel :

« $[\ldots]$ regulative ethics $[\ldots]$ are in fact inverifiable and, therefore, unscientific $»^{299}$

Si on dit par exemple qu'un empereur romain fut cruel, et si cruel est un mot qui sert à décrire ce personnage, alors il s'agit de la description d'un fait.

PUTNAM critique cette vision des choses. L'adjectif cruel peut en effet servir aussi à décrire un fait qu'à émettre un jugement de valeur sur la personne en question $^{300}$. Il faudrait, selon elle, reconnaître que nos jugements sont influencés par notre culture et par la situation dans laquelle nous nous trouvons lorsque nous l'émettons ${ }^{301}$. Ce débat peut sembler n'appartenir qu'au monde philosophique, mais il a des conséquences dans le domaine économique. Lorsqu'un individu affirme qu'un objet est capable de lui apporter un certain bien être à travers la satisfaction d'un besoin particulier, émet-il un jugement ou décrit-il une réalité ? Après nos propos sur la fragilité des hypothèses de rationalité, d'individualisme, de recherche des intérêts personnels, il nous faut mettre en cause celle de

\footnotetext{
${ }^{297}$ PUTNAM Hilary, 2002, p.56.

298 Idem.

${ }^{299}$ CARNAP Rudolf, « The Unity of Science ", 1934, p.26-27.

${ }^{300}$ PUTNAM Hilary, 2002, p.28.

${ }^{301}$ Idem, p.45.
} 
l'absence d'externalités, qui dit que l'individu, lorsqu'il prend une décision, ne serait pas influencé par autrui. L'individu face à un fait, c'est-à-dire un événement objectivement constatable, est supposé réagir de manière rationnelle. Il va choisir de se comporter de telle manière que les conséquences de son action vont lui apporter la plus grande satisfaction possible. A propos du plaisir éprouvé, John DEWEY affirme que :

«Agreeableness is precisely the agreeableness or congruence of some objective condition with some impulse, habit, or tendency of the agent. [...] of course, pure pleasure is a myth. Any pleasure is qualitatively unique, being precisely the harmony of one set of conditions with its appropriate activity. The pleasure of eating is one thing; the pleasure of hearing music, another; the pleasure of an amiable act, another; the pleasure of drunkness or anger is still another. [...] Hence the possibility of absolutely different moral values attaching to pleasures, according to the type or aspect of character which they express. But if the good is only a sum of pleasure, any pleasure as far as it goes, is as good as any other - the pleasure of malignity as good as the pleasure of kindness, simply as pleasure. ${ }^{302}$

Le plaisir est donc une émotion unique, lié à la fois à une situation, un fait, mais aussi à l'état d'esprit, pour ne pas dire à l'état émotionnel de l'individu. L'individu est donc une partie d'un tout - la situation dans laquelle il se trouve ou son environnement - et il réagit par rapport à celui-ci.

Pour Christine KORSGAARD, interprétant KANT, les valeurs ne proviennent pas du néant, au contraire, elles sont le résultat de nos émotions, c'est-à-dire qu'elles naissent dans notre psyché ${ }^{303}$. Pour PUTNAM, le fait de qualifier un acte de cruel, gentil, méchant, etc..., n'est possible que si l'individu a intégré auparavant de tels concepts. Il n'y a donc pas de séparation entre les valeurs et les faits, mais en plus, les valeurs constituent une base pour l'établissement de normes qui vont ensuite influencer la conduite des personnes, c'est-à-dire les faits ${ }^{304}$. Elle ajoute qu'un principe éthique peut être considéré comme vrai dans une culture, mais faux dans une autre ${ }^{305}$. Cette affirmation rejoint les propos d'EHRENFELS, pour qui, les règles qu'il a énoncées ne sont valables que dans son monde culturel. Les deux auteurs reprennent l'exemple de la chasteté, qui est un thème dont la valeur diffère véritablement selon les cultures. PUTNAM souligne d'ailleurs la nécessité d'une discussion et d'une explication afin d'arriver à une compréhension mutuelle.

De ce point de vue, il est difficile de répondre à la question de savoir quelle norme ou quel jugement éthique est vrai.

\footnotetext{
${ }^{302}$ DEWEY John, « The Middle Works », 1978, p.257-258.

${ }^{303}$ KORSGAARD Christine, "The Sources of Normativity », 1996, p.90-91.

${ }^{304}$ PUTNAM Hilary, 2002, p.118-119.

${ }^{305}$ Idem, p. 120.
} 
Les sciences économiques prétendent décrire les faits de manière objective et ces faits sont constatables sans l'intervention de valeur, que ce soit dans leur contenu ou dans la description de celui-ci. Nous avons vu que cette séparation n'est pas possible : les faits et les valeurs vont de pair et leur séparation est vaine. Nous allons voir à présent que les valeurs sont également présentes dans les théories économiques.

\subsection{3 - La présence des valeurs dans les théories économiques}

La position positiviste, la position normative et l'hypothèse de séparation entre valeur et fait ont conduit les sciences économiques à se prétendre libres de tout jugement de valeur. Pour les positivistes, nos expériences sensorielles nous donnent accès à la réalité objective. On reconnaît cependant aujourd'hui que les faits sont chargés de théorie et que les théories sont testées par les faits choisis en fonction de l'intérêt de la théorie ${ }^{306}$. La question qui se pose alors est celle de savoir si les théories contiennent des valeurs et si, par conséquent, les faits chargés de théories, sont également chargés de valeurs.

Les recherches scientifiques, selon WILBER, n'ont pas comme seul but la vérité, mais elles doivent être également intéressantes et valorisables, c'est-à-dire qu'elles doivent pouvoir trouver une application ultérieure ${ }^{307}$. Elles ont donc un but. De plus, le critère selon lequel ces recherches seraient " bonnes » ou " acceptables " ne peut s'établir par rapport à leur importance intrinsèque, mais selon le degré d'utilisation possible par la communauté scientifique ${ }^{308}$. Autrement dit, les théories scientifiques auraient une valeur d'effet par rapport à l'utilisation qui pourrait en être faite.

Thomas KUHN, entre autre, rejette le fait que les individus aient accès à la vérité à travers l'expérience de leurs sens, et qu'ils puissent voir le monde tel qu'il est réellement $^{309}$. Cela n'est possible qu'à travers le filtre des théories. Ces dernières

\footnotetext{
${ }^{306}$ WILBER Charles K., " Ethics and Economics », in WHALEN Charles J. (éd.) 1996, p.46 ; voir également THOMAS David, « Naturalism and Social Science: A Post-Empiricist Philosophy of Social Science », 1979 et CALDWELL Bruce J., " Beyond Positivism: Economic Methodology in the $20^{\text {th }}$ Century "), 1982.

${ }^{307}$ Idem, p.46-47.

${ }^{308}$ Voir également DWYER Larry, «The alleged Value-Neutrality of Economics: An Alternative View ", in Journal of Economics Issues, 1982, et WISMAN Jon D., " The Naturalistic Turn of Orthodox Economics : A Study of Methodological Misunderstanding », 1978.

${ }^{309}$ KUHN Thomas, « Die Struktur wissenschaftlicher Revolutionen ", 1976, p.45-48 ; SCHWEINZER Paul, « Wissenschaftlicher Fortschritt bei T.S. Kuhn am Beispiel zweier konkurrierender Paradigmen in der Theoretischen Nationalokonomie », 1997, p.9.
} 
contiendraient donc des valeurs. Pour compléter les propos de KUHN, WILBER rajoute :

"A world view greatly influences the scientific paradigm out of which one works; value judgments are closely associated with the world view; theories must remain coherent with the world view; facts themselves are theory-laden; therefore, the whole scientific venture is permeated by value judgments from the start. "

Les scientifiques sont avant tout des êtres humains qui vivent dans et avec leur environnement. Ils sont donc influencés par les valeurs qu'ils ont intégrées au cours de leur processus de socialisation et cela se répercute également sur leur travail.

Les théories économiques n'échappent pas à la règle, en ce sens où elles contiennent et impliquent aussi des jugements de valeur. Rappelons que pour le courant néolibéral dominant, l'individu est censé être rationnel, maximiser son bien être par la recherche de son intérêt personnel, et si tous les individus agissent de cette manière, alors ils conduiront l'économie à un point d'équilibre. Cette théorie implique les valeurs suivantes ${ }^{311}$ :

- d'abord les individus doivent être libres d'agir et de rechercher ce qu'ils veulent,

- ensuite l'équilibre du marché est perçu comme la situation économique idéale, ce qui implique de nouveau que les institutions du marché doivent être établies à tout moment et en tout lieu,

- les moyens et les buts doivent être rangés dans deux catégories différentes,

- les moyens et les buts doivent être mesurés quantitativement.

Le fait que le marché soit considéré comme une situation idéale doit être rapproché du fait que les individus doivent pour cela être libres, mais aussi que leurs préférences individuelles sont le seul critère pour pouvoir juger de leur bien être. Implicitement, il s'agit de deux normes (libertés et préférences comme critère de bien être) qui appartiennent également à la sphère des valeurs (liberté et bien être). L'équilibre de marché est la situation dans laquelle le plus grand nombre d'individus est satisfait, c'est-à-dire que leur bien être est supposé être maximum. Cet équilibre est donc un moyen d'atteindre un but qui est le bonheur des agents économiques, et présenté comme cela, il s'agit d'un but qui a une valeur éthique et d'un objectif impliquant un jugement de valeur. Si le marché est véritablement le meilleur moyen, alors il faudrait l'implanter partout où c'est possible.

\footnotetext{
${ }^{310}$ WILBER Charles K., in WHALEN Charles J. (éd.) 1996, p.47.

${ }^{311}$ Idem, p.48.
} 
La deuxième implication des théories économiques est que les moyens et les buts doivent être distingués, sans quoi il ne serait pas possible d'établir pourquoi les individus font ce qu'ils font. Les agents économiques vont acquérir des biens dans le but de satisfaire leurs besoins. La consommation de biens est donc un moyen et la satisfaction des besoins, un but. Le premier a donc une valeur d'effet tandis que le second a une valeur intrinsèque dérivée, qui peut être aussi une valeur intermédiaire par rapport au but de long terme qui est le bien être.

Pour WILBER ${ }^{312}$, l'individu qui consomme satisfait dans un premier temps ses besoins biologiques ou primaires, puis d'autres besoins dont l'importance dépend de l'environnement de l'individu. Celui qui par exemple exerce des activités sportives, devra d'abord apprendre les différents sports. Il aura également besoin d'instruments (pour celui qui pêche par exemple) ou de capacités (par l'entraînement). Enfin, pour exercer des activités les gens ont besoin d'un contexte social (danser le tango est possible que si l'individu a un ou une partenaire). Les agents économiques ne sont pas seulement consommateurs, ils sont également citoyens et travailleurs et ont à cet égard des obligations et des droits.

L'alternative proposée par WILBER est la suivante ${ }^{313}$ : si les sciences économiques veulent progresser dans leur compréhension du fonctionnement de l'économie, il leur faudrait améliorer leur conception du comportement humain. Concrètement, les individus agissent sur la base des valeurs morales intégrées, tout comme sur celle de l'intérêt personnel. Poussé à son extrême l'argument de recherche de l'intérêt personnel conduirait l'individu à ne pas respecter les règles qu'autrui s'impose. On a déjà vu que le résultat ne serait pas efficace. Qu'est-ce qui contraint les individus à respecter les règles de conduite?

Selon WILBER, notre volonté de satisfaire en priorité nos besoins est contrecarrée par notre sens moral, basé parfois sur nos convictions religieuses. Il donne l'exemple d'un employeur et d'un employé qui ne savent pas beaucoup l'un sur l'autre. Le premier va supposer le comportement de l'autre et réagir en fonction de ses suppositions. Le résultat d'un comportement non coopératif serait inefficace autant de manière générale que de manière individuelle (si chacun choisit de poursuivre ses propres buts alors on parlera plus d'un conflit entre les deux personnes, ce qui nuira au travail dans l'entreprise). Certes le fait de décider seul est moins cher et demande moins d'effort pour tout le monde, mais c'est aussi moins efficace. Le problème du "free rider» ou cavalier seul peut être résolu en ne lui faisant pas bénéficier des gains promis au groupe s'il ne le rejoint pas. En d'autres termes, le "free rider » est un comportement qu'il convient de

\footnotetext{
${ }^{312}$ Idem, p.51.

${ }^{313}$ Idem, p.52-61.
} 
réguler afin de revaloriser les actions de groupe. Réguler implique l'intervention des institutions et à travers elles, de l'Etat. Celui-ci peut encadrer le comportement des individus grâce à l'introduction de règles basées sur la morale qui, si elles sont respectées, permettront aux membres d'une société de mieux vivre ensemble. Autrement dit, la régulation du marché et des comportements n'est pas un obstacle à la maximisation du bien être, au contraire, elle est un moyen. Par exemple dans le cas de la pollution, l'intervention de l'Etat permet de réguler les rejets de gaz dans l'atmosphère, favorisant ainsi la bonne santé et donc le bien être des populations actuelles et à venir. Cependant pour WILBER, ce genre d'intervention a ses limites. Tout dépend de la volonté des individus à se laisser réguler. Il est possible alors de recourir à l'autorégulation au niveau du groupe, comme cela est le cas dans bien des pays pour les professions médicales.

Pour beaucoup d'individus également, leur comportement est influencé par les valeurs morales de la religion dont ils sont croyants. Un recul de l'importance des religions, tout comme celui de la régulation favorisent une perte d'importance des valeurs morales et un comportement, par conséquent, condamnable ${ }^{314}$.

La position de WILBER sur la régulation des comportements n'est pas nouvelle. Elle fait suite à celle d'Aristote sur le rôle des politiciens concernant la gestion de la cité et leur responsabilité quant à l'intériorisation par la population des valeurs morales, indissociables de la politique et de l'économie. De même, Thomas HOBBES dans " Le Leviathan » avait prédit que la présence de l'Etat garantissait une certaine harmonie entre les membres d'une même société. Enfin, la position d'EHRENFELS s'inscrit également dans ce courant. Il évoque l'importance des valeurs religieuses dans la régulation des comportements et déplore leur perte d'influence et les conséquences négatives d'un tel processus. Il étudie l'individu dans son environnement et ne fait pas abstraction des influences mutuelles. L'Etat se voit conférer un rôle non négligeable à travers la justice, dont les règles sanctionnent les comportements qui, de l'avis de la majorité, ont une valeur négative. En ce sens, l'utilisation de la théorie de Christian von EHRENFELS peut se révéler utile: elle propose une perspective toujours actuelle et qui permettrait de pallier quelques défauts ou quelques manques des sciences économiques modernes en matière de traitement du problème de la valeur.

${ }^{314}$ Idem, p.60. 
4.3 - A la recherche d'une alternative : pour une utilisation de la théorie de Christian von EHRENFELS dans les sciences économiques modernes

Dans les paragraphes précédents, nous avons montré la fragilité des hypothèses fondamentales du comportement individuel dans les sciences économiques modernes et cette perfectibilité conduit à vouloir rechercher une alternative. Nous avons également vu que dans la plupart des cas, la théorie du philosophe autrichien Christian von EHRENFELS est basée sur des arguments plus réalistes et qu'elle est plus à même de pouvoir expliquer la formation des valeurs. Elle aurait donc une place à prendre en tant qu'alternative, une place d'autant plus justifiée qu'une certaine tendance s'est dessinée ces dernières années appelant à une vision différente et plus réaliste que celle proposée par les néoclassiques.

Une première tendance est celle d'un regain d'intérêt pour les émotions. Cette tendance touche avant tout les philosophes et leur intérêt porte sur trois éléments en particulier ${ }^{315}$ :

- D'abord beaucoup d'auteurs affirment que le comportement humain ne se laisse souvent expliquer que par l'intermédiaire des sentiments. Les sentiments ne sont cependant pas censés appeler l'action, mais des souhaits ou des convictions qui précèdent une action.

- Les sentiments expriment bien souvent l'importance qu'un objet a pour nous. Il est donc important de les analyser afin de mieux comprendre les valeurs accordées.

- Les sentiments se laissent enfin combiner avec la rationalité individuelle dans la mesure où ils guident notre attention vers les objets qui sont censés nous être utiles et ainsi nous épargner des informations dont nous n'aurions pas besoin ${ }^{316}$. De ce point de vue, les sentiments constituent un complément à la rationalité.

Ces trois éléments semblent aller dans le sens de la théorie d'EHRENFELS qui nous permet de mieux comprendre les comportements des personnes vis-à-vis des objets qu'elles désirent. Les sciences économiques actuelles font abstraction de plusieurs éléments qui l'empêchent d'arriver à une conclusion réaliste en la matière. En particulier, elles omettent l'influence de l'environnement de l'individu sur ses décisions : dans les sociétés occidentales, les membres d'une société reçoivent tous une éducation qui inclut l'intériorisation de règles et de normes. Ces dernières vont influencer les préférences des personnes, c'est-à-dire l'importance qu'elles vont donner à un objet. Autre élément d'influence négligé

${ }^{315}$ HARTMANN Martin, 2005, p. 19.

${ }^{316}$ SOUSA Ronald De, « Die Rationalităt des Gefühls », 1997, p.319. 
dans les sciences économiques modernes : l'influence d'autrui sous quelque forme que ce soit (exemple, suggestion, ordre,...). On peut ainsi mieux expliquer les effets de mode où les individus vont chercher à acquérir des biens non pas pour satisfaire un besoin particulier, mais par rapport à autrui. A ce propos, EHRENFELS a élaboré deux concepts intéressants : ceux du "aktiven " et du " passiven Wertbildner ». On assiste effectivement à un transfert de valeurs entre les premiers et les seconds. Les modes sont souvent initiées par les premiers qui influencent ainsi le comportement des seconds. La valeur ou l'importance accordée à un objet est un phénomène dynamique, elle évolue non seulement avec le temps mais aussi en fonction de l'environnement des individus.

Enfin, les comportements individuels sont le fruit d'un héritage culturel : le philosophe a raison de limiter la validité de ses réflexions au monde qu'il connaît. Il est en effet illusoire de vouloir appliquer un même type de comportement à tous les individus. Les sciences économiques ne considèrent qu'un seul type d'agent économique. Or les valeurs qu'il accorde, dépendent aussi de l'intérêt que sa culture porte à un objet en particulier. Autrement dit sa décision d'agir sera influencée par le jugement qui sera fait par la suite de son comportement.

Parmi les alternatives proposées, nous allons en citer quelques unes dont le point commun est une vision moins restrictive et la volonté de réunir de nouveau l'économie et l'éthique.

Pour Bruno HOLLNAGEL, les contradictions entre théorie et pratique peuvent être résolues à travers un nouveau modèle ${ }^{317}$. Ce nouveau modèle permet une formalisation mathématique du comportement individuel mais HOLLNAGEL précise que pour autant les problèmes ne trouveront pas de solution mathématique exacte $^{318}$

Une première hypothèse qu'il pose est celle de la considération des influences extérieures sur les comportements individuels : l'individu est une partie d'un tout et il convient donc de ne pas négliger l'environnement dans lequel il vit. Il montre que la loi de l'offre et de la demande dans la détermination des prix ne se vérifie pas dans de nombreuses situations empiriques ${ }^{319}$. Le prix n'est qu'un des facteurs qui influencent la décision de l'individu. Aussi HOLLNAGEL considère que le prix d'un objet n'est pas fixé par l'acheteur, il est une donnée exogène et il entre en ligne de compte dans son évaluation de la situation. Il considère que l'individu, qu'il soit acheteur ou vendeur, n'aura de raison d'échanger que si l'utilité ("Nutzen ») qu'il en retire est supérieure aux privations ("Entbehrungen ») consenties $^{320}$. Les notions d'utilité et de privation s'entendent ici dans un sens

\footnotetext{
${ }^{317}$ HOLLNAGEL Bruno, « Wirtschaft ist anders! », 2000.

${ }^{318}$ Idem, p.10-11.

${ }^{319}$ Idem, p.33-36.

${ }^{320}$ Idem, p.37-38.
} 
large. Il définit l'utilité comme l'ensemble des avantages subjectifs en relation avec l'achat ou la vente de biens ou de services. L'utilité inclut des éléments matériels et immatériels (revenus, profits, reconnaissance, réputation, assurance personnelle, confort, joie, etc...). Elle inclut également des valeurs morales puisque la liste des avantages peut contenir des avantages liés à la morale intégrée lors du processus de socialisation ${ }^{321}$. Les privations sont l'ensemble des choses auxquelles l'individu renonce, accepte malgré lui ou risque dans l'acte d'achat ou de vente. Elles incluent l'argent, les incertitudes, l'effort, les risques, les charges physiques et psychologiques, les restrictions de tout ordre mais aussi la consommation de ressources naturelles (ressources matérielles ou encore les dommages créés) ${ }^{322}$.

Cette approche suggérée par HOLLNAGEL élimine les restrictions du modèle néo-classique mais pêche par ailleurs par une certaine imprécision. En effet, les notions d'utilité et de privation se définissent de telle sorte qu'elles puissent inclure un très grand nombre de facteurs sans préciser leur importance respective, ni le déroulement du processus de décision.

Enfin HOLLNAGEL conclut en affirmant que la valeur des choses n'est pas contenue dans les objets. C'est leur utilité (au sens où il l'entend) qui leur donne de la valeur et celle-ci est estimée subjectivement par chaque individu. Les objets n'ont de valeur qu'en tant que partie d'un tout. Ses propos se rapprochent de ceux du philosophe autrichien par bien des aspects, mais ce dernier reste néanmoins plus précis.

Une autre proposition allant dans le sens d'EHRENFELS est celle de Lawrence KOHLBERG $^{323}$ concernant le processus de développement et les facteurs de développement des individus. Ces derniers interagissent avec leur environnement et en retirent une certaine expérience ainsi qu'un certain nombre de valeurs, notamment des valeurs morales. Cette expérience et ces valeurs vont influencer leurs décisions et leur comportement. Certains facteurs environnementaux (culture, mode) peuvent en outre mener à un changement dans la prise de décision et il est donc important de ne pas les négliger.

Peter ULRICH aspire lui à une réconciliation entre les sciences économiques et l'éthique ${ }^{324}$. Son idée est d'abandonner les hypothèses selon lesquelles les sciences économiques ne contiendraient pas de jugement de valeur ou encore que

\footnotetext{
${ }^{321}$ Idem, p.39-40.

322 Idem, p.41.

${ }^{323}$ KOHLBERG Lawrence, « Die Psychologie der Moralentwicklung », 1995.

${ }^{324}$ ULRICH Peter, « Die Weiterentwicklung der ökonomischen Rationalităt - Zur Grundlegung der Ethik der Unternehmung ", 1987 ; ULRICH Peter, "Integrative Wirtschaftsethik. Grundlagen einer lebensdienlichen Ökonomie », 1997.
} 
la rationalité individuelle ne pourrait pas inclure une composante éthique. Il plaide donc pour la réintégration de l'éthique dans les sciences économiques et comme nous le dit Kersten PARCHE-KAWLIK :

«Es geht ihm um die philosophisch-ethische Erweiterung der ökonomischen Rationalität von innen her. $)^{325}$

La rationalité doit donc tenir compte des valeurs morales et on doit considérer que l'individu agit conformément aux règles éthiques qu'il a intégrées au cours de sa vie.

Peter KOSLOWSKI enfin, milite de la même manière pour un rapprochement entre économie et éthique, mais sa proposition va encore plus loin ${ }^{326}$. Il suggère de remplacer le concept de l'homo œconomicus par celui de "l'homo integralis". "L'économie éthique " met en avant l'image de l'homo integralis qui représente l'idéal d'un individu qui, au cours de son processus de décision et sous l'hypothèse d'une "rationalité intégrale ", évalue ses possibilités en considérant leurs aspects économiques, écologiques et esthétiques ${ }^{327}$. L'utilisation de ce concept permettrait, toujours selon KOSLOWSKI, de dépasser le cadre restreint de l'individu recherchant uniquement la maximisation de son utilité que les sciences économiques utilisent ${ }^{328}$.

Son approche est pour le moins interdisciplinaire et tente de sortir les sciences économiques de leur isolement en proposant d'intégrer des facteurs présents dans l'environnement de l'individu.

Cette volonté est également présente chez EHRENFELS : sa théorie de la valeur se révèle d'autant plus actuelle qu'elle intègre toutes les propositions que nous venons de mentionner et qu'elle milite avant tout pour une approche réaliste et non abstraite de la réalité. En ce sens, elle permet de mieux comprendre et de mieux expliquer le processus d'attribution des valeurs.

\footnotetext{
${ }^{325}$ PARCHE-KAWIK Kirsten, « Den homo oeconomicus băndigen? », 2001 , p.84.

${ }^{326}$ KOSLOWSKI Peter, « Prinzipien der ethischen Ökonomie - Grundlegung der Wirtschaftsethik und der auf die Ökonomie bezogene Ethik », 1988 ; KOSLOWSKI Peter, " Wirtschaftsethik in der Marktwirtschaft ", 1990 ; KOSLOWSKI Peter (éd.), " Neuere Entwicklungen in der

Wirtschaftsethik und Wirtschaftsphilosophie ", 1992

${ }^{327}$ KOSLOWSKI Peter (éd.), 1992, p.78-79.

${ }^{328}$ Idem, p.74-78.
} 
EHRENFELS évoque également à travers sa théorie l'importance de l'éthique. Les valeurs éthiques sont non seulement intégrées par les individus et jouent ainsi un rôle dans leur processus de valorisation; elles jouent également un rôle dans les sciences économiques, du point de vue par exemple du jugement de valeur qui est accordé à une politique économique.

En sciences économiques, les jugements de valeur interviennent lors de l'évaluation des politiques économiques. Dire qu'une politique est "bonne", c'est lui donner une telle valeur. Dire qu'une politique économique est la "meilleure" pour un pays est également un jugement de valeur. Comme l'a souligné EHRENFELS, une valeur accordée à un objet peut être différente selon les cultures. Ainsi une "bonne" politique économique dans un pays peut se révéler " inadaptée » dans un autre pays. Une politique économique adaptée est celle qui repense la notion de bien être. Le bien être, on l'a vu, est une notion complexe qui est le résultat d'un grand nombre de facteurs, plus ou moins subjectifs et relatifs selon les individus et les cultures.

L'intérêt d'une théorie générale de la valeur est qu'elle peut s'appliquer à la sphère économique avec ses lois de détermination de la valeur, mais aussi aux sciences économiques et au besoin d'une valorisation éthique des comportements des économistes dans certaines situations. On n'attribue pas simplement une valeur à un bien matériel ou à un service marchand. Un jugement de valeur est également porté sur les comportements et la valeur résultante est de nature éthique. Ce domaine, que l'on ne devrait pas négliger puisqu'il a des répercussions sur le bien être de tous, est pris en compte par la théorie de Christian von EHRENFELS. 


\section{CONCLUSION}

L'intérêt de ce travail a résidé dans le fait de redécouvrir et de montrer l'intérêt pour les sciences économiques actuelles de la théorie de la valeur de Christian von EHRENFELS. Le philosophe autrichien fait partie d'un mouvement intellectuel qui a imprégné les développements philosophiques et économiques dans l'Empire austro-hongrois de la fin du XIX ${ }^{\text {eme }}$. Issu d'une famille noble et aisée, il a montré très tôt de l'intérêt pour l'écriture et la musique. Il s'est intéressé à de nombreux domaines au cours de sa vie, parmi lesquels l'économie et le phénomène de la valeur. Son existence a été marquée par de grands bouleversements (tensions économiques et intellectuels avec le voisin allemand, la première Guerre Mondiale et son dénouement, le progrès technique changeant le quotidien, etc...) dont on retrouve l'influence dans ses discours. EHRENFELS est un aristotélicien, un adepte du discours de DARWIN et de celui des socialistes allemands. On retrouve en outre dans ses propos certains concepts ou certaines approches de l'Ecole Economique Autrichienne. EHRENFELS avait une personnalité conservatrice, que l'on remarque à travers son discours sur le déclin moral et la perte d'importance de la religion dans les sociétés occidentales; néanmoins il n'était pas réfractaire aux innovations, comme le prouve son utilisation de la théorie Mengerienne de la valeur marginale ou encore ses efforts après la première Guerre Mondiale pour une réconciliation entre la toute nouvelle République Tchécoslovaque et l'Allemagne. Sa vie a été marquée par de nombreuses déceptions, en particulier sur le plan personnel lorsqu'il constate par exemple qu'il n'a pas le talent nécessaire pour composer musicalement ou encore lorsque ses prévisions de réconciliation mentionnées plus haut, ne se sont pas réalisées. De ses travaux l'époque contemporaine n'aura retenu essentiellement que ses apports dans le domaine de la psychologie, et en particulier son développement de la théorie du Gestalt.

Cet intellectuel aux intérêts pluriels s'est consacré à la fin du XIX ${ }^{\text {eme }}$ au phénomène de la valeur. Son objectif était de développer une théorie générale, c'est-à-dire applicable à tous les domaines possibles. La définition du phénomène de la valeur qu'il nous propose n'est en fait pas très originale : considérée comme l'importance (subjective) accordée à un objet par un individu, sa définition se rapproche de celle de Carl MENGER. Les différences et l'originalité apparaissent lorsqu'il aborde les notions adjacentes à celle de la valeur, à savoir le désir, les dispositions émotionnelles ou encore le jugement de valeur. Le désir n'est plus assimilé seulement à l'utilité d'un objet, il peut être également fonction de son avantage. L'utilité et l'avantage sont liés tous les deux au bien être de l'individu, mais au bien être au sens large du terme. Le bien être décrit un état psychologique de l'individu lié à des émotions. Lorsque ce dernier considère un objet, il va 
éveiller certaines émotions en lui (qu'elles soient positives, négatives ou neutres). Les dispositions émotionnelles d'un individu face à un objet seront déterminantes par rapport au désir résultant. Ces émotions sont diverses, et variables d'un individu à l'autre. Au moment d'attribuer une valeur à un objet, quelque soit sa nature, l'individu peut subir des influences qu'elles soient intérieures ou extérieures. Une valeur attribuée peut donc également être le produit de l'influence d'autrui. Les valeurs apparaissent dans un contexte social, elles ont également, selon EHRENFELS, un cycle de vie. Autrement dit, elles sont dynamiques et évoluent avec le temps.

Pour EHRENFELS, la valeur accordée par un individu est donc fonction d'un processus psychologique comprenant la considération et la représentation de l'objet dans sa conscience, les émotions résultantes et le désir qui suit. Le désir ne dépend pas uniquement de l'utilité de l'objet. Le désir pousse l'individu à agir. La valorisation de l'objet peut être influencée par d'autres facteurs tels que l'éducation (intériorisation de valeurs à travers le processus de socialisation), la morale ou encore les valorisations des "aktiven Wertbildner ». L'individu vit dans un environnement dont on ne peut faire abstraction et qui joue un rôle dans sa prise de décision. Il est vain de vouloir donner une forme mathématique à cette théorie, d'autant que la solution ne pourrait être exacte.

Une place non négligeable est accordée par le philosophe autrichien aux valeurs éthiques. Elles sont considérées, au même titre que les valeurs économiques, comme un type particulier de valeur. EHRENFELS dénonce la baisse du niveau moral et celle de l'importance des valeurs correspondantes.

Dans le cadre de ce travail, nous avons procédé à une comparaison avec d'autres théories de la valeur du domaine économique. La théorie de la valeur d'Adam SMITH est celle qui se rapproche le plus de celle d'EHRENFELS, à condition de considérer également ses écrits sur les sentiments moraux. La théorie de David RICARDO qui prend pour point de départ celle d'Adam SMITH, établit la valeur comme un phénomène objectif qu'il assimile au prix des biens marchands. Sa théorie n'a que peu à voir avec celle d'EHRENFELS.

Enfin, le phénomène de la valeur considérée par Karl MARX offre un point de vue encore différent: il distingue la valeur du prix mais affirme qu'elle est objective et quantifiable. La valeur d'un objet est liée directement à la quantité de travail contenue dans cet objet, sachant que pour MARX les rapports de production (ceux entre ouvriers et leur patron) influencent ce processus de création de la valeur. Cette comparaison aura montré qu'une certaine diversité existe dans le domaine de la valeur : certains auteurs privilégient l'hypothèse de la subjectivité, d'autres de l'objectivité de la valeur; certains l'assimilent aux prix, d'autres différencient mécanismes de détermination du prix et ceux de la valeur. 
Dans les sciences économiques modernes, la valeur n'est pas véritablement considérée en tant que telle. Elle reste le plus souvent assimilée au prix des biens et services échangés sur un marché.

Leur théorie de la valeur est en fait une théorie des prix qui est soumise à un certain nombre d'hypothèses sur le comportement des agents économiques.

Nous avons montré qu'en réalité ces hypothèses ne se vérifient pas, ou peu dans le meilleur des cas. Si les individus ne sont ni rationnels, ni égoïstes, ni obstinés par la satisfaction de leurs besoins matériels à travers le marché, alors le développement d'une alternative devient nécessaire. Dans l'optique où la valeur n'est pas réduite au prix, la théorie d'EHRENFELS propose une alternative intéressante. Son utilisation est en outre possible à deux niveaux :

- au niveau de l'évaluation d'un objet pour une personne,

- au niveau de l'évaluation d'un comportement d'une personne, notamment en matière de politique économique.

Dans sa théorie, EHRENFELS avait déjà intégré des hypothèses que certains économistes contemporains voudraient voir réintégrées dans les sciences économiques. Ce sont par exemple les hypothèses de la non rationalité de l'individu, du rôle non négligeable des émotions, de l'influence de la société dans laquelle il vit, du rôle de l'éthique dans le processus de valorisation ou encore de la considération parallèle de la valeur et du fait.

Ce que la théorie du philosophe autrichien pourrait en outre nous apporter, est une nouvelle approche du phénomène de la valeur. On devrait différencier la valeur du prix, la première pouvant être assimilée à l'importance accordée à un objet par un individu et qui par la suite aura une influence sur son comportement en tant qu'agent économique; le second reflète non seulement les coûts de production mais aussi la valeur supposée qui est accordée par un individu à cet objet. Le prix d'un objet de luxe par exemple, tient compte de son coût de production, éventuellement des coûts engendrés par sa production (pollution) mais aussi du prestige qui en dérive. Le prestige a une valeur d'effet pour celui qui veut acquérir un tel objet. La valeur est un phénomène dont l'existence est liée à la société dans laquelle elle est utilisée et elle ne peut valoir dans toutes les sociétés. Autrement dit, elle est un élément culturel dont la validité ne peut être universellement reconnue. En ce sens la valeur revêt un aspect sociologique incontestable. Cependant, à l'image d'EHRENFELS, mais également de nombreux économistes du $X X X^{\text {ème }}$ siècle, la compréhension du processus de valorisation passe par une approche interdisciplinaire qui garde sa pertinence dans le cadre d'une analyse économique. Enfin, ce qui distingue particulièrement EHRENFELS est l'utilisation de la psychologie. Il affirme que les émotions jouent un rôle important dans le processus de valorisation et donc dans la façon d'agir de l'individu. Il suppose correctement que dans certaines situations les émotions 
l'emportent sur la raison. En ce sens, EHRENFELS fut un précurseur des recherches menées actuellement pour une utilisation en économie des connaissances acquises en psychologie sur le comportement humain :

"Grâce à des procédés modernes comme l'imagerie par résonance magnétique, les médecins sont parvenus à localiser les processus de l'activité cérébrale chez l'humain et à observer les réactions du cerveau dans des situations où les individus font appel à la réflexion. Ils ont notamment découvert que les zones d'activité qui peuvent être observés chez les personnes testées en laboratoire lors de décisions économiques ne sont pas compatibles avec le postulat du rationalisme. Au contraire, même les régions du cerveau responsables des émotions développent une activité extrême si, par exemple, les personnes sont amenées à prendre des décisions d'achat. Pour les stratèges du marketing, ce fait étaye des expériences fruits de longues années de pratique. ॥'

Certains organismes, comme la Réserve fédérale de Boston cherchent également à mettre en application ces nouvelles connaissances en les intégrant dans de nouveaux modèles économiques :

" Les modèles économiques standards du comportement humain, qui partent du principe que les individus sont des agents économiques bien informés, employés à maximiser un ensemble de préférences cohérentes, produisent très souvent des prévisions entachées d'erreurs grossières. Intéressée par des travaux récents sur l'économie du comportement, la Réserve fédérale de Boston a réuni des économistes, des spécialistes du comportement et des décideurs politiques à l'occasion de sa quarante huitième conférence économique en espérant que les économistes mettraient à profit les connaissances issues de la psychologie et d'autres sciences du comportement pour une meilleure compréhension du processus de prise de décision au niveau personnel et, ce qui préoccupe davantage les décideurs, au niveau global. L'objectif final étant d'exploiter ces connaissances pour améliorer les modèles économiques, les prévisions et les décisions de politique économique. $\|^{2}$

L'utilisation de la psychologie pour comprendre le comportement humain dans le cadre du processus de valorisation a été initiée par Christian von EHRENFELS et sa théorie offre le réalisme qui manque aux théories économiques actuelles et que quelques économistes tentent d'intégrer dans ces mêmes théories.

Ce travail a eu comme objectif non seulement de contribuer à la connaissance des phénomènes en sciences économiques, mais aussi de pouvoir en améliorer l'approche. Grâce à la redécouverte d'un philosophe et de son travail, un peu

\footnotetext{
${ }^{1}$ FISCHER Malte et KUTTER Susanne, « Comment la Recherche sur le Cerveau contredit les modèles économiques », 2005, p.2.

${ }^{2}$ KOPCKE Richard W., LITTLE Jane Sneddon et TOOTELL Geoffrey M.B., « Economie cognitive : les Implications pour le Marché du travail et les Politiques économiques », 2005, p.16.
} 
oubliés avec le temps, cet objectif a été réalisé. MENGER et l'Ecole Autrichienne avaient comme but, entre autre, la compréhension des essences ou "Wesen » de l'économie. On peut affirmer que le philosophe Christian von EHRENFELS y a contribué et qu'à ce titre il mérite de ne plus retomber dans l'oubli. Sa théorie générale de la valeur, de par son exhaustivité et sa complexité, pourrait par ailleurs donner lieu à des développements ultérieurs avec des approches différentes de celle qui est utilisée dans ce travail. 
Youri Tacoun - 978-3-631-75426-9

Downloaded from PubFactory at 01/11/2019 05:04:02AM

via free access 


\section{BIBLIOGRAPHIE}

ADORNO Theodor W., " Begriff der Soziologie », in Soziologische Exkurse, Institut für Sozialforschung, Francfort 1956.

AGLIETTA Michel, « Régulation et Crise du Capitalisme », Editions Odile Jacob, Paris 1997.

ALLPORT Gordon W., « Persönlichkeit », Hein, Meisenheim 1960.

ALTER Max, "Carl Menger and the Origins of the Austrian Economics ", Westview Press, Boulder, Colo 1990.

ANDERSEN Svend, " Einführung in die Ethik », Walter de Gruyter, Berlin \& New York 2003.

ARNOLD Wilhelm, EYSENCK Hans-Jürgen et MEILI Richard (eds), « Lexikon der Psychologie », Verlag Bechtermünz, Augsburg 1996.

ARROW Kenneth J., "Social Choice and Individual Values », John Wiley and Sons, $2^{\text {nd }}$ édition, New York, Londres et Sydney 1966.

AUER Ludwig von, " Dynamic Preferences, Choice Mechanisms, and Welfare ", Springer, Berlin \& Heidelberg 1998.

BACKHOUSE Roger E., « Explorations in Economic Methodology », Routledge, Londres \& New York 1998.

BANDURA Albert, « Lernen am Modell », Klett, Stuttgart 1976.

BANDURA Albert, "Self-regulation of Motivation and action through internal standards and goal systems ", in L.A. Pervin (éd.), " Goal concepts in personality and social psychology », Erlbaum, Hillsdale (New Jersey) 1989.

BARZ Heiner, KAMPIK Wilhelm, SINGER Thomas, TEUBER Stephan (eds), " Neue Werte, neue Wünsche », Metropolitan, Düsseldorf 2001.

BAUMOL William J., WILSON Charles (eds), "Welfare Economics ", collection en trois volumes, Elgar Reference Collection, Cheltenham (G-B) \& Northampton (USA) 2001. 
BECKER Gary S., "The Economic Approach to Human Behaviour », The University of Chicago Press, Chicago \& Londres 1976.

BECKER Gary S., " Human Capital, A Theoretical and Empirical Analysis with Special Reference to Education », The University of Chicago Press, Chicago 1993.

BENESCH Hellmuth (éd.), "dtv-Atlas zur Psychologie. Tafeln und Texte ", Volume 1, dtv, 4ème édition, Munich 1994.

BERGER Peter L. et BERGER Brigitte, «Wir und die Gesellschaft. Eine Einführung in die Soziologie - entwickelt an der Alltagserfahrung ", traduit de l'anglais par Monika Piessner, Rowohlt, Reinbeck 1976.

BERGER Peter R., « Der Donauraum im wirtschaftlichen Umbruch nach dem ersten Weltkrieg ", thèse en sciences économiques et sociales présentée à l'Université d'Economie de Vienne, Vienne 1979.

BIAUJEAUD Huguette, "Essai sur la Théorie Ricardienne de la Valeur ", Economica, Paris 1988 (A noter que ce livre était à l'origine une thèse présentée le 2/12/1933 à la Faculté de Droit de l'Université de Paris).

BIRBAUMER Niels et SCHMIDT Robert F. (eds), "Biologische Psychologie ", Editions Springer, $5^{\text {ème }}$ édition, Berlin, Heidelberg et New York 2003.

BIRNBACHER Dieter, « Analytische Einführung in die Ethik », Walter de Gruyter, Berlin \& New York 2003.

BLAUG Mark, «Economic Theory in Retrospect », $3^{\text {ème }}$ édition, Cambridge University Press, Cambridge 1978

BLAUG Mark, «The Economic Value of Education: Studies in the Economics of Education », Elgar, Aldershot Hants (G-B) 1992.

BLOCH Maurice, " Death, Women and Power », in " Death and the Regeneration of Life », Ed. M. Bloch et J. Parry, Cambridge University Press, Cambridge 1982.

BLUM Lawrence, « Compassion », in RORTY Amélie O. (éd.), « Explaining Emotions », The University of California Press, Berkeley \& Los Angeles 1980.

BLUM Lawrence, "Friendship, Altruism and Morality », Routledge et Paul Kegan, Londres 1980. 
BÖHM-BAWERK Eugen, « Grundzüge der Theorie des wirtschaftlichen Güterwerts ", extraits issus du " Jahrbücher für Nationalökonomie und Statisik ", volume XIII, Gustav Fischer, Jena 1886.

BONDOLFI Alberto et GROTEFELD Stefan (eds), " Ethik und Gesetzgebung. Probleme, Lösungsversuche, Konzepte ", Verlag Kohlhammer, Stuttgart, Berlin et Cologne 2000.

BOSKIN Michael J. (éd.), "Economics and Human Welfare ", Academic Press, New York, Londres, Toronto, Sydney et San Francisco 1979.

BOUDON Raymond, «L'Inégalité des Chances : la Mobilité Sociale dans les Sociétés Industrielles », $2^{\text {eme }}$ édition, Editions Colin, Paris 1978.

BOURDIEU Pierre, "La Noblesse d'Etat : grandes écoles et esprit de corps ", Editions de Minuit, Paris 1989.

BRAHM Otto (éd.), "Freie Bühne für modernes Leben », volume 1, Editions S. Fischer, Berlin 1891.

BRAHM Otto (éd.), "Freie Bühne für modernes Leben », volume 2, éditions S. Fischer, Berlin 1892.

BRAHM Otto (éd.), "Freie Bühne für modernes Leben ", volume 2, éditions S. Fischer, Berlin 1893.

BRAMOULLE Gérard, « Apriorisme et faillibilisme : en défense de Rothbard contre Popper ", in Journal des Economistes et des Etudes Humaines, volume 6, nr 1, mars 1995.

BRATMAN Michael, "Intentions, Plans, and Practical Reason », Harvard University Press, Cambridge (Massachussets) 1987.

BRAUN Edmund (éd.), « Wissenschaft und Ethik », Peter Lang, Bern 1986.

BRENNAN Teresa, "Globalization and its Terrors. Daily Life in the West ", Routledge, New York et Londres 2003.

BREDEKAMP Horst, «Thomas Hobbes. Der Leviathan », Akademie Verlag, Berlin 2003. 
BRENTANO Franz, " Grundlegung und Aufbau der Ethik », édité par Franziska Mayer-Hillebrand, Francke, Bern 1952.

BRENTANO Franz, "Grundzüge der Ästhetik », édité par Franziska MayerHillebrand, Francke, Bern 1959.

BRENTANO Franz, « Vom Ursprung sittlicher Erkenntnis », édité par Oskar Kraus, Hambourg, 1969.

BRENTANO Franz, « Deskriptive Psychologie », édité par R.M. Chisholm et W. Baumgartner, Hambourg, 1982.

BREWER Anthony, « A Minor Post-Ricardian? Marx as an Economist », in Revue History of Political Economics, 27:1, 1995.

BRIESKORN Norbert et WALLACHER Johannes (eds), « Homo oeconomicus: der Mensch der Zukunft ? », Kohlhammer, Suttgart 1998.

BROCH Hermann, « Nachruf au Robert Musil », in Schriften zur Literatur I : Kritik, Editions Paul Michael Lützeler, Francfort 1975.

BROOME John, «Ethics out of Economics », Cambridge University Press, Cambridge 1999.

BRUNI Luigino, "Vilfredo Pareto and the Birth of Modern Microeconomics », Edward Elgar, Cheltenham (G-B) \& Northampton (USA) 2002.

BRUNSTEIN J., LAUTENSCHLAGER U., NAWROTH B., PÖHLMANN K. et SCHULTHEISS O., "Persönliche Anliegen, soziale Motive und emotionales Wohlbefinden » in Zeitschrift für Differentielle und Diagnostische Psychologie, nr 16, 1995.

BRUNSTEIN Joachim \& MAIER Günter, « Persönliche Ziele: ein Überblick zum Stand der Forschung », in Psychologische Rundschau, nr 47, 1996.

BRZEZINSKI Zbigniew, " Macht und Moral : neue Werte für die Weltpolitik », Hoffmann und Campe, Hambourg 1994.

BURCHARDT Michael, « Marxistische Wirtschafstheorie », R. Oldenbourg Verlag, München 1997. 
CALDWELL Bruce J., « Beyond Positivism: Economic Methodology in the $20^{\text {th }}$ Century ", Allen and Unwin, Londres 1982.

ČAPEK Karel, " Gespräche mit Masaryk », traduit du tchèque par Camill Hoffmann et Eckhard Thiele, Deutsche Verlags-Anstalt, Stuttgart \& Munich 2001.

CARNAP Rudolf, " The Unity of Science », Kegan Paul, Trench Hubner, Londres 1934.

CASSEN Bernard, « Un Monde polyglotte pour échapper à la dictature de l'anglais ", in Le Monde Diplomatique, Janvier 2005, p.22-23.

COMETTI Jean Pierre et MULLIGAN Kevin (éd.), « La philosophie autrichienne de Bolzano à Musil », Librairie Philosophique J. Vrin, Paris 2001.

CUMMINGS Neil et LEWANDOWSKA Marysia, " The Value of Things ", Birkhäuser, Bâle, Boston et Berlin 2000.

CYSARZ Herbert, « Beiträge der Prager deutschen Universität zur Philosophie und Grundlagenforschung in der Zwischenkriegzeit ", in BOSL Karl et SEIBT Ferdinand (eds), "Kultur und Gesellschaft in der ersten tschechoslowakischen Republik », Oldenbourg Verlag, Munich et Vienne 1982, p.255-268.

DAAL Jan van et JOLINK Albert, « The Equilibrium Economics of Léon Walras ", Routledge, Londres 1993.

DAHMS Hans-Joachim, « Positivismusstreit », Suhrkamp Taschenbuch Wissenschaft, Francfort 1994.

DALHAUS Carl (éd.), " Richard Wagner - Werk und Wirkung », Bosse, Ratisbonne 1971.

DAVIDSON Richard J., "The functional neuroanatomy of affective style ", in LANE, NADEL et al. (eds), " Cognitive neuroscience of emotion. Series in affective science », Oxford University Press, New York 2000.

DEMPF Alois, " Der Wertgedanke in der Aristotelischen Ethik und Politik », VWGO, Vienne 1989.

DEWEY John, «The Middle Works ", vol. 5, Editions Jo Ann Boydston, University of Southern Illinois Press, Carbondale, 1978. 
DICKHAUT Hans, « Der Alkoholkranke Patient », Birkhäuser, Bâle 1992.

DOBB Maurice, "Theories of Values and Distribution since Adam Smith ", Cambridge University Press, Cambridge 1973.

DONALSON Thomas et WERHANE Patricia (eds), « Ethical Issues in Business. A Philosophical Approach ", $5^{\text {eme }}$ édition, Prentice Hall, Upper Saddle River (New Jersey) 1996.

DÖHRING Sieghart, « Berlioz, Wagner und die Deutschen », Dohr, Cologne 2003.

DROVER Glenn et KERANS Patrick (eds), « Welfare Theory », Edward Elgar, Aldershot \& Brookfield 1993.

DWYER Larry, «The alleged Value-Neutrality of Economics : An Alternative View », in Journal of Economics Issues, Nr 16, Mars 1982.

EATON Howard O., "The Austrian Philosophy of Values ", University of Oklahoma Press, Norman 1930.

EHRENFELS Bernhart von, « Geschichte der Schlösser und Güter Brunn am Walde, Lichtenau, Allentsgichwendt, Eppenberg und Raitbach », Leykam, Graz 1904.

EHRENFELS Christian von, « Die Brüder Von Hartenstein », Tragédie en cinq actes, Leykam, Graz 1885.

EHRENFELS Christian von, " Melusine », tragédie en vers, Carl Konegen, Vienne 1887.

EHRENFELS Christian von, " Metaphysische Ausführung im Anschlusse an Emil Du Bois-Reymond ", rapport de conférence à l'Académie impériale des Sciences, Phil.-hist. Klasse, Band 112, Vienne 1886.

EHRENFELS Christian von, « Über Fühlen und Wollen », sous-titre : « Une étude psychologique », Gerold, Vienne 1888.

EHRENFELS Christian von, « Richard Wagner und der Naturalismus », article paru dans le journal Freie Bühne für den Entwicklungskampf der Zeit, Jg. 2, Berlin 1891. 
EHRENFELS Christian von, «Über Werthaltung und Wert ", Archiv für systematische Philosophie, Band I, 1895.

EHRENFELS Christian von, «Zur Klärung der Wagner-Controverse », Konegen, Vienne 1896.

EHRENFELS Christian von, " Die musikalische Architektonik ", article paru dans la revue „Bayreuther Blätter“, Jg. 19, 1896.

EHRENFELS Christian von, " Sexualethik », Wiesbaden 1907.

EHRENFELS Christian von, "Richard Wagner und seine Apostaten. Ein Beitrag zur Jahrhundertsfeier », Heller, Vienne 1913.

EHRENFELS Christian von, " Wagner und seine neuen Apostaten », article paru dans le journal „Der Auftakt“, Jg. 11, nr 1, Prague 1914.

EHRENFELS Christian von, « Das Primzahlengesetz, entwickelt und dargestellt auf Grund der Gestalttheorie », Reisland, Leipzig 1922.

EHRENFELS Christian von, " Sieben Briefe an MEINONG ", in Philosophenbriefe (édité par R. KINDINGER), 1965.

EHRENFELS Christian von, "Werttheorie. Philosophische Schriften Band I ", édité par R. Fabian, Philosophia, Munich 1982.

EHRENFELS Christian von, "Werttheorie. Philosophische Schriften Band I ", édité par R. Fabian, Philosophia, Munich 1982.

EHRENFELS Christian von, "Kosmogonie », édité par Reinhard Fabian, Philosophia, München 1986.

EHRENFELS Joseph Michael Freiherr von, "Unterricht für Bauersleute von den Krankheiten der Pferde des Hornviehs der Schaafe und Schweine. Erdmann Hülfreichs Unterricht für Bauersleute von den Krankheiten der Pferde des Hornviehs der Schaafe und Schweine : Den Lesern des Noth- und Hülfbüchleins gewidmet ", Doll \& Schwaiger, Vienne 1790.

EIFLER Günter et SAAME Otto (eds), «Wissenschaft und Ethik », Mainzer Universitätsgespräche im Sommersemester 1991 und im Wintersemester 1991/1992. 
EISERMANN Gottfried, « Vilfredo Pareto. Ein Klassiker der Soziologie », Mohr, Tübingen 1987.

ERNST Petra, HARING Sabine A., SUPPANZ Werner (eds), « Aggression und Katharsis ", Passagen Verlag, Vienne 2004.

ESSBACH Wolfgang, « Die Junghegelianer », Fink, Munich 1988.

ETZIONI Amitai, «The Moral Dimension : Towards a New Economics », Collier MacMillan, Londres 1988.

EVANS, Richard, «Fakten und Fiktionen. Über die Grundlagen historischer Erkenntnis », Campus Verlag, Francfort 1998.

FABIAN Reinhard (éd.), "Christian Von Ehrenfels. Leben und Werk », Rodopi, Amsterdam 1986.

FANTASIA Rick, " Délits d'initiés sur le Marché Universitaire Américain », in Le Monde Diplomatique, Novembre 2004.

FARINA Francesco, HAHN Frank, VANNUCCI Stefano (eds), "Ethics, Rationality and Economic Behaviour ", Clarendon Press, Oxford 1996.

FASE Martin M.G., KANNING Walter et WALKER Donald A., " Economics, Welfare Policy and the History of Economic Thought », Edward Elgar, Cheltenham (G-B) \& Northampton (USA) 1999.

FEDERINI Fabienne, «L'abolition de l'esclavage de 1848 », L'Harmattan, Paris 1998.

FENICHEL Otto, «Psychoanalytische Neurosenlehre », volume 3, Walter Verlag, Freiburg 1977.

FERRO Marc (dir.), « Le Livre Noir de la Colonisation, XVIème-XXIème siècle : de l'extermination à la repentance », Robert Laffont, Paris 2003.

FISCHER Erika, « Moralische Einstellungsmuster und ihre Veränderbarkeit », S. Roderer Verlag, Regensburg 1989.

FISCHER Malte et KUTTER Susanne, « Comment la Recherche sur le Cerveau contredit les modèles économiques ", in Problèmes Economiques, $\mathrm{nr}$ 2883, 28.09.2005, p.2-4. 
FORRESTER Viviane, « L'horreur économique », Fayard, Paris 1996.

FOSTER John, « Valuing Nature? », Routledge, Londres \& New York 1997.

FRANK Robert H., « Passions within Reason: The Strategic Role of the Emotions », Norton, New York 1988.

FREUD Sigmund, "Vorlesung zur Einführung in die Psychoanalyse und Neue Folge ", volume 1, Oeuvres complètes, Fischer Taschenbuch Verlag, Francfort 2000.

FREUD Sigmund, " Schriften zur Behandlungstechnik », Oeuvres complètes, volume complémentaire, Fischer Taschenbuch Verlag, Francfort 2000.

FRÖHLICH Werner D. (éd.), «Wörterbuch der Psychologie », dtv, München 1998.

FROMM Erich, "Psychoanalyse und Ethik », traduit de l'anglais par Paul Stapf et Ignaz Müham, Deutscher Taschenbuch-Verlag, $4^{\text {eme }}$ édition, Munich 1992.

FULLER Steve, "Thomas Kuhn. A philosophical History of Our Times », The University of Chicago Press, Chicago \& Londres 2000.

GARRIGOU Alain, «Les Elites contre la République : Sciences Po et l'ENA », Editions La Découverte, Paris 2001.

GARZ Detlef, « Moral, Erziehung und Gesellschaft », Klinkhardt, Bad Heilbrunn 1998.

GERGER Silvia, "Chronischer Alkoholismus - ein Beitrag zur Geschichte der Pflege », Diplomarbeit, Université de Vienne, Vienne 2004.

GESANG Bernward, «Eine Verteidigung des Utilitarismus », Philipp Reclam, Stuttgart 2003.

GIBBARD Allan, "Wise choices, Apt Feelings: A Theory of Normative Judgement », Harvard University Press, Cambridge (Massachussets) 1990.

GILLIGAN Carol, "Moralische Orientierung und moralische Entwicklung ", in NUNNER-WINKLER Gertrud (éd.), « Weibliche Moral. Die Kontroverse um eine geschlechtsspezifische Ethik », Deutscher Taschenbuuch-Verlag, Munich 1991. 
GRAEBER David, « Dancing with Corpses Reconsidered : an Interpretation of Famadihana », in American Ethnologist nr 22, 1995.

GRAEBER David, « Toward an Anthropological Theory of Value. The False Coin of our Own Dreams“, Palgrave, New York 2001.

GRASSL Wolfgang et SMITH Barry (eds), " Austrian Economics: Historical and Philosophical Background », Croom Helm, Londres 1986.

GRAVOT Pierre, « Economie de l'Education », Economica, Paris 1993.

GREENSPAN Patricia, " Emotions and Reasons. An Inquiry into Emotional Justification », Routledge, New York 1988.

GROENEWEGEN Peter (éd.), « Economics and Ethics? », Routledge, Londres et New York 1996.

GRÜNDBICHLER Alois, "Werte und Preise in der Theorie von Karl Marx », Diplomarbeit an der Wirtschaftsuniversität Wien, Vienne 1986.

GUTSCHE Jens, « Produktpräferenzanalyse », Duncker \& Humblot, Berlin 1995.

HABERMAS Jürgen, « Erkenntnis und Interesse », Suhrkamp, Francfort 1991.

HALLER Rudolf, « Neopositivismus. Eine historische Einführung in die Philosophie des Wiener Kreises », Wissenschaftliche Buchgesellschaft, Darmstadt 1993.

HART James G. et EMBREE Lester, " Phenomenology of Value and Valuing ", Kluwer, Dordrecht 1997.

HARTMANN Hans Albrecht, « Wirtschaft und Werte - eine menschheitsgeschichtliche Mésalliance », discours prononcé à l'occasion du 65ème anniversaire du Professeur Reinhard Blum, Université d'Augsbourg, Augsburg 1998.

HARTMANN Martin, " Gefühle. Wie die Wissenschaften sie erklären », Campus Verlag, Francfort 2005.

HATZIMOYSIS Anthony (éd.), " Philosophy and the Emotions », Royal Institut of Philosophy Supplement nr 52, Cambridge University Press, Cambridge 2003. 
HAUSMAN Daniel M. et Mc PHERSON Michael S., « Taking Ethics seriously: Economics and contemporary Moral Philosophy », in Journal of Economic Literature, nr 31, 1993, p.671-731.

HAUTMANN Hans, « Karl Marx - Friedrich Engels : ein Vedemekum über ihr Leben und Werk ", Alfred Klahr Gesellschaft Quellen und Studien Sonder Band 1 , Vienne 2001.

HEGEL Georg Wilhelm Friedrich, " Reason in History », traduit de l'allemand par R. Hartman, Library of Liberal Arts, MacMillian, New York 1953.

HELLER Agnes, « A Radical Philosophy », VSA, Hambourg1978.

HELLER Agnes, « General Ethics », Blackwell, Oxford 1988.

HENRICH Dieter, « Die Einheit der Wissenschaft Max Webers », Mohr, Tübingen 1952.

HENRICH Dieter, « Hegel im Kontext », Suhrkamp, $3^{\text {eme }}$ édition, Francfort 1981.

HENNINGS Klaus H., "The Austrian Theory of Value and Capital », Elgar, Cheltenham 1997.

HERBART Johann-Friedrich, dans "Sämtliche Werke ", Band VI, édité par G. Hartenstein, Voss, Leipzig 1850-1852.

HERF Markus, « Wert- und Preismodell als Koordinationsmechanismen bei Arbeitsteilung : das Konzept von Karl Marx », Diplomarbeit, Universität Vienne 1988.

HERMANN et LANTERMANN (eds), " Persönlichkeitspsychologie : Ein Handbuch in Schlüsselbegriffe », Urban \& Schwarzenberg, Munich 1985.

HILDENBRAND Werner, "Equilibrium Analysis. Variations on Themes by Edgeworth and Walras ", North-Holland, Amsterdam 1988.

HILLINGER Claude, "Applied Cardinal Welfare Economics : Conceptual Foundation, Empirical Measurement, and Aggregation ", édité par la Faculté de Politique Economique de l'Université Ludwig-Maximilian de Munich, Munich 1994. 
HOBBES Thomas, « Leviathan oder Stoff, Form und Gewalt eines kirchlichen und bürgerlichen Staates ", édité par Iring Fetscher, traduit par Walter Euchner, Suhrkamp, Francfort 1984.

HODGSON David.H., " Consequences of Utilitarism », Clarendon Press, Oxford 1967.

HOFER Wolfgang, « Vergleichende Darstellung der Wirtschaftskreislauftheorien von Quesnay, Marx und Keynes unter Berücksichtigung ihrer Bedingtheit aus der Entwicklung der kapitalistischen Wirtschaftsweise », Diplomarbeit, Wirtschaftsuniversität, Vienne 1987.

HÖFFE Otfried (éd.), « Einführung in die utilitaristische Ethik », Francke Verlag, Tübingen 1992.

HÖFLER Alois, « Grundlehren der Psychologie. Lehrtext und Übungen für den Unterricht an Gymnasien », Tempsky, Freytag, Vienne \& Prague 1897.

HOLLANDER Samuel, « Economics of Adam Smith », Heinemann, Londres 1973.

HOLLANDER Samuel, « Classical Economics », University of Toronto Press, Toronto, Buffalo \& Londres 1992.

HOLLNAGEL Bruno, « Wirtschaft ist anders! Überraschende Einsichten in ökonomische Zusammenhänge », Verlag Wissenschaft \& Praxis, Sternenfels 2000.

HOLLSTEIN Bettina, "Wirtschaftsethik und Umwelt. Deutsche und französische Ansätze im Vergleich », Deutscher Universitätsverlag, Wiesbaden 1995.

HOMANN Karl et LÜTGE Christoph, « Einführung in die Wirtschaftsethik », LIT Verlag, Münster 2004.

HOOKER Brad, MASON Elinor et MILLER Dale E. (eds), « Morality, Rules and Consequences », Edinburgh University Press, Edinburgh 2000.

HULL Clark L., " Principles of Behaviour », Appleton Century Crofts, $7^{\text {eme }}$ edition, New York 1966.

HURRELMANN Klaus, " Einführung in die Sozialisationstheorie ", Beltz Verlag, $7^{\text {ème }}$ édition, Weinheim \& Bâle 2001. 
HUSSERL Edmund, « Erinnerung an Franz Brentano ", 1919, extrait de FISCHER Kurt Rudolf (éd.), „Das goldene Zeitalter der österreichischen Philosophie“, WUV Universitätsverlag, Vienne 1995.

JASPERS Karl, « Kant. Leben, Werk, Wirkung », Piper, $3^{\text {ème }}$ édition, Munich 1985.

JOLINK Albert, "The Evolutionist Economics of Léon Walras », Routledge, Londres \& New York 1996.

JOHANSSON Per-Olov, " An Introduction to Modern Welfare Economics ", Cambridge University Press, Cambridge 1991.

JONAS Hans, « Das Prinzip Verantwortung. Versuch einer Ethik für die technologische Zivilisation », Insel Verlag, Francfort 1979.

KAMLEITNER Bernadette, " Psychologische Erkenntnisse und die Annahmen der neoklassischen Ökonomie », Diplomarbeit, Université de Vienne, Vienne 2003.

KANN Robert A., " Geschichte des Habsburgerreiches 1526-1918 », Herman Böhlaus Verlag, Vienne, Cologne et Graz 1977.

KATZENSTEIN Alfred, "Suggestion und Hypnose in der psychotherapeutischen Praxis », Fischer Verlag, léna 1978.

KEREKES Amália, MILLNER Alexandra, OROSZ Magdolna et TELLER Katalin (eds), " Mehr oder Weininger ", sous-titre: « Eine Textoffensive aus Österreich/Ungarn », Braumüller, Vienne 2005.

KEREKES Lajos, « Von St Germain bis Genf. Österreich und seine Nachbarn 1918-1922 », Hermann Böhlaus Verlag, Vienne, Cologne et Graz 1979.

KERSTING Wolfgang, "Thomas Hobbes zur Einführung », Junius, Hambourg 2002.

KING Brett D. et WERTHEIMER Michael, « Max Wertheimer \& Gestalt Theory », Transaction Publishers, New Brunswick (USA) 2005.

KLUCKHOHN Klyde dans " Values and Value-orientations in the Theory of Action : an Exploration in Definition and Classification ", article paru dans "Towards a General Theory of Action », T. Parsons and E. Shils, Harvard University Press, Cambridge 1951. 
KOCH Claudia « Die ökonomische Werttheorie aus philosophischer Perspektive ", Aachen 1995.

KOHLBERG Lawrence, « Die Psychologie der Moralentwicklung ", édité par Wolfgang Althof, Suhrkamp, Francfort 1995.

KONEN Georg et WILHELMS Günther (eds), « Bleibt die Ethik auf der Strecke? ", Lit Verlag, Münster 2001.

KOPCKE Richard W., LITTLE Jane Sneddon et TOOTELL Geoffrey M.B., « Economie cognitive : les Implications pour le Marché du travail et les politiques économiques ", in Problèmes Economiques, nr 2883, 28.09.2005, p.16-30.

KOPPEN Erwin, « Dekadenter Wagnerismus. Studien zur europäischen Literatur des Fin de siècle », Gruyter, Berlin \& New York 1973.

KORSGAARD Christine, "The Sources of Normativity ", édité par Onora O’Neill, Cambridge University Press, Cambridge 1996.

KOSLOWSKI Peter, " Prinzipien der ethischen Ökonomie - Grundlegung der Wirtschaftsethik und der auf die Ökonomie bezogene Ethik », Mohr, Tübingen 1988.

KOSLOWSKI Peter, «Wirtschaftsethik in der Marktwirtschaft. Ethische Ökonomie als Theorie der ethischen und kulturellen Grundlagen des Wirtschaften ", in MATTHIESSEN Christian (éd.), "Ökonomie und Ethik. Moral des Marktes oder Kritik der reinen ökonomischen Vernunft », Hochschulverlag, Freiburg 1990, p.9-30.

KOSLOWSKI Peter (éd.), « Neuere Entwicklungen in der Wirtschaftsethik und Wirtschaftsphilosophie », Springer, Berlin 1992.

KRAFT Michael G., «Ökonomie zwischen Wissenschaft und Ethik. Eine dogmenhistorische Untersuchung von Léon Walras bis Milton Friedmann », Peter Lang, Francfort 2005.

KRAUS Karl, « Les derniers Jours de l'Humanité », traduit de l'allemand par Jean-Louis Besson et Henri Christophe, Agone, Paris 2005.

KRELLE Wilhelm et RECKTENWALD Horst C., " Gossen und seine « Gesetze » in unserer Zeit », Verlag Wirtschaft und Finanzen, Düsseldorf 1987. 
KRELLE Wilhelm, "Economics and Ethics 1. The Microeconomics Basis ", Springer, Berlin \& Heidelberg 2003.

KROEBER-RIEL Werner, " Werbung - Steuerung des Konsumentenverhaltens ", Physica Verlag, Würzburg \& Vienne 1982.

KRUSE Michael, « Zukunftsutilitarismus. Überlegungen zu einer Ethik für das globale Zeitalter », Tenea Verlag, Berlin 2002.

KUHN Thomas, « Die Struktur wissenschaftlicher Revolutionen“, Suhrkamp, $2^{\text {eme }}$ édition retravaillée, Francfort 1976.

KÜNG Hans, « Projekt Weltethos », Piper, Munich 1990.

KUNZMANN Peter, BURKARD Franz-Peter et WIEDMANN Franz, " dtv-Atlas Philosophie ", Deutscher Taschenbuch Verlag, $9^{\text {eme }}$ édition actualisée, Munich 2001.

LARDELLIER Pascal, « Le Steak caché des Fast-Foods », in Le Monde Diplomatique, Novembre 2003, p.32.

LeDOUX Joseph, « Das Netz der Gefühle. Wie Emotionen entstehen », Hansen, Munich et Vienne 1998.

LEHMANN Alfred, « die Hauptgesetze des menschlichen Gefühlens », Leipzig 1892.

LEUBE Kurt (éd.), « Die österreichische Schule der Nationalökonomie », Tome 1: Von Menger bis Mises, Manzverlag, Vienne 1995.

LIESSMAN Konrad Paul, « Karl Marx 1818-1989. Man stirbt nur zweimal », Sonderzahl, Vienne 1992.

LITSCHKA Michael, « Die österreichische Schule der nationalökonomie, normative ökonomik und integrative Wirtschaftsethik », Dissertation an der Wirtschaftsuniversität, Vienne 1999.

LIVET Pierre, «Emotions et valeurs chez Meinong et Ehrenfels » in COMETTI Jean-Pierre et MULLIGAN Kevin (eds), 2001.

LOCKE Edwin A. et LATHAM Gary P., " A Theory of Goal setting and Task performance », Prentice Hall, Englewood Cliffs (New Jersey) 1990. 
LÖSCHNIGG Martin, « Der Erste Weltkrieg in deutscher und englischer Dichtung ", Winter, Heidelberg 1994.

McADAMS Dan P., " Intimacy: the need to be close », Doubleday, New York 1989.

McCLELLAND David C., " Human Motivation », Cambridge University Press, Cambridge (Massachussets) 1985.

McCLENNEN Edward F., « Rationality and Dynamic Choice: Foundational Explorations », Cambridge University Press, Cambridge 1990.

MACH Ernst, « Die Analyse der Empfindungen und das Verhältnis des Physischen zum Psychischen ", éditions Gustav Fischer, 2nd édition, Iéna 1900.

MADER Melitta, « 650 Jahre Prager Universität », Diplomarbeit, Université de Vienne, Vienne 1999.

MAHESHWARI P.D. (éd.), « Towards the Theory of Value. Focus on David Ricardo », Progress Publishers, Bhopal (Inde) 1978.

MANSTETTEN Reiner, « Das Menschenbild der Ökonomie », Verlag Karl Alber, Freiburg \& Munich 2000.

MARGGRAF Rainer et STREB Sabine, «Ökonomische Bewertung der natürlichen Umwelt », Spektrum Akademischer Verlag, Heidelberg \& Berlin 1997.

MARX Karl, « Das Kapital. Erster Produktionsprozeß des Kapitals », in Karl MARX-Friedrich ENGELS-Werke, volume 23, Berlin 1962.

MARZEN Walter, « Marketing der Handelsbetriebe », Orac, Vienne 1986.

MASNE Pierre Le, "Carl Menger et la séparation entre économie et éthique ", Document de Travail, Janvier 2000, disponible sur internet http://sceco.univpoitiers.fr/gedes/docs/menger.pdf .

MEEK Ronald.L., " Studies in the Labour Theory of Value ", Lawrence \& Wishart, Londres 1956.

MEIER-SEETHALER Carola, « Gefühl und Urteilskraft. Ein Plädoyer für die emotionale Zukunft », Verlag C.H. Beck, Munich 1998. 
MEIKLE Scott, « Aristotle's Economic Thought », Clarendon Press, Oxford 1995.

MEINONG Alexius « Über Werthaltung und Wert », édité par Rudolf Kindinger in „Gesamtausgabe“ Band III : « Abhandlung zur Werttheorie », Akademische Druck- und Verlaganstalt, Graz 1968.

MEINONG Alexius, «Psychologisch-ethische Untersuchung zur WerthTheorie ", discours prononcé à la Karl-Franzens-Universität le 15 novembre 1894, Leuschner et Lubensky, Graz 1894.

MENGER Carl, « Untersuchungen über die Methoden der Sozialwissenschaften und der politischen Ökonomie insbesondere ", Duncker \& Humblot, Leipzig, 1883.

MENGER Carl, «Zur Theorie des Kapitals », Article paru dans le « Jahrbuch für Nationalökonomie und Statistik », 17ème bande, 1888.

MENGER Carl, " Grundsätze der Volkswirtschaftslehre », Braumüller, Vienne, première édition 1871 et seconde édition élaborée à partir des notes laissées par Carl Menger, Hölder-Pichler-Tempsky, Vienne 1923.

MILL John Stuart, "Der Utilitarismus ", traduction de Dieter Birnbacher, titre original : « Utilitarianism » (1871), Philipp Reclam, Stuttgart 2000.

MIROSHIMA Michio, "Walras'economics. A pure Theory of Capital and Money ", Cambridge University Press, Cambridge 1981.

MISES, Ludwig Von, " The historical Setting of the Austrian School of Economics », Arlington House, New Rochelle \& New York 1969.

MI-WON Kim, " Grundlegung der Werte in der Lebenswelt des Menschens », Tectum Verlag, Marburg 2000.

MULLIGAN Kevin, "The Expression of Exacteness: Ernst Mach, the Brentanists and the Ideal of Clarity ", in Robert PYNSENT (éd.), " Decadence and Innovation. Austro-Hungarian Life and Art at the Turn of the Century ", Weidenfeld \& Nicholson, Londres 1989, p.33-48.

MUNN Nancy, "The Frame of Gawa : A Symbolic Study of Value Transformation in a Massim Society ", Cambridge University Press, Cambridge 1986. 
MUSCHKA Wilhelm, « Der Legionär », Lang, Francfort et Vienne 1995.

MÜSSELER Jochen et PRINZ Wolfgang (eds), « Allgemeine Psychologie », Spektrum Akademischer Verlag, Heidelberg \& Berlin 2002.

NEILL Robin, «A New Theory of Value. The Canadian economics of H.A. Innis », University of Toronto Press, Toronto \& Buffalo 1972.

NG Yew-Kwang, "Welfare Economics. Towards a More Complete Analysis ", Palgrave McMillan, Houdmills \& New York 2004.

NUSSBAUM Martha, « Upheavals of Thoughts. The Intelligence of Emotions », Cambridge University Press, Cambridge 2001.

OAKLEY Allen, « The foundations of Austrian Economics from Menger to Mises », Edward Elgar Publishing, Cheltenham (R-U), Lyme (E-U), 1997, p.3233.

O'DONNELL Rory, "Adam Smith's Theory of Value and Distribution ", MacMillian Press, Houndmills \& Londres 1990.

PARCHE-KAWLIK Kirsten, « Den homo oeconomicus bändigen? », Peter Lang, Francfort 2003.

PARETO Vilfredo, « Cours d'économie politique », vol. I, Rouge, Lausanne 1896.

PARETO Vilfredo, "Cours d'économie politique », vol. II, Rouge, Lausanne 1897.

PARETO Vilfredo, « Allgemeine Soziologie », Mohr, Tübingen 1955.

PARETO Vilfredo, "Manuel d'économie politique ", traduit de l'italien par Alfred Bonnet, Pichon et Durand-Auzias, Paris 1963.

PARK Chan-Young, " Untersuchungen zur Werttheorie bei Franz Brentano ", Verlag Dr. JH Röll, Dettelbach, 1991.

PAUER-STUDER Herlinde (éd.), " Norms, Values ans Society », Kluwer Academic Publishers, Dordrecht, Boston et Londres 1994.

PAUER- STUDER Herlinde, « Einführung in die Ethik », WUV, Vienne 2003. 
PAULRE Bernard, " Genèse et Enjeux de l'Economie Cognitive », in Problèmes Economiques, $\mathrm{nr} 2883,28.09 .2005$, p.5-15.

PAZOS Manuel García, « Die Moralphilosophie John Stuart Mills », Tectum Verlag, Marburg 2001.

PETERSON V. Spike, « A Critical Rewriting of Global Political Economy. Integrating reproductive, productive and virtual Economies ", Ripes series in Global Political Economy, Routledge, Londres \& New York 2003.

PIAGET Jean, « Le Jugement moral chez l'enfant », Alcan, Paris 1932.

PIETRI-TONELLI Alfonso de, et BOUSQUET Georges H., « Vilfredo Pareto. Neoclassical Synthesis of Economics and Sociology », McMillan Press, Houndmills \& Londres 1994.

PIES Ingo et LESCHKE Martin, " Gary Beckers ökonomischer Imperialismus ", Mohr Siebeck, Tübingen 1998.

PLETICHA Heinrich (éd.), «Weltgeschichte », Tome 10, Verlagsgruppe Bertelsmann, Gütersloh 1996.

POPPER Karl R. et ECCLES John C., « Das Ich und sein Gehirn », Piper, $6^{\text {eme }}$ édition, Munich 1997.

PRIBORSKY Gerold, " Das Nutzenprinzip in der ökonomischen Theorie ", Diplomarbeit, Université d'Economie de Vienne, Vienne 1984.

PUTNAM Hilary, " The Collapse of the Fact/Value Dichotomy and Other Essays ", Harvard University Press, Cambridge (Massachussetts, E-U) et Londres (G.B) 2002.

RAMB Bern-Thomas et TIETZEL Manfred (eds), « Ökonomische Verhaltenstheorie ", Verlag Franz Vahlen, Munich 1993.

RAMEDHAN Erwin, « La Société Indonésienne face à la pénétration du modèle occidental ", in Le Monde Diplomatique, Juin 1981, p.22.

RATTNER Josef, « Basiswissen Tiefenpsychologie. Die wichtigsten Neurosenarten », Bechtermünz Verlag, Berlin 1999. 
RAUPACH Hans, « Der Tschechische Frühnationalismus », Essener VerlagAnstalt, Essen 1939.

RAWLS John, " Geschichte der Moralphilosophie », traduction de Joachim Schulte, Suhrkamp, Frankfort 2002.

RICARDO David, «Des Principes de l'Economie politique et de l'impôt », 1 ère édition, traduit de l'anglais par Constancio F.S., Ailaud, Paris 1819.

RICARDO David, " Minors Papers on the Currency Question ", édité par Jacob Harry Hollander, The Johns Hopkins Press, Baltimore 1932.

RICARDO David, « Des Principes de l'Economie politique et de l'impôts », 3 ème édition de 1821, traduit de l'anglais par Cécile Soudan, Flammarion, Paris 1993.

RICARDO David, «Essay on the influence of a low price of corn on the profits of stocks », Verlag Wirtschaft und Finanzen, Düsseldorf 1996.

RIEDER Karl, « Zur Diskussion um Sachkonzept und Methodenkonzept der Marxschen Werttheorie », Diplomarbeit, Université de Vienne, Vienne 2003.

RIDER Jacques Le, « Le Cas Otto Weininger », sous-titre: « Racines de l'Antiféminisme et de l'Antisémitisme ", Presses Universitaires de France, Paris 1982.

RITTER Joachim, « Hegel und die französische Revolution », Suhrkamp, 3 ème édition, Francfort 1989.

ROBBINS Lionel, " On the Nature and Significance of Economic Science ", MacMillan, Londres 1932.

ROGIN Leo, "The Meaning and Validity of Economic Theory », Harper, New York 1956.

ROSEN Frederick, "Classical Utilitarianism from Hume to Mill », Routledge, Londres \& New York 2003.

ROTHE Friedrich, « Karl Kraus. Die Biographie », Piper, Munich 2003.

RYSAVY Karin, «Ethik, Utilitarismus, Politik », Diplomarbeit, Université de Vienne, Vienne 2002.

SABOOGLU Müfit, « Rationalité et déterminisme », Peter Lang, Berne 1994. 
SANDVOSS Ernst R., "Immanuel Kant. Leben, Werk, Wirkung », Kohlhammer, Stuttgart, Berlin, Cologne et Mainz 1983.

SANDVOSS Ernst R., " Geschichte der Philosophie. Mittelalter, Neuzeit, Gegenwart », Deutscher Taschenbuch Verlag, édition actualisée, Munich 2001. SCHELLING Thomas. C., "The Strategy of Conflict ", Harvard University Press (1 ${ }^{\text {ere }}$ édition 1960), Cambridge (Massachussetts) 1997.

SCHLEICHERT Hubert (éd.), " Logischer Empirismus - der Wiener Kreis ", Wilhelm Fink Verlag, Munich 1975.

SCHOLL Alice, « Einfluß der Marketingintrumente auf die Kaufentscheidung und das damit verbundene Kaufverhalten ", Diplomarbeit, Université d'économie de Vienne, Vienne 1989.

SCHORSKE Carl E., "Wien. Geist und Gesellschaft im Fin de Siècle », Fischer, Francfort 1982.

SCHÜLER Winfried, « Der Bayreuther Kreis von seiner Entstehung bis zum Ausgang der Wilhelminischen Ära. Wagnerkult und Kulturreform im Geiste völkischer Weltanschauung », Aschendorff, Münster 1971.

SCHULTZ Theodore William, "Education and Economic Growth », in NELSON Henry B., " Social Forces Influencing American Education », Chicago University of Chicago Press, Chicago 1961, p.46-88.

SCHUMPETER Joseph A., " History of Economic Analysis », George Allen \&Unwin, Londres 1954.

SCHUSTER Peter et SPRINGER-KREMSER Marianne, " Anwendungen der Psychoanalyse », WUV Universitätsverlag, $2^{\text {ème }}$ édition, Vienne 1998.

SCHWEINZER Paul, « Wissenschaftlicher Fortschritt bei T.S. Kuhn am Beispiel zweier konkurrierender Paradigmen in der theoretischen Nationalökonomie ", Diplomarbeit, Université de Vienne, 1997.

SCHWINGHAMMER Melitta, " Die Diskussion des "Geschlechterproblems " in der Wiener Moderne - am Beispiel Otto Weininger (1880-1903) und Rosa Mayreder (1858-1938) », Diplomarbeit, Université de Vienne, Vienne 1995.

SEIERSTAD Åsne, « Le Libraire de Kaboul », J-C Lattès, Paris 2003. 
SEN Amartya, " On Ethics and Economics », Basil Blackwell, Oxford \& New York 1987.

SEN Amartya, "Ökonomie für den Menschen », traduction de Christiana Goldmann, Carl Hanser, Munich \& Vienne 2000.

SHAPIRO Michael J., " Reading Adam Smith: Desire, History and Value ", Collection Modernity and Political Thought, volume 4, Sage Publications, Newbury Park, Londres \& New Delhi 1993.

SHELDON Ken M. et KASSER Tim, " Pursuing personal goals: Skills enable progress, but not all progress is beneficial », in Personality and social Psychology Bulletin, nr 24, 1998.

SHELDON Ken M. et ELLIOT Alison J., " Goal Striving need satisfaction, and longitudinal well-being. The self-concordance model », in Journal of Personality and Social Psychology, nr 76, 1999.

SKINNER Andrew Stewart, "A System of Social Science. Papers relating to Adam Smith ", Clarendon Press, $2^{\text {eme }}$ édition, Oxford 1996.

SIDGWICK Henry, "The Methods of Ethics ", réimpression de l'édition de 1907, Thoemmes Press, Bristol (G.B) 1996.

SIDGWICK Henry, "The Principles of Political Economy », réimpression de l'édition de 1901, Thoemmes Press, Bristol (G.B) 1996.

SMITH Adam, "An Inquiry into the Nature and Causes of the Wealth of Nations ", édition Cannan, The Modern Library, New York 1937.

SMITH Adam, "The Works of Adam Smith », Volume 1: "The Theory of Moral Sentiments ", réimpression de l'édition de 1811-1812, Otto Zeller, Aalen 1963.

SMITH Barry, «Foundations of Gestalt Theory », Philosophia Verlag, Munich \& Vienne 1988.

SMITH Barry, " Austrian Philosophy: The Legacy of Franz Brentano », Open Court Publications, Chicago 1994.

SMITH Barry, « Aristoteliarism, Apriorism, Essentialism », in Boetkle P.J. (Ed.), "Elgar Companion to Austrian Economics", 1994. 
SOKOLOWSKI Kurt, "Wille und Bewußtheit » in KUHL J. et HECKHAUSEN H. (éds), " Enzyklopädie der Psychologie, Motivation und Emotion », Tome 4: Motivation, Volition und Handlung, Hofgrefe, Göttingen 1996.

SOUSA Ronald De, « Die Rationalität des Gefühls », Suhrkamp, Francfort 1997.

SRAFFA Piero, "Works and Correspondance of David Ricardo ", University Press, Cambridge (G.B) 1951.

STREISSLER Erich, HAX Herbert (eds), « Friedrich Von Wiesers wissenschaftliche Grundperspektive », Verlag Wirtschaft und Finanzen, Dusseldorf 1999.

SUCHANEK Andreas, "Ökonomische Ethik », Mohr Siebeck, Tübingen 2001.

SZADAI Annamaria, " Die Lausanner Schule ", Thèse, Hochschule für Welthandel in Wien, Vienne 1963.

SZOSTAK Rick, " Econ-Art: Divorcing Art from Science in Modern Economics », Pluto Press, Londres 1999.

SZOSTAK Rick, " Unifying Human Ethics ", manuscrit datant de 2002 et dont nous avons pu obtenir une copie avec l'accord cordiale de l'auteur. Le livre est paru en octobre 2005 sous le titre : "Unifying Ethics », University Press of America, Lanham (Maryland, Etats-Unis) 2005.

SZOSTAK Rick, " Toward a Unified Human Science », Article paru dans la revue Issues in Integrative Studies, Nr 18, p.115-157, 2000.

TEICHOVA Alice, MATIS Herbert (eds), « Österreich und die Tschechoslowakei 1918-1938 ", Studien zur Wirtschaftsgeschichte und Wirtschaftspolitik volume 4, Böhlau Verlag, Vienne, Cologne et Weimar 1996.

THEOBALD Werner, «Integrative Umweltbewertung », Springer, Berlin et Heidelberg 1998.

THEUER Rolf, « Enthumanisierung der Sozialwissenschaften », Diplomarbeit, Université d'économie de Vienne, 1986.

THOMAS David, « Naturalism and Social Science: A Post-Empiricist Philosophy of Social Science », Cambridge University Press, Cambridge 1979. 
TIMMERMANN Manfred, « Die ökonomischen Lehren von Marx, Keynes, Schumpeter ", Kohlkammer, Stuttgart 1987.

TIMMS Edward, ROBERTSON Ritchie (eds), « Vienna 1900. From Altenberg to Wittgenstein ", Austrian Studies volume 1, Edinburgh University Press, Edinburgh 1990.

TIMMS Edward, ROBERTSON Ritchie (eds), « Psychoanalysis in its Cultural Context ", Austrian Studies volume 3, Edinburgh University Press, Edinburgh 1992.

TOMAN Walter, " Introduction to Psychoanalytic Theory of Motivation ", Pergamon Press, Oxford (G.B) \& New York 1960.

TOMO Shigeki, « Eugen Von Böhm-Bawerk. Ein großer österreichischer Nationalökonom zwischen Theorie und Praxis ", Dissertation, Université d'Innsbruck, Innsbruck 1991.

ULRICH Peter, « Die Weiterentwicklung der ökonomischen Rationalität - Zur Grundlegung der Ethik der Unternehmung », in BIERVERT Bernd et HELD Martin (eds), «Ökonomische Theorie und Ethik », Campus, Francfort \& New York 1987, p.122-149.

ULRICH Peter, «Integrative Wirtschaftsethik. Grundlagen einer lebensdienlichen Ökonomie », Haupt, Berne Stuttgart et Vienne 1997.

WACQUANT Loïc, «Fermons les Prisons ! », in Le Monde Diplomatique, septembre 2004.

WALKER Donald A., "Walras's market models », Cambridge University Press, Cambridge 1996.

WALRAS Léon, "Eléments d'économie pure ou théorie de la richesse sociale ", édition définitive revue et augmentée par l'auteur, Pichon et Durand-Auzias, Paris 1952.

WALTER Hans Jürgen, " Gestalttheorie und Psychotherapie », Westdeutscher Verlag, $3^{\text {ème }}$ édition, Opladen 1994.

WEBER Max, « Wirtschaft und Gesellschaft », Mohr, Tübingen 1972. 
WEININGER Otto, « Geschlecht und Charakter. Eine prinzipielle Untersuchung », Matthes \& Seitz Verlag, Munich 1980.

WEININGER Otto, « Über die letzten Dinge », Matthes \& Seitz Verlag, Munich 1997.

WENTZEL Bettina, « Der Methodenstreit », Lang, Francfort \& Vienne 1999.

WHALEN Charles J. (éd.), « Political Economy for the $21^{\text {st }}$ Century.

Contemporary Views on the Trends of Economics ", M.E. Sharpe, Armonk (New York) et Londres 1996.

WHEEN Francis, « Karl Marx », Bertelsmann, München 2001.

WIESER Friedrich von, « Über den Ursprung und die Hauptgesetze des wirtschaftlichen Werthes », éditions Alfred Hölder, Vienne 1884.

WIESER, Friedrich von, « Der natürliche Werth », Editions Alfred Hölder, Vienne 1889.

WIESNER-PIHOFSKY Emmy, « Die Wiedergeburt der tschechischen Nation im 19. Jahrhundert », Diplomarbeit, Université de Vienne, Vienne 1994.

WILMES Eugen, «Friedrich Von Wieser », Dissertation, Université de Bonn, Bonn 1985.

WILSON James Q. et SKINNER Andrew S. (eds), " The Market and the State: Essays in Honour of Adam Smith », Clarendon Press, Oxford 1976.

WILSON James Q., « The Moral Sense », Free Press, New York 1993.

WISMAN Jon D., " The Naturalistic Turn of Orthodox Economics: A Study of Methodological Misunderstanding ", in Review of Social Economics, $\mathrm{Nr} 36$, Décembre 1978.

WOLFRAM Von WOLMAR Wolfgang, « Prag: Die älteste Universität des Reiches ", Arbeitsgemeinschaft Prager and Brünner Korporation, Berlin 1998.

WOOD Ellen Meiksins, « Empire of Capital », Verso, Londres 2003. 
WOOD John Cunningham (éd.), "Léon Walras. Critical Assessments », volume I : "The Life of Léon Walras and Perspectives on his Thought ", Routledge, Londres \& New York 1993.

WOOD John Cunningham (éd.), « Léon Walras. Critical Assessments », volume II : « Walrasian Economics », Routledge, Londres \& New York 1993.

WURM Wolfgang, " Abschaffung der Soziologie. Behaviourismus als Ideologie », Luchterhand, Darmstadt \& Neuwied 1974.

ZBINDEN Eric, "Werte und Macht in sozietalen Systemen ", thèse présentée à l'Université de Zurich, ADAG Administration \& Druck AG, Zurich 1984.

ZELINSKY Hartmut, « Richard Wagner - ein deutsches Thema 1876-1976. Ein Dokumentation zur Wirkungsgeschichte Richard Wagners ", Verlag Medusa, Francfort 1976.

ZIMBARDO Philip G., «Psychologie », Springer, $6^{\text {eme }}$ édition, Berlin \& Heidelberg 1995.

ZUMKLEY-MÜNKEL Cordula, « Imitationslernen. Theorien und empirische Befunde », Schwann, Düsseldorf 1976.

Dictionnaires:

Dictionnaire d'éthique et de Philosophie morale, éditions PUF, sous la direction de Monique CANTO-SPERBER, Paris 1996.

Dictionnaire Le Petit Larousse Compact 2004.

Le Grand Dictionnaire de la Langue Française, $2^{\text {ème }}$ édition dirigée par Alain REY, Dictionnaire le Robert, Paris 2001.

Dictionnaire Français-Allemand : Enzyklopedisches Wörterbuch, Langenscheidt, édition de 1933. 


\section{Forschungsergebnisse der Wirtschaftsuniversität Wien}

Herausgeber: Wirtschaftsuniversität Wien vertreten durch a.o. Univ. Prof. Dr. Barbara Sporn

Band 1 Stefan Felder: Frequenzallokation in der Telekommunikation. Ökonomische Analyse der Vergabe von Frequenzen unter besonderer Berücksichtigung der UMTS-Auktionen. 2004.

Band 2 Thomas Haller: Marketing im liberalisierten Strommarkt. Kommunikation und Produktplanung im Privatkundenmarkt. 2005.

Band 3 Alexander Stremitzer: Agency Theory: Methodology, Analysis. A Structured Approach to Writing Contracts. 2005.

Band 4 Günther Sedlacek: Analyse der Studiendauer und des Studienabbruch-Risikos. Unter Verwendung der statistischen Methoden der Ereignisanalyse. 2004.

Band 5 Monika Knassmüller: Unternehmensleitbilder im Vergleich. Sinn- und Bedeutungsrahmen deutschsprachiger Unternehmensleitbilder - Versuch einer empirischen (Re-)Konstruktion. 2005.

Band 6 Matthias Fink: Erfolgsfaktor Selbstverpflichtung bei vertrauensbasierten Kooperationen. Mit einem empirischen Befund. 2005.

Band 7 Michael Gerhard Kraft: Ökonomie zwischen Wissenschaft und Ethik. Eine dogmenhistorische Untersuchung von Léon M.E. Walras bis Milton Friedman. 2005.

Band 8 Ingrid Zechmeister: Mental Health Care Financing in the Process of Change. Challenges and Approaches for Austria. 2005.

Band 9 Sarah Meisenberger: Strukturierte Organisationen und Wissen. 2005.

Band 10 Anne-Katrin Neyer: Multinational teams in the European Commission and the European Parliament. 2005.

Band 11 Birgit Trukeschitz: Im Dienst Sozialer Dienste. Ökonomische Analyse der Beschäftigung in sozialen Dienstleistungseinrichtungen des Nonprofit Sektors. 2006

Band 12 Marcus Kölling: Interkulturelles Wissensmanagement. Deutschland Ost und West. 2006.

Band 13 Ulrich Berger: The Economics of Two-way Interconnection. 2006.

Band 14 Susanne Guth: Interoperability of DRM Systems. Exchanging and Processing XML-based Rights Expressions. 2006.

Band 15 Bernhard Klement: Ökonomische Kriterien und Anreizmechanismen für eine effiziente Förderung von industrieller Forschung und Innovation. Mit einer empirischen Quantifizierung der Hebeleffekte von F\&E-Förderinstrumenten in Österreich. 2006.

Band 16 Markus Imgrund: Wege aus der Insolvenz. Eine Analyse der Fortführung und Sanierung insolventer Klein- und Mittelbetriebe unter besonderer Berūcksichtigung des Konfigurationsansatzes. 2007.

Band 17 Nicolas Knotzer: Product Recommendations in E-Commerce Retailing Applications. 2008.

Band 18 Astrid Dickinger: Perceived Quality of Mobile Services. A Segment-Specific Analysis. 2007.

Band 19 Nadine Wiedermann-Ondrej: Hybride Finanzierungsinstrumente in der nationalen und internationalen Besteuerung der USA. 2008.

Band 20 Helmut Sorger: Entscheidungsorientiertes Risikomanagement in der Industrieunternehmung. 2008.

Band 21 Martin Rietsch: Messung und Analyse des ökonomischen Wechselkursrisikos aus Unternehmenssicht: Ein stochastischer Simulationsansatz. 2008. 
Band 22 Hans Christian Mantler: Makroökonomische Effizienz des Finanzsektors. Herleitung eines theoretischen Modells und Schätzung der Wachstumsimplikationen für die Marktwirtschaften und Transformationsökonomien Europas. 2008.

Band 23 Youri Tacoun: La théorie de la valeur de Christian von Ehrenfels. 2008.

Band 24 Monika Koller: Longitudinale Betrachtung der Kognitiven Dissonanz. Eine Tagebuchstudie zur Reiseentscheidung. 2008.

www.peterlang.de 